\title{
REAL TIME GRID RELIABILITY MANAGEMENT 2005
}

Prepared For:

\section{California Energy Commission}

Public Interest Energy Research Program

Prepared By:

Lawrence Berkeley National Laboratory

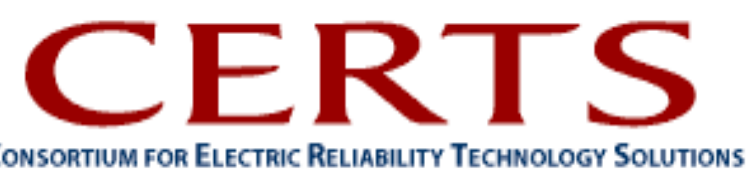




\section{Legal Notice}

This report was prepared as a result of work sponsored by the California Energy Commission (Energy Commission). It does not necessarily represent the views of the Energy Commission, its employees, or the State of California. The Energy Commission, the State of California, its employees, contractors, and subcontractors make no warrant, express or implied, and assume no legal liability for the information in this report; nor does any party represent that the use of this information will not infringe upon privately owned rights. This report has not been approved or disapproved by the Energy Commission, nor has the Energy Commission passed upon the accuracy or adequacy of this information in this report.

\section{Disclaimer}

This document was prepared as an account of work sponsored by the United States Government. While this document is believed to contain correct information, neither the United States Government nor any agency thereof, nor The Regents of the University of California, nor any of their employees, makes any warranty, express or implied, or assumes any legal responsibility for the accuracy, completeness, or usefulness of any information, apparatus, product, or process disclosed, or represents that its use would not infringe privately owned rights. Reference herein to any specific commercial product, process, or service by its trade name, trademark, manufacturer, or otherwise, does not necessarily constitute or imply its endorsement, recommendation, or favoring by the United States Government or any agency thereof, or The Regents of the University of California. The views and opinions of authors expressed herein do not necessarily state or reflect those of the United States Government or any agency thereof, or The Regents of the University of California.

Ernest Orlando Lawrence Berkeley National Laboratory is an equal opportunity employer. 


\section{Acknowledgements}

CERTS performers are grateful for the technical direction provided by The Public Interest Energy Research (PIER) Transmission Research Program (TRP) and Energy Commission PIER staff Jamie Patterson, Merwin Brown, Jim Cole, Virgil Rose and Larry Miller.

Task 2 was led by Manu Parashar, Electric Power Group (EPG), with assistance from research performers Abhijeet Agarwal, Matthew Varghese, and Jim Dyer, EPG; and consultants Ian Dobson, University of Wisconsin, and Yuri Makarov, Pacific Northwest National Laboratory (PNNL). California Independent System Operator (CA ISO) advisors were Soumen Ghosh, Patrick Truong, and Robert Sparks.

Task 3 was led by Manu Parashar with assistance from research performers Jim Dyer, Manu Parashar, Simon Mo, Peng Xiao, Jose Coroas, EPG; and consultants Ken Martin, Bonneville Power Administration (BPA), Dan Trudnowski, Montana State University, and Ian Dobson, University of Wisconsin. CA ISO advisors were Dave Hawkins, Jim Hiebert, Greg Tillitson, Paul Bleuss,NanLiu.

Task 4 was led by Bernard Lesieutre, Lawrence Berkeley National Laboratory (LBNL). Baj Agrawal, (Arizonia Public Service) APS, Jim Gronquist, Gerald Keenan, Dmitry Kosterev, Frank Puyleart, BPA, Irina Green, David Hawkins, Yuri Makarov, CA ISO, Anatoliy Meklin, Pacific Gas \& Electric (PG\&E), Abraham Ellis, Public Service Company of New Mexico (PNM), Henry Huang, Ning Lu, Pacific Northwest National Laboratory (PNNL), John Phillips, Puget Sound Energy (PSE), Garry Chinn, Southern California Edison (SCE), and Donald Davies, Western Elecricity Cooordinating Council (WECC) provided information for this work and reviewed the draft scoping study report.

This work was supported by the Office of Electricity Delivery and Energy Reliability, Office of Electric Transmission and Distribution, U.S. Department of Energy, under Contract No. DE-AC02-05CH 11231.

\section{Citation}

Eto, Joe, Manu Parashar, Bernard Lesieutre, and Nancy Jo Lewis, Consortium for Electric Reliability Technology Solutions (CERTS). 2007. Real Time Grid Reliability Management 2005. California Energy Commission, PIER Transmission Research Program. CEC-500-2007-XXX. 


\section{Preface}

The Public Interest Energy Research (PIER) Program supports public interest energy research and development that will help improve the quality of life in California by bringing environmentally safe, affordable, and reliable energy services and products to the marketplace.

The PIER Program, managed by the California Energy Commission conducts public interest research, development, and demonstration (RD\&D) projects to benefit the electricity and natural gas ratepayers in California. The Energy Commission awards up to $\$ 62$ million annually in electricity-related RD\&D, and up to $\$ 15$ million annually for natural gas RD\&D.

The PIER program strives to conduct the most promising public interest energy research by partnering with RD\&D organizations, including individuals, businesses, utilities, and public or private research institutions.

PIER funding efforts are focused on the following RD\&D program areas:

Buildings End-Use Energy Efficiency

Industrial/Agricultural/Water End-Use Energy Efficiency

Renewable Energy Technologies

Environmentally Preferred Advanced Generation

Energy-Related Environmental Research

Energy Systems Integration

Real Time Grid Reliability Management 2005 is the final report for the Real Time Grid Reliability Management 2005 project (contract number 500-02-004) conducted by the Consortium for Electric Reliability Technology Solutions (CERTS). The information from this project contributes to PIER's Transmission Research Program. For more information on the PIER Program, please visit the Energy Commission's Web site at http://www.energy.ca.gov/pier or contact the Energy Commission at (916) 654-5164. 


\section{Table of Contents}

Preface

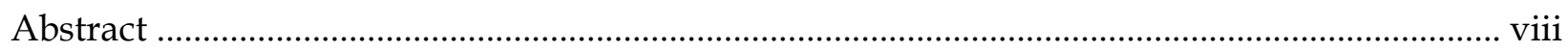

1.0 Task 2.0: Develop a Real-Time Voltage Security Assessment (VSA) Prototype Tool ............... 1

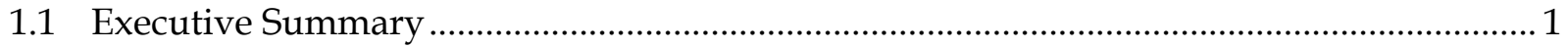

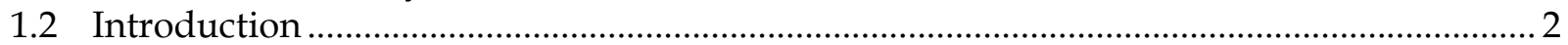

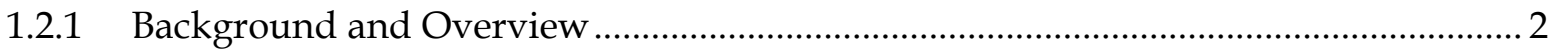

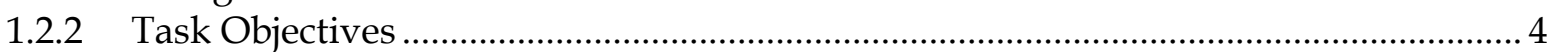

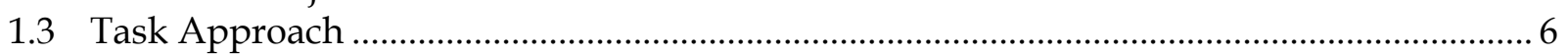

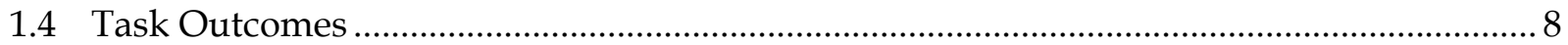

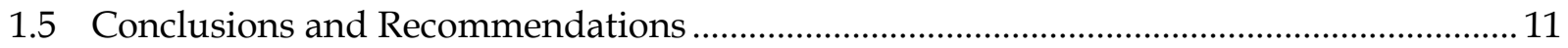

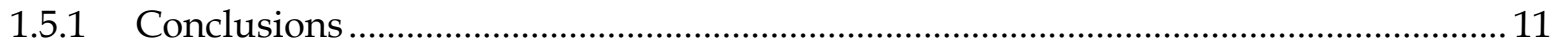

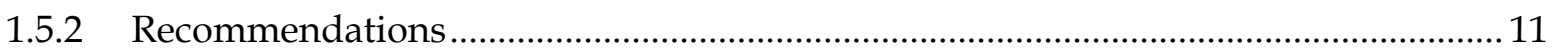

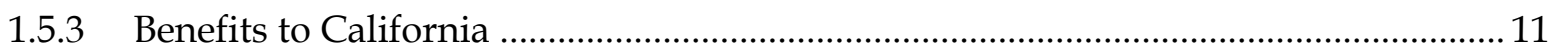

2.0 Task 3.0: Real-Time Applications of Phasors for Monitoring, Alarming and Control ............13

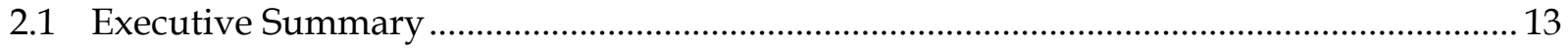

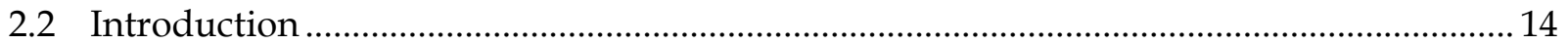

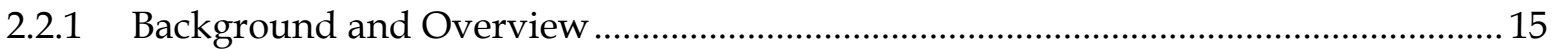

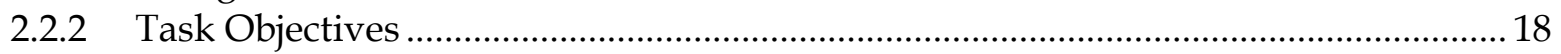

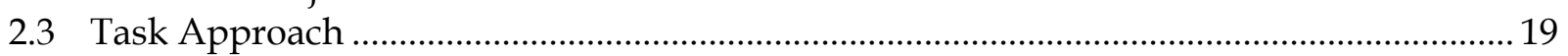

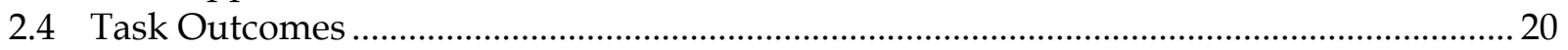

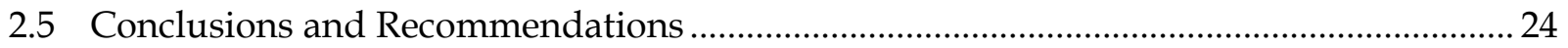

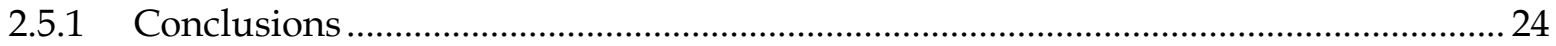

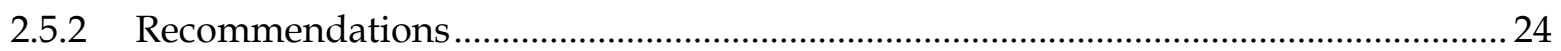

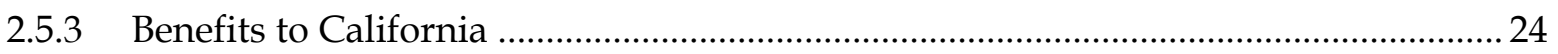

3.0 Task 4.0: Improving Dynamic Load and Generator Response Performance Tools.................. 26

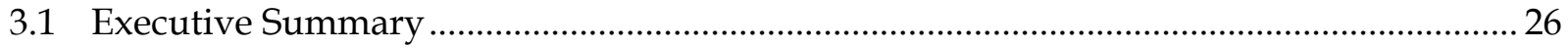

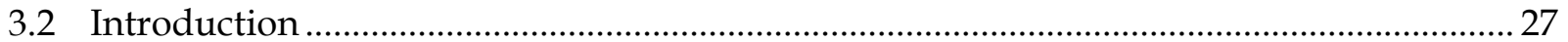

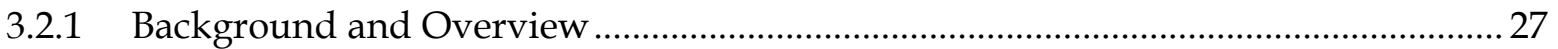

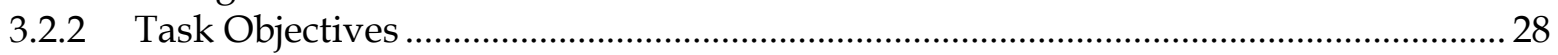

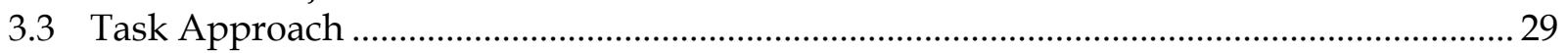

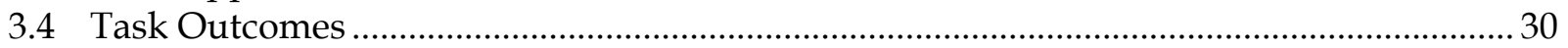

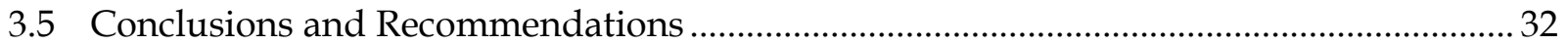

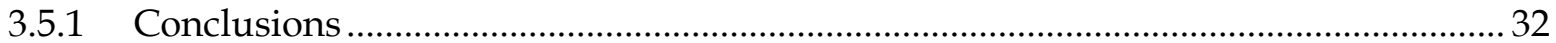

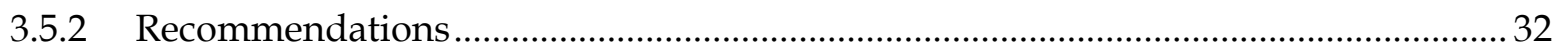

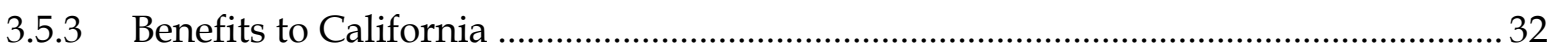

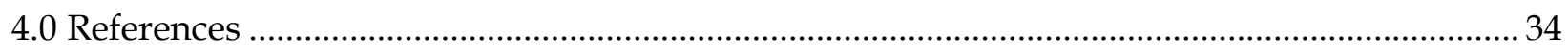

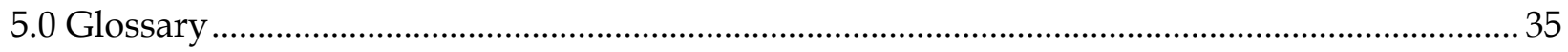




\section{List of Figures}

Figure 1. Multi Year Development Roadmap for CA ISO Voltage Security Assessment (VSA)

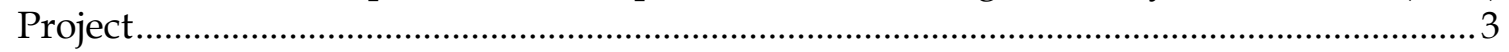

Figure 2. Task 3.0 Multi Year Research Roadmap for CA ISO Phasor Project ..............................16

Figure 3: CA ISO Phasor Network Diagram ............................................................................. 20

Figure 4: RTDMS Version 3 System Architecture .........................................................................22

\section{List of Tables}

Table 1: Summary of Research Recommendations ...... 


\section{Report Organization}

The Real Time Grid Reliability Management 2005 project consists of three parallel technical tasks:

- Task 2.0: Develop a Real-Time Voltage Security Assessment (VSA) Prototype Tool;

- Task 3.0: Real-Time Applications of Phasors for Monitoring, Alarming and Control;

- Task 4.0: Improving Dynamic Load and Generator Response Performance Tools.

The tasks funded under this work authorization were coordinated by Consortium for Electric Reliability Technology Solutions (CERTS) for the Energy Commission's PIER Transmission Research Program. Tasks 2 and 3 were the second phases of a multi-project ongoing RD\&D activity, while Task 4 was a new project. Earlier phases of the research on Tasks 2 and 3 were sponsored by PIER through a RD\&D contract with LBNL (Contract \#: 150-99-003) and through several task orders funded through the California Institute for Energy and Environment Contract \#500-24-004 (BOA\#20).

Additional funding through two separate subsequent work authorizations has been provided to build upon the work initiated in this work authorization. Follow-on work for Task 2: Develop a Real Time Voltage Security Assessment (VSA) Prototype Tool and Task 3: Real-Time Applications of Phasors for Monitoring, Alarming and Control has been funded under Contract \#500-02-004: MR-041, Real Time System Operations (RTSO) 2006-2007. Follow-on work for Task 4: Improving Dynamic Load and Generator Response Performance Tools has been funded under Contract \#500-02-004: MR-049, WECC Load Modeling Transmission Reliability Research Project.

An overview of Real Time Grid Reliability Management 2005 is provided in the Project Introduction. Additional reporting is organized separately for each technical task. 


\section{Project Introduction}

The increased need to manage the California electricity grid more actively in real time is a result of the ongoing transition from a system operated by vertically-integrated utilities serving native loads to one operated by an independent system operator supporting competitive energy markets. In effect, markets are replacing utilities in performing the match between generation and demand. System operators must both ensure reliability as they have always done and now also facilitate the operation of these newly created markets. This transition has confronted system operators with dramatic changes from past practices including new and greatly increased numbers of market participants and the resulting dramatic increase in the volumes of energy trades, often over long distances.

To meet these new challenges, operators have had, until now, only the previous generation of grid management tools. These tools were designed for a vertically-integrated and centrallycontrolled system whose relatively predictable conditions did not require the kind of minuteby-minute decision making resulting from the operation of today's electricity markets. For example, the traditional approach for managing reliability is to use models to analyze, months in advance, a pre-determined set of contingencies for peak load conditions and then set conservative operating limits for the system. Yet, managed and unmanaged power outages, transmission congestion, energy price spikes, frequency abnormalities, and voltage degradation routinely occur at times other than peak load and, sometimes, as a result of contingencies not previously considered. As a result, traditional management tools are increasingly inadequate and practices must be updated.

In light of these developments, the best strategy for managing reliability is to equip the system operator with better real-time information about actual operating margins so that he can better understand and manage the risk of operating closer to the edge. In some cases, these tools must be able to analyze geographically dispersed events in real-time, which require using timestamped data in order to conduct dynamic system analysis, alarm operators, and, in the future, enable dynamic response through automatic system controls. The strategic direction the Energy Commission is sponsoring for its research includes: (1) enhancement of tools that obtain and translate real-time data for analysis and operator actions, and (2) advanced monitoring tools with time synchronized phasor data. These tools and technologies will also set the stage for a future smart electricity grid that will be able to automatically sense and respond to system emergencies.

A companion strategy is to also address known deficiencies in the offline modeling tools that continue to be needed to ground the use of improved real-time tools. The risk of blackouts exacerbated by inaccurate models was clearly illustrated by the August 10, 1996 blackout (and others) in which simulations after the event were unable to replicate the event. Since that time, Western Electric Coordinating Council (WECC) members have improved some aspects of their modeling tools, yet it is well accepted that the models for load response remain deficient. Recent, close call events in the West, in which slow voltage recovery from faults has increased concern about uncontrolled, cascading voltage collapse (i.e., blackouts) continue to remind us of the need to improve these load models. 
The costs of blackouts are high. The August 14th 2003 blackout was estimated to have cost in the range of $\$ 4$ billon and $\$ 10$ billion in the United States (at least $\$ 2.3$ billion in Canada) (U.S.Canada Power System Outage Task Force 2004), and a recent report estimates that power system disturbances cost $\$ 80$ billion annually in the United States (LaCommare and Eto 2006).

The overall goals of this project are to:

- Improve the reliability and quality and cost/value of California's electricity through the use of new and better real-time operational tools.

- Develop two prototype real-time operational tools to meet California Independent System Operator (CA ISO) specifications.

- Initiate the transfer of these prototypes to a vendor for implementation as productiongrade operating tools.

- Prioritize options for data collection to improve the load and generator models used by CA ISO and WECC.

The objectives of this project are to:

- Develop and conduct first-ever demonstration of two prototype real-time software tools for CA ISO (Task 2.0 and 3.0); and

- Prepare a scoping study report on improving load and generator response models (Task 4.0).

The tasks funded under this work authorization represent the second phase of an ongoing multi-project RD\&D activity that is being coordinated by CERTS for the Energy Commission's PIER Transmission Research Program. Earlier phases of this research were sponsored by PIER through a RD\&D contract with LBNL (Contract \#150-99-003) and through several task orders funded through the California Institute for Energy and Environment (BOA\#20).

Additional funding through two separate subsequent work authorizations has already been provided to build upon the work initiated in this work authorization. Contract \#500-02-004, MR-041, Real Time System Operations (RTSO) 2006-2007 extends Task 2.0: Develop a Real Time Voltage Security Assessment (VSA) Prototype Tool and Task 3.0: Real-Time Applications of Phasors for Monitoring, Alarming and Control. Contract \#500-02-004: MR-049, WECC Load Modeling Transmission Reliability Research Project has initiated the research identified in Task 4.0: Improving Dynamic Load and Generator Response Performance Tools.

Tasks and deliverables described below refer solely to accomplishments that have been completed under the funding for this work authorization. 


\begin{abstract}
The increased need to manage California's electricity grid in real time is a result of the ongoing transition from a system operated by vertically-integrated utilities serving native loads to one operated by an independent system operator supporting competitive energy markets. During this transition period, the traditional approach to reliability management - construction of new transmission lines - has not been pursued due to unresolved issues related to the financing and recovery of transmission project costs. In the absence of investments in new transmission infrastructure, the best strategy for managing reliability is to equip system operators with better real-time information about actual operating margins so that they can better understand and manage the risk of operating closer to the edge. A companion strategy is to address known deficiencies in offline modeling tools that are needed to ground the use of improved real-time tools.

This project: (1) developed and conducted first-ever demonstrations of two prototype real-time software tools for voltage security assessment and phasor monitoring; and (2) prepared a scoping study on improving load and generator response models. Additional funding through two separate subsequent work authorizations has already been provided to build upon the work initiated in this project.
\end{abstract}

Keywords: Electricity grid, reliability, real-time operator tools, voltage security, time synchronized phasor measurements, dynamic load models, dynamic generation response 


\subsection{Task 2.0: Develop a Real-Time Voltage Security Assessment (VSA) Prototype Tool}

\subsection{Executive Summary}

California Independent System Operator (CA ISO) system operators need to know how to more effectively manage the grid and its reactive resources, including coordination with other organizations (interconnected system operators, load-serving entities, and generators), within today's changed operational environment, especially during periods of system stress. Conducting voltage security assessments is the principal means for achieving this end. The problem of voltage security assessment is exacerbated by the effects of multiple transfers through the network that result from the buying and selling of electric power across the boundaries of control areas in which the point of production and the point of delivery may be in geographically distant locations. CA ISO does not have a real-time dispatcher's tool for voltage security assessment (VSA). In this task, CERTS conducted a technical assessment of algorithms, developed a first prototype incorporating contingency ranking, continuation power flow and hyperplanes, and a prepared prototype functional specification. This task is the middle phase of a multi-project research activity through which CERTS is developing VSA prototype real-time operational tools that implement, test and demonstrate key CA ISO requirements for a future production-quality VSA tool. The VSA prototype tool implemented a traditional continuation power flow algorithm within a hyperplane framework that enabled contingency ranking. The accuracy of the VSA prototype tool was validated by comparing its results to those calculated by an industry standard off-line planning analysis tool. The VSA prototype tool also included wide-area visuals to support operators in managing voltage and Voltage-Ampere Reactive (VAR) resources on the transmission system. When completed in the final, subsequent phase of this research, CA ISO will use the results of this research to procure a production-quality tool that will become part of a suite of advanced computational tools for CA ISO congestion management, which is slated for implementation over the next few years. 


\subsection{Introduction}

California Independent System Operator (CA ISO) system operators need to know how to more effectively manage the grid and its reactive resources, including coordination with other organizations (interconnected system operators, load-serving entities, and generators), within today's changed operational environment, particularly during periods of system stress. The problem of voltage security assessment is exacerbated by the effects of multiple transfers through the network that result from the buying and selling of electric power across the boundaries of control areas in which the point of production and the point of delivery may be in geographically distant locations. CA ISO does not have a real-time dispatcher's tools for voltage security assessment (VSA). To meet CA ISO specifications, CERTS is developing a VSA prototype real-time operational tool that will implement, test and demonstrate the key elements of a future production-quality VSA tool.

The VSA Project consists of three tracks including data requirements, algorithms, and prototype development. There are four phases for each track. The phases include platform development and initial research, algorithm development and the proof-of-concept simulations, and data integration and project expansion. Figure 1 illustrates the multi-year research roadmap for the VSA project. The roadmap identifies the activities completed under this contract, which are identified as the second phase of research for each track. It also identifies earlier activities accomplished under \#BOA20 and activities on a subsequent phase that are being conducted through a follow-on contract, \#500-02-004, MR-041, Real Time System Operations (RTSO) 2006-2007.

\subsubsection{Background and Overview}

Over the past 40 years, more than 30 major blackouts worldwide have been related to voltage instability and collapse. Among them, at least 13 voltage-related blackouts occurred in the United States, including two major blackouts in the Western Interconnection in 1996 and a wide-scale blackout in the Eastern Interconnection in 2003. Several times, the blackout investigation teams have identified the need for on-line or real-time power flow and stability tools and indicators of voltage performance in a real-time operating environment in order to help prevent future blackouts.

Currently, CA ISO real time operations do not have a real-time dispatcher's VSA tool and corresponding wide-area visuals to manage the voltage and VAR resources on the transmission system, and to identify the following:

- Voltage security margin calculation.

- Worst-case contingencies leading to voltage collapse and/or contingencies with insufficient voltage stability margin.

- Abnormal reductions of nodal voltages.

- Contingency ranks according to a severity index for system problems.

- System conditions with insufficient stability margin. 
Phase 1: TO 21

\section{Input Data \\ Requirements}

Algorithms

Research

Prototype

Development

And Test

$$
\begin{gathered}
\text { Contract \# 500-99-013: } \\
\text { BOA \# } 20
\end{gathered}
$$

(1) VSA survey \& framework based on input in and utilities capabilities

(3) Prototype functional specification

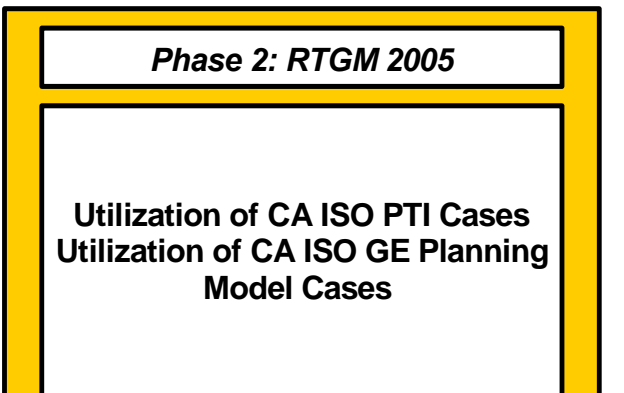

Survey recommended algorithms:

(1) Continuation Power Flow (2) Hyper planes

(3) Bus participation factors in voltage collapse to identify most affected points/regions

(4) Compute sensitivities with respect to voltage set points \& generator VAR limits

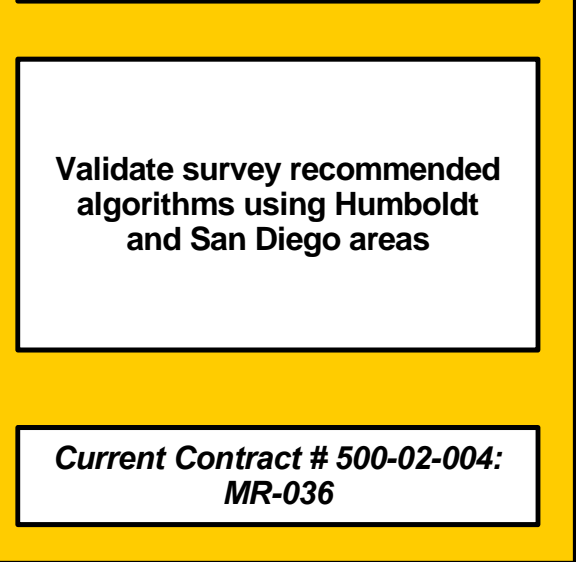

溇 Electric Power Group
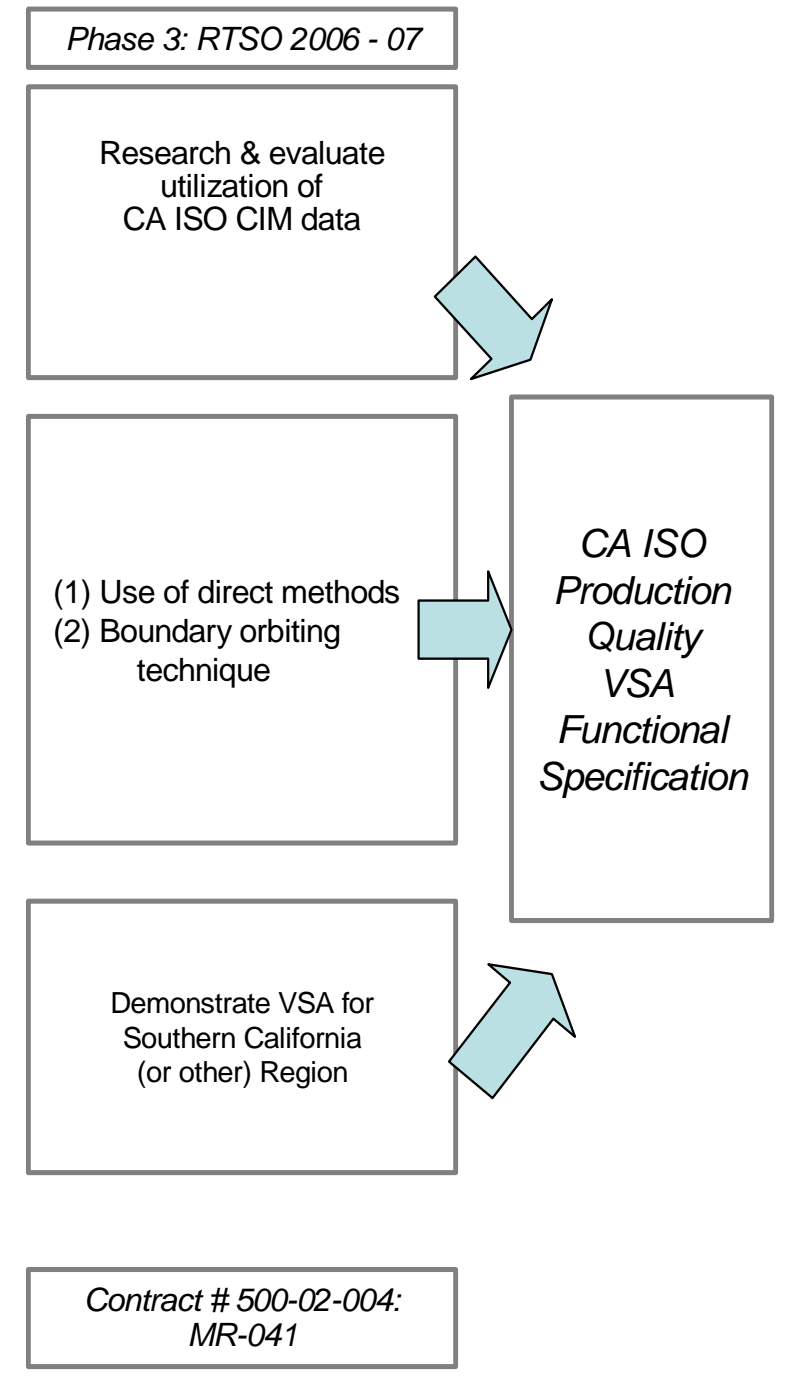

CA ISO

Production

Quality

VSA

Functional

Specification

CERTS

Figure 1. Multi Year Development Roadmap for CA ISO Voltage Security Assessment (VSA) Project 
- Weakest elements within the grid and the regions most affected by potential voltage problems

- A real-time dispatcher's dashboard-type of display providing information about voltage problems at the normal look-ahead operating conditions and for the worst-case contingencies (contingencies with large severity ranks) that may appear in the future.

CERTS development of an initial VAR-Voltage Management prototype tool was funded by the Department of Energy (DOE) Transmission Reliability program in 1999 and 2000 as a direct response to CA ISO's desire to improve procedures for implementing WECC's revised voltageVAR requirements. Prototype tailoring and enhancements for demonstration at CA ISO began under PIER support in 2001. Key project milestones under this earlier PIER funding have been: 1) in 2001-02, installation of initial prototype with snapshot displays of San Diego system, only; 2) in 2003, development of a full CA ISO system model, including incorporation of CA ISO user feedback, but still based on snapshots. In summer 2004, CA ISO developed a specification for a new real-time voltage security assessment tool that incorporates and extends the functionality of the original CERTS VAR-Voltage Management prototype for voltage security monitoring purposes. The prototype was enhanced to run contingency simulations and perform contingency ranking which is required for the Voltage Security Assessment prototype phase of the project, and these new features were tested on the CA ISO system.

In July of 2004, the TRP Program Review Committee recommended continued RD\&D in this area; focusing on what VAR margins are needed around the system, on what they are now, and on identifying options for addressing short-falls as they arise. This Committee also recommended research on identifying the information needed by operators to make better operating decisions, including improving comfort and confidence in new real-time operating tools.

Under a prior contract, CERTS formulated a survey (Appendix A. Summary of Survey Results on Methodologies for use in Real-Time Voltage Security Assessment) to reach out to experts in this field for comments, information, suggestions, and recommendations related to the VSA project. The survey was sent to 51 experts in universities and in the power industry worldwide. The consensus opinion was that the continuation method hyperplane approximation approaches are well suited for VSA.

\subsubsection{Task Objectives}

The overall technical objective is to conduct research that will assist CA ISO in acquiring a production-quality, real-time dispatcher's VSA tool, including wide-area visuals to help them manage the voltage and VAR resources on the transmission system. The tool includes the following features:

- Voltage security margin calculation,

- Worst-case contingencies leading to voltage collapse and/or contingencies with insufficient voltage stability margin,

- Abnormal reductions of nodal voltages,

- Contingency ranks according to a severity index for system problems, 
- System conditions with insufficient stability margin,

- Weakest elements within the grid and the regions most affected by potential voltage problems,

- A real-time dispatcher's dashboard-type of display providing information about voltage problems at the normal look-ahead operating conditions and for the worst-case contingencies (contingencies with large severity ranks) that may appear in the future.

Acceptance by CA ISO of a final functional specification for a production-quality VSA software tool is the principal evidence that this overall technical objective has been met.

The objective of Task 2.0 in support of this overall technical objective is to develop and conduct the first-ever demonstration of the first of two prototype real-time software tools for CA ISO (Task 3.0 is to conduct parallel work on the second of these prototype tools).

The specific objectives of Task 2.0 are to:

- Perform a technical assessment of algorithms: 1) for voltage security margin, the distance to instability determined for a selected loading of stress direction, and 2) to identify weak elements;

- Develop and test an initial VSA prototype software tool incorporating these features, and conduct a technical assessment of the continuation power flow algorithm; and

- Provide a functional specification for a next VSA prototype software tool to CA ISO that includes enhancements to these algorithms, among other features, such as contingency ranking. The actual development and testing of this prototype is being conducted under a separate contract \#500-02-004, MR-041, Real Time System Operations (RTSO) 20062007. 


\subsection{Task Approach}

The task approach involved research on each of the first phases of the three project tracks identified in Figure 1: (1) implementation and validation of suitable algorithms; (2) integration with CA ISO energy management system (EMS); and (3) creation of a platform for validating the tool through its development.

The project team conducted an extensive review of existing VSA approaches, and identified and selected a state-of-the-art combination of approaches and computational engines for implementation in this project. Key elements of the task approach are the use of 1) parameter continuation, 2) direct methods, and 3) hyperplane approximation of the voltage stability boundary.

1) Parameter continuation is also known as the predictor-corrector method. It involves finding a continuum of power flow solutions starting at some base load and leading to the steady state voltage stability limit (critical point) of the system. This method is quite robust and useful since it overcomes several mathematical obstacles.

2) Finding the exact critical point or the point of collapse using parameter continuation technique might require several iterations especially while operating close to the critical point. With the help of the direct method algorithm, one can avoid such multiple iterations and obtain the exact solution point directly. However, this method can only be applied when the operating point is in the vicinity of the point of collapse. Hence, VSA utilizes a parameter continuation-direct method hybrid approach to quickly find the point of collapse.

3) Hyperplane is a linear geometry in multi-dimensional space. In 1, 2, and 3 dimensions this happens to be a point, a line and a plane respectively. In power systems, a 2dimensional security region constructed by hyperplane approximation describes a region of safe operation (aka operating nomograms).

These elements were first approved by a panel of leading experts during the course of the survey described above. The elements were also verified in the course of face-to-face personal meetings with well-known university professors, industry experts, and software developers, and included email discussions, telephone exchanges, and feedback from industrial advisors and brainstorm meetings with the projects' consultants.

CERTS industrial advisors reviewed these developments during two Technical Advisory Committee (TAC) meetings conducted in 2005. The TAC, consisting of representatives from the CA ISO, California utilities, Bonneville Power Authority (BPA), DOE and other organizations, provided strategic guidance for this Real-Time Operations RD\&D program to foster rapid adoption of RD\&D results. At the TAC meetings, the California utilities shared their plans on various applications of phasor measurements such as local remedial control interests by SCE, state estimation improvements by SDG\&E, and critical path monitoring by PG\&E.

And, finally, CA ISO staff was consulted extensively throughout the project. 
The approach is based on the following principles and algorithms:

- Concepts of local voltage problem areas and descriptive variables influencing the voltage stability problem were used in each area. Information about the known voltage problem areas was utilized, and formal screening procedures to periodically discover new potential problem areas and their description parameters, were developed

- The voltage stability boundary was calculated and approximated. The approximated voltage security conditions in real time for a fast VSA, and hyperplanes to approximate the voltage stability boundary were used.

- To calculate the approximating hyperplanes, and reactive margins, the project team used the parameter continuation techniques and introduced a sufficient additional security margin to account for inaccuracies of approximation and uncertainties of the power flow parameters.

- A list of abnormal reductions in nodal voltages that highlighted the elements and regions most affected by potential voltage problems was compiled.

- The parameter continuation predictor corrector methods were implemented by identifying and developing necessary improvements.

- Motivated by survey results, the project team used the PSERC parameter continuation program and MATLAB programming language as a basis for building the VSA prototype functional specifications.

The project team prototyped the proposed algorithms on a platform developed by the team for validating the tool and tested it with a CA ISO-provided test case. The project team validated the VSA algorithm results through numerous meetings and correspondence with CA ISO staff, who helped identified test cases and appropriate stressing directions. The project team used the GE PSLF power flow tool (a well-accepted off-line planning tool currently used by CA ISO) to simulate the VSA algorithms and compare output results, and the CA ISO system planning model (in GE PSLF form). 


\subsection{Task Outcomes}

The project team completed research on each of the first phases of the three project tracks identified in Figure 1: (1) implementation and validation of suitable algorithms; (2) integration with CA ISO energy management system (EMS); and (3) creation of a platform for validating the tool through its development.

With respect to the project objectives, the project team:

- Performed a technical assessment of algorithms: (1) for voltage security margin, the distance to instability determined for a selected loading of stress direction, and (2) to identify weak elements.

- Developed and tested an initial VSA prototype software tool incorporating these features, and conduct a technical assessment of the continuation power flow algorithm and the hyperplane approach.

- Provided a functional specification for a next VSA prototype software tool to CA ISO that includes enhancements to these algorithms, among other features, such as contingency ranking. The actual development and testing of this prototype is being conducted under a separate contract \#500-02-004, MR-041, Real Time System Operations (RTSO) 2006-2007.

What follows in this sub-section is a summary of the task accomplishments and research findings. A complete discussion of the research findings is contained in Appendix B, CA ISO Real-Time Voltage Security Assessment Summary Report.

The proposed algorithm was successfully implemented on the VSA platform and tested on the Humboldt and San Diego areas. The efficacy of using hyperplanes to approximate the voltage stability boundaries, as well as identifying the associated weak elements and the 'controllable' elements for these boundaries were corroborated.

The approximated voltage stability boundary was compared to results obtained from the GE PSLF program which is commonly used in the Western Interconnection. The results of the comparison of the approximated voltage stability boundary to the results obtained from the GE PSLF program were within a few percent of each other. The main contributing factor to these discrepancies was the CA ISO state estimator model, which had deficiencies that required manual modifications to get it to solve. The GE PSLF handles such scenarios differently, and these differences show up in the stability boundary calculations. The project team expects that, with improvements to the CA ISO state estimator model, these differences will disappear.

A first prototype was developed and tested. Among other features, it incorporated a contingency ranking feature that had been previously requested by CA ISO, the continuation power flow algorithm as recommended by the academic experts, and the hyperplane concept. The release was preceded by factory testing, and presentation of results to CA ISO. 
The VSA prototype functional specifications (Appendix C) included:

- Expansion of VAR Management application to include contingency simulation and ranking capabilities.

- Calculation of sensitivity factors of the security margin with respect to dispatchable and non-dispatchable parameters in order to determine the reasons for potential instability and to identify the best actions to increase the security margin.

- Findings from the analysis of algorithms for assessing voltage security margins.

- Findings from the analysis of algorithms for weak elements calculation.

- Execution results from field test of prototype voltage security software.

- Displays of the most limiting overloads and voltage deviations restricting the current and future operating conditions.

- Remedial actions pre-calculated for the worst contingencies in order to effectively support the real-time dispatcher's need to respond quickly to dangerous contingencies once they occur.

Our research suggests that while the continuation method worked well in reaching the proximity of the collapse point in a particular stressing direction, several iterations of the algorithm and associated step-halving within the vicinity of the point-of-collapse were required to obtain the functions needed to extract accurate information about the hyperplane boundaries, weak elements and control elements.

The project team discovered the advantage of applying the direct method at this point is that it is a one step approach of finding the collapse point within a predefined tolerance, and therefore overcomes accuracy limitation in the continuation method. Once having accurately reached a point on the stability boundary, it is also theoretically feasible to apply the underlying continuation method framework to the direct method equations (as apposed to the powerflow equations as in the traditional continuation powerflow) and systematically trace the voltage stability boundary (i.e. Boundary Orbiting Method). This adaptation is believed to further reduce the computational time because there is no longer a need to return back to the operating point and move in a different stress direction to find a second point on the stability boundary. These enhancements will be incorporated into the VSA platform and this overall hybrid algorithmic approach will be validated under the follow-on contract.

Additionally, the efficacy of using hyperplanes (i.e., linear planes defined in multidimensional space) to approximate the voltage stability boundaries as well as identifying the controllable elements in the space of power injections was corroborated. In particular, the project team demonstrated that the attributes of hyperplanes (i.e. coefficients of the hyperplane) can be interpreted as the parametric sensitivities of the margin to power injections and therefore are particularly useful in ranking the most appropriate corrective actions to steer away from the stability boundary. Similarly, the participation factors at the various buses in the voltage collapse also fall out of the proposed methodology and aid in identifying the various weak areas with the worst degradation in voltages during a voltage collapse situation. 
In summary, the validation process confirmed that: 1) these hyperplanes, or piecewise linear approximations, can be extracted from the solution at the point of collapse in a particular stressing direction, 2) using piecewise linear approximations for the stability boundary are in fact appropriate, 3) the properties of these boundaries (e.g. the orientation of the hyperplanes) also offered valuable information on the 'control' elements which represent the optimal points in the system for corrective action to steer away from a dangerous condition, or the weak elements which are the areas where the impact of the voltage collapse phenomenon is the most severe. The findings also suggested that the continuum power flow algorithm on its own lacked the accuracy and speed required for a real time security assessment tool, and a hybrid approach, wherein the continuum power flow algorithm augmented with direct methods, could meet these performance requirements. 


\subsection{Conclusions and Recommendations}

\subsubsection{Conclusions}

The project team concluded that the continuation powerflow had limitations. It was not able to achieve the execution speed and accuracy required from a real time voltage security assessment tool to provide the level of detailed information required by CA ISO. However, the project team established that a hybrid approach, wherein the continuation powerflow is augmented by other algorithms such as the direct method, which are more suitable for calculating these margins near the voltage collapse point, could meet the desired performance requirements for a real time tool.

\subsubsection{Recommendations}

The project team recommends additional work to enhance the functionality of the VSA RealTime tool by conducting RD\&D on algorithms, such as the direct methods, which have the potential to overcome the limitations of the continuation powerflow, validating these methods on the CA ISO system, and finally providing a functional specification for a production-quality VSA tool to CA ISO.

This additional functionality and the projection quality functional specifications are being developed under the subsequent Work Authorization, Contract \#500-02-004, MR-041, RTSO 2006-2007.

\subsubsection{Benefits to California}

The benefit to California is the enhanced reliability of the CA ISO and interconnected Western Interconnection by providing reliability coordinators and control area operators at CA ISO, California's major utilities, and Bonneville Power Administration (BPA) with the latest technology in VAR management tools. Most of the voltage security assessment tools that are currently available commercially are well-suited for the planning environment, where they are used in an offline mode to conduct studies and define safe operating regions and margins (or nomograms). However, these nomograms, which are utilized to operate the grid in real time, tend to be conservative in order to accommodate unforeseen uncertainties, worst case conditions, and any discrepancies between the real time operating conditions and those used in the offline planning studies. Therefore, the ability to dynamically adjust the voltage security regions to changing system conditions, and compute margins in real time that accurately reflect the true system conditions, will have the following benefits to CA ISO and the California utilities:

- CA ISO will immediately benefit from increased reliability;

- Improved voltage monitoring may also improve the accuracy of locational marginal pricing (LMP) calculations that will accompany the roll-out of CA ISO's Market Redesign Technology Update (MRTU) initiative. MRTU will address problems in California's electricity markets that contributed to the market disruptions experienced in 2000 and 2001, and translate into better management, potentially at lower cost, of congestion on the CA ISO system. It builds on three foundational designs - a full 
network model of the electricity grid, an integrated day ahead forward market and LMP. The LMP is the result of the integrated forward market which provides nodal prices so that all market participants know the cost of generating power, serving load and resolving congestion at each location on the system. LMPs reflect physical constraints under all load and system conditions and offer better economic measures and signals with which to manage the system. These pricing patterns also indicate where additional generation and transmission upgrades are needed in the future; and

- A successful demonstration at the CA ISO will likely accelerate market acceptance of this and similar operating tools by the industry, leading to a promulgation of these first two benefits to other regions of the country. 


\subsection{Task 3.0: Real-Time Applications of Phasors for Monitoring, Alarming and Control}

\subsection{Executive Summary}

Electric industry restructuring in California has led to the formation of larger control areas with correspondingly larger areas of reliability oversight, as well as increased energy transactions over long, region-wide transmission paths. These developments have introduced greater uncertainty into real-time grid operations, which, in turn, has led to the need for better real-time information on actual conditions that can supplement traditional operating guidelines based on off-line studies. Currently, control areas depend on static nomograms produced from off-line simulations conducted several months in advance of the operating season to manage power flows on critical transmission paths. Because actual operating conditions may differ significantly from those assumed in preparing the off-line simulations, the California Independent System Operator (CA ISO) system may be operated (unknowingly) without adequate reliability margins. This project is the second phase of a multi-year program of research in which CERTS is conducting research on and developing prototypes for real-time applications of phasors for monitoring, alarming and control to accelerate the adoption and foster greater use of new, more accurate, time-synchronized phasor measurements by CA ISO reliability coordinators and control area operators, as well as by California and WECC utility transmission dispatchers. The project significantly leverages companion efforts, also managed by CERTS for the U.S. Department of Energy (DOE), to promote the use of phasor measurements nationally. For this project, version 3 of the CERTS Real-Time Dynamics Monitoring System (RTDMS) was developed and delivered for testing and feedback from the CA ISO. Version 3 includes real-time alarming and event detection, and event archiving and playback. In addition, training on the use of the tool was provided along with a user guide. Version 3 incorporated visualization concepts that were developed initially under DOE funding for the prototype RTDMS that has been released for evaluation by reliability coordinators in the Eastern Interconnection. Ultimately, research in this area will lead to the development of functional specifications for a production quality system that would be acquired by CA ISO and other entities with operating reliability responsibilities in the Western Interconnection. 


\subsection{Introduction}

Electric industry restructuring in California has led to the formation of larger control areas with correspondingly larger areas of reliability oversight, as well as increased energy transactions over long, region-wide transmission paths. These developments have introduced greater uncertainty into real-time grid operations, which, in turn, has led to the need for better real-time information on actual conditions that can supplement traditional operating guidelines based on off-line studies. Currently, control areas depend on static nomograms produced from off-line simulations conducted several months in advance of the operating season to manage power flows on critical transmission paths. Because actual operating conditions may differ significantly from those assumed in preparing the off-line simulations, the CA ISO system may be operated (unknowingly) without adequate reliability margins. CERTS is conducting research on real-time applications of phasors for monitoring, alarming and control to accelerate the adoption and foster greater use of new, more accurate, time-synchronized phasor measurements by CA ISO reliability coordinators and control area operators, as well as by California and WECC utility transmission dispatchers.

Applications based on phasor measurements will provide the real-time operating staff with the previously unavailable, yet greatly needed, tools to avoid voltage and dynamic instability, and monitor generator response to abnormal significant system frequency excursions. Perhaps of equal or greater importance, in the near term, the measurement infrastructure will provide CA ISO with an alternate, independent real-time monitoring system that could act as an end-of-line backup for failures affecting CA ISO's current Supervisory Control and Data Acquisition/Energy Management System (SCADA/EMS). In the long term, the infrastructure is expected to become a key element of CA ISO's next generation monitoring system for advanced real time control.

- SCADA is a category of software application program for process control, the gathering of data in real time from remote locations in order to control equipment and conditions. SCADA systems include hardware and software components. The hardware (sensors) gathers and feeds data into a computer that has SCADA software installed. The computer then processes this data and presents it in a timely manner. SCADA also records and logs all events into a file stored on a hard disk or sends them to a printer. SCADA warns when conditions become hazardous by sounding alarms.

- EMS is a system of computer-aided tools used by operators of electric utility grids to monitor, control, and optimize the performance of the generation and/or transmission system. The monitor and control functions are known as SCADA; the optimization packages are often referred to as "advanced applications."

This project is the second phase of an ongoing RD\&D activity coordinated by CERTS for the Energy Commission's PIER Transmission Research Program. Earlier phases of this research were sponsored by PIER through a RD\&D contract with LBNL (Contract \#150-99-003) and through several task orders funded through the California Institute for Energy and Environment (BOA\#20). Additional funding, through a separate subsequent work 
authorization, Contract \#500-02-004: MR-041, has already been provided to continue the work initiated in this task (see Figure 2, Multi Year Research Roadmap for CA ISO Phasor Project).

\subsubsection{Background and Overview}

Currently, the CA ISO and other WECC control areas depend on static nomograms to manage power flows on critical transmission paths. These nomograms are produced from off-line simulations that are conducted several months in advance of the operating season. Because actual operating conditions may differ significantly from those assumed in preparing the offline simulations and because the simulations, themselves, may be based on incorrect models (which is the topic addressed in Task 4.0), the CA ISO system may be operated (unknowingly) without adequate reliability margins.

Due to the long-distance nature of transmission in the west, there is a special need to ensure the dynamic stability of the grid. The information required to assess reliability margins in real-time must be: a) collected over a wide area; b) sampled at high frequency (30 times or more per second, compared to traditional monitoring at once every 4 seconds); and, most important of all, c) precisely time-stamped so that information collected from different locations can be synchronized with each other. Phasor measurement units are a leading example of the new generation of advanced measurement technology that has these capabilities. Currently, there is reasonable deployment of these units throughout the west; however, to date, they have only been used to support off-line applications, such as model validation and post-disturbance analysis.

The first research grade demonstration of phasor technologies was undertaken by Department of Energy (DOE), Electric Power Research Institute (EPRI), Bonneville Power Administration (BPA), and Western Area Power Administration (WAPA) in the early 1990s. The investment was paid in full when data recorded by the system was effectively used to investigate causes of the major 1996 west coast blackouts. DOE has continued to support outreach for these technologies and has provided technical support to the WECC committees that rely on these data for off-line and model validation reliability studies. 
INPUT DATA REQUIREMENTS

\section{CA ISO}

APPLICATIONS

RTDMS

Visualization

RTDMS

Monitoring

Small Signal Stability Monitoring

Frequency Data

Collection for

Analysis

NEW SYSTEM

CAPABILITIES

Wide-Area Real

Time Control

Applications

Using Phasors

SYSTEM NTEGRATION

\& SUPPORT

Support to Utility

Phasor Applications

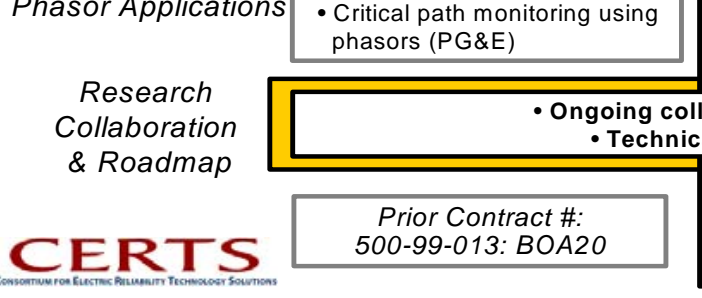

Functional specification

- Feasibility \& functional

specification
- Integration of CA ISO PDC

with Arbiters

Support California Utilities for: - Local real time remedial action - Lontrol using phasors (SCE) - State estimation improvements using phasors (SDG\&E) - Critical path monitoring using phasors (PG\&E)

CERTS

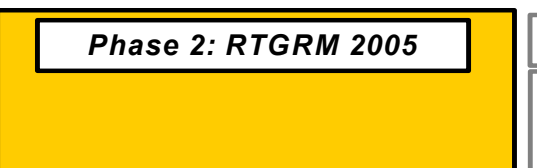

Phase 3: RTSO 2006-07

-RTDMS integration with CA ISO PI server for long term

replay

liance with new IEEE

C37.118 Standard

-RTDMS Version 4 (dashboard display, clutter management,

- Incorporate new user feedback

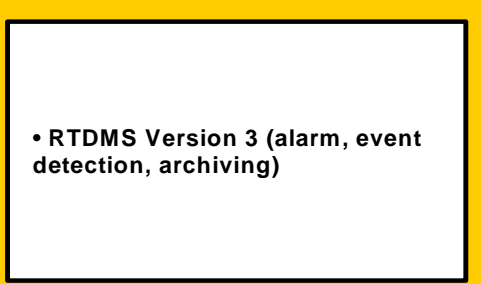

Feasibility Assessment Study Report:

- Frequency response analysis - Small Signal Stability

- Stability Nomograms

-Sensitivity analysis (voltage

angle, droop)

- Signature recognition

for event identification \&

Classification

Small Signal Stability Prototyp

Enhancements to improve
integration with WECC-NERC
standards

standards

Support California Utilities for:

- Local real time remedial action

control using phasors (SCE)

Collaboration with equivalen

Eritis state estimator project

Critical path monitoring using

phasors (PG\&E)

Support CA ISO phasor applica-

Lgration \& dissemination:

-

State estimal

- State estimation improvements
- Critical path monitoring

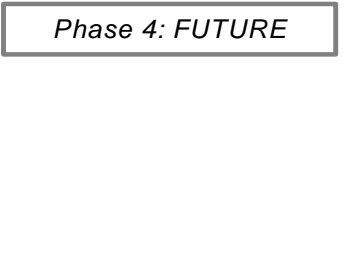
-Frequency response \& sensitivity analysis:

prototype developmen -Small signal stability: production quality commercial functional

California

Real-Time

Phasor

System for:

- Visualization r ----------

- Simulate and design

prototype

commercial functiona specification

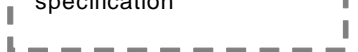

- Monitoring

- Measuremen

- Control \&

Protection

tions for real time control

\section{- Prediction}

Research • Ongoing collaboration and consultation with industry and academic experts (e.g. PSERC,

Figure 2. Task 3.0 Multi Year Research Roadmap for CA ISO Phasor Project
Current Contract \#
$500-02-004: M R-036$

500-02-004: MR-036

Follow-on Contract \#:

500-02-004: MR-041 
PIER, through CERTS, supported installation of an initial data connection and workstation to support offline analysis by CA ISO staff in 2002. In 2003 and 2004, PIER, through CERTS, supported the deployment of a real-time application, using phasor technology, to monitor actual grid conditions, a phasor-data link to BPA and WAPA for real-time data.

In July of 2004, the TRP Program Review Committee recommended that the Energy Commission and CA ISO continue the research, development and application of this technology including research to determine what phase angles and rates of change of phase angles are appropriate for various locations in system, what actions should be taken by operators or automatic control equipment, if there are major deviations, and the desired location for additional phasor monitoring equipment around the WECC. The proposed approach, developed in conjunction with CA ISO staff, represents a phased approach toward addressing these issues, centered around introducing phasor information to operators and working closely with them to modify and enhance the applications (including training) to increase their confidence in relying on this information to support their day-to-day activities.

During 2004 and 2005, the CERTS Real-Time Dynamics Monitoring System (RTDMS) phasor visualization prototype, which was initially developed as a stand-alone application, was transformed into a phasor technology development platform with the underlying functionalities to support a longer-term goal to use phasor technology for wide-area monitoring, alarming and control. In particular, the RTDMS platform now supports a serverclient architecture with the central server responsible for data management functions (such as data acquisition, filtering and caching), and multiple client applications installed on different machines can simultaneously access data from the central server.

In July of 2005, CERTS worked with CA ISO in developing an RD\&D Roadmap to guide the Phasor Applications technology research and development effort. Figure 2 is the Multi Year Research Roadmap for CA ISO Phasor Projects. It outlines the research and development plans by the various task activities that need attention, as well as through the various phases of the development cycle including research, development, demonstration, and technology transfer, and the intermediate milestones and deliverables. Potential applications include the use of phasor measurements for wide-area visibility, real-time monitoring and alarming, small-signal stability assessment, frequency data collection, nomogram validation and improvements, improved state estimation, and real-time control.

These applications will provide the operators with the previously unavailable, yet greatly needed, tools to avoid voltage and dynamic instability, and monitor generator response to abnormal significant system frequency excursions. Perhaps of equal or greater importance, in the near term, the measurement infrastructure will provide CA ISO with an alternate, independent real-time monitoring system that could act as an end-of-line backup for failures affecting CA ISO's current SCADA/EMS. In the long term, this infrastructure a key element of CA ISO's next generation monitoring system for advanced real time control.

The project significantly leverages companion efforts, also managed by CERTS for the U.S. Department of Energy (DOE), to promote the use of phasor measurements nationally. Starting in 2003, DOE has tasked CERTS to provide technical support to the industry-led development of a phasor-based measurement network in the Eastern Interconnection. CERTS has provided 
technical support for the creation of a phasor data concentrator hosted by Tennessee Valley Authority and developed a prototype platform and visualization application for evaluation by reliability coordinators in the east. The experience and concepts developed through these DOEsupported activities contributes directly to the research undertaken in this task.

\subsubsection{Task Objectives}

The overall technical objective is to accelerate the adoption and foster greater use of new, more accurate, time-synchronized phasor measurements by CA ISO reliability coordinators and control area operators as well as by California and WECC utility transmission dispatchers. The research required includes:

- Providing real-time operators with new tools that provide previously unavailable widearea visibility and information on the dynamic stability of the grid;

- Designing the conceptual look of operator displays for phasor applications;

- Defining functional specifications such that, upon completion, the tools can be transferred to a vendor (selected by CA ISO) for implementation as a production-grade operating tools; and

- Providing technical support to and assist in coordinating phasor applications being researched and developed by CA utilities.

The objective of Task 3.0 in support of this overall technical objective is to develop and conduct the first-ever demonstration of the second of two prototype real-time software tools for CA ISO (Task 2.0 is to conduct parallel work on the first of these prototype tools).

Delivery of the RTDMS Version 3, the user guide, and CA ISO staff training is the principal evidence that this objective has been met. 


\subsection{Task Approach}

The task approach involved research on the first phases of each of the four project tracks identified in Figure 2: a) input data requirements; b) CA ISO applications; c) new system capabilities, and d) system integration and support.

CERTS utilized and expanded the current WECC and CA ISO phasor infrastructure as the input data source for the real-time applications.

CERTS conducted a survey to reach out to experts in the field for their comments, suggestions, and recommendations on wide area security assessment. The survey was sent to 51 academic and power industry experts worldwide with sixteen responses received; eight of the respondents were from the power industry and eight from academia. The consensus from the joint CERTS-CA ISO survey (Appendix A. Summary of Survey Results on Methodologies for use in Real-Time Voltage Security Assessment) was that the use of phasor measurements for modal estimation to assess small signal stability was an ideal initial step towards achieving the CA ISO's objectives for wide area security assessment with phasors.

As a result of the survey, the project team began to collaborate with Bonneville Power Administration (BPA) and others to develop a small signal stability application on the RTDMS platform. The small signal stability application and the proposed visualization solution are being implemented on the RTDMS platform under Contract \#500-02-004: MR-041.

The project team developed a phasor technology platform for research and prototyping new tools, called the Real-Time Dynamics Monitoring System (RTDMS). The project team developed RTDMS with a server-client architecture to accommodate the high data rates associated with phasor measurements. This is a system design where the data management (i.e. RTDMS Server) and the algorithmic and visualization components (i.e. RTDMS Clients) are separate and independent functions in order to ensure that the computational burden of algorithms does not affect real time data management and flow, and cause data loss. The server-client architecture also offers the flexibility of having a single central data server, and multiple monitoring clients which could then simultaneously access the same central server and present the corresponding results within their individual visualization displays.

CERTS also provided assistance and support to California utilities in their efforts to use phasor measurements for local remedial action control (SCE), state estimation improvement (SDG\&E), and critical path monitoring (PG\&E). CERTS provided technical assistance to CA ISO in preparing their multi-year research roadmap, which included activities such as WECC-EIPP collaboration and knowledge exchange, and collaboration with industry and academic experts.

Throughout this task, the work built upon both off-line analysis and research conducted by CA ISO staff, as well as feedback from operators on the usability and usefulness of the information provided by the network and the means developed to present it. These interactive processes continued in parallel directly with the delivery of specific functionalities and prototype displays. 


\subsection{Task Outcomes}

CERTS produced task outcomes for each of the first phases of three of the four project tracks identified in Figure 2: a) input data requirements; b) CA ISO applications; c) new system capabilities, and d) system integration and support.

What follows in this sub-section is a summary of the task accomplishments and research findings. A complete discussion of the research findings is contained in Appendix D, CA ISO Phasor Applications Summary Report.

Initially the phasor network consisted of only 14 Phasor Measurement Units (PMUs) that gathered data at the sub-second resolution (30 samples/second) from two utilities and sent it in real time to CA ISO. Through this project, the network grew to 42 PMUs with expanded coverage including Western Area Power Administration (WAPA) and PG\&E regions. Presently, CA ISO Phasor Data Concentrator (PDC) receives data from PMUs in geographically distributed locations via the WECC Wide Area Network (WAN) which connects utilities' PDCs with the PDC at CA ISO. PMUs feed into their corresponding PDCs, and this data is further transmitted in real-time to the CA ISO PDC. Figure 3 shows the current synchronized data communication network and proposed expansions.

\section{CAISO PHASOR NETWORK DIAGRAM (PRESENT AND FUTURE)}

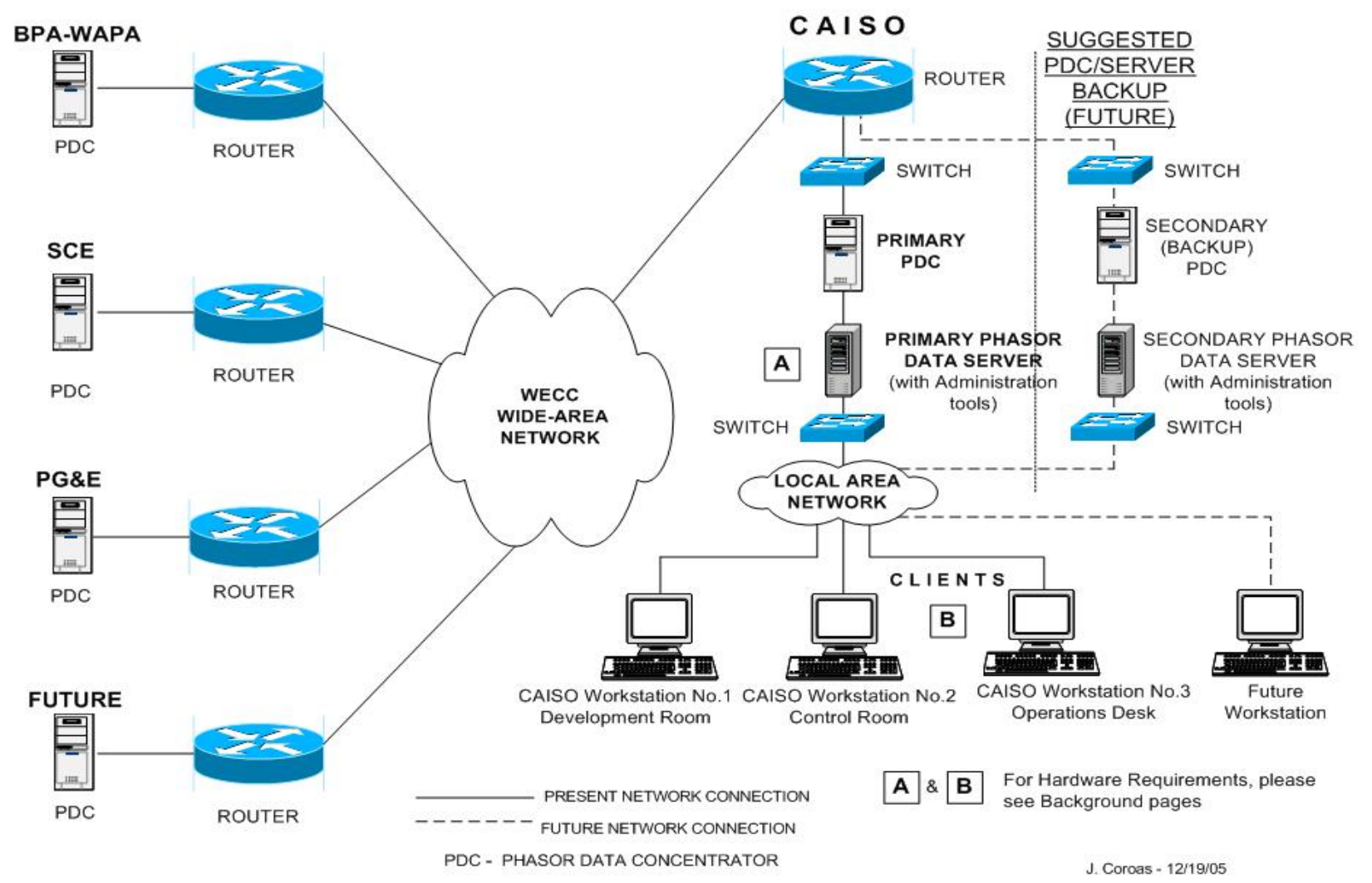

Figure 3: CA ISO Phasor Network Diagram 
CERTS developed and delivered RTDMS version 3 to CA ISO operators and reliability coordinators as a stand-alone application for real time monitoring. Version 3 includes applications for real-time alarming and event detection, and event archiving and playback. CERTS also provided training to CA ISO staff (Appendix E) and a users guide (Appendix F).

The current RTDMS application, version 3, at CA ISO reads the collective set of phasor data output from the CA ISO PDC and displays it in real-time. This complete set of timesynchronized high-resolution data provides the desired wide-area visibility across the western interconnection required for security monitoring by dispatchers including dynamic performance assessment and post disturbance analysis. See Figure 3: CA ISO Phasor Network Diagram.

Another initial application that was developed on the RTDMS platform includes the collection and archiving of sub-second frequency data from PMUs to meet new North American Electric Reliability Council (NERC)-WECC Western Interconnection frequency data collection requirements.

The RTDMS platform and visualization application tool is undergoing active evaluation by CA ISO operations staff. Operational versions of the prototype are located at both the CA ISO control room and operations desk. See Figure 3. Feedback from staff has been incorporated into specifications for future prototype releases and new avenues of basic research that is enabled by the availability of phasor measurement data in real-time.

The consensus from the joint CERTS-CA ISO survey was that the use of phasor measurements for modal estimation to assess small signal stability was an ideal initial step towards achieving the CA ISO's objectives for wide area security assessment with phasors. As noted earlier, the project team began conducting collaborative research with BPA and others in the Northwest to explore this application. Our initial research in this project concludes that it is possible to characterize the stability of the power system in real time and under ambient system conditions with respect to the low frequency oscillations that exist in the system. As a result, the project team began work to develop an application to monitor small signal oscillations in real time, and alert operators when the system is experiencing poor damping. CERTS began developing visualization enhancements to deal with the rapid growth in the numbers of PMUs within the WECC phasor network and the associated clutter. The small signal stability application and the proposed visualization solution are being implemented on the RTDMS platform under Contract \#500-02-004: MR-041, Real Time System Operations 2006-2007.

A wide-area research need that extended beyond the CA ISO, the use of phasor measurements for real-time wide-area control, was also addressed. As a first step towards achieving new system capabilities (see roadmap), the project team researched methods for utilizing phasor measurement to validate and possibly improve stability nomograms. This work is also continuing under Contract \#500-02-004: MR-041, Real Time System Operations 2006-2007.

Figure 4 provides a conceptual overview of the system hardware and software architecture for RTDMS Version 3. The RTDMS platform now supports a server-client architecture with the central RTDMS server responsible for data management functions such as data acquisition, 
filtering, data processing and caching, and a thin client layer where multiple RTDMS client applications installed on different machines can simultaneously access data from the central server. By centralizing the data management process, this architecture minimizes the necessary performance requirements for all client machines, which translates to a reduction in hardware cost. Finally, any new client applications that are developed in the future will be able to connect to the common RTDMS server for data access and retrieval.

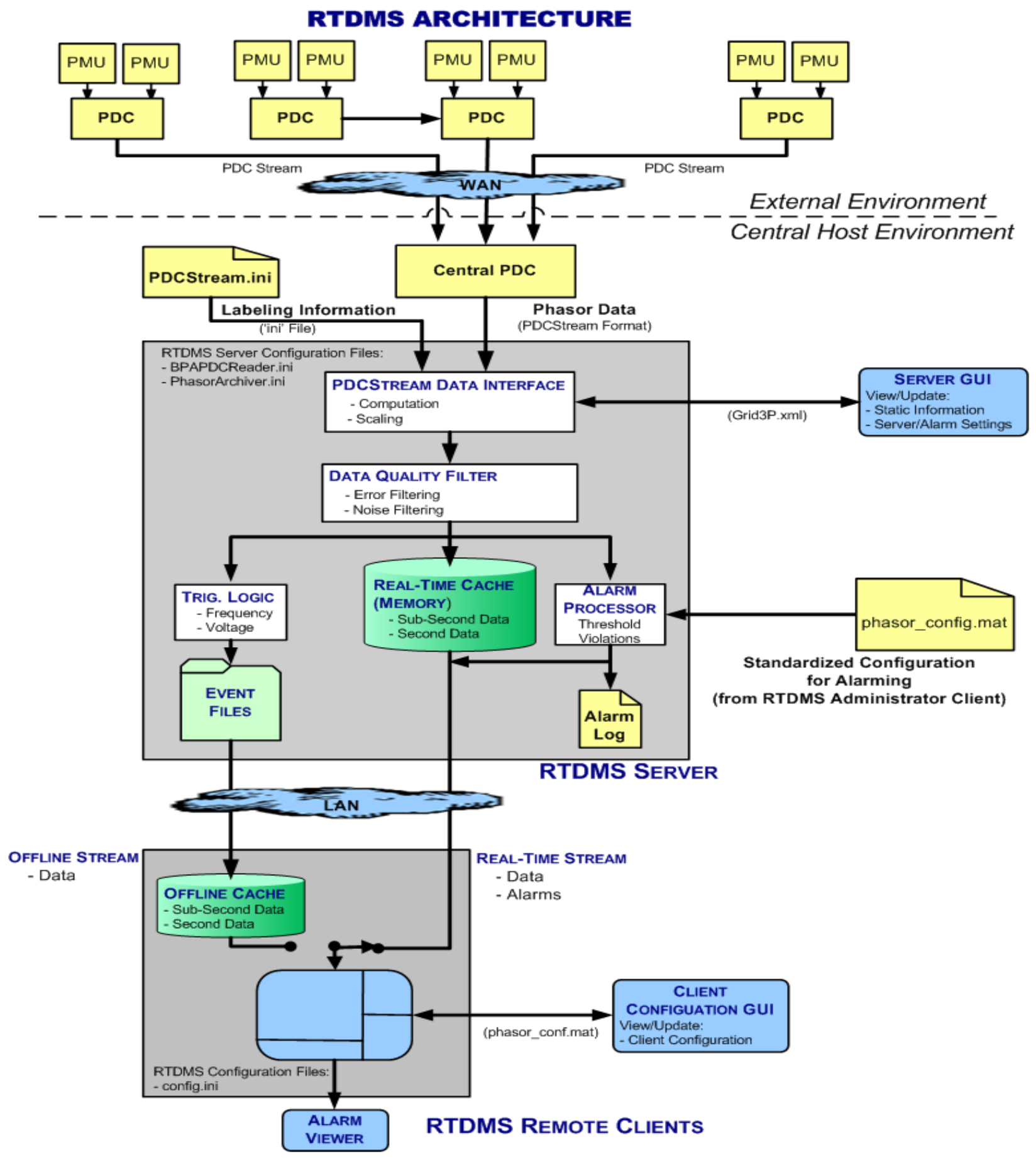

Figure 4: RTDMS Version 3 System Architecture 
CERTS provided system integration and technical support and assisted as needed in coordinating phasor applications being researched and developed by CA utilities (e.g. local remedial action controls with phasors at SCE, state estimation using phasor measurements at SDG\&E, and critical path monitoring by PG\&E) as well as WECC-EIPP collaboration and knowledge exchange, and collaboration with industry and academic experts. CERTS prepared and delivered a PMU Measurement Unit Installation and Cost Estimates document to SDG\&E, and have offered RTDMS to PG\&E to meet their respective objectives. 


\subsection{Conclusions and Recommendations}

\subsubsection{Conclusions}

The development and testing of a prototype RTDMS with CA ISO system operators has accelerated the adoption and use of time-synchronized phasor measurements for real-time applications in the Western Interconnection. Eventually, this wide-area, common view will allow operators to evaluate stability margins across critical transmission paths, detect potential system instability in real time, and, in the future, take manual or initiate automatic actions to mitigate or dampen these potential problems. It will also enable CA ISO, California and WECC utilities to explore closely related issues, such as use of phasor data to improve state estimation, to determine the optimal location of additional phasor measurements, and to gain the experience with the technology required to develop these advanced real-time control applications. Finally, it will facilitate technical exchange, collaboration, and resource leveraging with companion phasor measurement-based activities supported by the operating entities and DOE throughout North America.

\subsubsection{Recommendations}

CERTS recommend continued research and development of prototype applications that, ultimately, will provide the technical basis for preparation of functional specifications for a production-quality system that can be used by CA ISO to select a commercial vendor for the system. CERTS also recommend continued efforts through the WECC to expand and link phasor measurement units across the entire Western Interconnection.

Aspects of the additional RD\&D required have already begun under Contract \#500-02-004, MR-041, Real Time System Operations 2006-2007.

\subsubsection{Benefits to California}

The benefit to California is the enhanced reliability of the CA ISO and interconnected Western Interconnection by providing reliability coordinators and control area operators at CA ISO and California's major utilities with the latest advances in phasor measurement applications. Ultimately, system operators will be able to evaluate stability margins across critical transmission paths, detect potential system instability (pattern recognition) in real time, and then provide control signal(s) to devices or controls that will mitigate or dampen the instability. The wide-area, common view will also allow operators to detect unanticipated system limitations in real-time, even when the grid is operating within perceived safe portions of the existing operating nomograms. Thus, the system will serve a dual purpose to both improve transmission loadability from the point of view of transient stability and help operate the system within safe regions.

As a result, this research, ultimately, will have the following benefits for CA ISO and the California utilities:

- CA ISO will immediately benefit from increased reliability;

- Improved voltage monitoring may also improve the accuracy of locational marginal pricing (LMP) calculations that will accompany the roll-out of CA ISO's Market 
Redesign Technology Update (MRTU) initiative. MRTU will address problems in California's electricity markets that contributed to the market disruptions experienced in 2000 and 2001, and translate into better management, potentially at lower cost, of congestion on the CA ISO system. MRTU builds on three foundational designs - a full network model of the electricity grid, an integrated day ahead forward market and LMP. The LMP is the result of the integrated forward market which provides nodal prices so that all market participants know the cost of generating power, serving load and resolving congestion at each location on the system. LMPs reflect physical constraints under all load and system conditions and offer better economic measures and signals with which to manage the system. These pricing patterns also indicate where additional generation and transmission upgrades are needed in the future; and

- A successful demonstration at the CA ISO will likely accelerate market acceptance of this and similar operating tools by the industry, leading to a promulgation of these first two benefits to other regions of the country. 


\subsection{Task 4.0: Improving Dynamic Load and Generator Response Performance Tools}

\subsection{Executive Summary}

Secure operation of the electric grid relies on planning studies, conducted off-line, typically months in advance, that consider whether the grid is capable of withstanding credible contingencies (such as the unplanned loss of major generating plants or transmission inter-ties). These anticipatory (or what if) studies are used to establish operating limits for secure operation following criteria established by North American Electric Reliability Council (NERC) and Western Electricity Coordinating Council (WECC). The dynamic load models currently relied on to prepare these studies are known to be inadequate. They were first developed over 20 years ago and have rarely been updated to capture the dramatic changes that have taken place in the underlying composition of load (for example, increased saturation of power electronic devices, induction motors, etc). Similarly, dynamic models for governor frequency response control, until recently, have not kept pace with industry changes. Blackout risks, exacerbated by inaccurate models, were clearly illustrated in the August 10, 1996 blackout, when simulations, using these models, were unable to replicate blackout events. Recent disturbances in Southern California displaying delayed voltage recovery, and lightly damped oscillations on the California-Oregon Intertie are not captured by simulations using current models. This project prepared a scoping study to identify the research required to improve dynamic load and generator response models. The study was based on literature reviews of current state-of-the-art load modeling, active participation in the WECC Load Modeling Task Force, and discussions with leading researchers and practitioners. The project has already led to formal sponsorship by WECC of a follow-on, cost-shared PIER project to conduct research on the highest priorities identified in the scoping study. 


\subsection{Introduction}

Secure operation of the electric grid relies on planning studies, conducted off-line, typically months in advance, that consider whether the grid is capable of withstanding credible contingencies (such as the unplanned loss of major generating plants or transmission inter-ties). These anticipatory (or what if) studies are used to establish operating limits for secure operation following criteria established by NERC and WECC. The dynamic load models currently relied on to prepare these studies are known to be inadequate. They were first developed over 20 years ago and have rarely been updated to capture the dramatic changes that have taken place in the underlying composition of load (for example, increased saturation of power electronic devices, induction motors, etc). Similarly, dynamic models for governor frequency response control, until recently, have not kept pace with industry changes. Blackout risks, exacerbated by inaccurate models, were clearly illustrated in the August 10, 1996 blackout, when simulations, using these models, were unable to replicate blackout events.

This project is the first phase of an ongoing RD\&D activity coordinated by CERTS for the Energy Commission's PIER TRP. The purpose of this initial phase is to prepare a scoping study that identifies needed research to improve these dynamic models. The research identified in this scoping study is being conducted through a separate TRP contract \#500-02-004: MR-049, WECC Load Modeling Research Project.

\subsubsection{Background and Overview}

To study transient events, such as the sudden, unplanned loss of a major transmission line or generator, dynamic computer simulation models are used to predict the response of the grid in the first few cycles and seconds following an event. CA ISO and others have reported that these simulations are inaccurate and, sometimes, do not adequately predict the actual, observed response of the system to events, especially when the grid is under stressed conditions. A comparison of observed voltage and frequency transients to simulated responses suggests that there are inaccuracies both in load models and in generation plant control models used in these simulations.

The load models in use today were first developed over 20 years ago and have rarely been updated to capture the dramatic changes that have taken place in the underlying composition of load (for example, increased saturation of power electronic devices, induction motors, etc.). Moreover, the forms of the load models that are used are unrealistic because they are based on the estimated composition of loads during the summer peak period, while they are also used for studies of other time periods (such as other seasons and other times of day).

Similarly, until recently, the models for governor frequency response control have not kept pace with changes in the industry. CA ISO staff have noted decreased frequency response after large outages, and NERC has documented a persistent decline in frequency response in both the Eastern and Western Interconnections. As a result of restructuring, the actual settings on generator controls are not as well-known as they used to be by the transmission system operators because different firms now operate the generators. Previously, the same firm operated both the transmission system and the majority of generators, so information sharing on settings was easier. Newer generation technologies, especially, have plant controls that are 
believed to override the automatic governor controls on the generators. Yet, transmission operators have traditionally assumed that these controls are operating in order to ensure system reliability. Better information is required on the actual settings for these controls, as well as on the plant control systems than may override them. Recent WECC modeling work has resulted in new plant control models that should improve the simulations of plant response.

\subsubsection{Task Objectives}

The overall technical objective is to conduct research that will improve the accuracy of the dynamic models used in WECC to set reliability limits, retrospectively evaluate significant disturbances, and conduct additional planning studies.

Incorporation of research results into the models used by WECC to conduct these studies is the principal measure of success for this program of research.

The specific technical objective of Task 4.0 is to prepare a scoping study that identifies and prioritizes the research activities needed to improve the performance of computer-simulation models.

Follow-on work, which has been awarded through a separate TRP contract \#500-02-004: MR-049, WECC Load Modeling Research Project, to conduct the research identified in this scoping study, is the principal measure of success for this project. 


\subsection{Task Approach}

The task approach involved gathering information through literature reviews of current stateof-the-art load modeling, phone and electronic consultations with experts, and active participation in meetings of the WECC Load Modeling Task Force (LMTF).

The draft report of research findings and recommendations was submitted for review to a panel of seasoned and knowledgeable industry experts, including: Bob Cummings (NERC), Dimitry Kosterev (BPA), Irina Green (CA ISO), Anatoliy Meklin (PG\&E), Gary Chinn (SCE), Abraham Ellis (PNM), Henry Huang (PNNL), Donald Davies (WECC), David Hawkins (CA ISO); and Baj Agrawal (APS). In addition, presentations were made to the WECC LMTF, CA ISO, PIER and TRP.

A final report, Improving Dynamic Load and Generator Response Performance Tools, that responds to these reviewers was prepared and submitted to TRP/PIER/CIEE. (Appendix G. Scoping Study Report on Improving Load and Generator Response Models.) 


\subsection{Task Outcomes}

The literature reviews and interviews with staff in the West, documented the need for improved load generator governor modeling. CERTS paid special attention to WECC modeling activities, including the work of both the Model Validation Working Group and the Load Modeling Task Force. As a result, the project team learned that WECC research had neared completion of a project to improve models for generator governor controls, but that more work is required in the area of dynamic load modeling. Accordingly, the project team focused our efforts on RD\&D needs to improve dynamic load models.

The project team developed 16 recommendations to improve dynamic load models. See Table 1. The recommendations are grouped into three general areas (Policy, Load Modeling, Measurement and Validation, etc.), by level of effort, including time required, the need for/role of PIER support, and our assessment of the overall significance of each recommendation with respect to the others.

As part of the review process for the scoping study, the project team vetted its recommendations with the aforementioned WECC Working Group and Task Force. They were well-received. While the scoping study was in its final stages of development, the WECC Load Modeling Task Force sponsored a research project to implement the highest priority recommendations from the scoping study. The study is being cost-shared by industry participants and PIER. 
Table 1: Summary of Research Recommendations

\begin{tabular}{|c|c|c|c|c|}
\hline Recommendation & $\begin{array}{l}\text { Level of } \\
\text { Effort }\end{array}$ & $\begin{array}{l}\text { Time } \\
\text { Required }\end{array}$ & $\begin{array}{l}\text { PIER } \\
\text { Support }\end{array}$ & $\begin{array}{l}\text { Significan- } \\
\text { ce }\end{array}$ \\
\hline \multicolumn{5}{|l|}{ Load Model Development and Policies } \\
\hline Develop seasonal models. & Low & 1-year & Low & Moderate \\
\hline Validate with state estimator models. & Low & 1-year & Low & Moderate \\
\hline Review reliability criteria. & Low & Multi-year & Low & High \\
\hline \multicolumn{5}{|l|}{ Load Modeling } \\
\hline $\begin{array}{l}\text { Study motor mechanical load characteristics } \\
\text { and impact. }\end{array}$ & Moderate & 1-year & Moderate & High \\
\hline $\begin{array}{l}\text { Study impact of single-phase and three-phase } \\
\text { motors. }\end{array}$ & Moderate & 1-year & High & High \\
\hline $\begin{array}{l}\text { Model motor load shedding and Low-voltage } \\
\text { conditions. }\end{array}$ & Moderate & 1-year & Moderate & Low \\
\hline Improve Low-voltage protection. & Low & 1-year & Low & High \\
\hline \multicolumn{5}{|l|}{ Measurement and Validation } \\
\hline$\$ 10 \mathrm{~K}$ load monitor & Low & 1-year & Low & High \\
\hline $\begin{array}{l}\text { Scoping study: research needs for automatic } \\
\text { validation and dynamic state estimation. }\end{array}$ & Moderate & 1-year & Moderate & Moderate \\
\hline \multicolumn{5}{|l|}{ Load Monitoring } \\
\hline $\begin{array}{l}\text { Estimate load composition from } \\
\text { measurements. }\end{array}$ & High & Multi-year & High & High \\
\hline $\begin{array}{l}\text { Characterize model uncertainties using } \\
\text { measurements. }\end{array}$ & Moderate & Multi-year & High & High \\
\hline $\begin{array}{l}\text { Use harmonic information in measurements to } \\
\text { enhance load composition estimates. }\end{array}$ & Moderate & Multi-year & High & unknown \\
\hline \multicolumn{5}{|l|}{ Measurement-Only (Black Box) Models } \\
\hline FolLow research activities in this area. & Low & Multi-year & Low & Low \\
\hline \multicolumn{5}{|l|}{ Uncertainty Analysis } \\
\hline $\begin{array}{l}\text { Develop methods to assess the impact of load } \\
\text { model uncertainties on system studies. }\end{array}$ & High & Multi-year & High & High \\
\hline \multicolumn{5}{|l|}{ Generator Governor Models } \\
\hline $\begin{array}{l}\text { Support WECC activities to implement best } \\
\text { model and maintain data for generator } \\
\text { characteristics. }\end{array}$ & Low & Multi-year & Low & High \\
\hline $\begin{array}{l}\text { Develop tools to monitor individual generator } \\
\text { frequency response. }\end{array}$ & High & Multi-year & High & Moderate \\
\hline
\end{tabular}




\subsection{Conclusions and Recommendations}

\subsubsection{Conclusions}

CERTS corroborated industry sentiment that dynamic load models are the least accurate of all of the components in current dynamic system models. CERTS also develop support for the contention that air conditioner models, as a prominent class of dynamic load models, represent the most important area for improvements.

Alarmingly, evidence suggests that generator response to disturbances has been steadily decreasing. Studies conducted using traditional dynamic models do not reproduce these findings. A recent WECC project, however, has already produced an improved model that better represents actual generator governor behavior. Hence, additional, fundamental research is not required, as these efforts are already well-underway. Nevertheless, maintenance of the databases of generator characteristics remains important. In the future, there may be a need for tools to monitor generator frequency response, which is not a modeling issue, per se.

\subsubsection{Recommendations}

As noted in Table 1, above, the project team makes 16 recommendations to improve dynamic load models (Appendix G. pages 16-41). The recommendations are by general area (Policy, Load Modeling, Measurement and Validation, etc.), level of effort, time required, need for PIER support, and significance. CERTS recommend pursuit of research on these topics in a follow-on project.

CERTS also strongly recommend that this program of research be conducted in close collaboration with and, ideally, with support from the WECC Load Modeling Task Force. WECC already has a standing committee structure whose sole purpose is to review these models and seek to improve them as needed. Working directly with WECC is essential for ensuring that the results of this research can be implemented readily.

Note: based on the results of this task, a formal load modeling research program has been approved by the PIER TRP under Contract \#500-02-004: MR-049, WECC Load Modeling Research Project.

\subsubsection{Benefits to California}

California will benefit from improved dynamic models in several ways. First, improved models and a better understanding of the likely impacts of remaining uncertainties in these models will increase the reliability of grid operations by allowing operators to more accurately study system voltage problems and the dynamic stability response of the system to disturbances.

Second, improved models will increase operator confidence in operational limits and operator controls. Secure operation of the grid is maintained through planning for credible contingencies, including the specification of path ratings and the deployment of remedial action schemes. Improved models may identify the need to curb optimistic ratings or may allow increases for overly conservative ratings. In either case, confidence in grid security will increase. Confidence 
for operator actions will also increase with the ability to accurately predict system responses to events and actions.

Third, in the longer-term, improved models will benefit the decision process for capital investments, which must account for how operational limits value the benefit of a proposed resource. For example, load modeling studies will lead to better informed investments in components for remedial action schemes. Similarly since the models are used to set operational path ratings, they will impact decisions for transmission and generation investment. 


\subsection{References}

Electric Power Research Institute and PNM. 2002. Advanced Load Modeling. TR-1007318. Palo Alto, CA: September.

LaCommare, K. H., and J. H. Eto. 2006. "Understanding the Cost of Power Interruptions to U.S. Electricity Customers." Energy: The International Journal, 31 (12), 1509-1519.

U.S.-Canada Power System Outage Task Force. 2004. Final Report on the August 14, 2003 Blackout in the United States and Canada: Causes and Recommendations. April. 238 pages. 


\subsection{Glossary}

\begin{tabular}{|l|l|}
\hline Acronym & Definition \\
\hline APS & Arizona Public Service \\
\hline BPA & Bonneville Power Administration \\
\hline CA ISO & California Independent System Operator \\
\hline CERTS & Consortium for Electric Reliability Technology Solutions \\
\hline CIEE & California Institute for Energy and Environment \\
\hline DOE & Department of Energy \\
\hline EIPP & Eastern Interconnection Phasor Project \\
\hline EMS & Energy Management System \\
\hline EPG & Electric Power Group \\
\hline EPRI & Electric Power Research Institute \\
\hline LBNL & Lawrence Berkeley National Laboratory \\
\hline LMP & Locational Marginal Pricing \\
\hline LMTF & Load Modeling Task Force \\
\hline MRTU & Market Redesign and Technology Update \\
\hline NERC & North American Electric Reliability Council \\
\hline PDC & Phasor Data Concentrator \\
\hline PG\&E & Pacific Gas and Electric \\
\hline PIER & Public Interest Energy Research \\
\hline PMUs & Phasor Measurement Units \\
\hline PNM & Public Service Company of New Mexico \\
\hline PNNL & Pacific Northwest National Laboratory \\
\hline PSE & Puget Sound Energy \\
\hline PSERC & Power Systems Engineering Research Center \\
\hline PSLF & Positive Sequence Load Flow \\
\hline RD\&D & Research Development \& Demonstration \\
\hline RTDMS & Real Time Dynamics Monitoring System \\
\hline RTSO & Real Time System Operations \\
\hline SCADA/EMS & Supervisory Control and Data Acquisition/Energy Management System \\
\hline SCE & Southern California Edison \\
\hline
\end{tabular}




\begin{tabular}{|l|l|}
\hline SDG\&E & San Diego Gas and Electric \\
\hline TAC & Technical Advisory Committee \\
\hline TRP & Transmission Research Program \\
\hline VAR & Volt-Ampere Reactive \\
\hline VSA & Voltage Security Assessment \\
\hline WAN & Wide Area Network \\
\hline WAPA & Western Area Power Administration \\
\hline WECC & Western Electricity Coordinating Council \\
\hline
\end{tabular}


Appendix A

Task 2.0 CA ISO Real Time Voltage Security Assessment (VSA) Project: Summary of Survey Results on Methodologies for use in Real-Time Voltage Security Assessment 

Consortium for
CA ISO
Electric
Real Time
Reliability
Voltage Security
Technology
Solutions Assessment (VSA) Project

\section{Summary of Survey Results On Methodologies for use in Real Time Voltage Security Assessment}

For:

Energy Commission and CA ISO

April 2005 


\section{Introduction}

The CA ISO Real Time Voltage Security Assessment (VSA) project is designed to research methodologies and analytic approaches for utilization in developing tools for use by dispatchers that provide real time assessment of voltage margins and contingency rankings. As the first step to achieve this objective, CERTS/EPG formulated a survey to reach out to experts in this field for comments, information, suggestions, and recommendations. The Survey can be found in Appendix A. Two projects were suggested to the reviewers. The first was a proposal to develop a Real-Time Voltage Security Margin Assessment tool with the ability to identify abnormal reductions of nodal voltages, weak elements and regions most affected by voltage problems. The other was to develop a Real-Time Angle Stability Margin Assessment tool with the ability to use Phasor Measurement Unit (PMU) data to calculate "distance to instability".

\section{Survey Overview}

The surveys were sent to fifty-one experts in universities and in the power industry. Sixteen reviewers (Appendix D) responded and their responses are listed in Appendix B \& C. Eight of these respondents are from the power industry and eight are from academia. Four proposals for commercial software were received from Bigwood Systems Inc (BSI), Vaiman Research (V\&R), New England Transmission System Services (NETSS) and Energy Concepts International (ECl).

A summary of research issues, comments and conclusions from responses are provided in Table 1 (below)

Table 1: Summary of Issues, Responses and Conclusions

\begin{tabular}{|c|c|c|}
\hline ISSUE & RESPONSES / COMMENTS & CONCLUSION \\
\hline $\begin{array}{l}\text { Voltage Security Assessment (VSA) } \\
\text { (Hyper planes for security regions) }\end{array}$ & $\begin{array}{l}\text { Online hyper plane possible } \\
\text { Not as unproven as interior point methods. } \\
\text { Ideally suited for phenomena that are } \\
\text { local. }\end{array}$ & Hyper planes well suited for VSA \\
\hline Methodology for computing hyper planes & $\begin{array}{l}\text { Loading \& Generation Direction needed. } \\
\text { Stress path until voltage collapses. } \\
\text { At collapse, determine local boundary. }\end{array}$ & Use left eigenvector approach \\
\hline Direct versus Time-domain methods & $\begin{array}{l}\text { Time domain iterative methods are proven } \\
\text { and robust, capable of handling } \\
\text { intermediate discrete actions/events. } \\
\text { Example: Generator limits being reached } \\
\text { Direct methods rely on simplistic models }\end{array}$ & $\begin{array}{l}\text { Direct Method could be used for fine- } \\
\text { tuning the security boundaries after an } \\
\text { iterative set of continuation power flows }\end{array}$ \\
\hline Weak elements identification & $\begin{array}{l}\text { Voltage collapses are concentrated in } \\
\text { certain regions in the sense that the } \\
\text { voltage falls more in those regions. There } \\
\text { is no single element that collapses. That } \\
\text { is, voltage collapse occurs system wide } \\
\text { with varying participation from all the } \\
\text { system buses. }\end{array}$ & $\begin{array}{l}\text { The participation is computed from the } \\
\text { right eigenvector of the Jacobian evaluated } \\
\text { at voltage collapse corresponding to the } \\
\text { zero eigenvalue. }\end{array}$ \\
\hline $\begin{array}{l}\text { Transient Security Assessment (TSA) } \\
\text { (Hyper planes for security regions) }\end{array}$ & $\begin{array}{l}\text { Need detailed dynamical model. } \\
\text { Controlling UEP is hard to calculate. } \\
\text { Limited observability with PMUs. }\end{array}$ & $\begin{array}{l}\text { Hyper planes not yet validated for } \\
\text { application in TSA }\end{array}$ \\
\hline $\begin{array}{l}\text { Use of phasor measurements for security } \\
\text { assessment }\end{array}$ & Limited observability & Start with small signal stability assessment \\
\hline
\end{tabular}




\section{Summary of Responses for Real Time Voltage Security Assessment}

The responses led to the conclusion that the hyper plane approach to defining security regions is ideally suited for voltage instability assessment. Voltage instability is more of a local area/region phenomenon. Several participants in the survey felt that the algorithms that utilize Direct Methods should be augmented with time domain algorithms to capture system topology change. It was suggested that without time domain simulations, it is not clear how switching conditions could be revealed. The computational methods to be used in VSA could be grouped into 2 broad classes - the Iterative Approach using Continuation Power Flows and the Direct Method. The Direct Method does not provide information on any discontinuous events when the stress parameter is increased. These discontinuous events occur when thermal, voltage or reactive limits are reached.

\section{Summary of Responses for Real-Time Angle Stability Assessment}

The majority of respondents felt that transient stability assessment requires very detailed dynamical modeling to be a successful tool in wide area security assessment. A representative from a utility in the East listed concerns about the practical implementation of wide area monitoring such as the validity of the system model to capture phenomena of interest and also the accuracy of angle difference from PMUs of different vendors. Several respondents flagged the issue of un-observability in PMU based measurements and the subsequent problem of determining optimal PMU placement locations. A proposal addressing this item was received from NETSS that focuses on the economic assessment of voltage support measurements known as pilot points.

\section{Conclusions}

The majority of responses favor the use of the hyper plane approach in determining Voltage Security Assessment. Also, the majority of responses do not see hyper planes suitable for determining Dynamic Voltage Assessment at this time. Small Signal Stability Analysis is considered to be a good first step for Wide Area Stability Monitoring and assessment using phasor measurements.

\section{Recommendations}

1. The primary recommendation is to use the hyper plane approach in computing security regions for Voltage Security Assessment.

- The Continuation Power Flow has been tested by several researchers in commercial and noncommercial software and is recommended as an initial step in the CA ISO VSA computational engine.

- An alternate recommendation is a hybrid approach, where a Direct Method could be used for finetuning the security boundaries after an iterative set of continuation power flows.

2. Continue research in the following areas:

(a) Existing approaches that can be applied for modal and coherency analysis (Small Signal Analysis)

(b) Methods to develop practical applications for Dynamic Security Assessment (DSA). 


\section{APPENDIX A - CA ISO RTWSA Survey}

\section{REAL TIME WDE-AREA SECURTY ASSESSMENT FOR CA ISO SYSTEM REQUEST FOR COMMENTS ANDFEEDBACK}

REQUEST OBJECTIVE The California Independent System Operator (CA ISO) and other Grid and Transmission System Operators in California are exploring avenues to better optimize utilization of the existing transmission system via development of state-of-the-art real time wide-area security assessment applications using both traditional SCADA data and new phasor metering technologies providing sub-second measurements of relative phase angles, voltage magnitudes and current line flows. As the first step to achieve this objective, we are reaching out to you as experts in this field for comments, information, suggestions, and recommendations on the two applications and corresponding algorithms and methodologies described in the following sections. Additionally, alternate new, proven, methodologies to develop a state-of-the-art application to optimize the operating limits of the CA ISO system while maintaining adequate wide-area security and reliability are also welcome.

REAL TIME APPUCATIONS BENG CONSIDERED: The CA ISO is interested in real time system security via two projects. Both projects will be based on similar advanced methods for calculating angle, voltage and transient stability limits. In order to obtain fast security margin evaluation in real-time, the security regions will be calculated offline as a set of periodically updated (interconnection wide) constraints. The CA ISO proposal is aimed at developing a real-time dispatcher's security system display that will include the results of the two applications described below:

PROJECT 1: Develop a Real-Time Angle Stability Margin Assessment tool with the following characteristics:

$>$ Real-time wide area monitoring using Phasor Measurement Unit (PMU) data to calculate "distance to instability".

$>\quad$ The security margin for the current moment and system configuration is estimated.

$>$ The approximated stability boundaries are determined in coordinates of relative phase angles.

$>$ The system is unobservable because the interconnection state cannot be determined based on PMU data.

$>$ The analysis is conducted for the entire interconnection based on available geographically dispersed measurements.

PROJECT 2: Develop a Real-Time Voltage Security Margin Assessment tool with the following characteristics:

$>$ Real-time voltage security margin determination by (offline) operating constraints calculation.

$>$ Identification of abnormal reductions of nodal voltages, weak elements and regions most affected by voltage problems.

$>$ The analysis is conducted for local control areas, e.g. California ISO area.

$>\quad$ The boundaries are in coordinates of nodal power injections and power flows in selected lines such as cut-sets \& paths.

$>$ The project deals with the observable part of the system, such as the control area and potentially its closest vicinity.

METHODOLOGIES AND TECHNOLOGES BEING CONSIDERED: The focus would be on calculating global "metrics" for the stability margin instead of "bus oriented" indices. It is hoped that Direct Methods for calculating security regions offline will be used to obtain fast stability margin evaluation in real-time. Some publications on Direct Methods \& Phasors have been listed as a sample of the ideas being investigated by CA ISO.

a A. G. Phadke, J. S. Thorp, K. J. Karimi: "State Estimation with Phasor Measurements", IEEE Transactions on Power Systems, Vol. PWRS-1, No. 1, February 1986.

- Y. V. Makarov, D. J. Hill, and Z. Y. Dong, "Computation of bifurcation boundaries for power systems: a new Deltaplane method" IEEE Trans. on Circuits and Systems - I: Fundamental Theory and Applications, Vol. 47, No. 4, April 2000, pp. 536-544.

- Hsiao-Dong Chiang, Chia-Chi Chu, Gerry Cauley, "Direct stability analysis of electric power systems using energy functions: Theory, applications, and perspective", Proceedings of the IEEE, 13, pp. 1497-1529, 1995.

- F. Alvarado, I. Dobson \& Y. Hu, "Computation of Closest Bifurcations in Power Systems", IEEE Transactions on Power Systems, Vol 9, No 2, May 1994, pp. 918-22. 


\section{APPENDIX B - Survey Responses Specific to VSA \& TSA}

\begin{tabular}{|c|c|c|c|c|}
\hline Reviewer & \multicolumn{2}{|c|}{ VSA using Hyperplane Methods } & \multicolumn{2}{|c|}{ TSA using Hyperplane Methods } \\
\hline [1] & 口 & $\begin{array}{l}\text { Recommends hyperplane approach for VSA. } \\
\text { Furthermore suggests online hyperplane } \\
\text { computation if loading directions and } \\
\text { generating unit dispatch vectors are known a } \\
\text { priori. Needs only up to } 10 \text { load flow runs with } \\
\text { to compute "weak" elements. }\end{array}$ & $\begin{array}{l}\square \\
\square\end{array}$ & $\begin{array}{l}\text { Data are more demanding than VSA } \\
\text { Recommends using PMU measurements for modal } \\
\text { estimation (model independent technique) to } \\
\text { assess small signal stability }\end{array}$ \\
\hline [2] & $\square$ & $\begin{array}{l}\text { Adopted similar direct methods for } \\
\text { contingency ranking and also in hybrid system } \\
\text { aimed to give a measure of angle as well as } \\
\text { voltage stability. These experiences } \\
\text { demonstrate the applicability of the proposed } \\
\text { methodologies. } \\
\text { Practical security boundary must account for } \\
\text { grid topology changes (implying online } \\
\text { security assessment). }\end{array}$ & $\square$ & $\begin{array}{l}\text { Methods like the one proposed in this project have } \\
\text { not been implemented by any grid operator, even if } \\
\text { monitoring of system variables for angle and } \\
\text { voltage stability assessment has been a concern in } \\
\text { several research activities. } \\
\text { Optimal number \& placement of PMUs is required } \\
\text { to correctly evaluate system stability (complete } \\
\text { observability). }\end{array}$ \\
\hline [3] & ם & $\begin{array}{l}\text { More information needed about the } \\
\text { hyperplane approach to VSA. }\end{array}$ & 口 & $\begin{array}{l}\text { Computational complexity in computing controlling } \\
\text { unstable equilibrium point is a big drawback }\end{array}$ \\
\hline [4] & ם & $\begin{array}{l}\text { Has real potential - and it is not as unproven } \\
\text { as some other concepts like interior point } \\
\text { optimization. }\end{array}$ & $\begin{array}{l}\square \\
\square\end{array}$ & $\begin{array}{l}\text { Problem is limited observability } \\
\text { Requires time domain verification }\end{array}$ \\
\hline [5] & ם & $\begin{array}{l}\text { Seems to be ideally suited for voltage } \\
\text { instability where the phenomena is more } \\
\text { localized and ideally suited for decision based } \\
\text { on measurements } \\
\text { Not convinced that Practical Dynamic Security } \\
\text { Region direct method has any particular } \\
\text { computational advantage over other methods }\end{array}$ & $\begin{array}{l} \\
\square \\
\square \\
\square\end{array}$ & $\begin{array}{l}\text { Recommends EPRI-Siemens Approach } \\
\text { Direct method used for ranking contingencies } \\
\text { Full blown time domain program with energy } \\
\text { indicators is used determine stability }\end{array}$ \\
\hline [6] & a & $\begin{array}{l}\text { Experience has shown that secure operating } \\
\text { space calculations done off line rarely match } \\
\text { exact real time conditions, which may well be } \\
\text { away from design conditions, implying online } \\
\text { security assessment or adequate safety } \\
\text { margins. }\end{array}$ & $\square$ & $\begin{array}{l}\text { Investigated various grid margin indices with the } \\
\text { EPRI-Siemens product but were unable to develop } \\
\text { a suitable production metric. Integration of both } \\
\text { continuous (load growth) and non-continuous } \\
\text { (contingency) factors into a single metric was a } \\
\text { major challenge. }\end{array}$ \\
\hline [7] & ם & $\begin{array}{l}\text { Least squares approximation of hyper planes } \\
\text { with load flow simulations is prone to error } \\
\text { enhancement for bad state estimator } \\
\text { measurements. }\end{array}$ & $\square$ & $\begin{array}{l}\text { It is not clear how switching conditions can be } \\
\text { revealed without "time-domain" simulations. }\end{array}$ \\
\hline [8] & $\square$ & $\begin{array}{l}\text { Proposes New Electricity Transmission } \\
\text { Software Solutions (NETSS) for voltage } \\
\text { optimization, and the economic assessment of } \\
\text { voltage support measurements (known as pilot } \\
\text { points). } \\
\text { It is important to determine the right locations } \\
\text { to measure. Results depend on voltage } \\
\text { dispatch strategies, loading conditions and } \\
\text { system-specific equipment status. }\end{array}$ & $\square$ & Pilot points will help in PMU placement. \\
\hline
\end{tabular}




\section{APPENDIX C - Survey Responses related to General Issues}

\begin{tabular}{|c|c|c|}
\hline Reviewer & & General comments on tools and methodologies that were discussed in the Survey \\
\hline [9] & 口 & $\begin{array}{l}\text { Advises to use equations like } J^{\prime}(X) F(X)=0 \text { to search for the closest points of the steady-state stability boundary. } \\
\text { He also warns that the thermal constraints are often more limiting than stability constraints. }\end{array}$ \\
\hline [10] & & $\begin{array}{l}\text { Methods not yet been utilized by grid operators. } \\
\text { Emphasizes mode meter and system stiffness. } \\
\text { Refers to WACS paper by Carson Taylor. }\end{array}$ \\
\hline$[11]$ & $\begin{array}{l}\square \\
\square\end{array}$ & $\begin{array}{l}\text { Suggested the advantages of the following V\&R products: } \\
\text { For off-line computations the exact boundary of dynamic security region (security nomogram) is automatically } \\
\text { constructed using V\&R's Boundary of Operating Region (BOR) software. } \\
\text { For on-line computations, sensitivity-based } n \text {-dimensional boundary of operating region can be computed using } \\
\text { BOR. The approximated boundary may be computed using Direct methods. }\end{array}$ \\
\hline [12] & a & $\begin{array}{l}\text { Visualization of voltage stability region in cut-set space has been implemented and a visualization system of } \\
\text { dynamic security region in injection space to guarantee transient stability is in development for Henan Power } \\
\text { System of CHINA } \\
\text { It might be used in monitoring, assessment and optimization of security. "Up to now almost all research results of } \\
\text { mine are about the dynamic security region in power injection space and the voltage stability region in cut-set } \\
\text { power space. I think it might be used not only in security monitoring and control, but also in probabilistic security } \\
\text { assessment." }\end{array}$ \\
\hline [13] & $\square$ & $\begin{array}{l}\text { Submitted a Proceeding of IEEE paper on WACS accepted for publication in May 2005. This paper co-authored } \\
\text { by Taylor describes an online demonstration of a new response based wide area control system with } \\
\text { discontinuous actions for power system stabilization. }\end{array}$ \\
\hline [14] & $\square$ & Submitted a product overview of Energy Concepts International software "QuickStab". \\
\hline [15] & $\begin{array}{l}\square \\
\square\end{array}$ & $\begin{array}{l}\text { Submitted a company overview and product list of Bigwood systems. } \\
\text { This included information that showed partnerships with ABB to install BCU-DSA at the EMS of three power } \\
\text { companies. BCU method is the only method used in EPRI Direct } 4.0 \text { and BCU method has been implemented by } \\
\text { Siemens, at the Northern Power Company. }\end{array}$ \\
\hline [16] & $\begin{array}{l}\square \\
\square \\
\square \\
\square \\
\square\end{array}$ & $\begin{array}{l}\text { Provided areas of concern in the implementation of wide area monitoring such as: } \\
\text { Validity of the system model to capture the phenomena of interest. } \\
\text { Accuracy of angle measurements by PMUs. } \\
\text { Accuracy of angle differences from PMUs of different vendors. } \\
\text { Determining acceptable vs. unacceptable levels of angular separations among various pairs of PMU }\end{array}$ \\
\hline
\end{tabular}




\begin{tabular}{|c|}
\hline APPENDIXD \\
UST OF SURVEY RESPONDENTS \\
\hline Ian Dobson \\
(Wisconsin-Madison \\
\hline Enrico De Tuglie \\
(Politechnico di Bari) \\
\hline M. Anantha Pai \\
(University of llinois) \\
\hline Gerald T. Heydt \\
(Arizona State) \\
\hline Vijay Vittal \\
(Arizona State) \\
Raymond Vice \\
(Southern Co) \\
\hline Anatoliy Meklin \\
(PG\&E) \\
\hline Marija D. llic \\
(Carnegie Mellon) \\
\hline Alexander Kontorovich \\
(Israel) \\
\hline Bill Mittelstadt \\
(BPA) \\
\hline Marianna Vaiman \\
(V\&R Company) \\
\hline Yixin Yu \\
(Tianjin University) \\
\hline Carson Taylor \\
(BPA) \\
\hline Savu Savulesco \\
(ECI) \\
\hline H-D Chiang \\
(Cornell) \\
\hline Navin Bhatt \\
(AEP) \\
\hline
\end{tabular}




\section{APPENDIX E.1 - Literature Survey on Stability}

[1] C Taylor, "CIGRE Task Force 38.02.17 Advanced Angle Stability Controls"Dec 99.

[2] Y. Zeng, J. C. Fan, and Y. X. Yu, "Practical Dynamic Security Regions of Bulk Power Systems," Automation of Electric Power System, vol.25, No.16, pp.6-10, 2001.

[3] Y. X. Yu and W. P. Luan, "Practical Dynamic Security Regions of Power Systems," Proceedings of the CSEE, vol.10, Electrical Mathematics Supplement, pp.22-28, 1990.

[4] F. Feng and Y. X. Yu, "Dynamic Security Regions of Power Systems in Injection Spaces," Proceedings of the CSEE, vol.13, No.3, pp.14-22, 1993.

[5] Chadalavada, V., V. Vittal, et al., "An On-Line Contingency Filtering Scheme for Dynamic Security Assessment," IEEE Transactions on Power Systems, Vol. 12, No. 1, pp. 153-161, February 1997.

[6] Ilic-Spong, M., J. Christensen, and K.L. Eichorn, "Secondary Voltage Control Using Pilot Point Information," IEEE Transactions on Power Systems 3, 660-668, May 1988

[7] Ilic, M.D., S.X. Liu, G. Leung, C. Vialas and M. Athans, "Improved Secondary and Tertiary Voltage Control," IEEE Transactions on Power Systems, 10, November 1995, pp. 1851-1862.

[8] V. A. Mateev, "A Method of Numerical Solutions of Sets of Nonlinear Equations", Zhurnal Vychislitelnoi Matematiki Matematicheskoi Fiziki, vol.4, \# 6, pp983-994,1964.

[9] Yixin Yu, Yuan Zeng, Chunhua Huang, Stephen T. Lee and Pei Zhang. "A Practical Direct Method for Determining Dynamic Security Regions of Electrical Power Systems by Power Perturbation Analysis" International Conference on Electrical Engineering 2004 (ICEE2004), July 4-8, 2004, Sapporo, Japan.

[10] Hsiao-Dong Chiang, Chia-Chi Chu, Gerry Cauley, "Direct stability analysis of electric power systems using energy functions: Theory, applications, and perspective", Proceedings of the IEEE, 13, pp. 1497-1529, 1995.

[11] Hsiao-Dong Chiang, Cheng-Shan Wang and Hua Li, "Development of BCU classifiers for on-line dynamic contingency screening of electric power systems", IEEE Transactions on Power Systems, 14, pp. 660-666, 1999.

[12] James Momoh, Yuri V Makarov, William Mittelstadt, "A Framework of Voltage Stability Assessment in Power System Reliability Analysis", IEEE Transactions on Power Systems, Vol. 14, No.2, May 1999.

[13] Ilic, M.D. and J. Zaborszky, Dynamics and Control of Large Electric Power Systems, Wiley Interscience, May 2000

[14] F. Alvarado, I. Dobson \& Y. Hu, "Computation of Closest Bifurcations in Power Systems", IEEE Transactions on Power Systems, Vol 9, No 2, May 1994, pp. 918-22.

[15] Voltage Stability Analysis Program (VSTAB), Version 2.1, User's Manual, EPRI Research ProjectRP30400-01, August 1992.

[16] Chih-Wen Liu Thorp, J.S "A novel method to compute the closest unstable equilibrium point for transient stability region estimate in power systems" IEEE Trans. on Circuits and Systems - I: Fundamental Theory and Applications, Volume: 44, \# 7, July 1997 pp. 630-635

[17] A. M. Kontorovich and A. V. Krukov, "Definition of Power Flow Limit Conditions by the Permanent Loading Method", Proceedings of the Leningrad Polytechnic Institute, vol. 380, 1981, pp. 104-108.

[18]Y. V. Makarov, A. M. Kontorovich, D.J.Hill and I.A.Hiskens, "Solution Characteristics of Quadratic Power Flow Problems", Proceedings of the $12^{\text {th }}$ Power Systems Computation Conference, Vol. 1, Dresden, Germany, August 1996, pp. 460-467.

[19] ] P.W.Sauer and B.C.Lesieutre, "Power System Load Modeling", J.H.Chow, P.V.Kokotovic and R.J.Thomas, (edit) Systems and Control Theory for Power Systems, Springer-Verlag 1995, pp. 283-313.

[20] Y. V. Makarov, D. J. Hill, and Z. Y. Dong ,"Computation of bifurcation boundaries for power systems: a new Delta-plane method" IEEE Trans. on Circuits and Systems - I: Fundamental Theory and Applications, Vol. 47, No. 4, April 2000, pp. 536-544. 


\section{APPENDIX E.2 - Literature Survey on Phasors in SPS}

[1] Niusha Rostamkolai, Ph.D. dissertation, Virginia Tech 1986, Adaptive Optimal Control of AC/DC Systems.

[2] N. Rostamkolai, A.G. Phadke, J.S. Thorp, W.F. Long, Measurement based optimal control of high voltage AC/DC systems, IEEE Trans. on Power Systems, Vol. 3 No. 3, August 1988, pp 1139-1145.

[3] Edgardo Manansala, Ph.D. dissertation, Virginia Tech August 1989, Adaptive power system control.

[4] Edgardo C. Manansala, A.G. Phadke, An optimal centralized controller with nonlinear voltage control, Electric Machines and Power Systems, 19, 1991, pp 139-156.

[5] L. Mili, T. Baldwin, A.G. Phadke, Phasor measurements for voltage and transient stability monitoring and control, Workshop on Application of advanced mathematics to Power Systems, San Francisco, Sept. 4-6, 1991.

[6] A.F. Snyder, N. Hadjsaid, D. Georges, L. Mili, A.G. Phadke, O. Faucon, S. Vitet, Inter-area oscillation damping with power system stabilizers and synchronized phasor measurements, PowerCon 1998, China.

[7] M.A. Smith, Improved Dynamic Stability Using FACTS devices with phasor measurement feedback, MS Thesis, VTech, 1994.

[Extracted from Marek Zima, Special Protection Schemes in Electric Power Systems Literature survey ETH, 2002.]

The best trend, which has appeared in the last decade, is Synchronized Phasor Measurement Technology. The main idea is to measure the voltage and current phasors in the same time at the selected locations in the network, transmit them into a central point, where they can be compared, evaluated and further processed. The devices performing the measurements are called PMU (Phasor Measurement Unit). PMU is basically a conventional RTU (Remote Telemetry Unit) equipped with the receiver of GPS signal synchronizing the measurements and tagging the time stamp to them. PMU is also capable of pre-processing of data (Fourier transformation etc.). The PMU technology was originally developed in eighties by Thorp, Phadke and others at Cornell, Virginia Polytechnic Institute and American Electric Power. Since the quantities measured by PMUs are voltage and current phasors, the linear relation between them holds when modelling the branches in the network (i.e. $\pi$ - equivalent of line and transformer). This feature/property permits linear State Estimation process, thus avoiding repetitive manipulations with large matrices in iterative procedure as it is in the traditional case. This significantly reduces the computational time and errors level. This approach has probably been derived first time in [Phadke, 1986] where the authors have formulated the linear State Estimation equations and applied them on 118 buses IEEE test system. The authors assume that all substations are equipped with PMUs measuring all voltages and some selected currents. The high cost of both PMUs themselves (although nowadays this is not as critical issue as it used to be, the price of PMU is approximately 3500 USD) and the communication links to all substations force to keep the number of installed PMUs to a minimum.

[Phadke 86] A. G. Phadke, J. S. Thorp, K. J. Karimi: "State Estimation with Phasor Measurements", IEEE Transactions on Power Systems, Vol. PWRS-1, No. 1, February 1986 


\section{APPENDIX E.3 - Definition of Instability Phenomena}

- Frequency Instability - is inability of a power system to maintain steady frequency within the operating limits.

- Voltage Instability - is the inability of a power system to maintain steady acceptable voltages at all buses in the system under normal operating conditions and after being subjected to a disturbance. A system enters a state of voltage instability when a disturbance, increases in load demand, or change in system conditions causes a progressive and uncontrollable drop in voltage. A system is voltage unstable if, for at least one bus in the system, the bus voltage magnitude decreases as the reactive power injection in the same bus is increased.

- Transient Angular Instability (also called Generator's Out-of-step) - is the inability of the power system to maintain synchronism when subjected to a severe transient disturbance. The resulting system response involves large excursions of generator angles and is influenced by the nonlinear power-angle relationship.

- Local mode of Small-signal Angular Instability (also mentioned as Generator's Swinging or Power Oscillations) - is the inability of the power system to maintain synchronism under small disturbances. Such disturbances occur continually on the system because of small variations in loads and generation. The disturbances are considered sufficiently small for linearization of system equations to be permissible for purposes of analysis. Local modes or machine-system modes are associated with the swinging of units at a generating station with respect to the rest of the power system. The term local is used because the oscillations are localized at one station or small part of the power system.

[Extracted from Marek Zima, Instability Phenomena in Electric Power Systems Literature survey ETH, 2002]

An idea of preventive analysis conducted in regular on-line cycles adopting N-1 rule and applying the results immediately after the detected contingency, is probably the only solution for the phenomena on very fast time scale. In the voltage instability case it means calculations of a minimal load shedding necessary to stabilize the power system subjected to any contingency from the selected range. Thus, an optimization problem can be formulated, where the function to be minimized is the amount of load shedding subject to the following constraints: solvability of static power flow equations (this essentially means, that minimal feasible solution can be found in the maximum loadability point), allowed voltage limits, angle stability inequality constraints and dynamic equality constraints . Continuation Power Flow (CPF) can overcome the numerical problems indicated above. In principle, it is slightly reformulated conventional power flow. The equations are augmented by the term quantifying the load increase and containing new variable - load parameter. A new equation is introduced, which basically forces a continuation parameter chosen in the predictor step to hold its value in the iterative correction process. This continuation parameter is optimally loading in the beginning of the PV -curve and when approaching to "nose", voltage. Various techniques have been developed for predictor step in order to speed up the computations and increase the accuracy. Very good explanatory example of the tangent method and the secant predictor (in fact linear approximation estimate) can be found in [Chiang, 1999]. CPFLOW has probably become the most widely accepted tool for the voltage instability assessment/evaluation and a huge number of papers have been written about it.

[Chiang 99] Hsiao-Dong Chiang, Cheng-Shan Wang and Hua Li, "Development of BCU classifiers for on-line dynamic contingency screening of electric power systems", IEEE Transactions on Power Systems, 14, pp. 660-666, 1999. 
Appendix B

Deliverable Task 2.0 CA ISO Real-Time Voltage Security Assessment Summary Report 
Consortium for

Electric

Reliability

Technology

Solutions
Real-Time

Voltage Security

Assessment

(RTVSA)

\section{CA I SO Real-Time Voltage Security Assessment Summary Report}

Funded by the

California Public I nterest Energy Research

Transmission Research Program

February 9, 2006 


\section{TABLE OF CONTENTS}

1 INTRODUCTION ……...............................................................

1.1 Description of the Voltage Stability Problem ............................................... 6

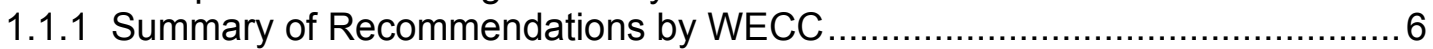

1.1.2 WECC voltage stability criteria................................................................. 9

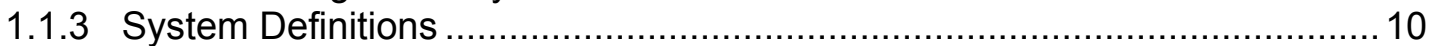

1.1.4 Representational Issues ........................................................... 10

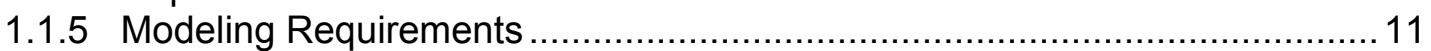

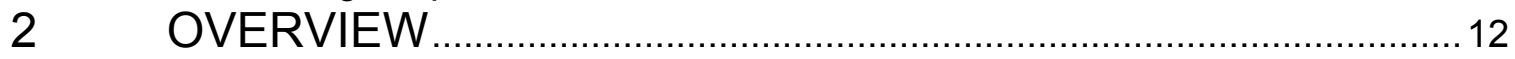

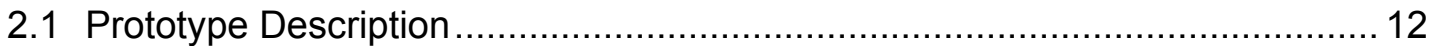

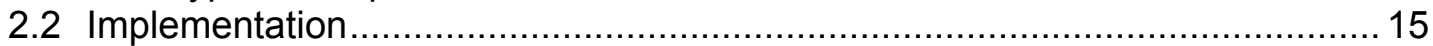

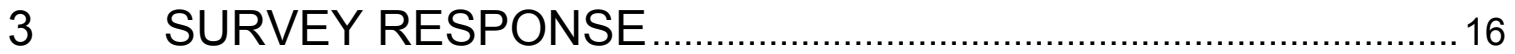

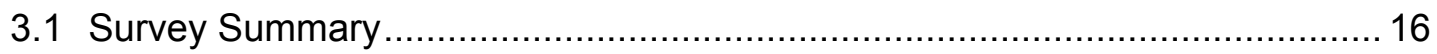

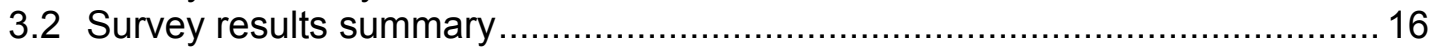

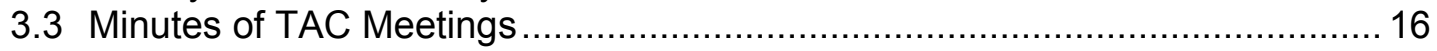

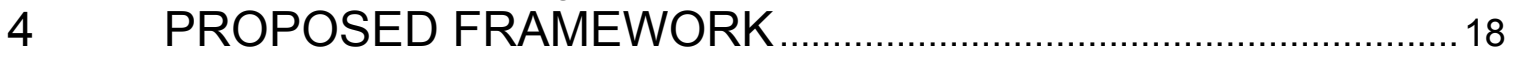

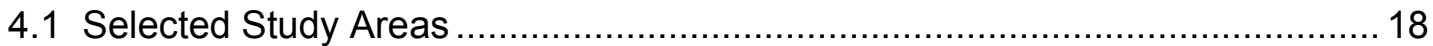

4.2 Description of Proposed Framework ...................................................... 18

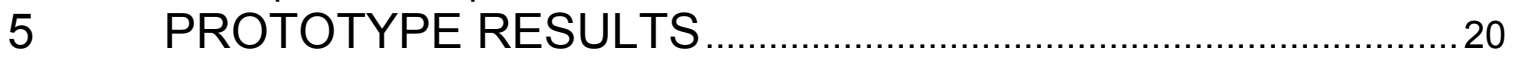

5.1 Description of the San Diego transmission system ..................................... 20

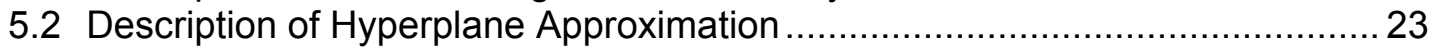

5.3 On the orientation of 2D hyperplane slices for CHILLS and MSSION ............ 24

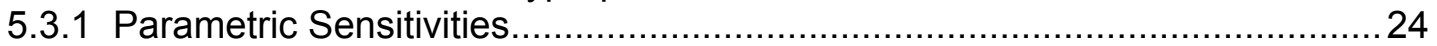

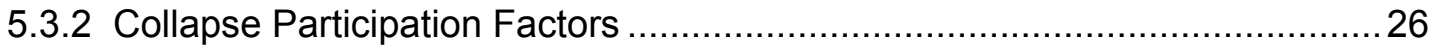

5.4 On the effects of adding a sink in a congested load pocket ........................... 28

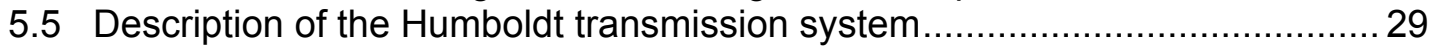

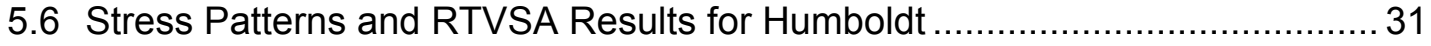

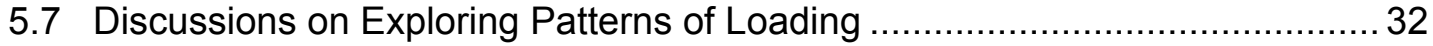

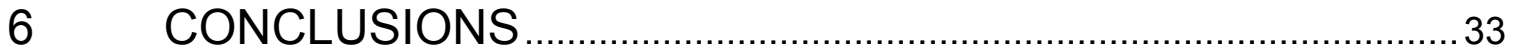

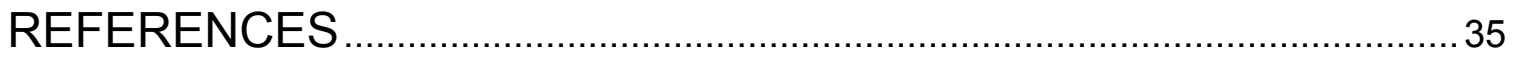




\section{TABLE OF FIGURES}

Figure 1 Multi-Year Development Roadmap for the CA ISO RTVSA ....................... 12

Figure 2 Block Diagram of RTVSA Prototype .................................................... 13

Figure 3 The proposed integration scheme for the VSA project ............................ 14

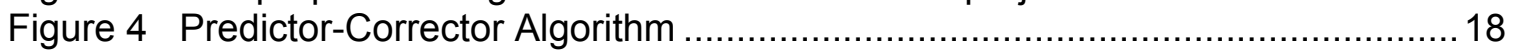

Figure 5 Major Import Paths in the SDGE System.......................................... 20

Figure 6 Map showing the two arms of the SWPL corridor in relation to SDGE .........21

Figure 7 Location of Songs and South Bay in the CA EHV Transmission Network ...... 21

Figure 8 One-Lines for the SOBAY-MIGUEL-SONGS area ................................. 22

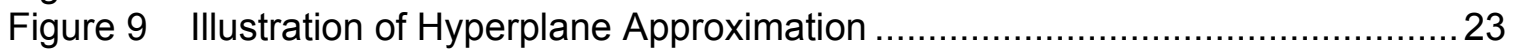

Figure 10 Lagrangian Multipliers for SDGE cases ........................................... 24

Figure 11 RTVSA Output: Hyperplane slices at Carlton Hills and Mission ...................25

Figure 12 RTVSA Output: PoC in MW for Carlton Hills and Mission .........................25

Figure 13 Top Eight Voltage Collapse Participation Factors for Stressing Pattern I ....26

Figure 14 Top Eight Angle Collapse Participation Factors for Stressing Pattern I.......26

Figure 15 PV curve for Carlton Hills with Stress Pattern III showing collapse ...............27

Figure 16 PV curve for Mission with Stress Pattern III showing collapse .....................27

Figure 17 P Angle curve for Mission with Stress Pattern III showing collapse ...............27

Figure 18 RTVSA Output: Hyperplane slices for Granite and Mission.......................28

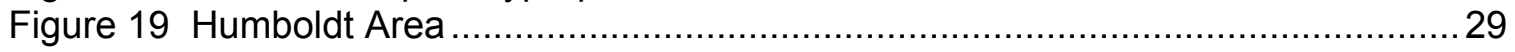

Figure 20 Location of Cottonwood in the CA EHV Transmission Network ....................29

Figure 21 One Lines for the HUMBOLDT CTTNWD SHASTA area ............................. 30

Figure 22 RTVSA Output: Hyperplane slices at Humboldt 1276 and Shasta 2590 .......32

Figure 23 RTVSA Output: Lagrangian Multipliers for Humboldt .................................. 32

\section{TABLE OF TABLES}

Table 1 WECC Voltage Stability Requirements ................................................. 10

Table 2 Bus Index and Color Code for the SOBAY-MIGUEL-SONGS area ..............22

Table 3 Patterns of SINK PF (Participation Factors) ........................................... 24

Table 4 Generator PF at the 3 Units of South Bay (SB) for all Vectors ......................24

Table 5 Patterns of SINK PF (Participation Factors) ......................................... 28

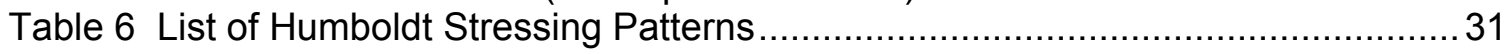

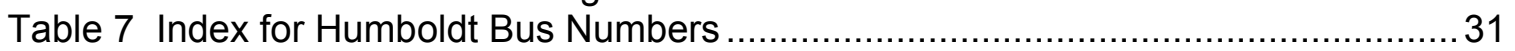




\section{ACKNOWLEDGEMENTS}

Special thanks to California ISO engineers Mr. Catalin Micsa and Mr. David Le for their consultations and help with selecting the study areas in California. The project team appreciate contributions from Mr. Sirajul Chowdhury, Mr. Eric Whitley, and Dr. Enamul Haq.

Mr. Dave Hawkins (California ISO) for his expertise, comprehensive support, and advices.

Dr. Yuri V. Makarov (PNNL), project consultant, for his role in suggesting the framework of the project, developing and selecting the methodology, participation in the brainstorm meetings, organizing face-to-face interviews and other contacts with the leading experts, advices, troubleshooting, literature review, and report writing.

Prof. Ian Dobson, (University of Wisconsin-Madison), project consultant, for his role in suggesting and developing the methodology, participation in the brainstorm meetings and interviews, expertise and essential advices.

Dr. S. L. Greene (Price Waterhouse Coopers) for his help with the PSERC software.

Mr. Jim Cole (California Institute for Energy Efficiency) for initiating and support of this project.

Mr. Joseph Eto (Lawrence Berkeley National Lab) for his support.

Participants of the EPG Methodology Survey for their expertise and advices:

Prof. M. Anantha Pai (University of California - Berkeley)

Mr. Raymond L. Vice (Southern Company Services, Inc.)

Dr. Savu Savulescu (Energy Concepts International Corp.)

Dr. Michael Y. Vaiman and Mrs. Marianna Vaiman (V\&R Energy Systems Research)

Dr. Alex M. Kontorovich (Israel)

Dr. Anatoliy Meklin (Pacific Gas and Electric)

Prof. Marija D. Ilic (Carnegie Mellon University)

Prof. Enrico De Tuglie (Politecnico di Bari, Italy)

Prof. Gerald T. Heydt (Arizona State University)

Mr. William Mittelstadt (Bonneville Power Administration)

Prof. Yixin Yu (Tianjin University, China)

Mr. Carson W. Taylor (Bonneville Power Administration)

Prof. H.-D. Chiang (Cornell University)

Dr. Navin Bhatt (American Electric Power)

Participants of the face-to-face interviews for their evaluation of the project and advices:

Prof. Ian Dobson (University of Wisconsin - Madison)

Prof. Vijay Vittal (Arizona State University)

Prof. Venkataramana Ajjarapu (lowa State University)

Dr. Zhao Yang Dong (University of Queensland, Australia)

Dr. Anatoliy Meklin (Pacific Gas and Electric)

Dr. Vitaliy Faybisovich (South California Edison)

Dr. Michael Vaiman and Dr. Marianna Vaiman (V\&R Energy Systems Research)

Participants of the TAC Meetings:

Dr. Jim Gronquist (Bonneville Power Administration)

Dr. Steve Lee (Electric Power Research Institute) 


\section{INTRODUCTION}

The Voltage Security Assessment (VSA) project is designed to be part of the suite of advanced computational tools for congestion management that is slated for practical applications in California in the next few years. Modern voltage assessment methods include the development of such advanced functions as identification of weak elements, automatic selection of remedial actions and automatic development of composite operating nomograms and security regions. Real-time production-grade VSA tools are becoming increasingly available nowadays. These tools are integrated with EMS/SCADA systems and use results from the state estimator.

Some advanced contemporary real-time applications already promote the idea of using the security regions determined in parameter space with the composite boundaries limited by stability, thermal, and voltage constraints. At the same time, the majority of the tools are still based on the static system power flow models and implement such traditional approaches as sink-source system stressing approach, $P-V$ and $V-Q$ analyses, V-Q sensitivity and modal analysis. Unfortunately, many of the most promising methods suggested in the literature have not been implemented yet in the industrial environment, including the state-of-the-art direct method to finding the exact Point of Collapse. Currently there exists no real-time monitoring tool for voltage security assessment. The problems of voltage security will be exacerbated by the effects of multitransfers through the network. These sets of simultaneous transfers are manifest because of the buying and selling of electric power across the boundaries of control areas. Moreover the point of production and the point of delivery may be in geographically distant locations.

An extensive analysis of existing VSA approaches was conducted. This included research by EPG, surveys from the leading experts' opinion worldwide, feedback from industrial advisors and brainstorm meetings with the projects' consultants. A state-of-the-art combination of approaches and computational engines was identified and selected for implementation in this project. The suggested approach is based on the following principles and algorithms:

- Use the concepts of local voltage problem areas and descriptive variables influencing the voltage stability problem in each area. Utilize information about the known voltage problem areas and develop formal screening procedures to periodically discover new potential problem areas and their description parameters.

- Calculate and approximate the voltage stability boundary off real time, and apply the approximated voltage security conditions in real time for a fast VSA. Use hyperplanes to approximate the voltage stability boundary.

- Apply the dual space concept while developing the sets of approximating hyperplanes. Use the descriptive variable space to determine the sequence of stress directions to approximate and visualize the boundary. Perform all the rest of the required computational work in the parameter space. A mapping between the dual spaces can be established based on predetermined generation dispatches and load scaling patterns and linearization.

- To calculate the approximating hyperplanes, apply a combination of the parameter continuation techniques and direct methods as suggested in this report. Introduce a sufficient additional security margin to account for inaccuracies of approximation and uncertainties of the power flow parameters.

- Produce a list of abnormal reductions in nodal voltages and highlight the elements and regions most affected by potential voltage problems. The list of most congested corridors in the system will be ranked by the worst-case contingencies leading to voltage collapse.

The initial framework of this project was originally formulated by California ISO. The key elements of the suggested approach which are the use of parameter continuation, direct methods and the hyperplane approximation of the voltage stability boundary were approved by a panel of leading experts in the area in the course of a survey conducted by EPG in 2005. These concepts were also verified in the course of face-to-face personal meetings with well-known university 
professors, industry experts, software developers and included email discussions and telephone exchanges. CERTS industrial advisors approved these developments during two TAC meetings conducted in 2005.

In 2005 , the project development team successfully implemented the parameter continuation predictor-corrector methods. Necessary improvements were identified and developed. The PSERC parameter continuation program and MATLAB programming language were used in the project. The techniques were tested for the California San Diego problem areas suggested for these purposes by California ISO. The approximated voltage stability boundary was compared to the results obtained from the GE PSLF program commonly used in the Western Interconnection.

Work in future years will include Direct Methods and the investigation of descriptive variables. This will further develop the concept of utilizing the dual space approach to choose axis sets that are needed for visualization purposes. Direct Method algorithms will be developed for finding the exact PoC (Point of Collapse). In addition, procedures will be implemented for selecting the minimum number of approximating hyperplanes. Other items in the scope of future work are techniques for analyzing margin sensitivities, coping with parameter uncertainties, and selecting remedial actions. The prototype tool will be tested for some additional problem areas in California, and then expanded to the entire CA ISO system.

\subsection{Description of the Voltage Stability Problem}

A system enters a state of voltage instability when a disturbance, increase in load demand, or change in system condition causes a progressive and uncontrollable decline in voltage. The main factor causing voltage instability is the inability of the power system to meet the demand for reactive power. Voltage stability is the ability of a power system to maintain acceptable voltages at all buses in the system under normal operating conditions and after being subjected to a disturbance. Voltage stability margin is the distance to instability determined for a selected loading or stress direction in parameter space. It is known that voltage magnitudes alone are poor indicators of voltage stability or security. Voltages can be near normal with generators, synchronous condensers, and SVCs near current limiting levels, thus resulting in a possible voltage collapse. Therefore, it is prudent to specify a MVAR margin or MW margin [20].

Venikov and co-authors in [41] first demonstrated that under certain model assumptions, such as constant voltages of the generator buses, matching load models and the same slack bus model, the static aperiodic stability limit or the saddle node bifurcation coincides with the power flow feasibility limit. This allows analyzing aperiodic stability limits using conventional power flow models under these modeling assumptions. Generally speaking, for the voltage stability problem, the assumptions do not hold, but nevertheless, the power flow model, and the power flow Jacobian matrix singularities are frequently used for an approximate description of the static aperiodic voltage stability problem.

\subsubsection{Summary of Recommendations by WECC}

The following is a list of blackouts and near blackouts related to voltage collapse. Many system blackouts and "near misses" were related to voltage instability and voltage collapse problems. See [1] to [19] for more details and among these, the following major events can be mentioned.

1. 1965: Eastern US and Canada

2. 1970: Japan and New York Power Pool

3. 1977: New York City and Jacksonville, Florida

4. 1978: France

5. 1982: Belgium and Florida

6. 1983: Sweden 
7. 1987: Tokyo, Japan, Western Tennessee, France

8. 1989, 1993-1994: Italy

9. 1996: US Western Interconnection (2 events)

10. 1997: The Netherlands

11. 1998: Atlanta, San Francisco Area, Upper Midwest U.S.

12. 1999: Northeast U.S. (2 events)

13. 2003: Croatia and Bosnia Herzegovina, Eastern U.S. and Canada, Denmark and Sweden, and Italy

14. 2004: Western Norway and Southern Greece

15. 2005: Moscow region, Russia

These also include the following blackouts or "near blackouts" related to voltage collapse:

1. Severe blackout in Eastern US (including NYC) and Canada on November 9, 1965 was initiated by disconnection of one of the five 230-kV lines from Beck power plant going north to Toronto. The blackout affected $\sim 30$ million people in Canada and USA, including New York City and lasted 13 hours. [2,3]

2. New York Power Pool disturbances of September 22, 1970. [1,18]

3. New York City blackout on July 13, 1977 was caused by a thunderstorm and heavy winds and rain. It affected 3 million people and lasted 22 hours. [2,3]

4. Jacksonville, Florida on September 22, 1977.

5. France December 19, $1978[1,19]$.

6. Northern Belgium, August 4, $1982[1,19]$.

7. Florida, September 9, November 26, December 28 and 30, 1982.[1,18]

8. Voltage collapse in Sweden on December 27, 1983, was a result of a breaker failure at a $400 \mathrm{kV}$ substation. The blackout affected 4.5 million people and lasted 5.5 hours. $[1,2,4]$

9. Tokyo, Japan, July 23, $1987[1,5]$

10. Western Tennessee, USA, August 22, 1987 [6]

11. France, January 12, $1987[1,19]$.

12. Italy, 1989.

13. Italy, 1993 and 1994.

14. US Western Interconnection Blackout, July 2, 1996, started with loss of two $345 \mathrm{kV}$ transmission lines Jim Bridger - Kinport and subsequent tripping of two Jim Bridger units in the southern Idaho-Montana region. This initiated a blackout in 14 western states, two Canadian provinces, and the northern portion of Baja California, Mexico. [7]

15. US Western Interconnection Blackout on August 10, 1996. This disturbance effectively began with the loss of the Keeler-Allston $500 \mathrm{kV}$ line in the Portland area. WECC system was separated into four islands, interrupting service to 7.5 million customers for periods ranging from several minutes to about nine hours. [8]

16. The Netherlands, 1997.

17. Atlanta, a near blackout situation with prolonged voltage reductions to 0.95 p.u., on June 2, 1998.

18. California ISO system disturbance (in San Francisco Area), December 8, 1998. The disturbance was caused by a PG\&E construction crew and by the Substation 
Operators failing to follow standard procedures at San Mateo substation. The blackout affected more than 456,000 customers and nearly one million people. [9]

19. Upper Midwest Blackout on June 25, 1998 load and affected 152,000 people in Minnesota, Montana, North Dakota, South Dakota, and Wisconsin in the United States; and Ontario, Manitoba, and Saskatchewan in Canada. Outages lasted up to 19 hours. The blackout was initiated by a lightning storm in Minnesota [3]

20. Northeast U.S. non-outage Disturbances on July 6 and 19, 1999, were caused by above-expected load in the PJM system. A near blackout situation with prolonged voltage reductions to 0.95 p.u. A voltage collapse was barely averted through the use of emergency procedures. [3]

21. Blackout in Croatia and Bosnia Herzegovina on January 12, 2003 was triggered by a short-circuit and circuit breaker malfunction on the $400 \mathrm{kV}$ transmission line Konjsko - Velebit. [10]

22. Blackout in the Eastern U.S. and Canada, August 14, 2003, was initiated by subsequent tree contacts of sagging conductors on the Stuart-Atlanta, HardingChamberlin, Hanna-Juniper, and Star - South Canton 345-kV transmission lines. The blackout affected up to 50 million people and caused shutdowns of more than 250 power plants with the total generation capacity loss reaching $61,800 \mathrm{MW}$. The estimated economic damage was $\$ 4.5-\$ 10$ billion. $[3,12]$

23. Denmark and Sweden, September 23, 2003, was caused by a short-circuit that affected two busbar sections at the Horred $400 \mathrm{kV}$ substation in western Sweden. About 2.4 million people were left without electricity for several hours. [12,13]

24. Italy, September 28, 2003 was triggered by tree flashover of the $380 \mathrm{kV}$ transmission lines Mettlen - Lavorgo and Sils - Soazza (in Switzerland). 57 million people were affected, restoration took about 4 hours. [14,15]

25. Western Norway, February 13, 2004. The blackout was caused by tripping the Nesflaten - Sauda $300 \mathrm{kV}$ line and malfunctions of the distant fault protection system. This 0.5 hours affected almost 500,000 people. [4]

26. Southern Greece and Athens blackout on July 12, 2004 was caused by the loss of unit $2(300 \mathrm{MW})$ at the Lavrio power station in the Athens area. [16]

27. May 24-25, 2005, Blackout on the Moscow region, Russia was caused by a combination of factors including severe equipment damages at the Chagino substation, short-circuits due to sagging of overheated conductors on the 110 and $220 \mathrm{kV}$ lines, reactive power shortage and voltage decline in the southern part of the Moscow Power System, and others. The Moscow blackout left at least 4 million people without electricity supply for more than 24 hours. [17]

Analysis of these blackouts indicated a need in developing real-time tools for monitoring system security margins including voltage stability margins under the normal and contingency conditions.

In connection to the June 2-3, 1996, major blackout in the WECC system, the Department of Energy recommended, among the others, the following measures to be implemented by WECC members to prevent future voltage collapse incidents [7] "WECC shall review the need for a security monitor function in the Western Interconnection to monitor operating conditions on a regional scale. WECC shall review the need for tools such as on-line power flow and stability programs and real time data monitors that can assess primary reliability indicators for frequency and voltage performance system-wide on a real-time".

These tools should have a look-ahead capability to leave time for real-time dispatchers to recognize the situation and apply preventive remedial actions. On October 18, 1996, the WECC investigation team analyzing August 10, 1996, blackout strongly recommended the following 
measure [8] "WECC shall pursue implementation of on-line power flow and security analysis, and recommend appropriate actions to increase the monitoring of key system parameters that would allow operators to identify potential problem outages and take corrective actions". Similar recommendations were formulated by the analytical teams investigating voltage collapse related blackouts in the other countries.

Summarizing the outcome of the previous blackout investigations, the U.S.-Canada Power System Outage Task Force that conducted the analysis of the August 14, 2003, blackout reiterated that "The current processes for assessing the potential for voltage instability and the need to enhance the existing operator training programs, operational tools, and annual technical assessments should be reviewed to improve the ability to predict future voltage stability problems prior to their occurrence, and to mitigate the potential for adverse effects on a regional scale". The Task Force also indicated that the blackout on August 14, 2003, had several causes or contributory factors in common with the earlier outages, including:

- Failure to ensure operation within secure limits

- Failure to identify emergency conditions and communicate that status to neighboring systems

- Inadequate regional-scale visibility over the power system

Despite the long history of similar requests to develop real time dispatcher tools to monitor the available voltage security, voltage stability and reactive power margins, those recommendations are not completely met by the majority of the US power system control centers. The CERTS survey [20] revealed that the "voltage collapse issues were only evaluated when the full AC power flow indicated an unusual operating condition, such as questionable voltage profiles. When voltage collapse or transient stability problems were suspected, the analysis was transferred to operations planners for detailed analysis. Some operators were guided by seasonal nomograms. In some cases this lack of dynamic security analysis activity was due to a lack of problems, or of interest in these phenomena. In other cases, it was due to a lack of efficient and integrated tools".

Besides the dispatchers' situation awareness and advisory purposes, there is a potential to develop automatic systems to prevent voltage instability and restore depressed voltages in the grid. This opportunity becomes especially important when the voltage collapse development process does not leave the human dispatchers to recognize the situation and apply adequate control measures. Controllable system separation could prevent a widespread system failure. Such a challenging task may be targeted by future R\&D projects.

\subsubsection{WECC voltage stability criteria}

The WECC voltage stability criteria [20], [24] are specified as real and reactive power margins and are apparently oriented mainly to the offline studies. At a minimum, each WECC member system shall conduct $P-V$ and $V-Q$ analyses to ensure that the minimum required margins are met. Sole reliance on either P-V or V-Q analysis is considered as not sufficient to assess voltage stability and proximity to voltage collapse. Each analysis is needed to confirm the results of the other. P-V analysis is needed to confirm the results of $\mathrm{V}-\mathrm{Q}$ analysis.

Member systems may use either method for general voltage stability evaluation and contingency screening. However, voltage stability margins must be demonstrated by both $P-V$ and $V-Q$ analyses. Table 1 lists the voltage stability criteria and the minimum margins for each disturbance level specified in [27]. From Table 1, the performance level "A" margin requirement is $5 \%$, decreasing by one half for subsequent performance levels " $\mathrm{B}$ " and " $\mathrm{C}$ ".

The percent quantity refers to the point of collapse value of the loading parameter in a given study. Thus, if the study considers a transfer across a key interface, then it is the percent of that flow that establishes the margin. 


\begin{tabular}{|c|c|c|c|}
\hline $\begin{array}{l}\text { Performance } \\
\text { Level }\end{array}$ & $\begin{array}{l}\text { Disturbance: } \\
\text { - Fault or No Fault } \\
\text { - DC Disturbance }\end{array}$ & MW Margin & MVAR Margin \\
\hline A & $\begin{array}{l}\text { Any element such as: } \\
\text { One Generator } \\
\text { One Circuit } \\
\text { One Transformer } \\
\text { One reactive Power Source } \\
\text { One DC Monopole } \\
\end{array}$ & $\geq 5 \%$ & $\begin{array}{l}\text { Worst Case } \\
\text { Scenario }\end{array}$ \\
\hline B & Bus Section & $\geq 2.5 \%$ & $\begin{array}{l}50 \% \text { of Margin } \\
\text { Requirement in } \\
\text { Level A }\end{array}$ \\
\hline $\mathrm{C}$ & $\begin{array}{l}\text { Any combination of two elements such as: } \\
\text { A Line and a Generator } \\
\text { A Line and a Reactive Power Source } \\
\text { Two Generators } \\
\text { Two Circuits } \\
\text { Two Transformers } \\
\text { Two Reactive Power Sources } \\
\text { DC Bipole }\end{array}$ & $\geq 2.5 \%$ & $\begin{array}{l}50 \% \text { of Margin } \\
\text { Requirement in } \\
\text { Level A }\end{array}$ \\
\hline $\mathrm{D}$ & $\begin{array}{l}\text { Any combination of three or more } \\
\text { elements } \\
\text { such as: } \\
\text { Three or More Circuits on ROW } \\
\text { Entire Substation } \\
\text { Entire Plant Including Switchyard }\end{array}$ & $>0$ & $>0$ \\
\hline
\end{tabular}

Table 1 WECC Voltage Stability Requirements

\subsubsection{System Definitions}

Voltage collapse is the process or sequence of events accompanying voltage instability that leads to unacceptable voltage profiles in a significant part of the system [1]. Many voltage collapses are not concentrated in a few power system buses. Rather the collapse is regional or involves the entire system. Utilities spend considerable resources in assembling sets of patterns of loading that the power system could actually experience in the next few hours. The ability of VSA to predict voltage profiles will benefit utilities by pruning the worst-case patterns of loading.

The power flow problem is described by a set of nonlinear algebraic equations that are generally different compared to the set of nonlinear algebraic equations obtained from the differential equations and describing the static voltage stability phenomena. The power flow calculations normally use simplified component models and involve secondary control models such as generation dispatch, multi-area power flow and switching capacitors. At the same time, while varying power flow parameters, the power flow equations may become inconsistent, and because of this a physical power flow solution ceases to exist. Such situations correspond to the power flow feasibility limits or power transfer limits. The power flow feasibility limit is determined by points where the power flow Jacobian matrix ${ }^{1}$ becomes singular or has a zero eigenvalue, or has a zero determinant, or can be nullified by multiplying by a nonzero vector.

\subsubsection{Representational Issues}

The number and selection of states in the system state vector allow a complete description of the dynamical behavior of the system. If the system has only three states, then the state vector has three components and can be visualized as a point in three-dimensional space, and the state space is three-dimensional. A similar concept applies when there are more than three states, say $n$ states. The state vector is thought of a point in an $n$-dimensional state space. An example of

${ }^{1}$ The Jacobian matrix of a set of $n$ equations in $n$ variables is an $n \times n$ matrix of partial derivatives whose entries are the derivatives of each equation with respect to each variable [31]. 
state space is the space of nodal voltage magnitudes and angles [31]. The parameter space can include any parameters that influence voltage stability. One of the examples of parameter space is the space of nodal active and reactive power injections, such as loads and generation. A system operating point can be represented both in state space and parameter space.

Power Injection Space: A representation of the Point of Collapse boundary can include coordinates of voltage magnitudes, voltage angles, real power injections and reactive power injections. These co-ordinates are indexed by buses. The bus power injections completely specify the power system state via the power system static equations. The various limits on power system operation can therefore be specified in power injection space. The position of the power injections of an operating point relative to these limits gives a representation of the power system security that is complete in the sense that all other representations of the power system security can be computed from it. It is also natural to use power injection space to specify transfers, load changes, redispatch, VAR support, generator and line flow limits. This justifies the basic hyperplane representation of the Point of Collapse boundary in power injection space.

Descriptive Variable Space : It is often useful to augment the set of power injections with other parameters. Then the hyperplanes in the augmented space indicate how the power injection hyperplanes change with parameters. This is used to compute the variation or sensitivity of the hyperplanes to input data and control parameters. Augmentation with states such as voltage angles and magnitudes gives a space containing both parameters and states and corresponding hyperplanes. The co-ordinates used in such a hyperplane representation of the Point of Collapse boundary have been named descriptive variables. Descriptive variables are usually a set of parameters describing cutset flows or flowgate flows. More generally, descriptive variables can be regarded as sets in parameter/state space of interest that are incomplete in the sense that these sets do not completely specify the power system state. For example, interarea flows are descriptive variables. In the simplest of cases, these descriptive variables may be linear combinations of the co-ordinates that are available at each bus. There are more complicated representations of these co-ordinates in which the power injections may be mapped to descriptive variables in a fashion that is not one-to-one. The descriptive variables are typically much fewer than the parameters, so many different parameters sets are mapped to a smaller set of descriptive variables. For example, if the descriptive variables are selected cutset flows, then many different sets of power injections yield the same cutset flows.

\subsubsection{Modeling Requirements}

The complete understanding of voltage collapse [31] requires a dynamic model in order to explain why the voltages fall dynamically. However, some computations concerning voltage collapse require only a static model. If a dynamic model is required, the power system is modeled by a set of differential equations with a slowly changing parameter. Computations which only require static models are advantageous because the results do not require load dynamics and other dynamics to be known. When using static models to obtain practical results, there is a caveat that there must be a way of identifying the stable operating equilibrium of the power system. In principle, this requires a dynamic model, but the stable operating point is often known by observing the real power system, or by experience, or by knowing the stable operating equilibrium at lower loading and tracking this equilibrium by gradually increasing the loading. Computations associated with voltage collapse that require dynamic models include (a) predictions of the outcome of the dynamic collapse, (b) any problem involving significant step changes in states or parameters, (c) any computations involving eigenvalues or singular values away from the voltage collapse. 


\section{OVERVIEW}

\subsection{Prototype Description}

The purpose of this section is to provide a description of the RTVSA prototype tool and demonstrate how the prototype tool captures the main functionalities of the VSA project and helps ts implementation. The Electric Power Group in cooperation with CA ISO, ENERGY COMMISSION, and LBNL has developed a Multi-Year Development Roadmap for the VSA project shown in Figure 1. The roadmap consists of three task tracks including data requirements, algorithms and prototype development. It is systemized in four phases starting from the current phase 2.

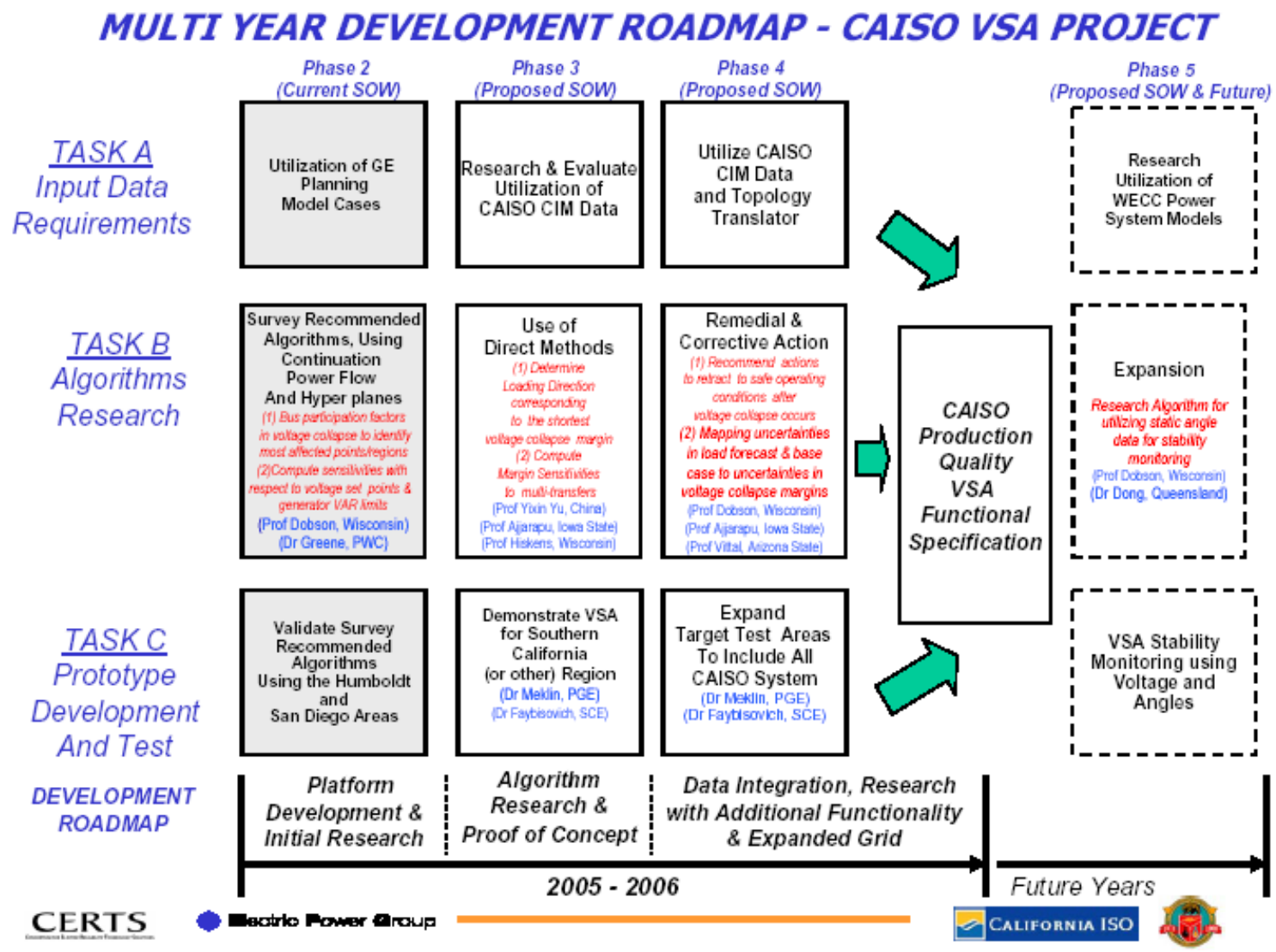

Figure 1 Multi-Year Development Roadmap for the CA ISO RTVSA

The phases include platform development and initial research, algorithm development and the proof-of-concept simulations, and data integration and project expansion. The project time span is two years with the potential expansion for the future years. For each track and each phase, the tasks are formulated, and the suggested project support teams are provided. The original VSA Prototype Functional Specification Summary is described by Figure 2. 


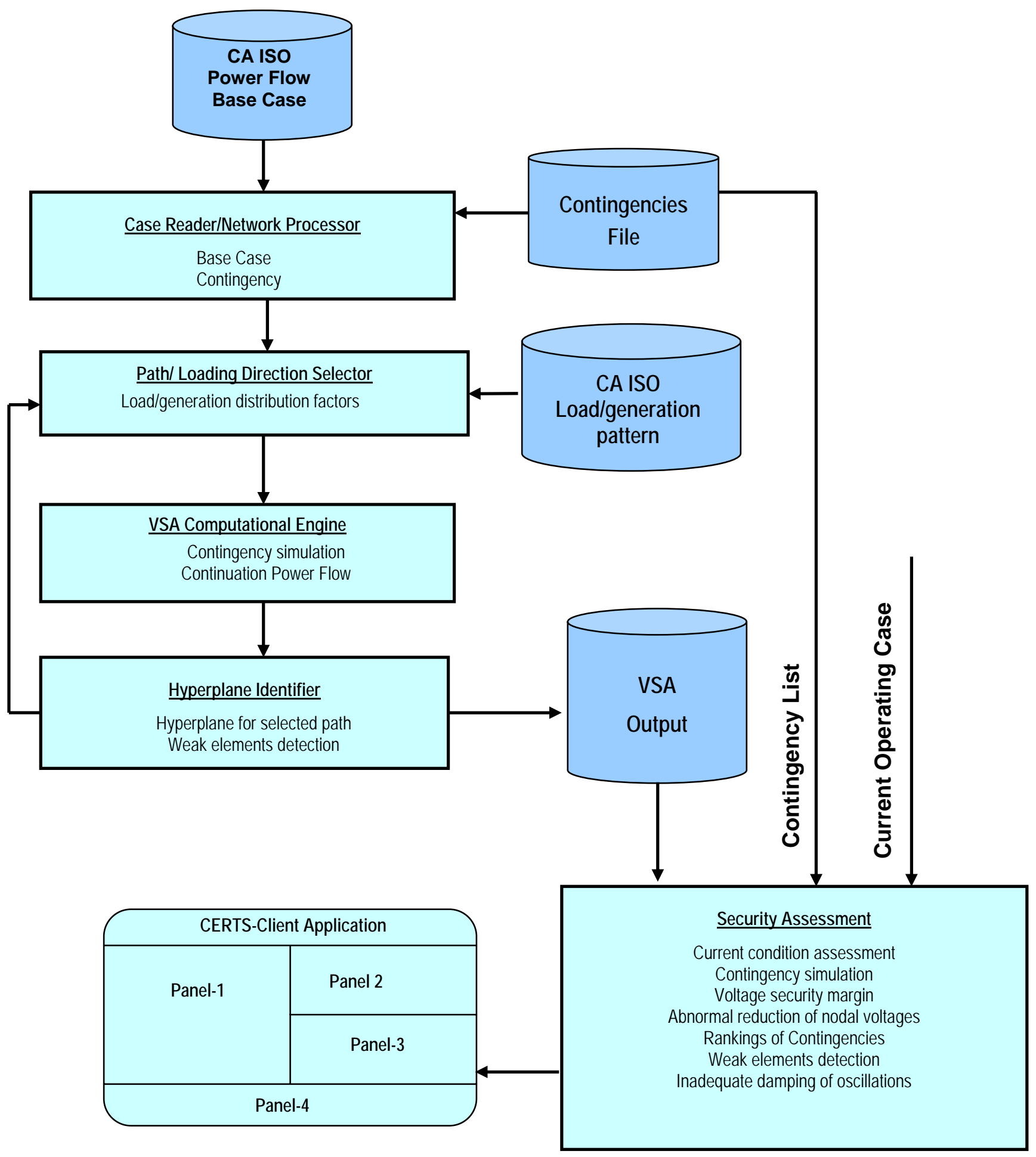

Figure 2 Block Diagram of RTVSA Prototype 


\section{What Will The VSA Application Do?}

The VSA application under development will be linked to the CA ISO EMS system model and data, develop and approximate voltage security regions, which is a form of multi-dimensional nomograms, using hyperplanes, calculate voltage stability indices and identify and display abnormal low voltages and weak elements and places in the system most vulnerable to voltage and voltage stability related problems. This application will also perform contingency analysis and provide the system operators with the voltage problem contingency rankings for the purposes of system monitoring and selecting preventing and emergency corrective actions. This application will be running in real time with the five-minute periodicity and provide the look-ahead capability, for up to two hours ahead, to allow the dispatchers to evaluate the situation, select and apply adequate remedial actions.

The prototype tool will implement, test and demonstrate the key elements of the future industrial grade VSA tool. The key elements of the prototype are described below in Figure 3.

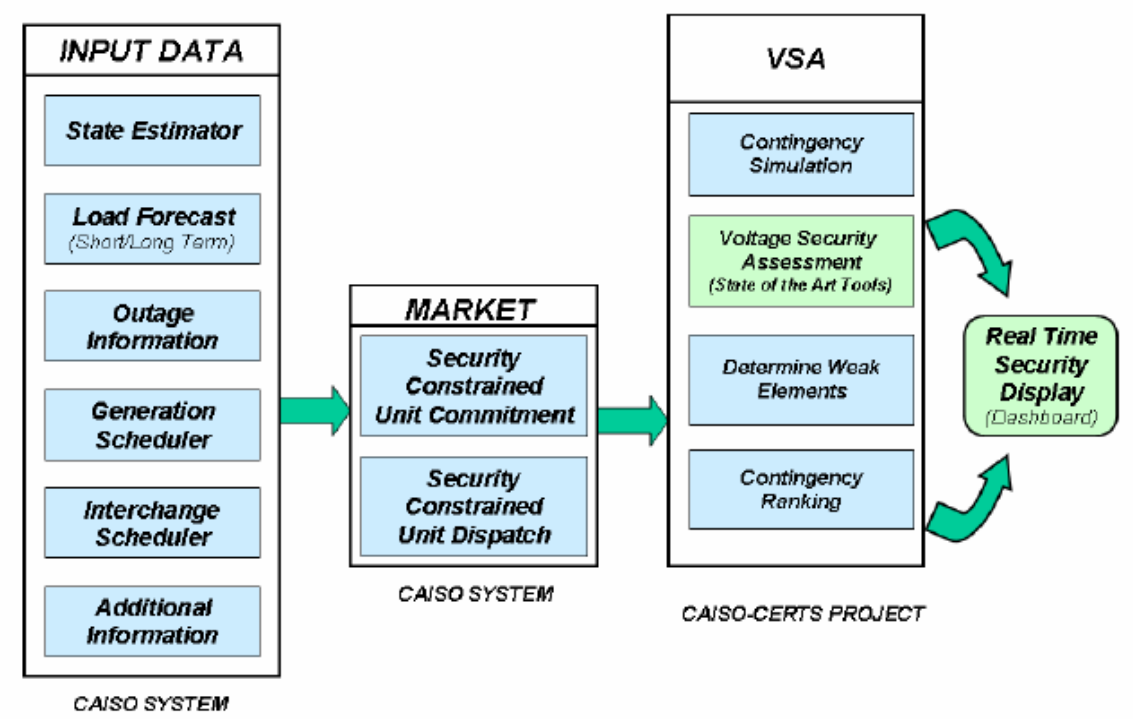

Figure 3 The proposed integration scheme for the VSA project

\section{Project grid monitoring visualization solution}

The VSA application will be designed for use by the CA ISO operators in monitoring and predicting system performance two hours ahead. EPG will work with the CA ISO to design a data display format suitable to CA ISO operators. The real time display will have the following information:

- The current operating condition

- Contingencies with insufficient stability margin

- Contingency ranks based on severity indices

- Abnormal reductions of nodal voltages

- Voltage security margins 


\subsection{Implementation}

The project was split into 3 tracks and these tracks were further subdivided into yearly goals (see Figure 1). The end product of these set of goals will be a phased implementation for the complete functional specifications for a production quality VSA system based on the results and benchmarking abilities of the prototype developed in concert with external advisors. Some of the items below have already been completed in 2005.

- Conduct an extensive review of the existing methods, algorithms, and production-grade VSA tools on the global scale, analyze the evolution and competition of the ideas in the area, identify the most promising proven approaches, and based on this analysis select state-of-the art methods and algorithms for this project.

- Review the voltage collapse blackouts for the last 40 years and summarize relevant recommendations of the blackout investigation teams.

- Contact the leading experts in the VSA area worldwide and conduct a survey summarizing their collective opinion on the selected candidate approaches; modify the selected framework based on the expert feedback. Present and discuss the project at the industrial and CERTS advisory meetings. Organize a series of face-to-face interviews and brainstorm meetings with the University professors, project consultants, CA ISO staff and utility engineers engaged in the area, Identify a group of potential contributors for the project. Cooperate with the other CERTS members and PSERC in order make this project successful.

- Work out a step-by-step design of the project based on the selected state-of-the art algorithms. Develop new approaches whenever it is necessary. Evaluate, test and select the existing software tools that can be incorporated in the prototype software.

- In cooperation with the CA ISO personnel, select areas with the known voltage stability related problems, design and conduct verification procedures for the prototype tool using the standard power flow computation tools such as PSLF.

- Develop design specification and produce a comprehensive report.

- Pre-calculation and approximation of the voltage stability boundary in the off-line mode

- Calculation of the voltage stability margin in real time using the pre-calculated approximations of the voltage stability boundary

- Identification of abnormal reductions of nodal voltages, weak elements and regions most affected by voltage and voltage stability problems.

- Transfer the conceptual design to CA ISO, other California Control Areas and Utilities and to the Vendors selected by these organizations for the further implementation of the design as production-grade operating tools.

- Cooperate with CA ISO-selected vendor in development of the production-quality VSA. 


\section{SURVEY RESPONSE}

The formulation of the CA ISO sponsored survey that inquired about the existing state-of-the-art algorithms for VSA from among experts in the industry and in the universities will be described. Further individual feedback from experts who had responded were obtained. These were tabled at the Technical Advisory Committee (TAC) Meeting in Pasadena on February 25, 2005. The Survey, the Survey Results and the TAC Meeting Minutes will be summarized.

\subsection{Survey Summary}

A survey was sent to experts in the power system field for comments, information, suggestions, and recommendations on the algorithms and methodologies for a state-of-the-art RTVSA application. The California Independent System Operator (CA ISO) and other Grid and Transmission System Operators in California need such a tool to explore avenues to better optimize utilization of the existing transmission system via development of state-of-the-art real time wide-area security assessment applications.

\subsection{Survey results summary}

CERTS/EPG formulated a survey to reach out to experts in this field for comments, information, suggestions, and recommendations related to the VSA project.

Survey Overview. The survey was sent to 51 experts in universities and in the power industry worldwide. Sixteen reviewers responded. Eight of these respondents are from the power industry and eight are from academia.

Summary of Responses. The consensus opinion was that the hyperplane approximation approach is well suited for VSA. Voltage instability is more of a local area/region phenomenon. Some participants felt that time domain simulations should augment the direct methods. One participant from the industry mentioned that it was not clear how switching conditions could be revealed without time domain simulations. Another respondent from the industry shared his difficulties with developing suitable VSA metrics because of the presence of both continuous, such as load growth and non-continuous, such as contingency, factors.

Main conclusions. The majority of responses favored the use of the hyperplane approach in determining voltage security assessment. Also, the majority of responses did not see hyperplanes suitable for determining dynamic voltage assessment at this time.

Recommendations.

- Use the hyperplane approach to approximate security regions for the VSA purposes.

- The computational engine for the VSA project is recommended to be the continuation power flow. This tool has been tested and proven by several researchers in commercial and noncommercial software.

- Pursue a hybrid approach, where the direct method is used for fine-tuning the security boundaries after an iterative set of continuation power flows.

\subsection{Minutes of TAC Meetings}

February 25, 2005, CERTS Technical Advisory Committee Meeting.

The CERTS TAC meeting in October was attended by the following technical advisors: lan Dobson (University of Wisconsin-Madison, PSERC), Joe Eto (LBNL), Bernie Lesieutre (LBNL), Yuri Makarov (CA ISO), Jim Dyer (EPG), Manu Parashar (EPG), Carlos Martinez (EPG), Matthew Varghese (EPG) and Jim Cole (CIEE). The following recommendations and comments were made by the Advisors:

- The overall consensus was that the hyperplane approach was well suited for VSA

- It was agreed that the security boundaries are intended to be defined offline with the possibility of extending this to online if found feasible 
- Existing nomograms could be improved by the VSA results

- Some filtering scheme would be required to ignore known weak elements/points in the system which aren't of concern, such as low voltage areas and radial networks.

- The general consensus was that transient stability assessment was infeasible at this time. Concerns were expressed that the real-time transient analysis is not practical with the current dynamic models.

- The next steps of the plan should include:

o Share the feedback received with those who provided input

o Utilize the experience of expert advisors during the project development, such as Prof. Ian Dobson (University of Wisconsin) and Dr. Anatoliy Meklin (PG\&E)

o Develop a detailed project plan, including the necessary methods of evaluating and testing the various functionality of this application

o Develop a detailed functional specification to include description of the overall process of how the VSA will integrate and work with CA MRTU and ADS systems.

o We should quickly move to evaluate the types of power flows needed, such as continuation power flows. Run simulations to find critical points.

o Document planned development for the near future.

- Jim Cole encouraged the group to investigate ways in which these projects can be related to congestion reduction. Congestion management costs the CA ISO some 500 million dollars and any project that helps reduce this congestion by $10 \%$ will be very appealing.

\section{October 16, 2005, CERTS Technical Advisory Committee Meeting.}

The TAC meeting in October was attended by the following technical advisors: Jim Gronquist (BPA), Bernie Lesieutre (LBNL), Irena Green (CA ISO). Manu Parashar (EPG) made presentation on the current CA ISO projects including the VSA project.

- The main concepts of the VSA project were supported by the meeting participants

- The only concern was expressed about the convergence of power flow GE-PSLF tool in the vicinity of power flow singularity conditions and its ability to find the PoC point. 


\section{PROPOSED FRAMEWORK}

The overall proposed framework for the year 2005 and beyond that was discussed and formulated with active participation by CERTS consultants Dr. Yuri Makarov and Prof. lan Dobson and EPG personnel Manu Parashar, Matthew Varghese and Jim Dyer during meetings in Pasadena (August 25-26, 2005) and in Madison (September 9, 2005).

\subsection{Selected Study Areas}

The selection of the critical parameters influencing the voltage stability margin and stress directions was conducted based on engineering judgment. The stress directions were defined using the sink-source and balanced loading principles. This means that the generators and the loads participating in each stress scenario are identified, as well as their individual participation factors; the participation factors are balanced so that the total of MW/MVAR increments and decrements is equal to zero. This allows avoiding re-dispatching of the remaining generation. Based on the California ISO recommendation, 2 study areas were selected for verifying the prototype VSA algorithms: the Humboldt and San Diego problem areas. The San Diego area was selected as the first to be studied with the RTVSA algorithm.

\subsection{Description of Proposed Framework}

For each stress direction in each problem area, execute the predictor-corrector procedure to find the Point of Collapse (PoC) in this direction. The selected platform for implementing the procedure includes the PSERC Continuation Power Flow program ${ }^{2}$ and MATLAB programming language. Major modifications have been made to the PSERC program to meet the objectives of the VSA project most efficiently. Details of the procedure are selected as follows:

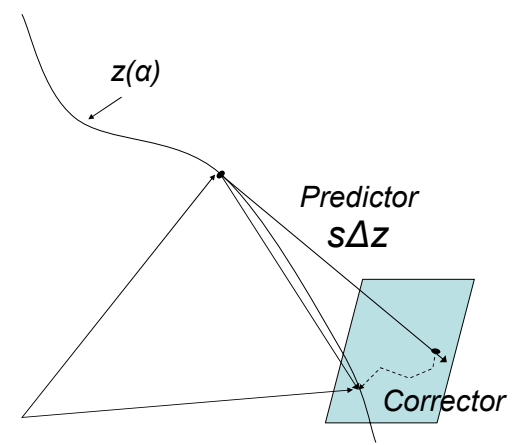

Figure 4 Predictor-Corrector Algorithm

a. Predictor steps are performed along the parametric trajectory $z(\alpha)$ in the extended state space that includes the power flow state space parameters $x$ plus an additional stress parameter $\alpha$ which defines the current position of power flow points along the stress direction $D$. The details of Figure 4 are as follows:

i. The direction of the step $\Delta z^{3}$ is selected by solving linearized predictor equations and by using one of the following approaches: (1) by fixing an extended state space parameter that experienced the most significant change at the previous predictor step, or (2) by analyzing the angle between the previous increment of state variables and the current direction of the predictor vector $\Delta z$ (the angle must not exceed certain specified threshold

\footnotetext{
2 Primary authors are S. L. Greene, R. Rajaraman, I. Dobson, F. Alvarado \& C. DeMarco

3 The increment vector $\Delta z$ consists of the increments of nodal voltages and angles $\Delta x$ and of the stress parameter $\Delta \alpha$.
} 
value). Measures (1) and (2) are designed to provide one-directional motion along the parametric trajectory.

ii. The size of the step $s$ is selected based on the maximum allowable changes of per unit nodal voltages and voltage angles.

iii. A dichotomy search procedure is implemented to correct (halve) the step size $s$ when the corrector step diverges or the PoC point is being approached. The entire predictor-corrector procedure stops when a specified accuracy (step size) is achieved.

b. Corrector step follows each predictor steps. The corrector step returns the predicted point to the parametric trajectory $z(\alpha)$ in the extended state space (the deviation occurs because of the linearization used at the preceding predictor step. This is done by fixing the stress parameter at the achieved value, and by solving the corrector equation with the help of the Newton-Raphson method.

c. The PoC criterion is based on sign change of the minimum eigenvalue. 


\section{PROTOTYPE RESULTS}

\subsection{Description of the San Diego transmission system}

The transmission tie-lines forming a boundary around San Diego include: San Onofre - San Luis Rey 1, 2, \& 3230 kV Lines - Path 44 lines- , San Onofre - Talega 1 \& 2230 kV Lines -Path 44 lines, Imperial Valley - Miguel $500 \mathrm{kV}$ line, Imperial Valley - La Rosita $230 \mathrm{kV}$ line, Imperial Valley - El Centro $230 \mathrm{kV}$ line. The San Diego Area boundary substations impacting the area can be defined by San Onofre, San Luis Rey, Talega, Imperial Valley, and Miguel. The diagrams in this section were extracted from R.01-10-024 "Direct Testimony of David Korinek" April 15, 2003.

SDG\&E's ability to import SONGS and other off-system generation is defined by two transmission import boundaries or constraints: the simultaneous import limit (SIL) and SDG\&E's nonsimultaneous import limit (NSIL). To help explain these two import conditions, a diagram showing the major import paths \& substations is shown in Figure 5. All SDG\&E customer load is located within the bubble labeled "Local SDG\&E Load Area." As shown on Figure 5, the NSIL is defined by SDG\&E's ability to import power into its local load area via the five $230 \mathrm{kV}$ lines which comprise the South-of-SONGS (SOS) WECC Path 44.

The SIL is defined by SDG\&E's ability to import power into its system via the Miguel $230 \mathrm{kV}$ bus plus the five $230 \mathrm{kV}$ lines SOS. Based on technical studies, the present NSIL and the SIL limits are $2500 \mathrm{MW}$ and $2850 \mathrm{MW}$, respectively. The $2850 \mathrm{MW}$ SIL limit applies when all transmission facilities are in operation. Conversely, the NSIL import limit only applies when SDG\&E's 500kV Southwest Power Link (SWPL) is out of operation. These limits define the maximum power imports presently available to serve SDG\&E's load area. If SDG\&E's customer load exceeds these import limits, it must be supplied by local generation within the service area.

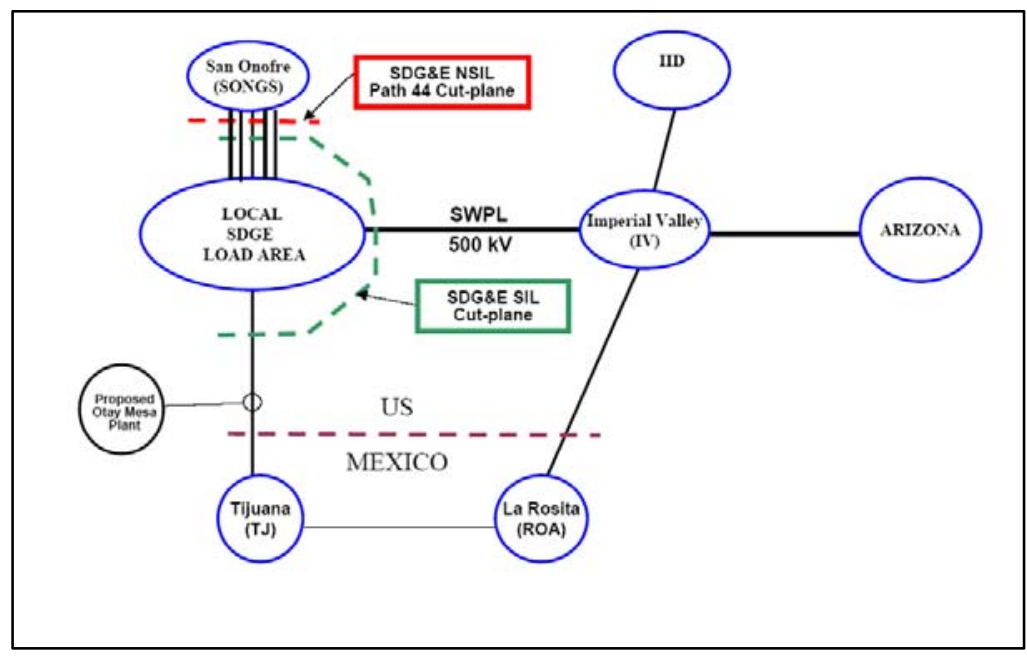

Figure 5 Major Import Paths in the SDGE System 
SDG\&E's electric transmission network is comprised of 130 substations with 884 miles of $69 \mathrm{kV}$ transmission lines, 265 miles of $138 \mathrm{kV}, 349$ miles of $230 \mathrm{kV}$, and 215 miles of $500 \mathrm{kV}$ transmission lines. Figure 6 shows a simplified diagram of the transmission system in San Diego County and southern portion of Orange County, excluding 69kV lines. Local "on system" generating resources are the South Bay Power Plant, connected at $69 \mathrm{kV}$ and $138 \mathrm{kV}$, and a number of combustion turbine facilities located around the service territory connected at $69 \mathrm{kV}$.

Imported resources are received via the Miguel Substation as the delivery point for power flow on the Southwest Power Link (SWPL), which is SDG\&E's $500 \mathrm{kV}$ transmission line that runs from Arizona to San Diego along the US/Mexico Border, and via the SONGS 230kV switchyard SDG\&E's only direct interconnection with the CA ISO controlled grid. Delivery of power from SDG\&E's share of the SONGS units flows into the service area as imports at 230kV.

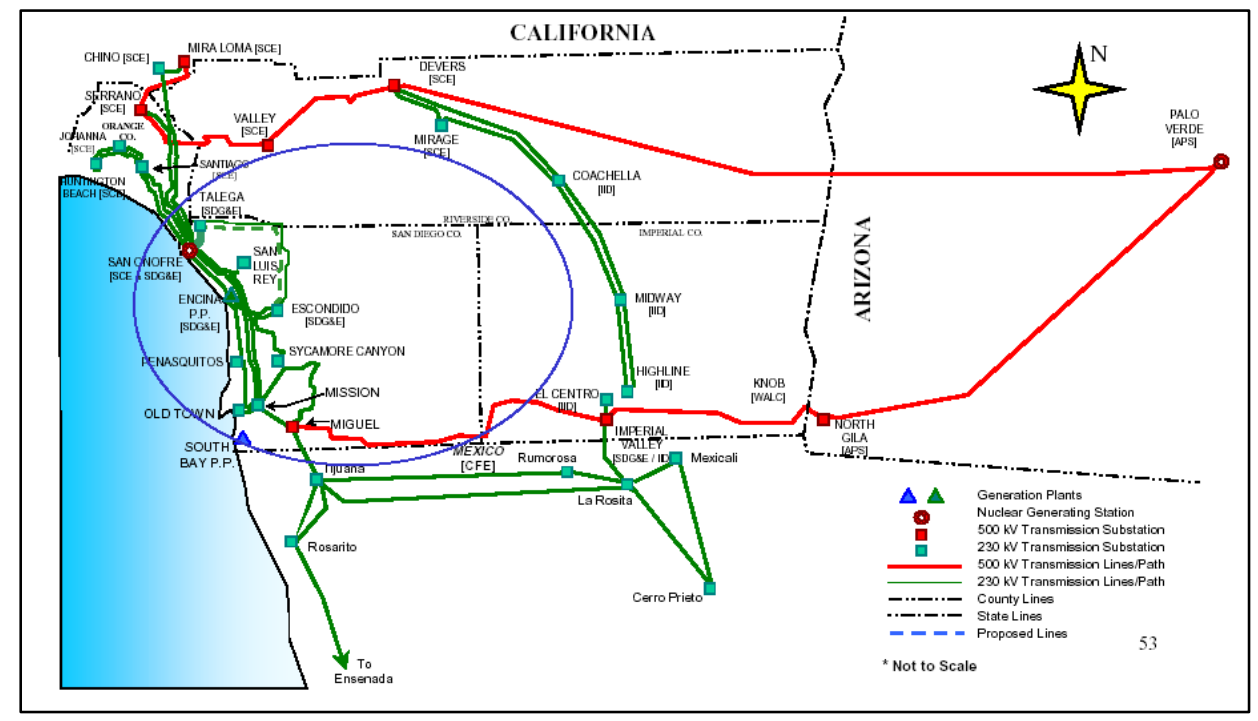

Figure 6 Map showing the two arms of the SWPL corridor in relation to SDGE

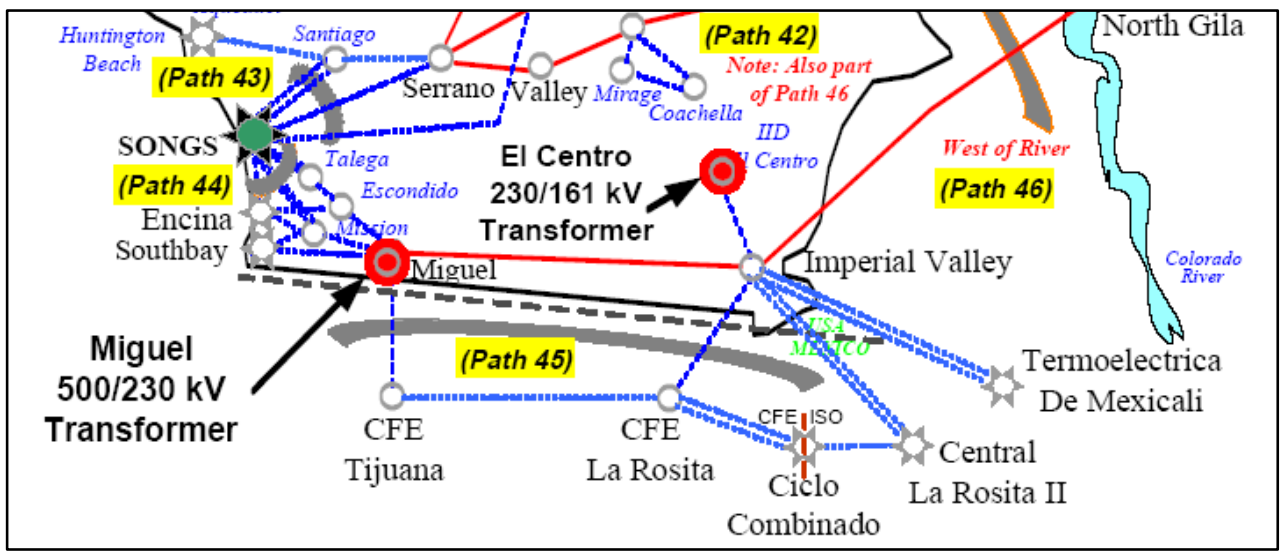

Figure 7 Location of Songs and South Bay in the CA EHV Transmission Network 


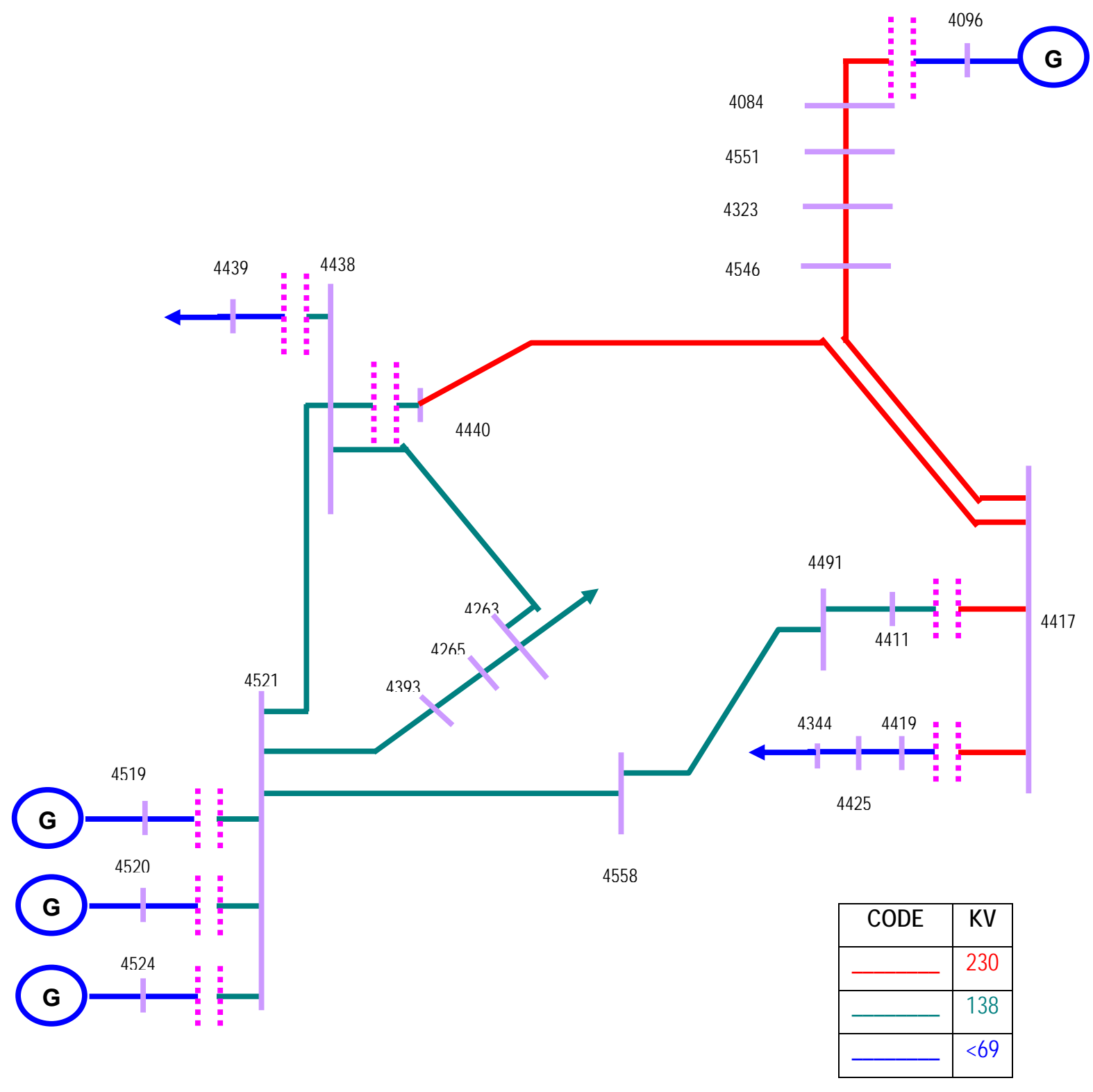

Table 2 Bus Index and Color Code for the SOBAY-MIGUEL-SONGS area

\begin{tabular}{|c|c|c|c|c|c|}
\hline BUS & GENERATOR & BUS & LOAD & BUS & LOAD \\
\hline 4519 & SOBAY & 4084 & SONGS & 4393 & LCOCHS \\
\hline 4520 & SOBAY & 4263 & CHILLS & 4439 & MSSION \\
\hline 4524 & SOBAY & 4323 & ESCNDO & 4491 & PRCTVY \\
\hline 4096 & SONGS & 4344 & GRNITE & 4558 & TLGCYN \\
\hline & & & & 4417 & MIGUEL \\
\hline
\end{tabular}

Figure 8 One-Lines for the SOBAY-MIGUEL-SONGS area 


\subsection{Description of Hyperplane Approximation}

The hyperplane approximation can be used as a part of the analytical boundary description or separately for the purposes of visualization. See Figure 9 for a geometrical illustration. The first known use of the approximation ideas was apparently related to the operating nomograms. See [78] for more details. The operating nomograms are usually represented visually as piecewise linear contours on a plane of two critical parameters. If three critical parameters are involved, the nomogram is represented by a number of contour lines. Each of them corresponds to a certain value of the third parameter. It becomes difficult to visualize nomograms for four or more critical parameters. The natural extension of the linearized stability nomograms for three or more critical parameters is based on the use of hyperplanes - the planes that are defined in multidimensional parameter space as approximations of the stability boundary.

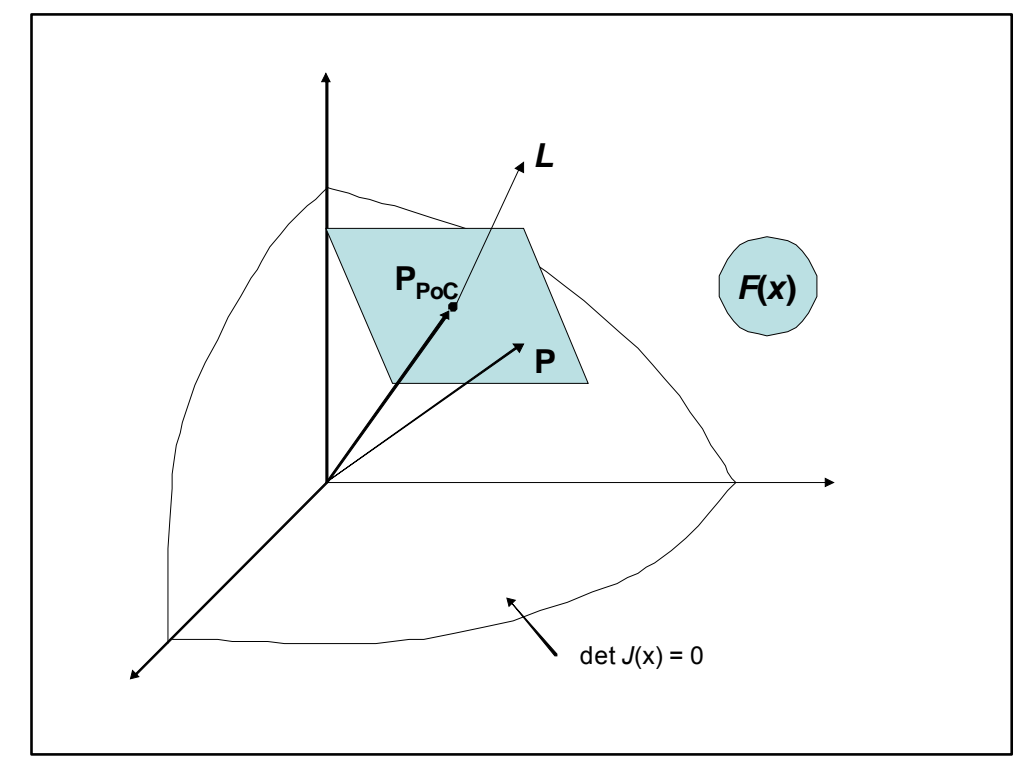

Figure 9 Illustration of Hyperplane Approximation

The RTVSA algorithm produces a tangent hyperplane $p=F(x)$ that can be described by the following formula ${ }^{4}$ :

$$
L^{t} \cdot[p-P o C]=0 \rightarrow p=F(x)
$$

Note that:

- It is a tangent plane to the load flow boundary if it is convex at the PoC (Point of Collapse).

- $L$ is the normal to the hyperplane and is identical to the set of Lagrangian multipliers at PoC The hyperplane is actually a $(n-1)$ subspace of the $n$-dimensional space $F(x)$.

\footnotetext{
${ }^{4}$ The notation $p$ denotes power injections and $x$ denotes states such as voltage magnitudes and angles.
} 


\subsection{On the orientation of 2D hyperplane slices for CHILLS and MSSION}

The following input data is used as a simple example to examine the RTVSA prototype. The stress parameters are sinks internal to SDGE. The sources have been constrained to be the set of three generating units at Southbay. This corresponds to a scenario with no import from SONGS or ENCINA or from units West of the River or from Mexico. The SINKS are Loads at Carlton Hills and Mission. The SOURCES are Generator Shifts at South Bay.

Table 3 Patterns of SINK PF (Participation Factors) ${ }^{5}$

\begin{tabular}{|c|c|c|c|c|c|}
\hline Pattern & Color & CHILLS & MSSN & \multicolumn{2}{|c|}{ Code } \\
\hline I & green & 0.99 & 0.01 & 1 & 0 \\
\hline II & red & 0.20 & 0.80 & 1 & 1 \\
\hline III & blue & 0.01 & 0.99 & 0 & 1 \\
\hline
\end{tabular}

Table 4 Generator PF at the 3 Units of South Bay (SB) for all Vectors

\begin{tabular}{|c|c|c|}
\hline SB 4519 & SB 4520 & SB 4524 \\
\hline 0.30 & 0.35 & 0.35 \\
\hline
\end{tabular}

\subsubsection{Parametric Sensitivities}

In Section 5.2, the set of Lagrange multipliers ${ }^{6}$ at $\mathrm{PoC}$ are stated to be identical to $L$, the hyperplane normal. Lagrangian Multipliers at the PoC can also be interpreted as the left eigenvector at the PoC.

Figure 10 shows the comparisons of Lagrangian Multipliers ${ }^{7}$ for the three stressing patterns. For example, Pattern II for CHILLS has a multiplier of 0.8 , which means that reducing the load at CHILLS by $1 \mathrm{MW}$ would help to increase the Margin to PoC by $0.8 \mathrm{MW}$. A bus with a very high Lagrangian Multiplier would signal congestion. Buses with very low Multipliers would be indicative of locations that are a large electrical metric away from the point of collapse. Indicators, such as the statistics of multipliers that are above a certain threshold, can be used for distinguishing the "non-locality" of the collapse phenomenon.
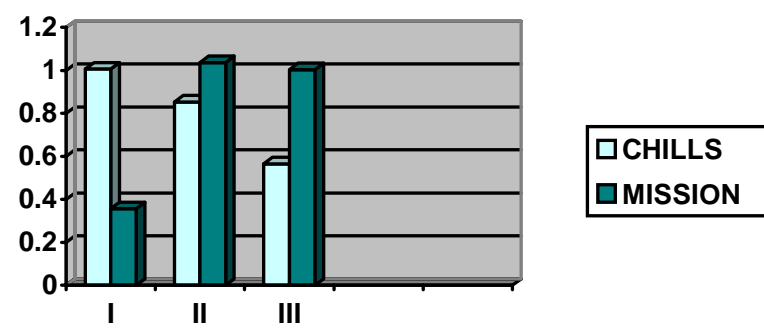

Figure 10 Lagrangian Multipliers for SDGE cases

\footnotetext{
${ }^{5}$ The load at Carlton Hills is approximately four times smaller than the load at Mission.

6 The coefficients of the hyperplane consist of elements of the left eigenvector which can be interpreted as the Lagrangian multipliers corresponding to the parametric sensitivity of the hyperplane. The hyperplanes can be visualized as the constraints in a traditional optimization problem. The intercept on the descriptive variable axis is inversely proportional to the Lagrangian multiplier associated with the descriptive variable.

${ }^{7}$ Local structure such as low voltage networks or radial lines or a persistently congested area may give rise to large spikes in the Lagrange Multipliers. For example, buses at BANING, CABZON, PANSEA, RENWD, SANVEN are at or near generators that are severely VAR limited in the base case. All of these buses are located near DEVERS. The Sensitivities for these buses were filtered out in the screening stage of the RTVSA algorithm.
} 
Figure 11 can be considered a geometric validation of the result. The intercepts on the $y$ axis (Mission) are smaller for patterns II and III because of the larger Lagrangian multipliers for Mission. Likewise, the intercept on the $x$ axis (Carlton Hills) is large for patterns II and III because of the small Lagrange multipliers at Carlton Hills. Stressing Pattern I has the opposite arrangement - a large Lagrangian multiplier for Carlton Hills and a small multiplier for Mission.

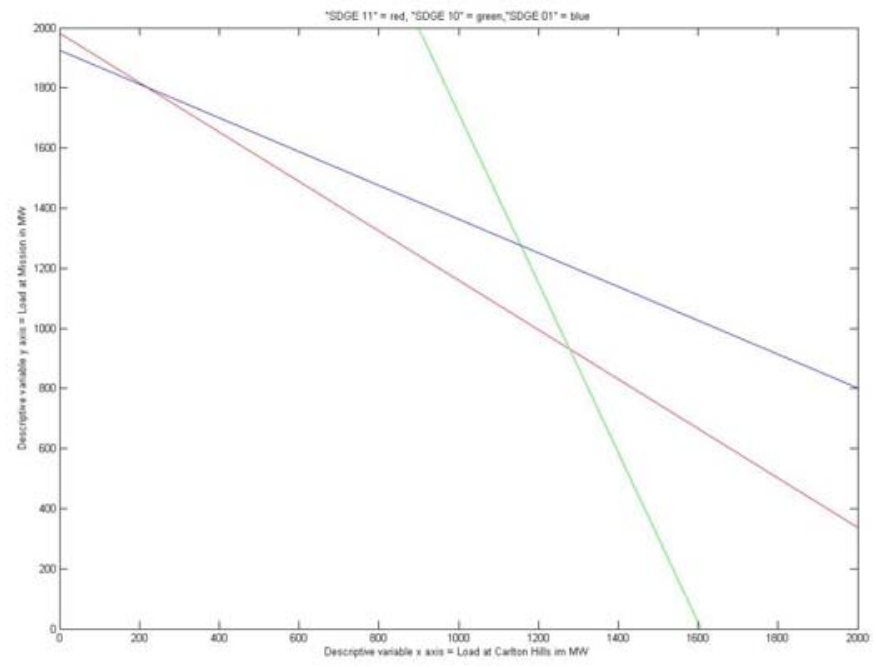

Figure 11 RTVSA Output: Hyperplane slices at Carlton Hills and Mission

The high values of PoC in the CHILLS MSSION case in Figure 11 are because the example was meant to illustrate the effects of electrical limits on the transmission of power from the source buses to a set of distributed sink buses. The effects of thermal limits have been temporarily neglected. The sources are also assumed to have an unlimited supply of reactive power. Both of these relaxations show the electrical capacity of the corridors of power flows from South Bay to CHILLS and MSSION. This capacity is far greater than when thermal and power injection limits are enforced. Such a study is useful in analyzing the effects of large power flows through corridors in the network that may actually occur in the event of an unexpected transfer or large power swings in a pre-collapse scenario.

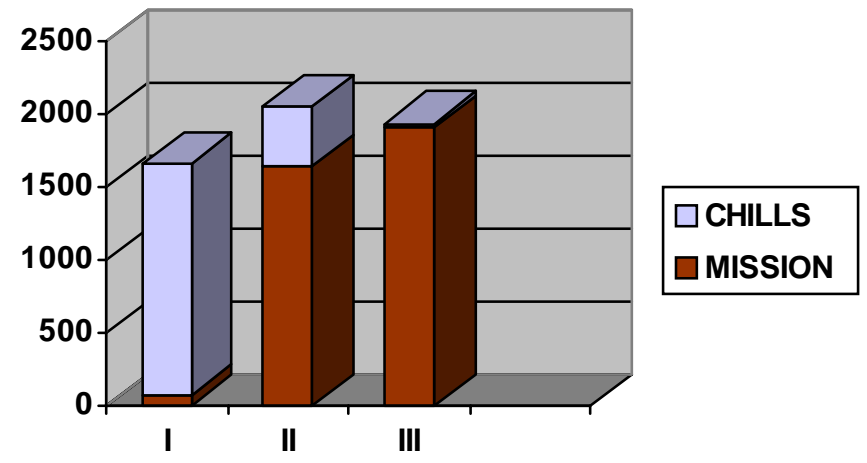

Figure 12 RTVSA Output: PoC in MW for Carlton Hills and Mission 


\subsubsection{Collapse Participation Factors}

The participation is computed from the right eigenvector of the Jacobian evaluated at voltage collapse corresponding to the zero eigenvalue. The right eigenvector provides information on the extent to which variables participate in the voltage collapse. This determines weak areas and whether the collapse is an angle collapse. Specifying to the operator which buses participate most in the voltage collapse is useful, but it should also be noted that the buses with the biggest falls in voltage in the collapse may not be the same as the most effective buses to inject reactive power. These Voltage Collapse Participation Factors can be expressed in terms of KV/(100 MW of the Margin to PoC). In other words, if the Margin to PoC decreases by $100 \mathrm{MW}$, then the Participation Factors will indicate the extent to which the voltages will recover. Figure 13 shows these for Stressing Pattern I.

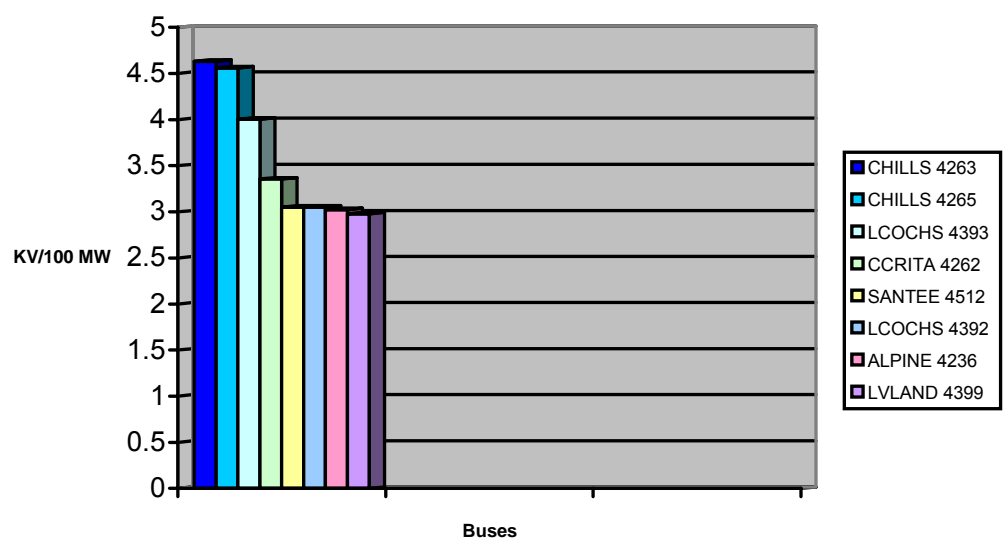

Figure 13 Top Eight Voltage Collapse Participation Factors for Stressing Pattern I

Similar to Voltage Collapse Participation Factors, one can examine the top ranked Angle Collapse Participation Factors. In order to translate between units of KV and units of degrees, the equivalence of 5 degrees and $7 \mathrm{KV}$ has been assumed ${ }^{8}$. See below for Stress Pattern I.

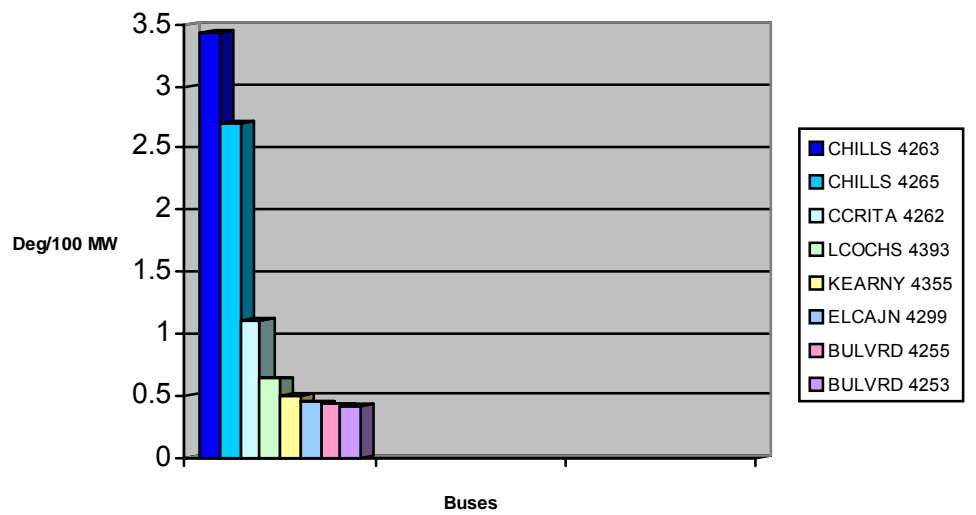

Figure 14 Top Eight Angle Collapse Participation Factors for Stressing Pattern I

\footnotetext{
${ }^{8}$ This is based on the original estimates that equates 0.08 radians to 0.05 pu voltage.
} 
In addition, the voltage magnitudes and angles can be plotted versus the stress magnitude to obtain the familiar "nose" curve that shows the sharpness of the collapse as the loading is increased. The Figures below show these for some typical buses.

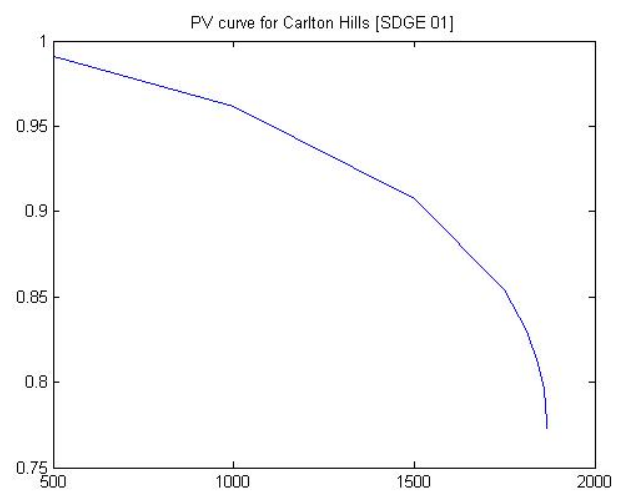

Figure 15 PV curve for Carlton Hills with Stress Pattern III showing collapse

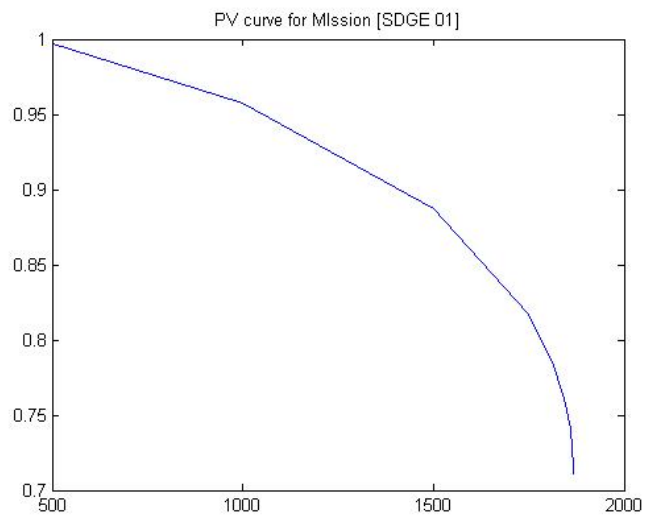

Figure 16 PV curve for Mission with Stress Pattern III showing collapse

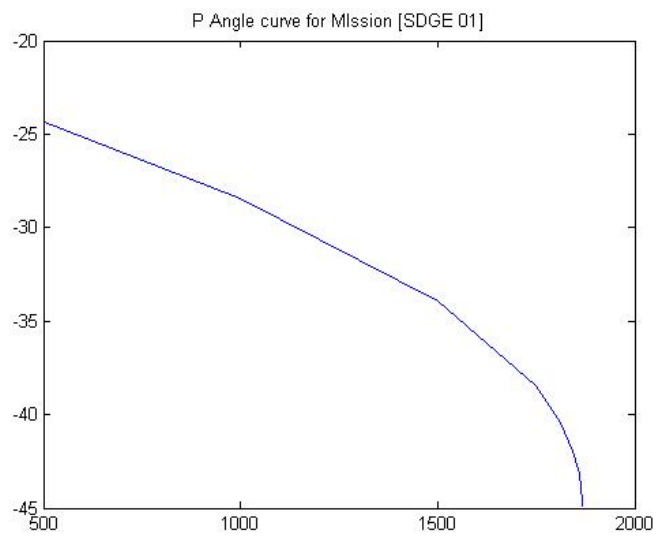

Figure $17 \mathrm{P}$ Angle curve for Mission with Stress Pattern III showing collapse 


\subsection{On the Effects of Adding a Sink in a Congested Load Pocket}

Granite is located a few buses away from Miguel. One of the end points of the South West Power Link (SWPL) is anchored at Miguel. Since Miguel does not have native load, the stressing of Granite as a sink can be viewed as an indirect perturbation of the power flows in the Miguel to Imperial Valley arm of the SWPL transfer corridor. The SINKS are Loads at Carlton Hills, Mission and Granite. The SOURCES are Generator Shifts at 3 units at South Bay. These units have equal PF (Participation Factors) for all Patterns.

Table 5 Patterns of SINK PF (Participation Factors)

\begin{tabular}{|l|l|l|l|l|l|l|}
\hline CHILLS & MSSION & GRNITE & Color & \multicolumn{3}{|l|}{ Code } \\
\hline 0.33 & 0.34 & 0.33 & green & 1 & 1 & 1 \\
\hline 0.02 & 0.49 & 0.49 & cyan & 0 & 1 & 1 \\
\hline 0.49 & 0.02 & 0.49 & magenta & 1 & 0 & 1 \\
\hline 0.49 & 0.49 & 0.02 & red & 1 & 1 & 0 \\
\hline
\end{tabular}

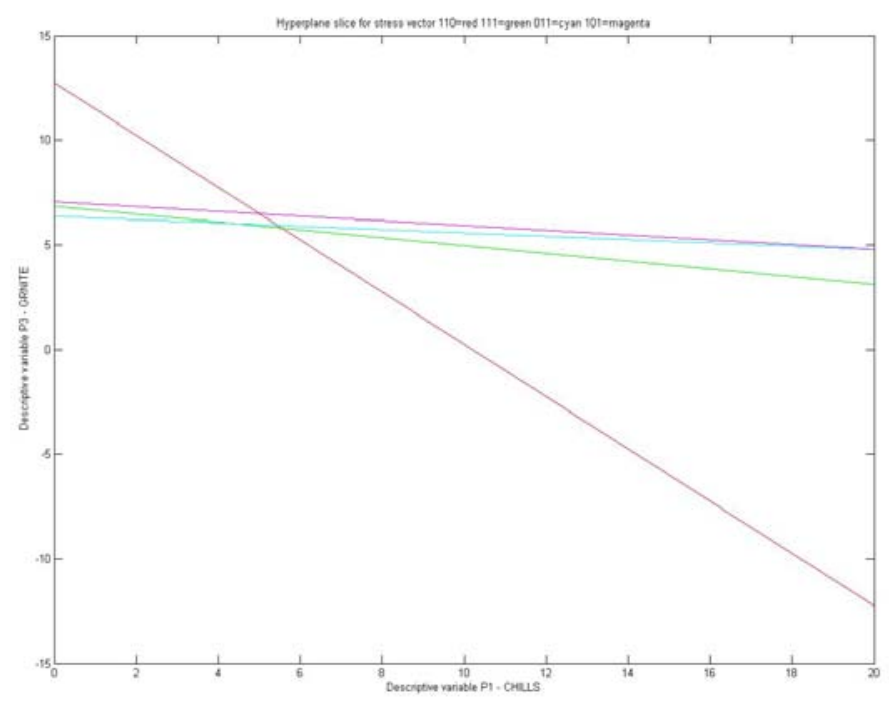

Figure 18 RTVSA Output: Hyperplane slices for Granite and Mission

The load at Granite Hills is located in a relatively congested area near Loveland and Los Coches. When the load at Granite is picked as one of the descriptive variables, the stressing patterns corresponding to $\{111\}\{011\}\{101\}$ are very different compared to the pattern $\{110\}$. This is the only pattern to have a very small stress at Granite. The "flatness" of the $\{111\}\{011\}\{101\}$ patterns can be attributed to the "easily congested paths" connected to the load at Granite. The smaller intercept on the Granite axis for the $\{111\}\{011\}\{101\}$ patterns correspond to larger Lagrangian multipliers which is an expression of greater systemic congestion. 


\subsection{Description of the Humboldt transmission system}

The Humboldt area is shown in the Figures below. The transmission tie lines into the area include Humboldt-Bridgeville 115 kV line 1, Humboldt-Trinity 115 kV line1, Willits-Garberville 60 line kV1, and Trinity-Maple Creek $60 \mathrm{kV}$. The substations that delineate the Humboldt Area are Low Gap $115 \mathrm{kV}$ and Humboldt $115 \mathrm{kV}$. The most critical contingencies for the Humboldt area involve (1) the loss of the Bridgeville-Cottonwood $115 \mathrm{kV}$ line along with one Humboldt Bay Power Plant and (2) the loss of the Humboldt-Trinity $115 \mathrm{kV}$ line along with one Humboldt Bay Power Plant. These contingencies are limited by the reactive power margin. They establish the target of $162 \mathrm{MW}$ as the minimum capacity necessary for the Humboldt area with $126 \mathrm{MW}$ of the Local Capacity requirement at $36 \mathrm{MW}$ of the municipal and Qualifying Facility (QF) generation.

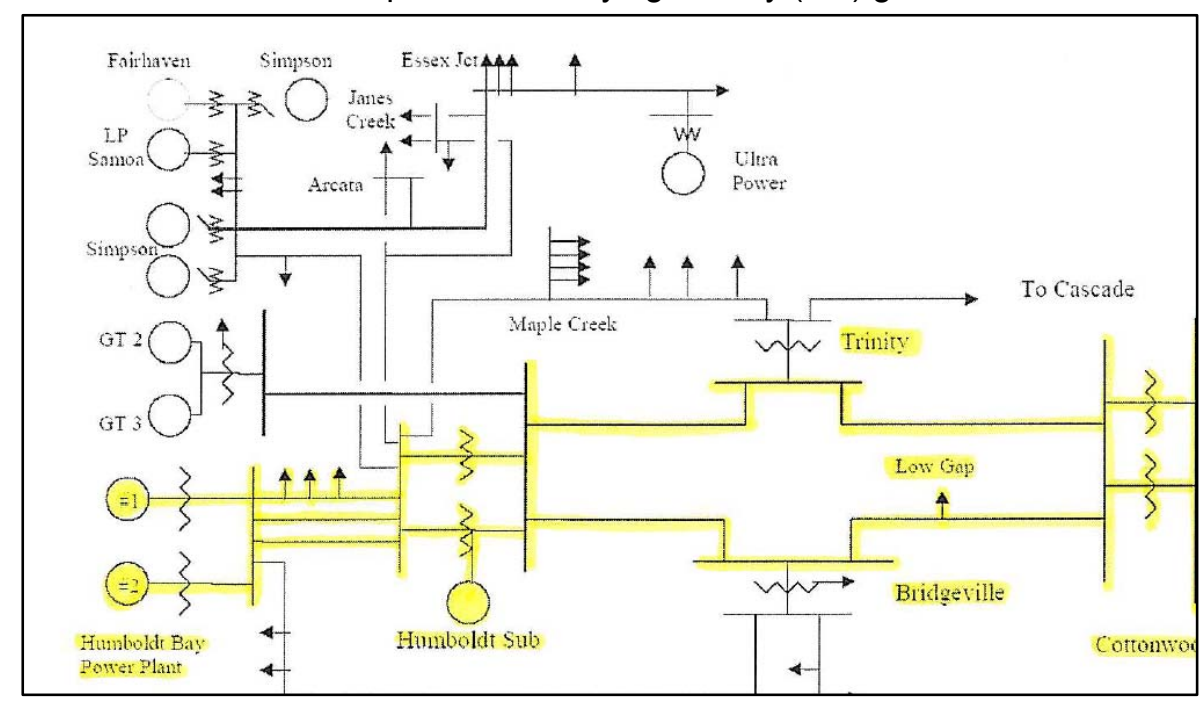

Figure 19 Humboldt Area

The Humboldt area covers most of Humboldt County. The grid is comprised of $60 \mathrm{kV}$ and $115 \mathrm{kV}$ transmission lines. Internal generation in the Humboldt area consists of two $53 \mathrm{MW}$ thermal generating units, two $15 \mathrm{MW}$ mobile gas turbines (GTs), one $25 \mathrm{MW}$ biomass self-generator and $36 \mathrm{MW}$ of QF generation.

Additionally, there is one off-line generator in the area having a capacity of $10 \mathrm{MW}$. The Humboldt Area includes the city of Eureka. This area has a winter peak load of approximately $190 \mathrm{MW}$. Pacific Gas \& Electric (PG\&E) owns the transmission and distribution systems in the Humboldt area. The major transmission lines serving this area are two $115 \mathrm{kV}$ lines from Cottonwood and one $60 \mathrm{kV}$ line from Trinity in the east and one $60 \mathrm{kV}$ line from Garberville in the south.

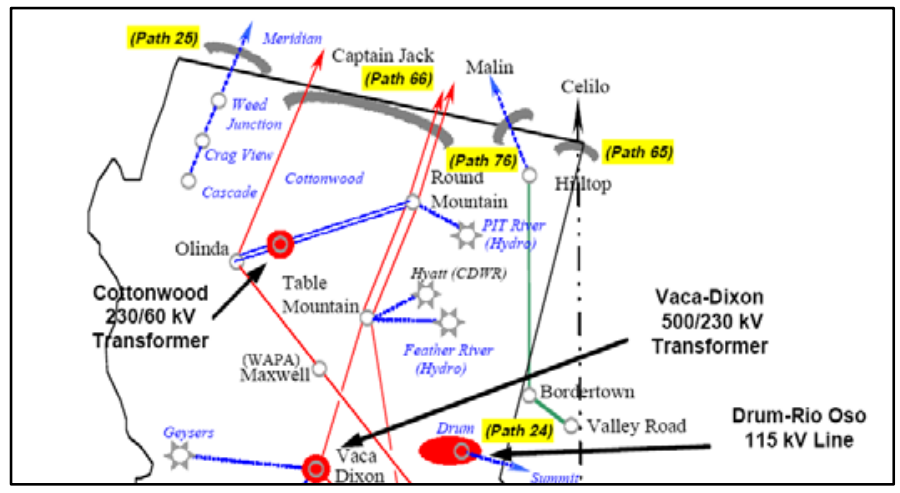

Figure 20 Location of Cottonwood in the CA EHV Transmission Network 
The import capability of the existing transmission system supplying the Humboldt Area is a function of the load in the Humboldt Area and the amount of internal generation on-line. The transmission system alone is capable of serving approximately $70 \mathrm{MW}$ of load. Internal generation in the Humboldt Area consists of two $53 \mathrm{MW}$ thermal generators, two 15MW mobile gas turbines (GTs), one $25 \mathrm{MW}$ biomass generator, as well as $46 \mathrm{MW}$ of Qualifying Facility (QF) generation. However there are currently other four off line generators in the area with a total of about $72 \mathrm{MW}$. All numbers are nameplate capacity.

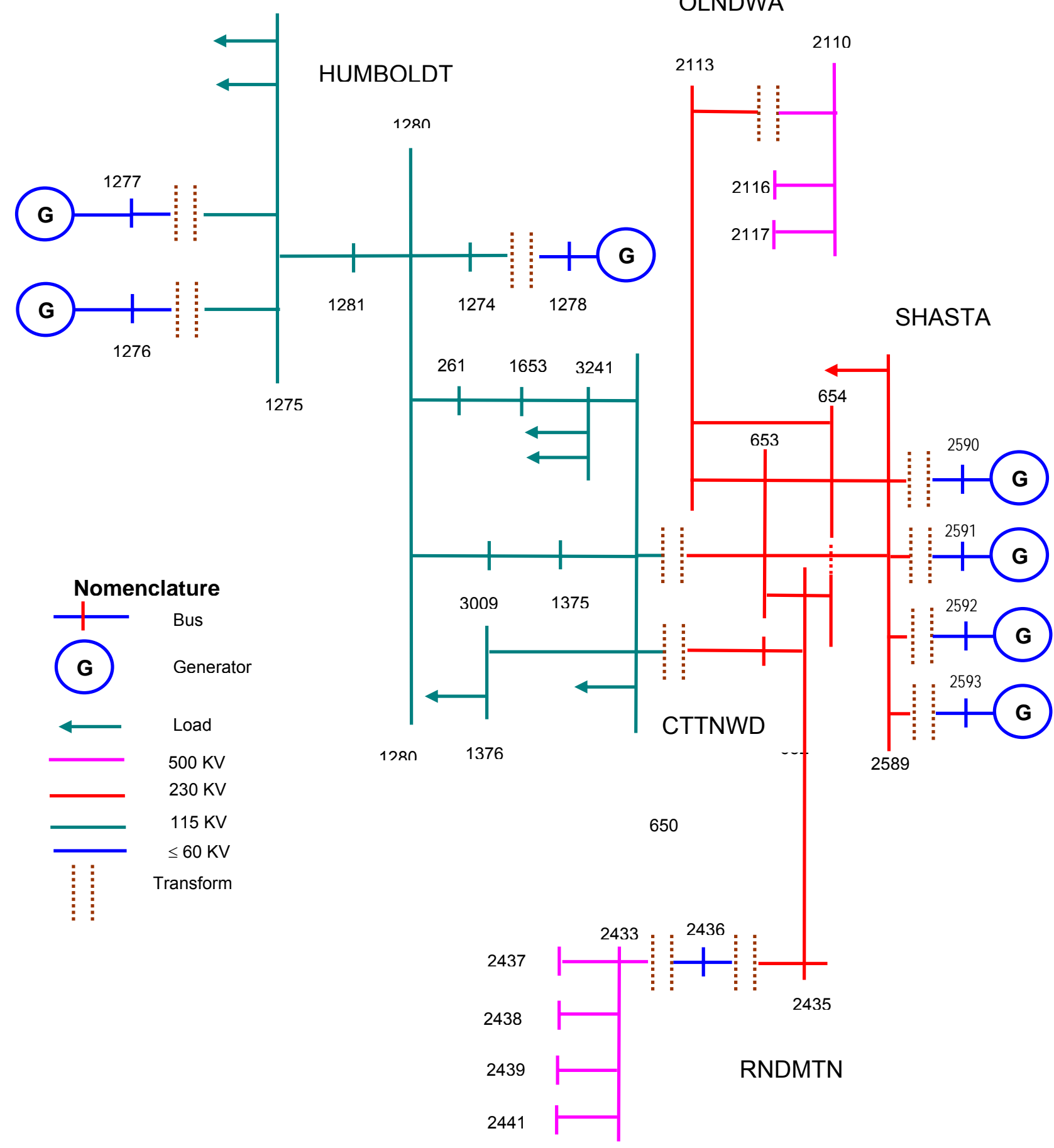

Figure 21 One Lines for the HUMBOLDT CTTNWD SHASTA area 


\subsection{Stress Patterns and RTVSA Results for Humboldt}

The stress patterns permuted the set of generation in Shasta and the Humboldt power plants. The sinks were kept unchanged at Cottonwood and Jessup. Shasta provides almost all of the generation in Pattern I. Humboldt provides one and a half times more generation than Shasta in Pattern II. The results in Figure 22 and Figure 23 are for these generator descriptive variables.

Table 6 List of Humboldt Stressing Patterns

\begin{tabular}{|c|c|c|l|c|c|c|c|}
\hline & & \multicolumn{3}{|c|}{ Source Vector } & \multicolumn{3}{c|}{ Sink Vector } \\
\hline Pattern & Color & $\begin{array}{c}\text { Bus } \\
\text { Number }\end{array}$ & $\begin{array}{l}\text { Bus } \\
\text { Name }\end{array}$ & $\begin{array}{c}\text { Participation } \\
\text { Factors }\end{array}$ & $\begin{array}{c}\text { Bus } \\
\text { Number }\end{array}$ & $\begin{array}{c}\text { Bus } \\
\text { Name }\end{array}$ & $\begin{array}{c}\text { Participation } \\
\text { Factors }\end{array}$ \\
\hline $\mathbf{I}$ & Red & 1276 & HUMBOLDT & 0.05 & 650 & CTTNWD & 0.6 \\
& & 1277 & HUMBOLDT & 0.05 & 1376 & JESSUP & 0.4 \\
& 2590 & SHASTA & 0.22 & & & \\
& & 2591 & SHASTA & 0.23 & & & \\
& & 2592 & SHASTA & 0.22 & & & 0.6 \\
\hline II & Blue & 2593 & SHASTA & 0.23 & & CTTNWD & \\
& & 1276 & HUMBOLDT & 0.30 & 650 & JESSUP & \\
& & 1277 & HUMBOLDT & 0.30 & 1376 & & \\
& & 2590 & SHASTA & 0.10 & & & \\
& & 2591 & SHASTA & 0.10 & & & \\
& & 2592 & SHASTA & 0.10 & & & \\
& & 2593 & SHASTA & 0.10 & & & \\
\hline
\end{tabular}

Table 7 Index for Humboldt Bus Numbers

\begin{tabular}{|c|c|c|c|c|c|}
\hline BUS & NAME & BUS & NAME & BUS & NAME \\
\hline 650 & CTTNWD & 261 & BRDGVL & 1653 & LOWGAP \\
652 & CTTNWD & 1375 & JESSTAP & 3009 & TRINITY \\
653 & CTTNWD & 1376 & JESSUP & 3241 & WILDWD \\
654 & CTTNWD & & & & \\
\hline 1274 & HUMBOLDT & 2589 & SHASTA & 2592 & SHASTA \\
1275 & HUMBOLDT & 2590 & SHASTA & 2593 & SHASTA \\
1276 & HUMBOLDT & 2591 & SHASTA & 1280 & HUMBOLDT \\
1277 & HUMBOLDT & 1278 & HUMBOLDT & 1281 & HUMBOLDT \\
\hline
\end{tabular}

The results in Figure 22 and Figure 23 emphasize the role of the generators at Shasta and Humboldt as descriptive variables. Negative Lagrangian Multipliers endow both hyperplane slices with positive slopes in the space of generator descriptive variable space.

A generator injection can be regarded as parameterizing a redispatch or transfer. Increasing a generator must be balanced by decreasing the distributed slack bus so what one is really looking at is the effect on voltage collapse of various transfers. A general transfer can either increase a voltage collapse margin or decrease it and this is what gives different signs of the hyperplane equation. On the other hand, loads are easier to model as descriptive variables because it is usually the case that increasing loads decrease voltage collapse margins. In particular, a negative multiplier means that increasing the transfer increases the margin, at least locally in the region of validity of the hyperplane. It is easy to construct such cases. Simply take a transfer that makes voltage collapse worse and reverse it so that source becomes sink and sink becomes source. This is the same as considering minus the transfer. For "nomogram-like" results one has to ensure that increasing all the descriptive variables make voltage collapse worse. One can probably do this by flipping the signs of some of the transfers. 


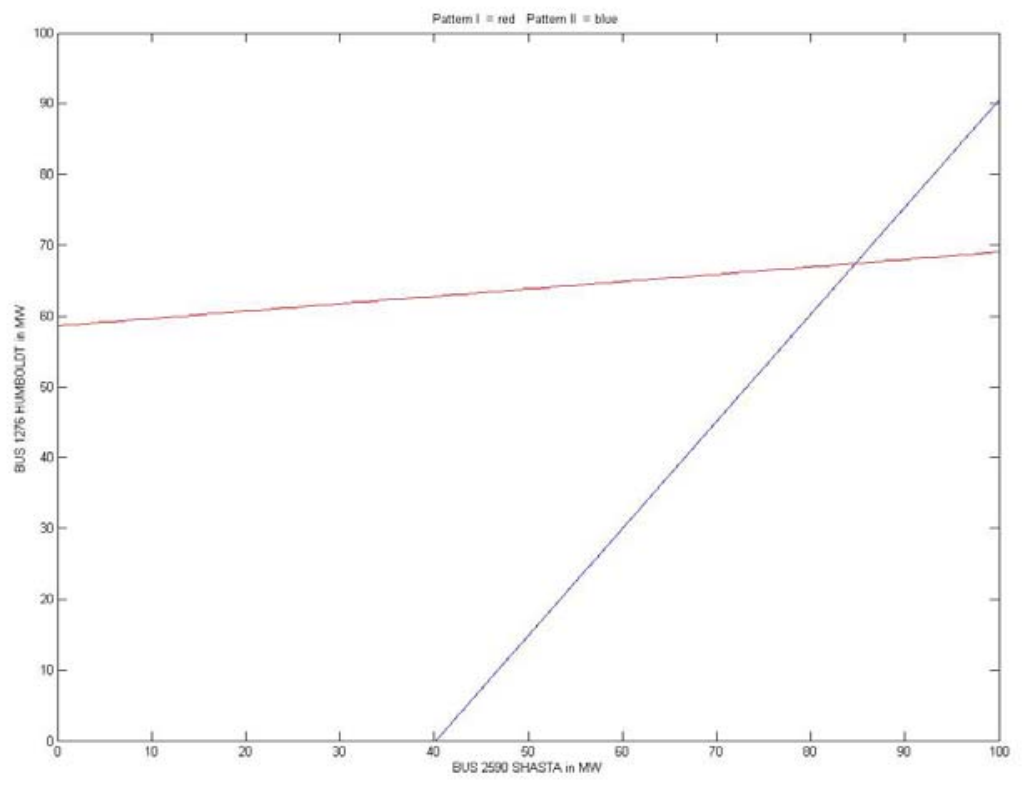

Figure 22 RTVSA Output: Hyperplane slices at Humboldt 1276 and Shasta 2590

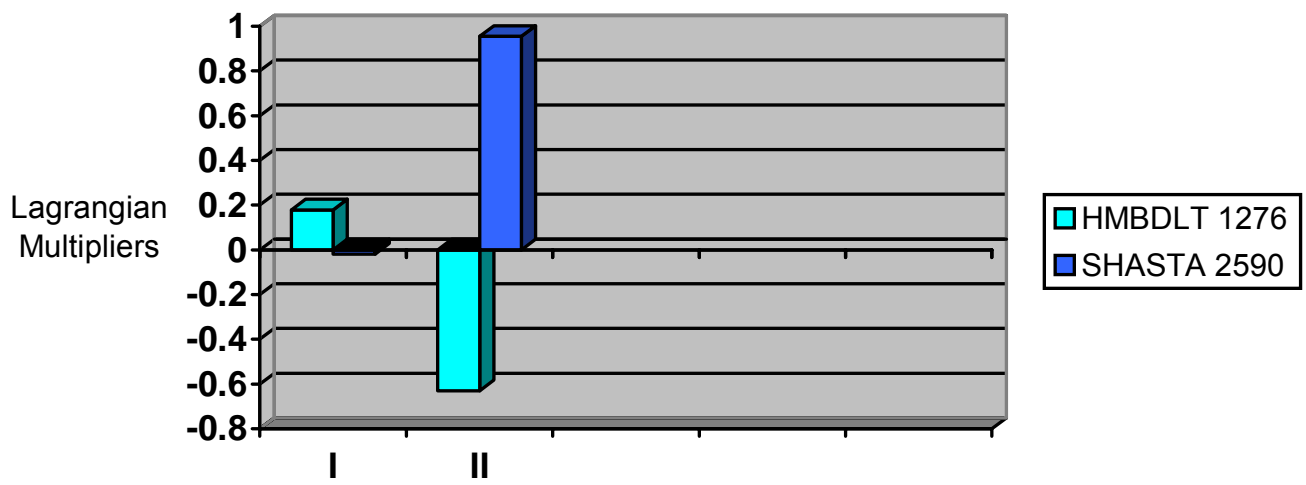

Figure 23 RTVSA Output: Lagrangian Multipliers for Humboldt

\subsection{Discussions on Exploring Patterns of Loading}

Considering all the theoretically possible patterns of loading, even if computationally feasible, is probably not desirable because it would imply reacting to voltage collapse situations that would not arise in practice. We are generally only interested in the voltage collapses that would result from the practically feasible patterns of loading that the power system could actually experience in the next few hours. Then a central question is how to specify the practically feasible patterns of loading. One can compute worst-case patterns of loadings to obtain worst-case margins. However, there can be several such locally worst-case patterns of loadings and some of these may correspond to unrealistic system loadings. The multiple worst-case loadings can correspond to voltage collapses concentrated in different areas. Some options are given below. 
- The patterns of loading can be predicted in real time. The customer load can be forecast and there should also be information about the likely bulk power transfers. However, the uncertainties and time frames of the predictions need to be managed.

- The operators may wish to specify patterns of loading that are meaningful or relevant to them. One can use past experience to select typical or worst-case patterns of loadings. Certain classes of patterns of loading can be associated with voltage collapses concentrated in different areas.

- Patterns of loading that give the best hyperplane approximations to the voltage collapse boundary for practically feasible loadings could be sought.

- Note that patterns of loading need to be fully defined in terms of injections at each generator and load bus. For example, in specifying a north-south bulk power transfer, the participation of each of the ramping up and ramping down generators needs to be specified. The choices of these participations can be determined from real-time data, from typical realistic cases, or from operator input.

\section{CONCLUSIONS}

The efficacy of using hyperplanes to approximate the boundary described by the Point of Collapse phenomenon is prone to a few caveats. The hyperplane approximation is only as good as the set of stressing vectors that are provided as input to the parameter continuation power flow. A set of "basis" vectors must be formulated that is "rich enough" to fully capture all possible stresses that the system might experience. Additionally, the Point of Collapse is usually indexed by a pre-determined set of contingencies. Each of these contingencies will spawn a parallel offline data structure to store the coefficients of the set of hyperplanes to be used in an on-line fashion when an outage is predicted.

Some of the intensive storage requirements for hyperplane coefficients along with the set of basis stress vectors may be relaxed if the boundary structure is observed to be cylindrical. The notion of such a "cylindrical" structure is manifested in the "flatness" of certain sets of stressing vectors. For example, the effect of adding a sink show that patterns $\{111\}\{101\}\{011\}$ are redundant in the descriptive variable space that includes Granite Hills. The "flatness" of these slices is because of the very small Lagrangian multipliers associated with Mission and Carlton Hills for the $\{111\}$ $\{101\}\{011\}$ stress patterns. The "flatness" of these hyperplane slices implies that it is safe to further load the system in the Carlton Hills and Mission direction. The overlaying of the $\{110\}$ stress vector does somewhat reduce the safe area of operation, which is precisely the same effect that an additional constraint would have on a standard nomogram application.

Determining the set of Lagrangian multipliers or parametric sensitivities at the exact PoC has the advantage in predicting precisely the controllable elements in the space of power injections that are responsible for the collapse. The next phase of this project will improve on the parameter continuation power flow by determining the exact $\mathrm{PoC}$ with a direct method. More meaningful descriptive variables such as cut-set flows should be used in determining the boundary of collapse. The on-line availability of distribution factors ${ }^{10}$ that map injections to cut-sets should help to improve the hyperplane identifier.

\footnotetext{
9 From Descriptive variables, operational hypersurfaces, and nomograms by lan Dobson Nov 25, 2005 - "Cylindrical view of the induced hypersurface" - To visualize the relation of the nomogram hypersurface Sn, the induced hypersurface Si and the security hypersurface S we change coordinates so that $\mathrm{Si}$ is a cylinder. Suppose there are $\mathrm{k}$ descriptive variables and $n$ parameters where $k<n$. The coordinates of parameter space are changed so that the first $k$ of the $n$ coordinates are the descriptive variables. For example, if $n=3$ and there are $k=2$ descriptive parameters and one other parameter and $\mathrm{Sn}$ is the arc of a circle, then $\mathrm{Si}$ is an arc of the surface of a cylinder. The issue of approximating the security hypersurface $\mathrm{S}$ by $\mathrm{Si}$ is how well $\mathrm{S}$ can be approximated by an Si of cylindrical form inside $\mathrm{S}$.

10 An example is the EPRI Interchange Distribution Calculator
} 
Note that while the worst case loading margin computation may well be useful, the worst case is sometimes an unrealistic pattern of loading that would not happen in practice. It is better to use the hyperplane methods to be restricted to practically feasible loadings. The experience seems to be that the closest bifurcation direction search should be restricted to feasible cones. The metric for the closest bifurcation should also be scrutinized according to the intended use of the closest bifurcation information: The Euclidean norm gives a measure of parameter space robustness but it does not give the increase in load or generator powers given by an L1 norm. 


\section{REFERENCES}

[1] P. Kundur, Power System Stability and Control, New York: McGraw-Hill, 1994.

[2] S. Lindahl, "Case studies of recent blackouts", CRIS International Workshop on Power System Blackouts Causes, Analyses, and Countermeasures, Lund, Sweden, May 3, 2004.

[3] Final Report on the August 14, 2003 Blackout in the United States and Canada: Causes and Recommendations, U.S. - Canada Power System Outage Task Force, April 5, 2004. Available online at http://www.nerc.com/ filez/blackout.html.

[4] G. Doorman, G. Kjǿlle, K. Uhlen, E. S. Huse, and N. Flatabǿ, "Vulnerability of the Nordic Power System", Report to the Nordic Council of Ministers, SINTEF Energy Research, May 2004. Available online at http://sparky.harvard.edu/hepg/Papers/Doorman.vul.nordic. system.0504.pdf

[5] A. Kurita and T. Sakurai, "The Power System Failure on July 23, 1987 in Tokyo", in Proceedings of the 27th Conference on Decision and Control, December 1988.

[6] G.C. Bullock, "Cascading Voltage Collapse in West Tennessee, August 22, 1987", Georgia Institute of Technology 44th Annual Protective Relaying Conference, May 2--4 1990.

[7] "The Electric Power Outages In The Western United States, July 2-3, 1996", DOE Report to the President, August 2, 1996. Available online at $\mathrm{ftp}: / / w w w . n e r c . c o m / p u b / s y s / a l l \_u p d l / d o c s / p u b s / d o e r e p t . p d f$.

[8] "Western Systems Coordinating Council Disturbance Report for the Power System Outage that Occurred on the Western Interconnection on August 10, 1996", October 18, 1996. Available online at ftp://www.nerc.com/pub/sys/all updl/docs/pubs/AUG10FIN.pdf.

[9] "California ISO System Disturbance Report: December 8, 1998 - San Francisco Area". Available online at http://www.CA ISO.com/docs/1999/03/31/1999033116341717931.pdf

[10] N. Dizdarevic, M. Majstrovic, S. Cudjic Coko, N. Mandic, and J. Bonovic, "Causes, Analyses and Countermeasures with Respect to Blackout in Croatia on January 12, 2003", CRIS International Workshop on Power System Blackouts Causes, Analyses, and Countermeasures, Lund, Sweden, May 3, 2004.

[11] "Technical Analysis of the August 14, 2003, Blackout: What Happened, Why, and What Did We Learn?", Report to the NERC Board of Trustees by the NERC Steering Group, July 13, 2004. Available online at ftp://www.nerc.com/pub/sys/all updl/docs/blackout/ NERC_Final_Blackout_Report_07_13_04.pdf

[12] "Power failure in Eastern Denmark and Southern Sweden on 23 September 2003", Final report on the course of events, Ekraft System, November 4, 2003. Available online at http://www.elkraft-system.dk

[13] "The Black-Out In Southern Sweden and Eastern Denmark", 23 September, 2003, Preliminary Report, PM 2003-10-02, Svenska Kraftnät. Available at http://www.svk.se/upload/3195/Disturbance Sweden DenmarkSept23.pdf

[14] "Interim Report of The Investigation Committee on the 28 September 2003 Blackout in Italy", Press Release, Union for the Coordination of Transmission of Electricity UCTE, Brussels, Belgium, October 3, 2003. Available online at http://www.energieschweiz.ch/imperia/md/content/medienmitteilungen/mm06-122003/82.pdf

[15] "Black-Out: The Events Of 28 September 2003", Press Release, Gestore Rete Transmissione Nazionale, Italy, October 1, 2003. Available online at http://www.grtn.it/eng/documentinewsstatiche/blackout28set03.pdf 
[16] C, Vournas, "Technical Summary on the Athens and Southern Greece Blackout of July 12, 2004". Available online at http://www.pserc.org/Greece_Outage_Summary.pdf.

[17] Отчет по расследованию аварии в ЕЭС России, происшедшей 25.05.2005. Председатель комиссии по расследованию аварии В.К.Паули, 18 июня 2005г. [Report on failure inquiry in UES of Russia that took place at May 25,2005 - in Russian]. Available online at: http://www.mosenergo.ru/download/r410 account.zip.

[18] "Voltage Stability of Power Systems: Concepts, Analytical Tools, and Industry Experience", IEEE Special Publication90TH0358-2-PWR, 1990.

[19] "Modeling of Voltage Collapse Including Dynamic Phenomena", CIGRE Task Force 38-0210, 1993.

[20] P. W. Sauer, K. Tomsovic, J. Dagle, S. Widergren, T. Nguyen, and L. Schienbein, "Integrated Security Analysis", Final Report, CERTS, July 2004. Available online at http://certs.lbl.gov/CERTS_P_RealTime.html.

[21] "Memorandum on the Development and the Reasons of the East Coast US Blackout on August 14, 2003", Department of Relay Protection and Automatics, SO-CDA, September 10, 2003 - in Russian.

[22] S. Vakhterov, "It Was Possible to Avoid the Blackout", Power Market, No. 1, 2004. - In Russian.

[23] Y. V. Makarov, V. I. Reshetov, V. A. Stroev, and N. I. Voropai, "Blackout Prevention in the United States, Europe and Russia", Proceedings of the IEEE - Special Issue on Power Technology \& Policy "Forty years after the 1965 Blackout", Vol. 93, No. 11, November 2005, pp. 1942-1955 (Invited paper).

[24] "Voltage Stability Criteria, Undervoltage Load Shedding Strategy, and Reactive Power Reserve Monitoring Methodology", Final Report, Reactive Power Reserve Work Group, Western Electricity Coordinating Council, May 1998. Available online at $\mathrm{http}: / /$ www.wecc. $\mathrm{biz} /$ modules.php?op=modload\&name=Downloads\&file=index\&req=getit\&lid= 1038.

[25] T. Van Cutsem and R. Mailhot, "Validation of a Fast Voltage Stability Analysis Method on the Hydro-Quebec System", IEEE Transactions on Power Systems, Vol. 12, No. 1, pp. 282-292, February 1997.

[26] B. Gao, G. K. Morrison, and P. Kundur, "Voltage Stability Evaluation Using Modal Analysis", IEEE Trans. on Power Systems, Vol. 7, No. 4, November 1992, pp. 1529-1542.

[27] "Reliability Criteria", Western Electricity Coordinating Council, April 2005. Available online at http://www.wecc. biz/modules.php?op=modload\&name=Downloads\&file=index\&req=getit\&lid= 1029

[28] C. W. Taylor, Power System Voltage Stability. New York: McGraw-Hill, 1994.

[29] T.J.E Miller, Editor, Reactive Power Control in Electric Systems. New York: John Wiley \& Sons, 1982

[30] H. K Clark, "More attention to reactive [power] and voltage will present opportunities to improve grid reliability", DOE Blackout forum, December 22, 2003. Available online at http://www.electricity.doe.gov/govforums/view_thread2.cfm?post_id=186.

[31] T. Van Cutsem, I. Dobson (Editor), C. DeMarco, D. Hill, I. Hiskens, T. Overbye, M. Venkatasubramanian, C. Vournas, Basic Theoretical Concepts, Chapter 2, IEEE Voltage Stability Work Group Report, April 1998. Available online at http://eceserv0.ece.wisc.edu/ dobson/WG/.

[32] B.M. Weedy and B.R. Cox, "Voltage stability of radial power links", Proc. IEEE, Vol.115, pp. 528-536, April 1968. 
[33] P. M. Anderson and A. A. Fouad. Power System Control and Stability, IEEE Press, New York 1994.

[34] A. M. Lyapunov, The General Problem of the Stability of Motion, London, Washington DC, Tayor \& Francis, 1992.

[35] R. Seydel, From Equilibrium to Chaos: Practical Bifurcation and Stability Analysis, SpringerVerlag, New York, 1994.

[36] S. Strogatz, Nonlinear Dynamics and Chaos: with Applications in Physics, Biology, Chemistry, and Engineering, Addison-Wesley, Reading MA, 1994.

[37] Thompson, J.M.T., Stewart, H.B., Nonlinear Dynamics and Chaos: Geometrical Methods for Engineers and Scientists, John Wiley, New York, 1986.

[38] E.H. Abed, P.P. Varaiya, "Nonlinear Oscillations in Power Systems", International Journal of Electric Energy and Power Systems, vol. 6, no. 1, January 1984, pp. 37-43.

[39] C.-W. Tan, M. Varghese, P. Varaiya, F.F. Wu, "Bifurcation, Chaos, and Voltage Collapse in Power Systems", Proceedings of the IEEE, Special issue on nonlinear phenomena in power systems, November 1995, vol. 83, no. 11, pp. 1484-1539.

[40] V. Ajjarapu and B. Lee, "Bibliography on Voltage stability", Available online at http://www.ee.iastate.edu/ venkatar/Biblio/biblio.html\#Group5.

[41] V. A. Venikov, V. A. Stroev, V. I. Idelchick, and V. I. Tarasov, "Estimation of electric power system steady-state stability in load flow calculation", IEEE Trans. on Power Apparatus and Systems, Vol. PAS-94, pp.1034 - 1041, May/June 1975.

[42] R. C. Hardiman, M. Kumbale, and Y. V. Makarov, "An Advanced Tool for Analyzing Multiple Cascading Failures", Proceedings of the 8th International Conference on Probabilistic Methods Applied to Power Systems, lowa State University, Ames, lowa, September 12-16, 2004.

[43] I. Dobson, "The Irrelevance of Load Dynamics for the Loading Margin to Voltage Collapse and its Sensitivities", in L. H. Fink, Editor, Bulk power system voltage phenomena III, voltage stability, security \& control, Proceedings of the ECC/NSF workshop, Davos, Switzerland, August 1994.

[44] C.A. Canizares, Conditions for saddle-node bifurcations in AC/DC power systems, Electrical Energy \& Power Systems, Vol. 17, No. 1, pp. 61-68, 1995.

[45] Y. V. Makarov, D. H. Popovic, and D.J. Hill, "Stabilization of Transient Processes in Power Systems by an Eigenvalue Shift Approach", IEEE Transactions on Power Systems, Vol. 13, Issue 2, May 1998, pp. $382-388$.

[46] Yu. E. Gurevich, L.E. Libova, and A.A. Okin, "Design of Stability and Emergency Control Automatic Devices in Power Systems", Energoatomizdat, Moscow, 1990 - in Russian.

[47] R. R. Austria, X. Y. Chao, N. P. Reppen, and D. E. Welsh, "Integrated Approach to Transfer Limit Calculations", IEEE Computer Applications in Power, January 1995, pp. 48-52.

[48] B. Gao, G.K. Morison and P. Kundur, "Towards the Development of a Systematic Approach for Voltage Stability Assessment of Large-Scale Power Systems", IEEE Transactions on Power Systems, Vol. PWRS-11, No. 3, August 1996, pp. 1314-1324.

[49] V.A. Matveev, "A Method of Numeric Solution of Sets of Nonlinear Equations", Zhurnal Vychislitelnoi Matematiki I Matematicheskoi Fiziki, Vol. 4, No. 6, pp. 983-994, 1964 - in Russian.

[50] V.I. Tarasov, "Implementation of a Permanent Loading Procedure to Definition of Load Flows on a Limit of Aperiodic Steady-State Stability", Voprosy primeneniya matematicheskih metodov pri upravlenii regimami i razvitiem elektricheskih sistem, Irkutsk, pp. 50-56, 1975 in Russian. 
[51] A.M. Kontorovich, Y.V. Makarov and A. A. Tarakanov, "Improvements of a Permanent Loading Technique to Compute Load Flows on Stability Margin", Trudy LPI, No. 380, pp. 3741, 1982 - in Russian.

[52] A. M. Kontorovich and Y. V. Makarov, "A permanent loading technique for fast analysis of power system stability limit load flows", USSR National Scientific and Technical Conference "Problems of Stability and Reliability of the USSR Power System", Dushanbe, 1989 - In Russian.

[53] P. Kessel and H. Glavitsch, "Estimating the voltage stability of a power system", IEEE Transactions on Power Delivery, Vol.PWRD-1, No. 3, July 1986, pp. 346-354.

[54] G. B. Price, "A generalized circle diagram approach for global analysis of transmission system performance", IEEE Trans. Power Apparatus and Systems, Vol. PAS-103, No. 10, pp. 2881-2890, October 1984, pp. 2881-2890.

[55] V. Ajjarapu and C. Christy, "The Continuation Power Flow : A Tool to Study Steady State Voltage Stability," IEEE Transactions on Power Systems, Vol. 7, No. 1, February 1992, pp. 416-423.

[56] T. Van Cutsem, C. Vournas, "Voltage Stability of Electric Power Systems", Kluwer Academic Publishers (Power Electronics and Power Systems Series), Boston, 1998

[57] A. Bihain, G. Burt, F. Casamatta, T. Koronides, R. Lopez, S. Massucco, D. Ruiz-Vega, and C. Vournas, "Advanced Perspectives and Implementation of Dynamic Security Assessment in the Open Market Environment", CIGRE 2002, France, August 2002.

[58] J. Jarjis and F.D. Galiana, "Quantitative Analysis of Steady State Stability in Power Networks", IEEE Trans. On Power Apparatus and Systems, Vol. PAS-100, No. 1, January 1981, pp. 318-326.

[59] A.M. Kontorovich, A.V. Kryukov, Y.V. Makarov, et al, Methods of stability indices computations for complicated power systems, Publishing House of the Irkutsk University, Irkutsk, 1988 (in Russian).

[60] C.A. Cañizares and F.L. Alvarado, "Computational Experience with the Point of Collapse Method on Very Large AC/DC Systems", Proceedings: Bulk Power System Voltage Phenomena - Voltage Stability And Security, ECC/NSF Workshop, Deep Creek Lake, MD, August 1991; published by ECC Inc., Fairfax, Virginia.

[61] T. Van Custem, "A method to compute reactive power margins with respect to voltage collapse", IEEE Trans. on Power Systems\} Vol. 6, No. 1, February 1991, pp. 145-156.

[62] C. A. Cañizares, , F. L. Alvarado, C. L. DeMarco, I. Dobson, W. F. Long, "Point of Collapse Methods Applied to AC/DC Power Systems", IEEE Transactions on Power Systems, Vol. 7, Issue 2, May 1992, pp. $673-683$.

[63] I. Dobson, "Observations on the Geometry of Saddle Node Bifurcation and Voltage Collapse in Electrical Power Systems", IEEE Transactions on Circuits and Systems I: Fundamental Theory and Applications, Vol. 39, Issue 3, March 1992, pp. 240 - 243.

[64] P. W. Sauer and B. C. Lesieutre, "Power System Load Modeling", J. H. Chow, P. V. Kokotovic and R. J. Thomas, (Editors), Systems and Control Theory for Power Systems, Springer-Verlag 1995, pp. 283-313.

[65] P. A. Löf, T. Smed, G. Andersson, and D. J. Hill, "Fast Calculation of a Voltage Stability Index," IEEE Trans. Power Systems, vol. 7, no. 1, pp. 54-64, February 1992.

[66] Y. Tamura, H. Mori, and S. Iwamoto, "Relationship Between Voltage Instability and Multiple Load Flow Solutions in Electric Power Systems,", IEEE Trans. on Power Apparatus and Systems, Vol.PAS-102, pp.1115 --1123, May 1983. 
[67] C.J. Parker, I.F. Morrison, and D. Sutanto, "Application of an Optimization Method for Determining the Reactive Margin From Voltage Collapse in Reactive Power Planning," IEEE Trans. Power Systems, Vol. 11, No. 3, August 1996, pp. 1473-1481.

[68] G.D. Irisarri, X. Wang, J. Tong, and S. Mokhtari, "Maximum Loadability of Power Systems Using Interior Point Non-linear Optimization Method," IEEE Trans. Power Systems, Vol. 12, No. 1, February 1997, pp. 162-172.

[69] "Operations Review of June 14, 2000 PG\&E Bay Area System Events Using AEMPFAST \& Software", Consultant Report P500-03-085F, Prepared by CERTS for the California Energy Commission, October 2003.

[70] I. Dobson and L. Lu, "Computing an Optimal Direction in Control Space to Avoid Saddle Node Bifurcation and Voltage Collapse in Electric Power Systems", IEEE Trans. on Automatic Control, Vol. 37, No. 10, October 1992, pp. 1616-1620.

[71] I. Dobson, L. Lu and Y. Hu, "A Direct Method for Computing a Closest Saddle Node Bifurcation in the Load Power Parameter Space of an Electric Power System", IEEE International Symposium on Circuits and Systems, Singapore, June 1991, pp. 3019-3022.

[72] I. Dobson and L. Lu, 'New Methods for Computing a Closest Saddle Node Bifurcations and Worst Case Load Power Margin for Voltage Collapse", IEEE Trans. on Power Systems, Vol. 8, No. 3, August 1993, pp. 905-913.

[73] F. Alvarado, I. Dobson and Y. Hu, "Computation of Closest Bifurcations in Power Systems", IEEE Trans. on Power Systems, Vol. 9, No. 2, May 1994, pp. 918-928.

[74] Y.V. Makarov, Q. Wu, D.J. Hill, D.H. Popovic and Z.Y. Dong, "Coordinated Steady-State Voltage Stability Assessment and Control", Proc. International Conference on Advances in Power System Control, Operation and Management APSCOM'97, Wanchai, Hong Kong, November 11-14, 1997.

[75] J. Kanetkar and S. Ranade, "Compact Representation of Power System Security - A Review," Proceedings of the North American Power Symposium, Reno, Nevada, 1992, pp. 312-321.

[76] F. D. Galiana, "Power Flow Feasibility and the Voltage Collapse Problem", Proceedings of 23rd CDC, Las Vegas, NV, December 1984, pp. 485-487.

[77] P. Kessel and H. Glavitsch, "Estimating the Voltage Stability Regions of a Power System," IEEE Transactions Power Delivery, Vol. PWRD-1, July 1986, pp. 346-354.

[78] J. D. McCalley, S. Wang, R. T. Treinen, and A. D. Papalexopoulos, "Security Boundary Visualization for Systems Operation", IEEE Transactions on Power Systems, Vol. 12, No. 2, May 1997, pp. 940-947.

[79] G. Zhou and J. D. McCalley, "Composite Security Boundary Visualization", IEEE Transactions on Power Systems, Vol. 14, No. 2, May 1999, pp. 725-731.

[80] J. Su, Y. Yu, H. Jia, P. Li, N. He, Z. Tang, and H. Fu, "Visualization of Voltage Stability Region of Bulk Power System", Proceedings of International Conference on Power System Technology PowerCon 2002, Vol. 3, 2002, pp. 1665-1668.

[81] M. A. Pai, "Power System Stability", Amsterdam, The Netherlands: North Holland, 1981.

[82] Y. Zeng and Y. Yu, "A Practical Direct Method for Determining Dynamic Security Regions of Electrical Power Systems", Proceedings of the International Conference on Power System Technology PowerCon 2002, Vol. 2, 2002, pp. 1270-1274.

[83] I. A. Hiskens and R. J. Davy, "Exploring the Power Flow Solution Space Boundary", IEEE Transactions on Power Systems, Vol. 16, No. 3, August 2001.

[84] Y.V. Makarov, V.A. Maslennikov, and D.J. Hill, "Calculation of Oscillatory Stability Margins in the Space of Power System Controlled Parameters", Proc. of the International Symposium 
on Electric Power Engineering Stockholm Power Tech, Vol. Power Systems, Stockholm, Sweden, June 1995, pp. 416-422.

[85] Y. V. Makarov and I. A. Hiskens, "A continuation method approach to finding the closest saddle node bifurcation point", Proceedings of the NSF/ECC Workshop on Bulk Power System Voltage Phenomena III, Davos, Switzerland, August 1994. Published by ECC Inc., Fairfax, Virginia.

[86] K. Huang and H. Yee, "Improved Tangent Hyperplane Method for Transient Stability Studies", Proceedings of the International Conference on Advances in Power System Control APSCOM-91, Vol. 1, 5-8 November, 1991, pp. 363 - 366.

[87] M. Djukanovic, D. J. Sobajic, and Y.-H. Pao, "Learning Tangent Hypersurfaces for Fast Assessment of Transient Stability, Proceedings of the Second International Forum on Applications of Neural Networks to Power Systems ANNPS '93, 19-22 April 1993, pp. 124 129.

[88] Y. Yu, Y. Zeng, C. Huang, S. T. Lee, and P. Zhang, "A Practical Direct Method for Determining Dynamic Security Regions of Electrical Power Systems by Power Perturbation Analysis", International Conference on Electrical Engineering ICEE2004, July 4-8, 2004, Sapporo, Japan.

[89] S. A. Sovalov and V. A. Semenov, Emergency Control in Power Systems, Energoatomizdat, Moscow, 1988 - in Russian.

[90] M. A. El-Sharkawi, R. J. Marks, M. E. Aggoune, D. C. Park, M. J. Damborg, and L. E. Atlas, "Dynamic Security Assessment of Power Systems Using Back Error Propagation Artificial Neuron Networks," Proceedings of the 2nd Symposium on Expert System Applications to Power Systems, Seattle, WA, July 1989.

[91] R. Kumar, A. Ipahchi, V. Brandwajan, M. A. El-Sharkawi, and G. Cauley, "Neuron Networks for Dynamic Security Assessment of Large Scale Power Systems: Requirements Overview," Proceedings of 1st International Forum on Applications of Neuron Networks to Power Systems, Seattle, WA, July 1991, pp. 65-71.

[92] A.A. El-Keib and X. Ma, "Application of Artificial Neural Networks in Voltage Stability Assessment," IEEE Transactions on Power System, Vol. 10, No.4, Nov. 1995, pp. 18901896.

[93] S. Chauhan and M.P. Dava, "Kohonen Neural Network Classifier for Voltage Collapse Margin Estimation," Electric Machines and Power Systems, Vol. 25, No. 6, July 1997, pp. 607-619.

[94] Y. Mansour, A. Y. Chang, J. Tamby, E. Vaahedi, B. R. Corns, and M. A. El-Sharkawi, "Large Scale Dynamic Security Screening and Ranking Using Neuron Networks," IEEE Transactions on Power Systems, Vol. 12, No. 2, May 1997, pp. 954-960.

[95] H B Wan and Y H Song, "Hybrid Supervised and Unsupervised Neural Network Approach to Voltage Stability Analysis", Electric Power System Research, Vol. 47, No. 2, 1998, pp.115122.

[96] V. R. Dinavahi and S. C. Srivastava, "ANN Based Voltage Stability Margin Prediction", IEEE Power Engineering Society Summer Meeting 2001, Vol. 2, 15-19 July 2001, pp. 1275-1280.

[97] A. Sittithumwat and K. Tomsovic, "Dynamic Security Margin Estimation Using Artificial Neural Networks", IEEE Power Engineering Society Summer Meeting 2002, Vol. 3, 2002, pp. 13221327.

[98] M. M. Salamaa, E. M. Saieda, M. M. Abou-Elsaada, and E. F. Gharianyb, "Estimating the Voltage Collapse Proximity Indicator Using Artificial Neural Network", Energy Conversion and Management, Vol. 42, No. 1, January 2001, pp. 69-79.

[99] H. Hakim, "Application of Pattern Recognition in Transient Security Assessment," Journal of Electrical Machines and Power Systems, Vol. 20, 1992, pp. 1-15. 
[100] L. Wehenkel, Automatic Learning Techniques in Power Systems, Kluwer Academic Publishers, MA, 1998.

[101] C. Aldea and S. C. Savulescu, "Evaluation of the Stability Reserve of Transelectrica's Transmission System by Using Quickstab Professional", WEC Regional Energy Forum FOREN 2004, Neptun, 13-17 June 2004.

[102] P. Dimo, “L’Analyse Nodale des Réseaux D'Énergie”, Eyrolles, 1971.

[103] T. Van Cutsem, C. Vournas, "Voltage Stability of Electric Power Systems", Kluwer Academic Publishers (Power Electronics and Power Systems Series), Boston, 1998.

[104] T. Van Cutsem and R. Mailhot, "Validation of a Fast Voltage Stability Analysis Method on the Hydro-Quebec System", IEEE Transactions on Power Systems, Vol. 12, No. 1, February 1997.

[105] F. Paganini, B.C. Lesieutre, "Generic Properties, One-Parameter Deformations, and The BCU Method", IEEE Transactions On Circuits And Systems I: Fundamental Theory And Applications", Vol. 46, No. 6, pp. 760-763, June 1999.

[106] H.-D. Chiang, C.-C. Chu, and G. Cauley, "Direct Stability Analysis of Electric Power Systems Using Energy Functions: Theory, Applications, and Perspective", Proceedings of the IEEE, 13, 1995, pp. 1497-1529.

[107] H.-D, Chiang, C.-S. Wang, and H. Li, "Development of BCU classifiers for on-line dynamic contingency screening of electric power systems", IEEE Transactions on Power Systems, 14,1999 , pp. 660-666.

[108] Y. V. Makarov, Z.-Y. Dong, and D. J. Hill, "A General Method for Small Signal Stability Analysis", IEEE Transactions on Power Systems, Vol. 13, Issue 3, August 1998, pp. 979 985.

[109] Z.-Y. Dong, Y.V. Makarov, and D.J. Hill, "Genetic Algorithms in Power System Small Signal Stability Analysis", Fourth International Conference on Advances in Power System Control, Operation and Management APSCOM-97, Vol. 1, November 11-14, 1997, pp. 342 - 347 (Conference Publication No. 450).

[110] A.A. Fouad and V. Vittal, Power System Transient Stability Analysis Using the Transient Energy Function Method, Prentice Hall, 1991.

[111] I. Dobson, "Computing a Closest Bifurcation Instability in Multidimensional Parameter Space", Journal of Nonlinear Science, Vol. 3, 1993, pp. 307-327.

[112] Y.V. Makarov, V. A. Maslennikov, and D. J. Hill, "Calculation of Oscillatory Stability Margins in the Space of Power System Controlled Parameters", Proc. International Symposium on Electric Power Engineering Stockholm Power Tech: Power Systems, Stockholm, Sweden, 18-22 June, 1995, pp. 416-422.

[113] Y. V. Makarov, D. J. Hill, and Z.-Y. Dong, "Computation of Bifurcation Boundaries for Power Systems: A New $\Delta$-Plane Method", IEEE Transactions on Circuits and Systems I: Fundamental Theory and Applications, Vol. 47, Issue 4, April 2000, pp. 536 - 544.

[114] Y.V. Makarov, D. J. Hill, and I. A. Hiskens, "Properties of Quadratic Equations and Their Application to Power System Analysis", Electrical Power and Energy Systems, 22 (2000), pp. 313-323.

[115] M. Fulczyk and M. Sobierajski, "Probabilistic assessment of power system voltage stability margin using P-Q curve", Proc. 8th International Conference on Probabilistic Methods Applied to Power Systems PSCE'2004, lowa State University, Ames, lowa, September 2004.

[116] "Voltage Stability/Security Assessment and On-Line Control", EPRI technical report TR101931-V1-4, Vol. 1-4, 1993. 
[117] “Voltage Stability Analysis Program (VSTAB)", Version 2.1, User's Manual, EPRI Research ProjectRP30400-01, August 1992.

[118] Xiaokang Xu, M. W. Gustafson, B.P Lam, J. D. Mountford., and S. L. Johnson, "Assessment of Voltage Stability and Real and Reactive Margins Using Advanced Analytical Tools", Proceedings of International Conference on Power System Technology PowerCon 2002, Vol. 4, 2002, pp. $2047-2051$.

[119] "VSAT - Voltage Security Assessment Tool", Available online: http://www.dsapowertools.com/downloads/VSAT Brochure.pdf.

[120] "PowerWorld Simulator - PVQV", Available online: http://www.powerworld.com/products/pvqv.asp.

[121] S. Lockwood, R. Navarro, E. Bajrektarevic, P. Burke, S. Kang, P. Ferron, V. Kotecha, S. Kolluri, M. Nagle, S. Lee, P. Zhang, S. K. Agarwal, M. Papic, J. Useldinger, P. C. Patro, L. Arnold, D. Osborn, L. Fan, L. Hopkins, Member, M. Y. Vaiman, and M. M. Vaiman, "Utility Experience Computing Physical and Operational Margins: Part I - Basic Concept and Evaluation", 2004 IEEE PES Power Systems Conference \& Exposition, New York, NY, October 10 - 13, 2004.

[122] S. Lockwood, R. Navarro, E. Bajrektarevic, P. Burke, S. Kang, P. Ferron, V. Kotecha, S. Kolluri, M. Nagle, S. Lee, P. Zhang, S. K. Agarwal, M. Papic, J. Useldinger, P. C. Patro, L. Arnold, D. Osborn, L. Fan, L. Hopkins, Member, M. Y. Vaiman, and M. M. Vaiman, "Utility Experience Computing Physical and Operational Margins: Part II - Application to Power System Studies", 2004 IEEE PES Power Systems Conference \& Exposition, New York, NY, October 10 - 13, 2004.

[123] "BOR-Transient Stability: Dynamic Security Region", Available online: http://www.vrenergy.com/docs/bor-ts.pdf

[124] M. Papic, M. Y. Vaiman, and M. M. Vaiman, "Determining a Secure Region of Operation for Idaho Power Company", IEEE Power Engineering Society General Meeting, June 12-16, 2005 , pp. $2720-2725$.

[125] "NEPLAN Electricity - Voltage Stability", Available online: http://www.neplan.ch/sites/en/neplan_elec_calcmod_voltage_stability.asp.

[126] "CYMVSTAB, Voltage Stability", Available online: http://www.cyme.com/software/cymvstab/.

[127] C.D. Vournas, "Interruptible Load as a Competitor to Local Generation for Preserving Voltage Security", Available online: http://www.transmission.bpa.gov/orgs/opi/power_stability/DirLdContVournas.pdf.

[128] "On-Line Application of DSATools", Available online: http://www.dsapowertools.com/html/prod_application.php

[129] "POM - Real Time (POM-RT)", Available online: http://www.vrenergy.com/pom-rt.htm.

[130] E. Hnyilicza, S. T. Y. Lee, F. C. Scbweppe, "Steady-State Security Regions: Set-Theoretic Approach," Proceedings of the IEEE PICA Conference, pp. 347-355,1975.

[131] S. T. Lee, "Community activity room as a new tool for transmission operation and planning under a competitive power market", IEEE Bologna Power Tech Conference Proceedings, Vol. 4, 23-26 June, 2003.

[132] "QuickStab at a Glance", Available online: http://www.eciqs.com/QuickStab_at_a_Glance.htm.

[133] T. Van Cutsem, F. Capitanescu, C. Moors, D. Lefebvre, V. Sermanson, "An Advanced Tool for Preventive Voltage Security Assessment", Proc. 7th Symposium of Specialists in Electric Operational and Expansion Planning (SEPOPE), Curitiba (Brazil), May 2000. 
Appendix C

Deliverable Task 2.0 CA ISO Voltage Security Assessment (VSA) Prototype Functional Specifications/Prototype Development 


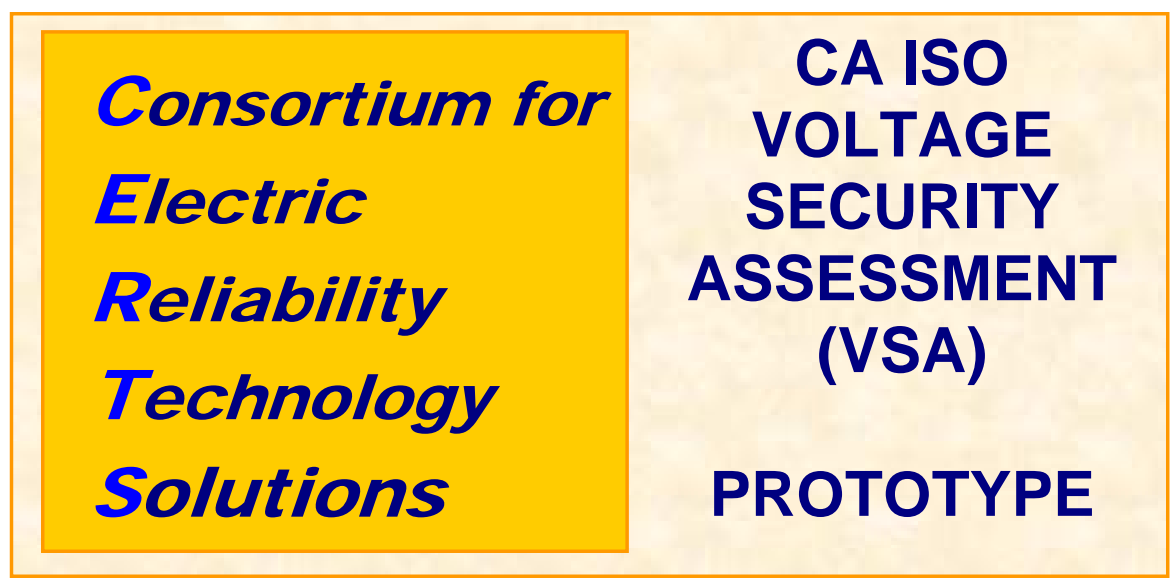

\section{FUNCTIONAL SPECIFICATION PROTOTYPE DEVELOPMENT}

\section{CALIFORNIA INDEPENDENT SYSTEM OPERATOR (CA ISO)}




\section{TABLE OF CONTENTS}

Page

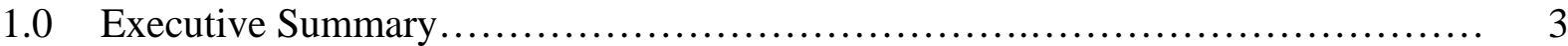

1.1 Objective.............................................................. 3

1.2 Technical Survey........................................................ 3

1.3 Project Summary.................................................. 3

1.4 Grid Monitoring Visualization Solution.................................. 4

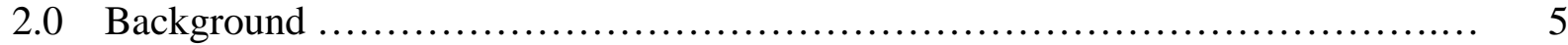

2.1 Key Definitions........................................................ 5

2.2 Voltage Security Assessment............................................ 5

3.0 Voltage Security Assessment Functionality .................................. 5

3.1 Contingency simulation..................................... 6

3.2 Voltage security regions and hyperplanes........................ 6

3.3 Voltage security margin assessment.............................. 6

3.4 Contingency ranking.......................................... 6

3.5 Identification of weak elements.............................. 7

4.0 Data Requirements.................................................... 7

5.0 Visualization Solution Approach and Architecture.............................. 8

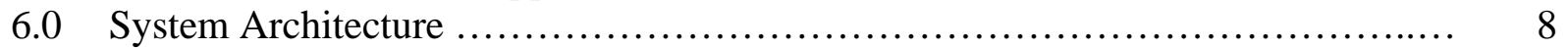

7.0 Implementation Schedule................................................ 11

\section{LIST OF FIGURES}

Figure 1: Voltage Security Assessments Prototype............................ 9

\section{LIST OF TABLES}

Table I: $\quad$ Implementation Schedule for CA ISO VSA Project....................... 11 


\subsection{EXECUTIVE SUMMARY}

\subsection{Objective}

Research and develop a Voltage Security Assessment (VSA) prototype to monitor system voltage conditions and provide real time dispatchers with reliability information related to reactive margin, abnormal nodal voltages, weak elements and contingency rankings.

\subsection{Technical Survey}

The CA ISO, the California Energy Commission (Energy Commission) and the Consortium for Electric Reliability Technology Solutions (CERTS) initially agreed to develop a real-time voltage and transient analysis security margin assessment tool using hyperplane technology. The CA ISO had proposed using the concept of hyperplane to implement this objective. A survey was conducted among experts to determine the feasibility of implementing this approach. The experts agreed that the hyperplane approach is well suited for voltage assessments but not yet proven to conduct transient analysis. Upon completion of the survey, the Energy Commission, CA ISO and CERTS agreed to implement a hyperplane based Voltage Security Assessment (VSA) prototype for the CA ISO control area. No transient analysis will be performed with hyperplanes at this time.

\subsection{VSA Project Summary}

Objective

Develop a prototype for Real-Time Voltage Security Assessment (VSA) application and prepare functional specifications to be used by CA ISO to select a vendor and for vendor to use it in the development of a production quality application for CA ISO.

Real-Time Voltage Security Assessment Project: Research and develop a Voltage Security Assessment (VSA) prototype to monitor system voltage conditions and provide system operators with real time reliability metrics related to reactive margin, abnormal nodal voltages, weak elements and contingency rankings.

What Will The Application Do?

The VSA application will take CA ISO system model and data, develop security regions (a form of nomogram) using hyperplanes, calculate security indices and identify and display abnormal low voltages and weak elements. This application will also perform contingency analysis and provide the system operators with contingency rankings for system monitoring and correction.

Why Is The Application Needed?

The CA ISO and neighboring systems are experiencing an increase in reactive power consumption. Inadequate reactive supply was identified as a cause of the August 
2003 blackout, and closer management of voltage is a key objective for reliability. This application will allow the system operators and reliability coordinators to monitor system voltage conditions and detect conditions that make the system vulnerable to voltage collapse and to take timely preventive actions. It will also provide operating staff with a more realistic security assessment with the potential of better utilization of the system facilities.

How Will The Application Benefit The Operators and Reliability Coordinators?

This application will provide near real-time assessment of voltage conditions and how they relate to safe and secure operating limits under normal and contingency conditions. System operators and reliability coordinators can use this information to evaluate and implement corrective actions such as generation re-scheduling, reactive VAR switching and/or load shedding.

What Are The Requirements To Implement This Application?

The VSA application will use data from the CA ISO state estimator and, therefore, will need to be connected to CA ISO system such that there is a continuous flow of model and data for analysis by the VSA application. The CA ISO suggested the Humbolt area be used in the development of the prototype. CA ISO will assist CERTS/EPG with the following:

- Initially provide a GE CA ISO system solved case with emphasis on the Humbolt area. Later, provide a solved case from the CA ISO State Estimator in PSLF bus/branch format.

- An algorithm to stress the system feeding the Humbolt area

- A contingency file selecting relevant contingencies to be analyzed in the prototype development

\subsection{Project Grid Monitoring Visualization Solution}

The VSA application will be designed for use by the CA ISO operators in monitoring and predicting system performance two hours ahead. CERTS-EPG will work with the CA ISO to design a data display format suitable to CA ISO operators. The real time display will have the following information:

- Security region whose security boundaries are determined in MW flows

- The current operating condition

- Worst case contingencies leading to voltage collapse

- Contingencies with insufficient stability margin

- Contingency ranks based on severity indices

- Abnormal reductions of nodal voltages

- Voltage security margins

- Weak elements and geographical locations

The Real Time Security Display will use a four panel display format developed by CERTS/EPG. This display will be designed taken into account the results of a yet to 
be performed Human Factor analysis.

\subsection{BACKGROUND}

\subsection{Key Definitions}

Security Region - Is the set of operating conditions where it's safe for the power system to operate. This region will be bounded by hyperplanes developed offline.

Hyperplane - A boundary established through offline analysis determined by stressing a cut set until the system reaches a state of voltage system instability.

Voltage Stability - The ability of a power system to maintain adequate voltages at all buses in the system under varying loading conditions.

Voltage Collapse - The condition where there is uncontrollable decay in system voltages at one or more load buses or even a significant portion of the network.

Security Margin - Is the available margin on a transmission path determined by subtracting the MW (or MVAR) flow on a transmission path from the MW (or MVAR) limit determined by the corresponding hyperplane.

Weak Element - Is defined as the power system location where voltage collapse has occurred upon directional stressing on a transmission path.

\subsection{Voltage Security Assessment}

The CA ISO, the California Energy Commission (Energy Commission) and the Consortium for Electric Reliability Technology Solutions (CERTS) initially agreed to develop a real-time voltage and transient analysis security margin assessment tool using hyperplane technology. Hyperplane technology consists in running a series of power flows with a specialized convergence technique to identify operating points in the system where a voltage limit has been reached.

The CA ISO had proposed using the concept of hyperplane to implement the objective outlined above. A survey was conducted among experts to determine the feasibility of implementing this approach. The experts agreed that the hyperplane approach is well suited for voltage assessments but not yet proven to conduct transient analysis. Upon completion of the survey, the Energy Commission, CA ISO and CERTS agreed to implement a hyperplane based Voltage Security Assessment (VSA) prototype for the CA ISO control area. No transient analysis will be performed with hyperplanes.

\subsection{VOLTAGE SECURITY ASSESSMENT FUNCTIONALITY}

The VSA will produce security regions periodically and perform voltage assessment every 5 minutes for the current system condition and for the next two hours in 5-minute increments. The VSA prototype will develop security 
regions and, using a post processing algorithm, perform an analysis of a given system conditions that will include the following functionality:

- Contingency simulation

- Determination of voltage security margins

- Contingency ranking

- Identification of weak elements

\subsection{Contingency simulation}

The prototype will have the ability to take a base case provided by the CA ISO, convert it to a format usable by the prototype, and run power flows until an acceptable solution is reached for the condition at hand. The prototype will also have the capability to take system elements such as predefined transmission lines, generators, and transformers out of service and run power flows without those elements.

The ultimate version of the VSA application shall have the ability to develop security regions and perform voltage security assessment on demand, for system conditions when a contingency has already occurred. The application shall also be able to, using the modified topology, perform contingency analysis in anticipation of the next contingency.

\subsection{Voltage security regions and hyperplanes}

The prototype will define the voltage security regions whose boundaries are piece-wise linear approximation (hyperplanes) in coordinates of nodal power injections and power flows. To determine the Hyperplane nomograms or security regions, the prototype shall perform a series of continuation power flows until the point of voltage collapse is reached; this point of voltage collapse will determine the hyperplane for the direction being stressed. Other limits reached during this stressing process such as thermal and voltage violations will be identified and reported. The user will specify a transfer or loading by either a vector indicating the participation of all the load and generator buses, or some other pattern that represents actual system loading conditions. The user will also specify the structural changes and/or system performance limits to be used during power flow runs.

\subsection{Voltage security margin assessment}

For the operating condition in question, the voltage security margins are determined from the voltage security regions developed offline for normal and contingency conditions. The distance from the current operating condition to the relevant edge of the security region will determine the voltage security margin for the condition at hand.

\subsection{Contingency rankings}


Contingency ranking will be determined by an algorithm that will sort the results of contingency analysis by either voltage security margin or by abnormal reduction of nodal voltages. Ranks will be specified with 1 as the contingency resulting in the smallest security margin or worst nodal voltage deviation.

\subsection{Identification of weak elements}

Weak elements, defined as those lines and or substations which are most affected by those contingencies causing worst damage to the reliability of the power system, will be determined by an algorithm based either on analysis of the direction of the critical vector or on analysis of the practical stability criteria (derivatives or sensitivities of active and reactive power with respect to variations of nodal voltage). CERTS/EPG and CA ISO will jointly decide on the most appropriate method. In addition the VSA application will produce scale-independent ranking using ratios for the most sensitive buses in the system.

CERTS/EPG and the CA ISO agreed to test this functionality focusing on the Humbolt area, where voltage problems are known to exist.

\subsection{DATA REQUIREMENTS}

The VSA tool will rely on state estimator data and will initially be used as an offline application. Later, this tool can be expanded for real-time use. When used as an off line application the prototype will require data from the CA ISO's State Estimator including the following:

- CA ISO control area model

- CA ISO control area solved power flows with state estimator data including MW and MVARS for load, generators and interchanges. These power flows should include heavy load conditions and heavily loaded transfer paths.

- Generator limits, voltage thresholds and line ratings.

In addition, the Voltage Security Assessment application will need the following information to determine hyperplanes and develop security regions:

- Transmission path loading vectors and any other system loading patterns relevant to the power system condition under review.

- Load and generation forecasts for two consecutive hours in intervals of five minutes.

- A list of relevant single outage contingencies and double element outages selected based on CA ISO's operational experience

When used as a real time application the tool will need direct input from the CA ISO state estimator to receive CA ISO control area real time data together with 
control area model, generator and thresholds limits and transmission path loading vectors.

CERTS/EPG and the CA ISO agreed to develop the VSA prototype using a continuation power flow engine provided by the Power System Engineering Research Center (PSERC). This application uses a bus/branch configuration and the input for this engine will be generated from a GE solved case to be provided by the CA ISO's planning group. When the CA ISO State estimator produces PSLF formatted cases, the prototype will be tested using these cases.

Additional data requirements will be identified once algorithms are selected and the corresponding design specifications are developed.

\subsection{VISUALIZATION SOLUTION APPROACH AND ARCHITECTURE}

The VSA real-time display will use a visualization solution based in a multi-view, geographic, approach that will include the following information:

- Security region whose security boundaries are determined in MW flows.

- The current operating condition

- The voltage security margin available with the system condition at hand

- Abnormal reduction of nodal voltages

- Contingency ranks based on severity indices

- Weak elements and geographical locations

- Worst case contingencies leading to voltage collapse

- Contingencies with insufficient stability margin

\subsection{SYSTEM ARCHITECTURE}

The Voltage Security Assessment prototype will be based on a power flow computational engine equipped to handle a continuation method algorithm necessary to solve numerous power flows quickly. Computational speed is necessary to solve many power flows in a short period of time to develop security regions based on piece-wise linear approximation (hyperplanes) of stability boundaries.

The VSA prototype system architectural overview is shown in Figure 1. Each of the different components of the application is described below:

Case Reader/Network Processor

The case reader component will be designed to interface with the state estimator CA ISO data source and to read a complete base case and translate into a format suitable for use with the VSA computational engine. It will also have the ability to read the contingencies files and modify the system topology to simulate outages.

Transfer Path/Loading Direction Selector

This application will select the path to be stressed and use the load and 
generation pattern provided by the CA ISO to in the directional stressing algorithm. This application will keep track of paths (cut sets) selected for directional transfer increases (path stressing). A new path will be selected for stressing only after the current path has been stressed to a point of voltage collapse and a hyperplane has been identified for that path.

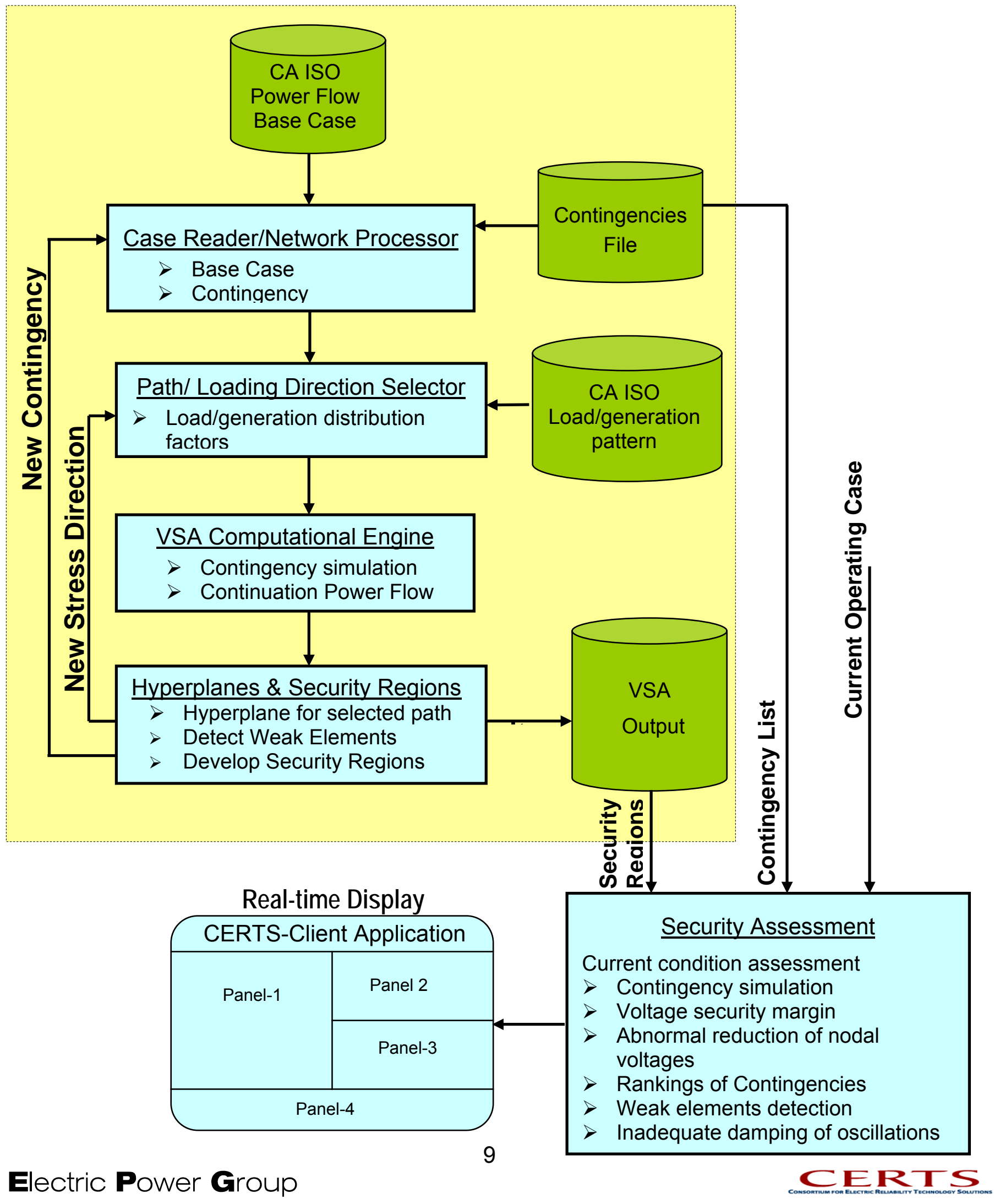


Figure 1: Voltage Security Assessment Prototype (System Architecture) 
VSA Computational Engine

At the center of the application is the VSA Computational Engine designed to take base case models and corresponding data from the CA ISO state estimator, run power flows, and determine the distance to voltage collapse. Abnormal reduction in nodal voltages will be identified during these runs.

Hyperplane \& Security Regions

At the voltage collapse point for a particular stressing direction, this function will use a linearization process to calculate the hyperplane and identify the weak elements. The set of hyperplanes developed for different transfer paths constitute the boundaries of the safe, stable, region and therefore define a security region. These security regions shall be defined for each contingency condition in a pre-determined contingency file. This file will be either provided by the CA ISO or will proceed from a contingency screening application.

\section{Security Assessment}

For the operating point in question, this component will perform the following:

- Analyze the current system conditions and map it to the security region.

- Access and display the security regions developed for the different system conditions including contingencies.

- Calculate the voltage security margin for the current system condition.

- Identify abnormal reduction of nodal voltages.

- Rank contingencies based on the security indexes obtained from contingency analysis.

- Identify the weak elements.

Client Application

The CERTS client application will read all the results obtained with the Voltage Security Assessment applications and display the following information for use by the dispatchers:

- Security region whose security boundaries are determined in MW flows

- The current operating condition

- Worst case contingencies leading to voltage collapse

- Contingencies with insufficient stability margin

- Contingency ranks based on severity indexes

- Abnormal reductions of nodal voltages

- Voltage security margins

- Weak elements and geographical locations 


\subsection{IMPLEMENTATION SCHEDULE}

Table I: Implementation Schedule for the CA ISO Voltage Security Assessment Project

\begin{tabular}{|l|c|c|}
\hline \multirow{2}{*}{ ACTION } & \multicolumn{2}{c|}{ Complion } \\
\cline { 2 - 3 } & Target & Done \\
\hline PHASE 1 & & \\
\hline Complete contingency analysis and ranking & $3 / 30 / 2005$ & $\sqrt{ }$ \\
\hline Factory test completed & $4 / 15 / 2005$ & $\sqrt{ }$ \\
\hline & & \\
\hline PHASE 2 & & $\sqrt{ }$ \\
\hline Project Executive Summary & $2 / 18 / 2005$ & $\sqrt{ }$ \\
\hline $\begin{array}{l}\text { Assess and research algorithms (Survey) to determine } \\
\text { security regions; prepare summary of responses }\end{array}$ & $3 / 15 / 2005$ & $\sqrt{ }$ \\
\hline Define algorithm to determine security regions for CA ISO & $3 / 25 / 2005$ & $\sqrt{ }$ \\
\hline Functional Specifications & $3 / 30 / 2005$ & $\sqrt{ }$ \\
\hline Complete Survey report for delivery & $4 / 15 / 2005$ & \\
\hline Customer Approval of Functional Specs & $4 / 15 / 2005$ & $\sqrt{ }$ \\
\hline Design Specifications & $5 / 20 / 2005$ & \\
\hline Prototype Development: & & \\
\hline$\bullet$ Complete Case Reader/Network Processor & $6 / 3 / 2005$ & \\
\hline$\bullet$ Complete Path/Loading Direction Selector & $6 / 3 / 2005$ & \\
\hline$\bullet$ Complete VSA Computational Engine & $6 / 10 / 2005$ & \\
\hline$\bullet$ Complete hyperplane identifier & $7 / 1 / 2005$ & \\
\hline$\bullet$ Complete security assessment application & $7 / 29 / 2005$ & \\
\hline Integration - VSA prototype & $8 / 19 / 2005$ & \\
\hline Validation using GE program & TBD & \\
\hline
\end{tabular}


Appendix D

Task 3.0 CA ISO Phasor Applications Summary Report 


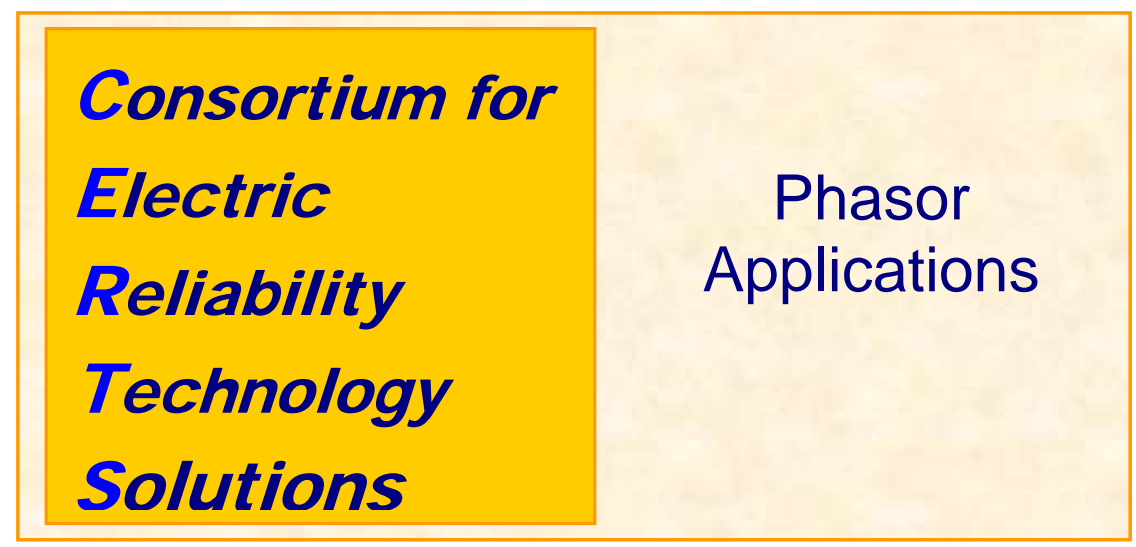

\section{CA I SO Phasor Applications Summary Report}

Funded by the California Public Interest Energy Research Transmission Research Program

Date: February 9, 2006 


\section{TABLE OF CONTENTS}

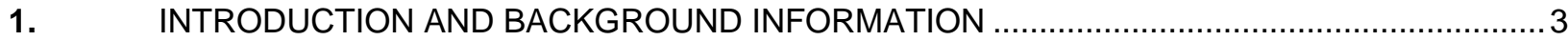

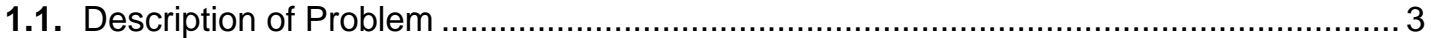

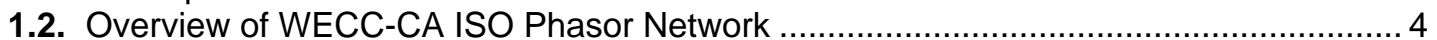

2. PROJECT OVERVIEW

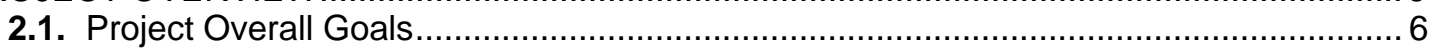

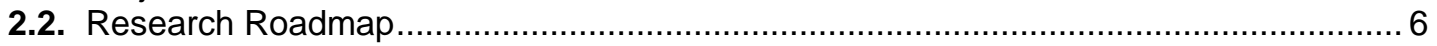

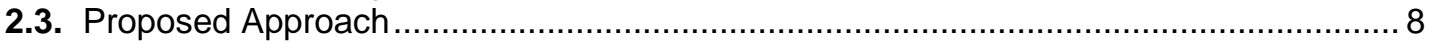

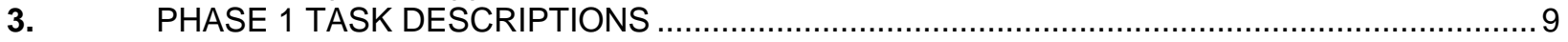

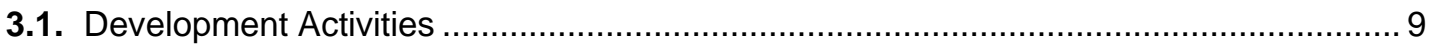

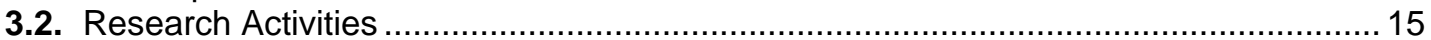

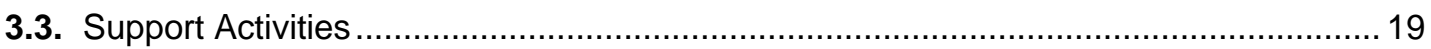

\section{LIST OF FIGURES}

Figure 1: Current and Future WECC-CA ISO Phasor Data Communication Network .............................. 5

Figure 2: Multi-Year Research and Development Roadmap for the Phasor Applications........................8

Figure 3: RTDMS Version 3 System Architecture ........................................................................ 10

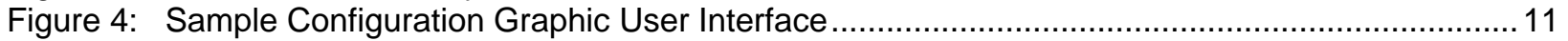

Figure 5: Real-Time Alarming on Monitored Metrics ..................................................................... 12

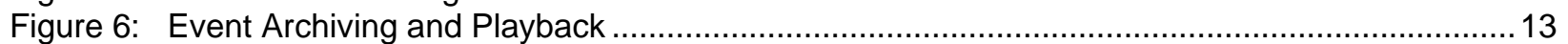

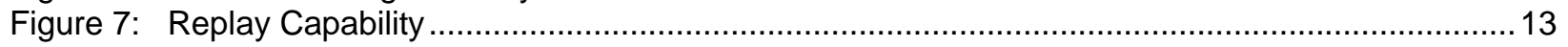

Figure 8: CA ISO Frequency Data Collection Redundant System ................................................ 14

Figure 9: Small-Signal Stability Monitoring Prototype Implementation Plan.......................................... 16

Figure 10: Using Phasor Measurements to Detect (a) Potential "Holes" and (b) Excessive

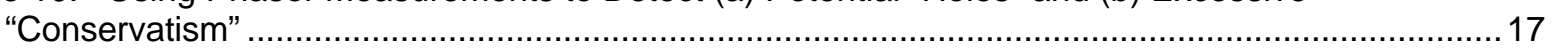

Figure 11: Conceptual View of Simple Angle Difference and Advanced Angle Nomograms.................. 19

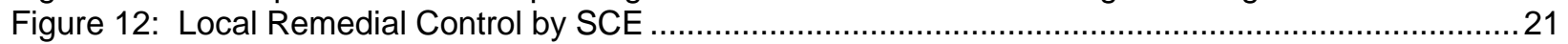

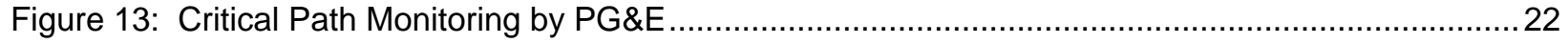




\section{INTRODUCTION AND BACKGROUND INFORMATION}

A PIER TRP funded multi-year project is currently being conducted by CERTS in cooperation with CA ISO aimed at research and demonstration activities of Real-Time Applications of Phasors for Monitoring, Alarming, and Control. The proposed applications of phasor measurements will provide the real-time operating staff with the previously unavailable, yet greatly needed, tools to avoid voltage and dynamic instability, and monitor generator response to abnormal significant system frequency excursions. Perhaps of equal or greater importance, in the near term, the measurement infrastructure will provide CA ISO with an alternate, independent real-time monitoring system that could act as an end-of-line backup for failures affecting CA ISO's current SCADA/EMS; in the long term, it would become a key element of CA ISO's next generation monitoring system necessary for advanced real time control. Some of the proposed applications include the use of phasor measurements for wide-area visibility, real-time monitoring and alarming, small-signal stability assessment, frequency data collection, nomogram validation and improvements, improved state estimation, and real-time control.

Phasor measurement technologies are a leading example of a new generation of advanced grid monitoring technologies that rely on high speed, time-synchronized, digital measurements. These characteristics are essential for monitoring real-time grid performance, validating (or replacing) off-line nomogram studies, providing advance warning of potential grid instabilities, and, ultimately, enabling the development and introduction of advanced automatic grid control approaches (such as adaptive islanding).

\subsection{Description of Problem}

CA ISO's traditional security assessment approach - based on SCADA data and off-line studies conducted long in advance of real time operations - are becoming increasingly unreliable for real time operations because they cannot fully anticipate all the conditions currently faced by operators. New technologies, which rely on accurate, high-resolution, real-time monitoring of actual (not hypothesized) system conditions, are needed to support the CA ISO's real-time operations. The purpose of these tools and systems is to monitor, assess, enable, and, ultimately, automatically take the necessary control action to prevent or mitigate problems in real time.

On August 10, 1996, a major disturbance separated the WECC system into four electrical islands (Northern Island, Southern Island, Northern California Island, and Alberta Island), interrupting service to 7.5 million customers for periods ranging from several minutes to about nine hours. This disturbance effectively began with the loss of the Keeler-Allston 500-kV line in the Portland area, due to inadequate right-of way maintenance, which overloaded parallel lines and depressed transmission voltages. These conditions led to subsequent tripping of additional lines and McNary generating units, triggering oscillations that increased in magnitude for approximately 70 seconds, until the protective devices tripped the three 500-kV California-Oregon Intertie (COI) lines due to low voltage (less than $315 \mathrm{kV}$ on the Malin $500 \mathrm{kV}$ bus) and subsequent islanding of the WECC system [1]. The growing oscillations can be attributed to an increased electrical angle between northwest generation and COI.

On October 18, 1996, the WECC investigation team analyzing August 10, 1996, blackout strongly recommended that "the WSCC CWG shall investigate the cause of the undamped oscillations..." and that "all WSCC owners of generators, in conjunction with the WSCC TSS and CWG shall assess whether installed excitation systems and PSS on units with capacity of $10 \mathrm{MW}$ or greater, are properly tested, tuned, and correctly modeled in transient stability studies." [1].

Summarizing the outcome of the previous blackout investigations, the U.S.-Canada Power System Outage Task Force that conducted the analysis of the August 14, 2003 which impacted 50 million people in the Eastern Interconnection, indicated several causes or contributory factors in common with the earlier outages including [2]"

- Inadequate situational awareness and regional-scale visibility over the bulk power system 
- Inability of system operators or coordinators to visualize events on the entire system

- $\quad$ Failure to ensure operation within secure limits

- Failure to identify emergency conditions and communicate that status to neighboring systems

- Inadequate training of operating personnel

The bi-national investigation team recommended the need for "wide-area visibility and situational awareness to address problems before they propagate..., use of time-synchronized data recorders..., improved system modeling, data quality and data exchange practices..., and better real-time tools for operators and reliability coordinators" [2].

Phasor technology is one of the key promising technologies on the horizon that offers new possibilities in providing the industry with new tools and applications to address these blackout recommendations and to tackle reliability management and operational challenges faced by CA ISO operators and WECC reliability coordinators. Such data that recorded during the 1996 blackout was invaluable in investigating the causes of the major 1996 blackout. It also complements existing SCADA systems by providing the high sub-second resolution and global visibility to address the new emerging need for wide area grid monitoring; real-time dynamics and stability monitoring; dynamic system ratings to operate system closer to the margin to reduce congestion costs and increased asset utilization; and improvements in state estimation, protection and control.

\subsection{Overview of WECC-CA ISO Phasor Network}

Under PIER support, CERTS and CA ISO have made significant progress towards accomplishing the CA ISO's goal of using phasor technology for wide-area real-time monitoring and control. On September 4, 2002, a Phasor Data Concentrator (PDC) was installed at CA ISO in Folsom, CA. At the start of the project almost 4 years ago, the initial phasor network consisted of only 14 Phasor Measurement Units (PMUs) gathering data at 30 samples/second and sending it in real-time to the PDC at Bonneville Power Administration (BPA). This setup included 12 PMU devices located at various substations within and around BPA's jurisdictional area that were sending data directly to the BPA PDC for time synchronization, and communication links between BPA and Southern California Edison (SCE) comprising of an analog microwave circuit between BPA in Vancouver (WA) and LADWP in Los Angeles (CA) and a digital link from there to SCE in Rosemead (CA) for real-time data exchange of selective signals (2 phasors and 1 frequency) from 2 of their respective PMUs between the two utilities. This collective set of timesynchronized phasor data from the BPA PDC was then forwarded to the CA ISO PDC in real-time using the existing WECC/CA ISO Wide Area Network (WAN) used to exchange control center data between utilities.

Subsequent expansion work on the network has resulted in greater wide-area visibility across the WECC. Presently, the CA ISO PDC receives data from 42 PMUs from geographically distributed locations via the WECC WAN which connects the BPA, SCE and PG\&E PDCs with the PDC at CA ISO. PMUs belonging to BPA feed directly to the BPA PDC while WAPA PMU data is fed from the WAPA PDC to the BPA PDC over the WECC/CA ISO WAN. The SCE and PG\&E PMUs feed into their corresponding PDCs, and this data is further transmitted in real-time to the CA ISO PDC. Figure 1 below shows the current Western Electricity Coordinating Council (WECC)-CA ISO synchronized data communication network and the proposed expansions. 


\section{CAISO PHASOR NETWORK DIAGRAM}

(PRESENT AND FUTURE)

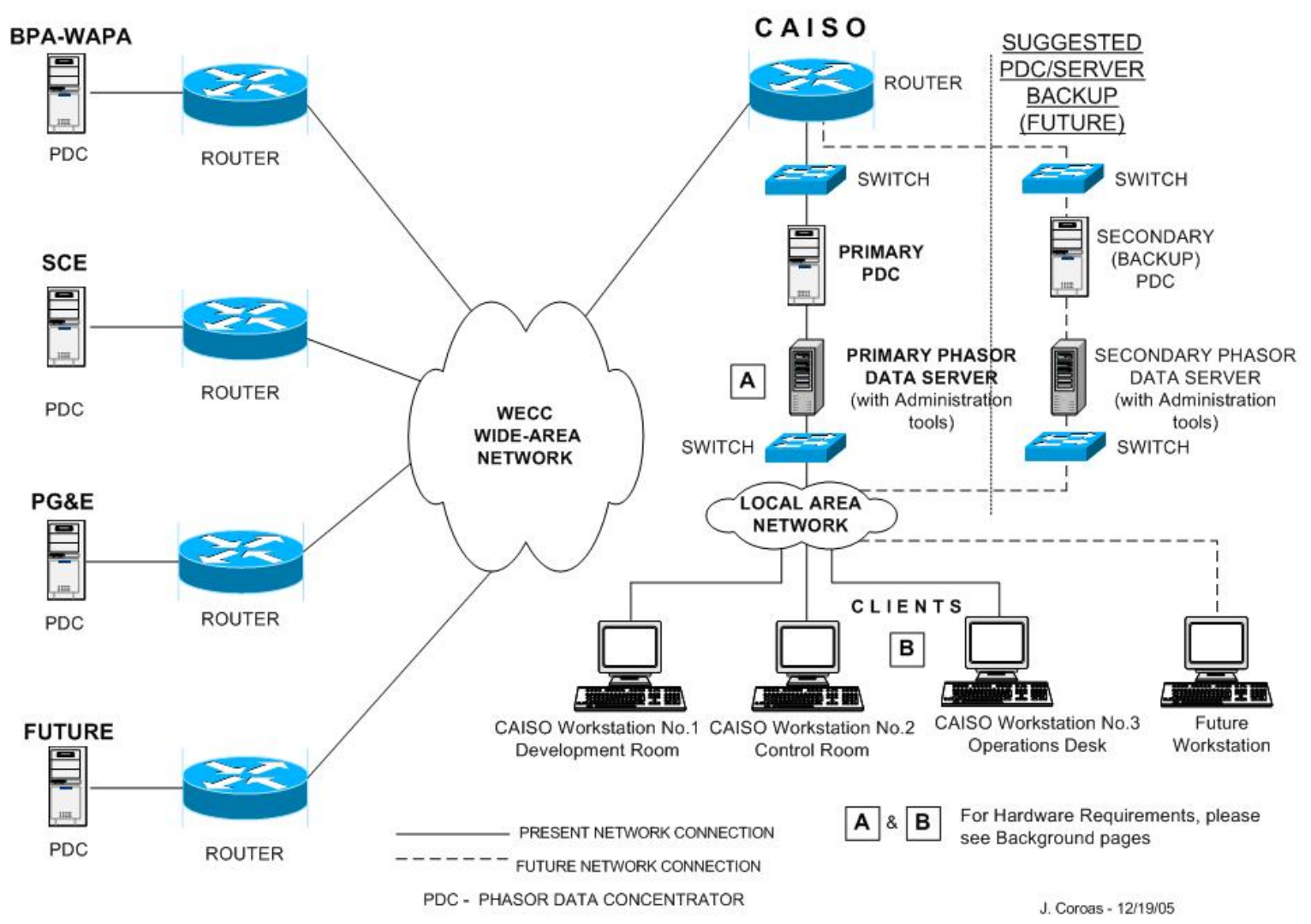

Figure 1: Current and Future WECC-CA ISO Phasor Data Communication Network

The current Real-Time Dynamics Monitoring System ${ }^{\text {TM }}$ (RTDMS) application at CA ISO reads the collective set of phasor data output from the CA ISO PDC and displays it in real-time. This complete set of time-synchronized high-resolution data provides the desired wide-area visibility across the western interconnection required for security monitoring by dispatchers including dynamic performance assessment and post disturbance analysis.

Future plans for the network include exploring the integration of phasor data from Arizona and British Columbia into the WECC phasor network which would close any observable gaps that currently exist in the network. Furthermore, it would also benefit the CA ISO to extend the phasor data stream all the way to CA ISO's Alhambra facility and create a secondary backup system there for redundancy.

The RTDMS phasor system display station which was initially installed at the CA ISO control room has recently been relocated to the WECC Reliability Coordinator's desk It will not be long before the longawaited phasor data could be presented to all the WECC reliability coordinators in real-time and within standardized displays. With this phasor network in place, reliability coordinators as well as system operators will be able to monitor over 250 voltage and current phasors at key $500 \mathrm{kV}, 345 \mathrm{kV}$ and $230 \mathrm{kV}$ substations throughout the WECC. 


\section{PROJECT OVERVIEW}

The current WECC and CA ISO phasor infrastructure has matured to the point that this infrastructure can be used as a new data source to support real-time system operations applications. The emphasis of this project over the next few years therefore is to focus on applications that are uniquely suited for phasors and will provide the real-time operating staff with the previously unavailable, yet greatly needed, tools to monitor grid reliability and avoid voltage and dynamic instability, as well as key metrics for tracking grid performance, such as generator response to abnormal significant system frequency excursions. It will also enable CA ISO, California and WECC utilities to explore closely related issues, such as use of phasor data to improve system planning models, to improve state estimation, to determine the optimal location of additional phasor measurements, and ultimately to gain the experience with the technology needed to begin developing real-time control applications.

In July of 2004, the CERTS Program Review Committee recommended that the Energy Commission and CA ISO continue the research, development and application of this technology including research to determine what phase angles and rates of change of phase angles (and associated ranges around these quantities, including appropriate boundaries or thresholds, recognizing inescapable uncertainties) are appropriate for various locations in system, what actions should be taken by operators or automatic control equipment, if there are major deviations, and the desired location for additional phasor monitoring equipment around the WECC. The proposed approach, developed in conjunction with CA ISO staff, represents a phased approach toward addressing these issues, centered around introducing phasor information to operators and working closely with them to modify and enhance the applications (including training) to increase their confidence in relying on this information to support their day-to-day activities.

\subsection{Project Overall Goals}

This overall objective of this project is to accelerate the adoption and foster greater use of new, more accurate, time-synchronized phasor measurements by CA ISO reliability coordinators and control area operators as well as by California and WECC utility transmission dispatchers towards achieving the longterm goal of using phasor technology for wide-area monitoring, alarming and control. The specific research objectives of this task are:

- To provide real-time operators with new tools which provide previously unavailable wide-area visibility and information on the dynamic stability of the grid.

- Design the conceptual look of operator displays for phasor applications.

- Define functional specifications such that, upon completion, the tools can be transferred to a vendor (selected by CA ISO) for implementation as a production-grade operating tools

- Provide technical support to and assist in coordinating phasor applications being researched and developed by CA utilities.

- In the long run, provide the basis for the introduction of a new generation of automatic grid controls.

At the conclusion of this project, prototypes for several CA ISO phasor applications will have been researched, developed, factory- and field-tested, all in close coordination with and oversight provided by CA ISO, such that the findings from these activities will be summarized in the functional specifications for commercial-grade, production-quality tools that can be acquired by CA ISO [3].

\subsection{Research Roadmap}

In July of 2005, CERTS worked with CA ISO in developing an R\&D Roadmap to guide the Phasor Applications technology research and development effort. This multi-year roadmap shown in Figure 1 layouts out the research and development plans by the various task activities that need attention as well as through the various phases of the development cycle including research, development, demonstration, and technology transfer, and the intermediate milestones and deliverables [3]. The end product of many of the activities listed in the tasks is to develop functional specifications for production quality applications 
that could be passed on to commercial vendors of CA ISO's choosing for implementation. The various tasks identified within the roadmap are summarized below:

Input Data Requirement: The present WECC-CA ISO Phasor Network uses the BPA developed proprietary PDCStream format for real-time data transfer between BPA, WAPA, SCE, PG\&E and CA ISO. The new industry phasor format standard for real-time data transfer, IEEE C37.118, has recently been approved. As the phasor devices migrate to the new standard, the various phasor prototype applications developed will have to comply with this new standard. Furthermore, as additional devices, such as relays or disturbance fault recorders, that have phasor measurement and GPS time synchronization capabilities (such as DFRs, relays) comply to the new standard, they could also be integrated into the phasor system to close any observability gaps that present exist.

CA ISO Applications: Various applications which are well suited for phasor measurements and that meet the CA ISO needs have been identified, all of which are planned for development on the existing RTDMS platform. These applications include identifying and addressing wide-area visualization needs on this phasor data within standardized displays and summary screens with special attention towards avoiding screen clutter, and real-time monitoring, alarming and reporting capabilities based on advanced monitoring metrics and indicators on this high resolution phasor data. Other applications include a SmallSignal Stability monitoring application to detect the low frequency electromechanical oscillations when observable in the power system and to closely monitor and track their oscillatory frequency and associated damping to provide early warning on poorly or lightly damped situations where there is a danger of the oscillations growing unstable. There is also a Frequency Data Collection project to collect and archive sub-second frequency data from PMUs to meet new NERC-WECC Western Interconnection frequency data collection requirements.

New System Capabilities: This task addresses a wide-area research need that extends beyond the CA ISO, which is the use of phasor measurements for real-time wide-area control. As a first step towards achieving this goal, the objective is to research and develop methods for utilizing phasor measurement to validate and possibly improve stability nomograms.

System Integration and Support: Each of the CA utilities has identified pilot projects to demonstrate the application of phasor technology to problems that are of interest to these utilities. In particular, SCE is interested in local remedial action controls with phasors, SDG\&E would like to demonstrate improvements in the state estimation results using PMU measurements, and PG\&E's interest lines in monitoring critical paths via PMUs. To the extent possible, CERTS will be providing assistance and support to each utility's undertaken projects. Additionally, CERTS will be coordinating collaboration activities with academic and industry experts as needs arise. 


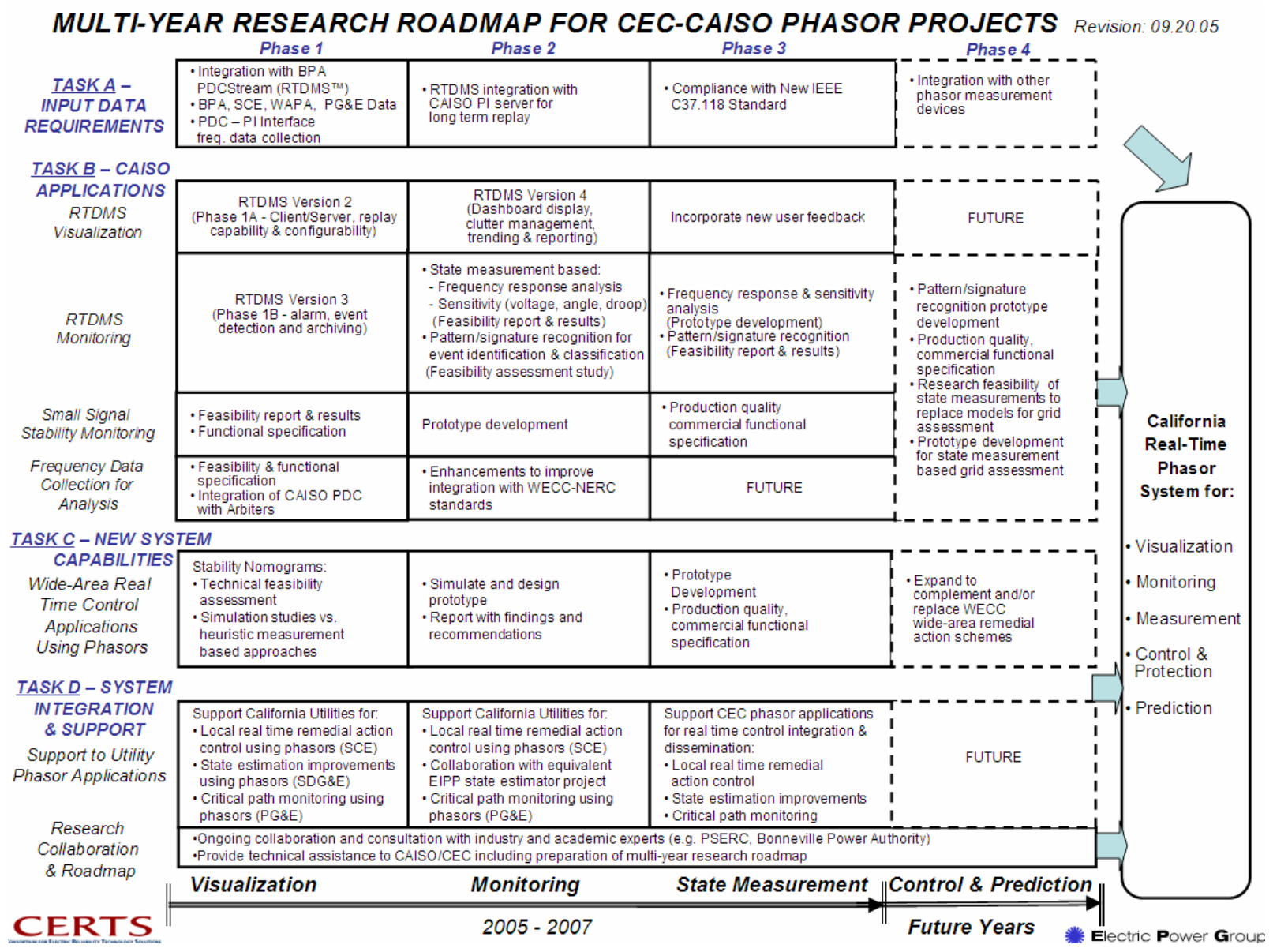

Figure 2: Multi-Year Research and Development Roadmap for the Phasor Applications

\subsection{Proposed Approach}

To successfully accomplish the tasks outlined within the roadmap, we propose a phased out approach for each of the various applications which would include the following stages:

- Conduct the initial research and feasibility assessment on the defined problem

- Perform an extensive review of existing methods and algorithms, and select the most appropriate algorithm(s) to be used in the prototype application

- Work closely with CA ISO staff to develop a functional specification for the prototype tool

- Implement and test the prototype in the field

- From the understanding gained with the prototype tool, develop a functional specification for the production quality application to be implemented by commercial vendors.

The level of research and development associated with the various applications would be a major contributing factor in defining the amount of effort spent with each of these different stages of the proposed approach.

A Technical Advisory Committee (TAC) consisting of representatives from the CA ISO, California utility, BPA, DOE and other organizations provides strategic guidance for this Real-Time Operations R\&D program and foster rapid adoption of R\&D results by these organizations. 


\section{PHASE 1 TASK DESCRIPTIONS}

The various activities within the research roadmap that were performed during Phase 1 of the project are described in greater detail within this section. Depending on where these activities stand within the proposed approach, the effort during this phase can be categorized as Development Activities (RTDMS Versions 2 and 3, Frequency Data Collection), Research Activities (Small-Signal Stability Monitoring), and Support Activities (Support to Utilities).

\subsection{Development Activities}

Real-Time Dynamics Monitoring System (RTDMS - Version 2 and Version 3): The Real-Time Dynamics Monitoring System (RTDMS - Version 1) was initially developed as a stand-alone visualization application. During Phase 1 of the project, this application was transformed into a phasor technology research and development platform, supporting a central server / multi-client architecture as well as additional underlying functionalities required to support the development of additional phasor applications as outlined within the roadmap. Some of the key functional enhancements include:

RTDMS Version 2 (Phase 1A) - Delivered to CA ISO in January 2005

- Implement multi-user capability

- Expand system wide-area visibility to include SCE phasor data

- Research and improve wide-area visuals through configurability

- $\quad$ Research and improve display for frequency response monitoring

- $\quad$ Research and implement replay capability

RTDMS Version 3 (Phase 1B) - Delivered to CA ISO in August 2005

- Research and incorporate transient detector

- $\quad$ Research and enhance alarming capability

- Research and add event archiving capability for transient events

- $\quad$ Expand system wide-area visibility to include PG\&E phasor data

- $\quad$ Research and improve wide-area visuals to reduce cluttering

A conceptual overview of the system hardware and software architecture is shown in Figure 3 [13]. The RTDMS platform now supports a server-client architecture with the central RTDMS Server responsible for data management functions such as data acquisition, filtering, data processing and caching, and a thin client layer where multiple RTDMS Client applications installed on different machines can simultaneously access data from the central server. By centralizing the data management process, this architecture minimizes the necessary performance requirements for all Client machines and this translates to a reduction in hardware cost. Additionally, any new Client applications that would be developed under this project could connect to the common RTDMS Server for data access and retrieval. 


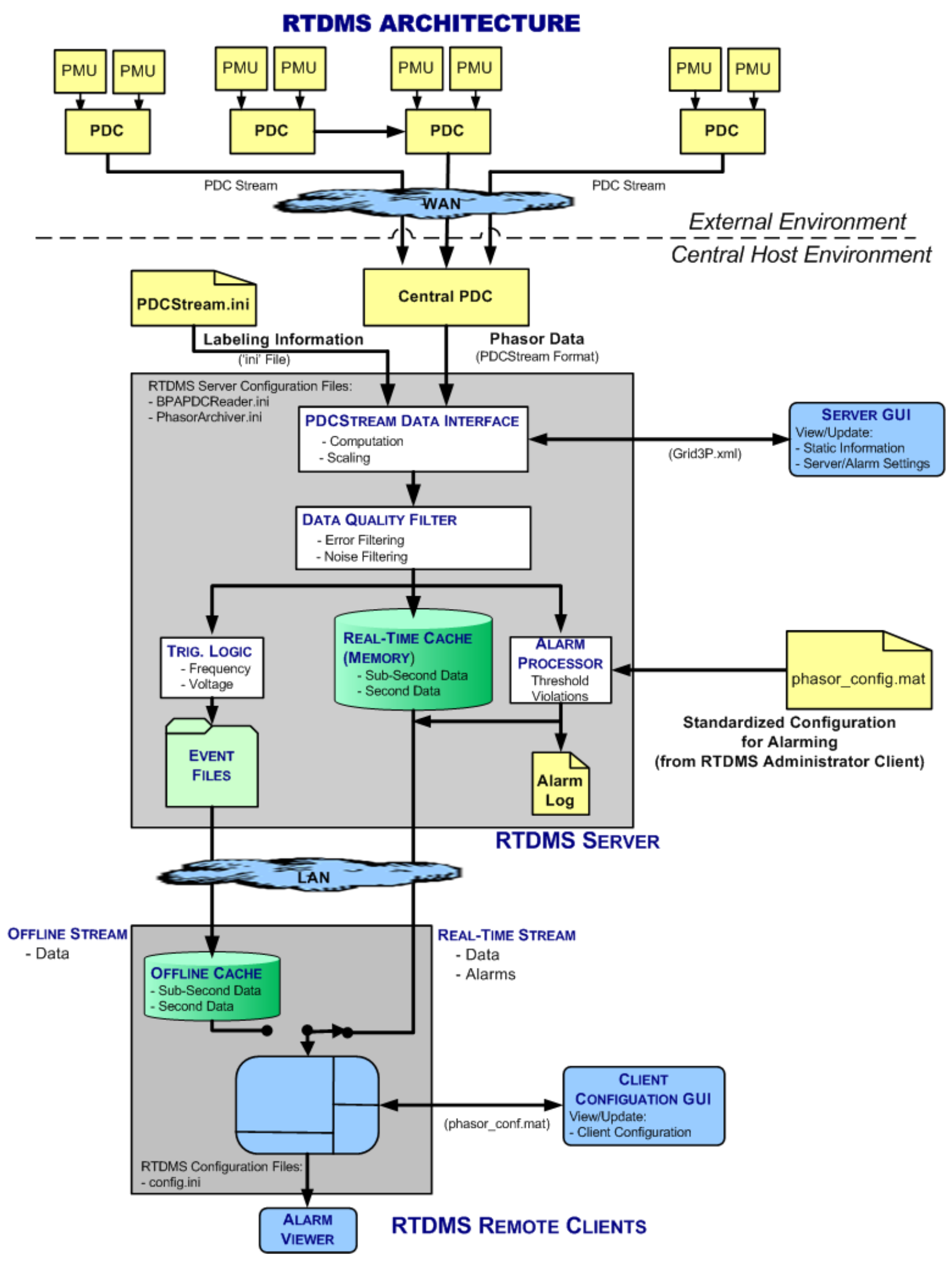

Figure 3: RTDMS Version 3 System Architecture 
Some of the additional enhancements include the configurability capabilities that allow the end user to grow and customize the overall RTDMS system and the visualization displays to reflect changes within the phasor network as new PMUs are added into the WECC phasor network and additional data from these new units is made available (see Figure 4).

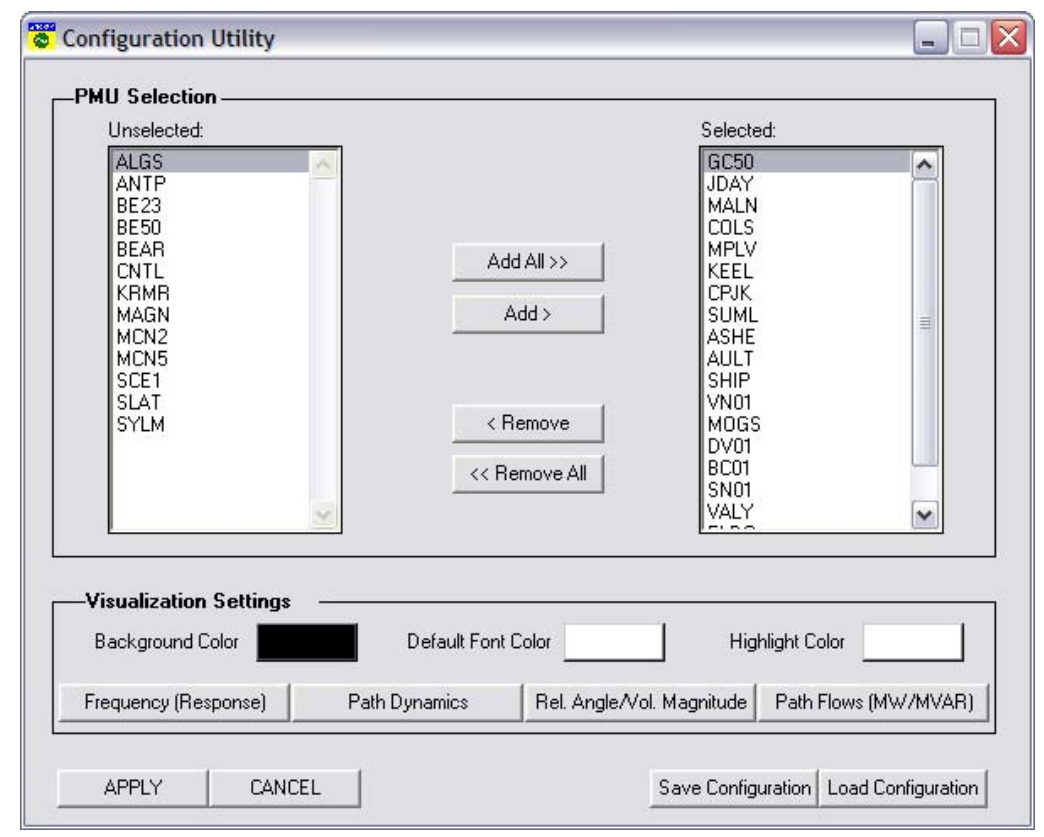

Figure 4: Sample Configuration Graphic User Interface

Alarming logic has also been built into the Server to analyze the streaming real-time data to detect and alarm on either threshold violations or significant rate-of-change (i.e. change within a second interval exceeding a specified threshold) on identified signals and metrics. Some of the alarming conditions presently set within the RTDMS Server include:

- System frequency exceeding specified threshold limits

- Sudden changes in system and local frequencies

- Voltage magnitudes exceed minimum or maximum thresholds (i.e. low or high voltages)

- $\quad$ Sudden changes in voltage magnitudes

- $\quad$ Angle difference across specified paths exceed defined threshold limits

- $\quad$ MW/MVAR flows across specified paths exceed defined threshold limits

Not only are the generated alarms permanently logged at the Server, these alarms are immediately made visible on all RTDMS Clients that are connected to the Server. All relevant information pertaining to these alarms (such as the metric associated with the violation, the start time of violation, the end time of violation, the violation value and corresponding threshold, the violation type, etc) are continuously shown in the alarming window (see Figure 5). The end user must acknowledge the alarm to make it disappear. 

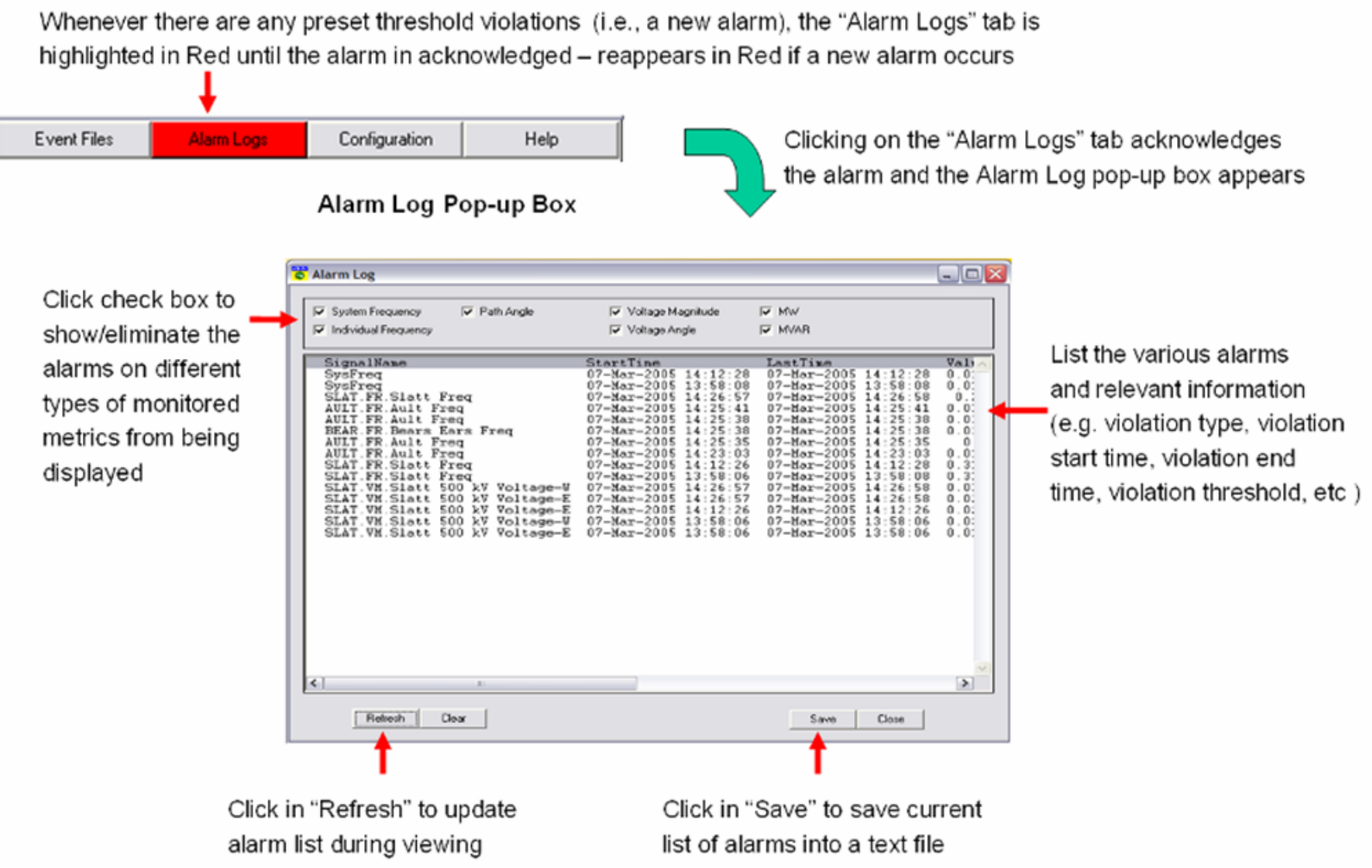

Figure 5: Real-Time Alarming on Monitored Metrics

Additionally, abrupt changes sensed in the voltage or frequency measurements may suggest that the system is undergoing a transient event such as a fault in the system or the loss of generation and/or load. Whenever a transient is detected, the application automatically saves, both, pre- and post-disturbance data and an alarm summary log into an event file that is labeled by the date and time of the event. This archived data can be loaded into RTDMS Clients in an Offline study mode and this set of data is adequate to recreate and payback all its visuals (see Figure 6). 


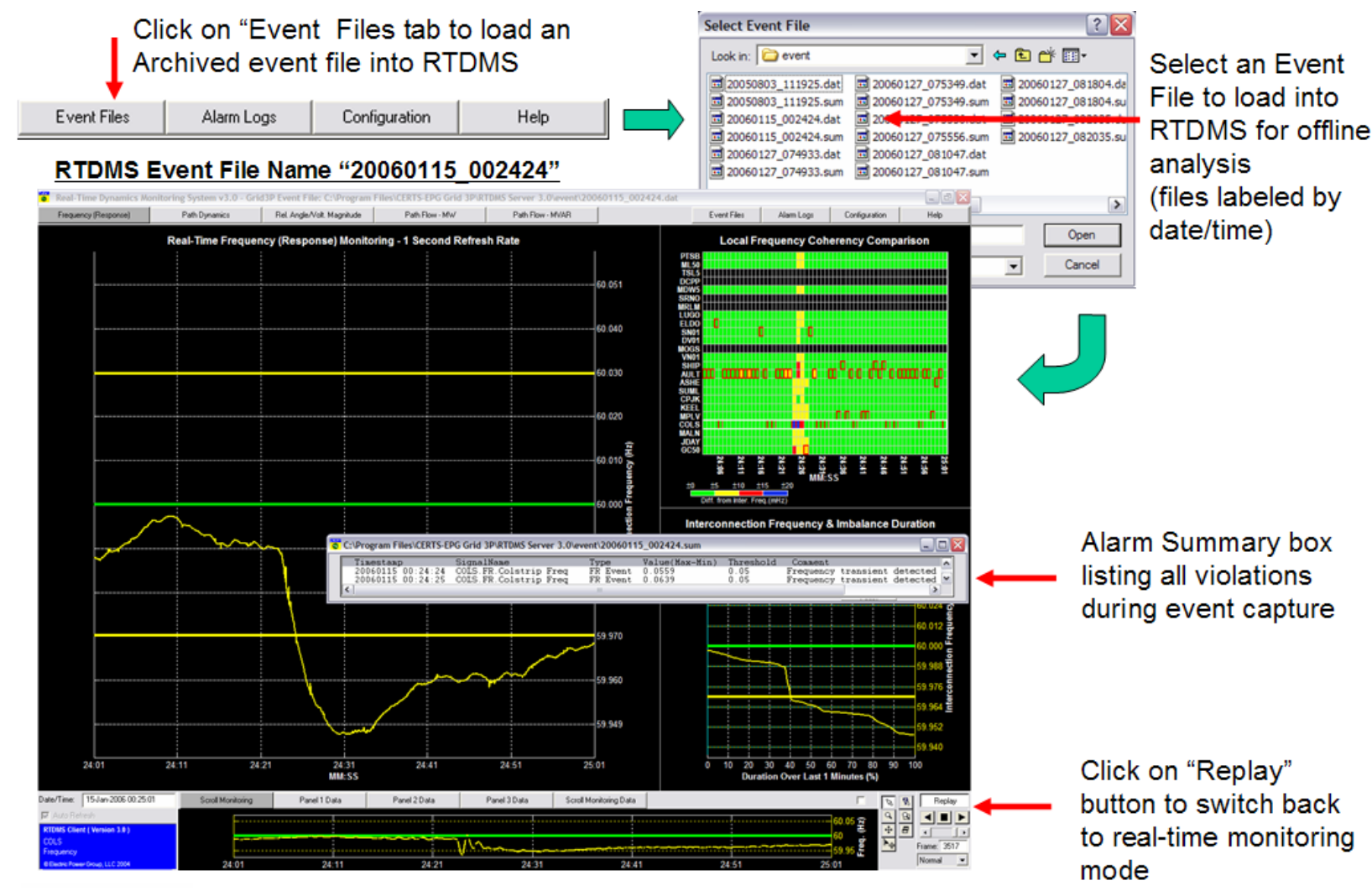

Figure 6: Event Archiving and Playback

During real-time operation, the Replay functionality (Figure 7) permits the user to switch from the RealTime monitoring mode to the Replay mode to view cached data for historical tracking and analyses. Once in the Replay mode, the auto-rewind/auto-forward buttons or the slider can be used to move back/ahead in time for closer inspection of the data and metrics.

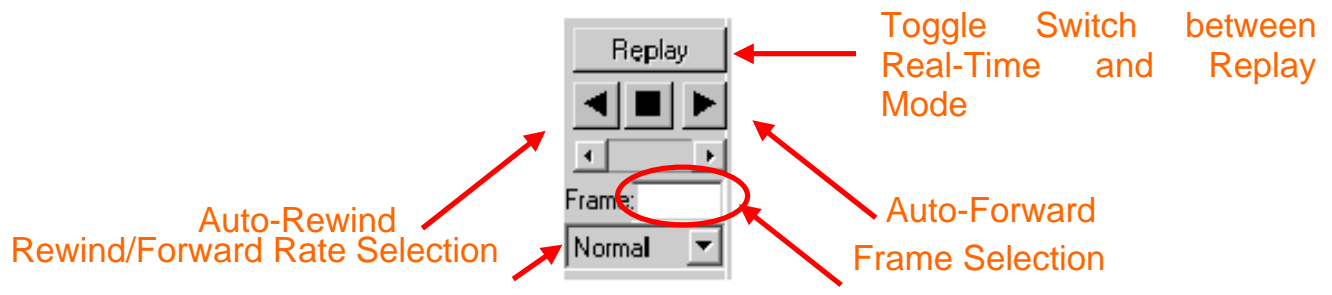

Figure 7: Replay Capability

The use of the replay capability as described above can also be used to interrogate event files when they are loaded into the RTDMS Client. Other enhancements included expanded wide-area visibility to include SCE and PG\&E phasor data.

The RTDMS phasor system with above mentioned features was delivered to CA ISO in 2Q 2005, and has been installed at the Reliability Coordinators desk.

Frequency Data Collection: In response to the USA-Canadian August $14^{\text {th }}, 2003$ Blackout Investigation Task Force blackout recommendations which emphasized the need for the collection and archiving of synchronized wide-area data, NERC-WECC and some of its subcommittees have been working during the last two years in defining functional requirements for the collection and archiving process. The CA 
ISO Frequency Data Collection project was defined to meet these new guidelines by acquiring subsecond frequency data from two main sources: the CA ISO Arbiter Frequency Monitors at Folsom and Alhambra, and the PMUs presently operational at BPA, SCE, WAPA, and PG\&E. Joint meetings were held between CA ISO and CERTS/EPG in January and February, 2005, to discuss and review proposed system architectures and cost estimates.

CA ISO management decided that for this project, the primary data source for CA ISO frequency data would be the two CA ISO Arbiter systems, and the secondary data source will be the from at least 6 geographically and electrically separated PMU devices within the Western Interconnection. CERTS/EPG would be responsible for integrating the secondary data source; i.e. the Phasor Data Services software running as a Windows NT service to collect GPS time-stamped frequency data from the CA ISO PDC with a rate greater than 10 samples/second and accuracy of $0.001 \mathrm{~Hz}$, data filtering to eliminate noise or data dropouts, and storing the collected data into the Folsom and alternate PI Historians [21]. The PI database deployment, integration and redundancy would be CA ISO's responsibility (see Figure 8).

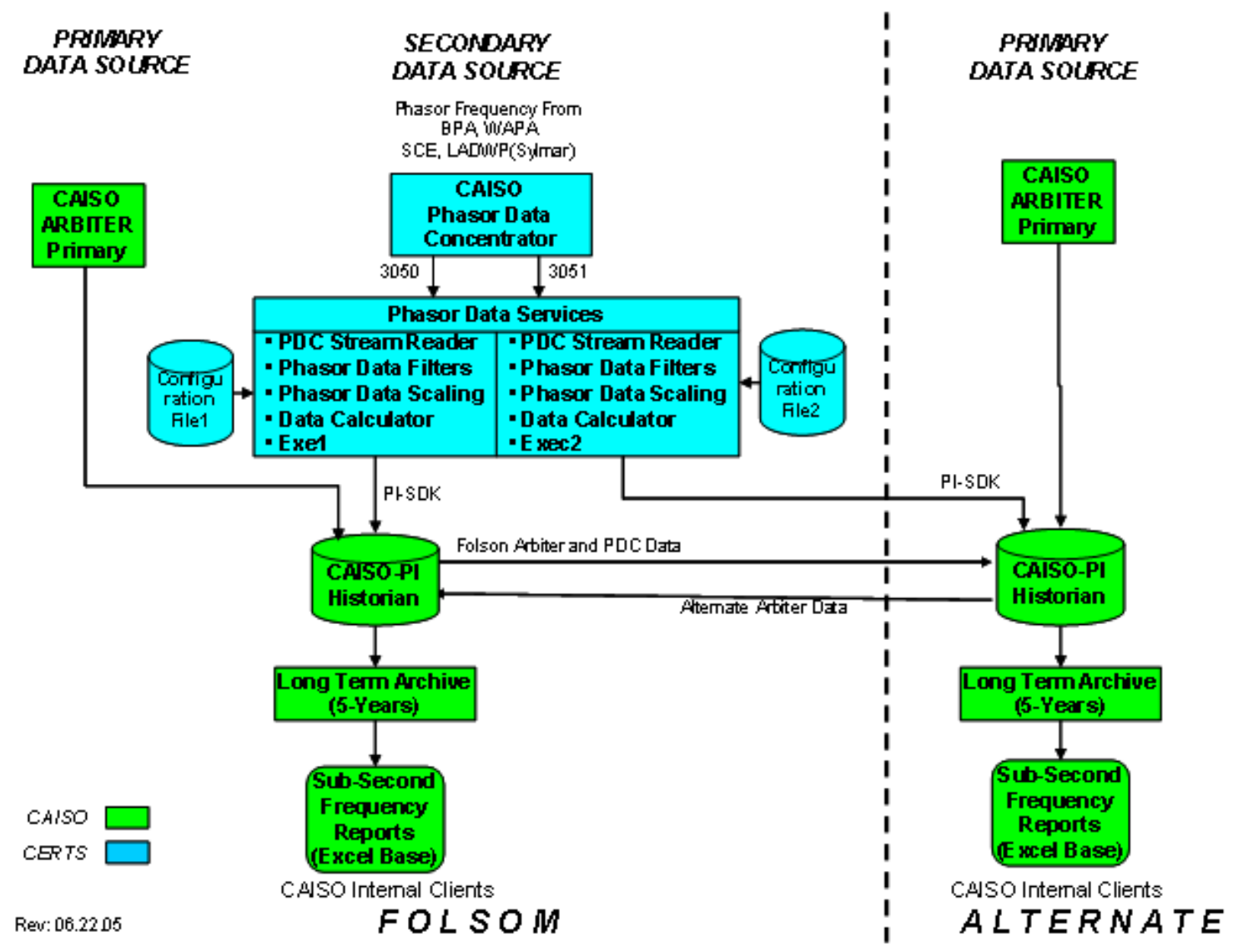

Figure 8: CA ISO Frequency Data Collection Redundant System

The project and system architecture was approved by CA ISO IT and management in April, and the functional specification was delivered to CA ISO in June. The development began shortly thereafter and the factory test was complete in July.

The field test and delivery to CA ISO on integrating the secondary data source was place on hold due to CA ISO restructuring. The primary system is presently operational at CA ISO and fulfills the immediate project needs of archiving sub-second frequency data. CA ISO management at this time has elected not 
to archive the PMU frequency data into their historian but are interested in archiving the PMU phasor data instead. The CERTS/EPG development on this project could easily be adapted to fulfill this objective.

\subsection{Research Activities}

In late 2004, CA ISO shared their interest in pursuing a Wide-Area Security Assessment (WASA) project to monitor security margins (i.e., "distance to instability") across the entire interconnection in real-time based on geographically dispersed PMUs, and voltage magnitudes and static angle measurements in particular. A key limitation here is lack of full observability by the PMU devices. In early 2005, CERTS/EPG formulated a survey to reach out to experts in the field for their comments, suggestions, and recommendations on the 2 projects: Voltage Security Assessment (VSA) and Wide-Area Security Assessment (WASA). The survey was sent to 51 academic and power industry experts worldwide with sixteen responses received; eight of the respondents were from the power industry and eight from academia. A common consensus was that the use of phasor measurements for modal estimation to assess small-signal stability was am ideal first step towards achieving the WASA project objectives. Mathematically, small-signal stability is a Hopf bifurcation where a stable equilibrium becomes oscillatory unstable and the consequence is either steady state oscillation or a growing oscillatory transient process. Hopf bifurcations are associated with a pair of complex conjugate eigenvalues whose real parts are changing their sign from negative to positive [20].

Small-Signal Stability Monitoring: Low-frequency poorly damped electromechanical oscillations that are observed in the power system are of interest as they characterize the stability of the power system and often limit power flows across the grid. While there is a danger that such modes can lead to instability following a sizable disturbance, there is also the risk of these modes becoming unstable (i.e., negatively damped) due to gradual changes in the system conditions. The ability to continuously track these modes in real-time would therefore be a valuable tool for dispatchers and power system operations engineers.

The focus of this project is to develop a prototype application that analyzes phasor data in real-time to identify dominant low-frequency electromechanical modes in the system and detect lightly damped oscillations under ambient conditions (i.e. mode frequencies, shapes, and damping). The idea is to alert operators when the system is experiencing poor damping.

Recently there have been multiple efforts to identify the dominating modes from phasor measurements. The underlying idea is that small fluctuations such as random load switching act as a constant low-level excitation to the electromechanical dynamics in the power system and are observed in the power-flows through, for example, voltage angle variations across transmission corridors $[8,9,10,16,17,18,19]$. Assuming that the random variations are white noise and stationary over the frequency range of interest (typically of $0.1+\mathrm{Hz}$ ), then the spectral content of phasor measurement signals , which is colored by power-system dynamics, can be used to estimate the inter-area modal frequencies and damping.

Bonneville Power Administration (BPA) also shares a common interest in the area of small-signal stability. BPA has been a pioneer in the phasor technology area and presently has over 15 of their own PMUs installed and connected to the WECC phasor network. They are very interested in extracting data both for real-time processing to provide alarms for system operators for impending trouble as well as observing the state of the system during staged system tests. Both CERTS/EPG and BPA have decided to collaborate on this small-signal stability prototype application research and development effort, thereby leveraging off each others expertise and eliminating unnecessary duplication. A Statement of Collaboration is presently in place between the two entities with the following key tasks identified (see Figure 9):

- Identify platform for algorithms evaluation and implementation plan

- Algorithm development and refinement including reviewing existing work to date, evaluating methodology alternatives on actual and model test data

- Prototype implementation and testing

- Online operation and evaluation of prototype 
- Application studies to determine relationships between observed modal behavior and power system stress conditions.

Prof. Dan Trudnowski, who is a leading expert in estimating mode properties from system measurements and has published several papers in this area, is also a key advisor on the project. Some of the proposed algorithms that shall be reviewed include but are not limited to:

- $\quad$ Yule Walker methods

- Autocorrelation with Prony Analysis

- $\quad$ Sub-space identification methods

- $\quad$ Least-squares ARMA methods

Monte Carlo simulations shall be used to evaluate the performance of each algorithm under consideration before incorporating them into the prototype application.

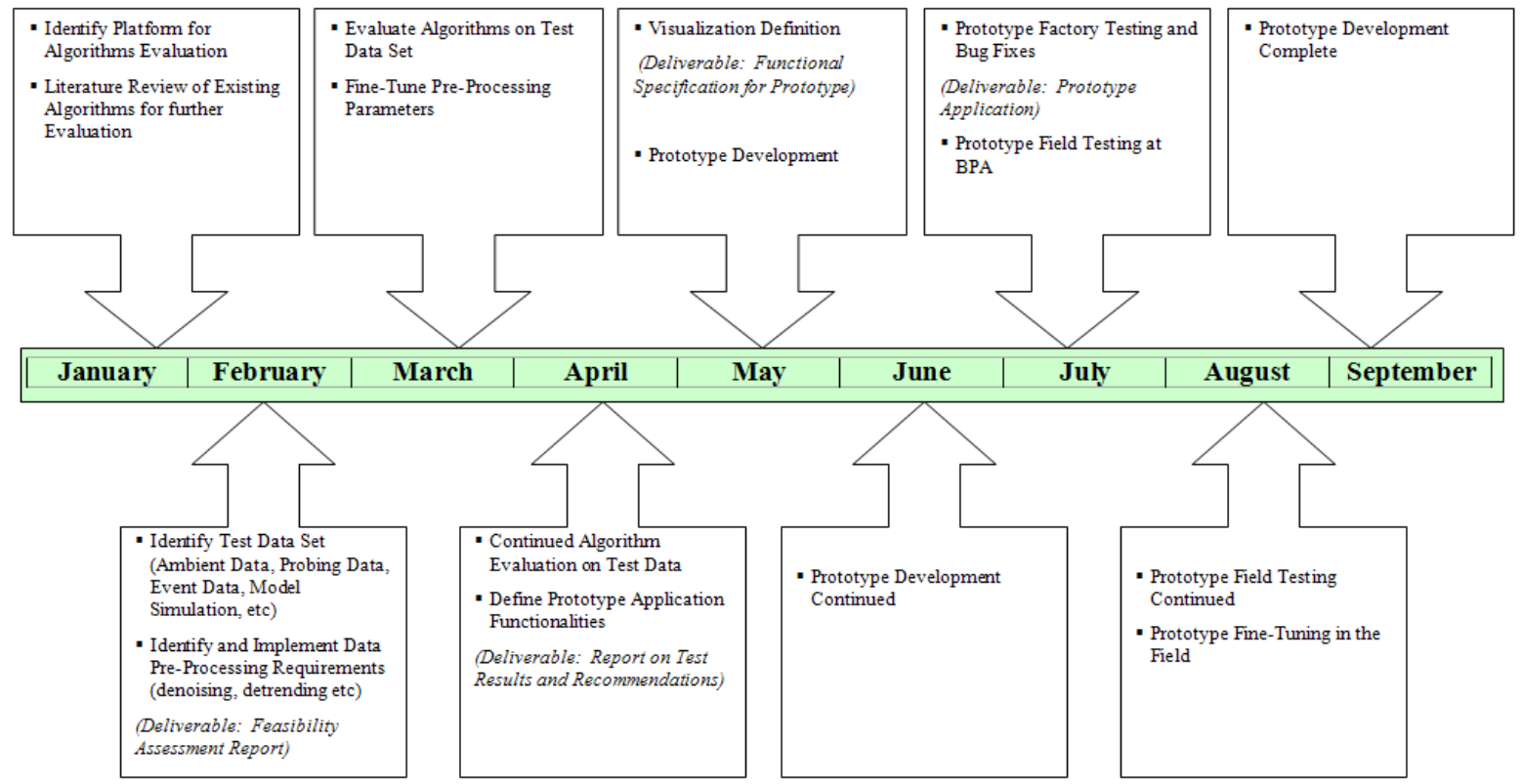

Figure 9: Small-Signal Stability Monitoring Prototype Implementation Plan

Stability Nomogram Validation: The objective during the current phase of the project was to conduct a feasibility assessment study to propose several approaches of using these time synchronized, high resolution PMU measurements, and possibly other EMS/SCADA data, for better assessment of the system operating conditions with respect to their stability limits, and consequently improve or validate existing nomograms.

The need for a more dynamically adjustable nomogram is well understood at the California ISO, and several ideas have been generated around the potential use of manually or automatically adjusted nomograms. In general terms, the proposed concept deals with the tradeoff between the pre-calculated fixed operating limits that are based on extensive computations (which tend to be more conservative due to the uncertainty about the applications) and the limits calculated in real time and adjusted to the current system conditions (which are computationally less expensive, but based on better knowledge of current conditions). Additionally, the real-time operating conditions can deviate from the simulated conditions that have been used to build the pre-calculated existing nomograms. The existing nomograms have been developed using a very limited number of critical parameters that can hardly reflect the changes of the remaining system parameters that are not included in the nomograms. Hence, these conservative limits 
adversely effect the definition of congestion costs on the one hand, and do not completely exclude system problems on the other hand.

By shifting the focus from some of the pre-calculated operating constraints to real-time calculations, it is possible to build more flexible nomograms. Specifically, the use of real-time measurements provided by PMUs and the results of real-time stability assessment applications can complement the existing nomograms by making the pre-calculated nomograms less conservative. These measurements can also provide data to select critical nomogram parameters for visualization based on real-time information and determine new areas and situations where additional nomograms may be required. Some of the ways in which phasor measurements can be used to validate or improve nomograms are [12]:

- Detection of potential "holes" in the existing nomograms (Figure 10a)

- Detection of excessive "conservatism" in the existing nomograms (Figure 10b)

- Use of reduced dynamic equivalents whose parameters are estimated by phasor measurements in both transient stability and small-signal oscillatory studies.

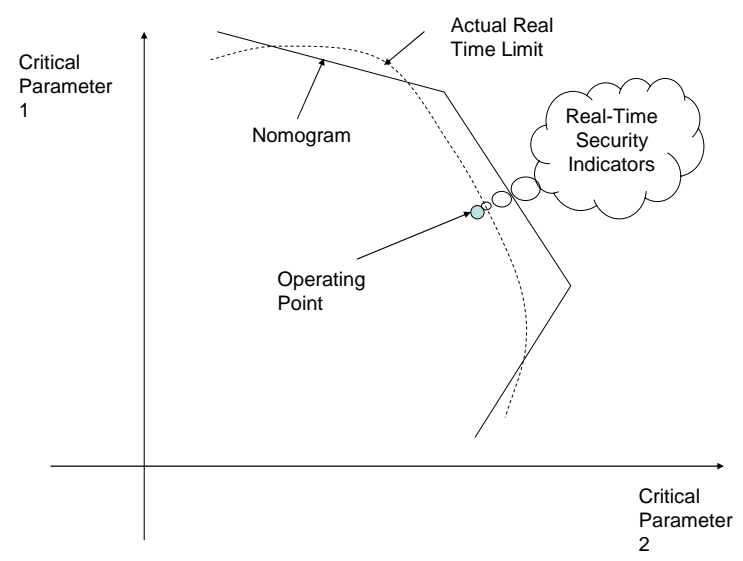

(a)

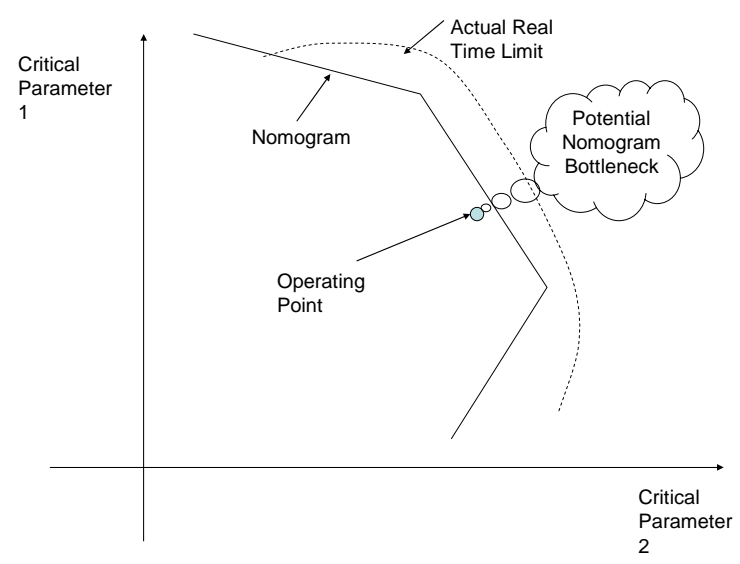

(b)

\section{Figure 10: Using Phasor Measurements to Detect (a) Potential "Holes" and (b) Excessive "Conservatism"}

At the same time, there are several limiting factors that need to be considered while addressing these tasks:

- The nomograms reflect various contingency and system conditions. The real-time measurements reflect just the current system state/contingency, and therefore are not indicative of potential stability problems that might happen for the same load and generation pattern under different contingency conditions or under heavier loading conditions.

- $\quad$ Although PMUs can track the dynamics of certain grid variables in real time, there are only a limited in number of PMUs distributed over a wide area. Since PMUs do not provide full observability of the system state - additional data from the state estimation and SCADA may be required.

- The number and location of the existing PMUs may not be adequate to the task of monitoring of local stability limits such as those induced by voltage stability problems.

Nevertheless, phasor measurements do provide wide area observability of system swing or oscillatory dynamics where the state estimator performance is too slow, and certain approaches that exploit these attributes can be suggested for nomogram validation purposes. 
Although the existing set of PMU measurements do not provide complete system observability, they could nevertheless provide wide-area visibility and one could conceptualize a completely new type of WideArea Nomograms for monitoring where nodal voltages (magnitude and/or angle) may provide a more convenient coordinate system for measuring certain stability margins when compared with nodal power injections that are traditionally used for this purpose. For example, angle differences may indicate more stress posed on the system, and that there are certain limits of this stress that make the system unstable or push it beyond the admissible operating limits such as thermal or voltage magnitude limits. At the same time, conditions applied to the angle differences are quite primitive and do not provide an acceptable accuracy of approximation of the power flow stability boundary, especially due to the nonlinear shape of this boundary. A hypothetical wide area nomogram for three angles (shown in Figure 11) could be described by the following set of inequalities [7]:

$$
\left\{\begin{array}{l}
\rho_{11} \delta_{1}+\rho_{12} \delta_{2}+\rho_{12} \delta_{2} \leq \delta_{1}^{\max } \\
\rho_{21} \delta_{1}+\rho_{22} \delta_{2}+\rho_{22} \delta_{2} \leq \delta_{2}^{\max } \\
\cdots \\
\rho_{m 1} \delta_{1}+\rho_{m 2} \delta_{2}+\rho_{m 2} \delta_{2} \leq \delta_{m}^{\max }
\end{array}\right.
$$

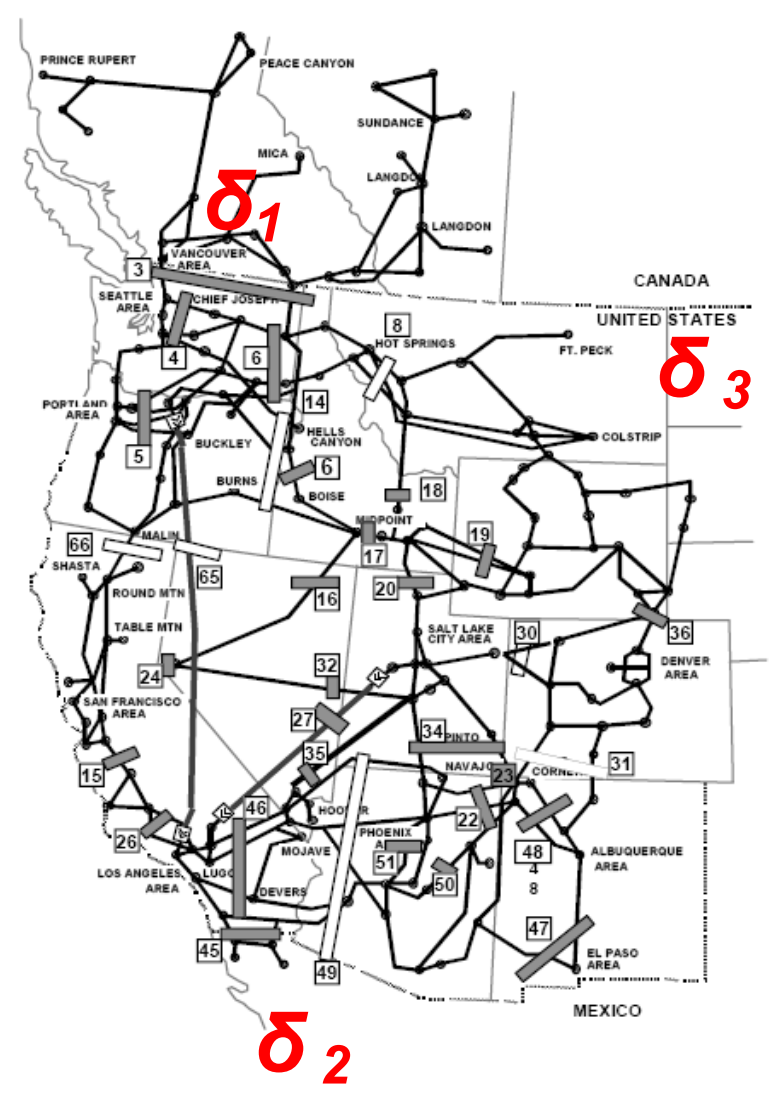




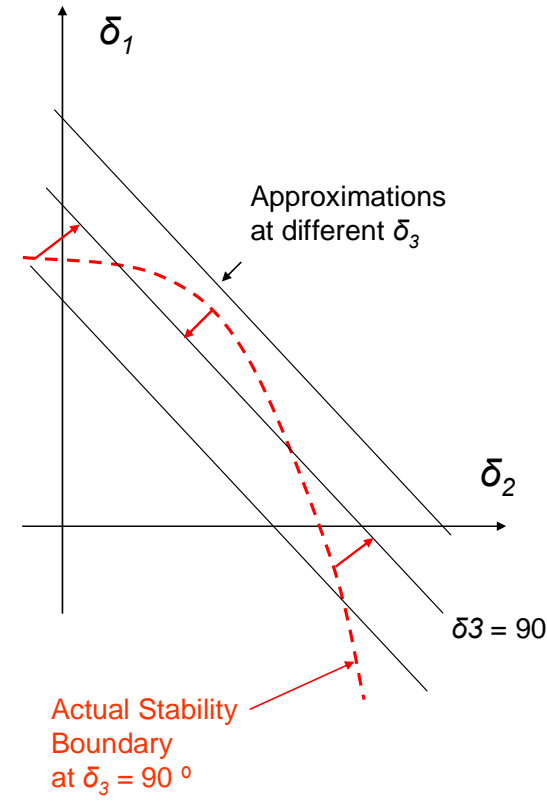

(a)

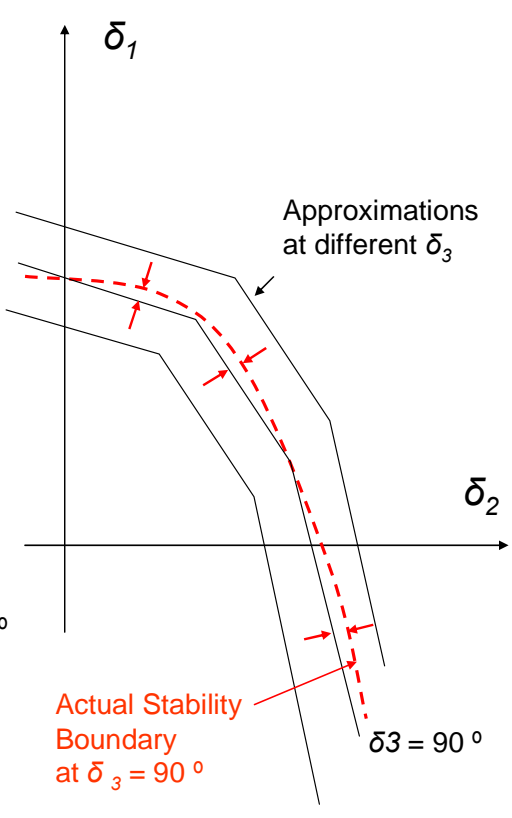

(b)

Figure 11: Conceptual View of Simple Angle Difference and Advanced Angle Nomograms

Additionally, a CA PIER funded parallel effort by CERTS is currently underway in developing a Voltage Security Application (VSA) that runs in real time and provides real time dispatchers with real time reliability metrics related to voltage stability limits. This VSA platform can easily be expanded to study wide-area voltage stability problems by selecting global stressing directions and developing the corresponding security regions. The algorithms being developed in the VSA application provide voltage magnitude and angle information as well as their corresponding sensitivities and participation factors in voltage collapse. Hence, while the proposed VSA framework uses data from the CA ISO state estimator and assumes full observability, this same VSA framework could also be used to develop wide-area nomograms whose coordinates would be nodal voltage magnitudes and angles, and the PMU measurements could directly be used to monitoring the system conditions with respect to these new nomograms for a wide-area security assessment.

A Feasibility Assessment Study describing the above mentioned approaches has been delivered to CA ISO. The next steps on this project are:

- Review this report with the CA ISO Planning and Operating Eng. staff

- Solicit their comments and feedback

- Determine if they support the continuation of research in this area

- Identify a specific transmission path and associated nomogram that would be well suited for this type of research.

- Review the above CA ISO feedback with CIEE/ Energy Commission for their approval.

\subsection{Support Activities}

At the Technical Advisory Committee (TAC) meetings, the California utilities shared their plans on various applications of phasor measurements such as local remedial control interests by SCE, state estimation improvements by SDG\&E, and critical path monitoring by PG\&E. 
Local Remedial Control (SCE): As a pilot project, Southern California Edison has suggested implementing a phasor based control scheme internal to the CA ISO grid, which would not require WECC review or approval and would also avoid impacts on other utilities.

The first suggestion is to utilize phasors data to enhance or replace a Remedial Action Scheme (RAS) for an outage of the two $115 \mathrm{kV}$ transmission lines (Control-Haiwee-Inyokern \# 1 and 2) south of Control Substation, located in Bishop, CA. SCE has generating resources (hydro and geothermal) in and north of the Bishop area that also serve the load in the Mammoth ski resort area (winter peaking). At times, the generation is greater than the load and the excess flows south on SCE's $115 \mathrm{kV}$ lines and to LADWP's system, via the Control-Inyo $115 \mathrm{kV}$ lines. For an outage of the two Control-Haiwee-Inyokern $115 \mathrm{kV}$ lines, SCE has a RAS to trip generation and avoid a total collapse of the remaining system north of Bishop and to avoid an out-of-step condition with LADWP. Currently, the arming of this RAS is a manual process by the operator located at Control Substation.

With PMUs located at Bishop and Kramer, the objective of the project is to perform studies of the south of Control system, and to use phasor (and possibly SCADA) data to clearly define the limits and thresholds for action including:

- suggesting arming and disarming points to the operator

- suggesting or selecting RAS tripping requirements

Eventually the phasor and SCADA data can automatically select tripping requirements and arm/disarm the RAS.

A second suggestion is to utilize the phasor data to automate the N-1 and N-2 RAS between SCE's Big Creek area and Magunden Substation in a phased approach similar to the suggestion above.

CERTS/EPG met with SCE engineers on December $16^{\text {th }}, 2005$ to discuss the status of this project. SCE informed that due to other priorities and limited resources, there hasn't been much progress on this activity. CERTS/EPG has offered to provide assistance in moving this project forward and will be meeting again with SCE's operations staff during 1Q 2006 to better understand the existing RAS at Bishop. SCE also informed that they will be look further into the possibility of sharing the SCE phasor data with other WECC members and the required Non-Disclosure Agreements (NDAs). 


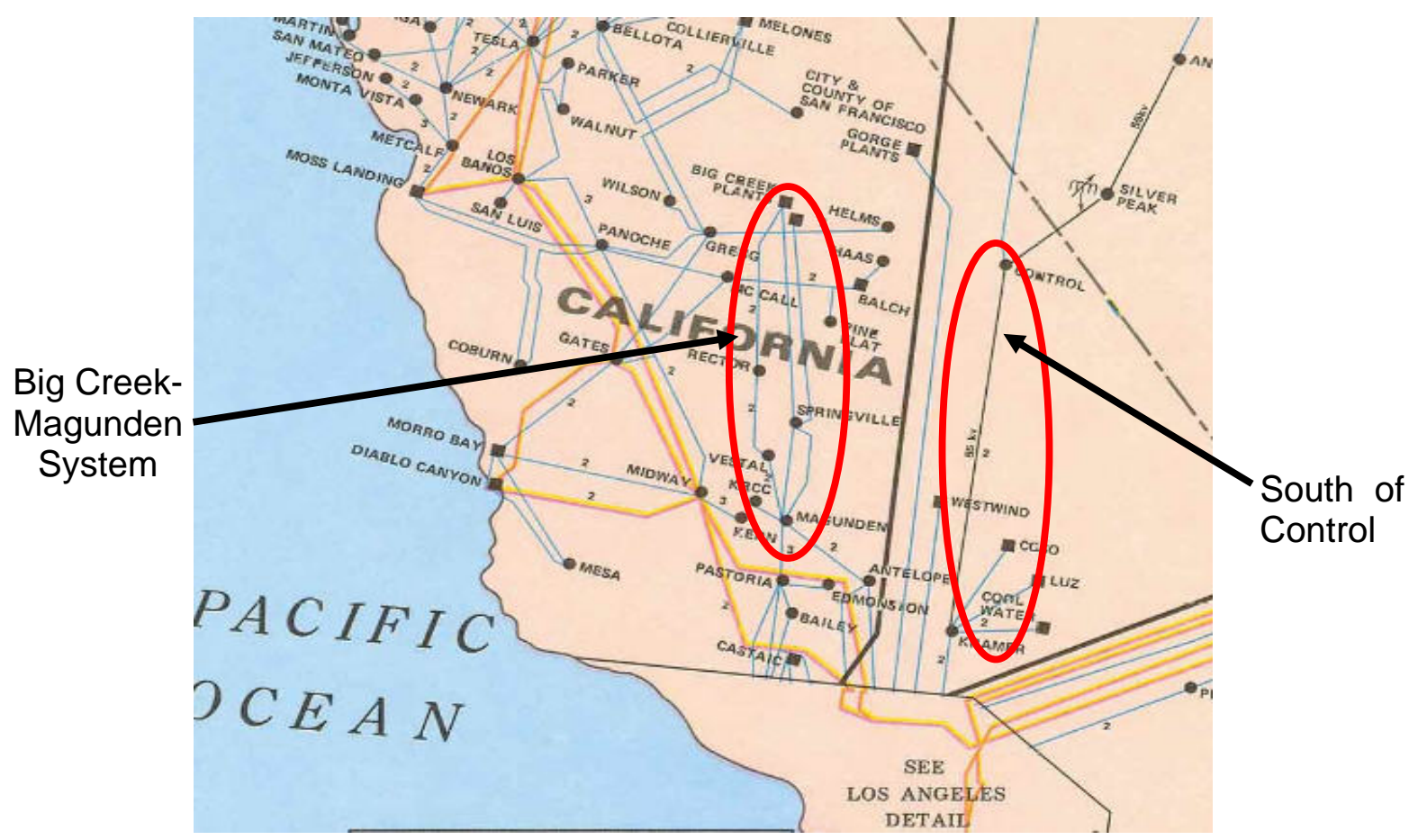

Figure 12: Local Remedial Control by SCE

State Estimation with Phasors (SDG\&E): San Diego Gas \& Electric (SDG\&E) has proposed a two year Research Development and Demonstration (RD\&D) project to integrate time-synchronized phasor measurement data into the WLS State Estimator on the EMS at SDG\&E. The successful completion of the project will be measured by demonstration of improved state calculations and enhanced congestion management as a result of the phasor measurement integration. Direct benefits to SDG\&E will include improved power system security assessment and congestion cost savings for ratepayers. Direct benefits to the CA ISO will include additional phasor visibility for use in its own suite of operator tools and software applications.

SDG\&E proposes to install approximately 4-6 PMU's at key locations, particularly SDG\&E's 500kV interconnection with Arizona. The South West Power Link (SWPL) is one of the two major interstate tie lines connecting Arizona and California. A PDC and associated software will be installed to aggregate this phasor data which shall be shared with CA ISO and other utilities to enhance the Wide Area Measurement System (WAMS) and Wide area Control system (WACS) initiatives in WECC.

SDG\&E also plan on evaluating the following three possible methods for incorporating phasor measurements from the PMUs into present state estimation calculations and a selection will be made [22]:

1. Using weighted least squares with significant weight on the PMU measurements

2. Eliminating the equations associated with the voltage phase angle measurements made by the PMU

3. Employing a hybrid estimation model that uses the measurements of PMU to modify the WLS estimates with the PMU

A technical report on the evaluation and selection will be part of this task.

Some of other objectives of the project include the use of phasor data and the improved estimates to optimize congestion management on SWPL and particularly at Miguel (western terminus of SWPL), demonstrate to what extent the improved estimates lead to better congestion management, financial benefits and improved reliability and to investigate related benefits such as improvement to the calculation of ATC. 
To assist SDG\&E with the project, CERTS/EPG has prepared and delivered a PMU Measurement Unit Installation and Cost Estimates document to SDG\&E [11], and is available to provide assistance as required.

Path Monitoring (PG\&E): PG\&E would like to use phasor measurements to monitor two of their critical paths: Path 15 and Path 26. Path 15 is composed of six transmission lines located near Fresno, California. There are two $500 \mathrm{kV}$ lines and four $230 \mathrm{kV}$ lines (Figure 13). The flow on Path 15 is normally South-to-North, except for heavy exports from Northern California to Southern California, which may create North-to-South flow on Path 15 during normal system conditions. The maximum South-to-North Operating Transfer Capacity (OTC) of this path is $3950 \mathrm{MW}$, and the maximum North-to-South OTC is $1275 \mathrm{MW}$ with normal system conditions. Path 26 is composed of three $500 \mathrm{kV}$ transmission lines between Northern and Southern California (Figure 13). The flow on Path 26 in normally North-to-South, except during high South-to-North flows on Path 15. With high South-to-North flows on Path 15, Path 26 is normally lightly loaded during normal system. Path 15 and Path 26 are electrically in series - one path may restrict the flows on the other due to a constraint.

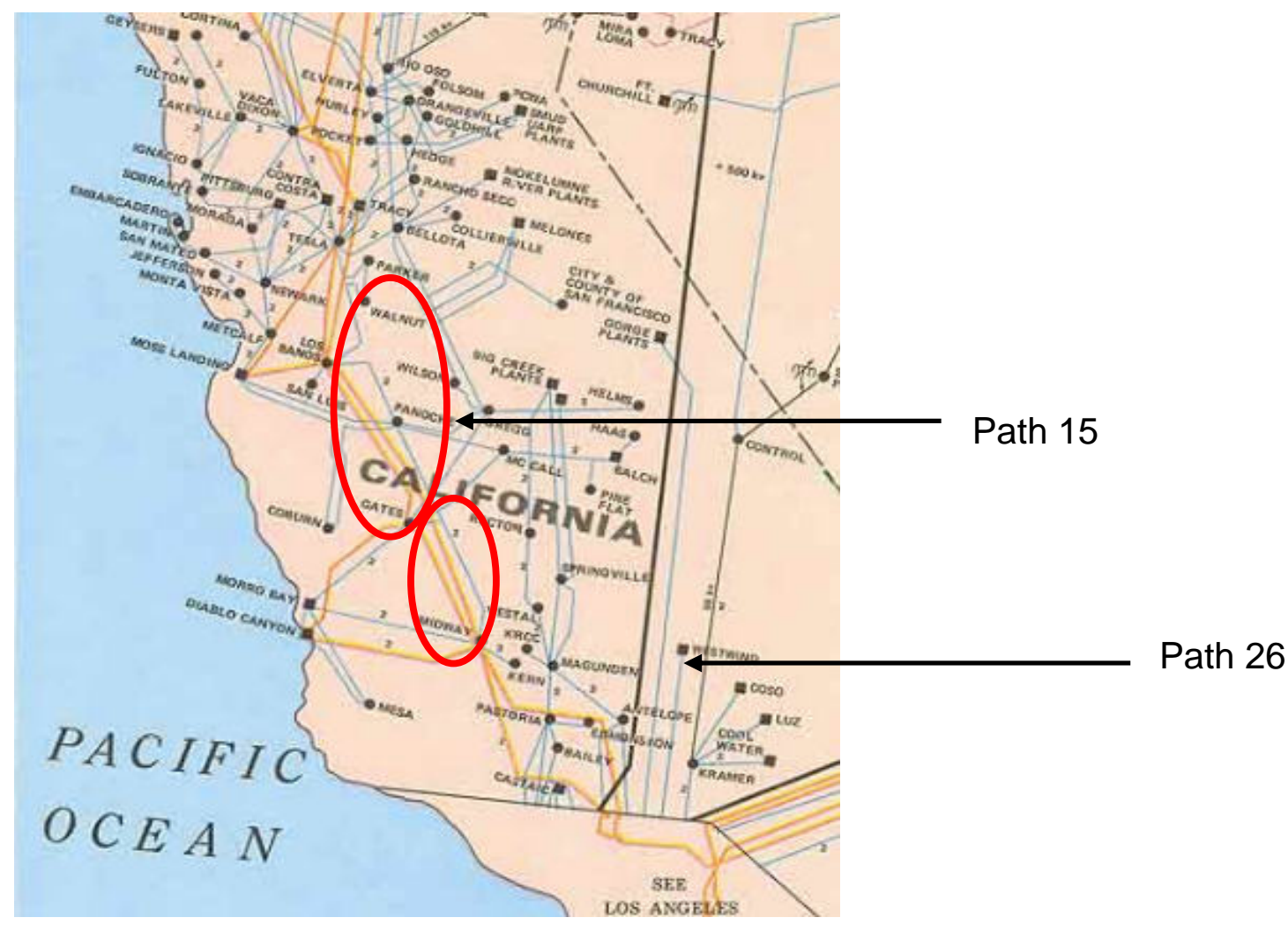

Figure 13: Critical Path Monitoring by PG\&E

PG\&E has PMUs installed at Midway, Moss Landing, Pittsburgh, Diablo Canyon, and Tesla, and intend to instal PMUs at Gates and Los Banos during 1Q 2006. CERTS met with PG\&E staff on December 8, 2005 to share the CA ISO research roadmap, and demonstrate the RTDMS application. They are presently working towards real-time sharing of phasor data with BPA, and the RTDMS system will be installed at PG\&E as soon as this data exchange is established. 


\section{References}

[1] "Western Systems Coordinating Council Disturbance Report for the Power System Outage that Occurred on the Western Interconnection on August 10, 1996", October 18, 1996. Available online at ftp://www.nerc.com/pub/sys/all updl/docs/pubs/AUG10FIN.pdf.

[2] Final Report on the August 14, 2003 Blackout in the United States and Canada: Causes and Recommendations, U.S. - Canada Power System Outage Task Force, April 5, 2004. Available online at http://www.nerc.com/ filez/blackout.html.

[3] "CERTS PIER TRP Real-Time System Operations (RTSO) 2006-2007 Project Plan", November 29, 2005.

[4] A.A. Fouad and V. Vittal, Power System Transient Stability Analysis Using the Transient Energy Function Method, Prentice Hall, 1991.

[5] Y. V. Makarov, Z.-Y. Dong, and D. J. Hill, "A General Method for Small Signal Stability Analysis", IEEE Transactions on Power Systems, Vol. 13, Issue 3, August 1998, pp. 979 - 985.

[6] Z.-Y. Dong, Y.V. Makarov, and D.J. Hill, "Genetic Algorithms in Power System Small Signal Stability Analysis", Fourth International Conference on Advances in Power System Control, Operation and Management APSCOM-97, Vol. 1, November 11-14, 1997, pp. 342 - 347 (Conference Publication No. 450).

[7] Y. V. Makarov, D. J. Hill, and Z.-Y. Dong, "Computation of Bifurcation Boundaries for Power Systems: A New $\Delta$-Plane Method", IEEE Transactions on Circuits and Systems I: Fundamental Theory and Applications, Vol. 47, Issue 4, April 2000, pp. $536-544$.

[8] J.W. Pierre, D.J. Trudnowski, M.K. Donnelly, "Initial Results in Electromechanical Mode Identification from Ambient Data," IEEE Transactions on Power Systems, Vol. 12, No. 3, Aug. 1997, pp. 1245-1251.

[9] R.W. Wies, J.W. Pierre, D.J. Trudnowski, "Use of ARMA Block Processing for Estimating Stationary Low-Frequency Electromechanical Modes of Power Systems", IEEE Transactions on Power Systems, Vo. 18, N0. 1, Feb. 2003, pp. 167-173.

[10] D. Trudnowski, "Estimating Swing Mode Properties using Spectral Analysis of Time-Synchronized Signals", EIPP Working Note 2005.

[11] J. Coroas, C. Martinez, J. Dyer, "Phasor Measurement Unit (PMU) Installation Process and Cost Estimates", Report prepared for San Diego Gas \& Electric and California Energy Commission, May 2005.

[12] CERTS/EPG, "Nomogram Validation using Phasor Measurements: Feasibility Assessment Report", Report funded by PIER-TRP prepared for CA ISO, Jan 2006.

[13] CERTS/EPG, "Real-Time Dynamics Monitoring Systems (RTDMS) Version 3 Users Guide, Document prepared for CA ISO, Aug 2005.

[14] CERTS/EPG, "Functional Specifications for CA ISO RTDMS Version 4.0", Document prepared for CA ISO, Dec 2005.

[15] G. E. P. Box, G. M. Jenkins and G. C. Reinsel, "Time Series Analysis Forecasting and Control," Prentice-Hall Inc., 1994. 
[16] N.G. Bretas and A.G. Phadke, "Real Time Instability Prediction Through Adaptive Time Series Coefficients", 1999 IEEE Power Engineering Society Winter Meeting, Vol. 1, 31 Jan.- 4 Feb. 1999, pp. 731-736.

[17] R. Betancourt, "Estimation of Parameters in Transient Stability Output Swings Using Autoregressive Models", 1990 IEE Proceedings - Generation, Transmission and Distribution, Vol. 137, July 1990, No. 4, pp. 315-320.

[18] G. Ledwich and E. Palmer, "Modal Estimates from Normal Operation of Power Systems", 2000 IEEE Power Engineering Society Winter Meeting, Vol. 2, 23-27 Jan. 2000, pp. 1527 -1531.

[19] M. Hemmingsson, O. Samuelsson, K.O.H. Pedersen, A. Nielsen, "Estimation of Electro-Mechanical Mode Parameters Using Frequency Measurements", 2001 IEEE Power Engineering Society Winter Meeting, Vol. 3 , 28 Jan.-1 Feb. 2001, pp. 1172-1177.

[20] T. Van Cutsem, I. Dobson (Editor), C. DeMarco, D. Hill, I. Hiskens, T. Overbye, M. Venkatasubramanian, C. Vournas, Basic Theoretical Concepts, Chapter 2, IEEE Voltage Stability Work Group Report, April 1998. Available online at http://eceserv0.ece.wisc.edu/ dobson/WG/.

[21] C. Martinez, S. Mo, "Functional Specification for Phasor Based Secondary Data Source", Document prepared for CA ISO, May 2005.

[22] L. Kondragunta, "Enhancement of State Estimation Results Using Real Time Phasoe Measurement Data", Draft Report for Review by CIEE, Aug 15, 2005. 
Appendix E

Task 3.0 Prototype Phasor-Based Real-Time Monitoring Software Tool Training Presentation 
Consortium for

Electric

Reliability

Technology

Solutions
CA ISO

Real-Time

Dynamics

Monitoring

System $^{\mathrm{TM}}$

(RTDMS)

\title{
USER GUIDE
}

\section{Version 3}

August 2005

\author{
Prepared by: \\ 敞 Electric Power Group \\ for




\section{Preface}

Electric Power Group (EPG), under the auspices of CERTS, has developed the CA ISO Real-Time Dynamics Monitoring System (RTDMS). RTDMS was built on the Grid-3P TM technology platform and uses high resolution synchronized data from Phasor Measurement Units (PMUs) for monitoring grid dynamics. The application is for the real time monitoring and tracking of phase angles between sources and sink buses within the Western Interconnection, local and system frequencies at a high resolution as well as MW and MVAR flows computed from the voltage and current phasor measurements.

The flow of real-time data and information from the substation PMUs to the utility Phasor Data Concentrator (PDC), through the WECC Wide-Area Network and into the CA ISO's PDC and visualization monitor is depicted in Figure 1. The Real Time Dynamics Monitoring System is used to visualize phasor measurement data and provide the user with displays to monitor real-time dynamics and system events within the WECC grid.

\section{CAISO PHASOR NETWORK DIAGRAM (PRESENT AND FUTURE)}

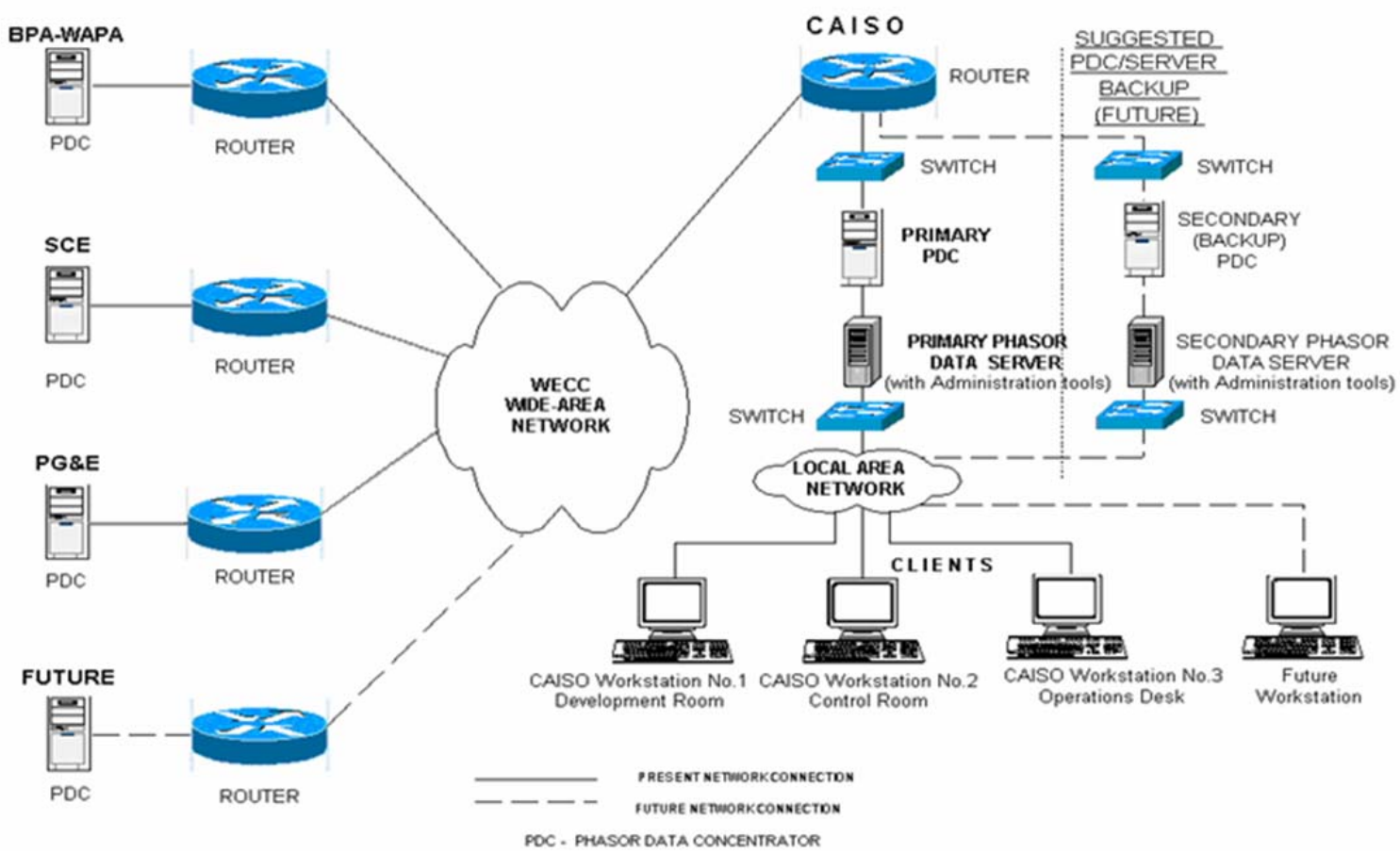

Figure 1: Data Flow for the Real-Time Dynamics Monitoring System Prototype

\section{License Agreements and Copyrights / Non-Disclosure Agreements}

The Real-Time Dynamics Monitoring System technology platform was developed by EPG under the auspices of CERTS and funded by the Department of Energy. Adaptation of RTDMS for CA ISO use was funded by the Energy Commission.

RTDMS is intended for the exclusive use by the CA ISO and the technology is protected by copyright granted by the US Department of Energy to Electric Power Group. No duplication or commercialization of this application is permitted without the written consent of Electric Power Group.

This application is for authorized users only subject to Non-Disclosure Agreement between data providers and CA ISO. 


\section{Table of Contents}

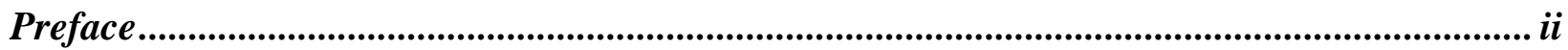

License Agreements and Copyrights / Non-Disclosure Agreements ............................................. ii

Table of Contents ....................................................................................................................................iii

List of Figures ..................................................................................................................................

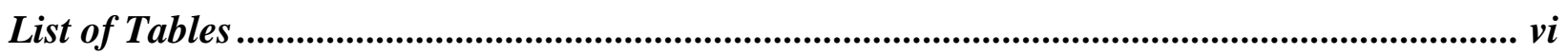

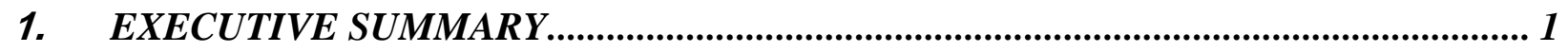

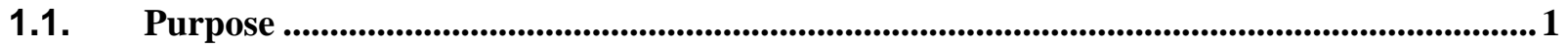

1.2. Intended Users........................................................................................................................... 1

1.3. Functional Overview............................................................................................................... 1

1.4. RTDMS Client Application Requirements..............................................................................

1.5. Monitoring Performance..........................................................................................................

2. INTRODUCTION ............................................................................................................. 4

2.1. Real Time Wide Area Performance Monitoring........................................................................ 4

2.2. RTDMS - Functional Overview ………………….....................................................................4

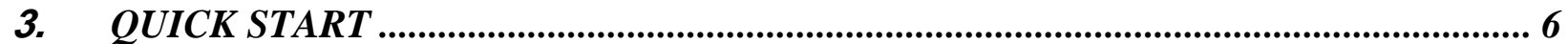

4. REAL-TIME DYNAMICS MONITORING SYSTEM OVERVIEW .............................. 7

4.1. Application Architecture................................................................................................................ 7

4.1.1. Data Retrieving, Alarming and Storage - RTDMS Server ...............................................................

4.1.2. RTDMS Client Monitoring Visualization Application - RTDMS Clients.........................................

4.2. Visualization Architecture ............................................................................................................ 9

4.2.1. Date/Time \& Auto Refresh Window ………………………………………………………………....9

4.2.2. Configuration Utility.................................................................................................................

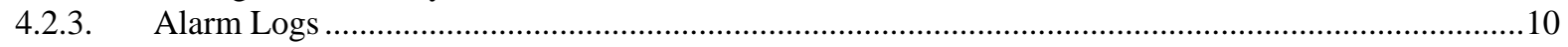

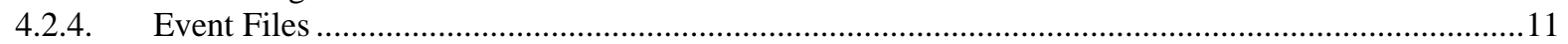

4.2.5. Navigational Tools.......................................................................................................................12

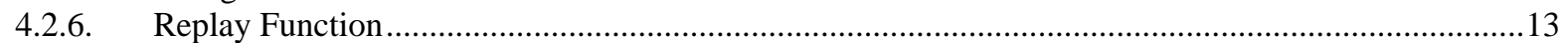

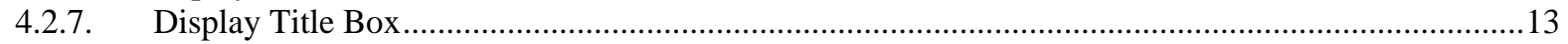

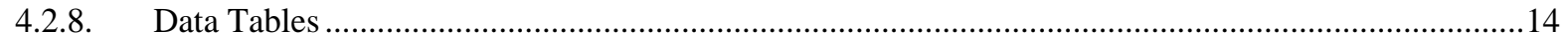

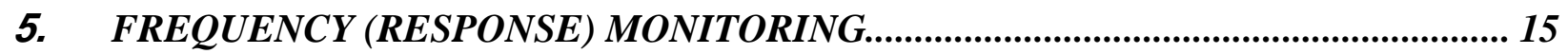

5.1. Overview .................................................................................................................................. 15

5.2. Frequency (Response) Monitoring One-Panel Display ………………………........................16

5.3. Frequency (Response) Monitoring Four-Panel Display ......................................................... 19

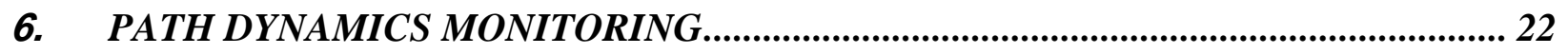

6.1. Overview ....................................................................................................................... 22

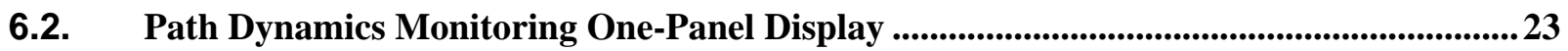

6.3. Path Dynamics Monitoring Four-Panel Display ......................................................................... 25

6.3.1. Path Dynamics - Wide Area Monitoring/Tracking ...............................................................25 
6.3.2. Path Dynamics - Local Area Monitoring/Tracking ............................................................................27

7. RELATIVE ANGLE|VOLTAGE MAGNITUDE MONITORING.............................. 30

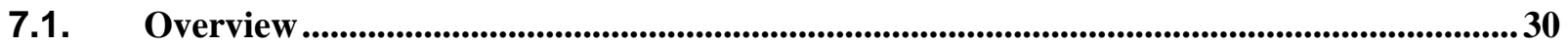

7.2. Relative Angle/Voltage Magnitude One-Panel Display …....................................................... 31

7.3. Relative Angle/Voltage Magnitude Four-Panel Display...................................................34

7.3.1. Relative Angle/Voltage Magnitude - Wide Area Monitoring/Tracking...............................................34

7.3.2. Relative Angle/Voltage Magnitude - Local Area Monitoring/Tracking ….........................................36

8. PATH FLOW (MW) MONITORING ................................................................ 38

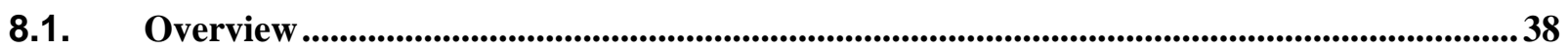

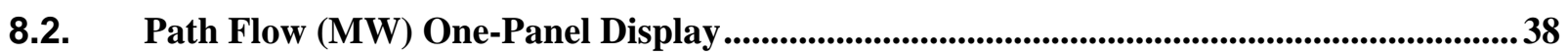

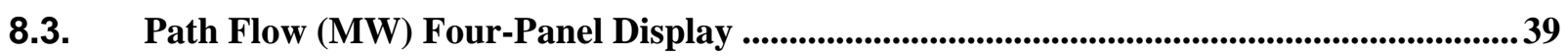

9. PATH FLOW (MVAR) MONITORING ............................................................. 41

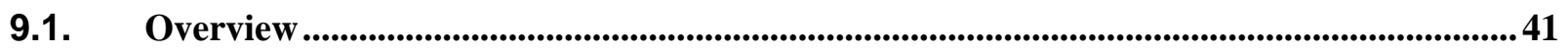

9.2. Path Flow (MVAR) One-Panel Display …....................................................................................41

9.3. Path Flow (MVAR) Four-Panel Display.................................................................................... 42

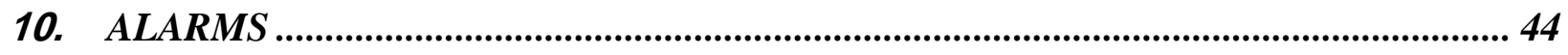

11. EVENT FILES AND OFFLINE ANALYSIS ................................................... 46

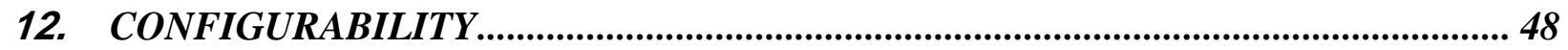

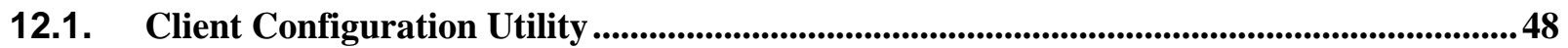

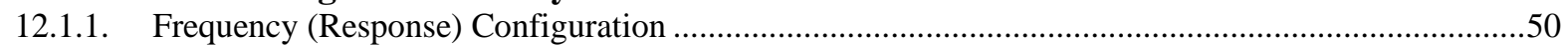

12.1.2. Path Dynamics Configuration....................................................................................................51

12.1.3. Relative Angle/Voltage Magnitude Configuration ............................................................................52

12.1.4. Path Flow (MW/MVAR) Configuration............................................................................................52

13. PROPERTY EDITOR ....................................................................................................... 54

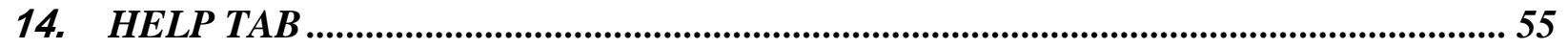

CUSTOMER SUPPORT CONTACT INFORMATION ................................................. 56

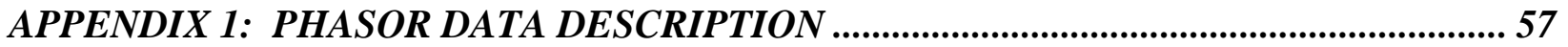

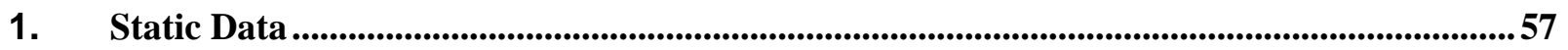

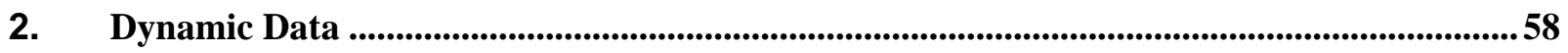




\section{List of Figures}

Figure 1: Data Flow for the Real-Time Dynamics Monitoring System Prototype ................................... ii

Figure 2: RTDMS Client Monitoring Application - Sample Display (Path Dynamics) ................................. 2

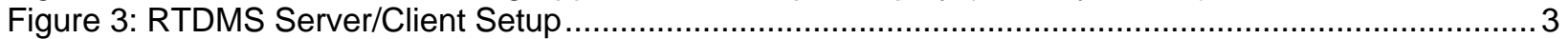

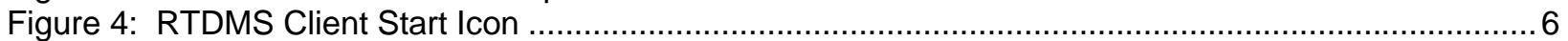

Figure 5: RTDMS Default Display - Frequency (Response) Monitoring ........................................... 6

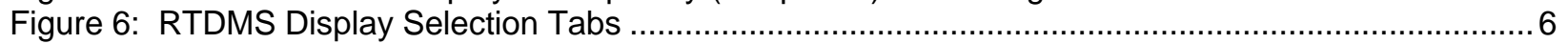

Figure 7: Real-Time Dynamics Monitoring System Architecture ..................................................... 8

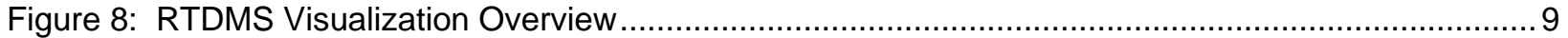

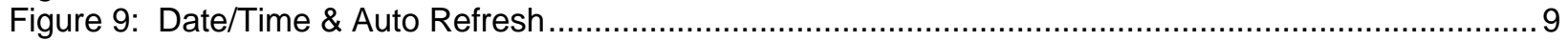

Figure 10: Date Selection Graphic User Interface for Replay .......................................................... 9

Figure 11: Configuration Utility Graphic User Interface ............................................................... 10

Figure 12: "Alarm Logs" Tab Highlighted in Red to Alert RTDMS User of New Alarm............................ 10

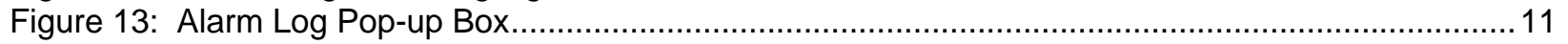

Figure 14: Navigation Window to Load Event Files .................................................................. 11

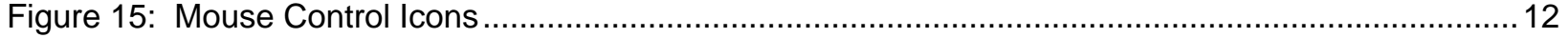

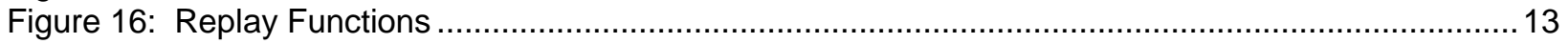

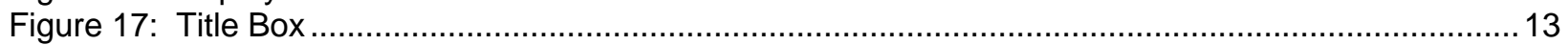

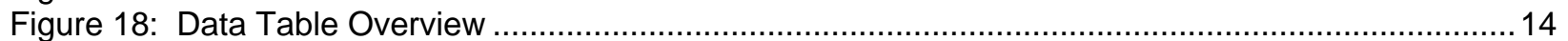

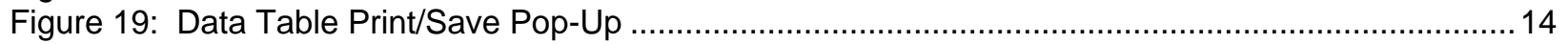

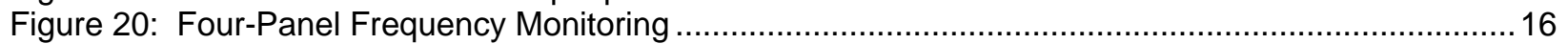

Figure 21: Generation-Load Imbalance Display/Duration Pop-Up .................................................... 16

Figure 22: Four-Panel Frequency/Generation-Load Imbalance Monitoring ...................................... 17

Figure 23: Soft/Hard Threshold Selection Pop-Up ....................................................................... 18

Figure 24: Frequency /Imbalance Duration Selection Pop-Up ...................................................... 18

Figure 25: Scroll Monitoring Graph (Enlarged) - (a) delta interconnection frequency (b) local frequency

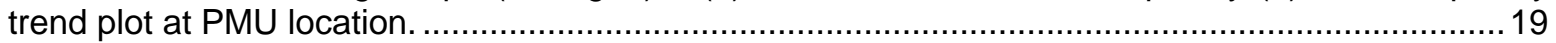

Figure 26: Local Frequency Coherence Comparison (Panel 2) ........................................................20

Figure 27: Local Frequency Coherence Comparison Duration Selection Pop-Up ................................20

Figure 28: Local Frequency Coherence Comparison Legend ........................................................20

Figure 29: Interconnection Frequency \& Imbalance Duration Curves (Panel 3) ...................................21

Figure 30: Interconnection Frequency \& Imbalance Duration Curves Display Pop-Up..........................21

Figure 31: Interconnection Frequency \& Imbalance Duration Curves Duration Selection Pop-Up............21

Figure 32: Four-Panel Path Dynamics Monitoring (Wide Area)........................................................ 22

Figure 33: Four-Panel Path Dynamics Monitoring (Local Area) ........................................................23

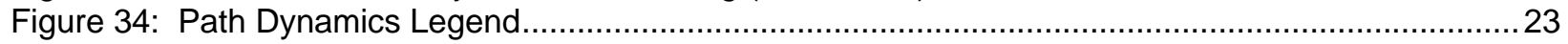

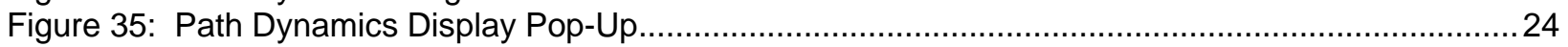

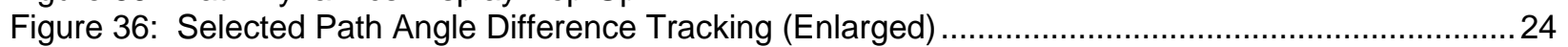

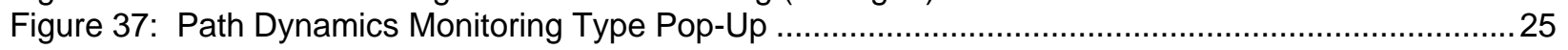

Figure 38: Four-Panel Path Dynamics - Wide Area Monitoring/Tracking ...........................................25

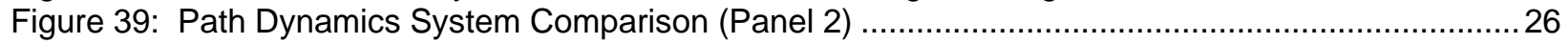

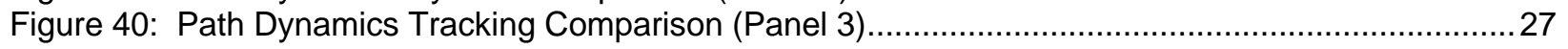

Figure 41: Path Dynamics Tracking Comparison Duration Pop-Up ..............................................2 27

Figure 42: Four-Panel Path Dynamics - Local Area Monitoring/Tracking ..........................................2 28

Figure 43: Four-Panel Path Dynamics - Local Area Monitoring/Tracking (Four Paths Selected) .............29

Figure 44: Four-Panel Relative Angle/Voltage Magnitude Monitoring (Wide Area) ................................. 30

Figure 45: Four-Panel Relative Angle/Voltage Magnitude Monitoring (Local Area) ................................ 31

Figure 46: Relative Angle/Voltage Magnitude - Relative Angle and Voltage Magnitude Legends........... 31

Figure 47: Voltage Monitoring Reference Selector .......................................................................... 32

Figure 48: Relative Angle/Voltage Magnitude Display Options Pop-Up............................................ 32

Figure 49: One-Panel Relative Angle/Voltage Magnitude with Voltage Magnitude Information ................33

Figure 50: Relative Angle/Voltage Magnitude Voltage Levels Pop-Up ............................................... 33

Figure 51: Selected Relative Angle/Voltage Magnitude Tracking...................................................... 34

Figure 52: Relative Angle/Voltage Magnitude Monitoring Type Pop-Up ............................................ 34

Figure 53: Four-Panel Relative Angle/Voltage Magnitude - Wide Area Monitoring/Tracking.................... 35 
Figure 54: System Voltage Angle Tracking/Comparison Duration Pop-Up .......................................... 35 Figure 55: Four-Panel Relative Angle/Voltage Magnitude - Local Area Monitoring/Tracking ...................36

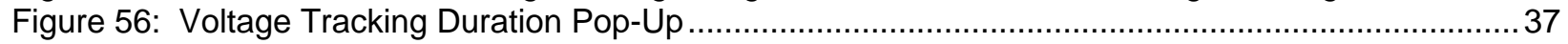

Figure 57: Voltage Tracking Including Voltage Magnitude (Panel 3) .................................................... 37

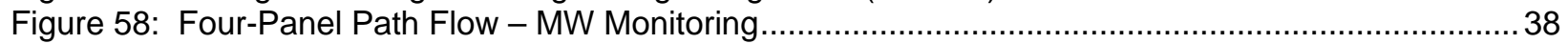

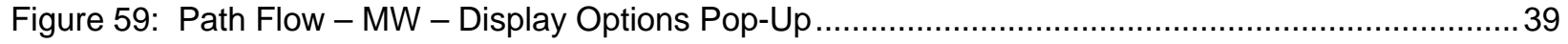

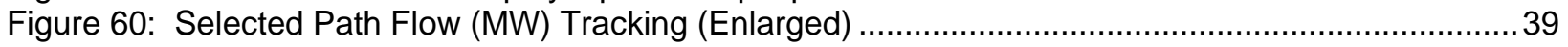

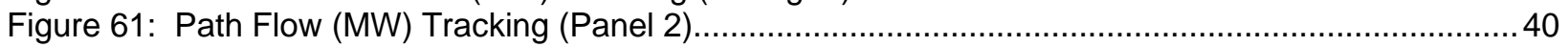

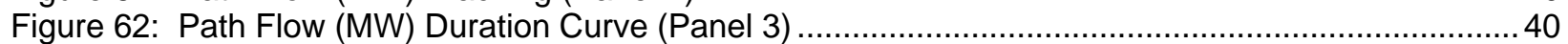

Figure 63: Four-Panel Path Flow - MVAR Monitoring .................................................................... 41

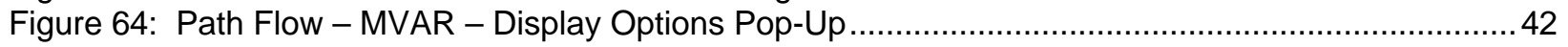

Figure 65: Selected Path Flow (MVAR) Tracking (Enlarged) …....................................................... 42

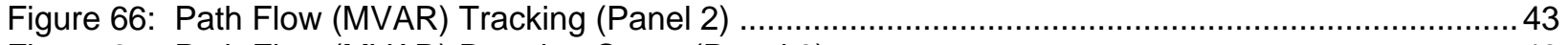

Figure 67: Path Flow (MVAR) Duration Curve (Panel 3) …........................................................... 43

Figure 68: "Alarm Logs" Tab Highlighted in Red to Alert RTDMS User of New Alarm........................... 44

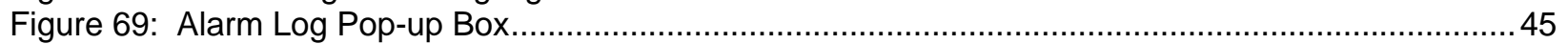

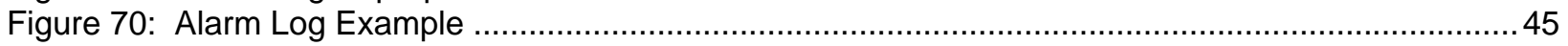

Figure 71: "Event Files" Tab for Loading Disturbance Files ......................................................... 46

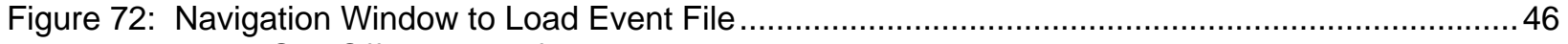

Figure 73: RTDMS in Offline Mode for Post-Disturbance Analysis ................................................. 47

Figure 74: Configuration Utility Master Graphic User Interface ..................................................... 48

Figure 75: Warning Pop-Up during PMU Removal ........................................................................ 49

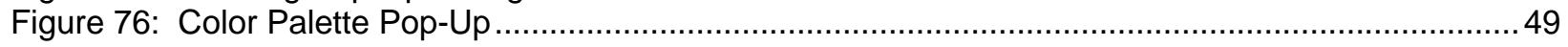

Figure 77: Frequency (Response) Configuration Graphic User Interface …....................................... 50

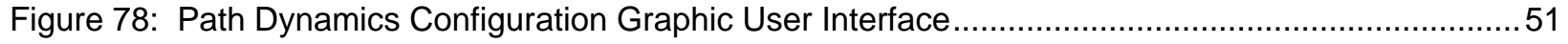

Figure 79: Rel. Angle / Volt. Magnitude Configuration Graphic User Interface .....................................5 52

Figure 80: MW / MVAR Path Configuration Graphic User Interface ....................................................5 53

Figure 81: Property Editor Sample Graphic User Interface (Path Dynamics - Panel 1) ........................5 54

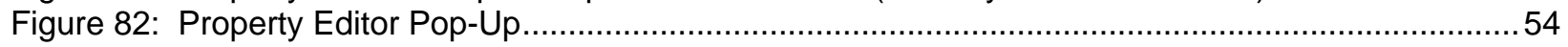

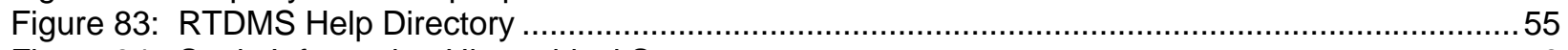

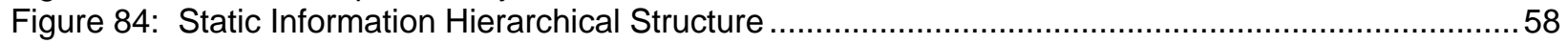

\section{List of Tables}

Table 1: RTDMS Visualization Client Application - Recommended Hardware Requirements ................... 3

Table 2: Current and Future PMUs to be Incorporated into the WECC Phasor Network .........................5

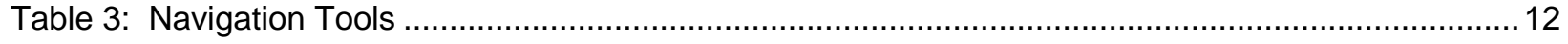




\section{EXECUTIVE SUMMARY}

\subsection{Purpose}

The CA ISO Real-Time Dynamics Monitoring System is designed to monitor the dynamics within the WECC grid and assess the system behavior during normal and disturbance conditions.

\subsection{Intended Users}

Operating authorities such as Independent System Operators (ISOs), Regional Transmission Organizations (RTOs), Reliability Coordinators, and Control Area Dispatchers in their management of grid reliability.

\subsection{Functional Overview}

- Real-Time Phasor Data Access in BPA's PDCStream Format - The CA ISO RTDMS system reads phasor data in the BPA defined industry standard PDCStream format. Phasor data input for this prototype can originate from the Phasor Measurement Units (PMUs) directly or from collection devices such as Phasor Data Concentrators (PDCs) or data repositories. This includes synchronized, highly accurate and precisely time tagged measurements of voltage phase angle and magnitude, current phase angle and magnitude, frequencies and MWs and MVARs

- Information Dissemination from the Server to Client Over LAN - The system architecture relies on prevalent Microsoft technologies to disseminate the data cached in memory at the host RTDMS Server to multiple RTDMS Client Applications over a Local Area Network (LAN).

The flow of data is as follows:

- The PDCStream Data Reader within the RTDMS Server reads the real-time data stream (Voltages, Currents, Frequency) and computes derived quantities (such as MW and MVAR flows).

- The RTDMS Server archives both the raw and derived quantities in memory within a rolling cache, the most recent alarms, and permanently archives event files, and makes this data and information available to all the RTDMS Client applications.

- The RTDMS Clients are the visualization applications that can access the phasor data and alarms from the Server over the LAN.

- Remote Client Monitoring Applications - The RTDMS Clients are stand-alone monitoring applications that connect to the RTDMS Server and acquire phasor data from the Server.

- Multiple Visualization Displays - The application will have multiple displays such as the one shown in Figure 2 for monitoring:

Interconnection Frequency

- Identify approximate point of acceleration or deceleration (loss of load or generation) within the WECC system.

- Assess system coherency and its dynamic stress under normal operating conditions.

Voltage Magnitude and Relative Angles

- Comprehensive profile of WECC voltage angles and magnitudes.

- Identify the high and low voltage regions within the WECC grid.

- Monitor angles relative to a specific location, utility or regions.

- Operating organizations ability to set threshold limits.

Angle Differences across Identified Transmission Paths 
- Provides a birds-eye view of the WECC sources and sinks of power.

- Monitor phase angle differences across key WECC paths.

- Operating organizations ability to set threshold limits.

Real and Reactive Power Flows Across Monitored Lines

- Monitor actual MW and MVAR flows at key paths.

- Track flows with respect to predefined thresholds.

- Operating organizations ability to set threshold limits.

- Configurability - A configuration tool provides a friendly graphic user interface to specify visualization subsets, and update the displays with new paths, thresholds etc, as new PMUs are added to the system. The defined configuration settings are portable and can be transferred with ease to other monitoring Clients. This procedure with preserve display standardization across the Western Interconnection.

- Alarms - The application uses hard limits on monitoring metrics (frequency, angle difference pairs, MW, MVAR) and rate of change for visual alarming. The hard limits are defined by the users based on offline studies, simulation and operating experience. All relevant information pertaining to these alarms (such as the metric associated with the violation, the start time of violation, the end time of violation, the violation value and corresponding threshold, the violation type, etc) are continuously shown in the alarming display and are also logged into a 'txt' file.

- Event Files - In addition to alarming, event files consisting of all monitored data over specified time duration (default duration is 5 minutes) are automatically stored whenever a rate of change alarm is generated. These event files can be loaded into the application for offline analysis.

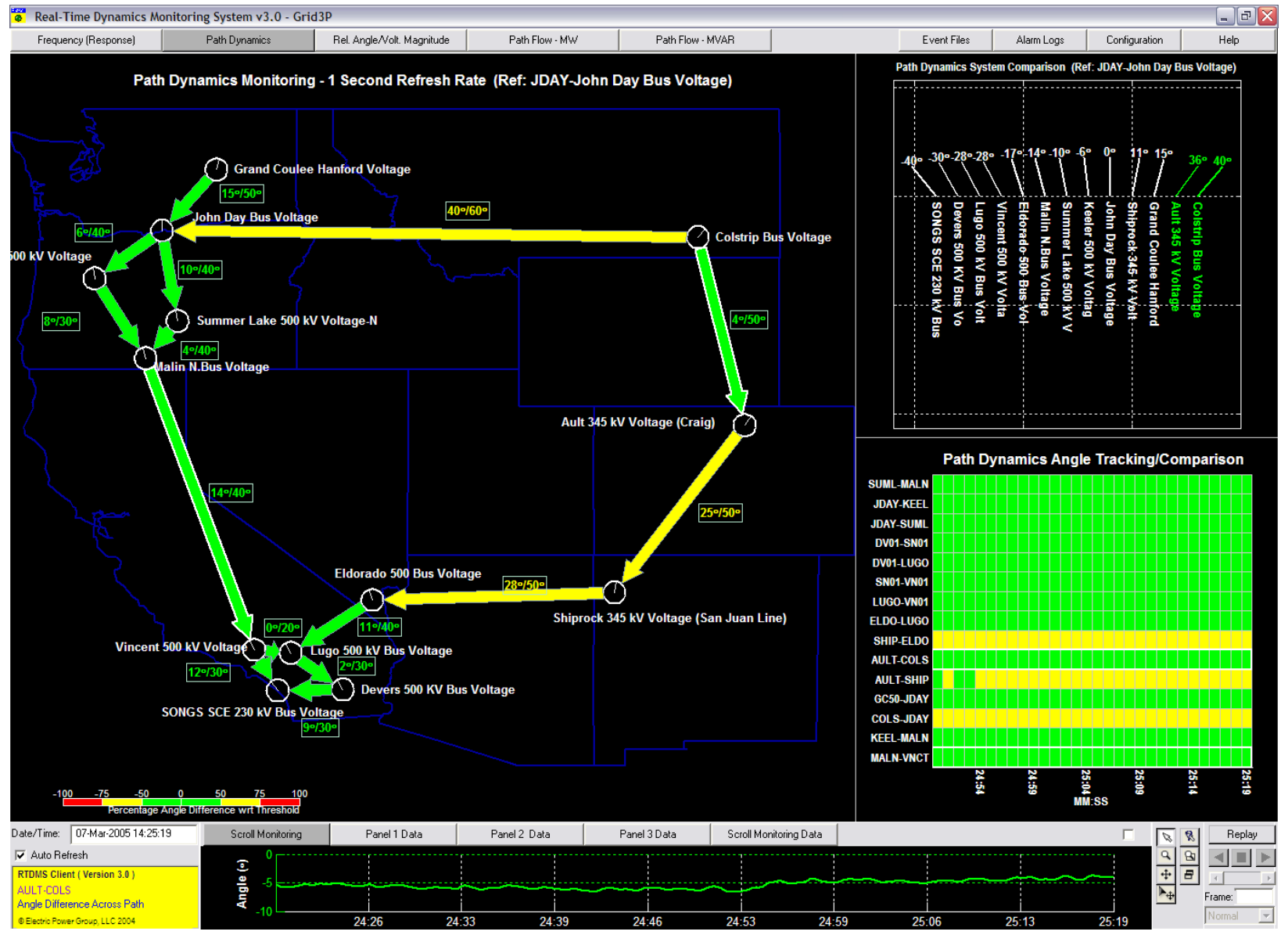

Figure 2: RTDMS Client Monitoring Application - Sample Display (Path Dynamics) 


\subsection{RTDMS Client Application Requirements}

The user can install the RTDMS Clients on a desktop computer and remotely connect to the RTDMS Server for accessing, displaying and monitoring real time phasor data (Figure 3).

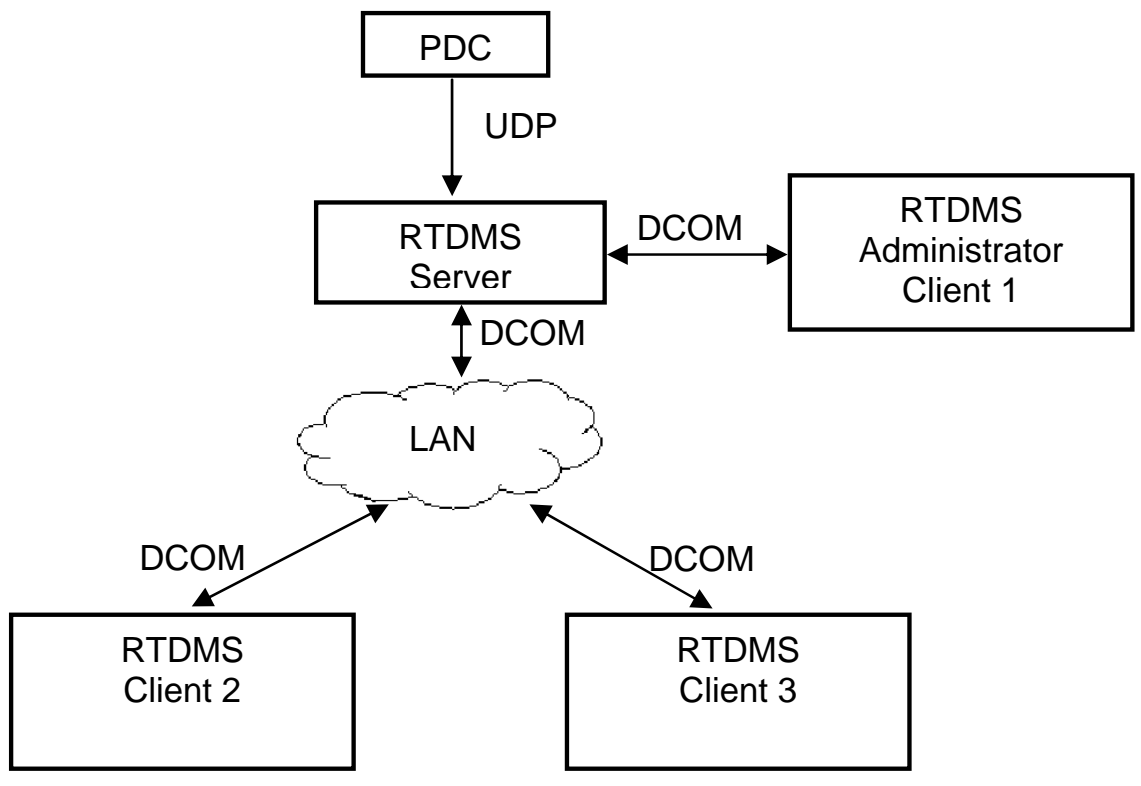

Figure 3: RTDMS Server/Client Setup

Requirements: The following is required to run the RTDMS Client application:

Table 1: RTDMS Visualization Client Application - Recommended Hardware Requirements

\begin{tabular}{|l|l|}
\hline Memory & $512 \mathrm{MB}$ or greater \\
\hline CPU & Pentium III processor or up (1.0 G Hz or higher) \\
\hline Video & $64 \mathrm{MB}$ graphic memory \\
& AGP 8X or PCl express graphic card such as nVidia Quadro \\
& FX 3000 and up. \\
\hline Storage & $20 \mathrm{~GB}$ hard disk and up \\
\hline Operating System & Windows 2000, Windows XP Professional \\
\hline Display & 1280 by 1024 Pixels or better \\
\hline
\end{tabular}

\subsection{Monitoring Performance}

The goal for the RTDMS system is to make information available to users in the shortest possible time. The performance of the application (such as refresh rates and resolution, replay time, etc) will be determined by the supporting system architecture. Some of the factors influencing system performance are:

- data resolution at the source

- communication latencies

- $\quad$ number of PMUs to be included in the visualization displays

In this version of the application, users may experience a delay of a few seconds from the time the data is read by the measurement units and when it is displayed within the RTDMS Client's visualization displays. 


\section{INTRODUCTION}

\subsection{Real Time Wide Area Performance Monitoring}

Investigation of the August 14, 2003 blackout concluded the need for wide-area monitoring to maintain the integrity of the grid through continuous checking of important metrics during normal and emergency conditions. The RTDMS application provides a tool to meet these new monitoring requirements. It collects phasor data procured by Phasor Measurement Units (PMUs) in a synchronized manner to provide real time information about the status of the system. The high resolution data offers wide-area visibility of system dynamics and real-time grid reliability assessment by utilities, ISOs, RTOs and Reliability Coordinators during normal and disturbance conditions. Details on how this application will accomplish this goal are provided in this document.

\subsection{RTDMS - Functional Overview}

This "User Guide" has been developed for the Real-Time Dynamics Monitoring System - a phasor monitoring system developed on the Grid-3Р тм Platform, and designed to utilize the true potential of phasor technology to closely track grid dynamics and assess the health of the grid. In particular, the ability of phasor measurements to directly and accurately measure system states (voltages/current magnitudes and angles), and to have them available precisely time-tagged at a very high sub-second rate offers a wide-area snapshot of system dynamics in real time. This kind of observability isn't available with conventional SCADA data (i.e., SCADA data is not capable of capturing swing dynamics). Furthermore, algorithms within the system also use the precise timing information to continuously track phasor data and perform real-time estimates of crucial network parameters and selected metrics to provide the most accurate up-to-date assessment of the system limits.

The phasor measurement units at a substation collect a set of data at a sub-second rate (30 times a second, 60 times a second, etc). Included in this set are voltage phasor measurement(s), current phasor measurements on each of the lines connected to the substation, a local frequency measurement as well as an accurate time stamp of when this set of measurements was made. The data from each one of the PMUs is then sent to a local Phasor Data Concentrator (PDC) where the received data is synchronized based on the time stamps and is forwarded to Central Data Repository (CA ISO PDC) as close to real time as possible (Figure 1). Currently, there are about twenty data collection locations as indicated in Table 2. In the future, additional data is expected from new PMU installations and from other utilities.

The RTDMS Client applications, through their various geographic and graphic displays, provide operators and dispatchers both real-time and historical information, and alarms on:

- Local and interconnection frequency, as well as changes in frequency that can be mapped to precise generation-load imbalances within the interconnection. The local frequency measurements can be used to assess system coherency and its dynamic stress under normal operating conditions.

- Phase angle differences across different utilities with respect to their alarming thresholds to assess the static stress across the system and proximity to instability.

- Comprehensive voltage angle and magnitude profiles offer a birds-eye view of the sources and sinks of power and identify the high and low voltage regions within the grid.

- The MW and MVAR flows, computed from the voltage and current phasors, and track these flows with respect to predefined thresholds.

In addition to real-time monitoring capabilities, the application also stores short duration data files whenever transient events are detected, which can be loaded into the application for offline postdisturbance analysis.

All the displays in the application have been designed bearing the end user in mind and the information presented in the displays is pertinent to what would be valuable to them. It has been demonstrated that the more effective operational displays are those that follow a hierarchical approach to presenting operational data. In this hierarchical approach, very critical data is presented at the high level on a very 
simple system display. From the high-level system display the users can navigate to a lower level and more detailed displays.

Table 2: Current and Future PMUs to be Incorporated into the WECC Phasor Network

\begin{tabular}{|c|c|}
\hline PMU Location & Owner \\
\hline 1. Slatt & BPA \\
\hline 2. Summer Lake & BPA \\
\hline 3. Captain Jack & BPA \\
\hline 4. Keeler & BPA \\
\hline 5. Maple Valley & BPA \\
\hline 6. Sylmar & BPA \\
\hline 7. Big Eddy 500 kV & BPA \\
\hline 8. Big Eddy 230 kV & BPA \\
\hline 9. Colstrip & BPA \\
\hline 10. Malin & BPA \\
\hline 11. John Day & BPA \\
\hline 12. Grand Coulee & BPA \\
\hline 13. McNary $230 \mathrm{kV}$ & BPA \\
\hline 14. McNary 500kV & BPA \\
\hline 15. Ashe 500 kV & BPA \\
\hline 16. Ault & WAPA \\
\hline 17. Bears Ears & WAPA \\
\hline 18. PMOC UPS (Spare) & WAPA \\
\hline 19. Shiprock & WAPA \\
\hline 20. Vincent ${ }^{1}$ & SCE \\
\hline 21. Devers $500 \& 230 \mathrm{kV}$ & SCE \\
\hline 22. Mohave & SCE \\
\hline 23. Devers $115 \mathrm{kV}$ & SCE \\
\hline 24. Big Creek & SCE \\
\hline 25. Alamitos & SCE \\
\hline 26. San Onofre & SCE \\
\hline 27. Kramer & SCE \\
\hline 28. Valley & SCE \\
\hline 29. Antelope & SCE \\
\hline 30. Control & SCE \\
\hline 31. Lugo & SCE \\
\hline 32. Eldorado & SCE \\
\hline 33. Magunden & SCE \\
\hline 34. Midway & PG\&E \\
\hline 35. Diablo Canyon & PG\&E \\
\hline 36. Moss Landing & PG\&E \\
\hline 37. Tesla & PG\&E \\
\hline 38. Pittsburg & PG\&E \\
\hline 39. Los Banos & PG\&E \\
\hline 40. Metcalf & PG\&E \\
\hline 41. Table Mountain & PG\&E \\
\hline 42. Gates & PG\&E \\
\hline
\end{tabular}




\section{QUICK START}

The RTDMS Server 3.0 must be installed and running on a PC machine to which the PDC is sending data to, and RTDMS Client 3.0 should be configured to receive data from the RTDMS Server 3.0, before the RTDMS Client 3.0 can be launched. Please refer to the 'RTDMS Installation and Support Guide' for detailed step-by-step instructions on the installation and setup process.

To start the RTDMS Client application, click on the "CA ISO RTDMS Client 3.0" shortcut icon located on the desktop (Figure 4).

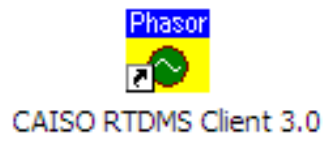

\section{Figure 4: RTDMS Client Start Icon}

Clicking on the icon will launch the application. When the application starts, the default "Frequency (Response)" monitoring display is shown (Figure 5). The chart presented in this display is the interconnection frequency plot with up to a one second refresh rate with the most current time being to the right along the horizontal axis. The frequency range being graphed is shown along the vertical axis to the right of the plot.

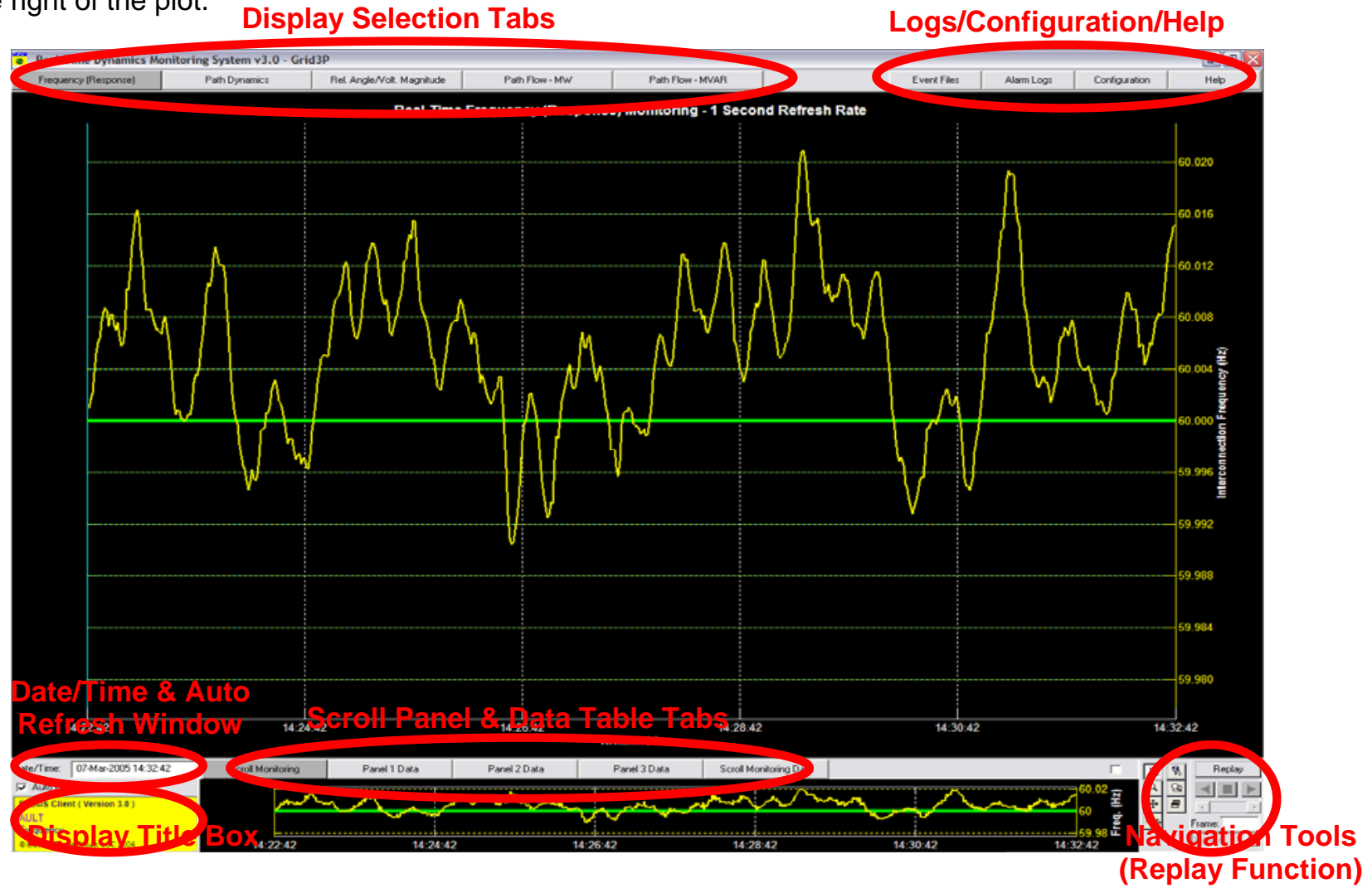

Figure 5: RTDMS Default Display - Frequency (Response) Monitoring

There are a total of five display selection tabs located at the top left corner of the application (Figure 6) corresponding to each of the five (5) different monitoring functions of the application: "Frequency (Response)", "Path Dynamics", "Relative Angle/Voltage Magnitude", "Path Flow-MW" and "Path FlowMVAR". The user can use these tabs to navigate between various functional displays by clicking on the appropriate tab.

\begin{tabular}{c|c|c|c|c|c|}
\hline Frequency (Response) & Path Dynamics & Rel. AngleNolt. Magnitude & Path Flow - MW & Path Flow - MVAR \\
\hline \hline
\end{tabular}

Figure 6: RTDMS Display Selection Tabs 


\section{REAL-TIME DYNAMICS MONITORING SYSTEM OVERVIEW}

This section of the user's guide will include a brief view of the system architecture, a description of the navigational tools, and a detailed description of the individual monitoring tabs offered by the application.

\subsection{Application Architecture}

Figure 7 shows the architecture of the RTDMS System. The system adheres to a Client/Server architecture where the Server, connected to the host data source (CA ISO Phasor Data Concentrator), will distribute the information to the multiple Client monitoring applications over a secure LAN connection. This straightforward configuration will facilitate a seamless, open and flexible integration with the Client for distribution and visualization of the data. Each of the different components of the application is described below:

\subsubsection{Data Retrieving, Alarming and Storage - RTDMS Server}

The PDCStream data reader component within the Server is designed to interface directly with the PDC over a local area network, and to read, in real-time, the complete phasor data stream. In addition to the data stream, the reader also reads all supplemental static information such as PMU, their coordinates, signal names, signal types, etc (Refer to Appendix 1). The phasor data read by PDCStream data reader from the PDC is pre-processed and cached at the RTDMS Server and is available for real time visualization via the applications running on multiple Client terminals. In addition, the data is also analyzed in real-time to detect any predefined threshold violations or transient events, which are both logged into text file as well provide real-time alarming at all RTDMS visualization Clients. In the case of transient event detection, the disturbance data is also permanently stored into event files of pre-specified duration (default is 5 minutes) and can be loaded into the RTDMS visualization Clients for postdisturbance analysis.

\subsubsection{RTDMS Client Monitoring Visualization Application - RTDMS Clients}

The RTDMS visualization displays, running on the Client terminals, is delivered as a stand-alone application designed to connect to the RTDMS Server over a LAN connection. It has the ability to acquire phasor data from the host, to process this data, and to display it in geo-graphic displays. The displays shall enable the user to monitor and track meaningful performance metrics with respect to predefined thresholds and will be alarmed whenever these thresholds are violated. These Clients may also be used to load event file data saved into the local offline cache for post-disturbance analysis within the application. This User's Guide elaborates on the use of the RTDMS Client application. 


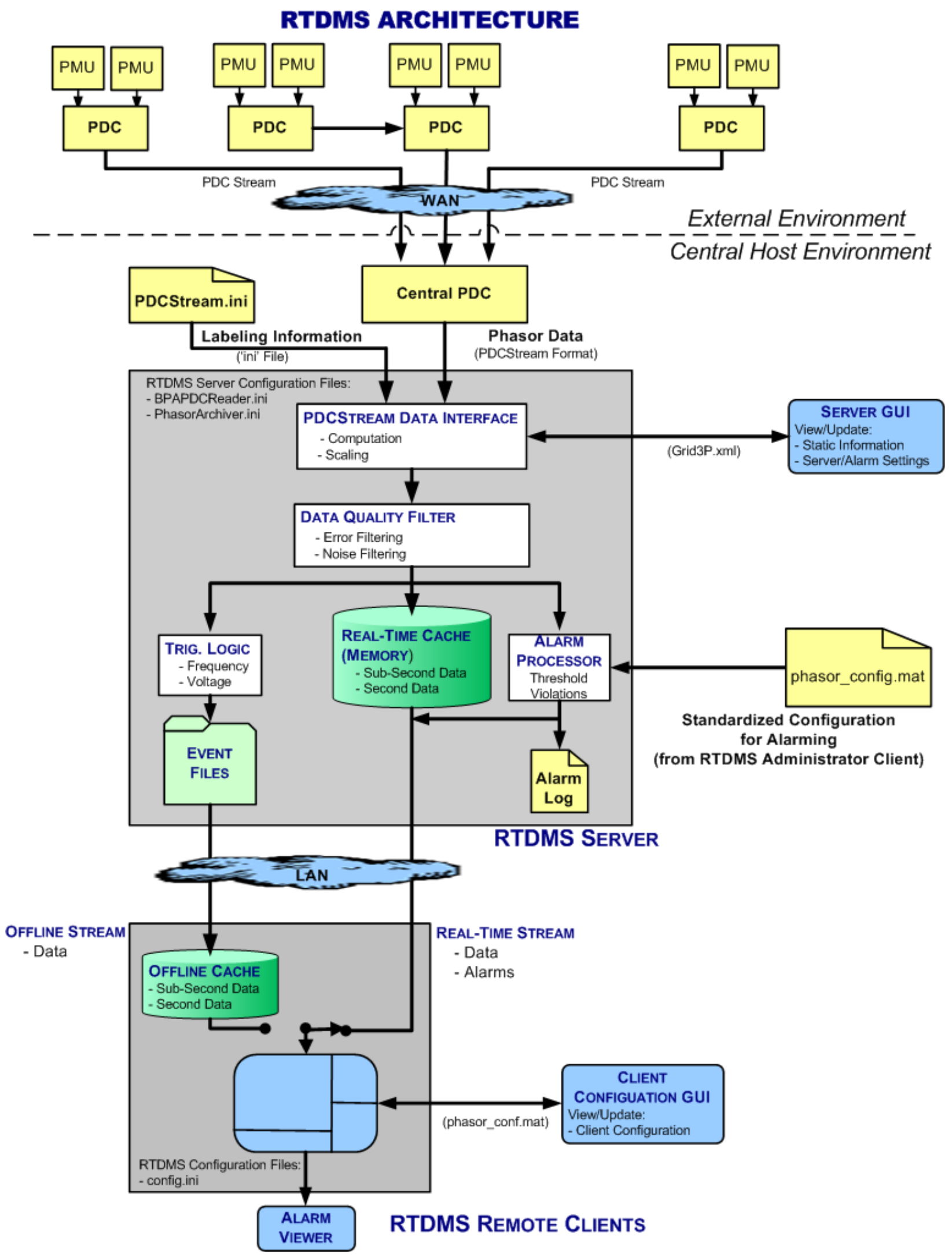

Figure 7: Real-Time Dynamics Monitoring System Architecture 


\subsection{Visualization Architecture}

The visualization overview shown in Figure 8 lays out the displays available to the user. Whether in RealTime (i.e., streaming data) or Offline (i.e. archived event files) monitoring modes, each of the following five (5) displays: "Frequency (Response)", "Path Dynamics" (Angle Differences Across Regions), "Relative Angle/Voltage Magnitude", "Path Flow - MW" and "Path Flow - MVAR" are available within the application and can be utilized by the user by selecting the applicable data visualization. The "Wide Area" and "Local Area" monitoring capabilities available for some of the displays present the data in different forms and therefore allow for better analysis of the data.

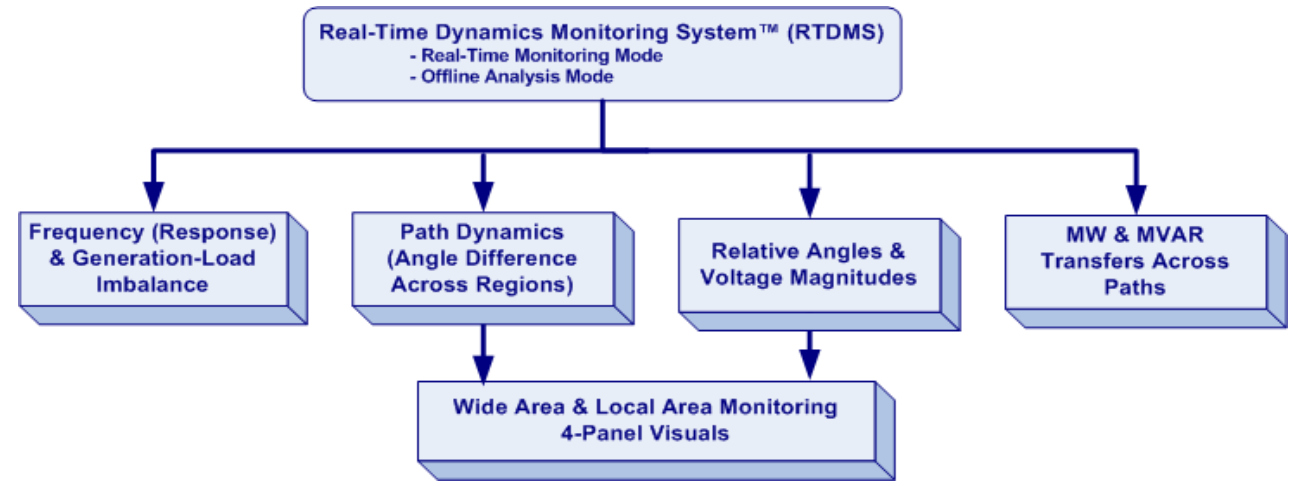

Figure 8: RTDMS Visualization Overview

In addition to the geo-graphical plots and multi-panel views (One-Panel or Four-Panel) within the various displays, the displays (and the platform in general) offer configuration, alarming and navigational capabilities, through control icons, selection tabs, and information windows located within the display.

\subsubsection{Date/Time \& Auto Refresh Window}

In real-time operation, the date and time of the most recent data available for visualization within the application are displayed in a date/time window located in the lower left corner of the screen (Figure 9). The application uses the 24 hour clock and automatically refreshes with new data once every second and this date/time window is updated accordingly. In offline mode, the date and time during the event capture is shown in the date/time window.

Date/Time: 07 Jul-2004 11:53:40
$\sqrt{\checkmark}$ Auto Refresh

Figure 9: Date/Time \& Auto Refresh

Note: During real-time operation, the automatic refreshing of the data can also be stopped by clicking off the "Auto Refresh" check box. This will freeze the data and hold the displays for closer interrogation.

Both, real-time and event data can be replayed with the application for close interrogation. When in Replay mode (Refer to Section 4.2.6), the user may manually alter the time within the Date/Time window and rewind to a desired point in time. To do this, either type in the desired time into the Date/Time window or right click within this window to launch the "Date Selection" graphic user interface (Figure 10).

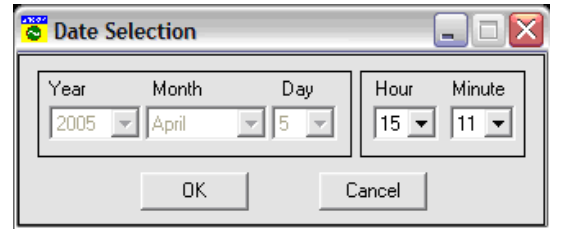

Figure 10: Date Selection Graphic User Interface for Replay 


\subsubsection{Configuration Utility}

The "Configuration Utility" lets the user customize the functionality of the visualization application. With the utility, the user has the ability to select a subset of PMUs from those available to the application, and customize each of the displays based on signals belonging to the subset of PMUs. The configuration utility therefore allows the user to update the application as PMUs are added/removed from the phasor network. The details on how to use this feature are included in Section 12.

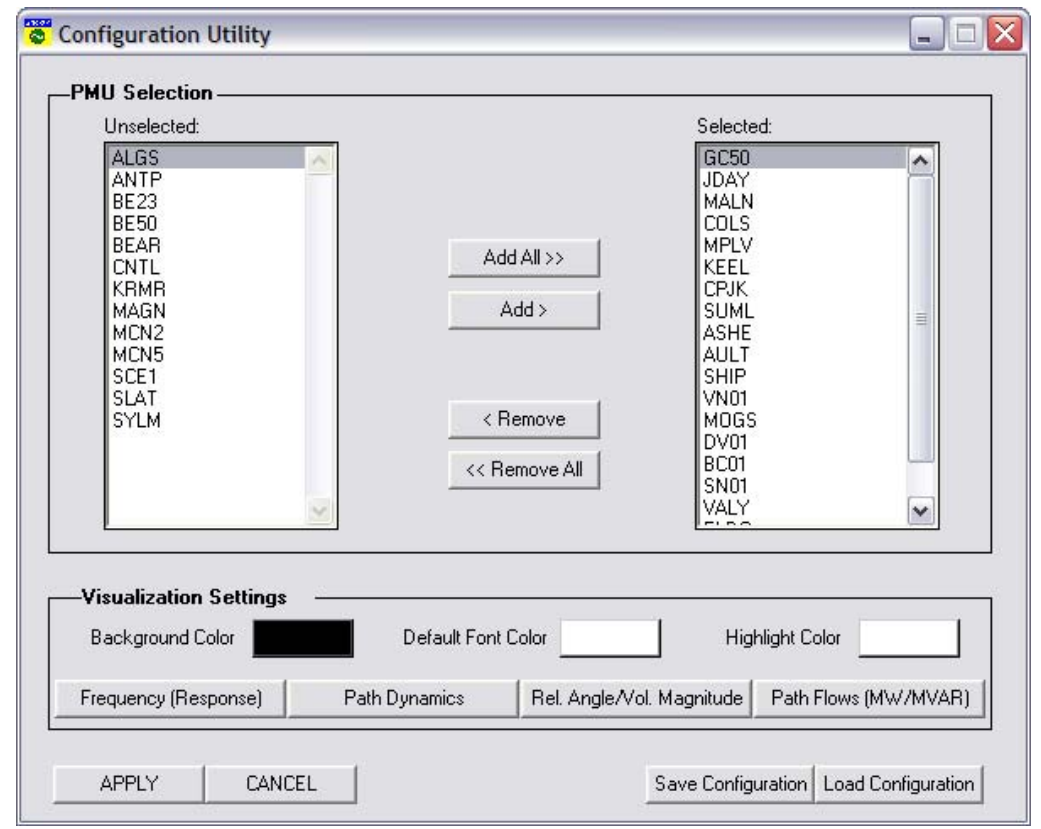

Figure 11: Configuration Utility Graphic User Interface

\subsubsection{Alarm Logs}

The "Alarm Log" pop-up box keeps track of the most recent violations under any/all of the following conditions:

- System frequency exceeding specified threshold limits

- Local frequency variations exceed specified thresholds limits

- Sudden changes in system and local frequencies

- Voltage magnitudes exceed minimum or maximum thresholds (i.e. low or high voltages)

- Sudden changes in voltage magnitudes

- Relative voltage angles exceeding specified thresholds limits w-r-t chosen reference

- Angle difference across specified paths exceed defined threshold limits

- MW/MVAR flows across specified paths exceed defined threshold limits

Note: When sudden changes in voltage magnitude or frequency signals are detected, the phasor data is stored into event files for offline post-disturbance analysis (Refer to Section 4.2.4).

Whenever an alarm occurs, the "Alarm Logs" tab located in the top right corner of the RTDMS Clients is highlighted in red until the alarm is acknowledged by the user (Figure 12).

\begin{tabular}{|l||l|l|l||} 
Event Files & Alarm Logs & Configuration & Help \\
\hline
\end{tabular}

Figure 12: “Alarm Logs" Tab Highlighted in Red to Alert RTDMS User of New Alarm

To acknowledge the alarm, click on this tab and the "Alarm Log" box pops-up (Figure 13). Though all the alarms are logged at the RTDMS Server as a text file, the user may be selective on the alarms he wishes 
to see at the RTDMS Client. This is done by selecting/deselecting the appropriate alarm types within the "Alarm Log" box. For each alarm listed in the box, the alarm log provides information on the signal associated with the violation, the start time of the violation, the end time of the violation (or most current time during a violation), the violation value and corresponding threshold, and the nature of the violation (Refer to Section 10 for further details).

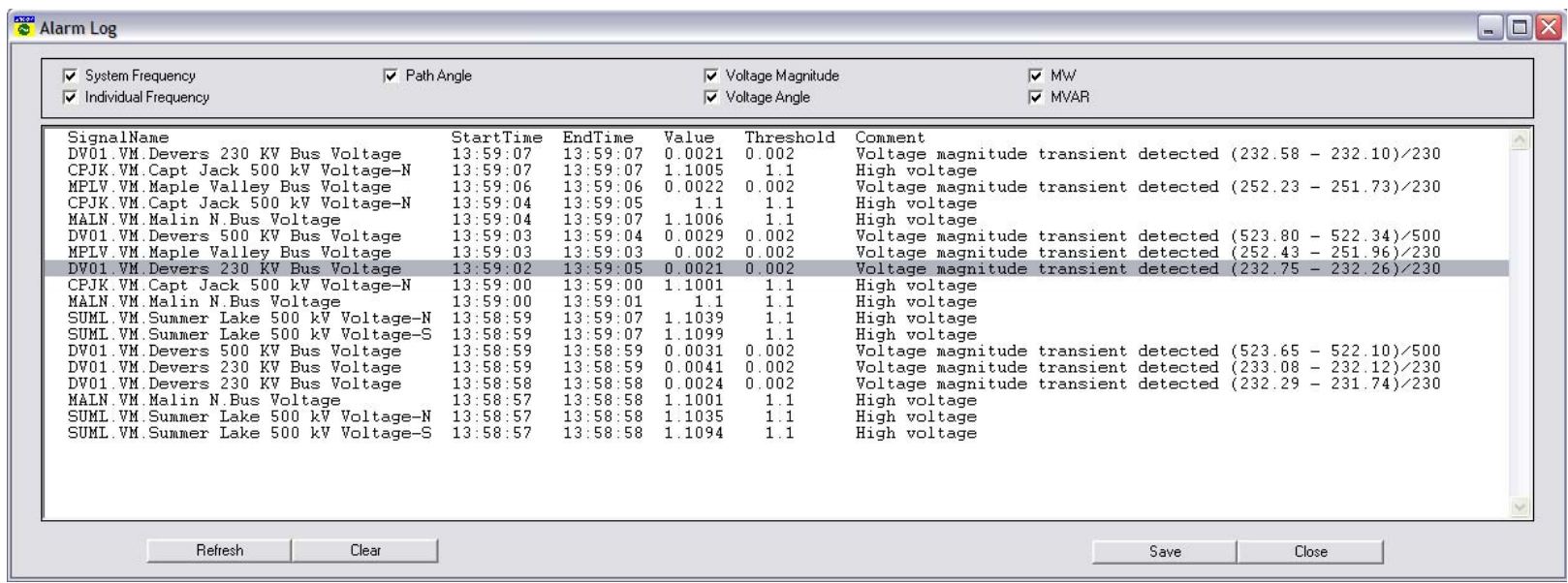

Figure 13: Alarm Log Pop-up Box

\subsubsection{Event Files}

Event detection logic built into the RTDMS Server identifies sudden changes in both frequency and voltage magnitude measurements and triggers data and alarm storage into event files over predefined time duration (default duration of these event files is 5 minutes). These event files can be loaded into RTDMS Clients for offline post-disturbance analysis (Figure 14). The details on loading these files and viewing them are provided in Section 11.

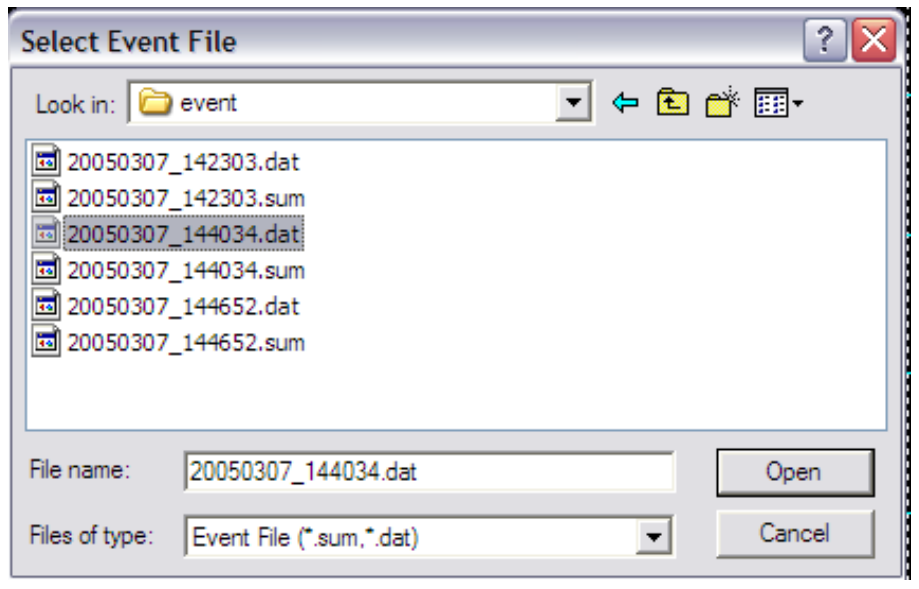

Figure 14: Navigation Window to Load Event Files 


\subsubsection{Navigational Tools}

The navigation tools provided to the user can be selected by clicking on one of the seven mouse control icons shown in Figure 15 located in the bottom right corner of all the displays. The functionality of these icons is briefly described in Table 3.

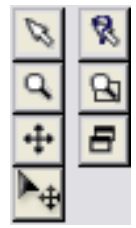

Figure 15: Mouse Control Icons

Table 3: Navigation Tools

\begin{tabular}{|c|c|c|}
\hline Function & Mouse Control Icon & What it does \\
\hline Selection Arrow & $\begin{array}{l}\text { - This is the default Navigation tool. } \\
\text { Selecting this tool allows the user to click } \\
\text { (either right or left) and move through the } \\
\text { different plots and data tables. }\end{array}$ & $\begin{array}{l}\text { Needs to be selected (clicked) } \\
\text { prior to selecting the desired } \\
\text { area to be viewed. }\end{array}$ \\
\hline Zoom In/Out & $\begin{array}{l}\text { Q - Click the magnifying glass to resize } \\
\text { the image. With the mouse on the } \\
\text { image, hold the button and slide the } \\
\text { mouse down or to the left to reduce the } \\
\text { size of the image or move the mouse up } \\
\text { or to the right to increase the size of the } \\
\text { image. }\end{array}$ & $\begin{array}{l}\text { Zoom In - Enlarge the details of } \\
\text { the selected area. } \\
\text { Zoom Out - Provides an aerial } \\
\text { view for a selected area. }\end{array}$ \\
\hline Reposition & $\begin{array}{l}\ddagger \text { - Click the cross arrows, move the } \\
\text { cursor to the viewing panel, click the left } \\
\text { mouse and drag the object to the desired } \\
\text { location in the panel }\end{array}$ & $\begin{array}{l}\text { Allows the user to reposition or } \\
\text { center objects being displayed } \\
\text { in the panel. }\end{array}$ \\
\hline Pick to Move Text & $\begin{array}{l}\text { - Click on the cross arrows with the } \\
\text { pointer, move the cursor to the text label, } \\
\text { click the left mouse and drag the text } \\
\text { label to the desired location in the panel. }\end{array}$ & $\begin{array}{l}\text { Allows the user to reposition } \\
\text { text labels being displayed in } \\
\text { the panel. }\end{array}$ \\
\hline Pick to Display Data & $\begin{array}{l}\text { - Click on the arrow within the } \\
\text { question mark to select data within the } \\
\text { plot or diagram. }\end{array}$ & $\begin{array}{l}\text { Brings hidden data associated } \\
\text { with an object into view. }\end{array}$ \\
\hline Rubber Band Zoom & $\begin{array}{l}\text { - Click the magnifying glass with the } \\
\text { box in the background to select the zoom } \\
\text { desired. }\end{array}$ & $\begin{array}{l}\text { Places a box around the } \\
\text { desired part of an area or plot } \\
\text { to be enlarged. When the Right } \\
\text { Mouse Bottom (RMB) is } \\
\text { released the selected area will } \\
\text { be enlarged. }\end{array}$ \\
\hline Restore to Original Size & $\begin{array}{l}\text { ㅍ. Click on the double plot icon to } \\
\text { restore the plot or diagram to its original } \\
\text { size. }\end{array}$ & $\begin{array}{l}\text { Restores a diagram to its } \\
\text { default size when utilized. }\end{array}$ \\
\hline
\end{tabular}




\subsubsection{Replay Function}

During real-time operation, the Replay functionality (Figure 16) permits the user to switch from the RealTime monitoring mode to the Replay mode to view cached data for historical tracking and analyses. Once in the Replay mode, the auto-rewind/auto-forward buttons or the slider can be used to move back/ahead in time and recreate the various RTDMS displays with the cached data.

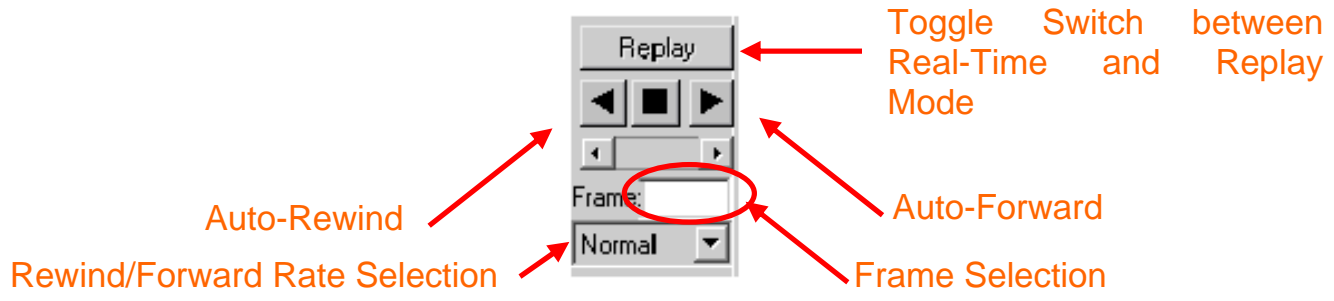

Figure 16: Replay Functions

Once in the Replay mode, the user can move back/ahead in time and recreate the various RTDMS displays using the cached data in one of the following ways:

- Use the Auto-Rewind/Auto-Forward ${ }^{2}$ buttons to automatically step back or forth in time. The Rate Selection for the auto-rewind (auto-forward) feature is set though a pull-down menu where "Slow", "Normal", and "Fast" options correspond to a incremental step size of 1 second, 10 seconds and 60 seconds respectively between screen refreshes.

- Use the Slider to manually interrogate the data within the cache.

- Enter the frame number directly into "Frame" box. Each frame index corresponds to a particular slice in time (in seconds). Hence for a 1 hour long cache, there are 3600 frames with the $3600^{\text {th }}$ frame being the most current set of data and the $1^{\text {st }}$ frame corresponding to one hour old data.

- Manually enter the Date/Time window to rewind/forward to a desired point in time (Refer to Section 4.2.1).

The use of the replay capability as described above can also be used to interrogate event files when they are loaded into the RTDMS Client. Once in Offline mode, clicking on the "Replay" button brings the user back to the Real-Time operating mode.

Note: When in Replay mode, the screen refreshes are no longer in real-time - their execute times are dependent on the data retrieval times between the Server and the Client.

Whether in Replay or Offline mode, the "Alarm Log" box continues to automatically update with the most recent alarms detected at the RTDMS Server.

\subsubsection{Display Title Box}

The Title box (Figure 17) identifies the information that is currently being displayed in the "Scroll Monitoring" panel (i.e., the Panel 4 at the bottom of the displays). A Title box with a yellow background implies that the application is being used in a Real-Time mode for viewing real-time streaming data, while a blue background indicates that the application is being used in an Offline viewing mode for analysis of previously saved event files. These example indicate that the the MVAR flows on Path 75 are being viewed in the "Scroll Monitoring" panel.

\begin{tabular}{|l|l|}
\hline RTDMS Client ( Version 3.0) & RTDMS Client ( Version 3.0) \\
Path 75 & Path 75 \\
Path Flow (MVAR) & Path Flow (MVAR) \\
eElectric Power Group, LLC 2004 & OElectric Power Group, LLC 2004 \\
\hline
\end{tabular}

Figure 17: Title Box

\footnotetext{
${ }^{2}$ When using the forward function with the slider positioned all the way to the right, the displays will automatically rewind to start of the cached data and progress forwards from that point onwards.
} 


\subsubsection{Data Tables}

All the visuals in the application are generated from the input phasor data. This input data is also available in textual format in the panel 4. In particular, the data associated with the graphics within a particular panel of a display can be examined by selecting the corresponding panel data tab within that display (Figure 18). These data tables can also be enlarged by clicking the box in the upper right hand corner of the data tables.

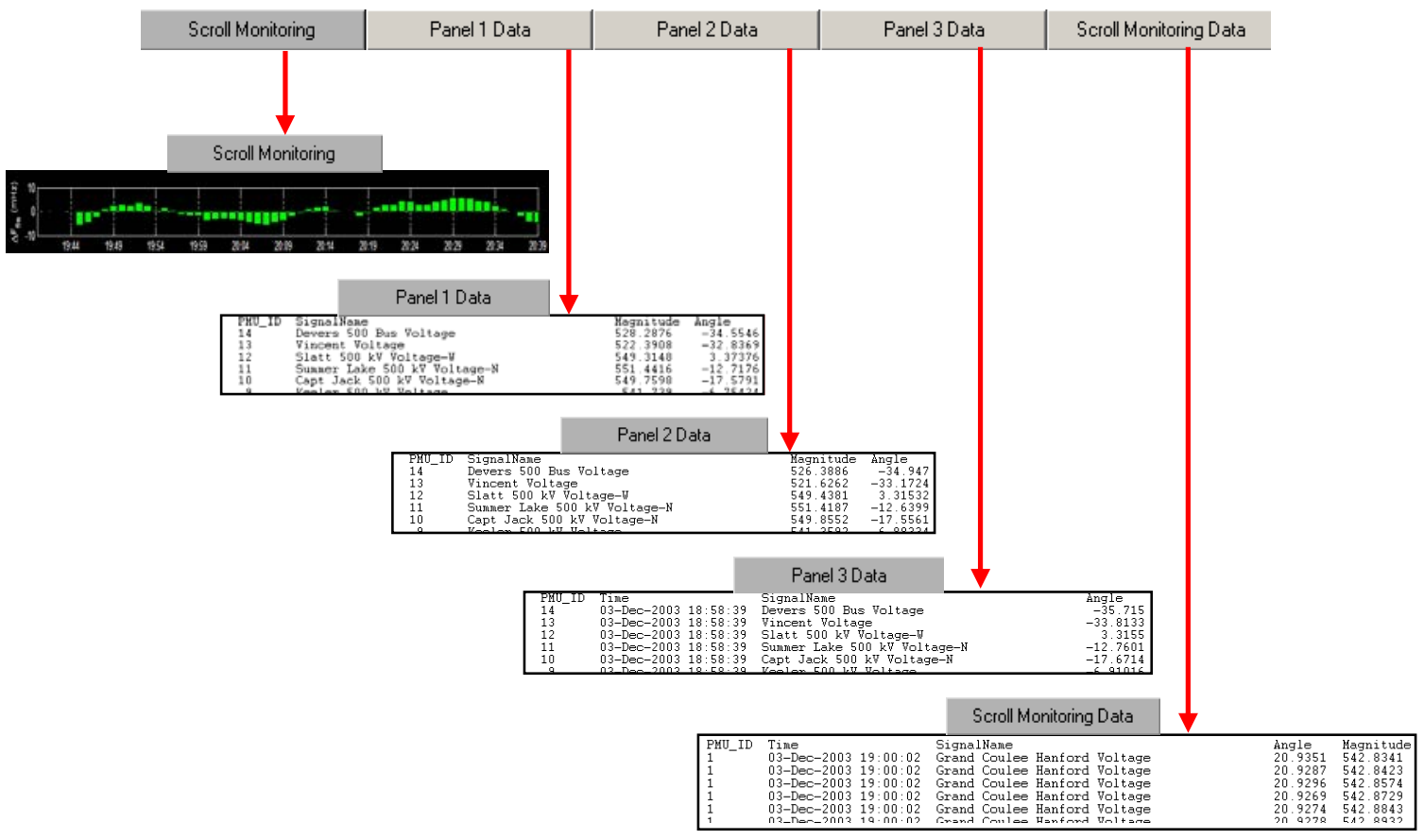

Figure 18: Data Table Overview

The textual information belonging to a particular panel can also be exported to any text editor and printed or saved as a text file. To do this, right click within a particular data panel of interest and the pop-up menu shown in (Figure 19) will appear.

\begin{tabular}{|c|c|c|c|c|c|c|}
\hline Scroll Monitoring & Pan & 11 Data & Panel 2 Data & Panel 3 Data & Scroll Monitoring Data & $\Gamma$ \\
\hline $\begin{array}{l}\text { Time } \\
28-S e p-2004 \\
28-S e p-2004 \\
28-S e p-2004 \\
28-S e p-2004 \\
28-S e p-2004\end{array}$ & $\begin{array}{l}13: 28: 10 \\
13: 28: 10 \\
13: 28: 10 \\
13: 28: 10 \\
13: 28: 10 \\
12: 10\end{array}$ & $\begin{array}{c}\text { Frequency } \\
60.005 \\
60.005 \\
60.0051 \\
60.0051 \\
60.0053\end{array}$ & $\begin{array}{l}\text { Load_Imbalance } \\
\mathrm{NaN} \\
\mathrm{NaN} \\
\mathrm{NaN} \\
\mathrm{NaN} \\
\mathrm{NaN} \\
\mathrm{T}-\mathrm{T}\end{array}$ & $\begin{array}{l}\text { Threshold } \\
200 \\
200 \\
200 \\
200 \\
200 \\
200\end{array}$ & $A^{\text {View/Print Panel Data }}$ & $\hat{\square}$ \\
\hline
\end{tabular}

Figure 19: Data Table Print/Save Pop-Up 


\section{FREQUENCY (RESPONSE) MONITORING}

\subsection{Overview}

Using phasor data, the application is capable of tracking the interconnection frequency in real time. In particular, the fine sub-second resolution associated with phasor technology enables the user to closely monitor and track the interconnection frequency during a disturbance. Furthermore, using a running window to dynamically track the change in interconnection frequency over a specified time interval (i.e., time scales associated with primary control); this frequency response can be accurately mapped to a generation-load imbalance in the system (in MW) through the natural frequency response coefficient for the interconnection.

Phasor data also has the capability of providing synchronized local frequency measurements across the interconnection. The application therefore can track the deviation in each of the local frequencies from the interconnection frequency as well. The maximum separation in local frequencies from the interconnection frequency is a measure of interconnection coherency at each instant in time. Moreover, in the event of a sudden generation-load imbalance, the local frequency having the greatest deviation during the transient timeframe provides a good estimate of the geographic location of the imbalance. All this information is conveyed through the various panels of the "Frequency (Response)" monitoring display.

The "Frequency (Response)" monitoring graphics are shown in Figure 20. In this configuration, the default graphics can be changed in two ways: the corresponding "Generation-Load Imbalance" graphic can be added or the user may go to a "Four-Panel" display where additional information is available. Here, each of the panels in the four-panel display can also be enlarged by double clicking with the left mouse button on the desired panel.

Double clicking in the "Frequency (Response) Monitoring" main panel will take the user to the four-panel display as seen in Figure 20. The four panels can either be viewed together or any one of them can be enlarged individually for easier viewing and analyzing. The user also has the ability to change the time duration in either of these panels. Below is a description of the four panels:

- Panel 1 - "Frequency (Response) Monitoring", default display.

- Panel 2 - Is the upper right display "Local Frequency Coherence Comparison".

- Panel 3 - Is the lower right graph "Interconnection Frequency \& Imbalance Duration Curves."

- Panel 4 - Is the bottom panel showing frequency change bar chart or local frequency trend plots.

Panels 2, 3 and 4 can be enlarged by double clicking within the desired panel with the left mouse button. To return to the four-panel display, double left click on the enlarged panel and the four-panel display will be restored. 


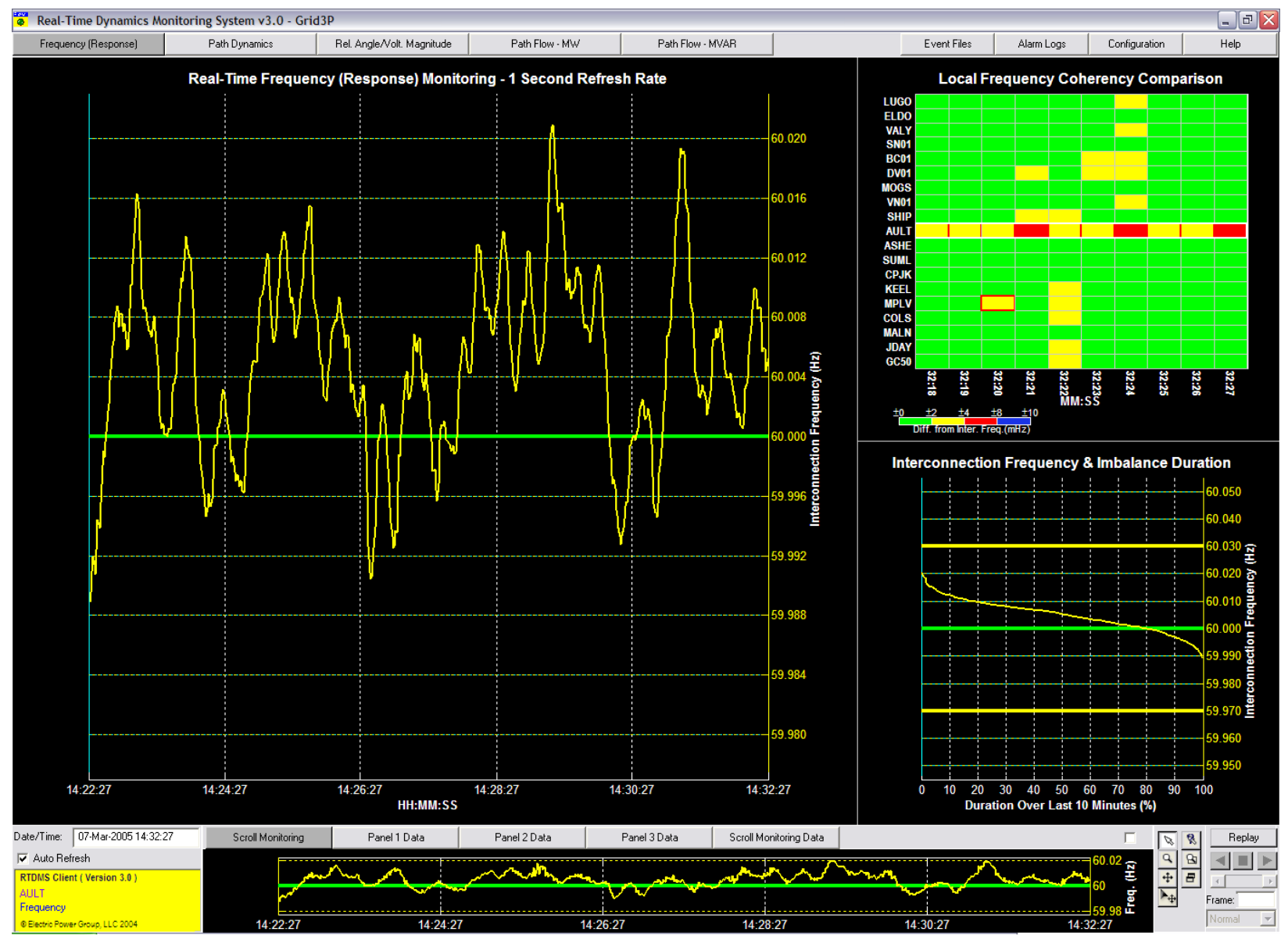

Figure 20: Four-Panel Frequency Monitoring

\subsection{Frequency (Response) Monitoring One-Panel Display}

The "Real-Time Frequency (Response) Monitoring" graph refreshes every second to give the user a real time view of the interconnection frequency ${ }^{3}$ at a default one second resolution ${ }^{4}$. However, the resolution may be changed to the higher sub-second resolution to accurately view the interconnection frequency changes in the event of a system disturbance. An additional graph (the "Generation-Load Imbalance (MW)" graph), which maps changes in Interconnection frequency to associated generation-load imbalances within the system, can be overlaid over the interconnection frequency graph.

To have the "Generation-Load Imbalance" graph displayed, right mouse click in the "Real-Time Frequency (Response) Monitoring" default panel. The Display/Duration pop-up box will appear as seen in Figure 21. Select "Generation-Load Imbalance" and this graph will be added to the Interconnection frequency graph.

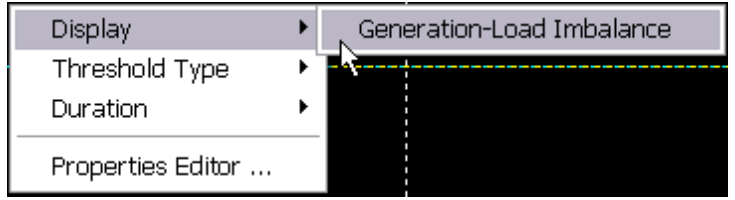

Figure 21: Generation-Load Imbalance Display/Duration Pop-Up

\footnotetext{
${ }^{3}$ The interconnection frequency is calculated by averaging the local frequencies at each of phasor monitoring points

${ }^{4}$ The one second resolution data is computed by averaging the sub-second resolution data over the second time interval. 
The generation-load imbalance computation is based on the frequency change between the present time and ten seconds in the past (time scale associated with primary control responsible for the system frequency response), and is appropriately scaled by the "Natural Frequency Response Coefficient" of the Interconnection and displayed as the "Generation-Load Imbalance". During a frequency excursion this graphic will identify both the amount of imbalance (MW) that caused the deviation as well as the recovery of the Interconnection. The "Natural Frequency Response Coefficient" for the Western Interconnection is $1500 \mathrm{MW} / 0.1 \mathrm{~Hz}$ and for the Eastern Interconnection is $3200 \mathrm{MW} / 0.1 \mathrm{~Hz}$. Both the time duration over which the frequency change is calculated and the imbalance mapping coefficient applied to this change are user configurable through the Configuration Utility (Refer to Section 10).

In Figure 22, the main display shows both Interconnection frequency and generation-load imbalance. The right axis, in yellow, demonstrates the "Interconnection Frequency $(\mathrm{Hz})$ ". The left axis, in cyan, gives the "Generation-Load Imbalance (MW)". Fixed monitoring thresholds for the interconnection frequency appear as bold yellow lines across the chart if the Interconnection frequency approaches the threshold. The default threshold values are set to $59.97 \mathrm{~Hz}$ and $60.03 \mathrm{~Hz}$ as outlined in the NERC Policy-9 frequency Monitoring criteria for Reliability Coordinators. "...Take remedial actions when interconnection frequency error is in excess of $0.07 \mathrm{~Hz}$ for more than 20 minutes...", but may be changed by the user through the configuration utility. Alternately, using an option in the pull-down menu (Figure 23), the user may replace the fixed thresholds by soft thresholds defined as a set number standard deviation of the mean value calculated from the Interconnection frequency data over the specified time duration within the chart (Refer to Section 10).

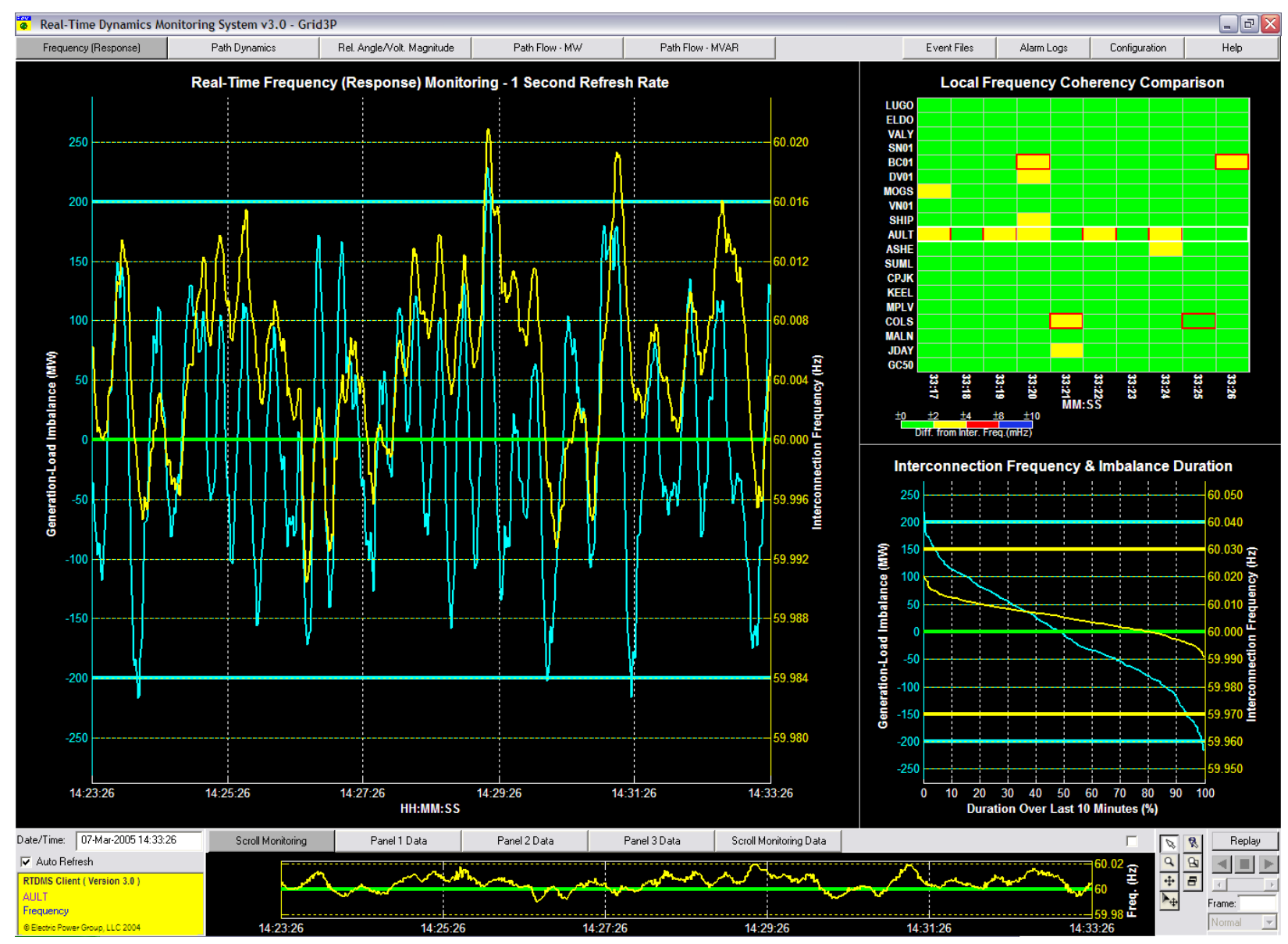

Figure 22: Four-Panel Frequency/Generation-Load Imbalance Monitoring

The "Generation-Load Imbalance" threshold lines (cyan) also appear when this plot is displayed. While the default thresholds for the generation-load imbalance (cyan) value are $\pm 200 \mathrm{MW}$, it can be changed through the configuration utility as well (Refer to Section 10). 


\begin{tabular}{|l|l|l|}
\hline Display & & - \\
\hline Threshold Type & $\bullet$ & $\checkmark$ Hard Limit \\
\hline Duration & & Soft Limit \\
\hline Properties Editor ... & \\
\hline
\end{tabular}

Figure 23: Soft/Hard Threshold Selection Pop-Up

The default duration time for the "Real-Time Frequency (Response) Monitoring" graph is 10 minutes. This duration can be changed by clicking the right mouse button within the panel; the Display/Duration pop-up box will appear as seen in Figure 24. The time duration desired can then be selected for the panel $^{5}$.

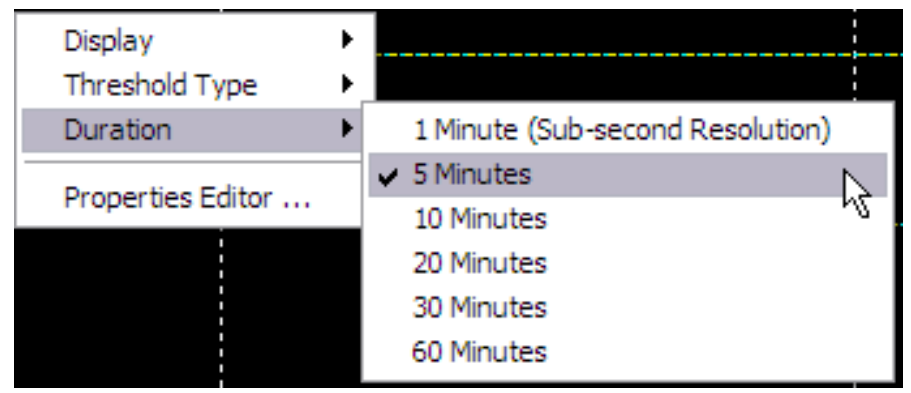

Figure 24: Frequency /Imbalance Duration Selection Pop-Up

The "Scroll Monitoring" panel button, highlighted in Figure 25, is one of five selections for the window beneath the main panel. As a default, here the change in frequency over the specified time duration is displayed on a bar chart. Alternately, through a pull-down menu option, this plot can be replaced by a trend plot of local frequency measurements at any of the available PMU locations. The PMU whose frequency measurements are to be displayed in this panel is chosen by clicking within the row corresponding to this PMU in the "Local Frequency Coherence Comparison" plot shown in panel 2 (see Section 5.3). In either case, time duration for these plot matches the duration set for the frequency monitoring display in panel 1.

The remaining four tabs "Panel 1 Data", "Panel 2 Data", "Panel 3 Data" and "Scroll Monitoring Data" provide data in tabular and textual format for the plots in each of the corresponding panels of the fourpanel display. Each of the five selections can also be enlarged by either clicking in the upper right hand corner box or double clicking on the display. Remove the check in the box or double click within the display to return the bottom panel to its default display.

${ }^{5}$ If a one minute duration is selected, then the data used in the plot for the frequency and the imbalance data are at its highest sub-second resolution which is needed for detailed visibility of transient frequency responses 


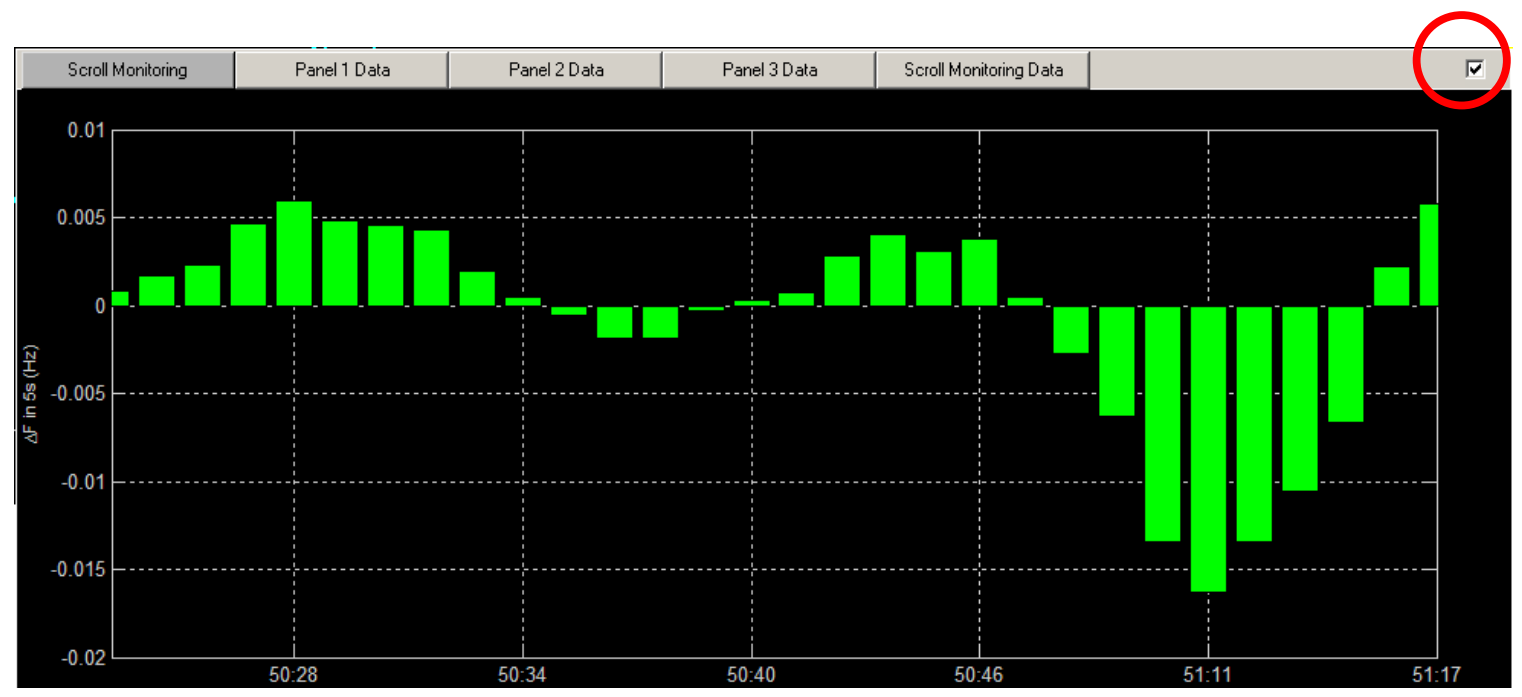

(a)

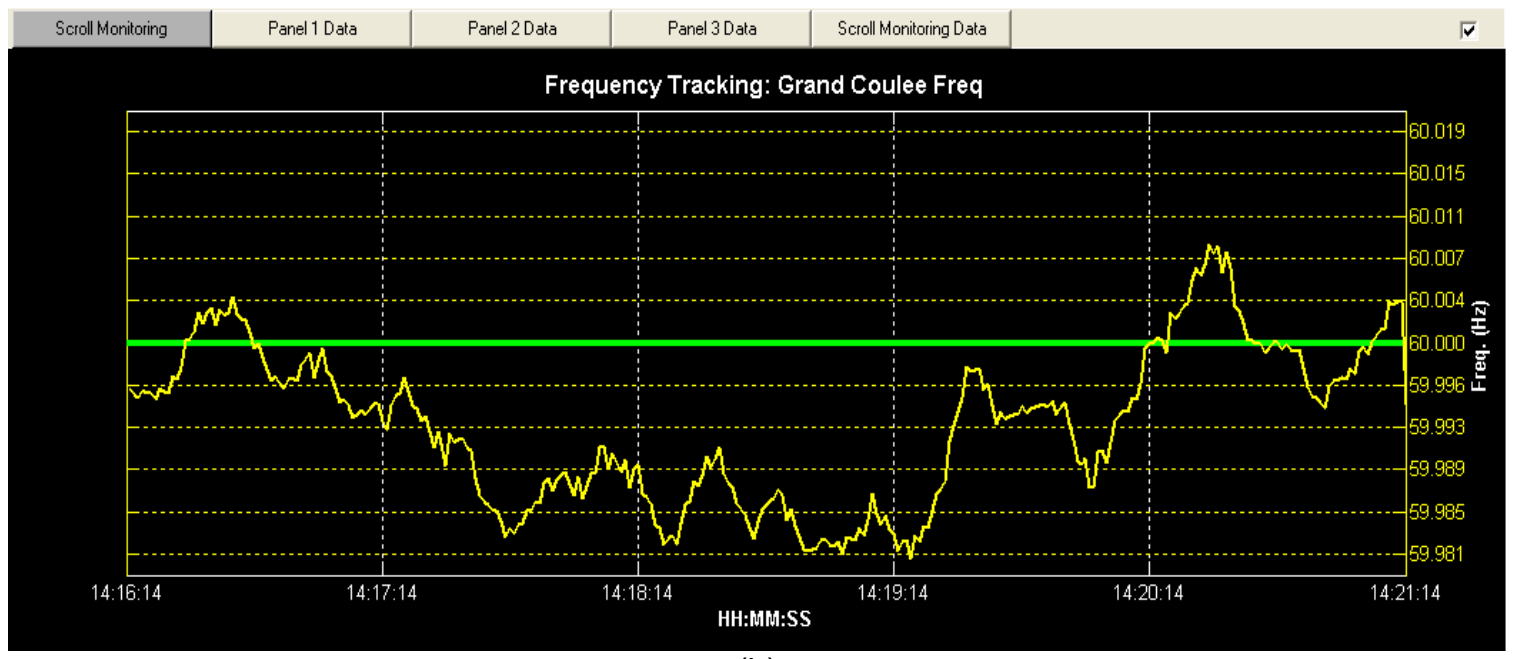

(b)

Figure 25: Scroll Monitoring Graph (Enlarged) - (a) delta interconnection frequency (b) local frequency trend plot at PMU location.

\subsection{Frequency (Response) Monitoring Four-Panel Display}

In the four-panel view, the "Real-Time Frequency (Response) Monitoring" graph described in section 5.2 is displayed as Panel 1. Panel 2 is the upper right display "Local Frequency Coherence Comparison" and Panel 3 is the lower right graph "Interconnection Frequency \& Imbalance Duration Curves." Panel 4 is the "Scroll Monitoring" frequency change bar chart explained in the previous section. Panels 2 and 3 can be enlarged by double clicking within the desired panel with the left mouse button. To return to the fourpanel display, double left click on the enlarged panel and the four-panel display will be restored. Panel 4 can be enlarged or reduced by clicking on the upper right hand corner box.

In Panel 2, the objective of the "Local Frequency Coherence Comparison" display (Figure 26) is to provide the operating staff with a quick source of information on the spread in local frequencies as a measure of dynamic stress being exerted on the system under normal conditions. Furthermore, in the event of an outage, it will help identify which area of the Interconnection experienced a loss of load or generation causing a noticeable local frequency deviation over the transient timeframe. 


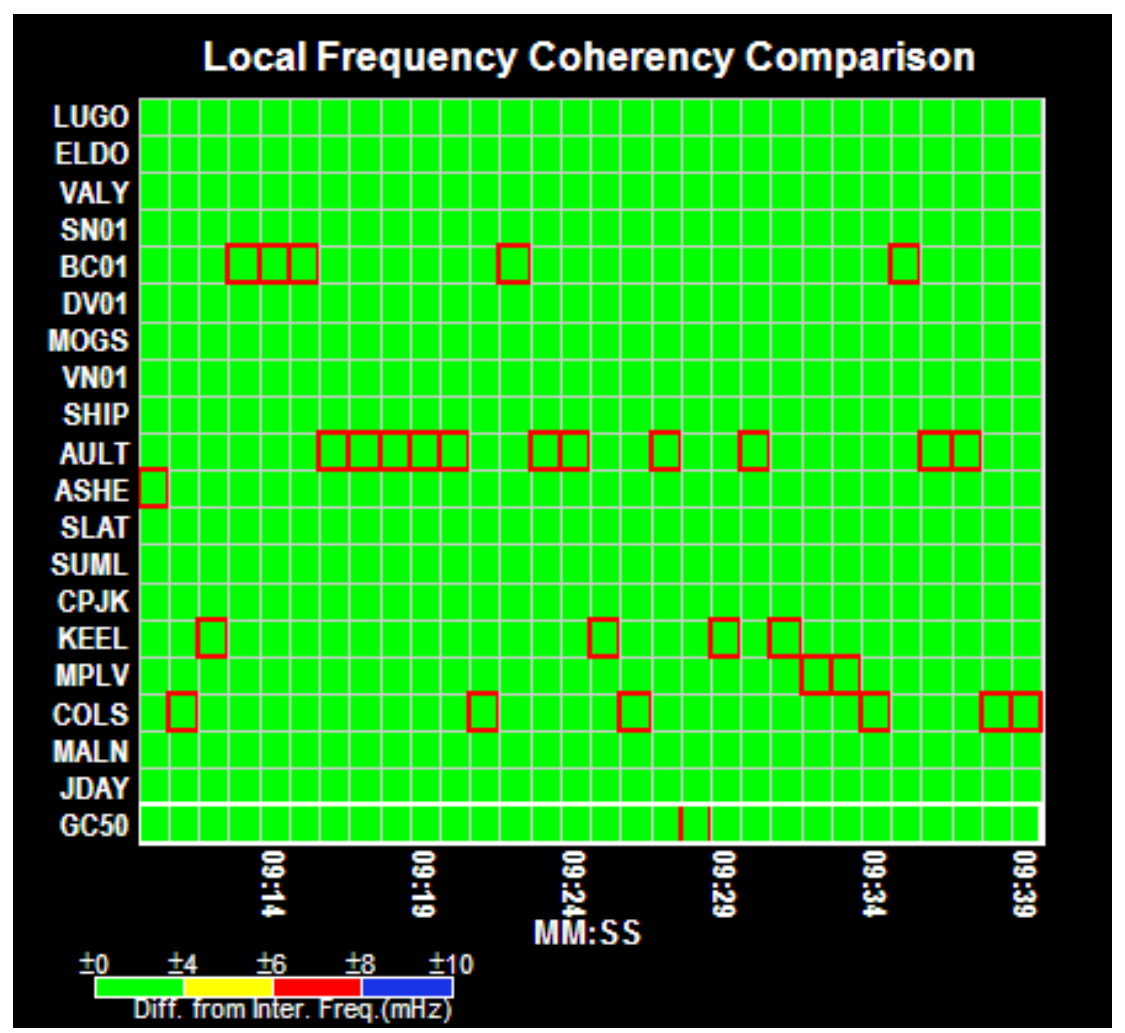

Figure 26: Local Frequency Coherence Comparison (Panel 2)

The $x$-axis of the plot is the time in minutes and seconds (MM:SS). The duration time for the plot can be changed by the user. To change the time (x-axis), right click in Panel 2. Figure 27 will appear and it lists the selectable time periods available for the panel.

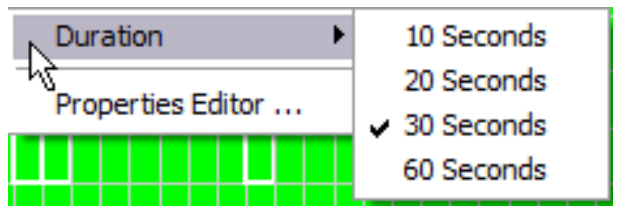

Figure 27: Local Frequency Coherence Comparison Duration Selection Pop-Up

The color legend represents the four (4) levels of deviation from the Interconnection frequency (Figure 28).

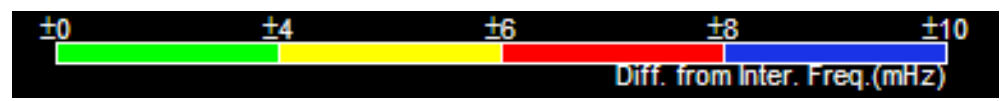

Figure 28: Local Frequency Coherence Comparison Legend

The y-axis lists the PMU locations where local frequency data is available for comparison. To the right of each PMU is a series of rectangles along the time axis that will indicate (using color) its frequency deviation from the system frequency at a particular instance in time.

In addition, at each slice in time, the one cell associated with the PMU measuring the largest local frequency deviation for that time instance will be highlighted with a border around it as well. In the event

${ }^{6}$ Any black (blank) cells in the plot indicate absent phasor data. 
of a major frequency excursion, the PMU reporting this greatest frequency deviation is most likely to be in the close proximity of the area that experienced the problem.

The "Interconnection Frequency \& Imbalance Duration Curves" in Panel 3 (Figure 29) presents a statistical distribution of the interconnection frequency as the default graph.

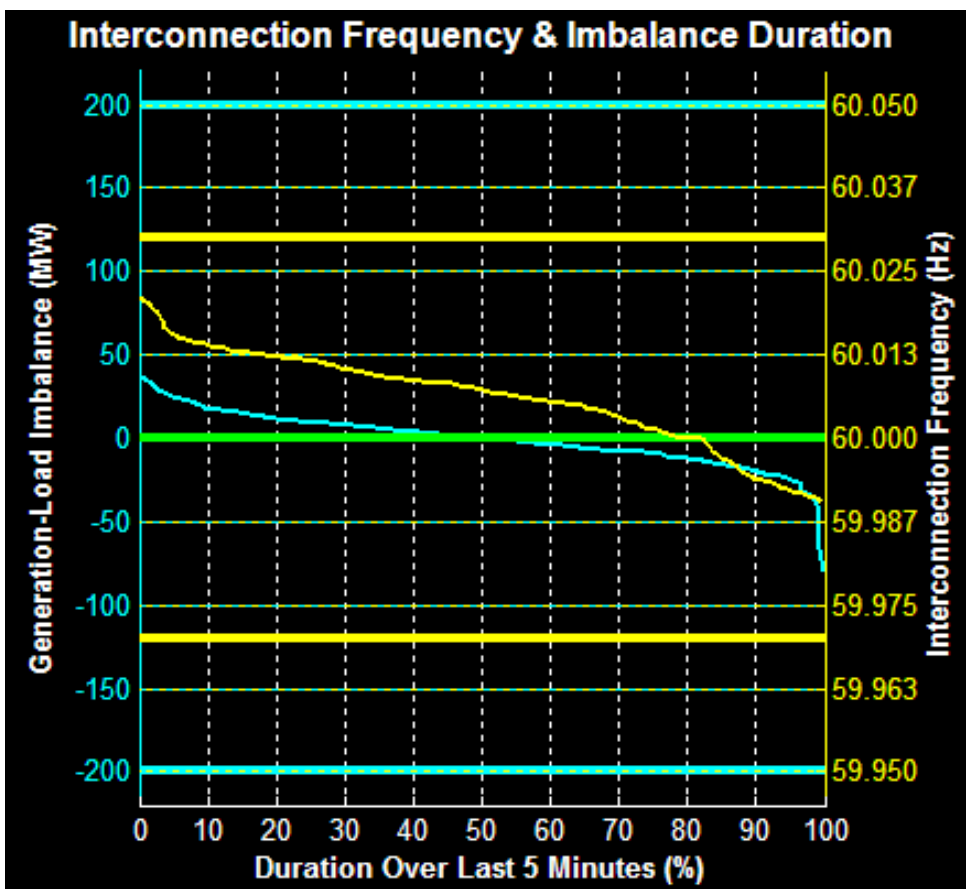

Figure 29: Interconnection Frequency \& Imbalance Duration Curves (Panel 3)

The statistical distribution for the generation-load imbalance graph can be added by right clicking in the panel and selecting "Generation-Load Imbalance" (Figure 30). The thresholds and the y-axis are the same as for Panel 1. The $\mathrm{x}$-axis shows the percent of the time that the interconnection frequency was above/below nominal frequency. The generation-load imbalance line demonstrates the percent of time that the interconnection imbalance was above/below zero. As with other graphs, the duration over which the distribution is computed can be changed through a pull-down menu made available through a right click within the panel (Figure 31).

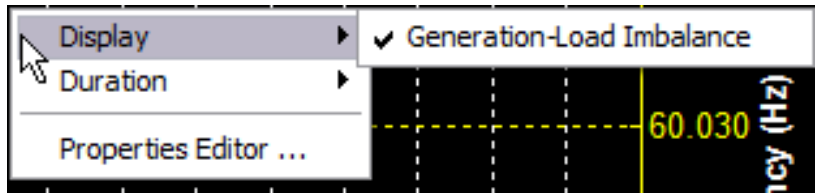

Figure 30: Interconnection Frequency \& Imbalance Duration Curves Display Pop-Up

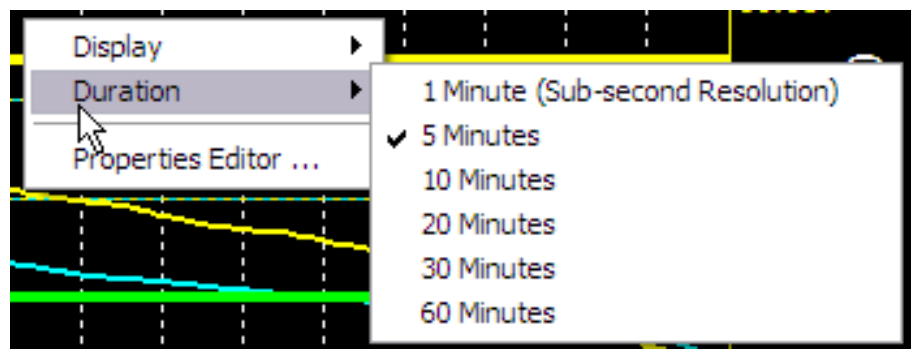

Figure 31: Interconnection Frequency \& Imbalance Duration Curves Duration Selection Pop-Up 


\section{PATH DYNAMICS MONITORING}

\subsection{Overview}

The "Path Dynamics" monitoring display will allow the dispatcher to monitor voltage angle differences between key monitoring points in the power system and track associated dynamics in real time with respect to predetermined thresholds. The identified "paths" (i.e., voltage phase angle differences between the two points in the system for which phasor measurements are available) and their corresponding thresholds are user defined and shall be obtained from offline stability studies. The application continuously tracks these angle differences across all the identified paths and displays them as colored arrows between the points defining the paths in the display. A change in the angle differences would be an indication of a change in the power transfer across the path or a change in system configuration, while the size of the angle difference would indicate the stress or lack of stress the transmission system is undergoing.

The "Path Dynamics" tab function is similar to the "Frequency (Response)" tab with the exception that it offers the ability to visualize the data and graphics in two views: a "Wide Area" view (Figure 32) and a "Local Area" view (Figure 33).

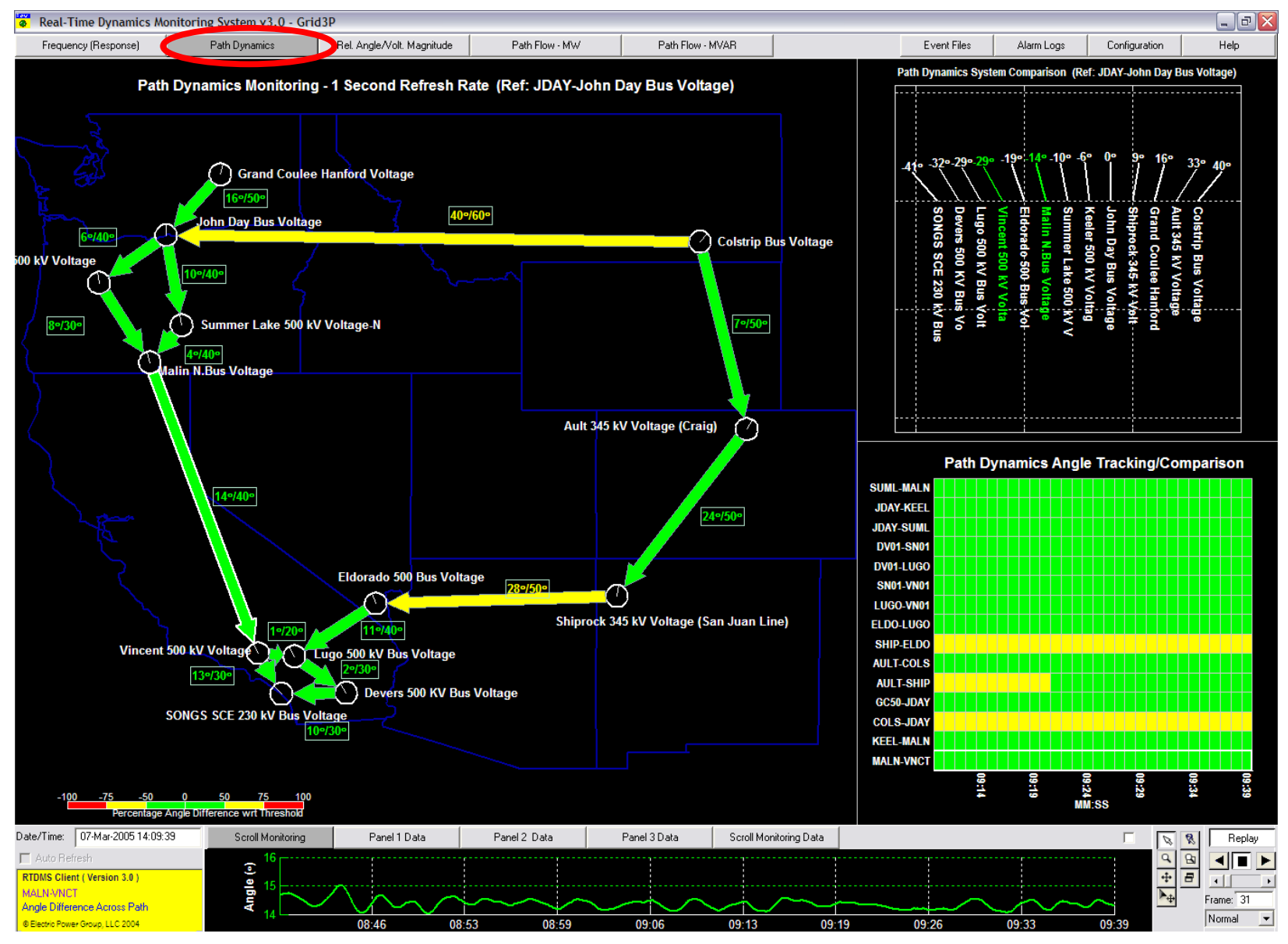

Figure 32: Four-Panel Path Dynamics Monitoring (Wide Area) 


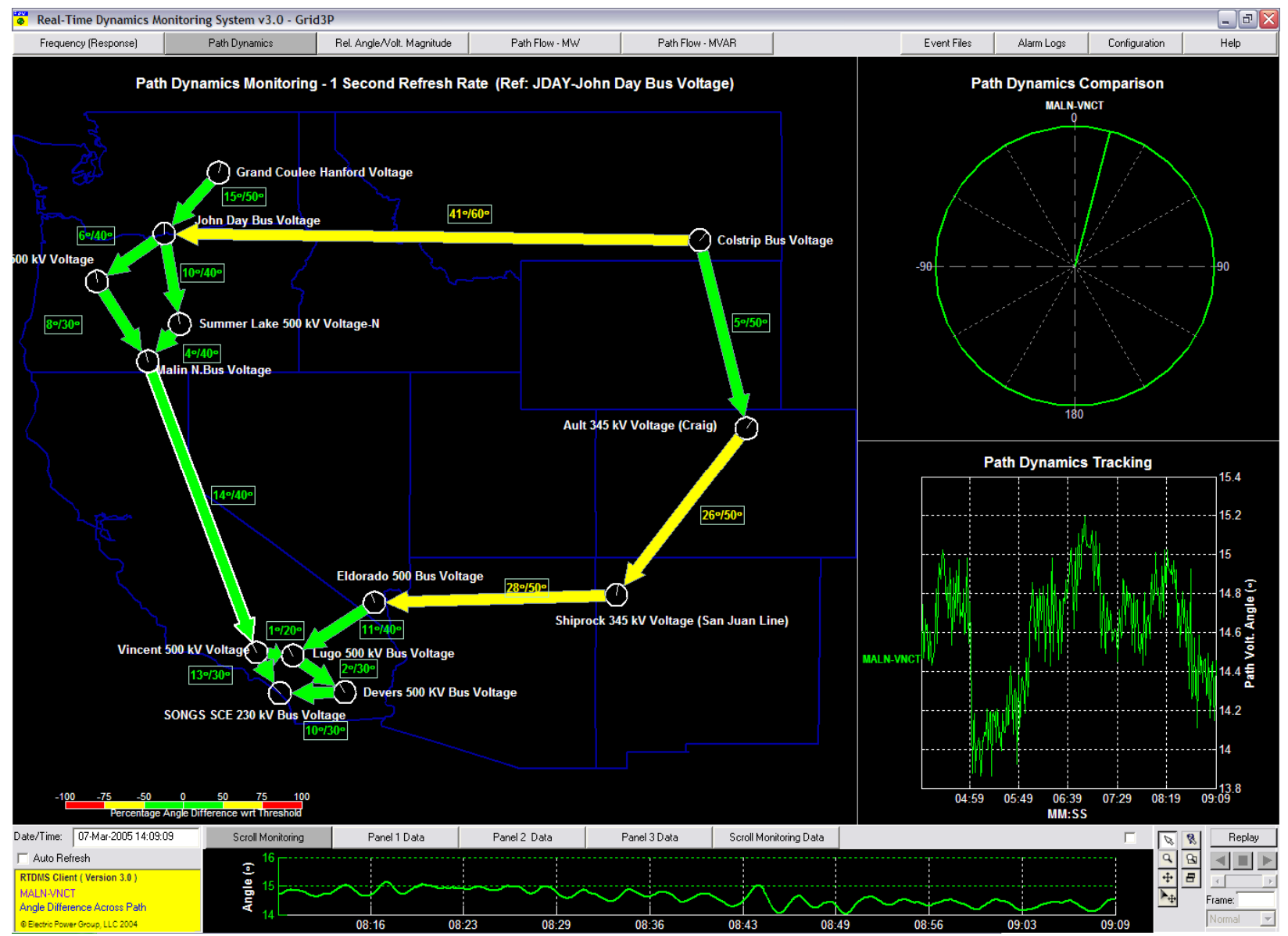

Figure 33: Four-Panel Path Dynamics Monitoring (Local Area)

\subsection{Path Dynamics Monitoring One-Panel Display}

The "Path Dynamics" one-panel display is selected by clicking on the "Path Dynamics" tab (red circle in Figure 32). The angle difference across a path is displayed as a color-coded arrow connecting the two phasor monitoring points that define the path. The direction of arrow is defined such that it points from the larger of the two angles to the smaller one to demonstrate the direction of the power flow, and can therefore change under varying system and market conditions.

The color of the arrow is based on a legend located in the bottom left hand corner of the display (Figure 34). Its colors indicate the proximity (in percentage) of the actual phase angle difference across the path to the predefined alarming threshold defined for it. This actual angle value (the number on the left) and the alarming threshold (the number on the right) are shown in a box adjacent to the colored arrow as well.

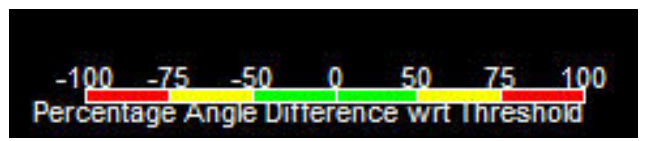

Figure 34: Path Dynamics Legend

Note: While the arrow and the adjoining text indicates the angle difference between the two monitoring points, the dials at the two ends of the arrow represent the measured angles at each of these points where the dial's needle orientation with respect to the vertical is representative of the angle being measured at that location with respect to a common reference angle (Refer to Section 7). 
Example: In Figure 33, the Malin-Vincent path has been selected and it has an actual voltage angle difference of $14^{\circ}$ (i.e., the difference between the voltage angle measured by the Malin PMU and the voltage angle measured by the Vincent PMU is $14^{\circ}$ ). This angle difference must not exceed a preset threshold value of $40^{\circ}$. The correct threshold limit for this path is derived by offline engineering studies, and updated into the application through the configuration utility (Refer to Section 10). An actual angle difference of $14^{\circ}$ implies that this angle is $35 \%$ of its $40^{\circ}$ threshold limit. From the color legend (Figure 34), the Malin-Vincent path is shown as a green arrow since this percentage value falls below $50 \%$.

The default setting for the "Path Dynamics Monitoring" panel is to display the actual angle difference values, station names, and thresholds next to the path arrow. The names and angle difference associated with the paths can be removed from the display, if desired. To do this, right mouse click in the "Path Dynamics Monitoring" panel and the pop-up menu box shown in Figure 35 will appear. Deselect the "Station Name" and "Path Angle Value" and the name and values will disappear.

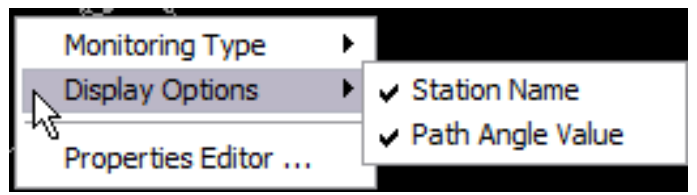

Figure 35: Path Dynamics Display Pop-Up

Under the "Scroll Monitoring" display, located below the main path display, (display is enlarged by clicking check box in upper right corner) user will find the phase angle difference for the last selected path (Figure 36). To select a specific path to be displayed on the scroll monitoring panel, first make sure you have clicked on the "Selection Arrow" (bottom right), and then point and click on the desired path to be tracked. The outline of the path arrow will turn white indicating it was selected. The path name on the scroll monitoring display should reflect the line selected. In this view the Malin-Vincent path has been selected. The $y$-axis on the left of the plot is the angle difference (in degrees), while the $x$-axis is time in minutes and seconds (MM:SS) with the most current time to the right of the axis. This plot allows the user to track the angle difference across the path over the past minute at the highest sub-second resolution.

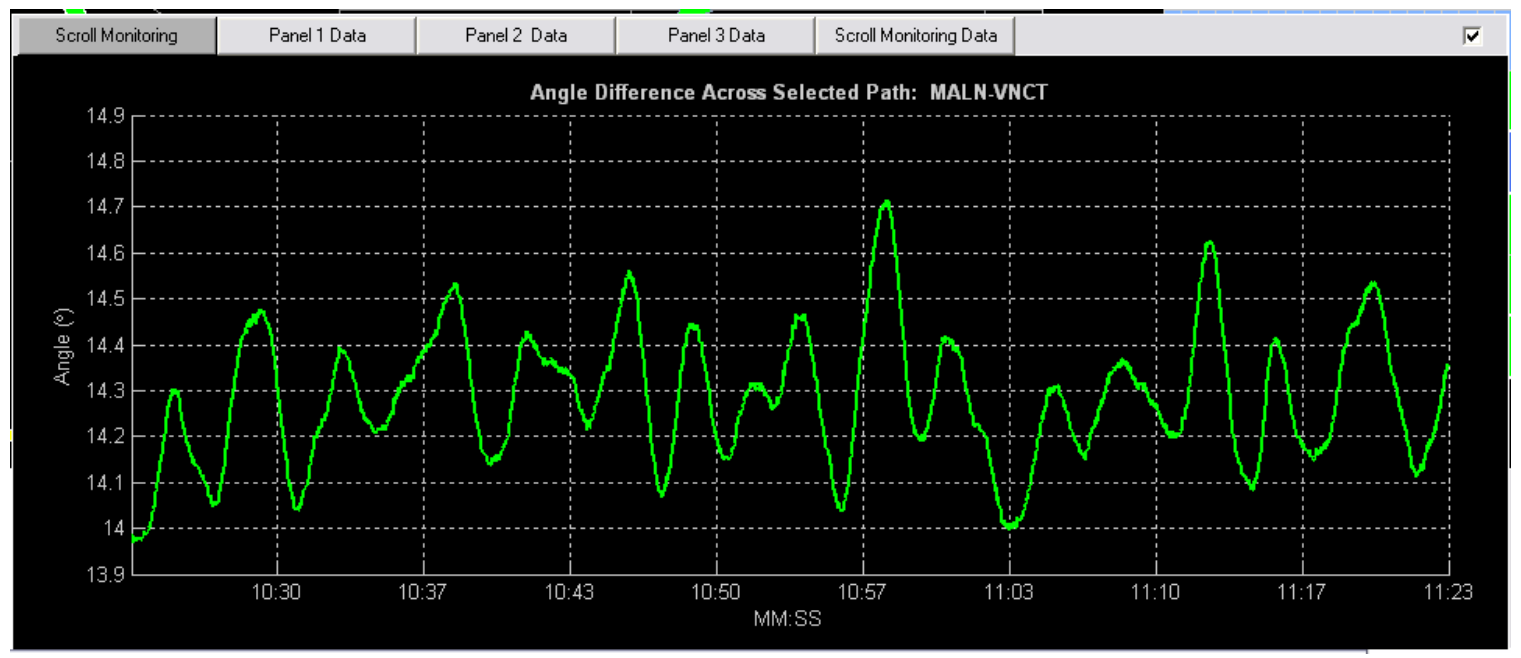

Figure 36: Selected Path Angle Difference Tracking (Enlarged) 


\subsection{Path Dynamics Monitoring Four-Panel Display}

A double left mouse click in the default one-panel will take the user to the four-panel display. This may also be done using a right mouse click within the one-panel display to show the pop-up menu box and to select the desired four-panel view ("Wide Area Monitoring/Tracking" or "Local Area Monitoring/Tracking") from the menu (Figure 37).

\begin{tabular}{|l|l|}
\hline $\begin{array}{l}\text { Monitoring Type } \\
\text { Display Options }\end{array}$ & $\begin{array}{l}\text { Wide Area Monitoring/Tracking } \\
\text { Local Area Monitoring/Tracking }\end{array}$ \\
\hline Properties Editor ... & \\
\hline
\end{tabular}

Figure 37: Path Dynamics Monitoring Type Pop-Up

\subsubsection{Path Dynamics - Wide Area Monitoring/Tracking}

Select the "Wide Area Monitoring/Tracking" and the view shown in Figure 38 will be presented. Each of the three panels (panel 1, panel 2 and panel 3) can be enlarged by double clicking with the left mouse button within the panel.

In this view, the "Path Dynamics Monitoring" display described in section 6.2 is shown in panel 1 of the four-panel display. Panel 2 in the upper right corner is the "Path Dynamics System Comparison" display and panel 3 in the lower right corner is the "Path Dynamics Angle Tracking/Comparison" display. Panel 4 is the "Scroll Monitoring" plot which was explained in the previous section.

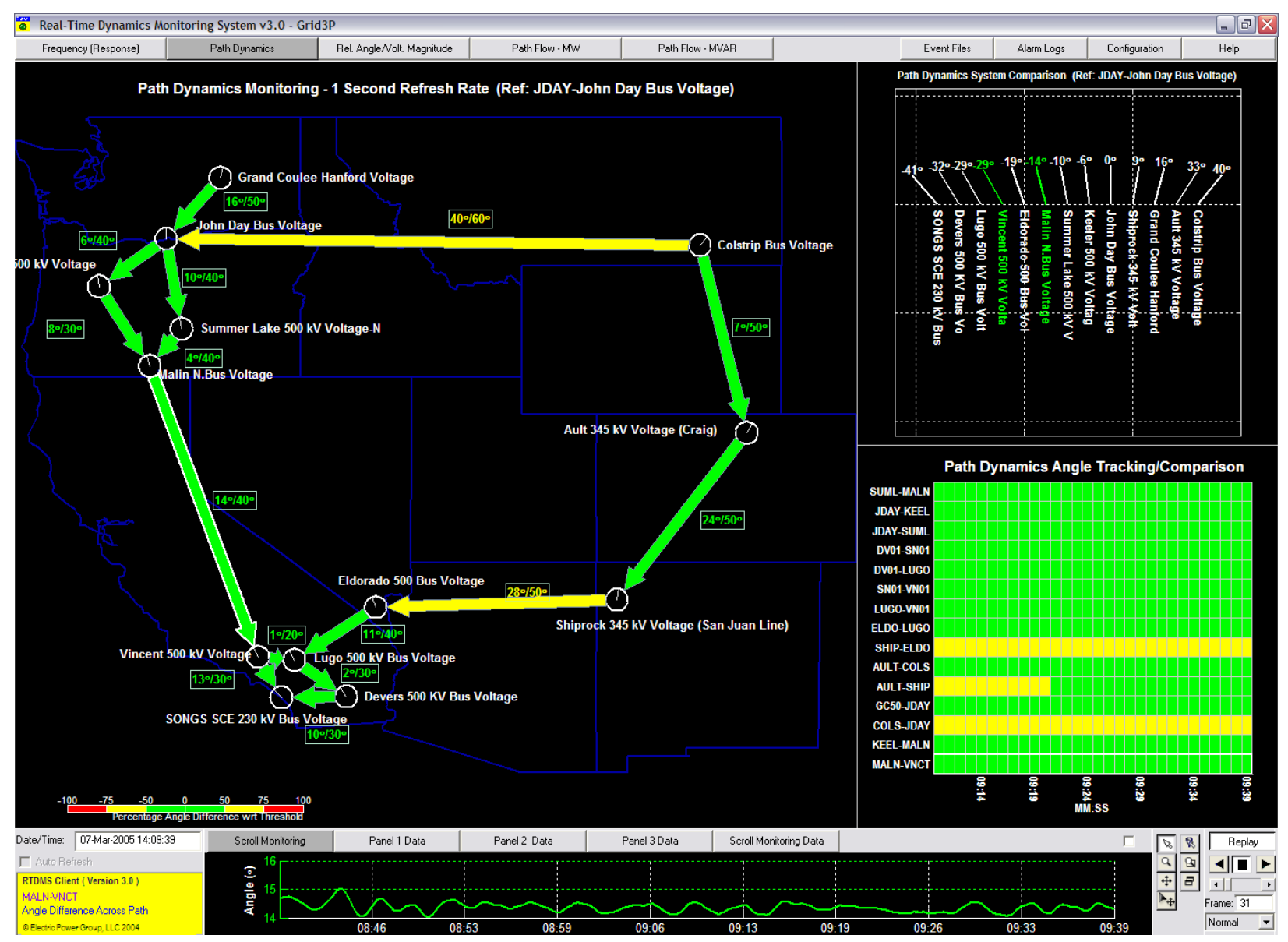

Figure 38: Four-Panel Path Dynamics - Wide Area Monitoring/Tracking 
The "Path Dynamics System Comparison" display uses a feather plot to let the user analyze the phase angle behavior at the source and sink of a selected path with respect to the angles at the sources/sinks of all the identified dynamics paths shown in panel 1. Each vector of this plot corresponds to a monitoring location and the orientation of this vector with respect to the vertical represents the voltage angle being measured at that point with respect to a common reference ${ }^{7}$ (Note: Increase in angles corresponds to a clockwise rotation with respect to the vertical). In the display above (Figure 38), John Day is the reference and will be displayed as a vertical vector (i.e., 0 degrees). Furthermore, the vectors in the feather plot are ordered such that the points to the right of reference will have a leading angle and the points to the left will have a lagging angle (Figure 39). As power flows from larger angles to smaller angles, this sorted plot offers a wide area view of the power flows within the system. Finally, the two vectors representing the source and sink of the selected path are color coded based on the legend in Figure 34.

Example: In the display below (Figure 39), the Malin-Vincent path has been selected. The path is shown by a solid yellow arrow in the geographic display (Figure 38, panel 1). In the feather plot, the vectors corresponding to the source and sink of this path (i.e., Malin and Vincent) also appear in the color of the path arrow (green in this example).

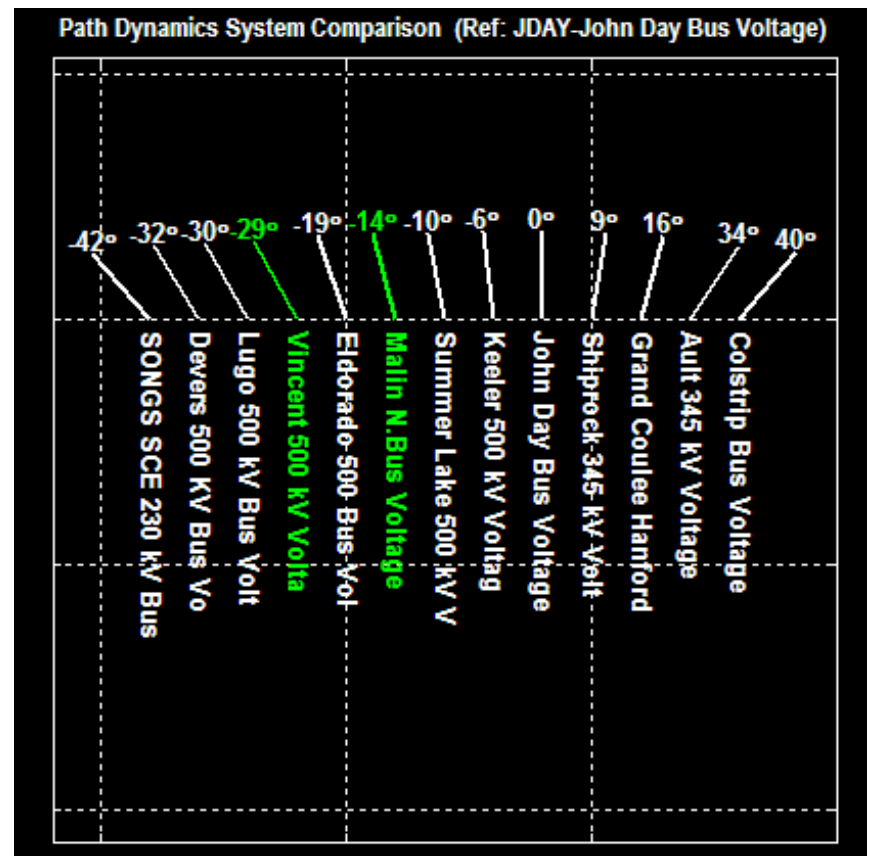

Figure 39: Path Dynamics System Comparison (Panel 2)

The graphics in the "Path Dynamics Tracking/Comparison" panel provides a birds-eye view of the phase angle difference across all the identified paths for tracking and comparison purposes (Figure 40). The $x-$ axis of the plot is time in minutes and seconds (MM:SS) and the $y$-axis lists the identified paths. Hence, each cell in the plot corresponds to a particular path and time, and the color coding of the cell, which represents the phase angle difference across the path, can be cross referenced to the color legend at the bottom of the geographic display in panel 1 (Figure 38). The default time duration for the plot is 30 seconds and may be changed by the user.

\footnotetext{
${ }^{7}$ The reference may be changed and is described in Section 7.2.
} 


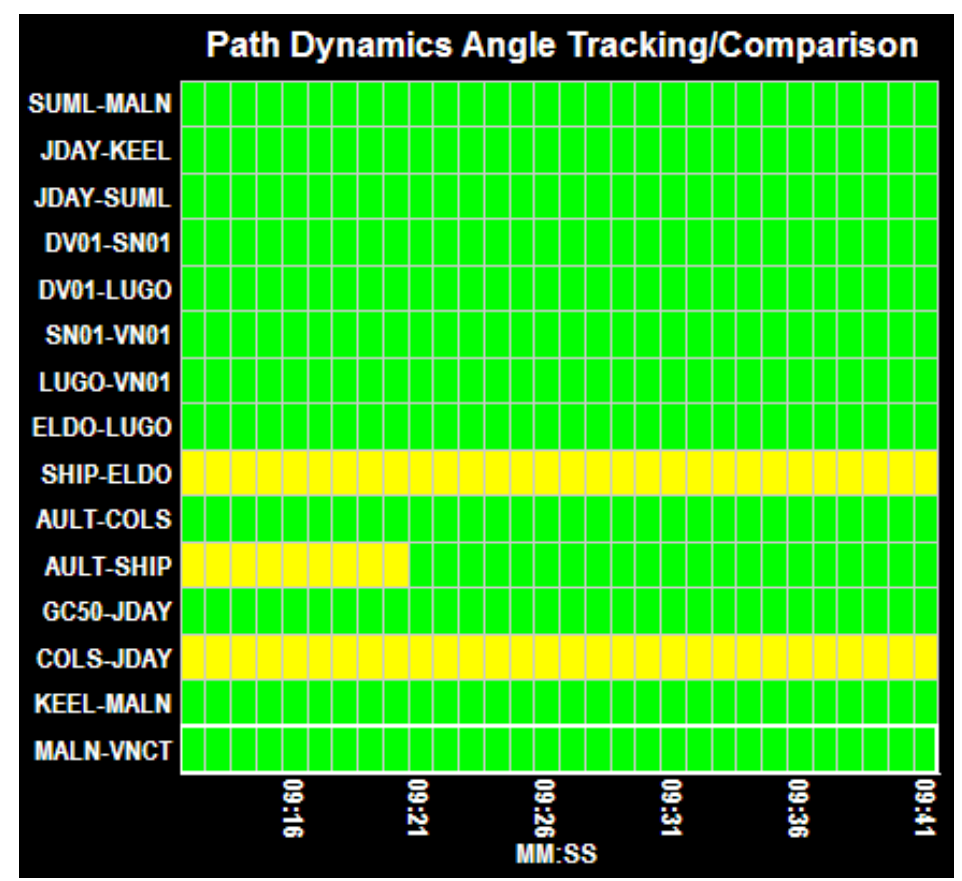

Figure 40: Path Dynamics Tracking Comparison (Panel 3)

To set the desired time duration, left click in the "Path Dynamics Tracking/Comparison" panel and the pull-down menu shown in Figure 41 will appear. Select the desired duration from the available options.

\begin{tabular}{|l|r|}
\hline Duration & 10 Seconds \\
20 Seconds \\
30 Seconds \\
60 Seconds
\end{tabular}

\section{Figure 41: Path Dynamics Tracking Comparison Duration Pop-Up}

\subsubsection{Path Dynamics - Local Area Monitoring/Tracking}

While the "Wide Area Monitoring/Tracking" view provided a macroscopic understanding of the angle differences across identified paths in relation to the overall system behavior, the purpose of "Local Area Monitoring/Tracking" view is to let the user get more specific data and information about specific selected paths.

Selecting "Local Monitoring/Tracking" option from the menu in Figure 37 will present the four-panel display as seen in Figure 42. Double left clicking either in panel 1, panel 2 or panel 3 of the display will enlarge the corresponding panel. 


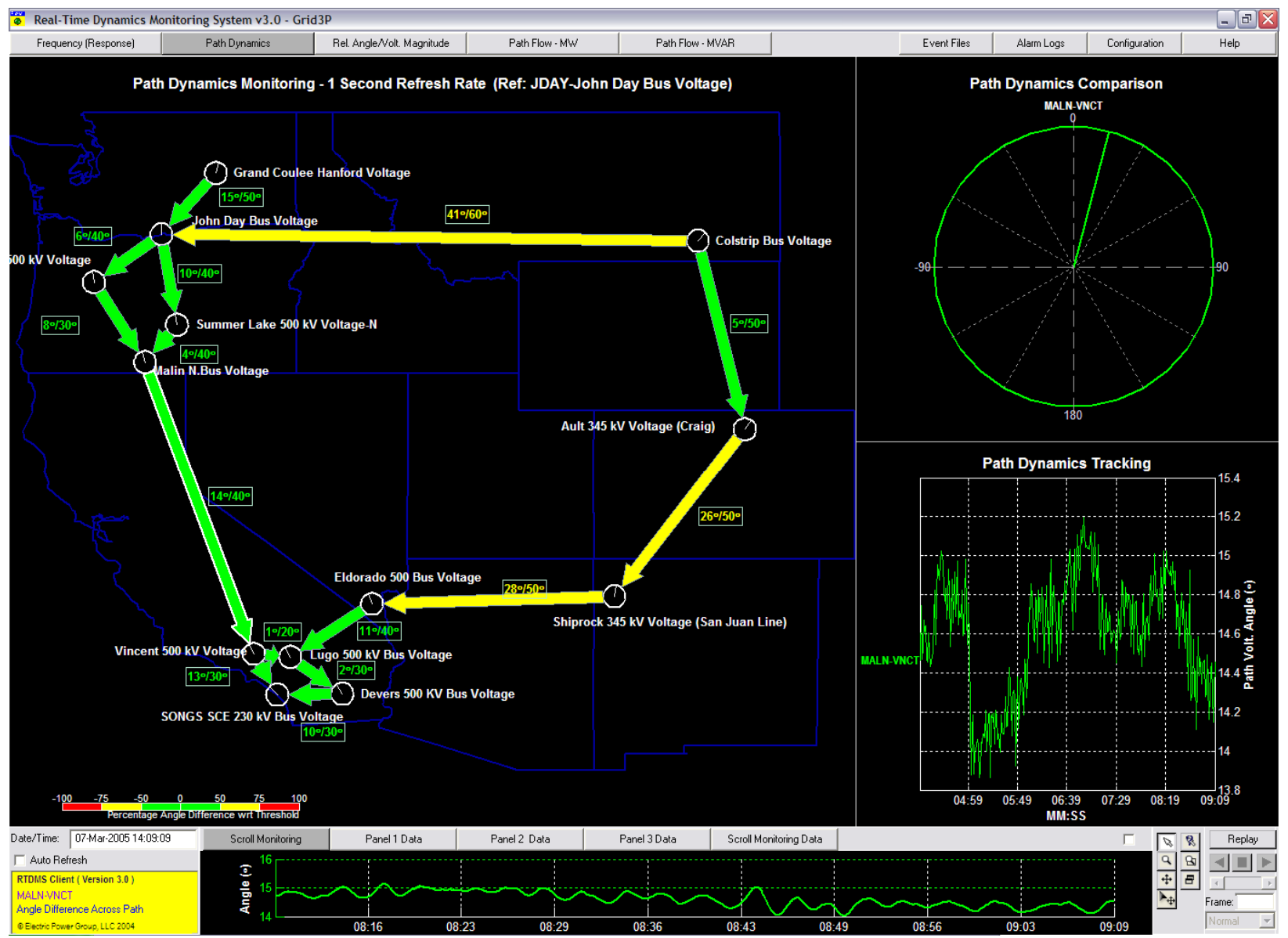

Figure 42: Four-Panel Path Dynamics - Local Area Monitoring/Tracking

The "Path Dynamics Comparison" in panel 2 allows the user to compare the phase angle differences across a set of selected paths. The user may left mouse click on any of the arrows shown in panel 1 to select/deselect a path and the phase angle difference across the path is added to panel 2 as a dial where the orientation of the dial with respect to the vertical is indicative of the angle. The color of the dial matches the color of the path arrow selected, both of which are determined by the color legend in the geographic panel (Figure 34).

The "Path Dynamics Tracking" in panel 3 provides a historical profile of the phase angle differences across the set of selected paths at the second resolution. The $x$-axis of the plot is time in minutes and seconds (MM:SS) and the $y$-axis is the angle difference in degrees. As with all other plots, the time duration of the plot may be altered via a pull-down menu made available by using the right mouse click within the panel.

Example: In Figure 42, the Malin-Vincent path has been selected and shows the corresponding graphics in panel 2 and panel 3 of the display. In panel 2, the angle across the path is shown as a dial where the needle of the dial makes a $14^{\circ}$ angle with the vertical axis in the clockwise direction to indicate the angle difference across the path. In panel 3 , the time history for the angle across the path is shown over the past five minute interval (default time duration) and at a second resolution.

Multiple paths (maximum 6) can be selected and displayed in the "Path Dynamics Comparison" and the "Path Dynamics Tracking" graphics. The selected paths will appear as seen in Figure 43. Note that each selected path arrow is outlined in white. 


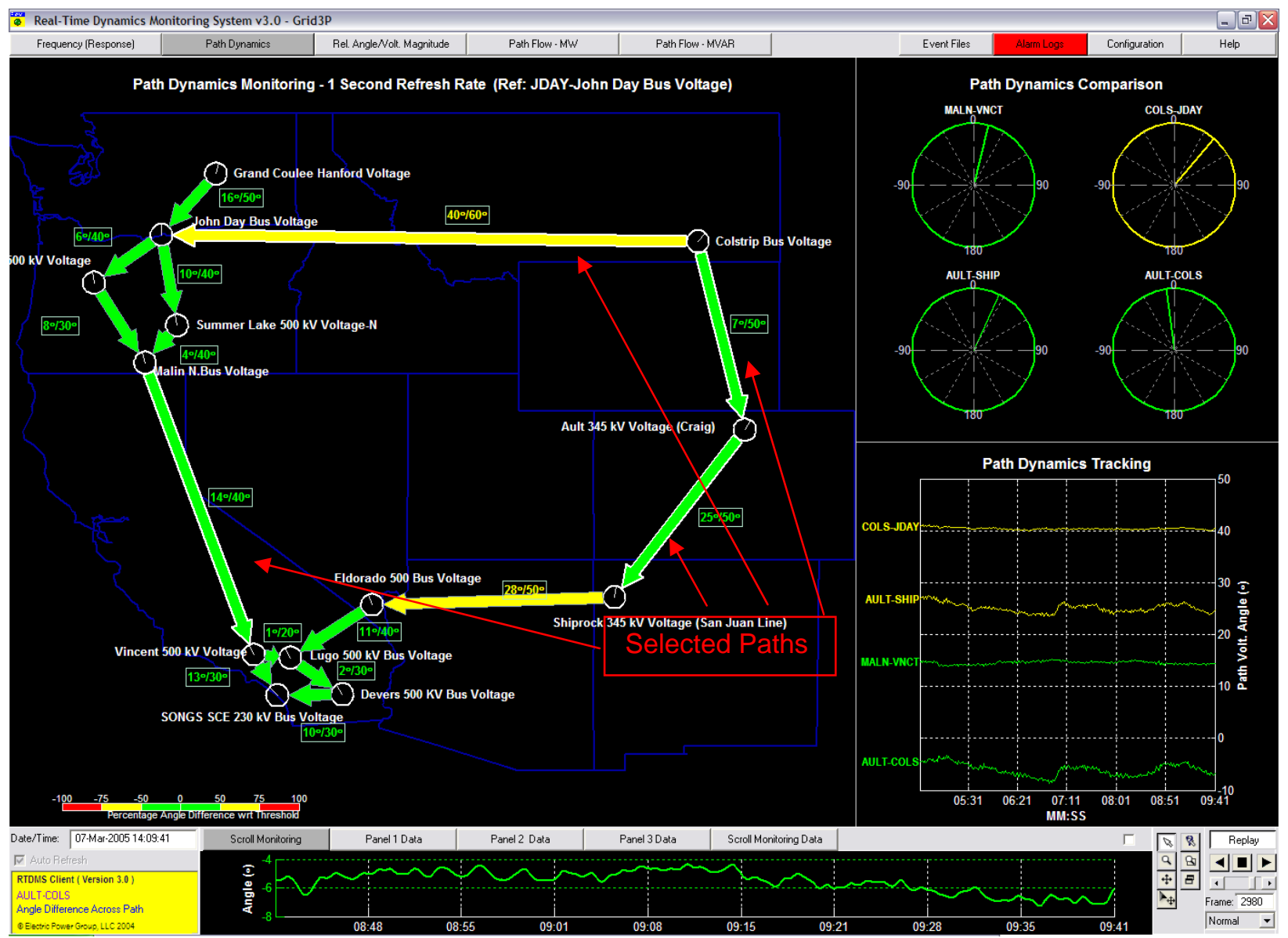

Figure 43: Four-Panel Path Dynamics - Local Area Monitoring/Tracking (Four Paths Selected) 


\section{RELATIVE ANGLEIVOLTAGE MAGNITUDE MONITORING}

\subsection{Overview}

The value of phasor technology lies in its capability to precisely and simultaneously measure voltage phasors (i.e., voltage angles and voltage magnitudes) at various points across the power system at a high rate and to provide accurate timing information along with these measurements. This display shows the voltage angle and voltage magnitude measured at each of the phasor monitoring points on a geographical map. The voltage angles are measured relative to a common selectable reference for the grid (i.e., the angle difference between a monitoring point and the reference point). Such a geographic angle and voltage magnitude profile provides a birds-eye view of the sources and sinks of power across the grid, the high and low voltage regions within the system, as well as helps identify regions that may be operating under stressed conditions.

Like the "Path Dynamics" monitoring display, the "Relative Angle/Voltage Magnitude" monitoring display also offers a "Wide Area" (Figure 44) and a "Local Area" (Figure 45) view and the user can select between the two views using options available within a pull-down menu.

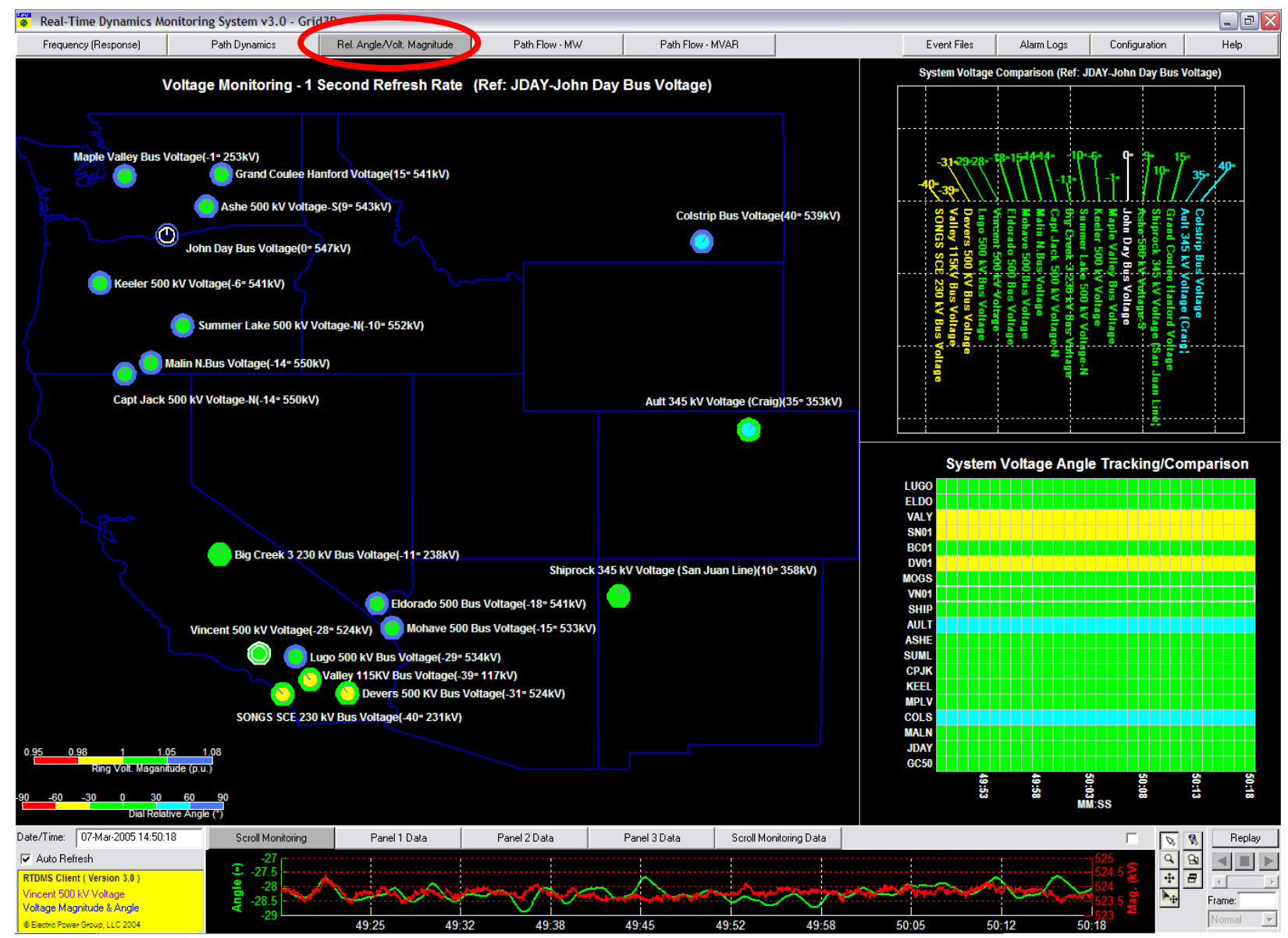

Figure 44: Four-Panel Relative Angle/Voltage Magnitude Monitoring (Wide Area) 


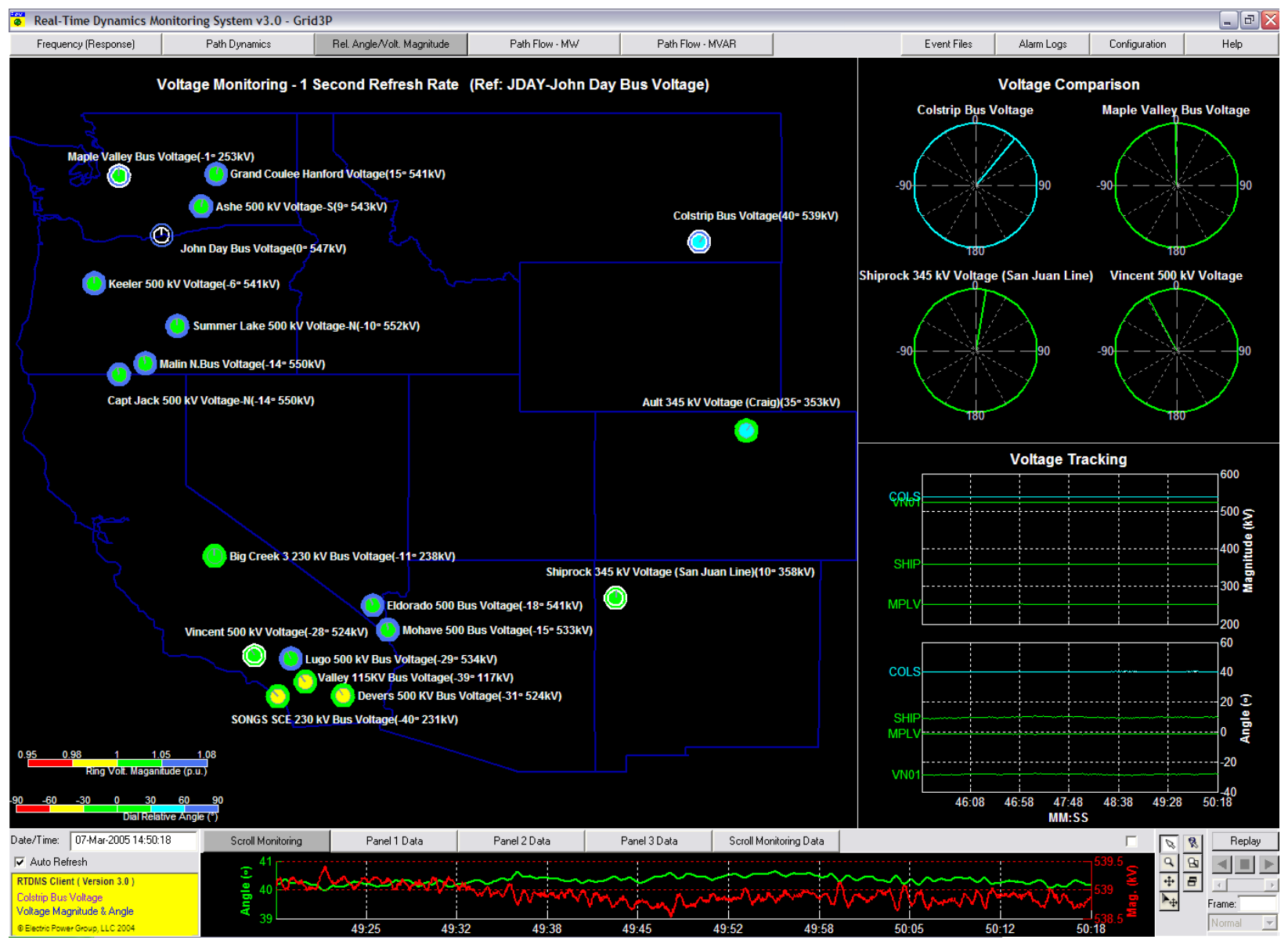

Figure 45: Four-Panel Relative Angle/Voltage Magnitude Monitoring (Local Area)

\subsection{Relative Angle/Voltage Magnitude One-Panel Display}

Selecting the "Relative Angle/Voltage Magnitude" tab at the top of any of the displays will bring up just Panel 1, the left panel in Figure 44. In Panel 1, the voltage angles with respect to a common reference are displayed as a dial at the corresponding monitoring location in a geographic display, while the voltage magnitudes are indicated by a color-coded ring around the corresponding dial within the geographic display. Both the orientation of the dial's needle with respect to the vertical and its color are representative of the angle being measured at that location (Note: Increase in angles corresponds to a clockwise rotation with respect to the vertical). The legends used this color-coding are located at the bottom left of the display (see Figure 44). The voltage angle and voltage magnitude legend is also colorcoded based on "per-unit" values (Figure 46).

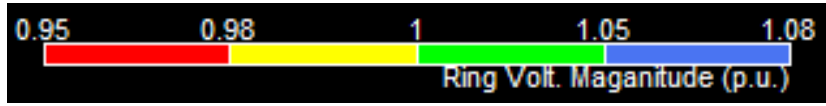

Figure 46: Relative Angle/Voltage Magnitude - Relative Angle and Voltage Magnitude Legends

Example: In Figure 49, the Vincent voltage is $524 \mathrm{kV}$ (outer ring is green) which is between 1.00 p.u. and 1.05 p.u. This second ring allows the user to visually scan for abnormal voltages both above and below the nominal values.

The reference for all the angles in the display is the one dial that is not colored (Grand Coulee in Figure 44). This reference can be changed by the user. 
To change the reference for the system angles, right mouse click on the monitoring location that is to set as the reference and the pop-up menu box shown in Figure 47 will appear. Select the "Set as Reference" option and the appropriate voltage from those available for that particular monitoring location.

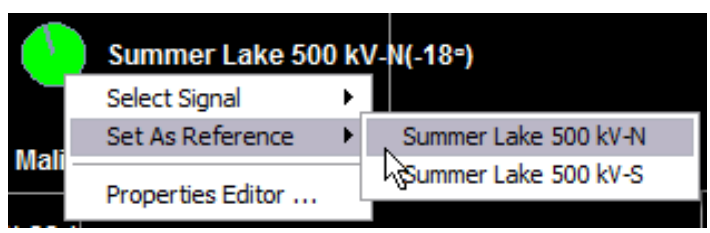

Figure 47: Voltage Monitoring Reference Selector

In addition to the angle information, the user may also choose to view the voltage magnitude information at each of the monitoring locations. This is done by right mouse clicking within the geographic display and selecting the "Include Volt. Magnitude" option in the pull down menu box. The same menu can be used to display the "Station Name" or "Signal Value" next to the monitoring location within the geographic display (Figure 48).

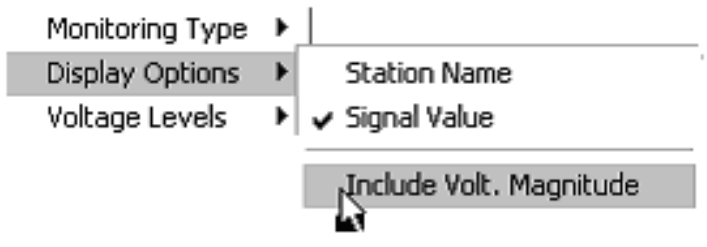

Figure 48: Relative Angle/Voltage Magnitude Display Options Pop-Up

When the voltage magnitude information is chosen, it is displayed along with angle information as a ring around the dials where the color and the size of the ring are indicative of the voltage magnitude (Figure 49). The voltage magnitude legend is color-coded based on "per-unit" values (Figure 46). 


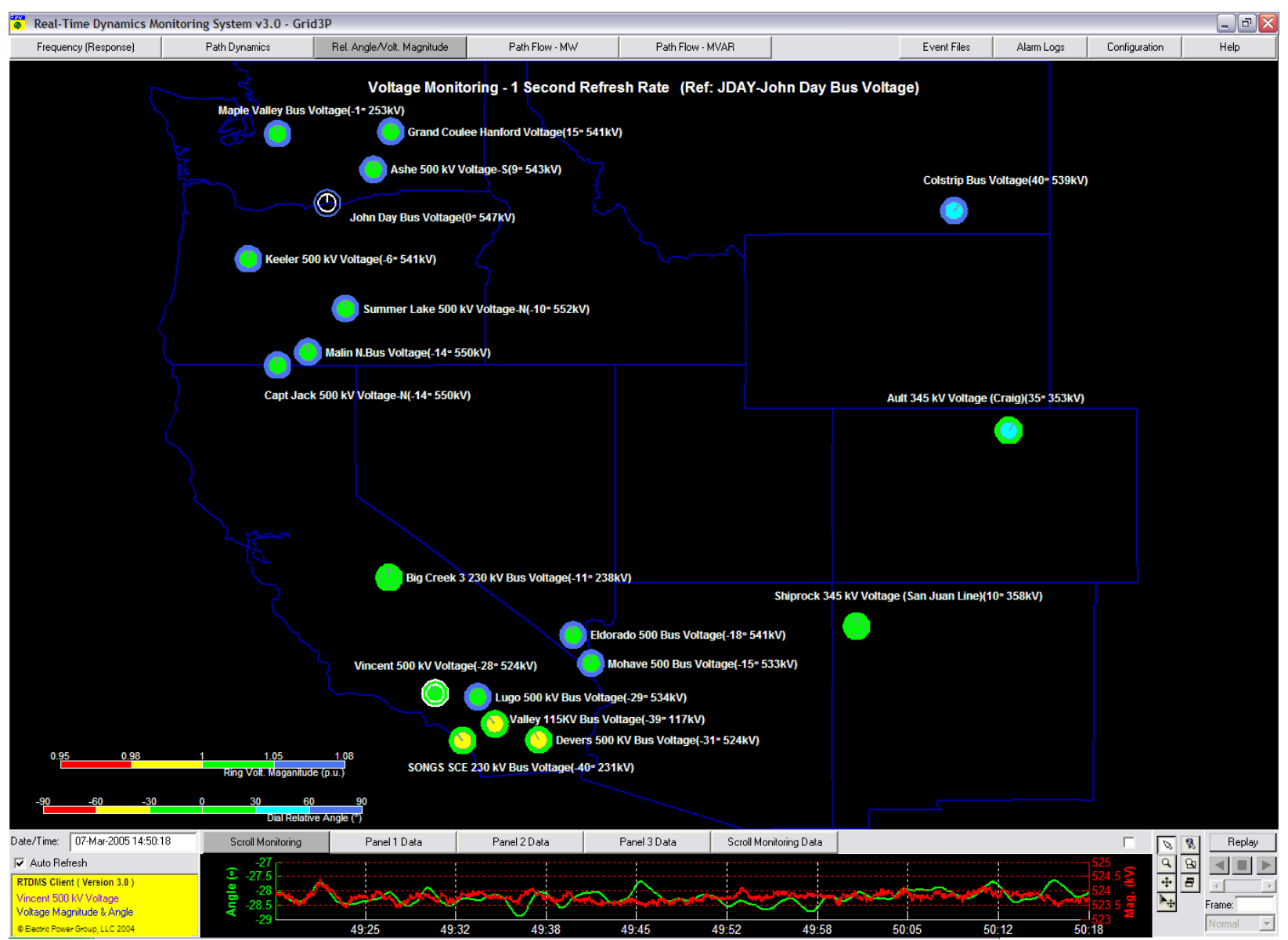

Figure 49: One-Panel Relative Angle/Voltage Magnitude with Voltage Magnitude Information

The display also allows the user to be selective in viewing the angles and voltage magnitudes being displayed based on their voltage levels. This is done via a right mouse click within the geographic display to show the pop-up menu box In Figure 50. The default setting is "All" for the "Voltage Levels". To change to a specific voltage level, right click on the desired voltage. In doing, only those monitoring points whose voltages belong to the selected voltage level will appear in the geographic panel.

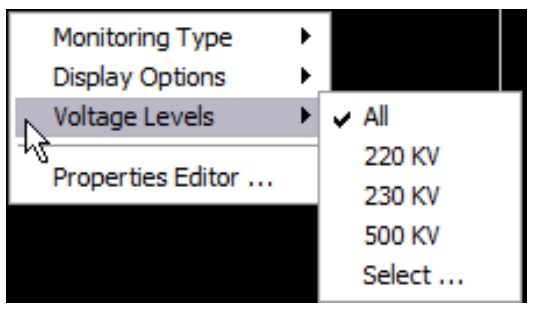

Figure 50: Relative Angle/Voltage Magnitude Voltage Levels Pop-Up

The "Scroll Monitoring" at the bottom of Panel 1 tracks the relative angle of the last selected monitoring location at the highest sub-second resolution over the past one minute (Figure 51). Furthermore, if the "Include Volt. Magnitude" option has been selected (Figure 48), then the voltage magnitude at this monitoring location is also shown here. 


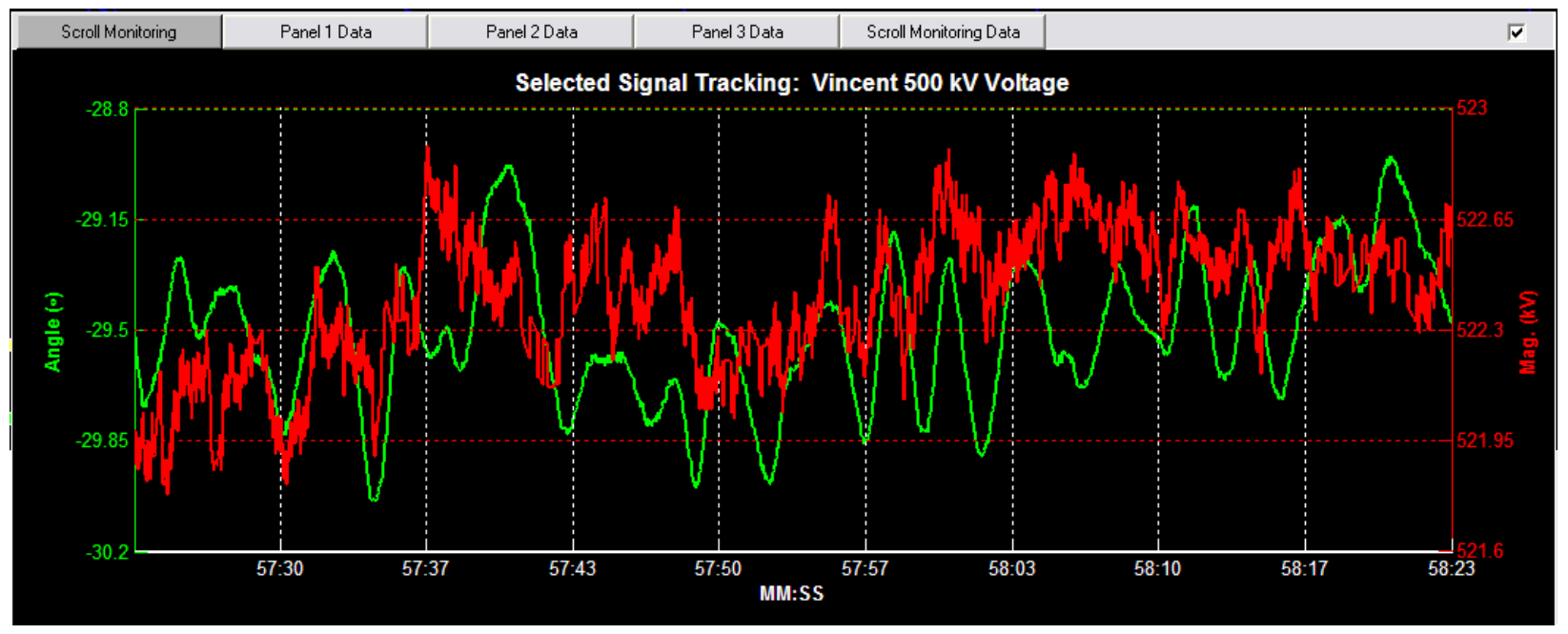

Figure 51: Selected Relative Angle/Voltage Magnitude Tracking

\subsection{Relative Angle/Voltage Magnitude Four-Panel Display}

As with all other displays, a double left mouse click in Panel 1 display will take the user to the corresponding four-panel display. This may also be done using a right mouse click within the one-panel display to show the pop-up menu box and to select the desired four-panel view ("Wide Area Monitoring/Tracking" or "Local Area Monitoring/Tracking") from the menu (Figure 54).

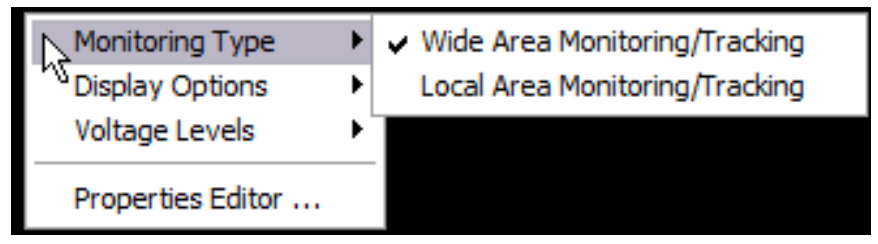

Figure 52: Relative Angle/Voltage Magnitude Monitoring Type Pop-Up

\subsubsection{Relative Angle/Voltage Magnitude - Wide Area Monitoring/Tracking}

The four panels within the "Wide Area Monitoring/Tracking" view are the "Voltage Monitoring - One Second Refresh Rate" display in panel 1 which was described earlier. The "System Voltage Comparison" is displayed in Panel 2 and the "System Voltage Angle Tracking/Comparison" is shown in Panel 3. The "Scroll Monitoring" Panel which is located at the bottom of the four-panel display was also described earlier. 


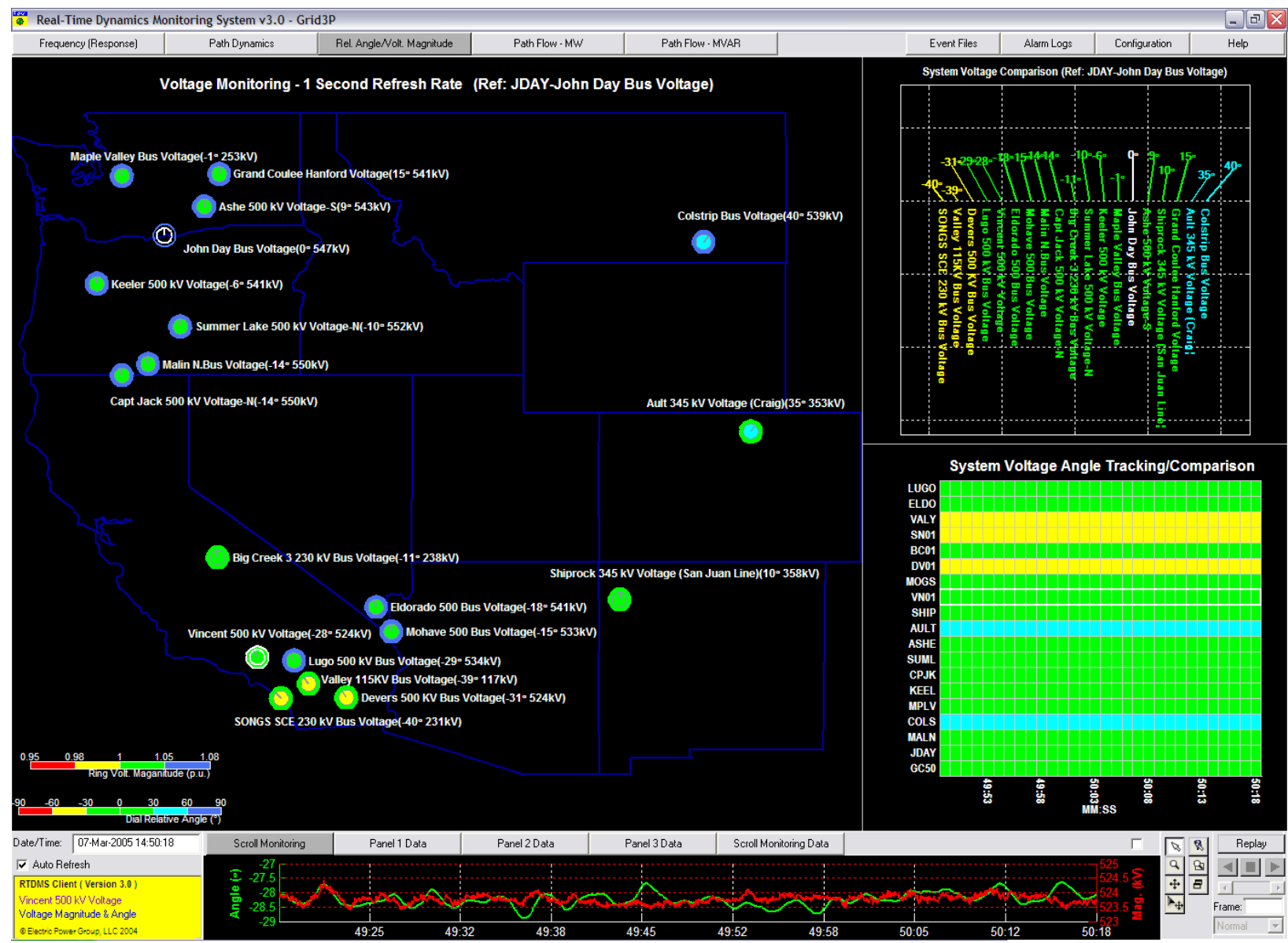

Figure 53: Four-Panel Relative Angle/Voltage Magnitude - Wide Area Monitoring/Tracking

The "System Voltage Comparison" panel uses a feather plot to compare all the voltage angles and magnitudes across the power grid (top right of Figure 53). Each vector of this plot corresponds to a monitoring location and the length and the orientation of this vector with respect to the vertical is indicative of the relative angle at that monitoring location. The reference for the angles is always a vertical vector (i.e., 0 degrees) and is shown in white (i.e., Grand Coulee). The vectors are also color coded according to the legend in the bottom left of Figure 53.

In the "System Voltage Angle Tracking Comparison" panel, the time history of all the relative angles is provided for tracking and comparison purposes (bottom right of Figure 49). The x-axis of the plot is time in minutes and seconds (MM:SS) and the $y$-axis lists the monitoring locations. Each cell in the plot, therefore, corresponds to a particular monitoring location and time, and the color coding of the cell, which represents the relative angle, can be cross referenced to the color legend at the bottom of the geographic display in panel 1 (Figure 49). The time duration for the plot is selectable.

To select a desired time, right click in the "System Voltage Angle Tracking/Comparison" panel. Figure 54 presents the selectable time available.

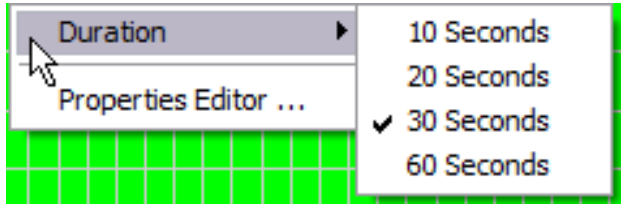

Figure 54: System Voltage Angle Tracking/Comparison Duration Pop-Up 


\subsubsection{Relative Angle/Voltage Magnitude - Local Area Monitoring/Tracking}

Figure 55 shows the four-panel graphics for the "Local Area Monitoring/Tracking" view. Each of panels can be enlarged by double left clicking on the desired panel.

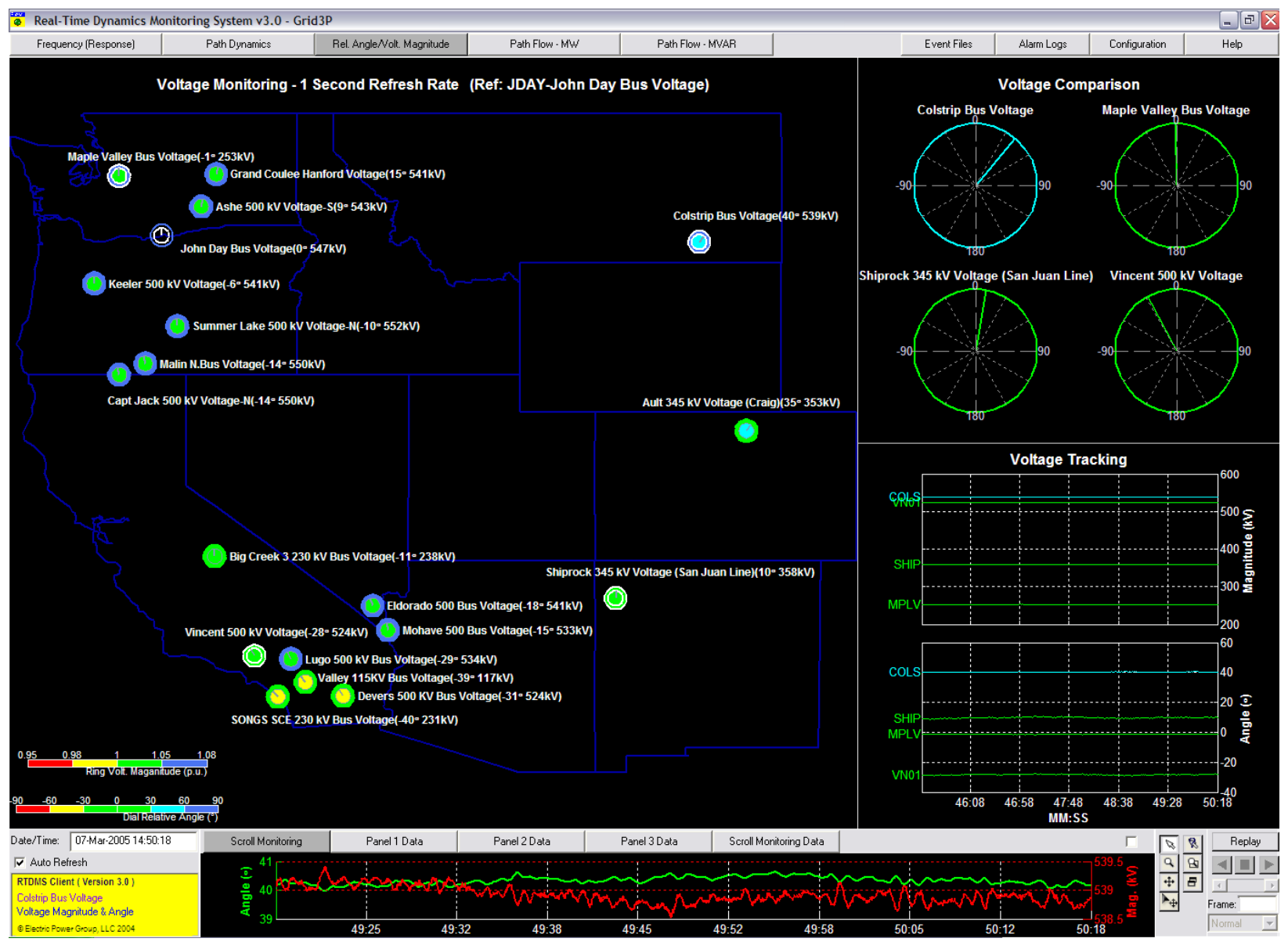

Figure 55: Four-Panel Relative Angle/Voltage Magnitude - Local Area Monitoring/Tracking

In Figure 55 displays, in the top right panel, the voltage angles for Grand Coulee, Colstrip, Vincent and Ault are viewed as dials on the "Voltage Comparison" panel. In this display, the angles for up to six monitoring locations may be added by left clicking on the corresponding dials in the geographic display. A selected location in the geographic panel will have a white border around it as an indicator of its selection. To deselect it, simply left click on it again and it will be removed from the "Voltage Comparison" panel.

The "Voltage Tracking" panel is shown in the bottom right of Figure 55. It provides a time history for the relative angles at the selected monitoring locations and the second resolution. The $x$-axis of the plot is time in minutes and seconds (MM:SS) and the $y$-axis is the angle in degrees. The time duration of the plot may be altered via a pop-up menu made available using the right mouse click within the panel (Figure 56).

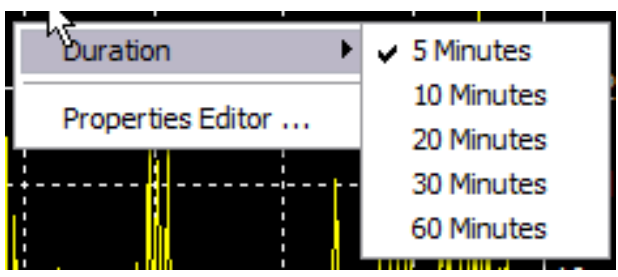




\section{Figure 56: Voltage Tracking Duration Pop-Up}

Finally, if the "Include Volt. Magnitude" option has been selected (Figure 48), then the time history for the voltage magnitudes at the selected monitoring location and the second resolution is shown as well (Figure 57). The upper plot tracks the voltage (in kV) and the lower tracks the relative angle (in degrees).

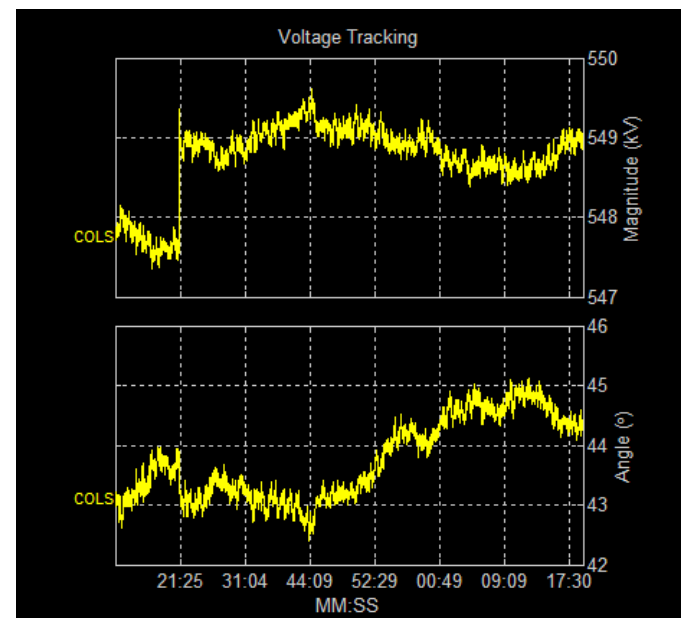

Figure 57: Voltage Tracking Including Voltage Magnitude (Panel 3) 


\section{PATH FLOW (MW) MONITORING}

\subsection{Overview}

The application receives voltage phasor measurements at each of the monitoring locations as well as current phasor measurement on lines connected to the monitoring location. These measurements are used to calculate the real and reactive power flows on the monitored line. The "Path Flow - MW" monitoring display tracks the real power flows across monitored transmission lines or paths (set of monitored lines for which limits have been defined) with respect to their predefined flow limits. Furthermore, as these values are obtained solely from phasor measurements, they offer redundancy to the existing SCADA system. Figure 58 outlines the graphics available within the "Path Flow - MW" monitoring display.

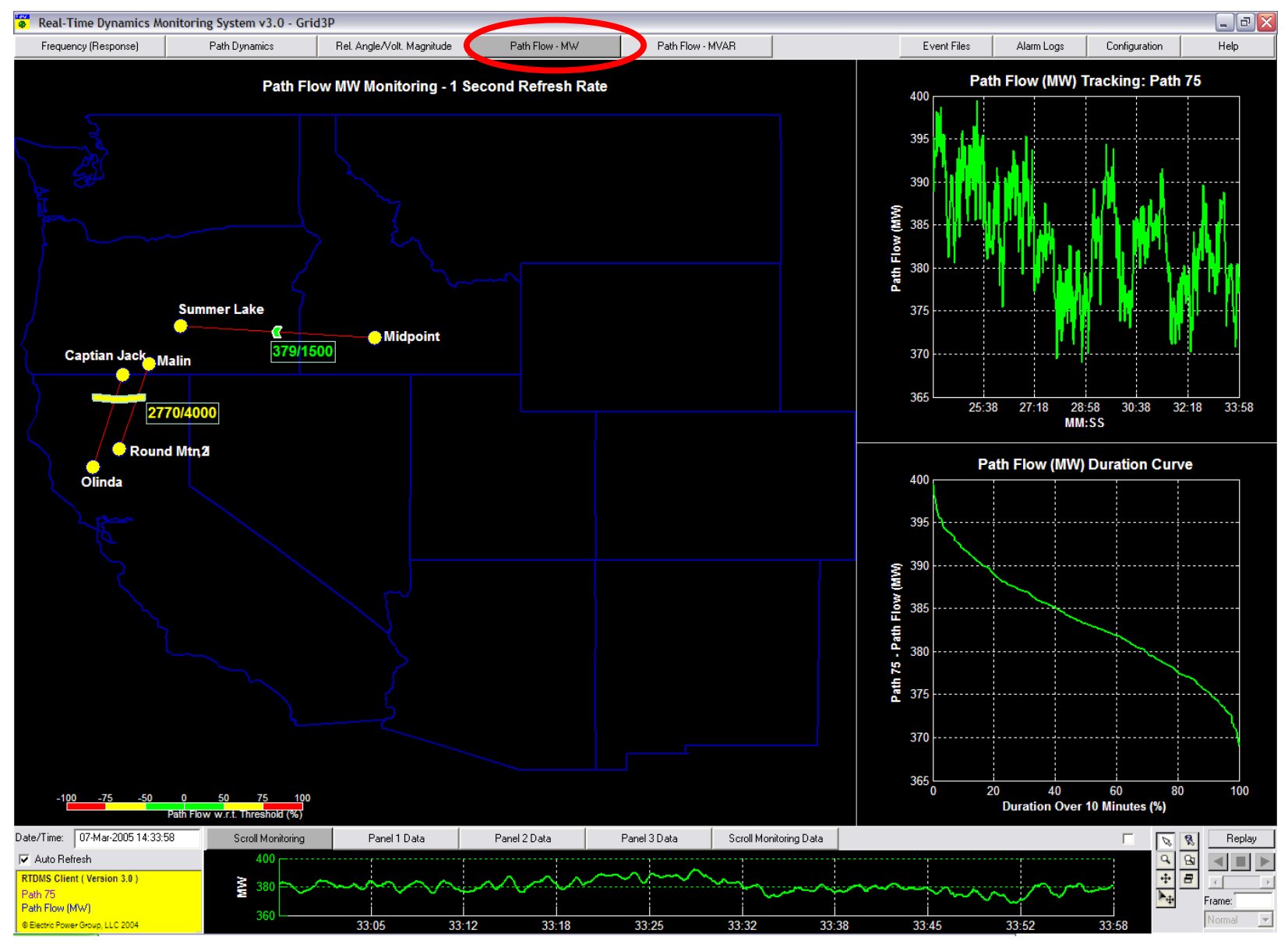

Figure 58: Four-Panel Path Flow - MW Monitoring

\subsection{Path Flow (MW) One-Panel Display}

Selecting the "Path Flow -MW" tab at the top of any of the displays will bring up just Panel 1, the left panel in Figure 58, where the various transmission paths are plotted on a geographical map in the "Path Flow MW Monitoring" panel of the display. The real flows through that path are shown as an arrowhead across all the lines that define the path. While the color of the arrowhead represents the proximity (in percentage) of the MW flows to their alarming thresholds (bottom left on Figure 58), the arrowhead indicates the directionality of these flows.

Under the default settings, the actual MW flows and their corresponding limits are shown next to the arrowhead. The actual flows are listed to the left and the thresholds are listed to the right. The station 
names at both ends of the monitored line are also shown. To remove the station names from the displays, right click within the panel and the pop-up menu will appear (Figure 59). Click on the "Station Name" and the names will be removed. The same pop-up menu can be used to eliminate the "Path Flow Value" from the displays.

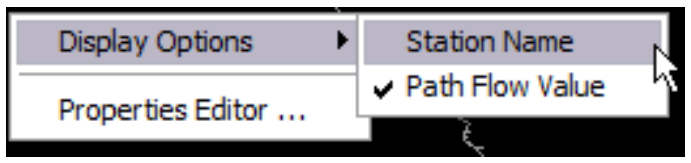

Figure 59: Path Flow - MW - Display Options Pop-Up

Figure 60 presents an enlarged view of Panel 4 "Scroll Monitoring" and the actual MW flow across the single selected path. A path is selected with a left mouse click on the arrowhead in the geographic display for the path and will be indicated by a white border around the arrowhead. The $y$-axis to the left of the plot is the MW flow and the $\mathrm{x}$-axis is time in minutes and seconds (MM:SS). This plot allows the user to track the MW flows across the selected path over the past minute at the highest sub-second resolution.

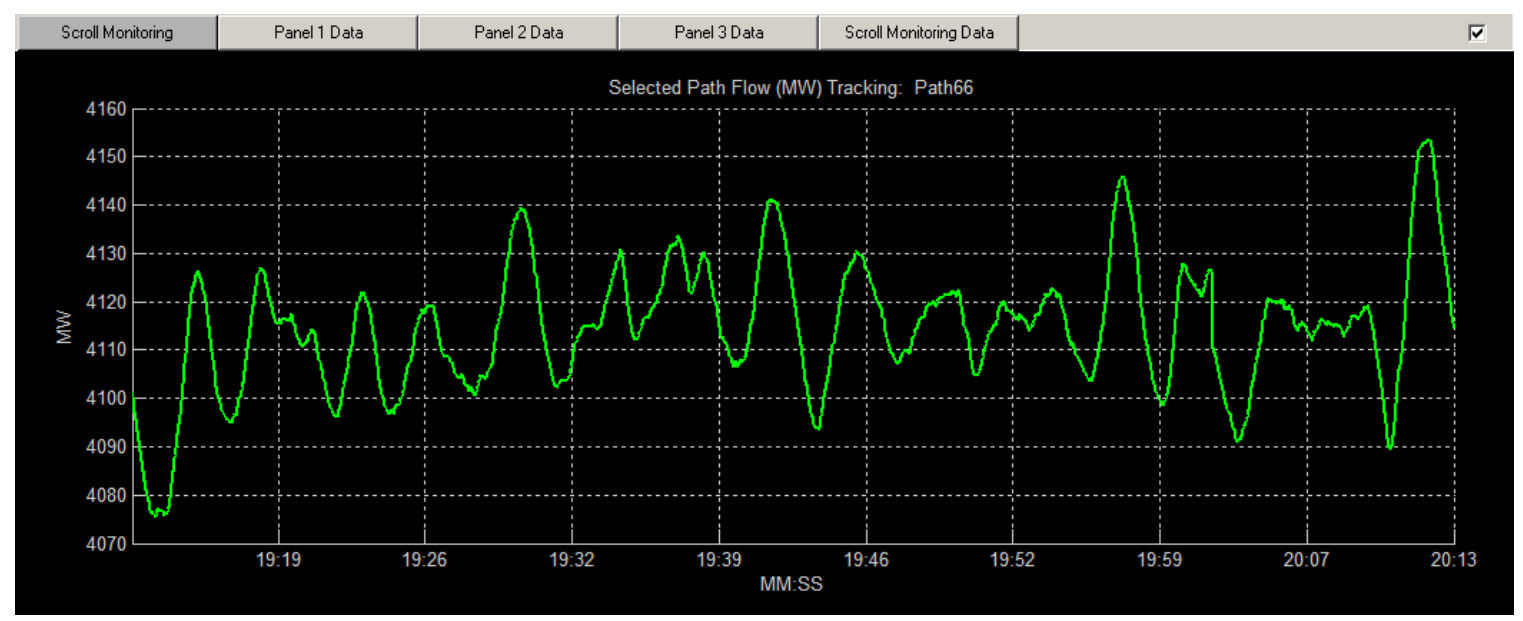

Figure 60: Selected Path Flow (MW) Tracking (Enlarged)

\subsection{Path Flow (MW) Four-Panel Display}

Left double clicks within Panel 1 will bring up the corresponding four-panel display (Figure 58). To enlarge any of the panels, double click with the left mouse button within the desired panel.

Panel 2 - the "Path Flow (MW) Tracking" - shows the actual MW flow over a specific path when selected (Figure 61). The panel provides a time history of the MW flows across the selected path at the second resolution. The x-axis of the plot is time in minutes and seconds (MM:SS) and the y-axis is the MW flow. A path is selected with a left mouse click on the arrowhead in the geographic display for the path and will be indicated by a white border around the arrowhead. The time duration of the plot may be altered via a pop-up menu made available using the right click within the panel. 


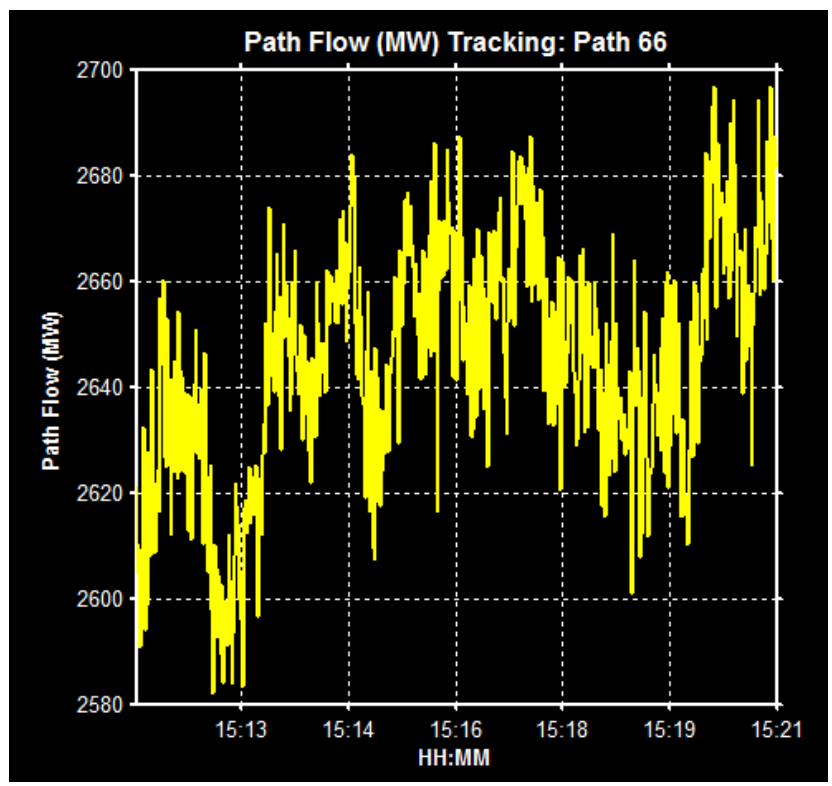

Figure 61: Path Flow (MW) Tracking (Panel 2)

Panel 3 - the "Path Flow (MW) Duration Curve" - displays a statistical distribution of the MW flows across the selected path (Figure 62). For each MW value along the $y$-axis, the corresponding $x$-axis along the curve shows the percentage time that the MW flows were above the value. In Figure 62, the path 66 had over $2665 \mathrm{MW}$ flowing for $20 \%$ of the time. The duration over which the distribution is computed is also selectable through a pull-down menu displayed via a right click within the panel.

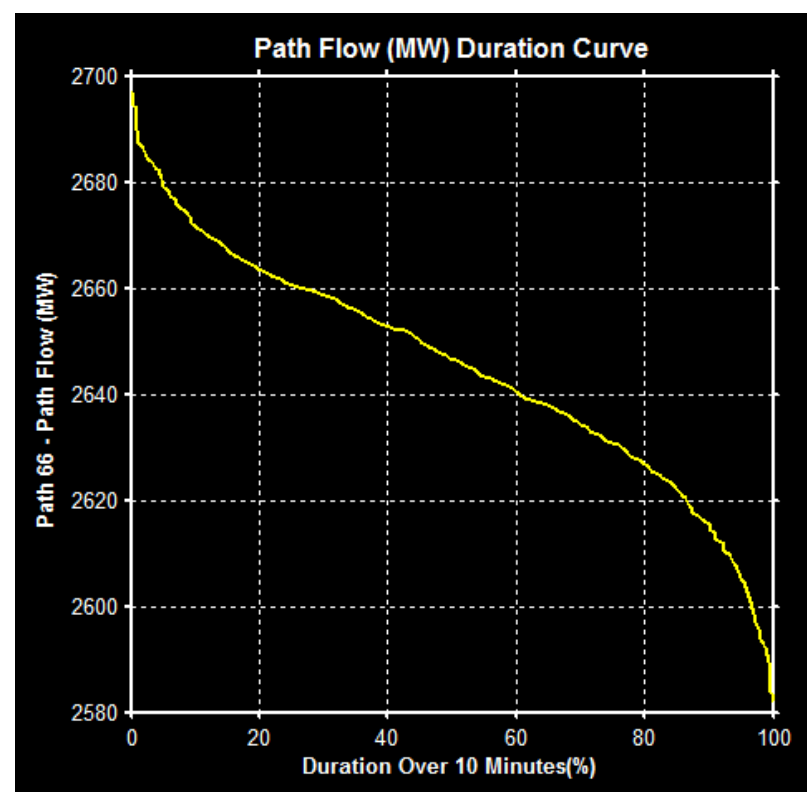

Figure 62: Path Flow (MW) Duration Curve (Panel 3) 


\section{PATH FLOW (MVAR) MONITORING}

\subsection{Overview}

The "Path Flow - MVAR" monitoring display tracks the reactive power flows across monitored lines or transmission paths (set of monitored lines for which limits have been defined) with respect to their predefined flow limits. Figure 63 outlines the graphics available within the "Path Flow - MVAR" monitoring display.

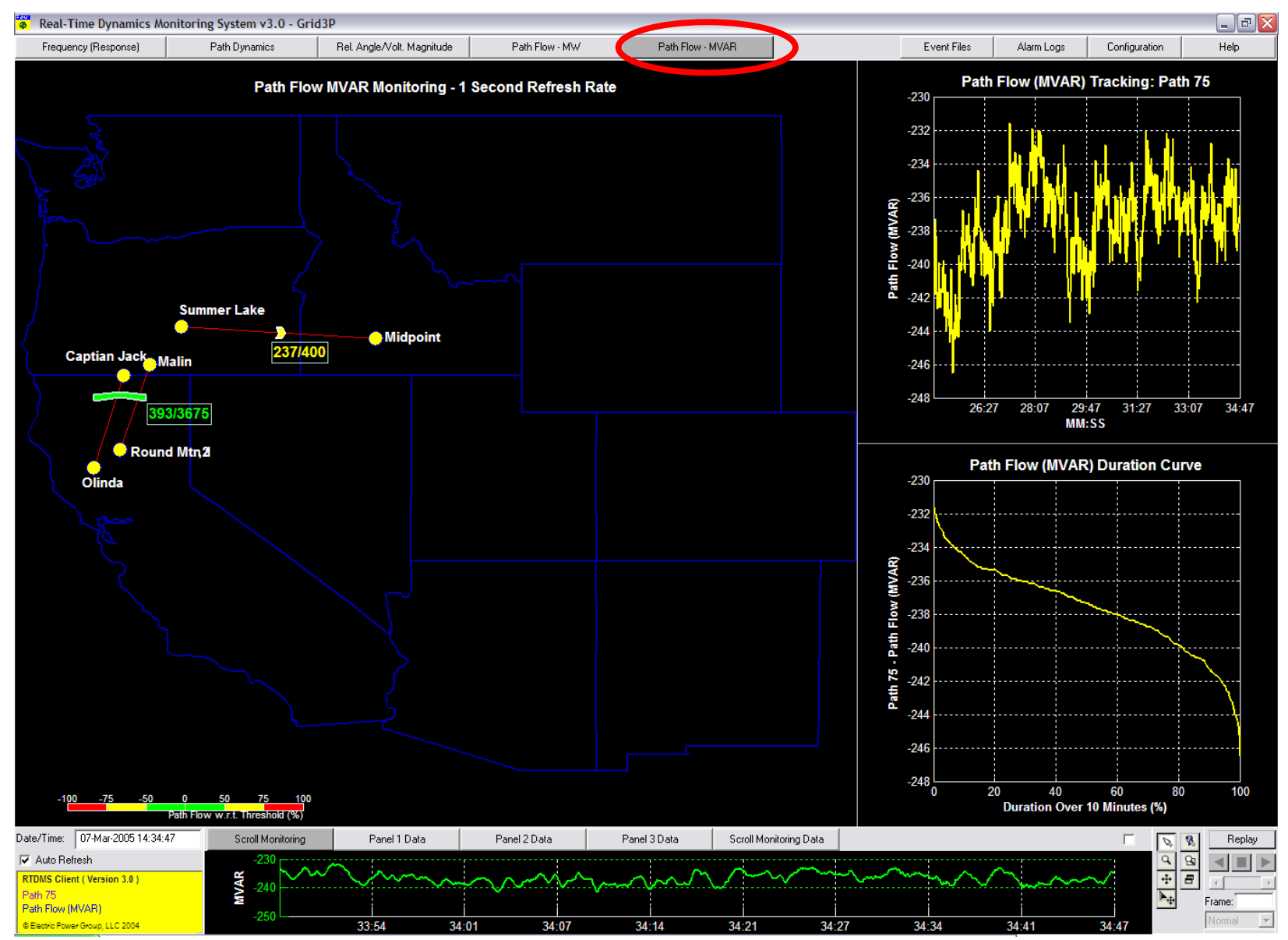

Figure 63: Four-Panel Path Flow - MVAR Monitoring

\subsection{Path Flow (MVAR) One-Panel Display}

Selecting the "Path Flow - MVAR" tab at the top of any of the displays will bring up just Panel 1, the left panel in Figure 63, where the various transmission paths are plotted on a geographical map in the "Path Flow - MVAR Monitoring" panel of the display. The reactive flows through that path are shown as an arrowhead across all the lines that define the path. While the color of the arrowhead represents the proximity (in percentage) of the MVAR flows to their alarming thresholds (bottom left on Figure 63), the arrowhead indicates the directionality of these flows.

Like the MW display, the actual MVAR flows and their corresponding limits are shown next to the arrowhead. The actual flows are listed to the left and the thresholds are listed to the right. The station names at both ends of the monitored line are also shown. To remove the station names from the display, right click within the panel and the pop-up menu will appear (Figure 64). Click on the "Station Name" and 
the names will be removed. The same pop-up menu can be used to add/remove the "Path Flow Value" and/or "Station Name".

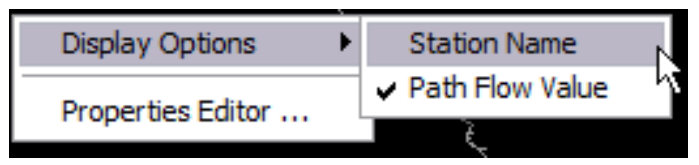

Figure 64: Path Flow - MVAR - Display Options Pop-Up

Figure 65 presents an enlarged view of the actual MVAR flow across the single selected path. As with the MW display, the path is selected with a left mouse click on the arrowhead in the geographic display for the path and will be indicated by a white border around the arrowhead. The y-axis to the left of the plot is the MVAR flow and the x-axis is time in minutes and seconds (MM:SS). This plot allows the user to track the MVAR flows across the selected path over the past minute at the highest sub-second resolution.

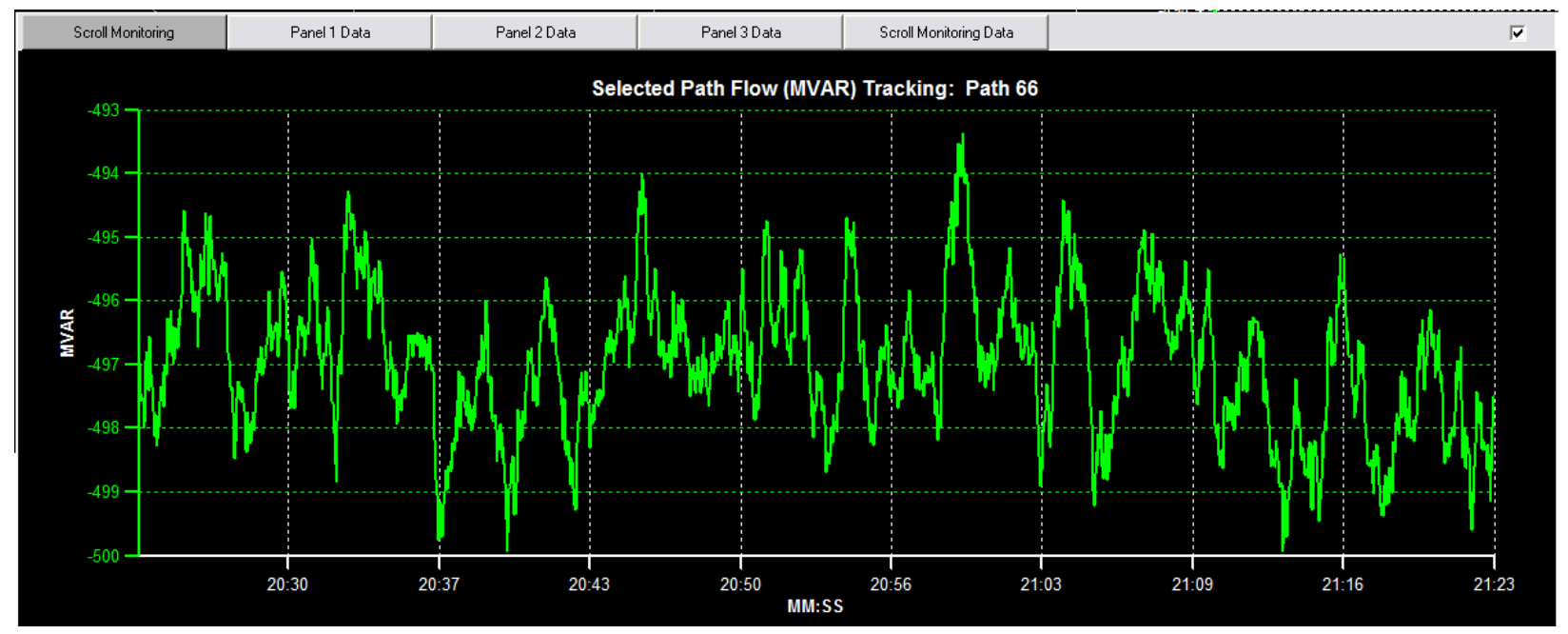

Figure 65: Selected Path Flow (MVAR) Tracking (Enlarged)

\subsection{Path Flow (MVAR) Four-Panel Display}

A left double click within panel 1 will bring up the corresponding four-panel display (Figure 63). To enlarge any of the panels, double click with the left mouse button within the desired panel.

Panel 2, the top right of Figure 63, is a view of the "Path Flow (MVAR) Tracking" plot where the time history of the MVAR flows on the selected path (Path 66) are being tracked. The panel provides a time history of the MVAR flows across the selected path at the second resolution (Figure 66). The x-axis of the plot is time in minutes and seconds (MM:SS) and the $y$-axis is the MW flow. The time duration of the plot may be altered via a pull-down menu made available using the right click within the panel. 


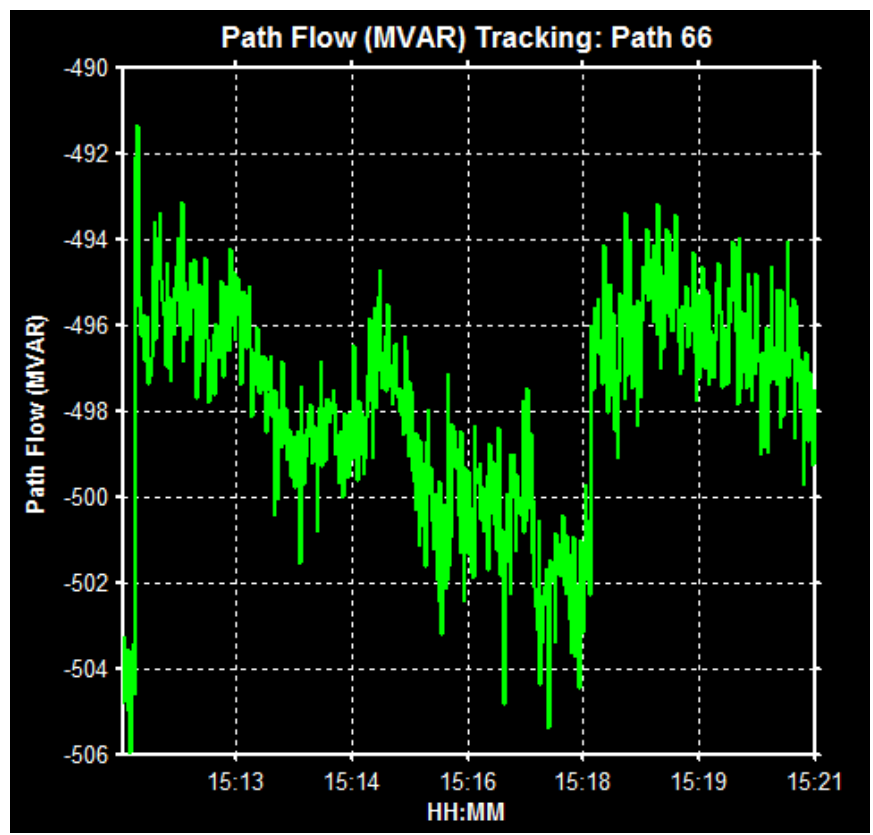

Figure 66: Path Flow (MVAR) Tracking (Panel 2)

Panel 3, the bottom right of Figure 63, is a view of the "Path Flow (MVAR) Duration Curve". Panel 3 displays a statistical distribution of the MVAR flows across the selected path (Figure 67). For each MVAR value along the $y$-axis, the corresponding $x$-axis along the curve shows the percentage time that the MW flows were above the value. In the figure, Path 66 had over 70 MVAR flowing from Cordova for 20\% of the time. The duration over which the distribution is computed is also selectable through a pull-down menu displayed via a right click within the panel.

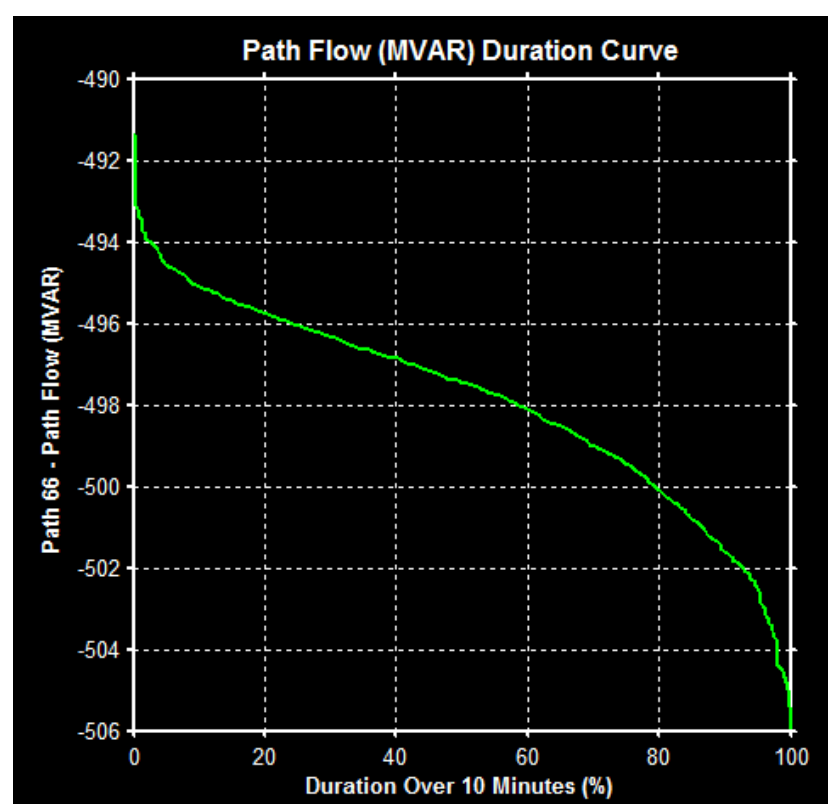

Figure 67: Path Flow (MVAR) Duration Curve (Panel 3) 


\section{ALARMS}

Alarming logic built into the RTDMS Server has the ability to analyze the streaming real-time data to detect and alarm on either threshold violations or significant changes (i.e. change within a second interval exceeding a specified threshold) on certain identified signals and metrics. In particular, the types of alarming logic within the RTDMS Server include:

- System frequency exceeding specified threshold limits

- Local frequency variations exceed specified thresholds limits

- Sudden changes in system and local frequencies

- Voltage magnitudes exceed minimum or maximum thresholds (i.e. low or high voltages)

- Sudden changes in voltage magnitudes

- Relative voltage angles exceeding specified thresholds limits w-r-t chosen reference

- Angle difference across specified paths exceed defined threshold limits

- MW/MVAR flows across specified paths exceed defined threshold limits

Note: When sudden changes in voltage magnitude or frequency signals are detected, the phasor data is stored into event files for offline post-disturbance analysis (Refer to Section 11).

The threshold parameters and signals/metrics used for alarming are specified in the 'PhasorArchiver.ini' file at the RTDMS Server (i.e. file resides within the 'RTDMS Server 3.0' directory). Having these alarming parameter definitions and the detection capability at the central RTDMS Server ensures consistency in the alarming process across all RTDMS Clients even when each RTDMS Client may differ in its particular configuration as defined through its "Configuration Utility" (Refer to Section 12).

(Note: The central alarming parameters may be changed by manually editing the 'PhasorArchiver.ini'. Alternately, the parameter settings within any RTDMS configuration file that can be created on the RTDMS Client using its "Configuration Utility", may be imported into the RTDMS Server to automatically update the parameters in the 'PhasorArchiver.ini' with the settings as defined within the imported configuration file. Please refer to the RTDMS Installation and Support Guide for further details on the RTDMS Server configurability).

Whenever a violation is detected in real time, it is automatically logged into text files at the RTDMS Server. For convenience, these alarm log files are named by the starting date and time of the alarm logging process into the file (e.g. '20050307_135259alarm.log' indicates that this file contains alarm logs beginning at 13:52:59 on the $7^{\text {th }}$ of March, 2005). All alarm logs at the Server are stored in the in an 'alarm' folder within the 'RTDMS Server 3.0' directory (when the RTDMS Server is installed in the default installation directory, these files reside in the 'C:IProgram FilesICERTS-EPG Grid 3PIRTDMS Server 3.0lalarml' directory).

Not only are the generated alarms permanently logged at the Server, these alarms are immediately made visible on all RTDMS Clients that are connected to the Server at the time. Whenever, a new violation is detected at the Server, the "Alarm Logs" tab located in the top right corner of all RTDMS Clients is highlighted in red to indicate the occurrence of a new alarm (Figure 68). This "Alarm Logs" tab remains red until the alarm is acknowledged by the user.

\section{Event Files}

Configuration

Help

Figure 68: “Alarm Logs” Tab Highlighted in Red to Alert RTDMS User of New Alarm

To acknowledge the alarm, click on this tab and the "Alarm Log" box pops-up (Figure 69). Though all the alarms are logged at the RTDMS Server as a text file, the user may be selective on the alarms he wishes to see at the RTDMS Client. This is done by selecting/deselecting the appropriate alarm types within the "Alarm Log" box. For each alarm listed in the box, the alarm log provides information on the signal associated with the violation, the start time of the violation, the end time of the violation (or most current time during a violation), the violation value and corresponding threshold, and the nature of the violation. 


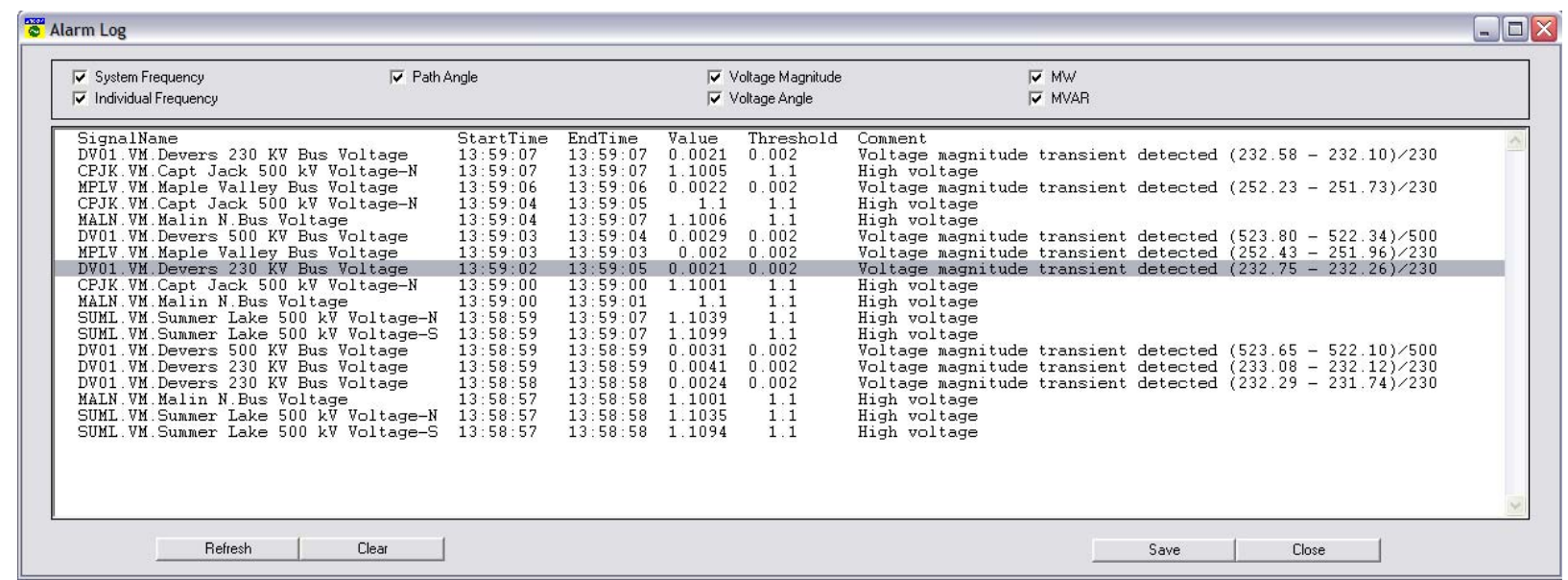

Figure 69: Alarm Log Pop-up Box

Example: Figure 70 shows an alarm logged into the "Alarm Log" box when a sudden changed in 'Devers 230kV Bus Voltage' voltage signal occurred on March $7^{\text {th }}, 2005$ at 13:59:02 that triggered the alarm. The voltage magnitude change within the first second was 0.0221 p.u. (i.e. $11 \mathrm{kV}$ at a $500 \mathrm{kV}$ base) which exceeded the alarming rate of change voltage threshold as specified in 'PhasorArchiver.ini' file at the RTDMS Server (i.e. 0.002 p.u. in this example), and this violation persisted though the next few seconds (i.e., 13:59:05).

SignalName StartTime EndTime Value Threshold Conment

DV01. WM. Devers $230 \mathrm{KV}$ Bus Voltage $13: 59: 02$ 13:59:05 $0.0021 \quad 0.002$ Voltage magnitude transient detected (232.75 - 232.26)/230

\section{Figure 70: Alarm Log Example}

If a new alarm occurs while viewing the alarm log, the "Alarm Logs" tab again switches color to red. Clicking on the "Refresh" button serves to acknowledge the alarm and updates the current list of alarms shown in the alarm log with the new alarms. Furthermore, the violations observed in the "Alarm Log" box can also be saved into a text file for future viewing by clicking on the "Save" button and specifying the save location. Alternately, the current list of alarms may be removed fom the box by clicking on the "Clear" button.

Note: Whether in Replay or Offline mode, the "Alarm Log" box continues to keep track of the most recent alarms detected at the RTDMS Server. 


\section{EVENT FILES AND OFFLINE ANALYSIS}

Whenever a rate of change alarm either in frequency or voltage magnitude measurements is encountered, all the streaming data and the subsequent alarm logs over a predefined time duration (default duration is 5 minutes of post-event data) are automatically stored at the RTDMS Server. In particular, for each captured event, a '.dat'. and a '.sum' file is created in the 'event' folder within the 'RTDMS Server 3.0' directory that stores the data and the alarm summary respectively (when the RTDMS Server is installed in the default installation directory, these files reside in the 'C:IProgram FilesICERTSEPG Grid 3PIRTDMS Server 3.0leventl' directory). Both the data and the alarm summary files are named by the event start times (e.g. '20050307_144034.dat' and '20050307_144034.sum' files contain the data and the alarm summary for an event that was captured on $7^{\text {th }}$ of March, 2005 at 14:40:34). As with alarming parameters, the event capture time duration and the rate of change thresholds can be modified by manually editing the 'PhasorArchiver.ini' file residing at the RTDMS Server. Please refer to the RTDMS Installation and Support Guide for further details on the RTDMS Server configurability.

Event files stored at the RTDMS Server are available for viewing by all RTDMS Clients that are connected to the RTDMS Server over a local area network. These event files can be loaded into RTDMS Clients for offline post-disturbance analysis. When a sudden change in frequency or voltage magnitude is detected, the corresponding alarm triggers the 'Alarm Logs' tab in the RTDMS Clients to turn red indicating the occurrence of a new alarm (Figure 68 ) and this alarm is automatically registered in the 'Alarm Log' box (Figure 69).

To switch out of real-time monitoring mode and to load a captured event file into the RTDMS Client, click on the "Event Files" tab located on the top right corner of the Client application.

\begin{tabular}{|c|c|c|c|}
\hline Event Files & Alarm Logs & Configuration & Help \\
\hline
\end{tabular}

Figure 71: "Event Files" Tab for Loading Disturbance Files

When the "Event Files" tab is clicked, the navigation window pops up allowing the user to find and select the event file of interest (Figure 72). By default, these files are stored in the 'event' folder within the 'RTDMS Server 3.0' directory. Select the file of interest as labeled by the start date/time of the event (select '.dat' file to load both the data and the alarm summary for the event or '.sum' file to only load the alarm summary for the event), and click on "Open" to being loading the file into the RTDMS Client for offline analysis. As an example, in Figure 72, the event captured on $7^{\text {th }}$ March, 2005 at 14:40:34 is being loaded into the application.

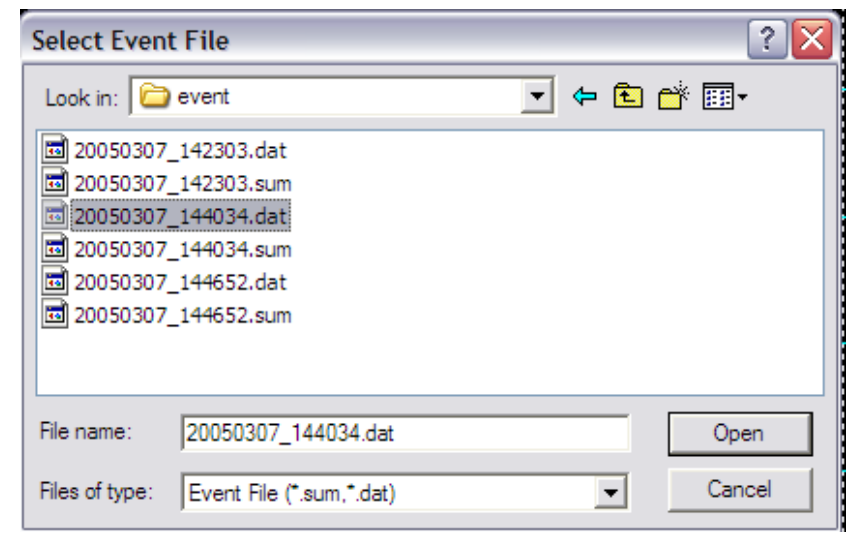

Figure 72: Navigation Window to Load Event File

All the functionality offered in the real-time monitoring mode (i.e., multi-panel displays, navigational tools, replay, data tables etc) is available to the user in this Offline mode. Hence, with the event file loaded into the RTDMS Client, the user can utilize these capabilities for a post-disturbance analysis of the event file. 
Example: An event that was captured on $7^{\text {th }}$ March 2005 at 14:40:34 has been selected (Figure 72) and loaded into the RTDMS visualization Client (Figure 73). When the application completes loading the data, it automatically switches into the Offline mode which is indicated by a switch in the Title box's background color from yellow to blue (Figure 17). Additionally, the alarm summary pop-up box also appears listing all the violations during the course of the event. In the example, one of the violations listed in the alarm summary for the event file is a sudden frequency change of $41.8 \mathrm{mHz}$ within a second as measured by the 'Slatt' PMU on $7^{\text {th }}$ March 2005 at 14:41:11. To further investigate this event alarm, the "Frequency (Response)" monitoring four-panel display was selected and the replay capability was used to rewind to the time in question within the event file. The time duration for the trend plot in Panel 1 was set to 1 minute to allow viewing of this data at the sub-second resolution. Additionally, the 'Slatt' frequency was selected within the "Local Frequency Coherency Comparison" mesh plot and automatically displayed in Panel 4. A look at the different panels of this display (Figure 73) confirms sudden transients in the 'Slat' frequency between 14:41:11 and 14:41:13 resulting in it having the biggest deviation from the interconnection frequency during the time in question.

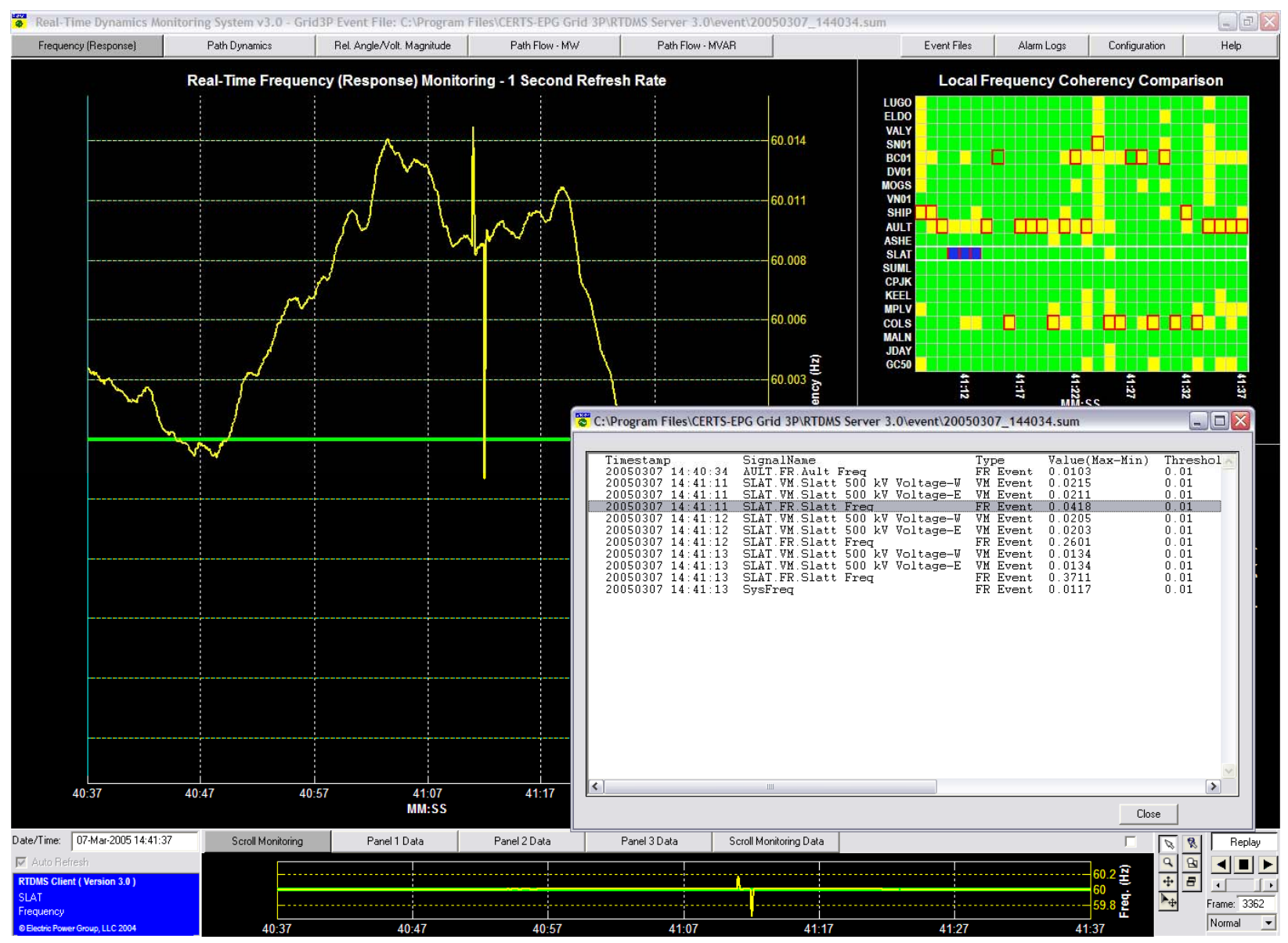

Figure 73: RTDMS in Offline Mode for Post-Disturbance Analysis

Once the offline analysis is complete, the user can switch back to "Real-Time" mode by clicking on the "Replay" button.

(Note: It is recommended that the user wait the predefined recording duration after the event has been detected before loading the event file to allow for the event recording completion. Attempting to load an event file that hasn't completed recording will only load partial data into the application). 


\section{CONFIGURABILITY}

The RTDMS Server communicates both the phasor data and static information to all the remote monitoring RTDMS Clients (Refer to Appendix 1). If there is a change in the static information (reflecting changes in the system configuration such as the addition or removal of PMUs), it is detected by each of the remote Client applications, and the configuration utility window automatically pops up with the latest signal information. The user can utilize this utility to update the visualization displays. Alternately, the user may manually launch the utility by clicking on the "Configuration" tab located on the top right corner of the display and reconfigure the display visuals. All the configuration settings are saved in a single file for easy portability. These configuration files have a '.mat' extension (e.g. 'phasor_conf_default.mat') and can be loaded in any RTDMS Client application. These settings include the newly defined set of monitoring preferences such as the monitoring points, paths, alarming thresholds as well as the visualization properties such as color schemes, font types and sizes, labels etc. The configuration details are described in this and the following section (Section 13).

(Note: RTDMS configuration files can not only be shared across different RTDMS Clients, they can also be imported into the RTDMS Server to specify alarming signals/metrics and threshold parameters).

\subsection{Client Configuration Utility}

The configuration utility graphic user interface (GUI) is shown in Figure 74. Under the "PMU Selection" section of the GUI, the most current set of PMUs which are available to the system fall either under the "Unselected" list or the "Selected" list (PMUs identified by a four letter PMU code - Refer to Appendix 1). The "Selected" list is the subset of PMUs whose monitored signals are to be used within the visualization. The "Unselected" list is the remaining set of PMUs.

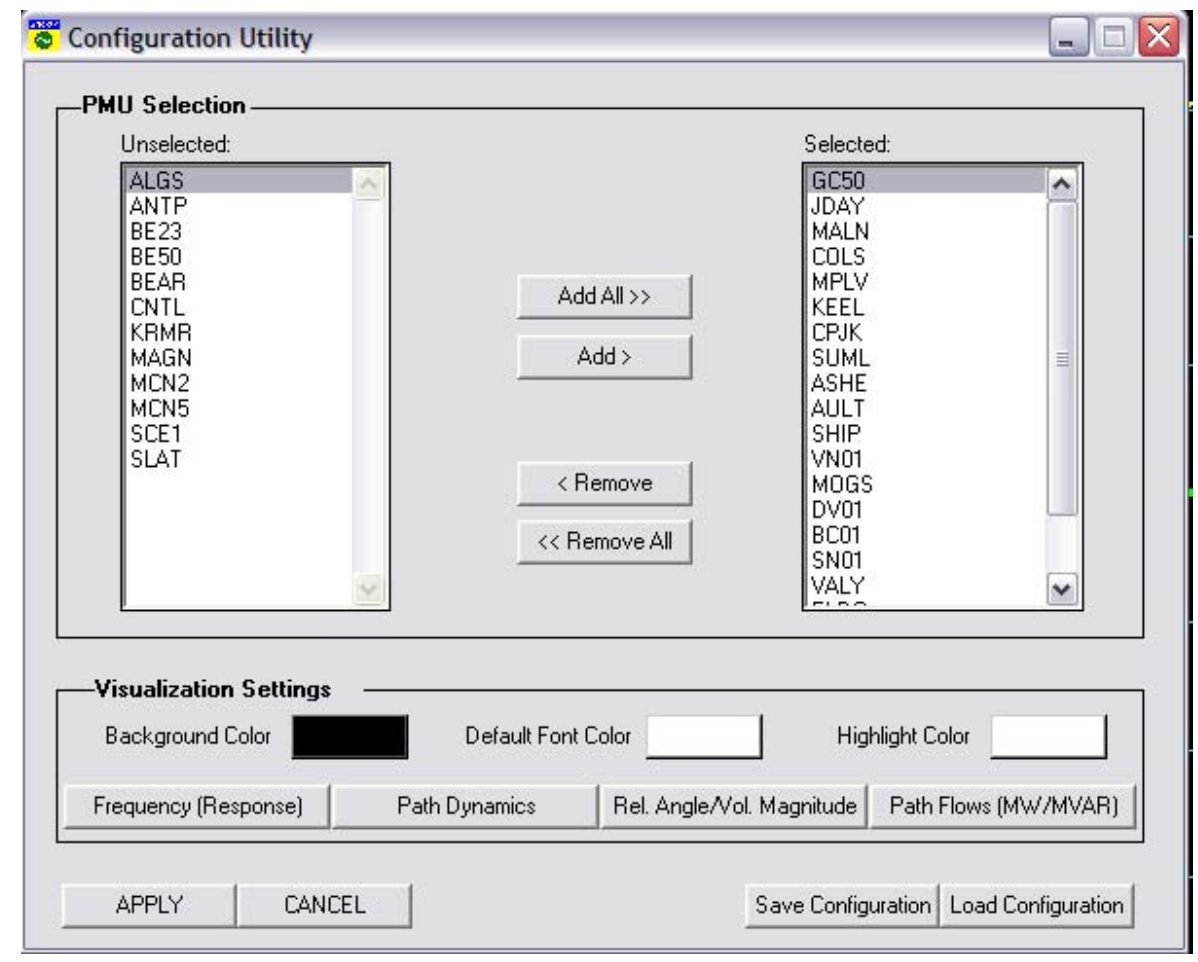

Figure 74: Configuration Utility Master Graphic User Interface

When a new PMU is installed and connected to the system and the corresponding static information is updated at the Server, the change is detected by all the monitoring Client applications and it is automatically added to the "Unselected" list. The user may now choose to include the information being 
monitored by the new PMU by selecting it under the "Unselected" list and clicking on the "Add" button. The new PMU is moved from the "Unselected" list into the "Selected" list.

The user can also remove PMU(s) by highlighting them in the "Selected" list and clicking on the "Remove" button. If the monitored signals from a PMU that are being removed are used in path definitions (either angle difference paths or MW/MVAR paths), then a warning box pops up to alert the user (Figure 75).

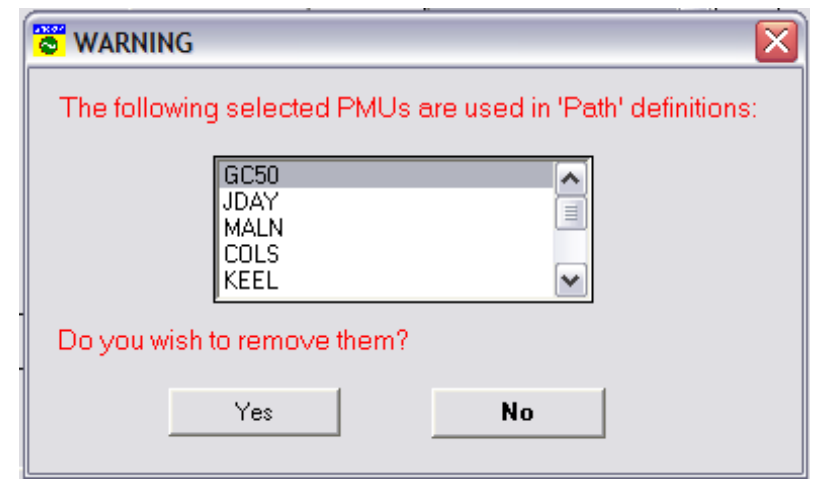

Figure 75: Warning Pop-Up during PMU Removal

If the user still wishes to remove the $\mathrm{PMU}(\mathrm{s})$, then the user can acknowledge the warning by clicking "Yes", in which case, the PMU(s) are removed from the "Selected" list and moved to the "Unselected" list. Any path definitions that utilized signals monitored by those de-selected PMUs are removed from the displays.

The "Visualization Settings" within the configuration GUI let the user configure the application displays based on the "PMU Selection" (shown under the "Selected" list) through the procedure outlined above. The user can change the background color of all the displays. To do this, click on the rectangular color box and the color palette is launched (Figure 76). The user can either choose from the available color selection by clicking on the color or define a customized color using the R-G-B sliders. The selected/defined color is shown in the rectangular box. Once a background color is selected, click on "OK" button. The User can also change the text and graphic highlight colors in a similar fashion.

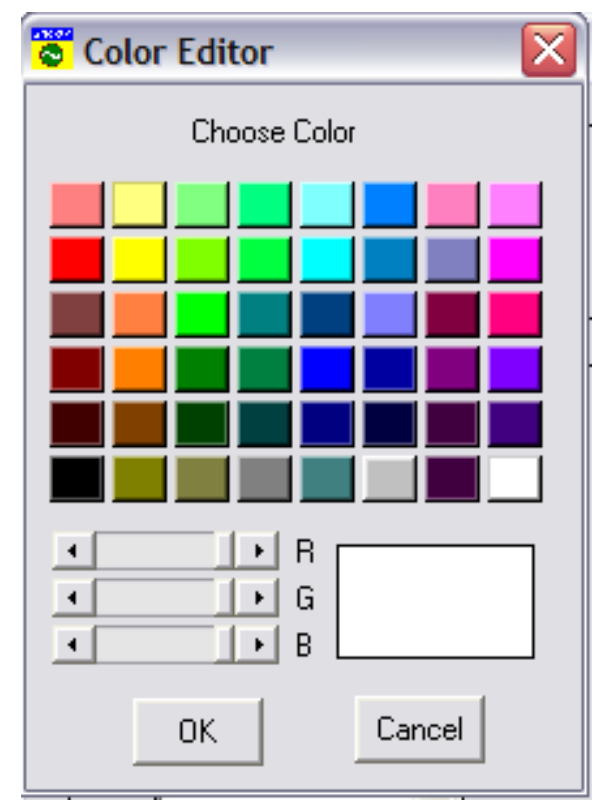

Figure 76: Color Palette Pop-Up 
Within the "Visualization Settings" are four (4) buttons corresponding to each of the monitoring functions and displays: "Frequency (Response)", "Path Dynamics", "Rel. Angle / Vol. Magnitude", Path Flows (MW/MVAR). Clicking on a button launches a configuration GUI for the corresponding display that lets the user configure the display. Once these configuration GUIs have been used to modify the display parameters, click on the "APPLY" button for the changes to take effect. The user can also save these latest set of configuration settings as a new configuration file. To do this click on "Save Configuration" and the user will be prompted to assign a name to the file. The user can later load this file or a previously saved configuration file by clicking on the "Load Configuration" and selecting it from the appropriate file list shown. Alternately, the user may click on the "CANCEL" button to revert to the earlier configuration (Note: The default configuration setting file is labeled as 'phasor_conf_default.mat').

\subsubsection{Frequency (Response) Configuration}

Click on the "Frequency (Response)" button within the Configuration Utility and the Frequency (Response) configuration GUI will pop-up (Figure 77). The user can use this GUI to update the frequency and the frequency response monitoring settings. For frequency monitoring, the user can set the scheduled frequency (shown as a solid horizontal green line in the frequency monitoring displays) and associated soft/hard alarming limits (shown as a solid yellow line in the frequency monitoring displays). While the hard limits are fixed upper and lower frequency limits with respect to the scheduled frequency, the soft limits are variable statistical limits determined by the set number of standard deviations from the mean as computed from the frequency data available within the frequency monitoring display.

For frequency response monitoring, the user can also change the "Natural Frequency Response Coefficient" that maps Interconnection frequency changes to generation-load imbalances within the system, by entering the new coefficient (in $\mathrm{MW} / 0.1 \mathrm{~Hz}$ ) under the "Imbalance Coefficient" field, the time duration (in seconds) over which the frequency change is calculated under the "Response Interval" field, as well as the alarming limits (shown as a solid cyan line in the frequency monitoring displays). Click on the "OK" button once the two fields have been updated (and the "APPLY" button within the Configuration Utility in Figure 74), and the changes are registered. The generation-load imbalance is calculated by multiplying the Interconnection frequency change over the specified time duration multiplied by the imbalance coefficient.

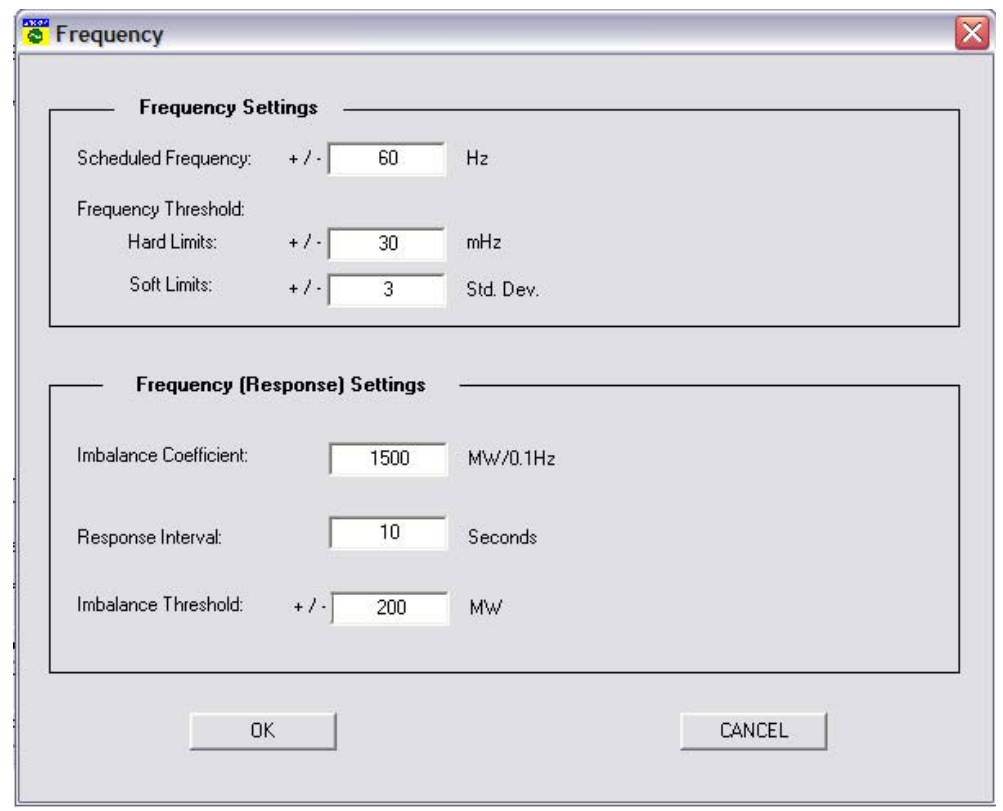

Figure 77: Frequency (Response) Configuration Graphic User Interface 


\subsubsection{Path Dynamics Configuration}

Click on the "Path Dynamics" button within the Configuration Utility and the Path Dynamics configuration GUI will pop-up (Figure 78). The user can use this GUI to define a new path and corresponding alarming thresholds, remove a existing path, or modify the path name or thresholds on an existing path.

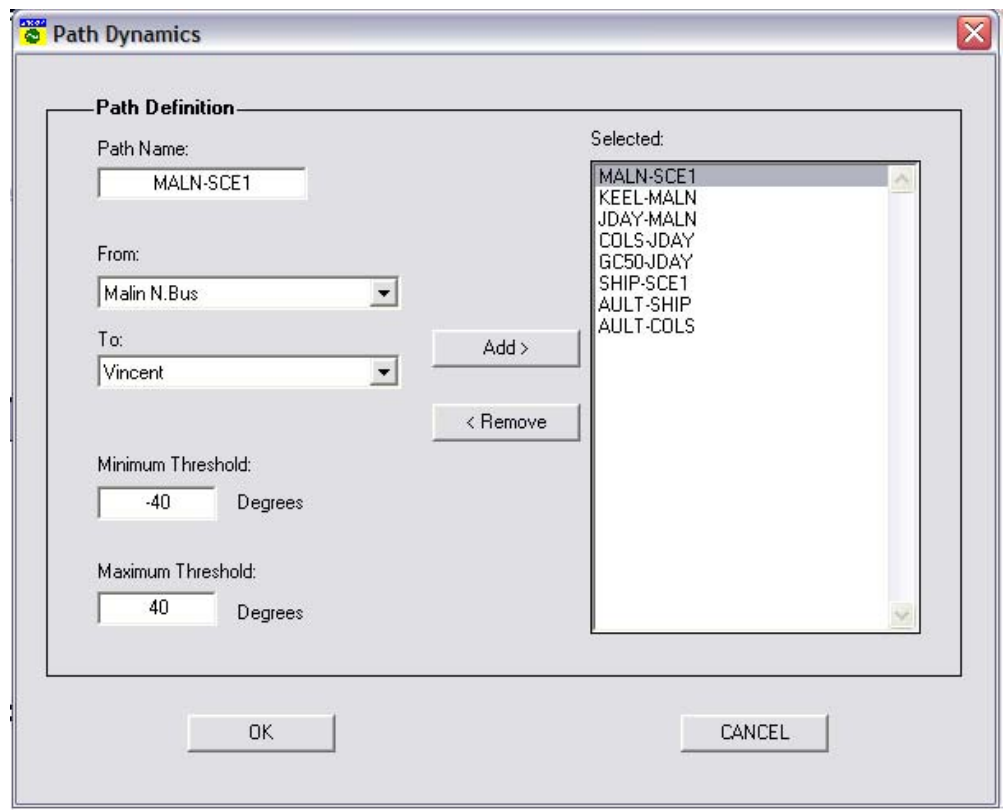

Figure 78: Path Dynamics Configuration Graphic User Interface

To add a new path to the "Path Dynamics" display, select the "from" voltage and the "to" voltage from those available in the pull-down list (these set of voltages are being monitored by the selected subset of PMUs defined in Section 12.1). The new path is then defined as the voltage angle difference between the "from" voltage angle and the "to" voltage angle and will be shown within the "Path Dynamics" display as an arrow between the two locations (the arrow in the display points from the larger voltage angle to the smaller voltage angle and therefore reflects the directionality of power flow from the source to the sink). The "Path Name" field is automatically filled according to the PMUs associated with the "from" and "to" voltages but can be altered by the user. The minimum and maximum alarming thresholds for the new path are entered under the "Minimum Threshold" and "Maximum Threshold" fields respectively. Once all the fields have been specified, click on the "Add" button and the new path is added to the set of existing paths shown under the "Selected" list.

To remove an existing path from the "Path Dynamics" display, choose the desired path from the "Selected" list and click on the "Remove" button.

To update the fields of an existing path within the "Path Dynamics" display, choose the desired path from the "Selected" list, modify the "Path Name", "Minimum Threshold" and "Maximum Threshold" fields, and click on the "Add" button.

Once the desired changes have been made, click on the "OK" button (and the "APPLY" button within the Configuration Utility in Figure 74) for the changes to take effect ${ }^{8}$.

\footnotetext{
${ }^{8}$ Once the a new path is added, use the "Pick to Move Text" icon under the navigation tools to reposition the associated text information within the display and avoid overlap.
} 


\subsubsection{Relative Angle/Voltage Magnitude Configuration}

Click on the "Rel. Angle / Volt. Magnitude" button within the Configuration Utility and the Relative Angle I Voltage Magnitude configuration GUI will pop-up (Figure 79). The user can use this GUI to modify the reference PMU, to adjust a PMU's coordinates within the geographic displays, and to set a voltage signal from $\mathrm{PMU}$ as the default monitoring voltage for that PMU.

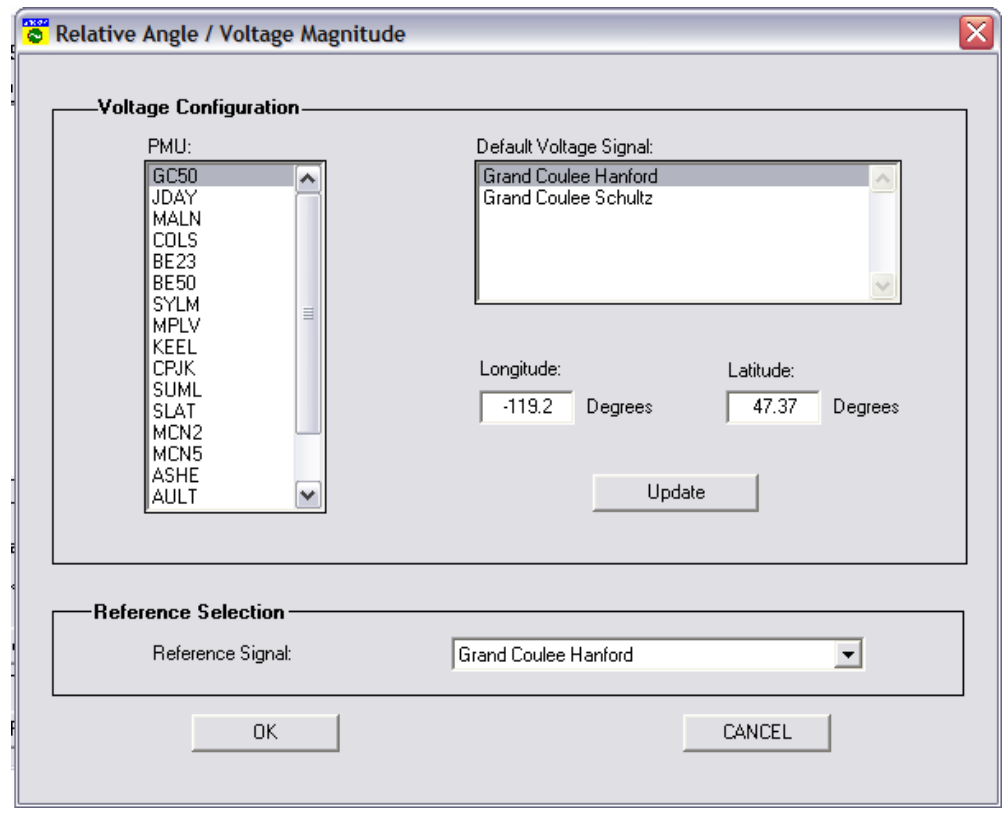

Figure 79: Rel. Angle I Volt. Magnitude Configuration Graphic User Interface

Some PMUs are configured to measure multiple voltages available at the PMU location (such as the high or low sides of transformers and series elements). Under such situations, the configuration utility allows the user to set one of these voltage signals as the default voltage to be shown within the "Rel. Angle I Volt. Magnitude" display. To do this, select a PMU from the "PMU" list and all its monitored voltages are shown in the "Default Voltage Signal" list. Select one of the available voltage signals from the list to set as the default voltage signal. Additionally, update the coordinates of the PMU in the "Longitude" and "Latitude" fields if desired. Click on the "Update" button to register the changes.

To change the reference used to compute the relative angles for the display, select the new "Reference Signal" from the list of available signals in the pull-down list.

Once the desired changes have been made, click on the "OK" button (and the "APPLY" button within the Configuration Utility in Figure 74) for the changes to take effect.

\subsubsection{Path Flow (MW/MVAR) Configuration}

Click on the "Path Flow (MW/MVAR)" button within the Configuration Utility and the MW/MVAR Path configuration GUI will pop-up (Figure 80). The user can use this GUI to define new MW/MVAR paths or flowgates (a single line or set of lines across which the current flows are being measured and the power flows can therefore be computed), remove existing MW/MVAR paths, or modify the thresholds on existing MW/MVAR paths. 


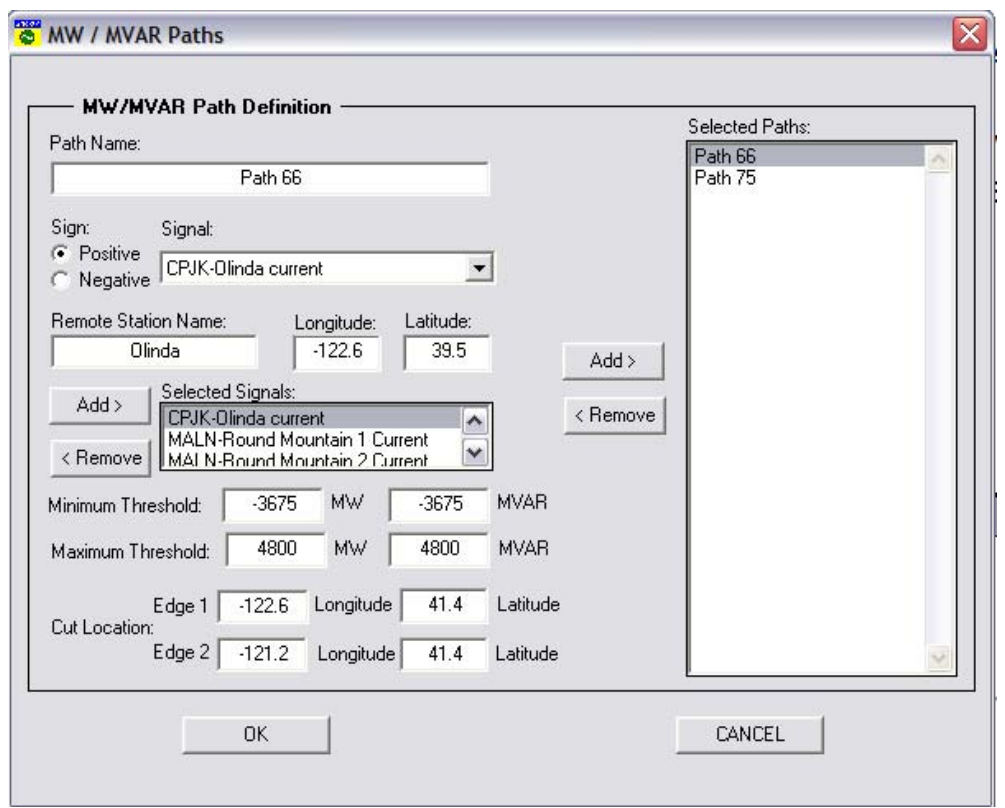

Figure 80: MW I MVAR Path Configuration Graphic User Interface

To add a new MW/MVAR path to the "Path Flow - MW (MVAR)" displays, enter its name under the "Path Name" field. Select the first "Signal" to be included in the path or flowgate definition from those available in the pull-down list - these are lines across which the MW and MVAR flows have been computed. Choose the "Sign" to be associated with the line flow to define the directionality of the flow (i.e., is the monitored power away from the PMU (positive sign) or towards the PMU (negative sign)). While the PMU information (such as its station name, latitude and longitude) is available to the Client applications and within the configuration utility from the host site, this information may not be available for the remote station (i.e., the station at the other end of the selected line). If this remote station information is available at the host site, the "Remote Station Name", "Longitude" and "Latitude" fields are automatically populated, else the user can manually enter them. Once the information has been entered, click on the "Add" button located to the left of the GUI, and the signal is added to the "Selected Signals" list. Repeat the above process to add any additional line flows. The net flows through the set of signals within the "Selected Signals" list will define the path. Next, specify the net "Minimum Threshold" and "Maximum Threshold" fields for the real and reactive flows across the defined path. Finally, the "Edge1" and "Edge2" fields define the corners of the arrowhead associated with this new path and will be shown within the display. These fields are automatically filled such that the arrowhead is appropriately centered and oriented across the $1^{\text {st }}$ line in the "Selected Signals" list. The user may change these coordinates to rescale the arrowhead. Once the information is entered, click on the "Add" button located in the center of the GUI and the new MW/MVAR path is added to the set of existing paths shown under the "Selected Paths" list.

To remove an existing path from the "Path Flow - MW (MVAR)" displays, choose the desired MW/MVAR path from the "Selected Paths" list and click on the "Remove" button located in the center of the GUI.

To update the fields of an existing path within the "Path Flow - MW (MVAR)" displays, choose the desired MW/MVAR path from the "Selected Paths" list, modify the appropriate fields, and click on the "Add" button located in the center of the GUI.

Once the desired changes have been made, click on the "OK" button (and the "APPLY" button within the Configuration Utility in Figure 74) for the changes to take effect" ${ }^{9}$.

\footnotetext{
${ }^{9}$ Use the "Pick to Move Text" icon under navigation tools to reposition associated text and avoid clutter.
} 


\section{PROPERTY EDITOR}

The visual properties of each of the panels within all five displays (5) can be customized by the user through a property editor GUI for that particular panel. A sample GUI is shown in Figure 81. Some of the capabilities of the property editor GUIs are:

- change the maps in geographic displays

- modify font settings of titles, axis labels, legend labels, identifiers, text values etc

- change properties of graphics such as the dials and arrows

- adjust legends - their thresholds and associated colors

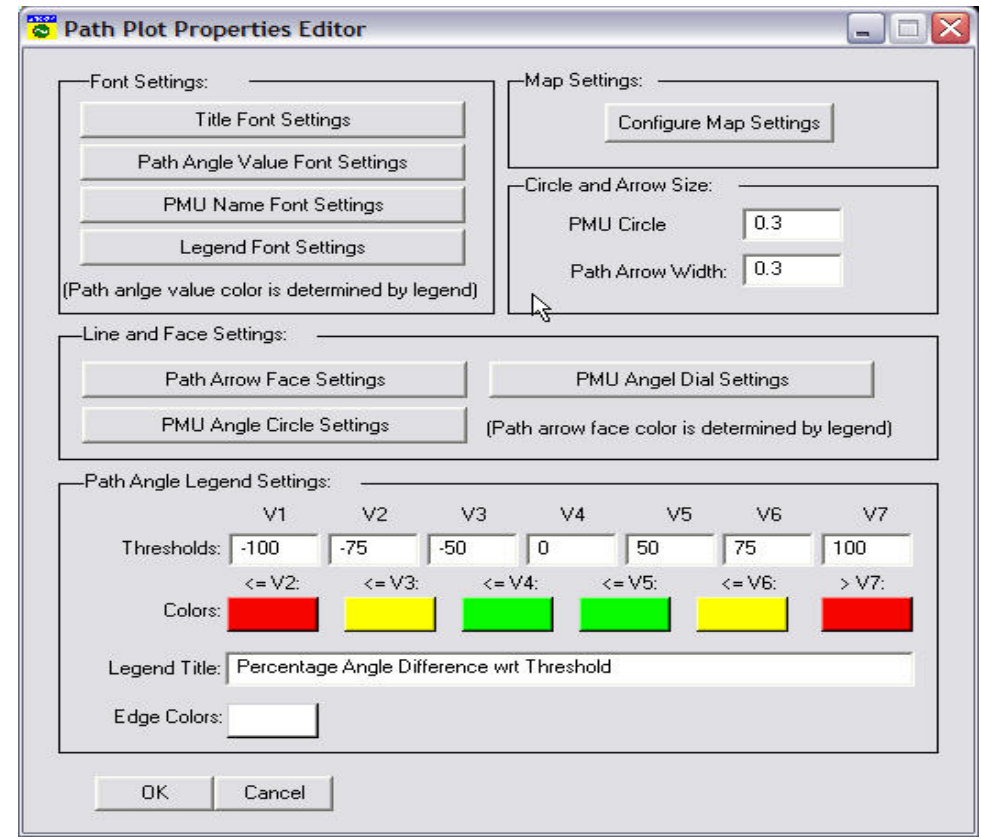

Figure 81: Property Editor Sample Graphic User Interface (Path Dynamics - Panel 1)

To open the property editor for a panel, right click within the panel and the pop-up menu shown in Figure 82 will appear. Select the "Property Editor..." option to launch the editor in for the particular panel.

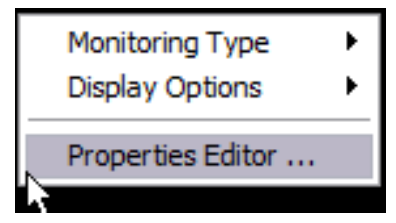

Figure 82: Property Editor Pop-Up

Note: The modifications made through the property editor for a panel are limited to the particular panel and do not affect the other panels. 


\section{HELP TAB}

This tab, located on the top right corner, brings the RTDMS help directory listing information about the application categorized by major topics and sub-topics along with search capability based in key words (Figure 83).

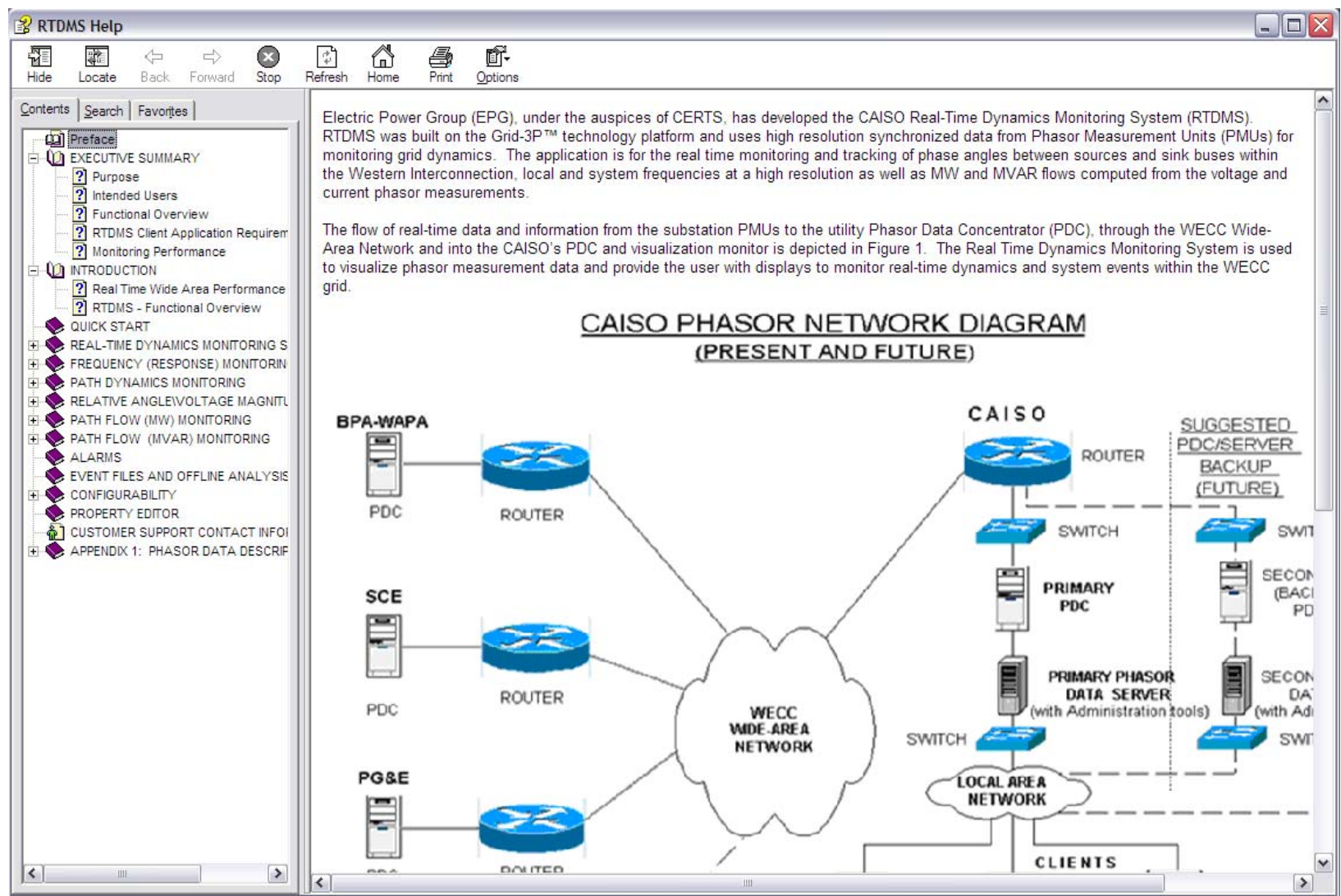

Figure 83: RTDMS Help Directory 


\section{CUSTOMER SUPPORT CONTACT INFORMATION}

Electric Power Group support information:

Manu Parashar

201 South Lake Avenue, Suite 400

Pasadena, California 91101

E-mail parashar@electricpowergroup.com

Tel. 626-685-2015 Ext. 130
Simon Mo

201 South Lake Avenue, Suite 400

Pasadena, California 91101

E-mail mo@electricpowergroup.com

Tel. 626-685-2015 Ext. 123 


\section{APPENDIX 1: PHASOR DATA DESCRIPTION}

Two types of data constitute the real-time phasor stream: static information about the PMU and the signal descriptions associated with each of its channels, and dynamic phasor values with timestamp information. This section outlines the data structure used within the RTDMS application.

\section{Static Data}

Version Information - Stores version and update information for static data.

Version: major and minor version information.

Update Date: the date the static information is updated.

\section{Example}

Version: 1.0

Update Date: 22-Aug-2004 16:07:44

PMU Description - Stores the static information of every PMU in the system to be monitored.

PMU ID: an integer to identify the PMU, unique.

PMU Code: short name, 4 characters string.

PMU Name: long name.

Longitude: location of PMU.

Latitude: location of PMU.

Vendor: Vendor of the PMU.

\section{Example}

PMU ID: 2

PMU Code: JDAY

PMU Name: John Day

Longitude: -119.2

Latitude: 47.37

Vendor: Macrodyne

Signal Description - Stores the static information signals provided by PMUs.

Signal ID: an integer to identify the signal, unique.

Signal Name: the description of the signal.

Signal Type: the code to describe the signal type such as

VA: voltage angle

VM: voltage magnitude

IA: current angle

IM: current magnitude

FR: frequency

PP: MW

PQ: MVAR

\#\#: Others

PMU ID: indicate the PMU with which the signal associates.

Base kV: an integer to present the voltage level of the signal (-1 if not applicable).

Item Name: the corresponding tag name in OPC/PI Server for this signal.

Remote Longitude: location of line current endpoint (optional, only applies to current type signal).

Remote Latitude: location of line current endpoint (optional, only applies to current type signal).

Remote Name: remote station name at the other end of this signal.

Example

Signal ID: 5

Signal Name: John Day-Big Eddy Current

Signal Type: IA 
PMU ID: 2

Base kV: 500

Item Name: JDAY.IA.johnday- Big Eddy Current

Remote Longitude:

Remote Latitude:

Remote Name:

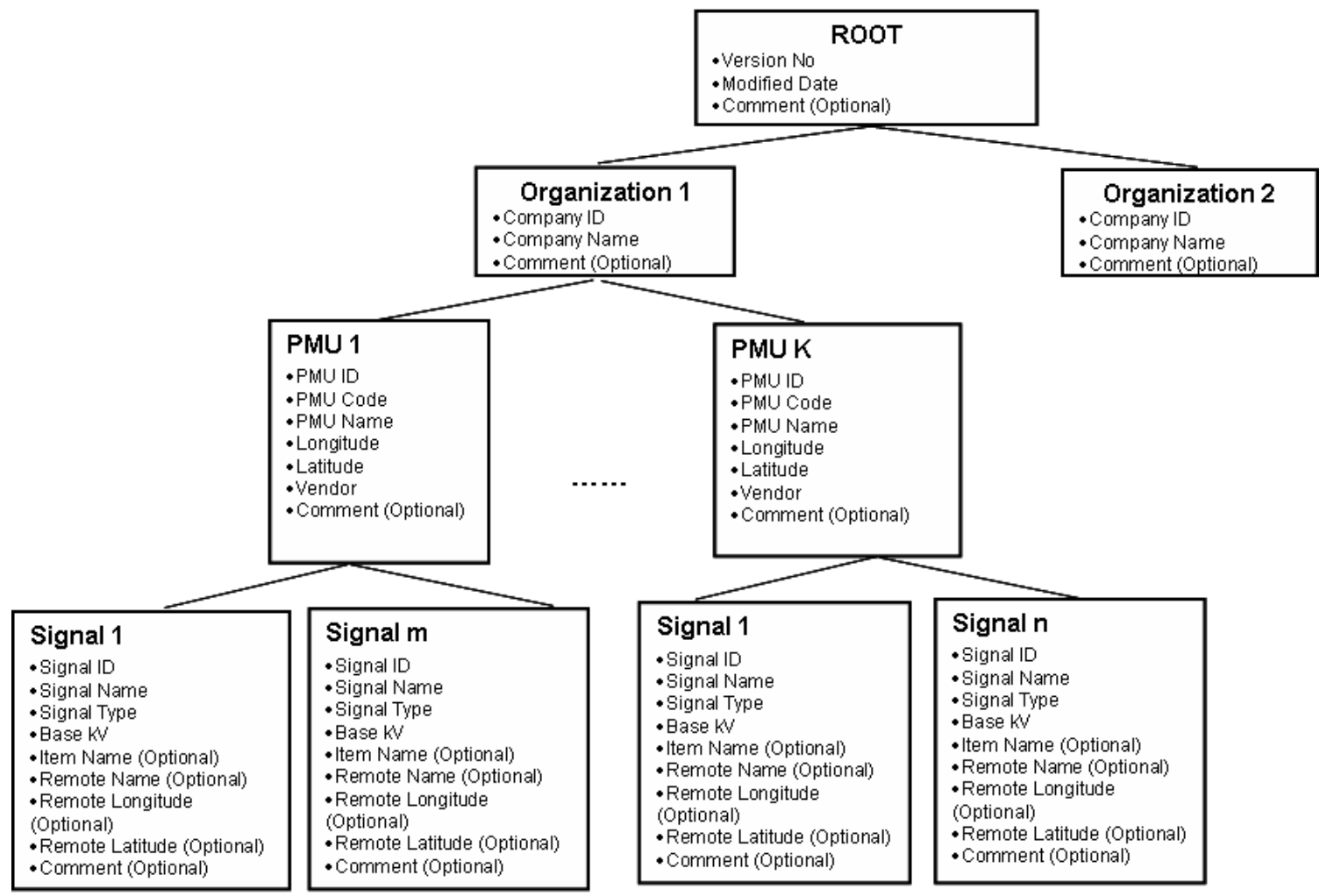

Figure 84: Static Information Hierarchical Structure

\section{Dynamic Data}

Time-stamped dynamic data of all signals are available for access. Dynamic data includes real-time data and historical data. Dynamic data can be accessed according to the provided signal ids and the timestamp. This data in the application is maintained as a two-dimensional array where the rows correspond to the signals and the signals correspond to different time instances.

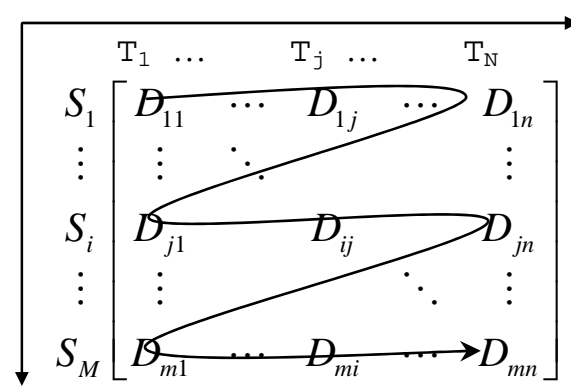

Signal 


\begin{tabular}{|l|c|}
\hline Consortium for & Real-Time \\
Electric & Dynamics \\
Reliability & Monitoring \\
Technology & System \\
Solutions & RTDMS $^{\mathrm{TM}}$ ) \\
\hline
\end{tabular}

\section{RTDMS \\ CA I SO TRAI NI NG SESSI ON \\ J anuary 31, 2006}

Manu Parashar \& Jim Dyer

Electric Power Group (EPG) 


\section{Agenda}

$>$ Phasor Technology - Overview

$>$ The Importance of Using Synchronized Data

$>$ Review of Some WECC Events

> CA ISO Real-Time Dynamics Monitoring System

$>$ Project Objectives

$>$ System Architecture

$>$ What the System Operator Will See

$>$ RTDMS Visualization

$>$ Architecture

$>$ Navigation Within RTDMS

$>$ Displays

$>$ Client Support

$>$ RTDMS Application Demo 


\section{Phasor Technology Overview}

CERTS 


\section{What Causes Power to Flow on the Grid}

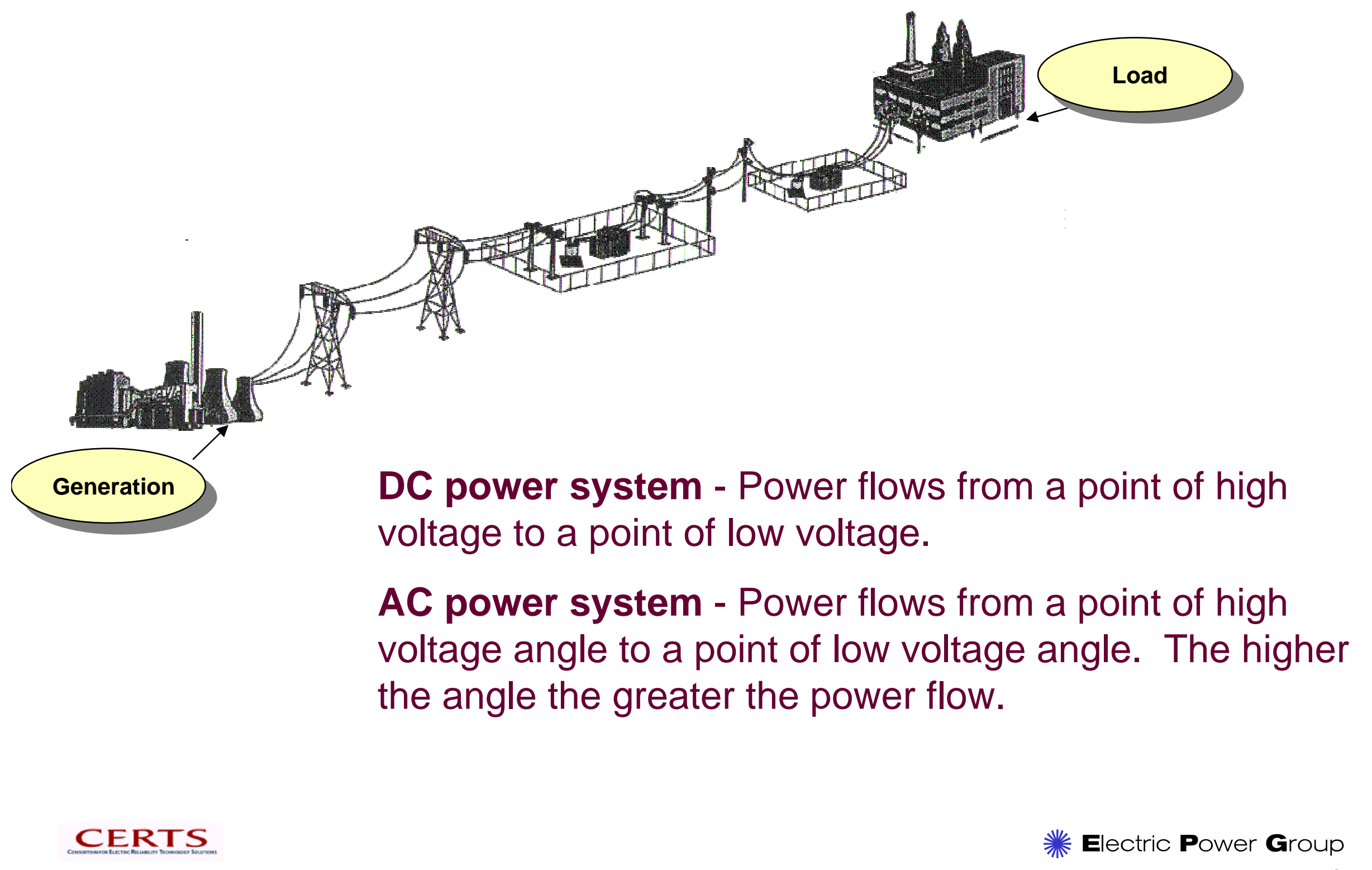




\section{What Causes Voltage Angle to Increase}

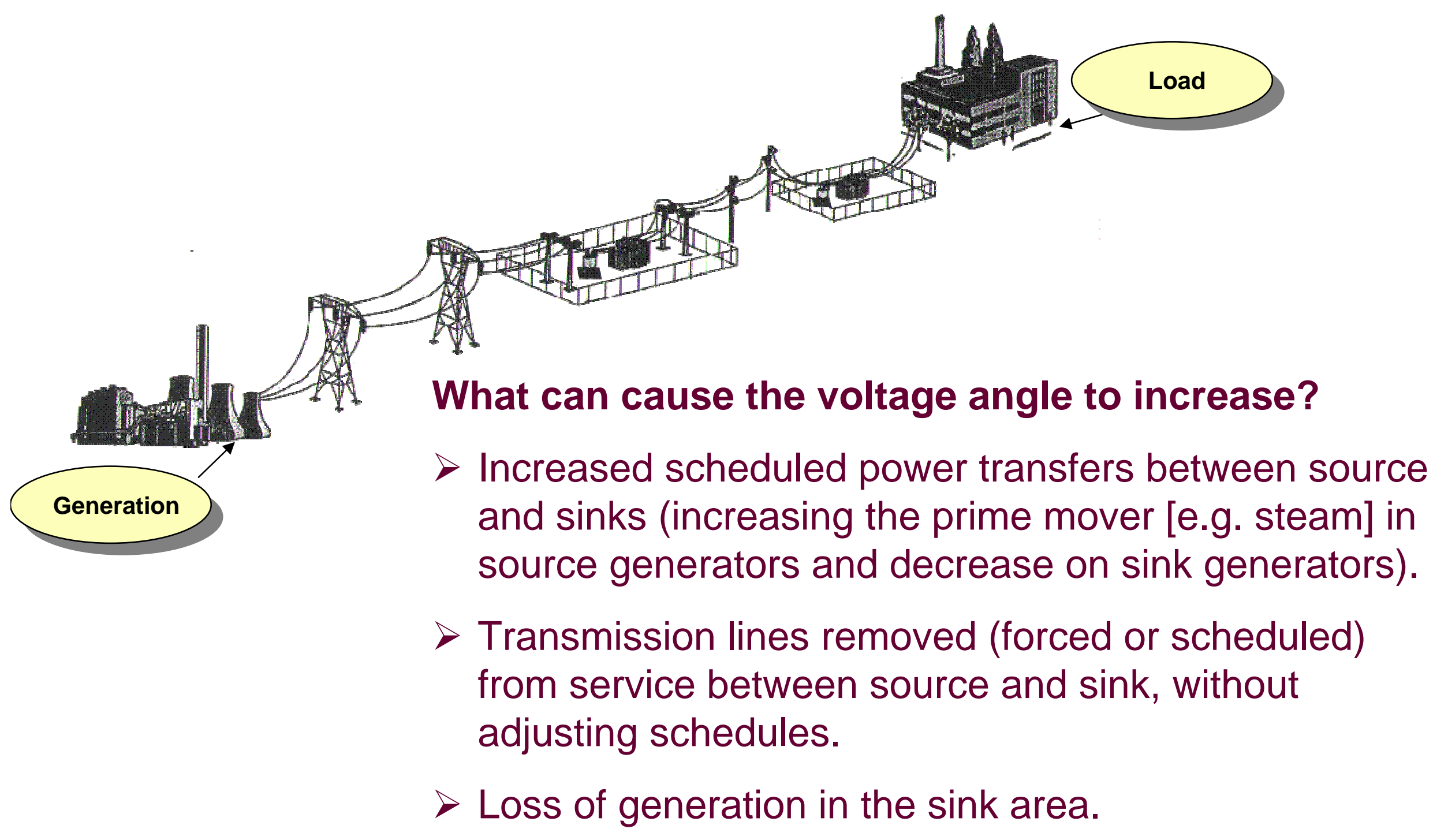




\section{Eastern Interconnection - Angle Separation on 8-14-03}

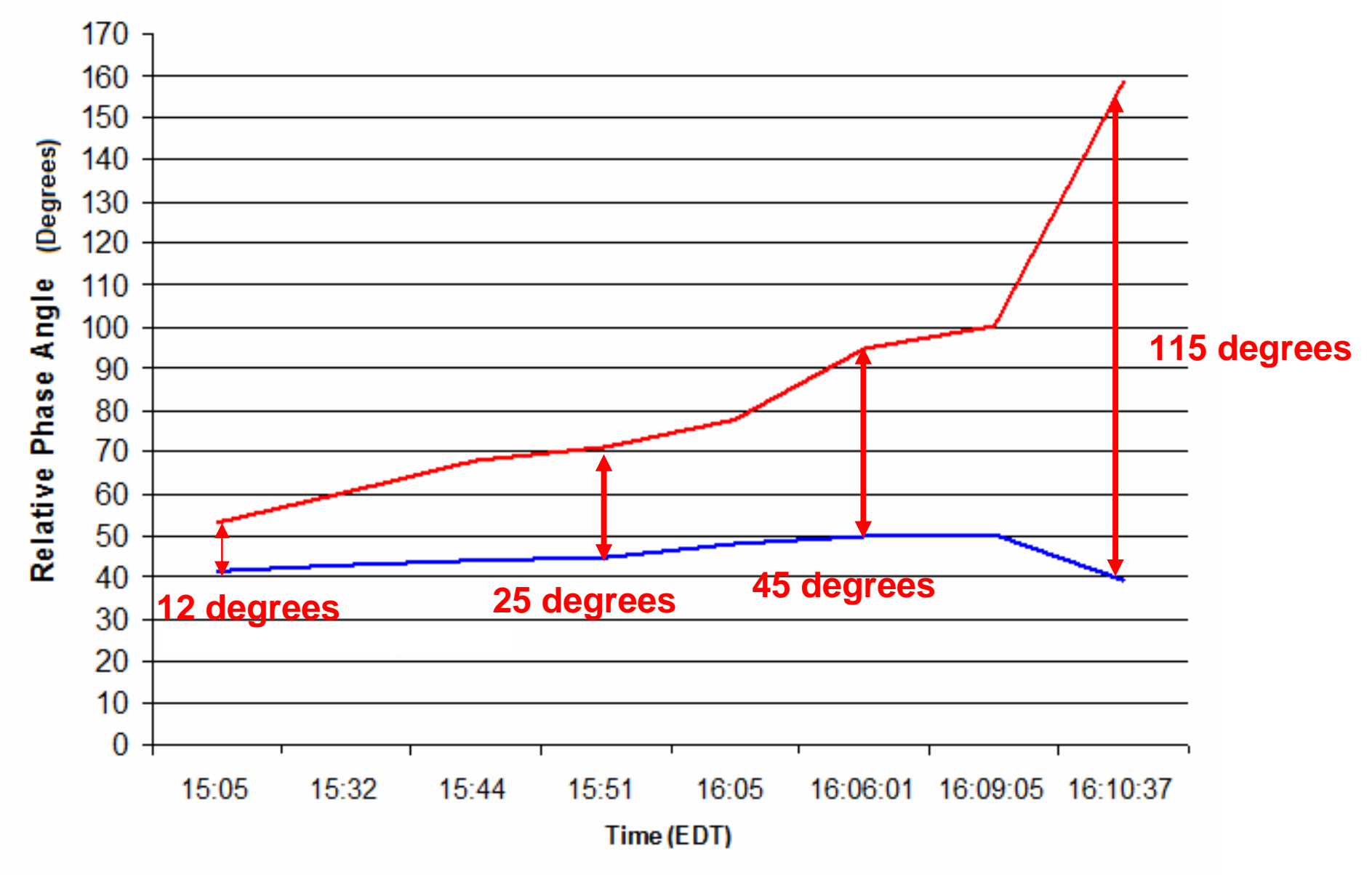

\section{- Cleveland - West MI}




\section{Power System Dynamics}

\section{Mechanical Analogy for Power System Dynamics}

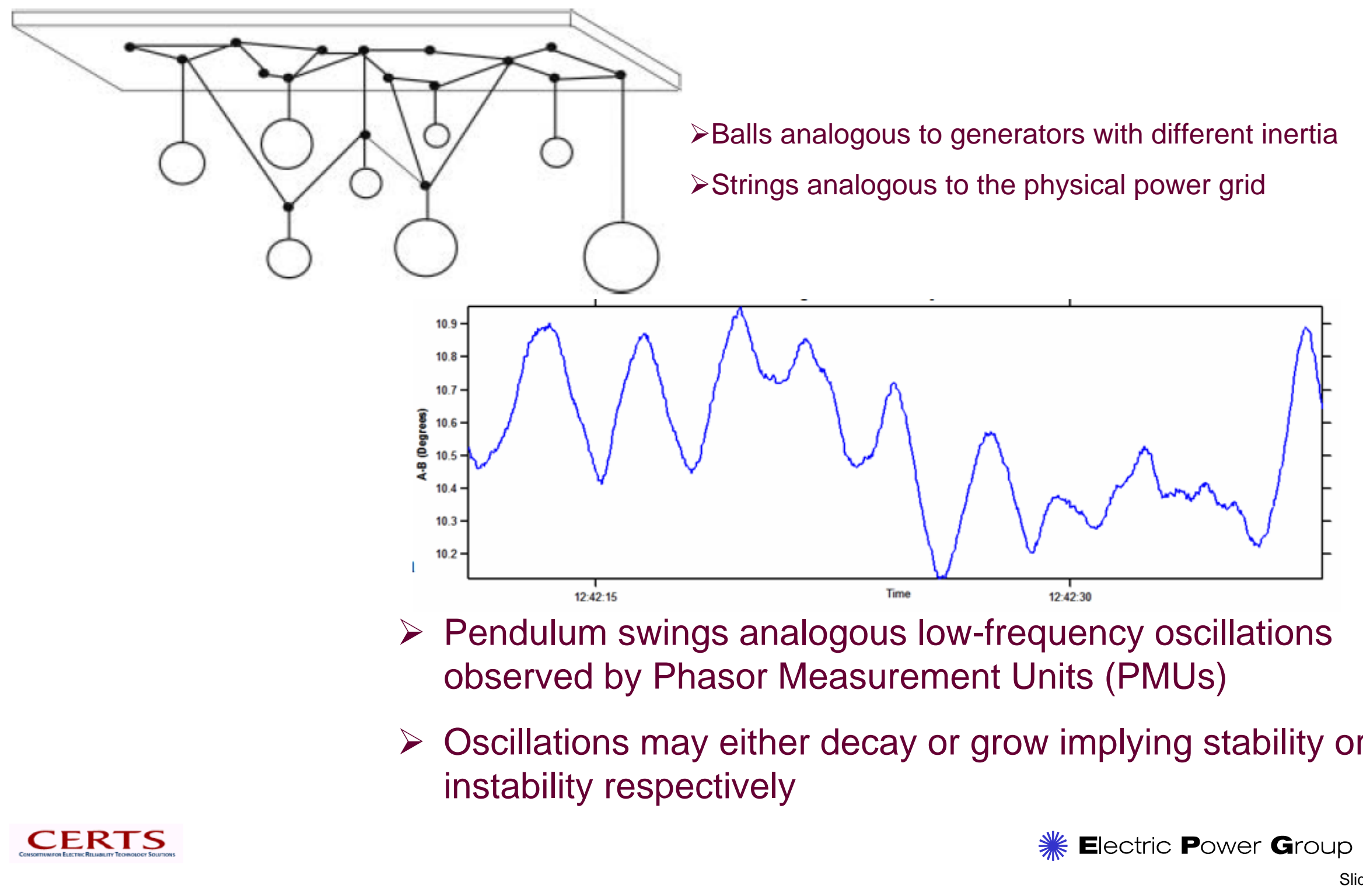




\section{Source of Phasor Measurements}

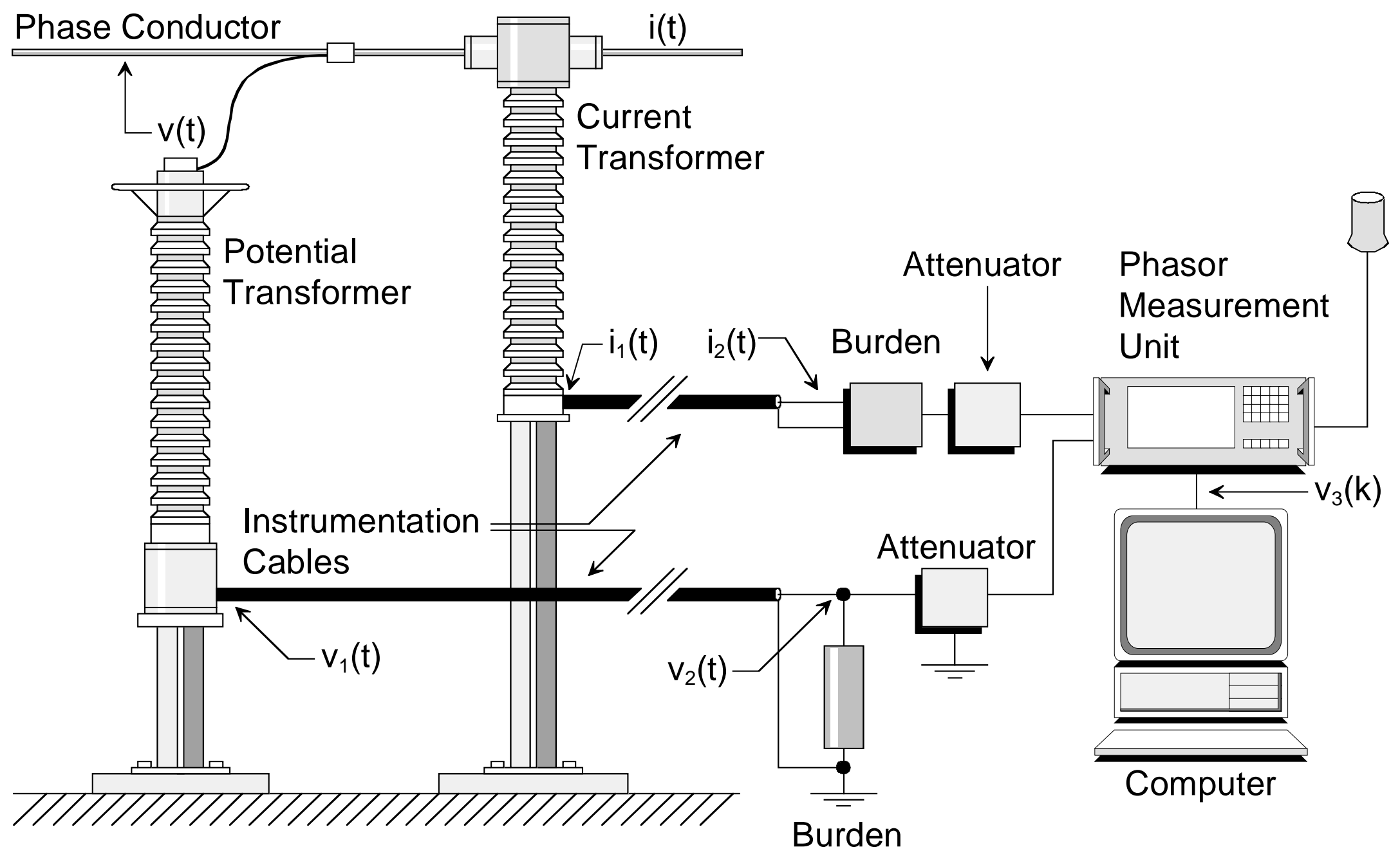




\section{What Phasor Measurements are all about}

\section{Phasor Overview}

"Reference $60 \mathrm{~Hz}$ Signal using GPS time signal"

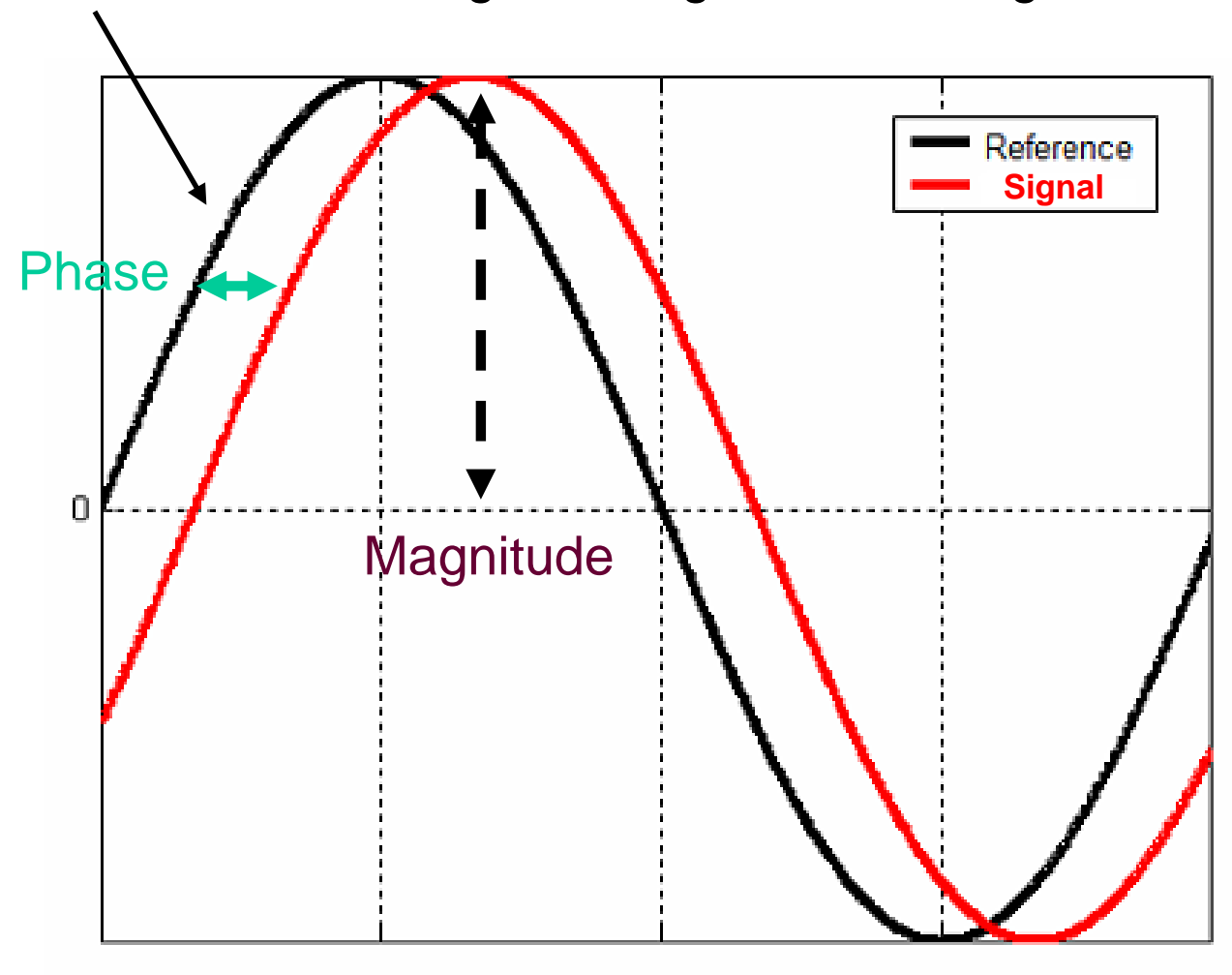

CERTS

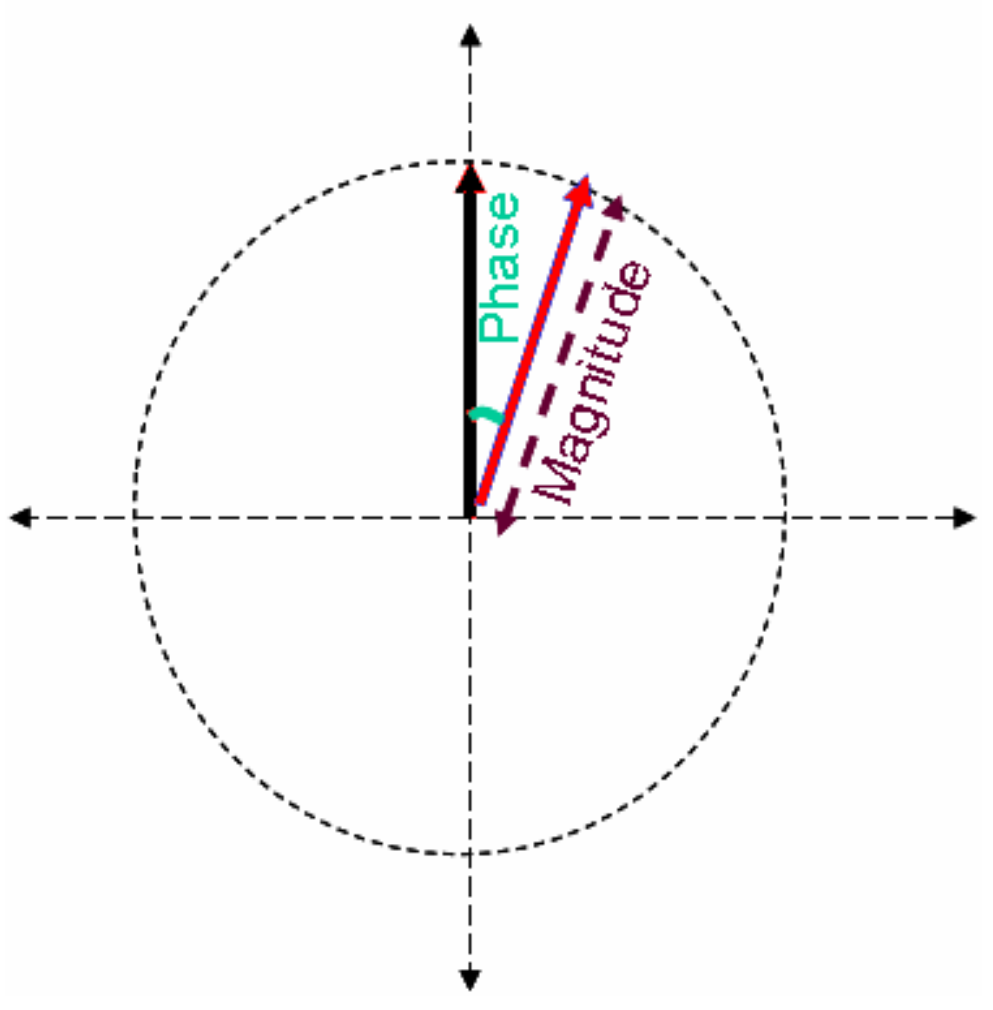

溇 Electric Power Group 


\section{What Phasor Measurements are all about (cont.)}

\section{Strobe Light Analogy}

Pure $60 \mathrm{~Hz}$ Signal

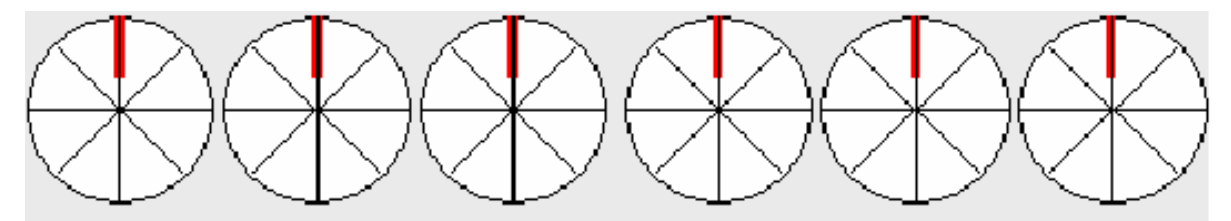

Decelerating System

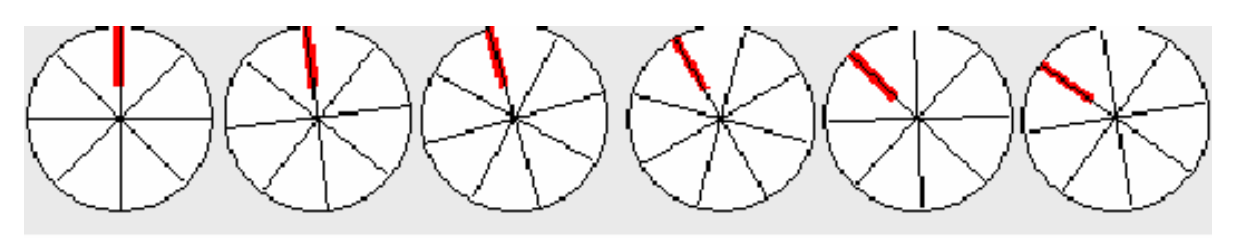

Accelerating System

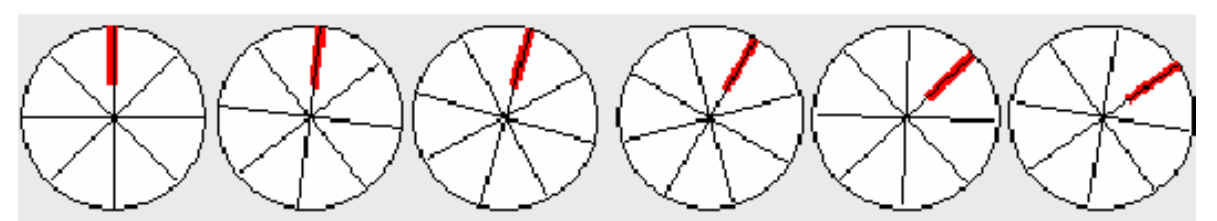




\section{What Phasor Measurements are all about (cont.)}

\section{System Wide “Snapshot" Across Power Grid}

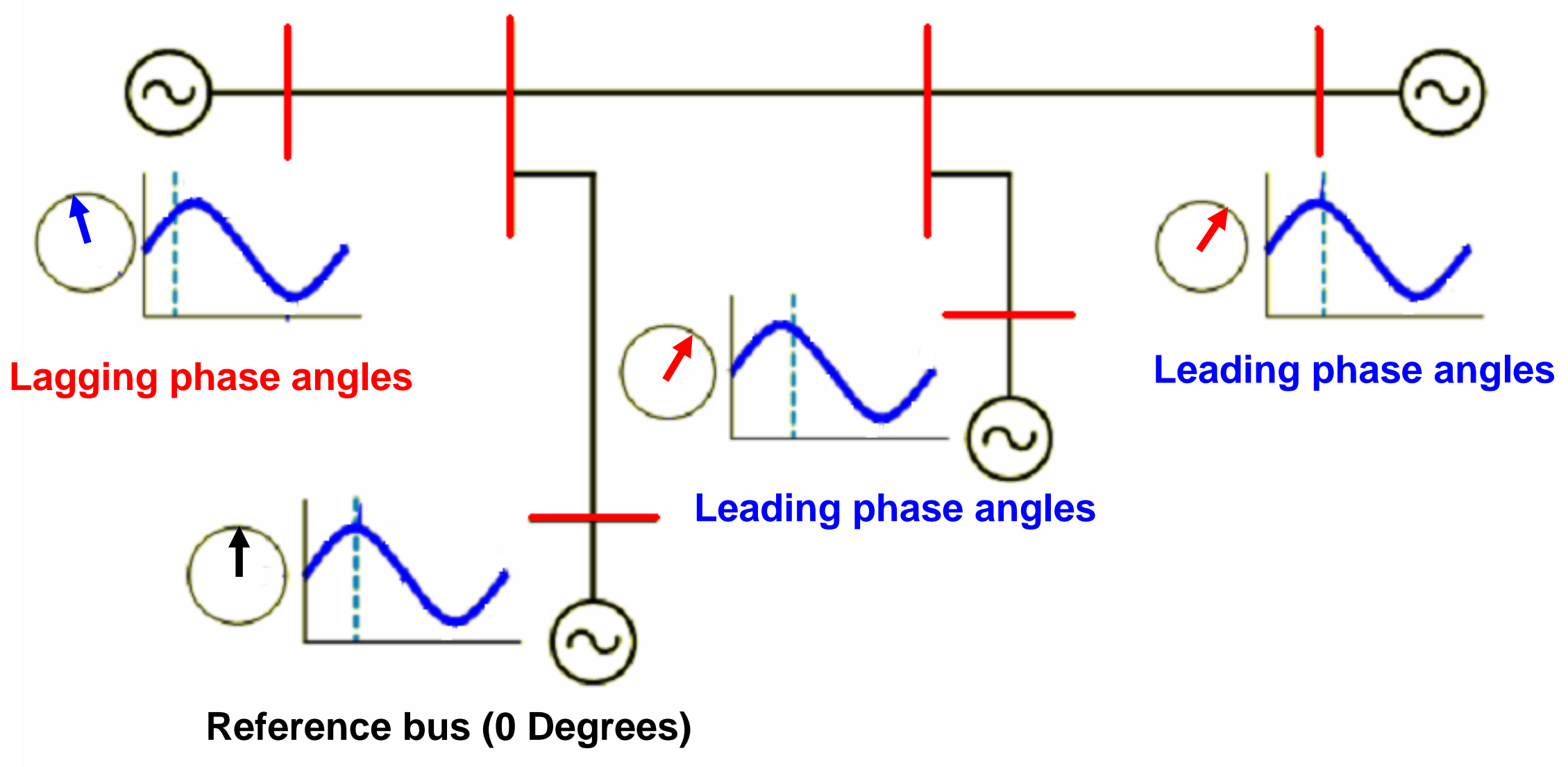




\section{Importance of PMU Data Synchronization}
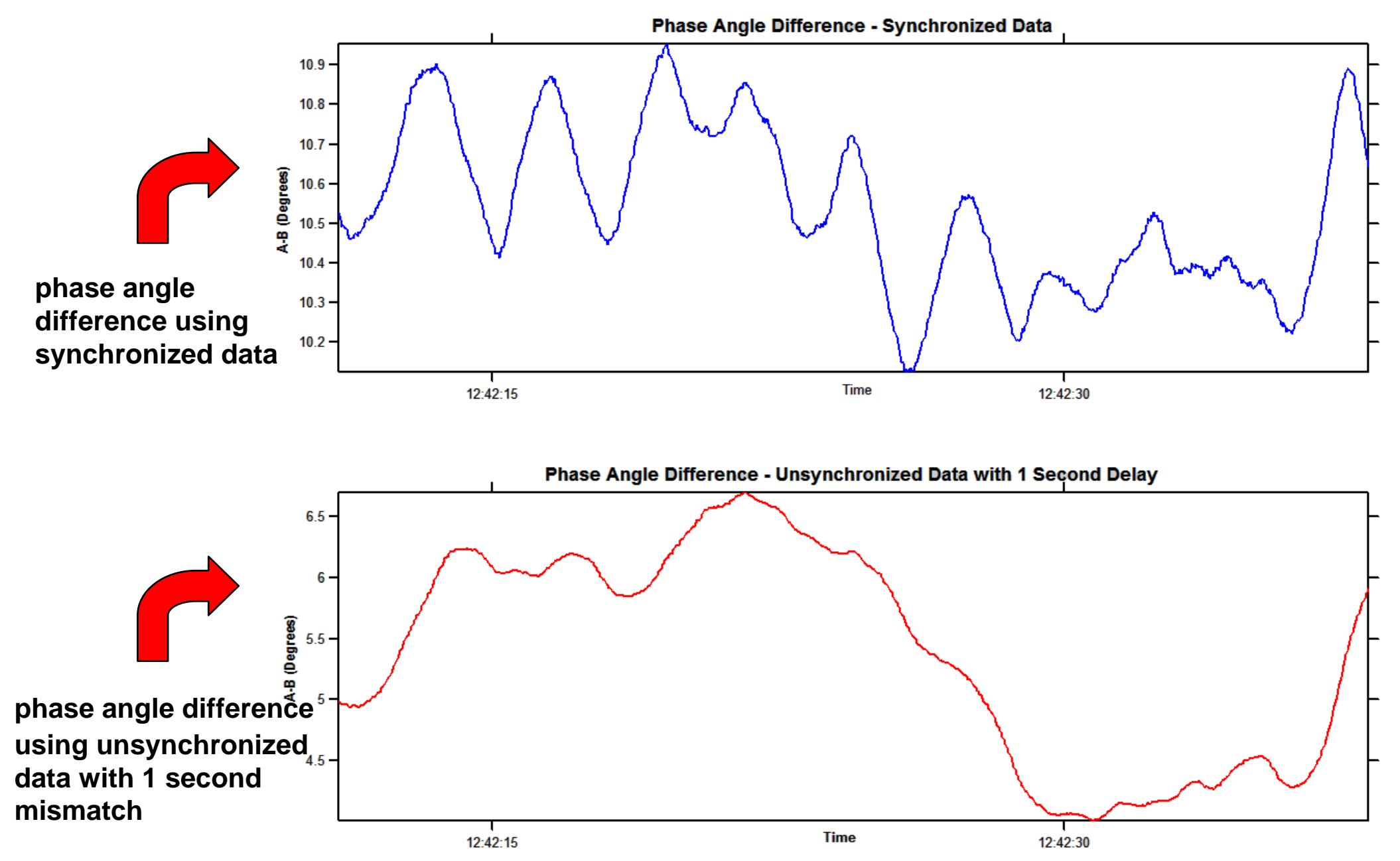

CERTS 


\section{What Phasor Measurements are all about (cont.)}

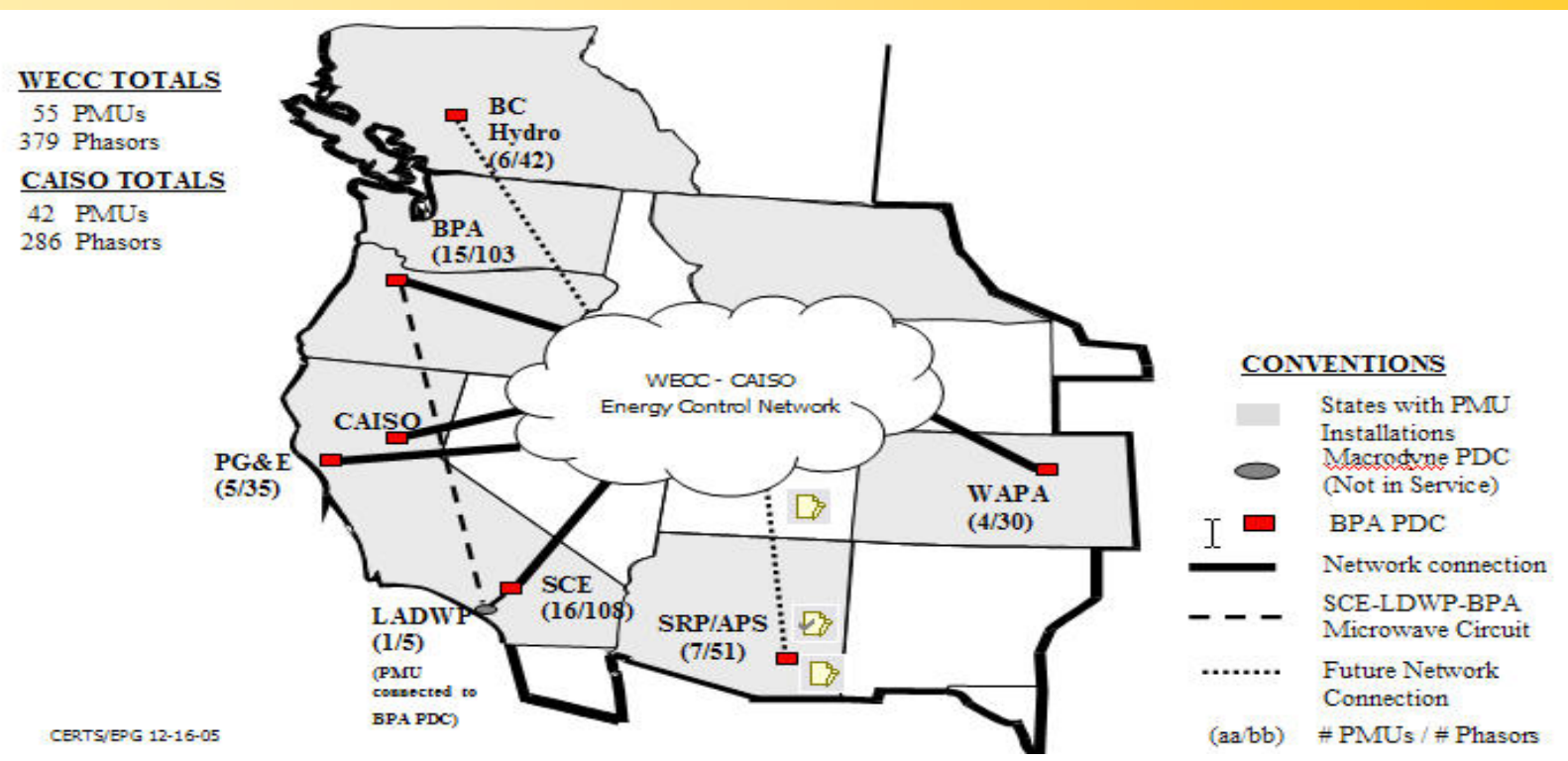

Networking - Most stability events, involve a widespread area, and involve oscillations and control interactions between neighboring utilities and geographic operational regions. This dictates the need for multiple recording devices across the transmission grid.

Time Synchronization - Phasor measurements are time-stamped using the global satellite positioning system so that measurements from across the interconnection can be precisely aligned for comparison against one another. 


\section{What Phasor Measurements are all about (cont)}

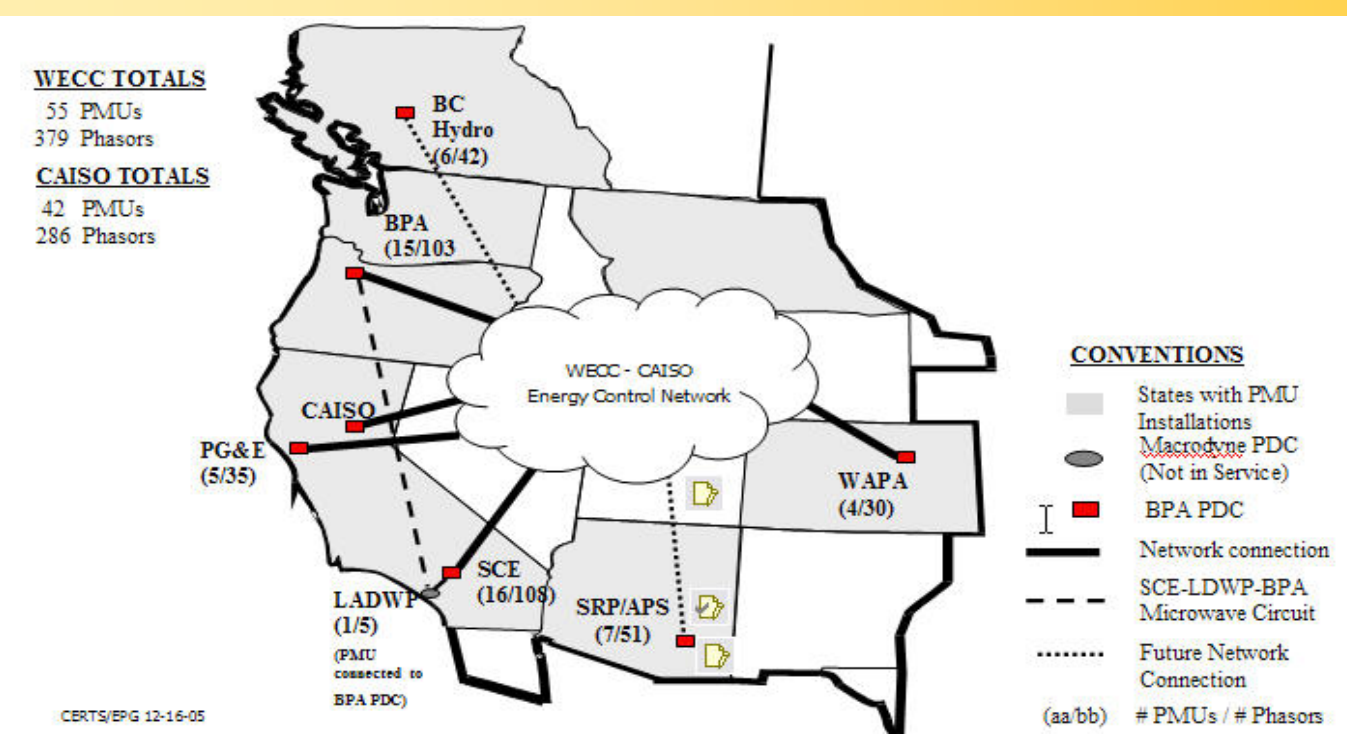

The Primary Hardware Elements in a Phasor Network are:

Phasor Measurement Unit (PMU) - PMUs are located at key substations and measures and are capable of gathering better data at higher sampling rates than analog monitoring devices. The PMU time stamps the local frequency, voltage and line currents at a rate of 30 to 60 times per second. The voltage and current data is used to calculate MW and MVAR flows on key lines. Substation PMU phasor data is transmitted to a PDC at a central location..

Phasor Data Concentrator (PDC) - Receives, integrates, and stores phasor signals from remote PMUs. Can also exchange records with PDCs at other locations. One of the primary functions of the PDC is to perform data synchronization. 


\section{Phasor Technology - Industry Uses}

$>$ The use of phasor technology allows the industry to take high resolution "snapshots" of what is happening throughout the Western Interconnection grid and evaluate the grids performance during system events.

$>$ System operators and planners can use data gathered by PMUs for a host of applications, including:

- State estimation

- Real-time wide area monitoring

- Validation of power system models

- Transient instability protection and fault location systems 


\section{Value of Phasor Technologies - Example}

\section{WECC's Experience}

Comparison of model simulation system performance predictions prior to the WECC's August 10, 1996 blackout (lower panel) and conditions actually recorded by phasor technologies (upper panel) showed that the planning models were not able to accurately capture underlying causes of the blackout

The WECC has since modified their simulation models to better represent actual system performance.
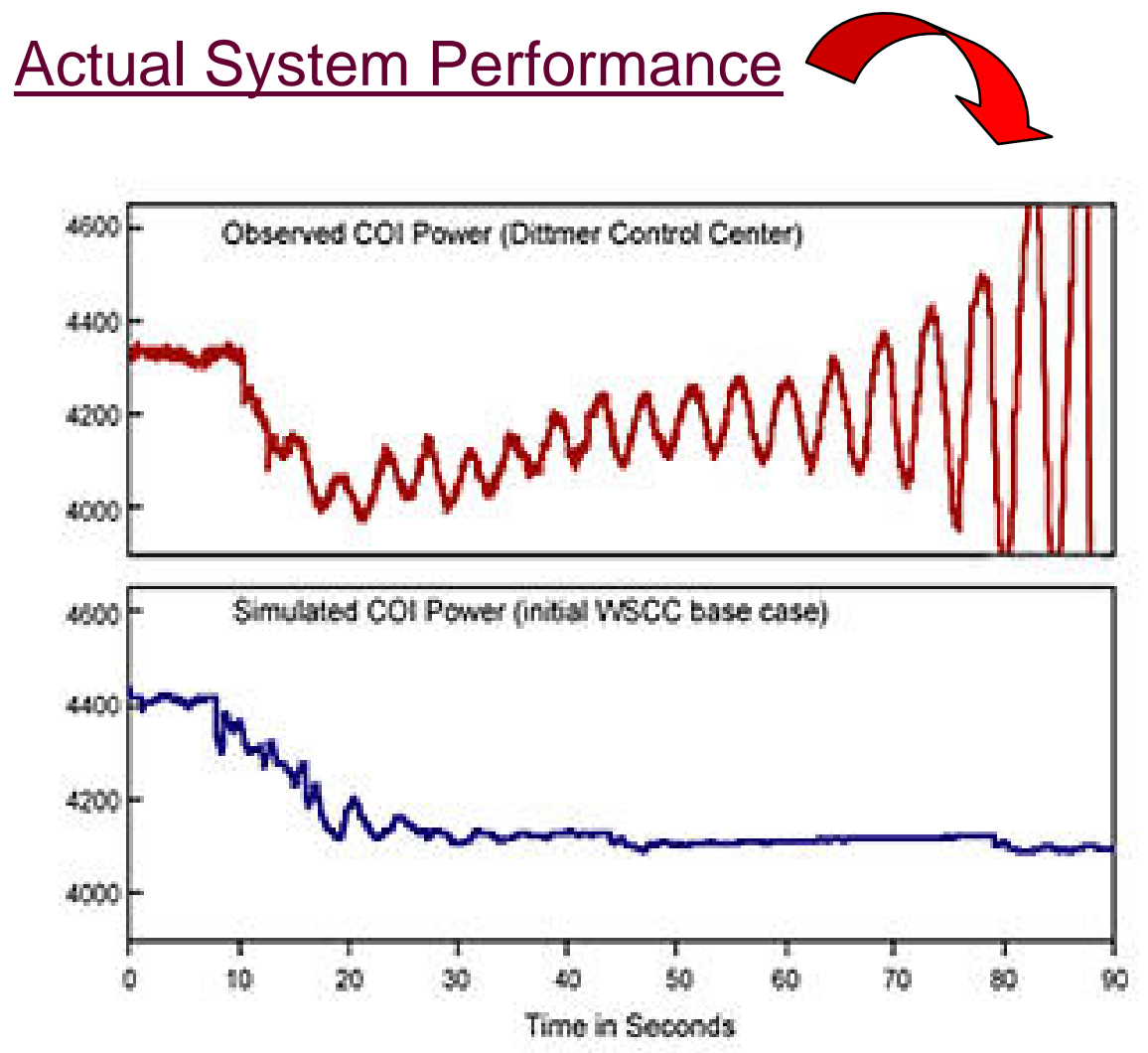

Model Simulation - Predicted System Performance 


\section{Actual WECC Phasor Data Events}




\section{WECC Event - \# 1}

- $\quad$ Location: Western Interconnection

- Date/Time: Friday, August 4, 2000, 7:56PM

- High Static Stress and Low Dynamic Stress

- $\quad$ System Conditions:

1) System was operating with an angle greater than 90 degrees between Devers Substation (Palm Springs, Ca.) and Grand Coulee Power Plant (near Spokane, Wa.), a distance of over 1,000 miles

2) A $500 \mathrm{kV}$ tie-line exporting power from British Columbia to Alberta, Canada tripped

3) Loss of line resulted in increased flows by $450 \mathrm{MW}$

4) The dynamic stress between Devers and Grand Coulee increased to 108 degrees (an 18 degree increase)

5) System oscillated for about 60 seconds showing low damping 


\section{WECC Event - \# 1}

\section{8/04/00 Event at 12:55 Pacific Time (08/04/00 at 19:55 GMT )}

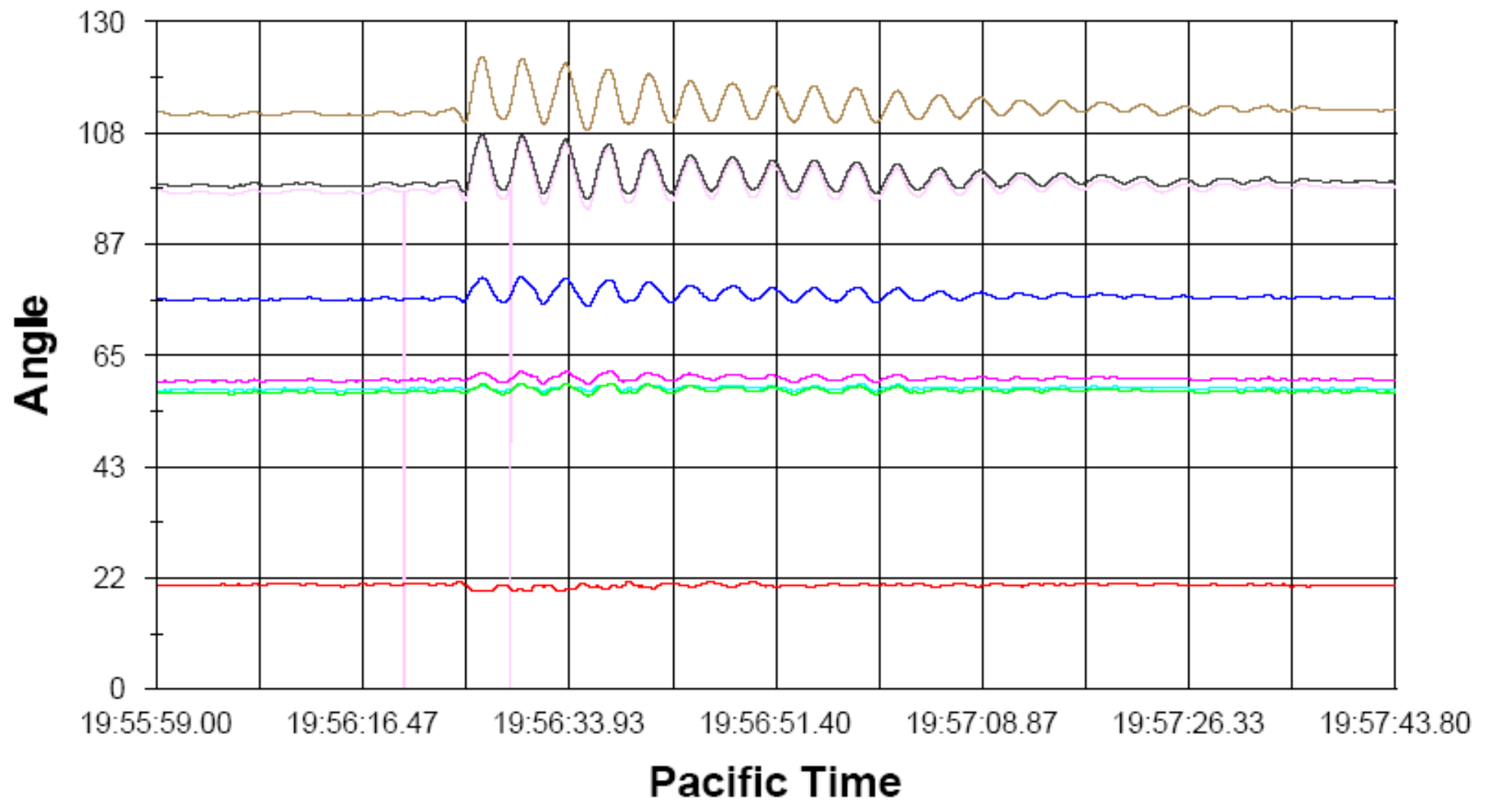

- Grand Coulee

- John Day

- Malin N Colstrip

- Big Eddy 500

- Keeler $500 \mathrm{kV}$

- Vincent

- Devers $500 \mathrm{kV}$

- Vincent $500 \mathrm{kV}$ Mohave 500kV

- Devers $500 \mathrm{kV}$ Grand Coulee 500kV

Angle Reference is Colstrip

J. Balance, B. Bhargava, G.D. Rodriquez, "Use of Phasor Measurement System for Enhancing AC-DC Power System Transmission Reliability and Capacity."

CERTS 


\section{Comparison with August $10^{\text {th }} 1996$}

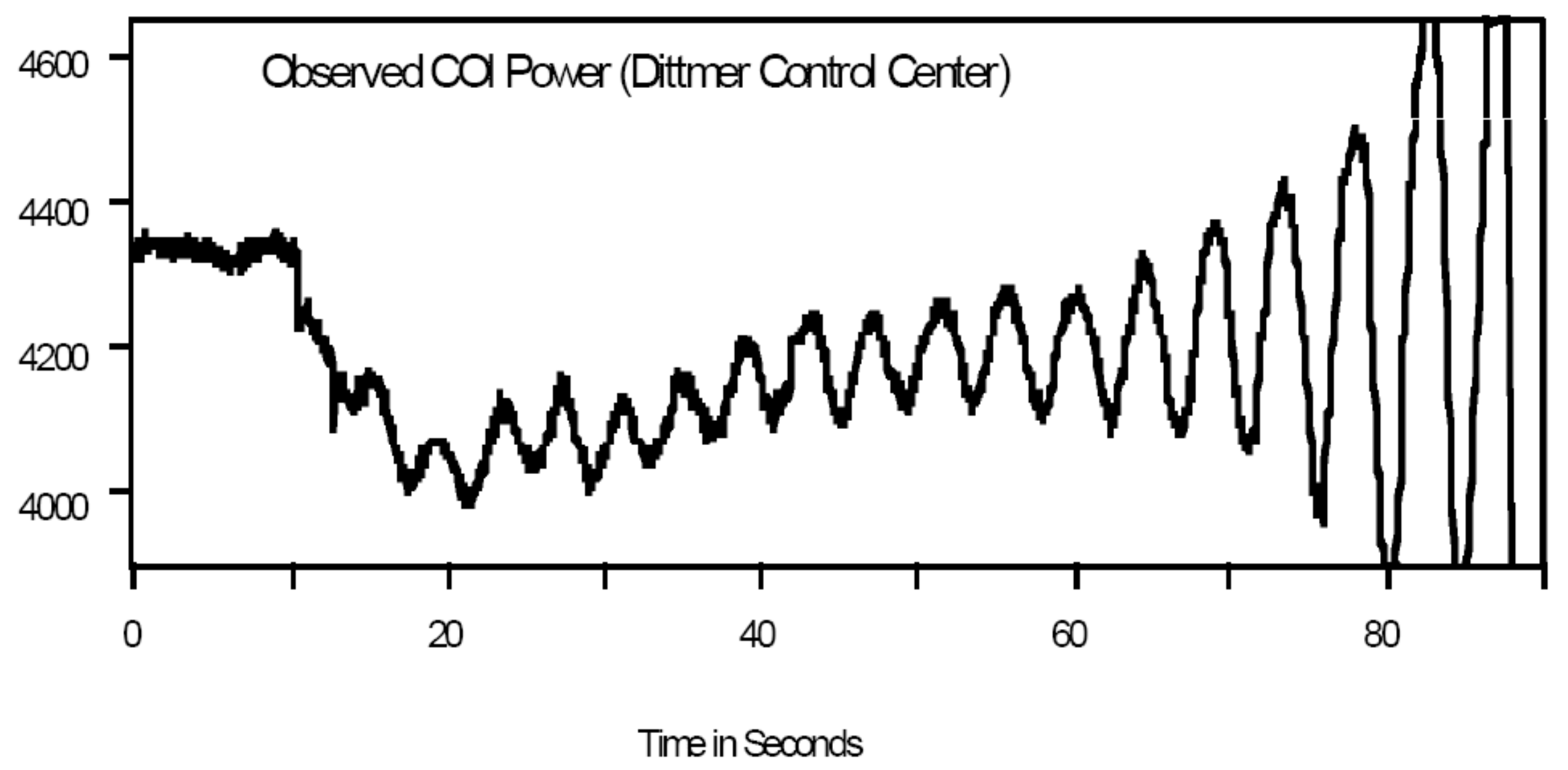

CERTS 


\section{WECC Event \# 2}

- Location: Western Interconnection

- Date/Time: Tuesday, October 8, 2002, 3:38PM

- $\quad$ Abnormal Interconnection Frequency: $59.62 \mathrm{~Hz}(380 \mathrm{mHz})$

- System Conditions:

1) An AC line fault occurred in the northwest tripping three $500 \mathrm{kV}$ lines

2) SPS operated by applying the $1400 \mathrm{MW}$ Chief Joseph break and tripping $2800 \mathrm{MW}$ of generation in northern WECC system.

3) The frequency dropped to $59.620 \mathrm{~Hz}$. 


\section{WECC Event \#2}

10/08/02 Event at 15:30 Pacific Time (10/08/02 at 22:30 GMT )

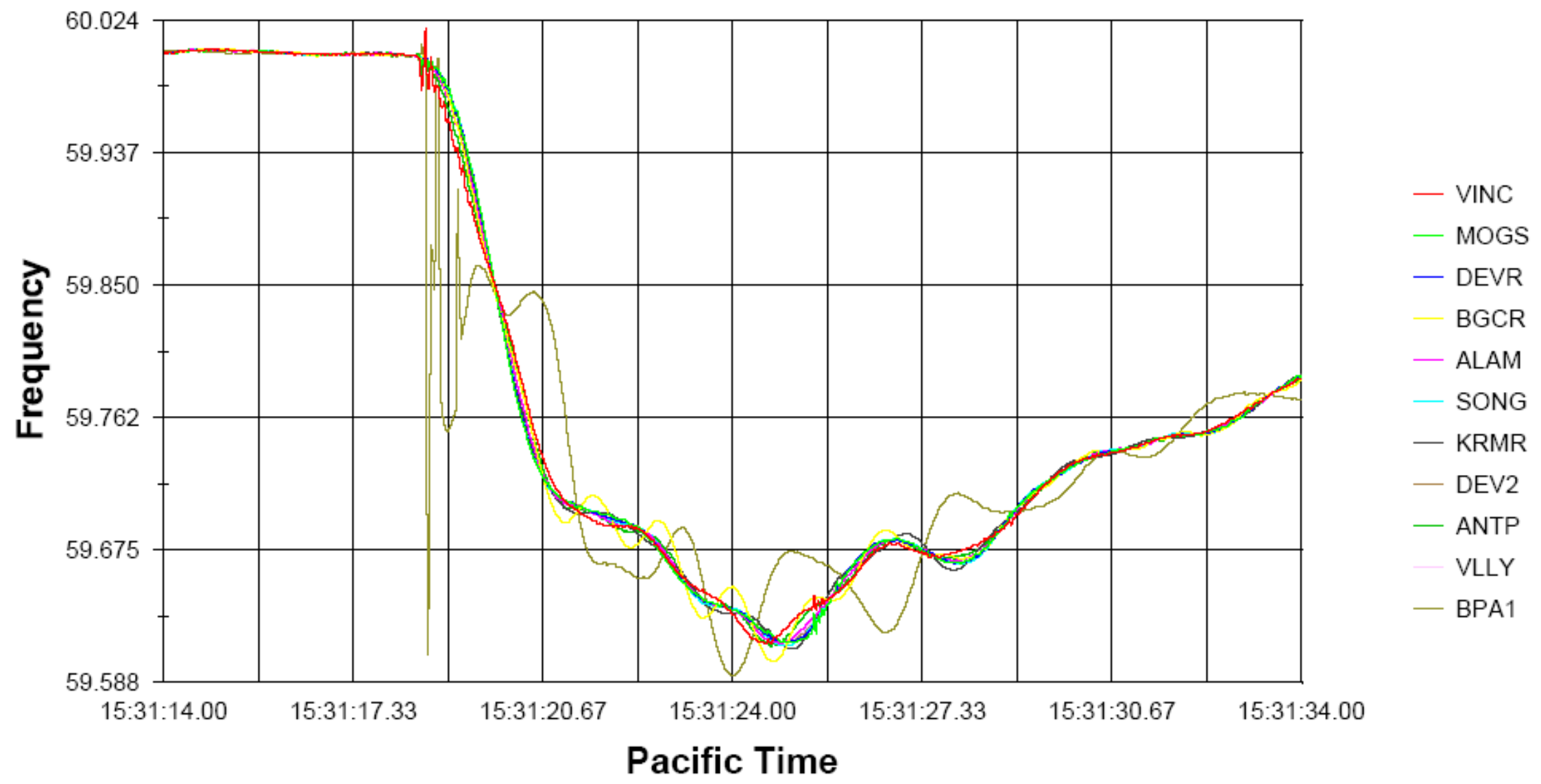




\section{WECC Event \# 3}

- Location: Western Interconnection

- Date/Time: Sunday, January 15, 2006, 00:24AM

- $\quad$ Generator Trip (System Frequency Response Captured by RTDMS)

- System Conditions:

1) NEW Colstrip Unit 1 relayed while carrying $240 \mathrm{MW}$

2) System frequency deviated from $59.995 \mathrm{~Hz}$ to $59.947 \mathrm{~Hz}$

3) Recovered to $59.961 \mathrm{~Hz}$ by governor action

4) Returned to pre-disturbance level at 00:29 


\section{WECC Event \#3}

\section{RTDMS Event File Name "20060115 002424"}

Real-Time Dynamics Monitoring System v3.0 - Grid3P Event File: C:IProgram FilesICERTS-EPG Grid 3PIRTDMS Server 3.0levent120060115_002424.dat \begin{tabular}{|l|l|l|l|l|}
\hline Frequency (Response) & Path Dynamics & Rel. AngleNolt. Magnitude & Path Flow - MW & Path Flow - MVAR \\
\hline
\end{tabular}

Real-Time Frequency (Response) Monitoring - 1 Second Refresh Rate
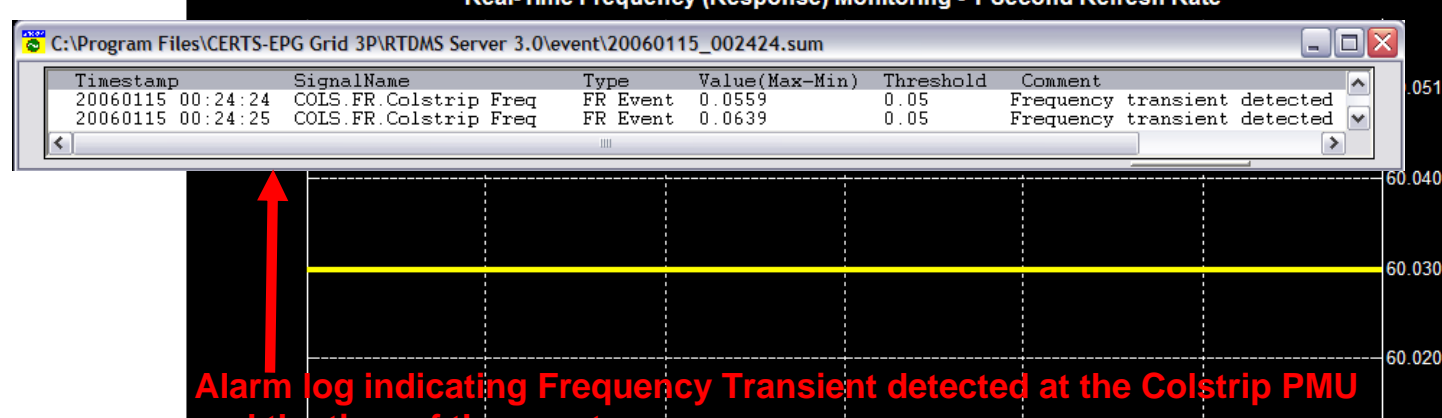

and the time of the event

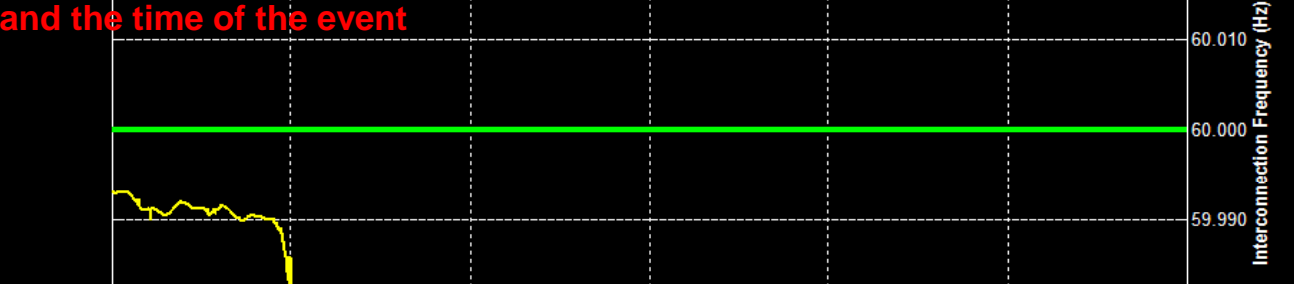

Systein Frequenç

Response
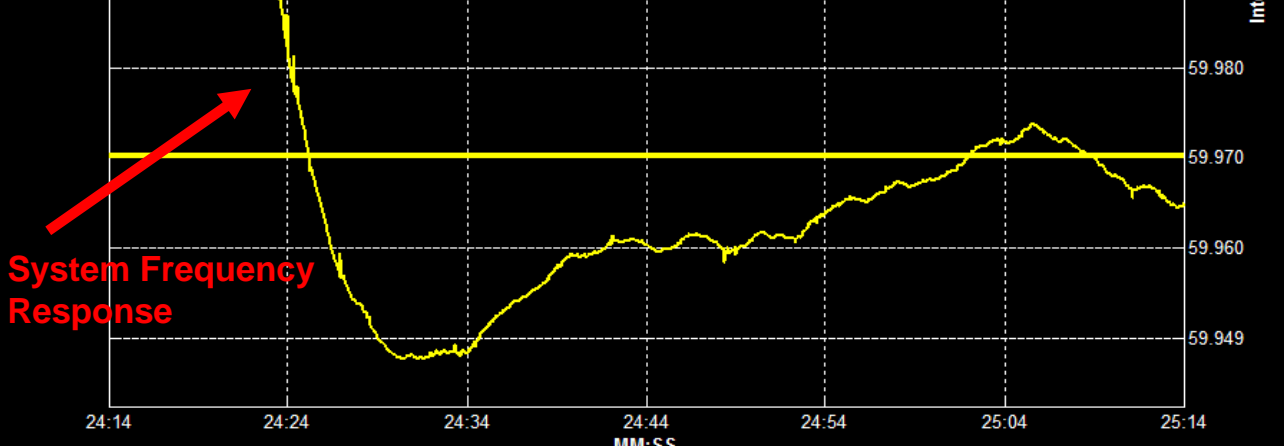

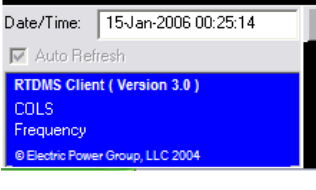

MM:SS

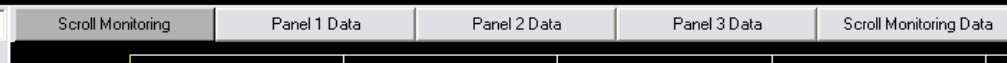

Panel 3Data

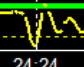

V1:24

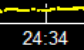

24:44

$24: 54$

Data
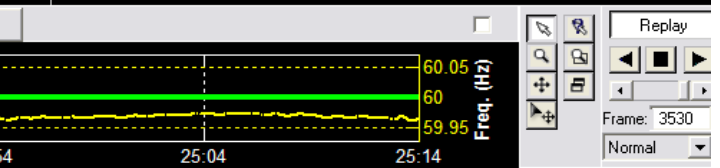

Local Frequency Coherency Comparison

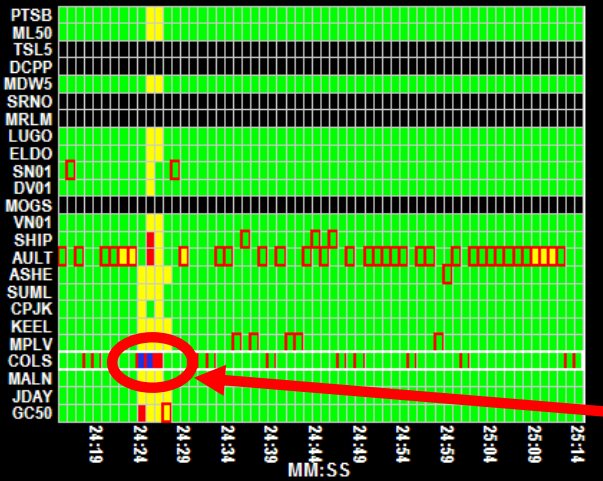

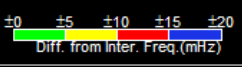

Interconnection Frequency \& Imbalance Duration

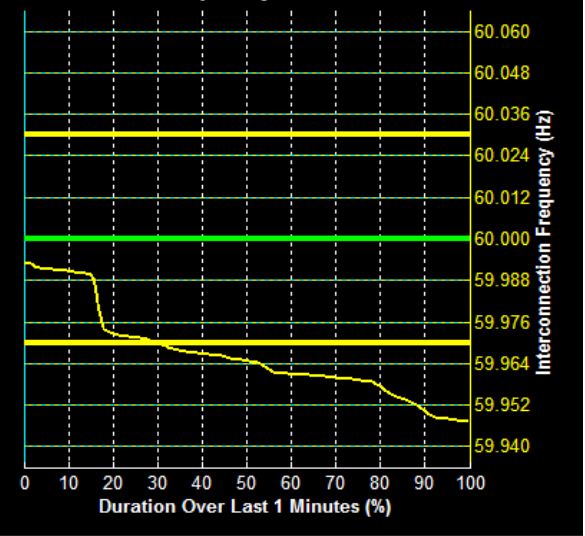




\section{WECC Event \#3 (Frequency Response)}

\section{January 15, 2006 (CA ISO Log)}

01/15/2006 - 00:24 System frequency deviated from $59.995 \mathrm{~Hz}$ to $59.947 \mathrm{~Hz}$ and recovered to $59.961 \mathrm{~Hz}$ by governor action when NWE Colstrip Unit 1 relayed while carrying 240 MW. System frequency returned to pre-disturbance level at 00:29.
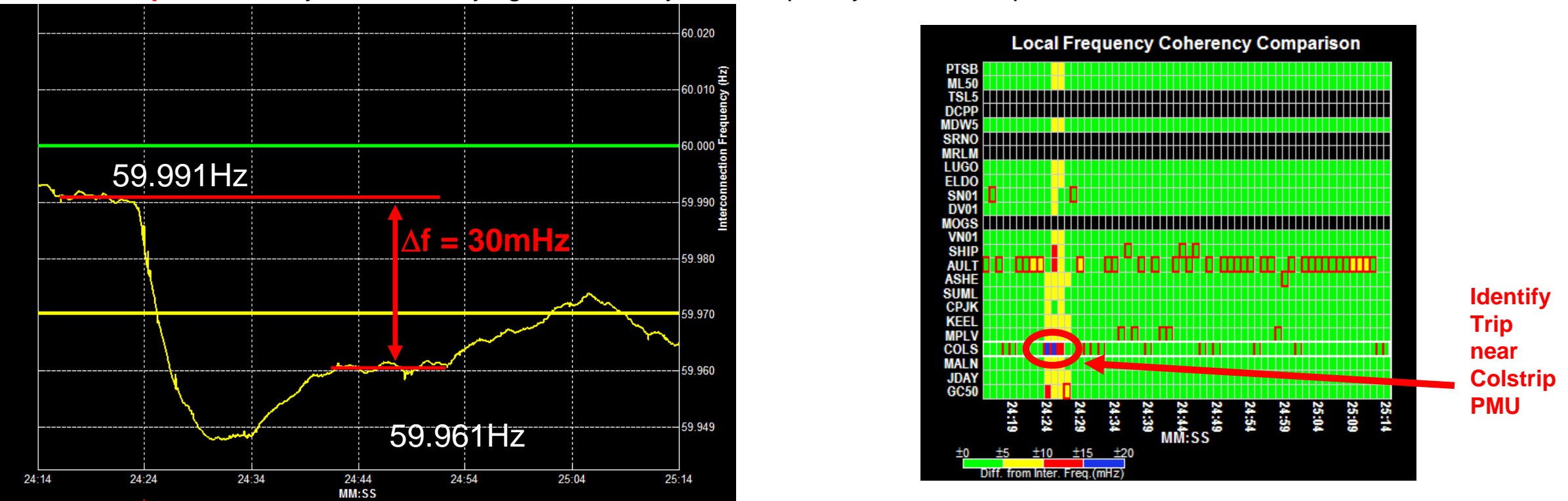

Jan 15, 2005 00:24:24 AM

$\beta=\Delta \mathrm{P} / \Delta \mathrm{f}=800 \mathrm{MW} / 0.1 \mathrm{~Hz}$

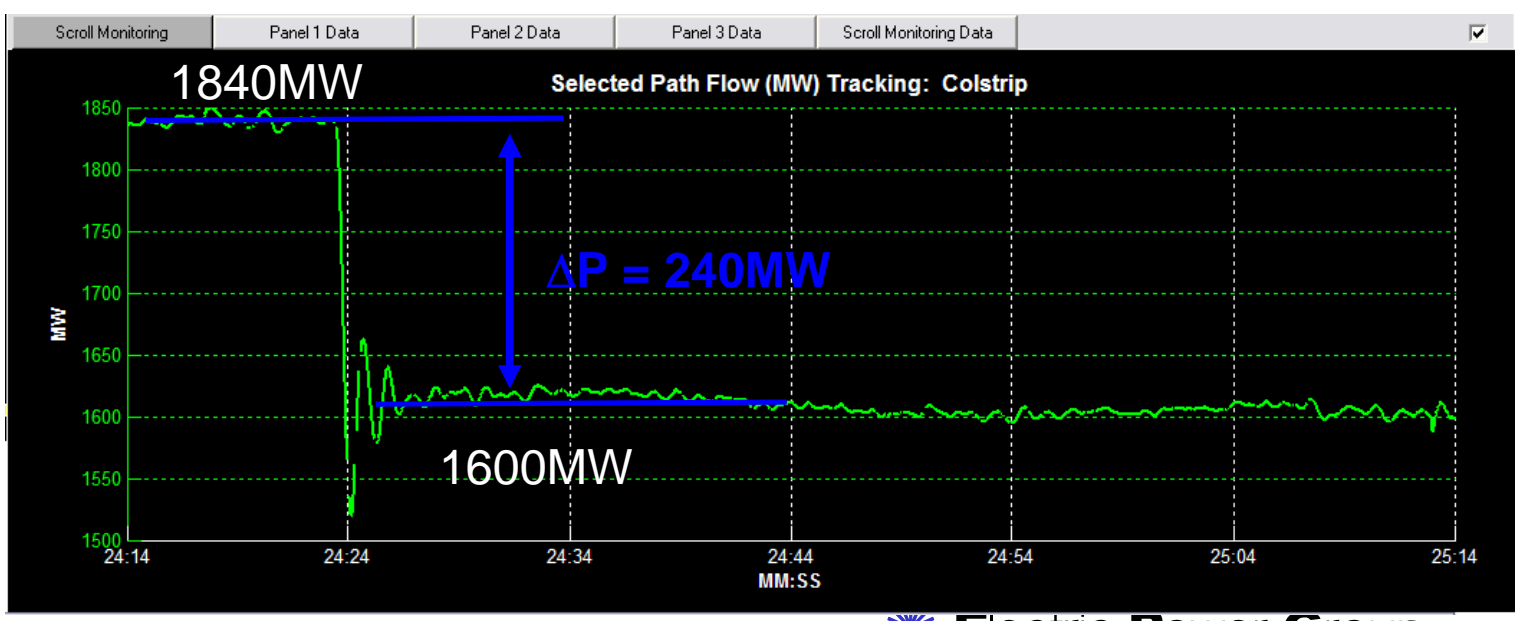

CERTS 


\section{Any Questions About Phasor Technology?}




\section{Real-Time Dynamics Monitoring System (RTDMS)}




\section{Project Objectives for RTDMS Applications}

$>$ Develop a Real-Time Phasor Monitoring Prototype System for use by system operators at utilities, ISOs and reliability coordination centers.

$>$ Enable system operators to gain familiarity with phasor technology for reliability monitoring and real-time operations.

$>$ Learn to utilize phasor data to recognize normal and abnormal conditions, and assess grid stress.

$>$ Provide system operators with real-time wide area information to increase situational awareness to avoid August 10, 1996 type blackouts.

$>$ Monitor across the entire Western Interconnection (WI) for reliability, stability, system dynamics, and other key metrics using time synchronized phasor data.

$>$ Enable system operators to evaluate and provide feedback on metrics monitored, visualization formats, functionality and displays. 


\section{CA ISO RTDMS System Architecture}

\section{CAISO PHASOR NETWORK DIAGRAM}

(PRESENT AND FUTURE)

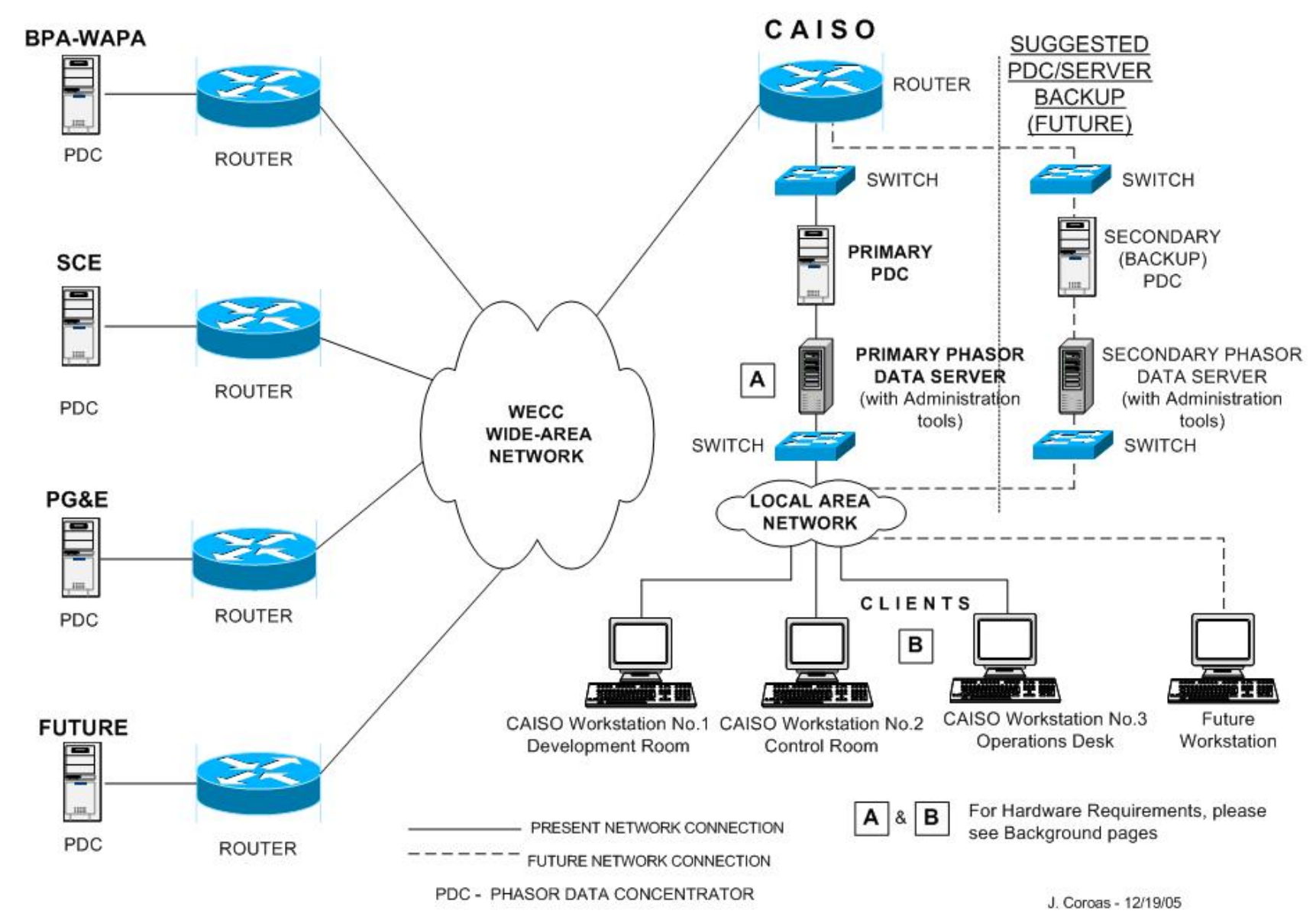




\section{CA ISO RTDMS System Architecture}

Data Acquisition

- Data Reading

- Data Cleansing

- Data Processing

Data Management

- Short-Term Buffer

for RT Monitoring

and Alarming

- Historical Data for

Trending/Reporting

Remote Monitoring

Clients

- Data Retrieval

- RT Monitoring \&

Alarming

- Trending \& Reporting

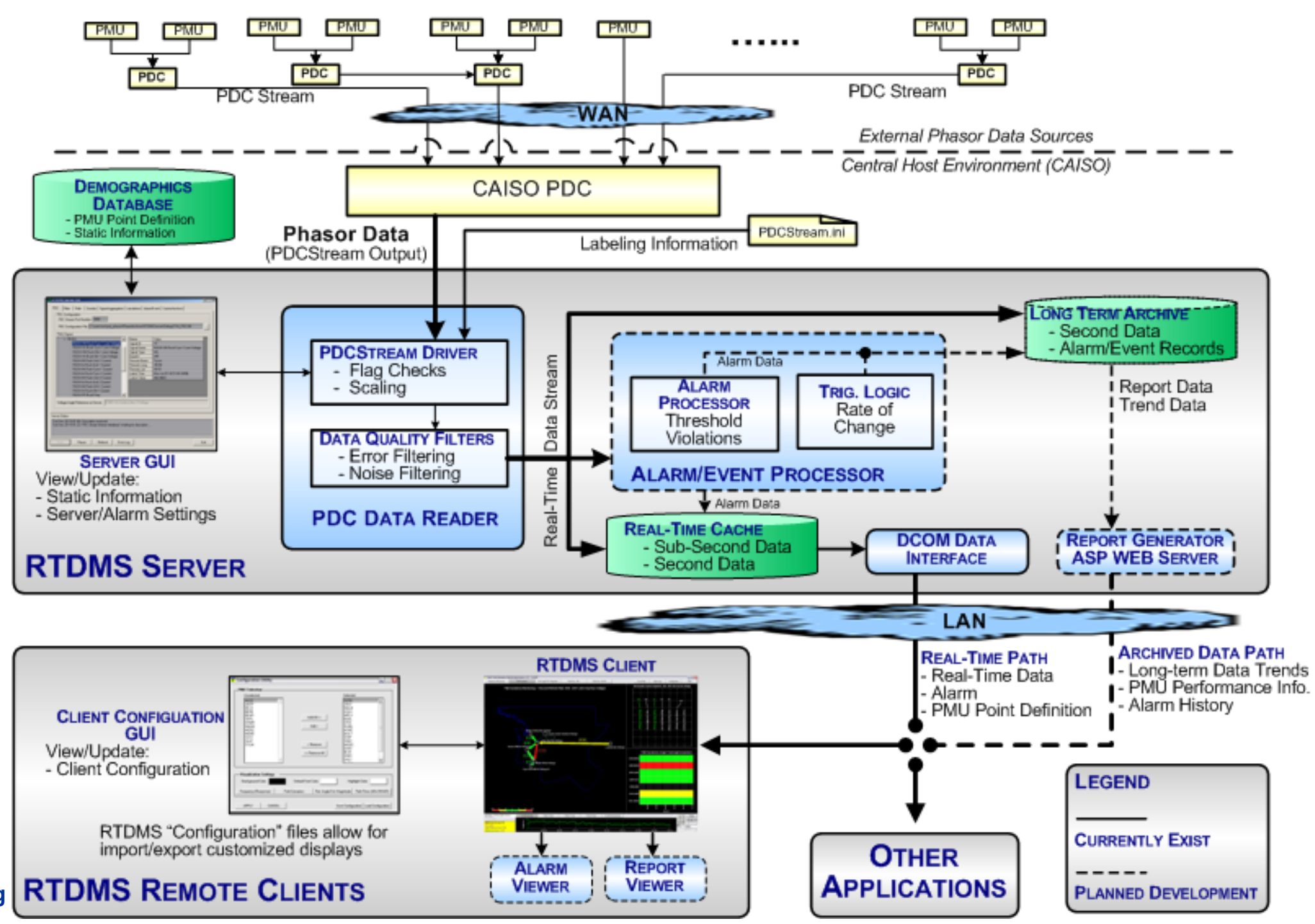

CERTS

溇 Electric Power Group 


\section{RTDMS - What will the System Operator See?}

$>$ Wide Area View of WECC - Key metrics at selected locations and transmission corridors.

$>$ Key Metrics Include - System frequency, voltages, phase angles and angle differences between major sources and sinks

$>$ Violation of Key Thresholds (defined limits) - Visual alarming (color coded)

$>$ Rapid Changes in Metrics - Visual alarming

$>$ Identify System Anomalies 


\section{How To Navigate Around The RTDMS Screens}




\section{RTDMS Visualization Architecture}
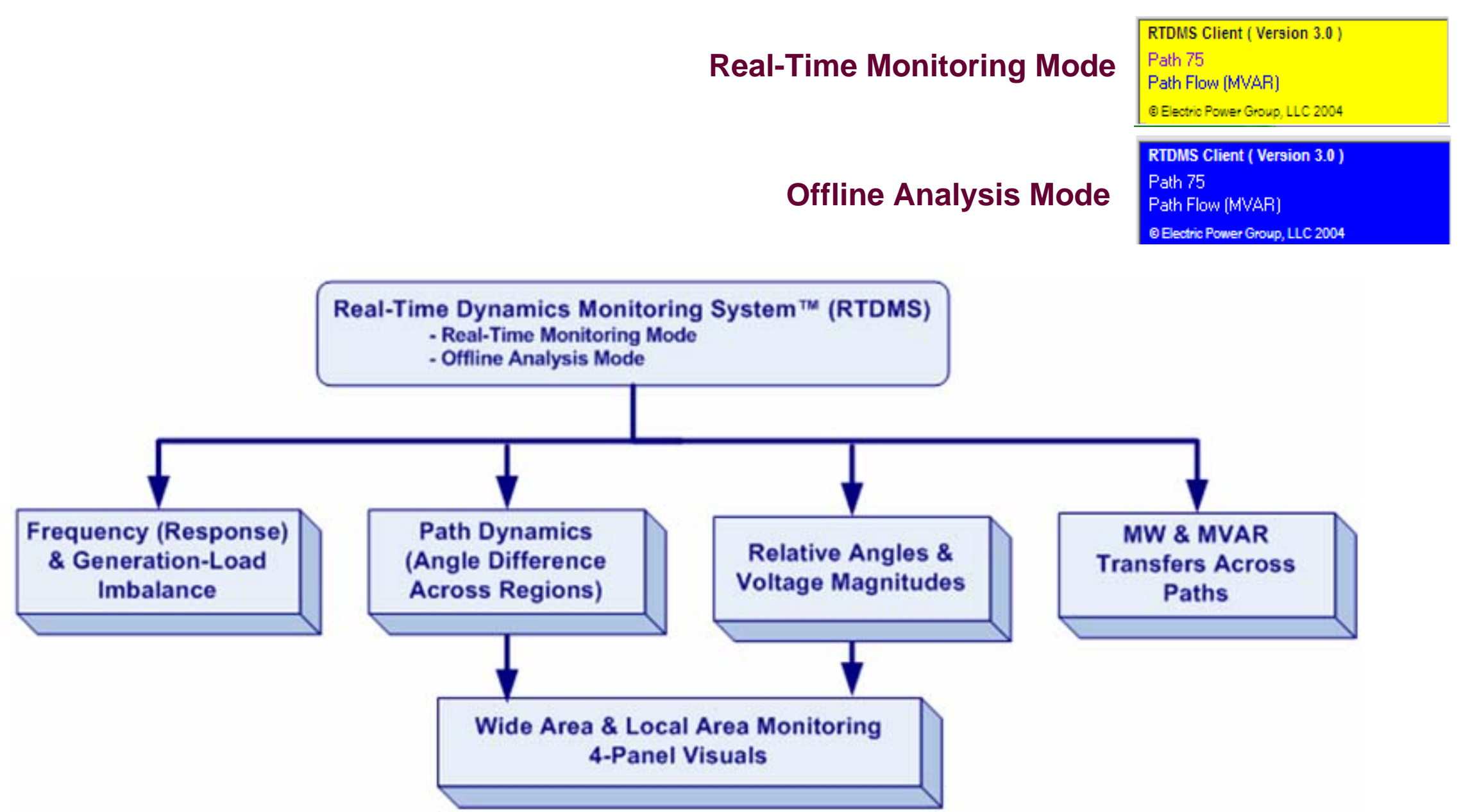


\section{Four-Panel Display Major Functions}

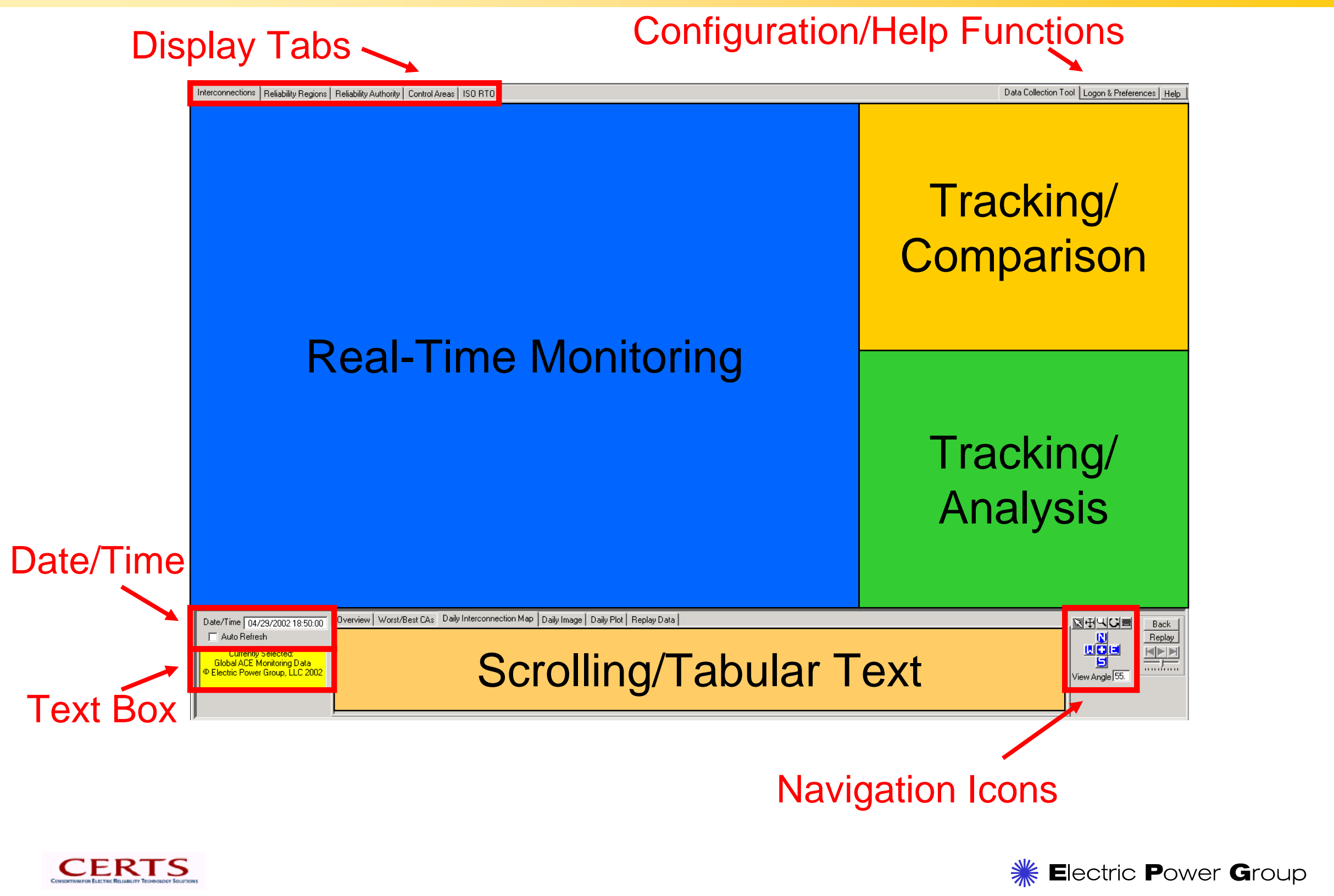




\section{Sample Four-Panel Display}

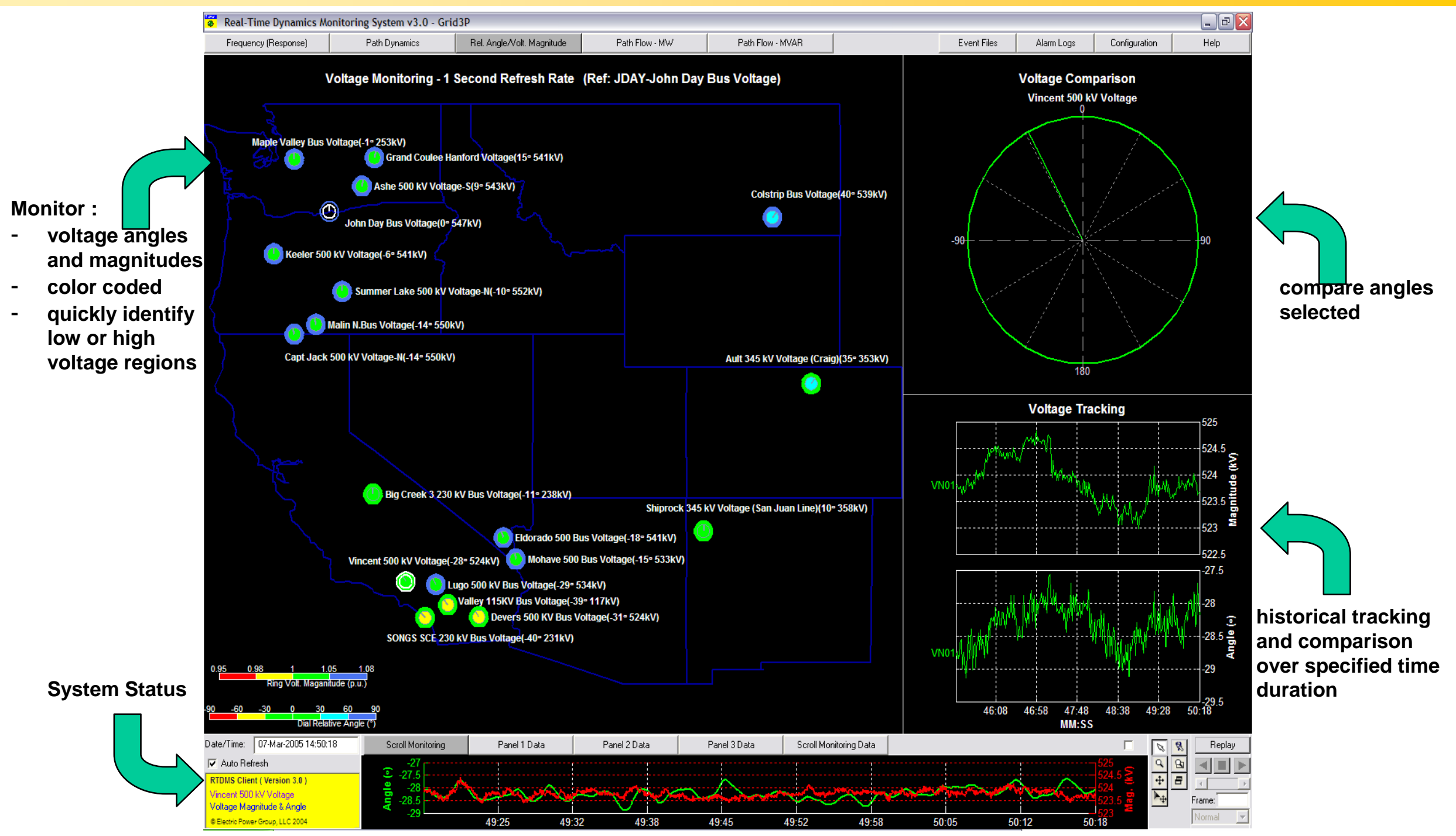




\section{Tabular Table}

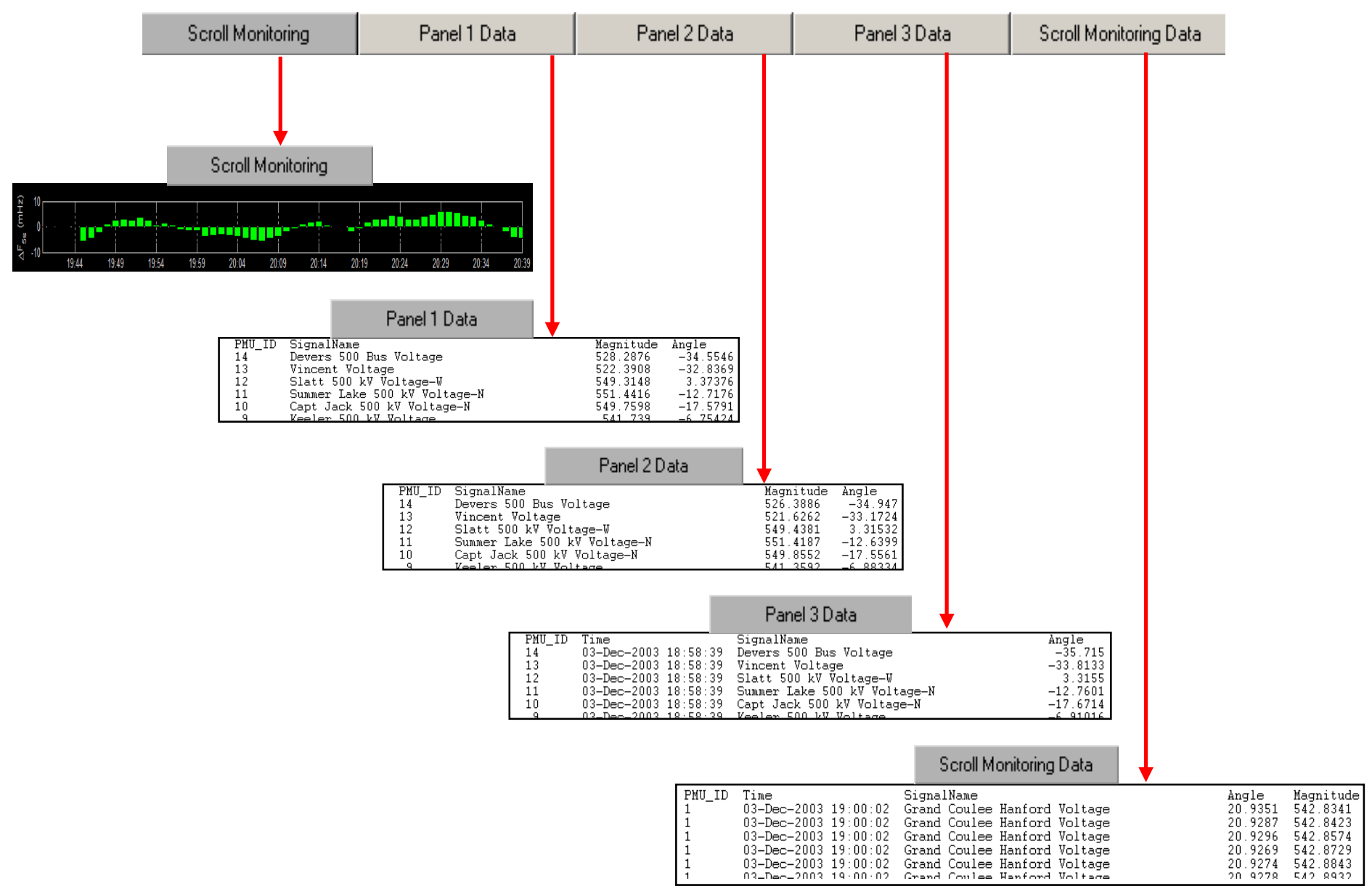

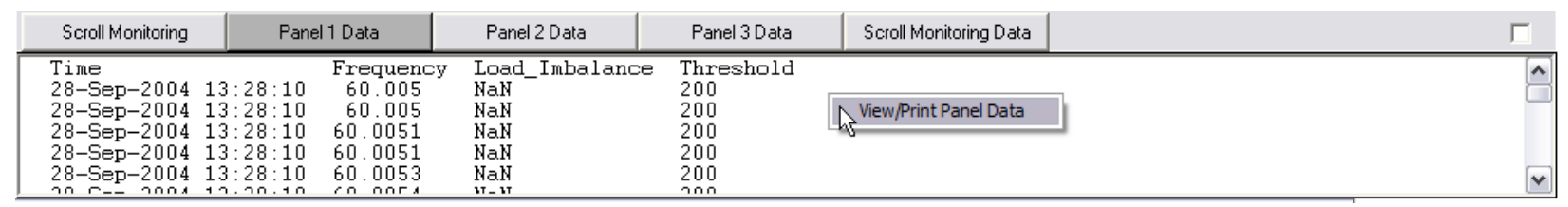




\section{Navigation Tools}

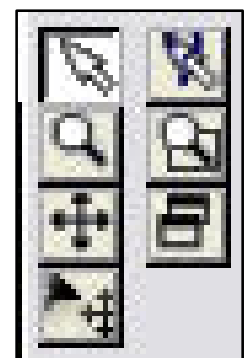

\section{Selection Arrow}

This is the default Navigation tool. Selecting this tool allows the user to click and move through the different plots and data tables

\section{Zoom In/Out}

With the mouse on the image hold the button and slide the mouse down or to the left to reduce the size of the image or move the mouse up or to the right to increase the size of the image.

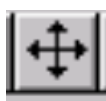

\section{Reposition}

Click the cross arrows, move the cursor to the viewing panel, click the left mouse and drag the object to the desired location in the panel

\section{Pick to Move Text}

Click on the cross arrows with the pointer, move the cursor to the text label, click the left mouse and drag the text label to the desired location in the panel

\section{\&}

\section{Pick to Display Data}

Click on the arrow within the question mark to select data within the plot or diagram

\section{Q Rubber Band Zoom}

Click the magnifying glass with the box in the background, place mouse within the plot or diagram and left click to select the zoom desired

\section{E Restore to Original Size}

Click on the double plot icon and click on the appropriate panel to restore the plot or diagram to its original size 


\title{
Auto Refresh, Freeze and Replay
}

\section{Real-Time Streaming Data (Auto Refresh)}

\author{
Date/Time: 03-Dec-2003 21:45:59 \\ ॠ Ruto Refresh \\ Click to remove "check" mark
}

\section{Freeze Data}

\section{Г Auto Refresh}

When "check" mark is removed the current data is held

\section{Replay Data}

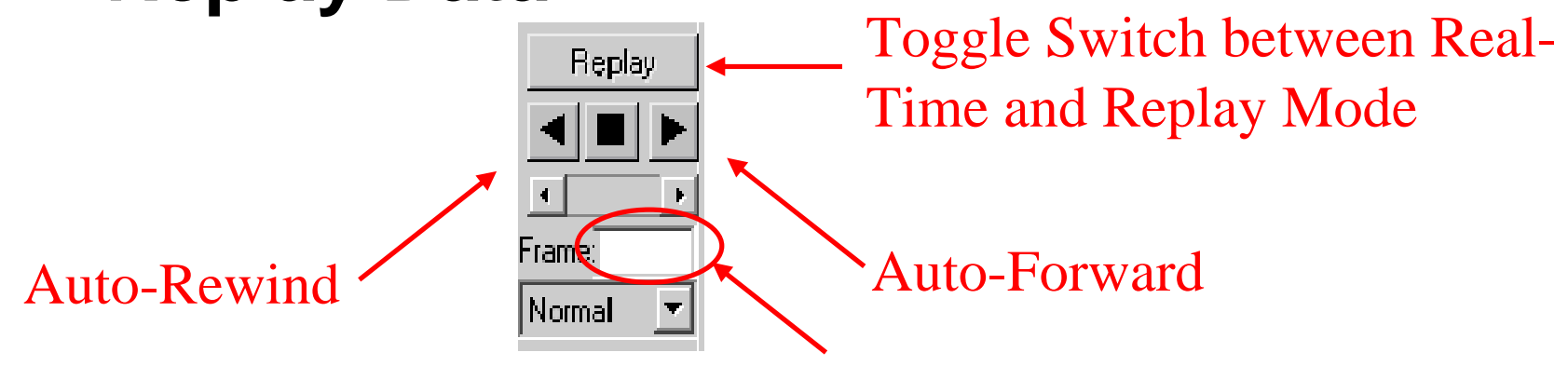




\section{RTDMS Client Configuration Utility}

Configuration menus allow easy scalability as new monitoring devices are added to the system

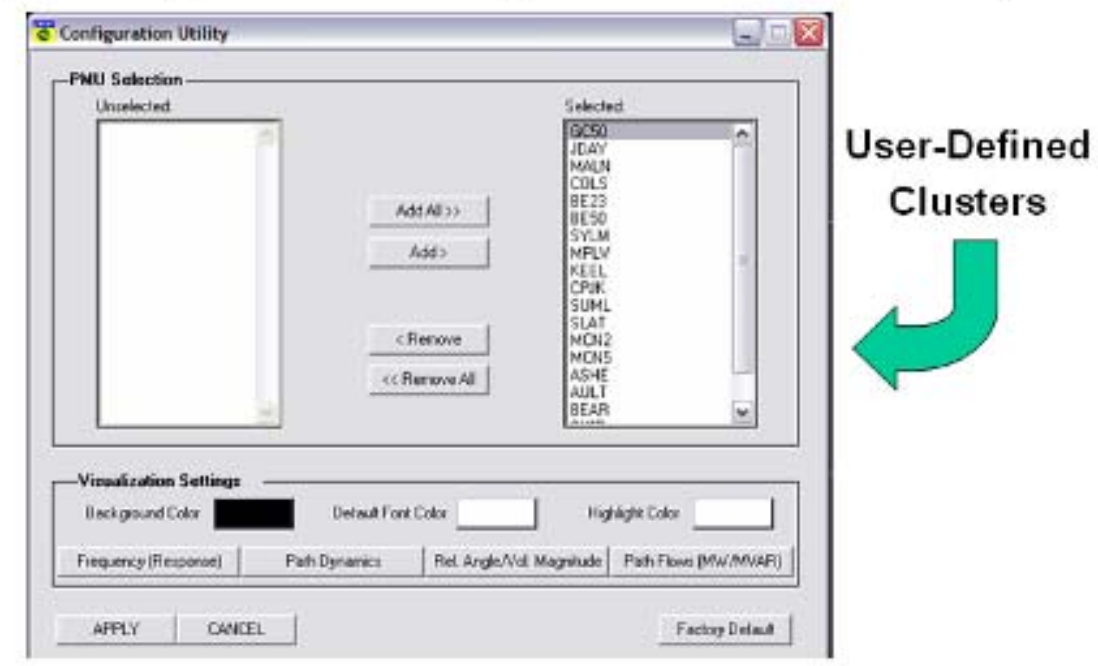

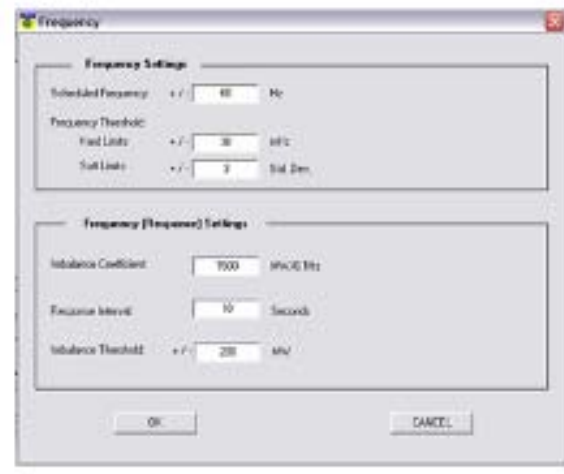

Frequency

Monitoring Display

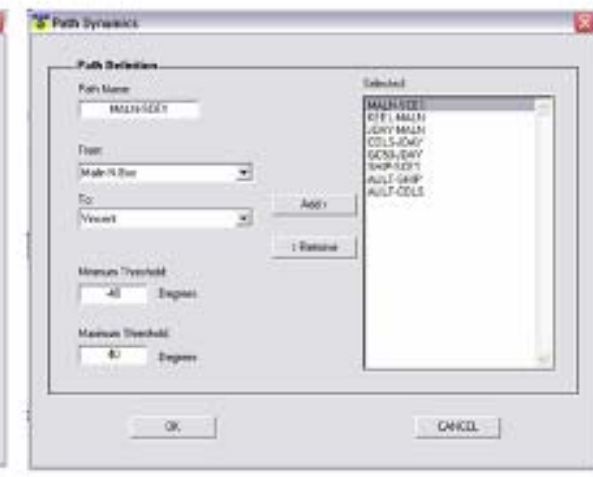

Path Dynamics Display

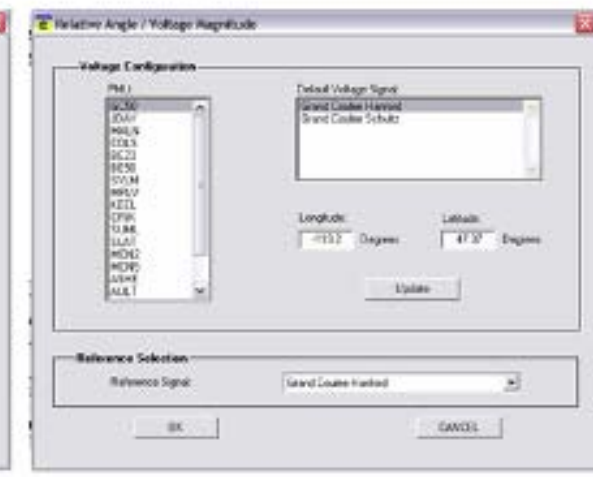

Relative Angle / Voltage Magnitude Display

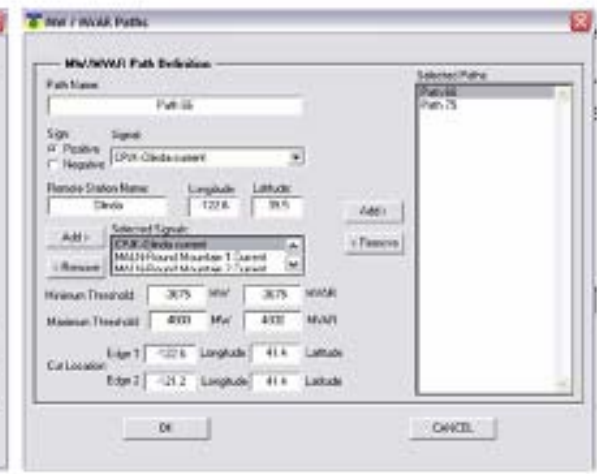

MW / MVAR Path Monitoring Display

䊝 Electric Power Group 


\section{RTDMS Property Editor}

- Change the maps in geographic displays

- Modify font settings of titles, axis labels, legend labels, identifiers, text values etc

- Change graphics Properties Adjust legends - their thresholds and associated colors

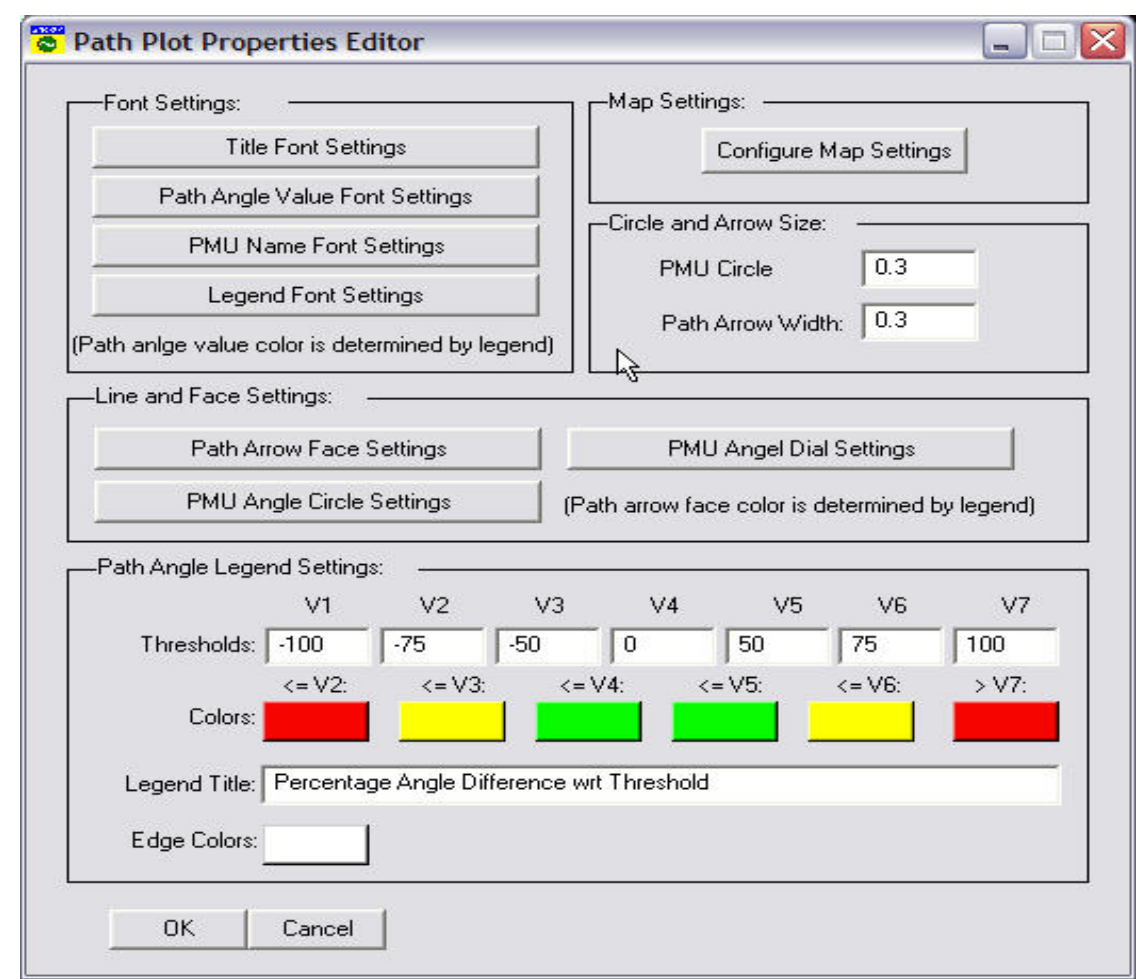




\section{Alarm Logs}

Whenever there are any preset threshold violations (i.e., a new alarm), the "Alarm Logs" tab is

highlighted in Red until the alarm in acknowledged - reappears in Red if a new alarm occurs

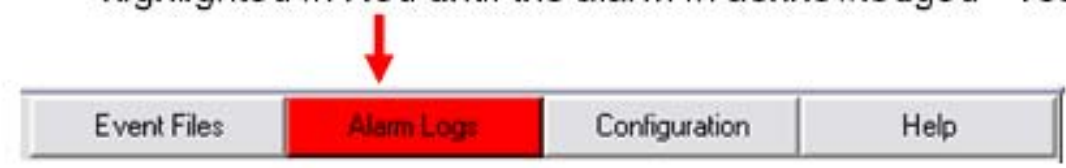

Alarm Log Pop-up Box

Clicking on the "Alarm Logs" tab acknowledges the alarm and the Alarm Log pop-up box appears

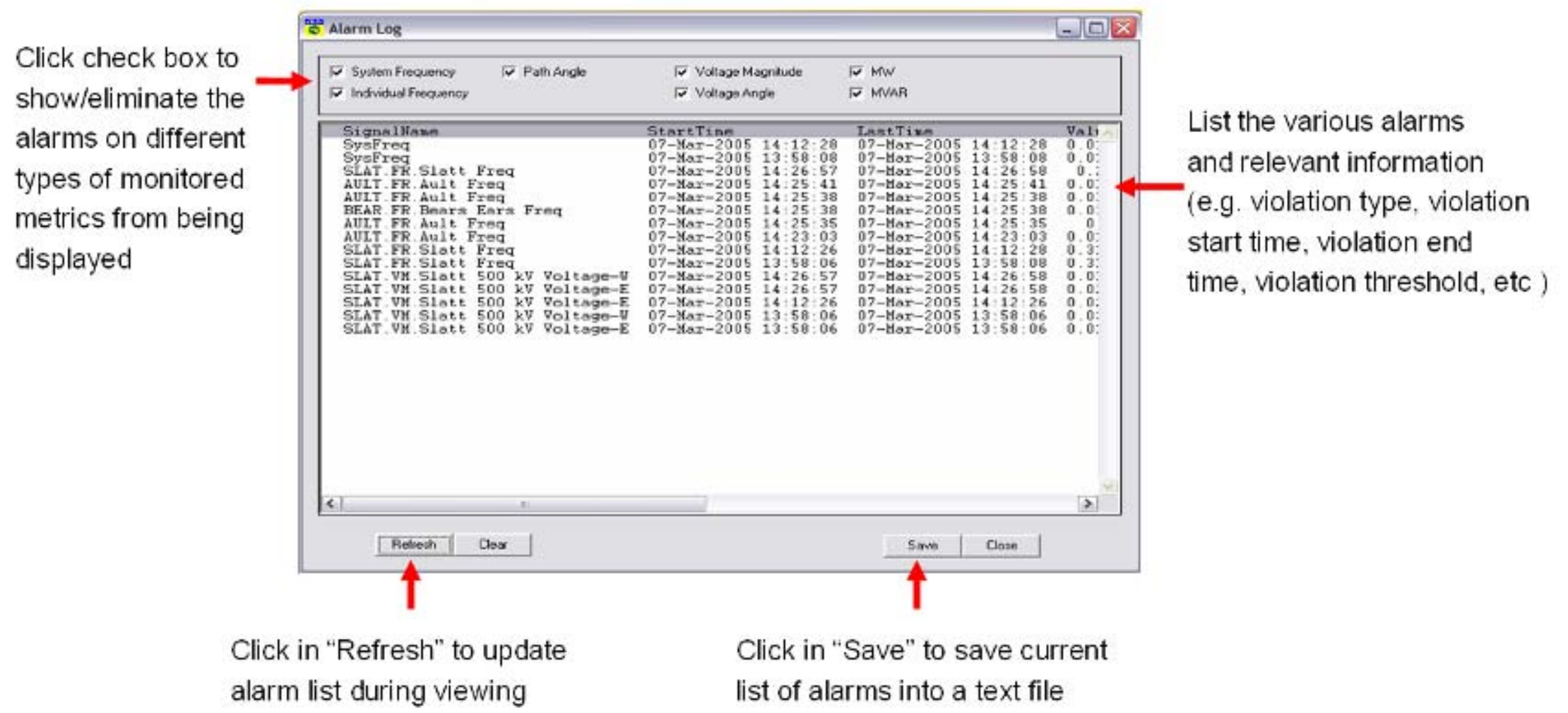

CERTS 


\section{Event Files (RTDMS Offline Analysis)}

Click on "Event Files" tab to load an archived event file into the RTDMS application

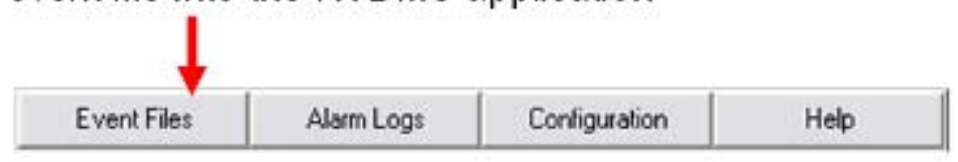

\section{RTDMS in Offline Mode}

Transient in frequency measurements triggered event capture

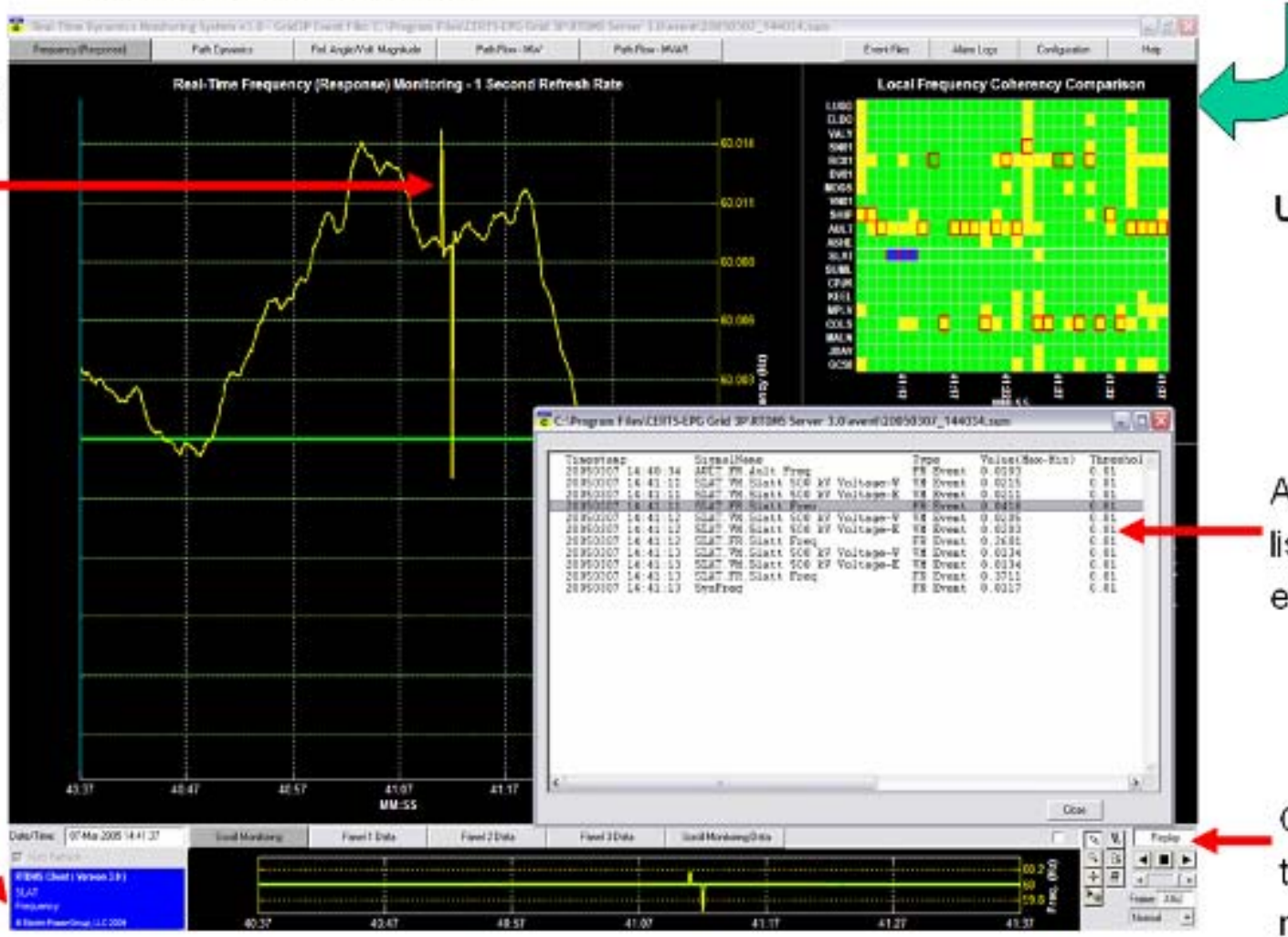

\section{User-Defined}

Clusters

Alarm Summary box

listing all violations during event capture

Title Box with blue background implies RTDMS is running in offline mode

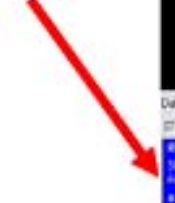

\section{CERTS}

Select an Event File to load into RTDMS for (files labeled by date/time) offline analysis
Click on "Replay" button to switch back to real-time monitoring mode 


\section{RTDMS - Frequency Transient Example}

Alarm log indicating Frequency Transient detected at the Colstrip PMU and
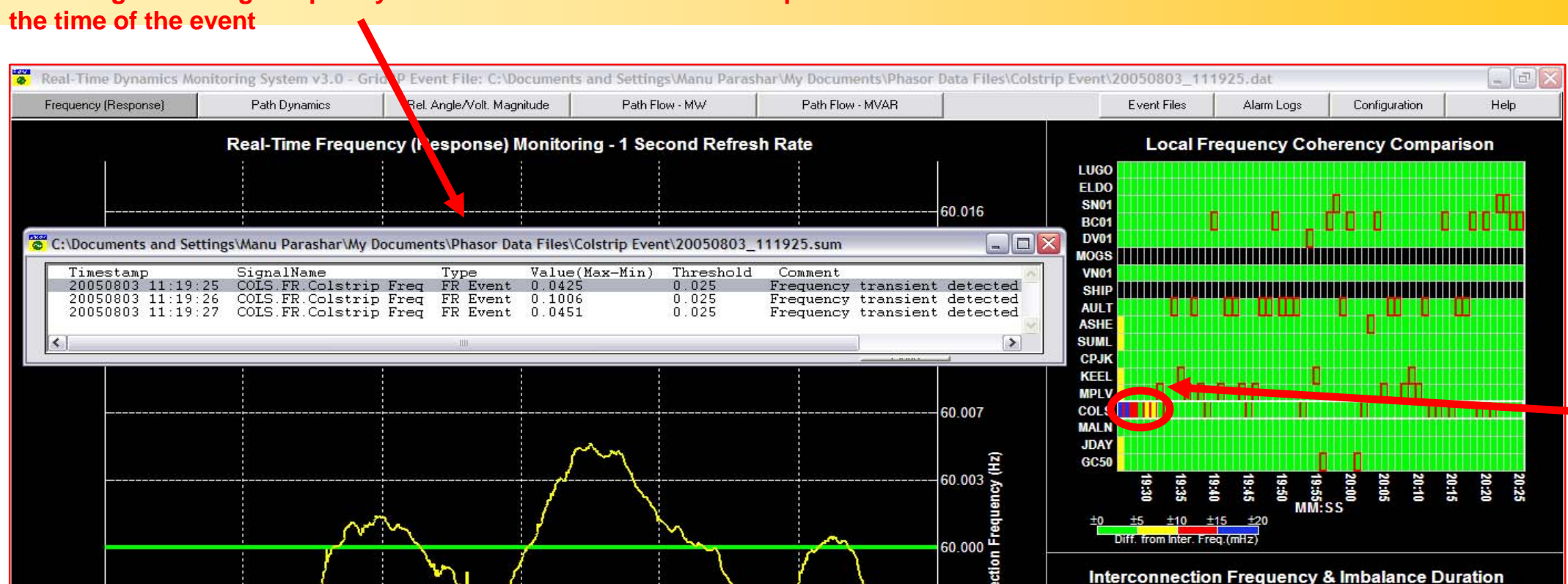

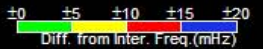

Trip

near

Colstrip

Interconnection Frequency \& Imbalance Duration

PMU
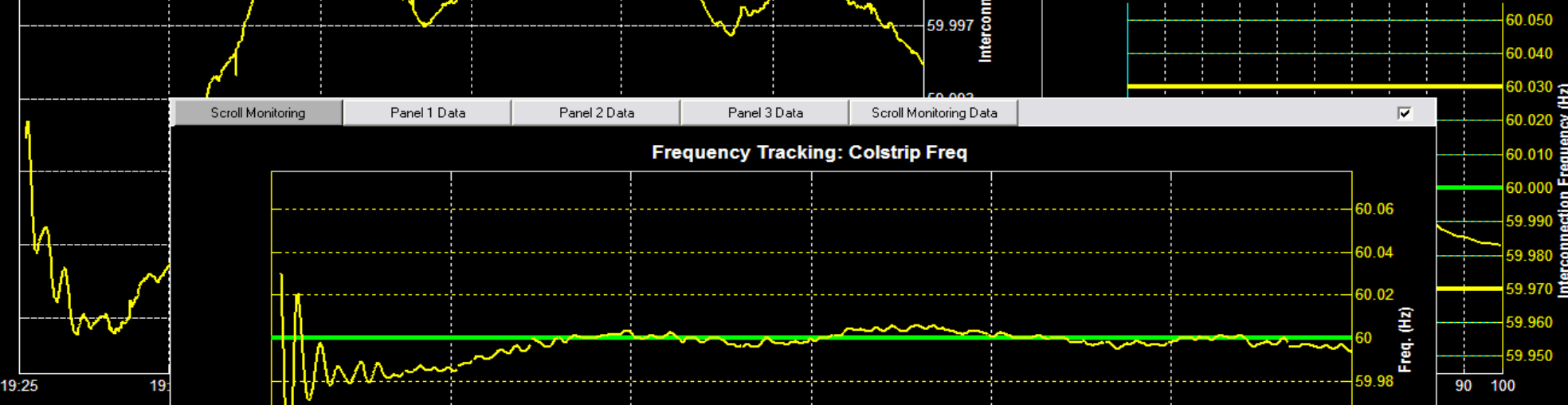

Date/Time: 03-Aug-2005 11:20:25

RTDMS Client ( Version 3.0)

ROLS
Frequency

)
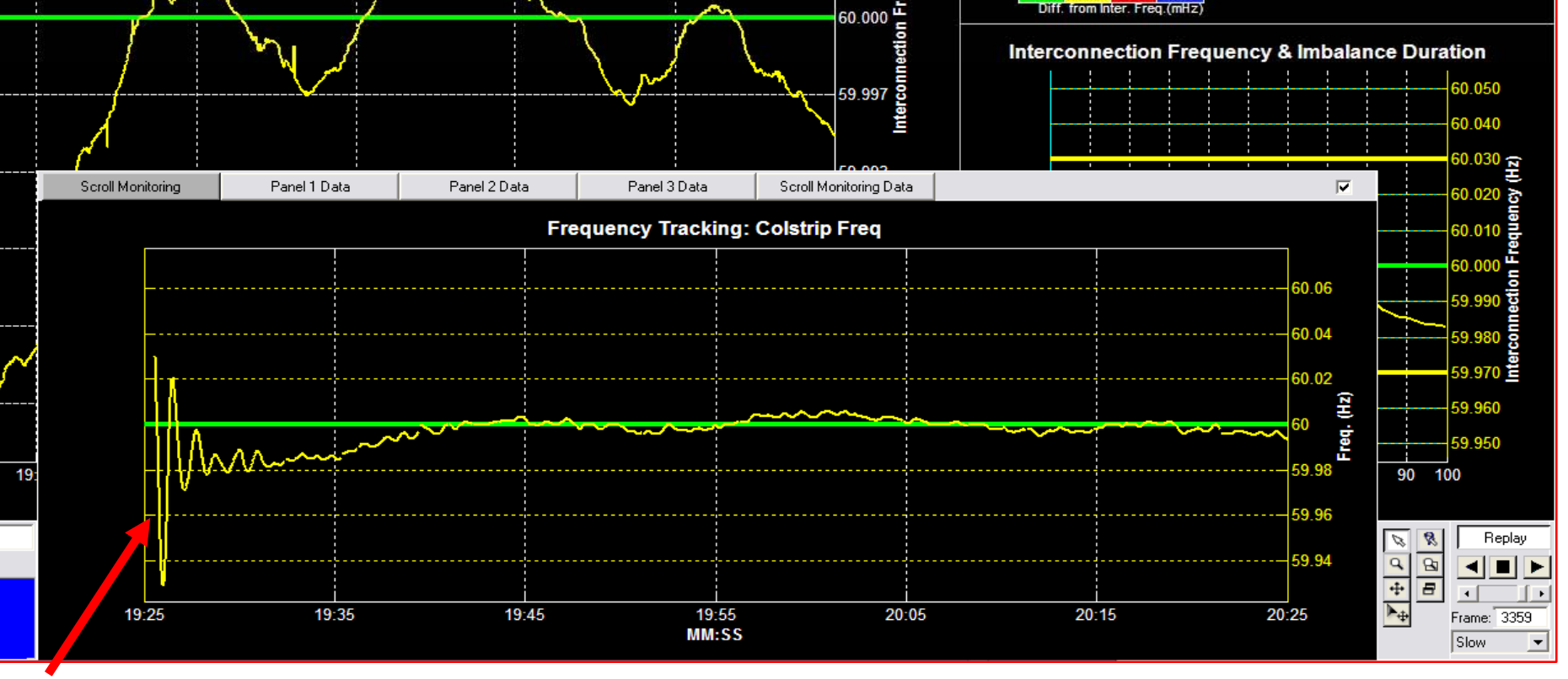

Panel 2 Data

Scroll Monitoring Data

Frequency Tracking: Colstrip Freq

Frequency response at Colstrip PMU CERTS

橉 Electric Power Group 


\section{CA ISO RTDMS Functionalities Summary}

RTDMS Functionalities at CA ISO:

$>$ Includes BPA, SCE, WAPA, and PGE phasor data shown in real-time

$>$ Server-Client architecture (Multi-user capability)

$>$ Geographic visualization on Voltages, Angle Differences, Frequency, MW \& MVAR

$>$ End user configurability

$>$ Replay capability

$>$ Real-time alarming and event detection

$>$ Event archiving and playback 


\section{System Support and Help Tab}




\section{Help Tab}

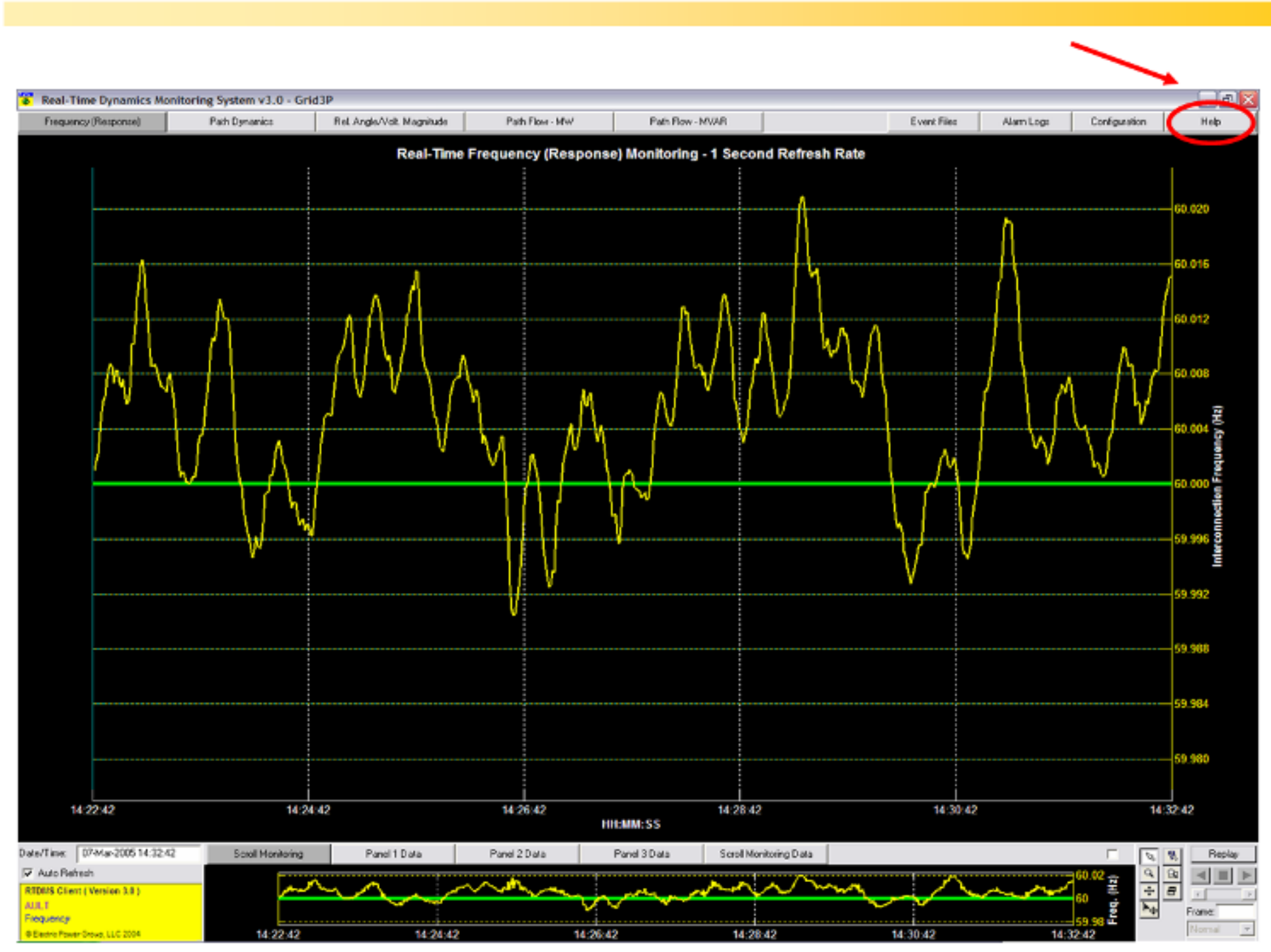

CERTS 


\section{Help Overview}

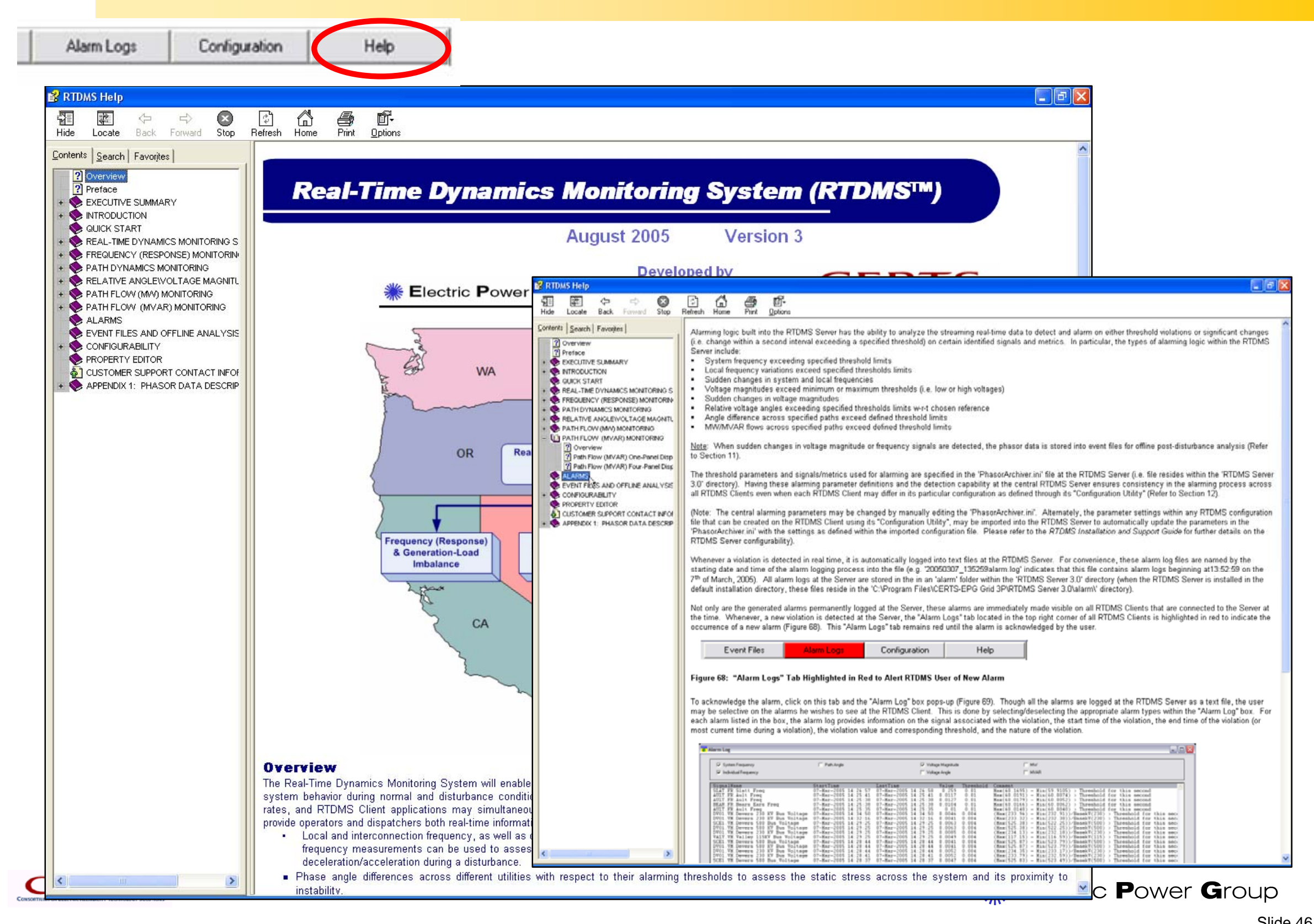




\section{Contact Information}

Manu Parashar

Office: (626) 685-2015 ext 130

parashar@electricpowergroup.com 


\section{Any Questions Before We Move On To The Demo?}




\section{Start RTDMS Client Application}

Phasor

10

CAISO RTDMS Client 3.0

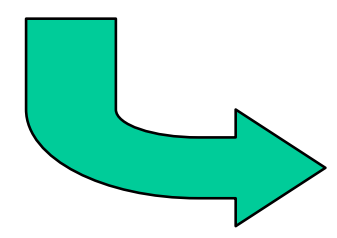

\section{Frequency (Response) Monitoring Display (default)}

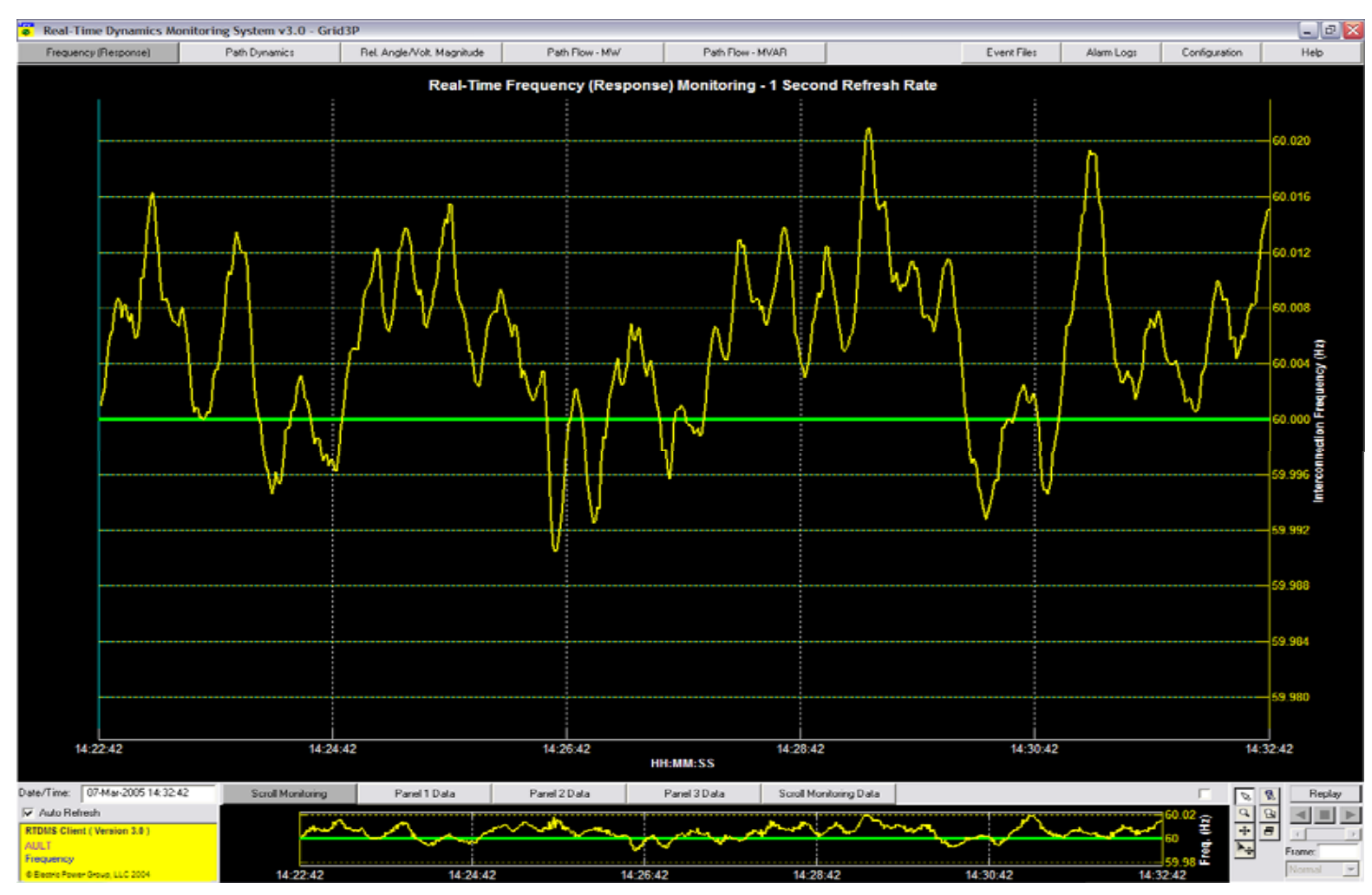




\section{Frequency Monitoring Display}

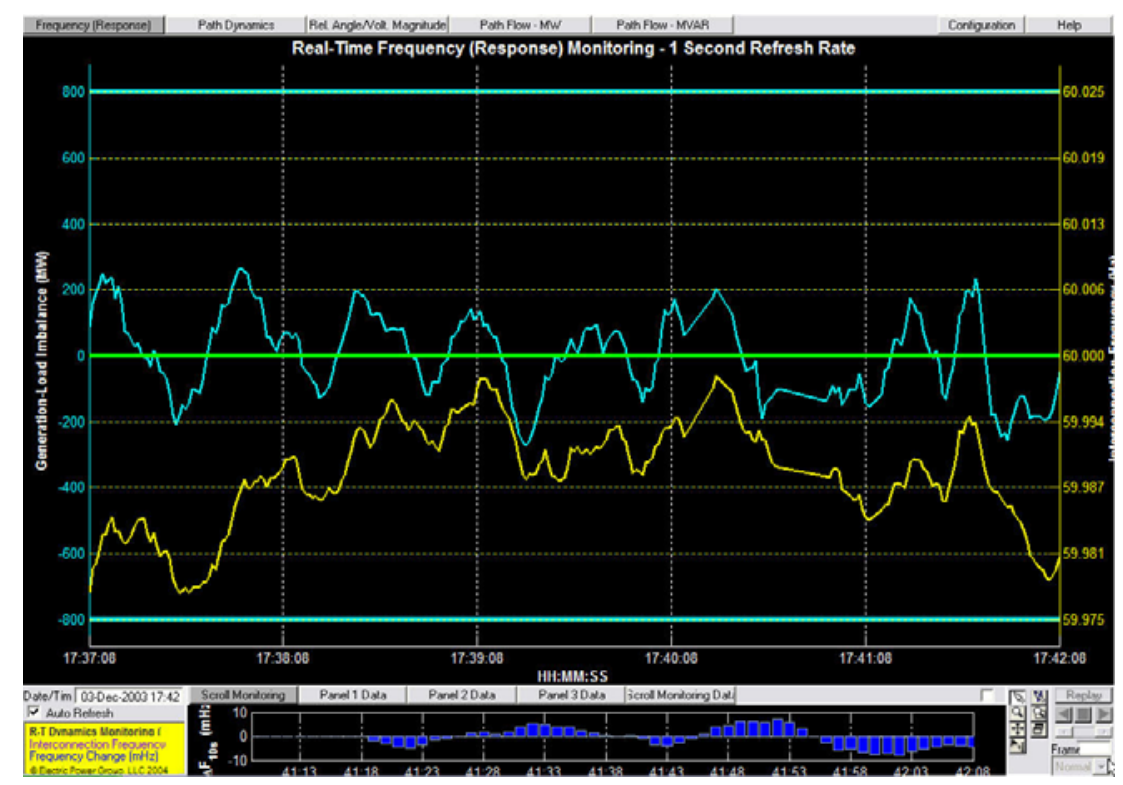

1-Panel Visual

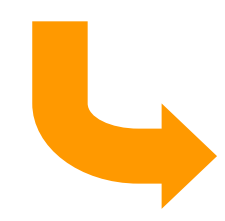

4-Panel Visual

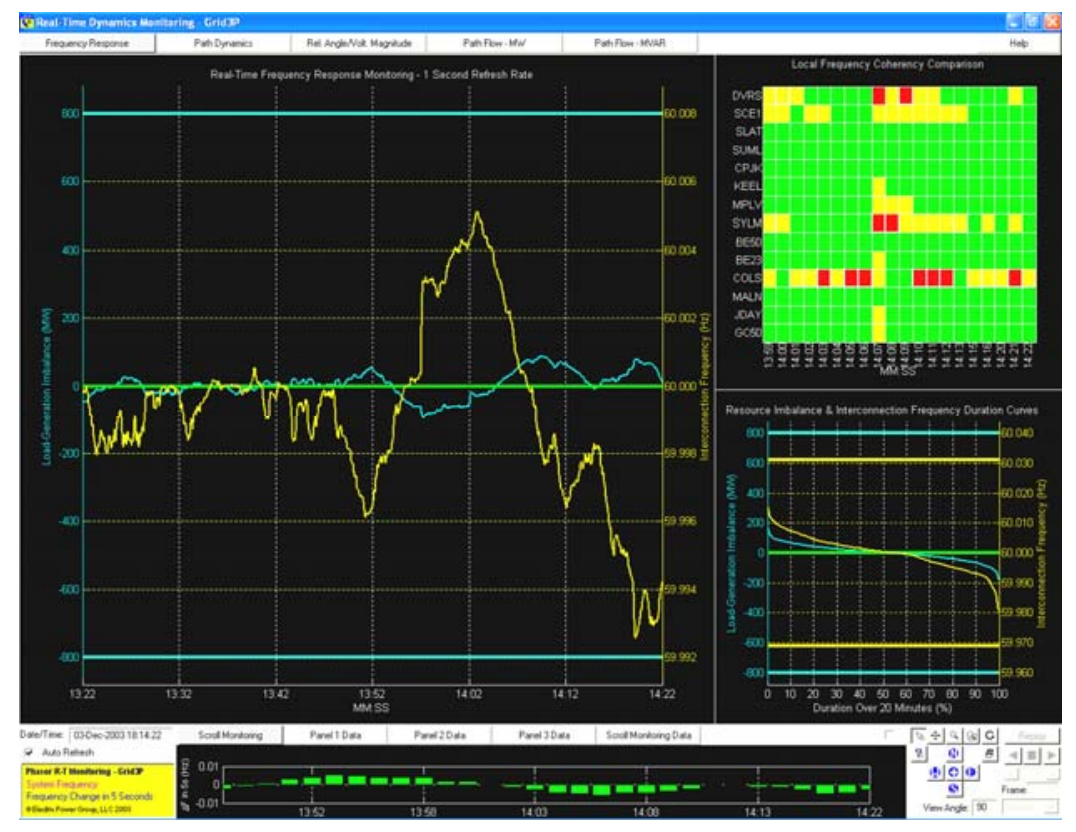

CERTS 


\section{Path Dynamics Monitoring Display \\ - Local and Wide-Area Views}

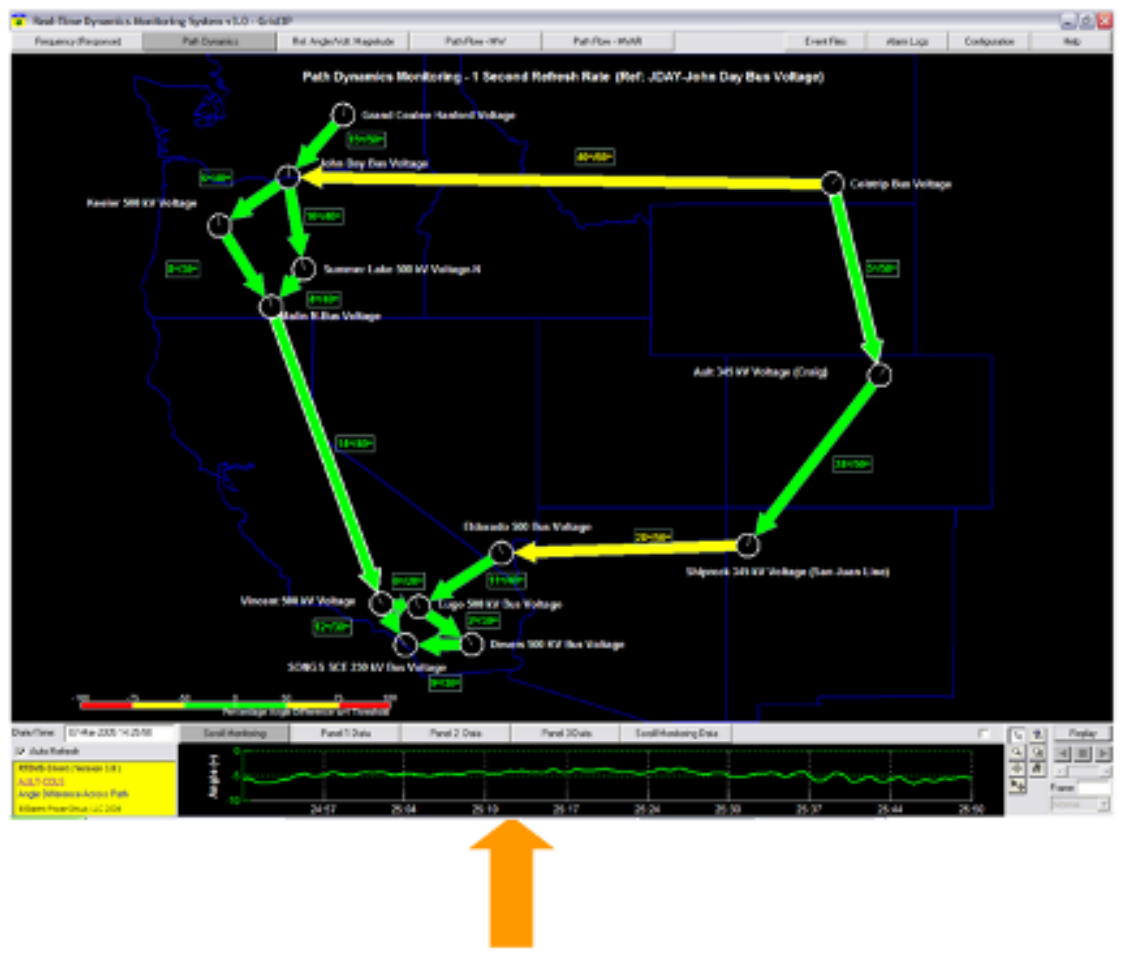

One Panel View

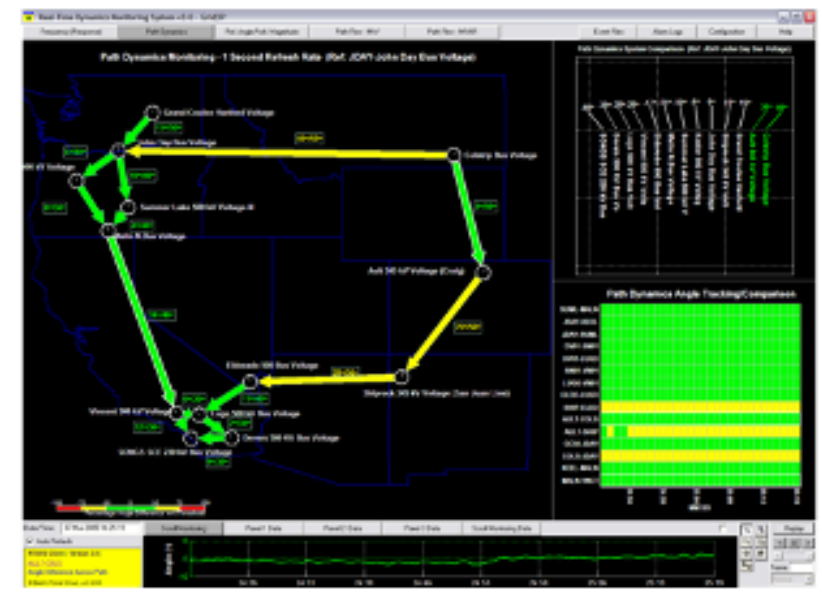

Wide-Area View
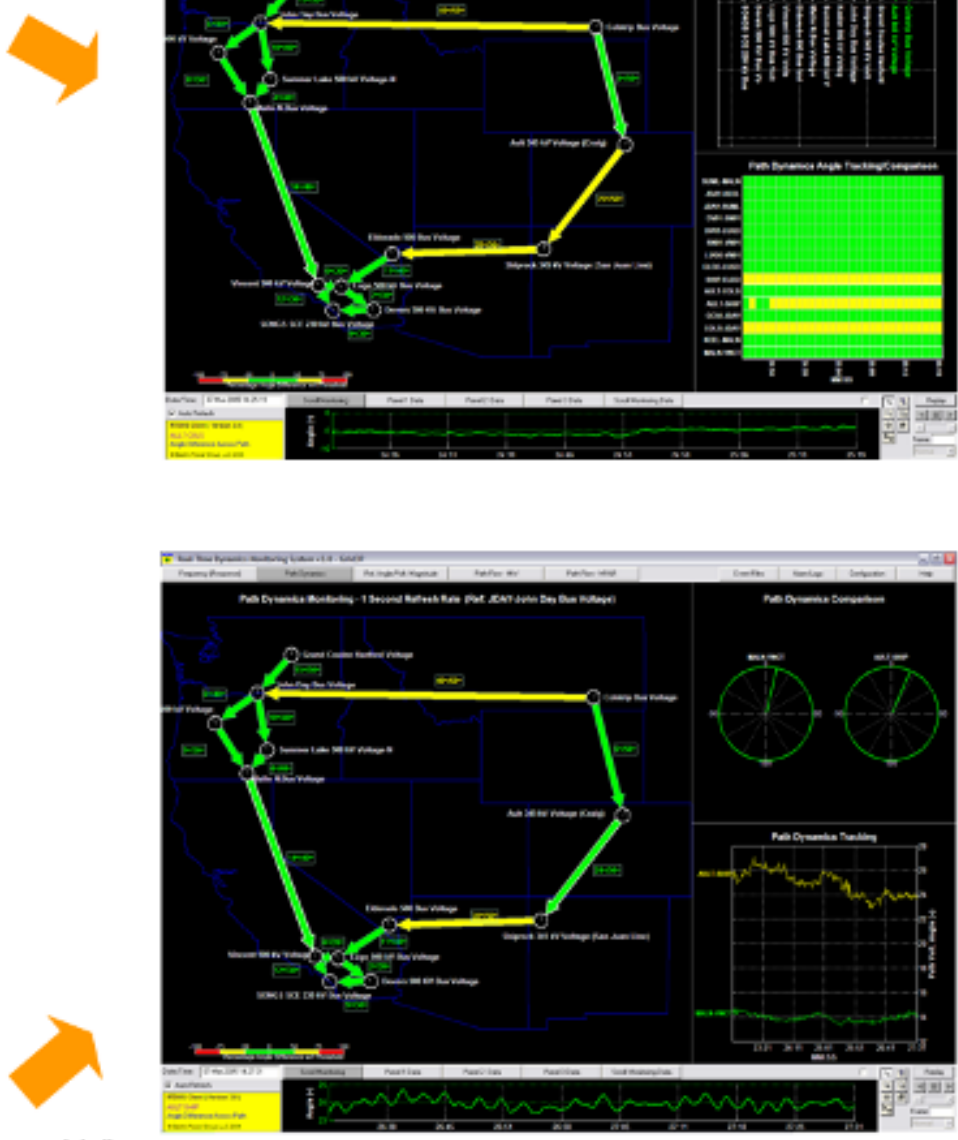


\section{Relative Angle/Voltage Magnitude Monitoring Display \\ - Local and Wide-Area Views}

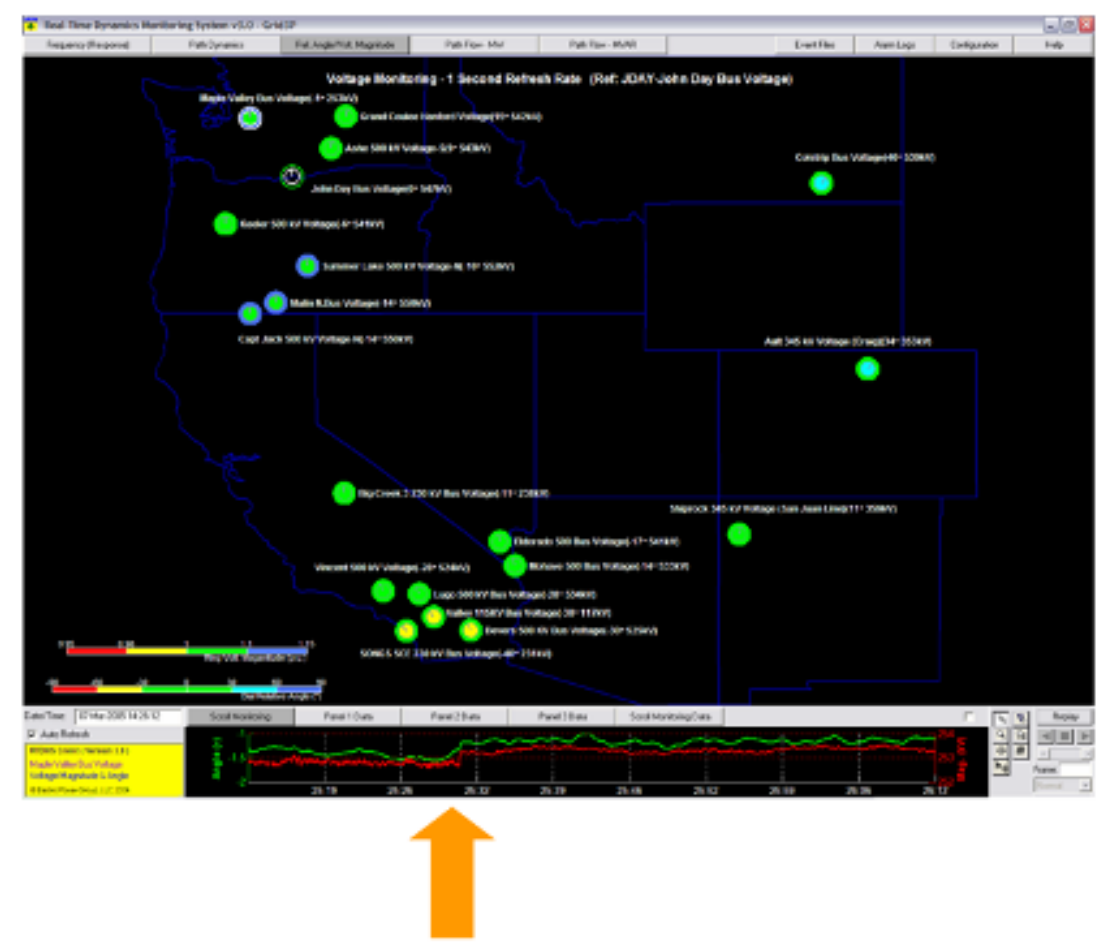

One Panel View
Wide-Area View
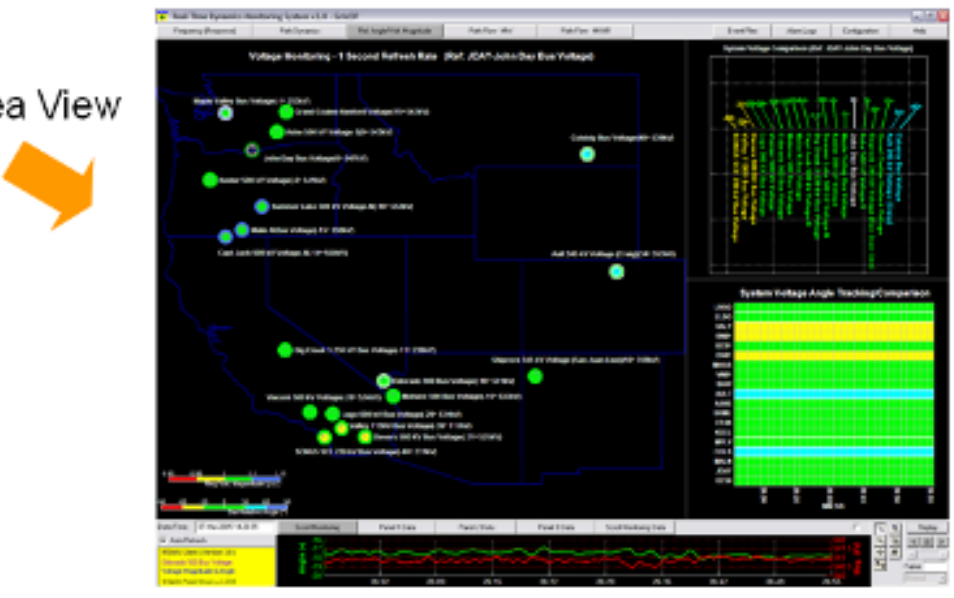

Local View

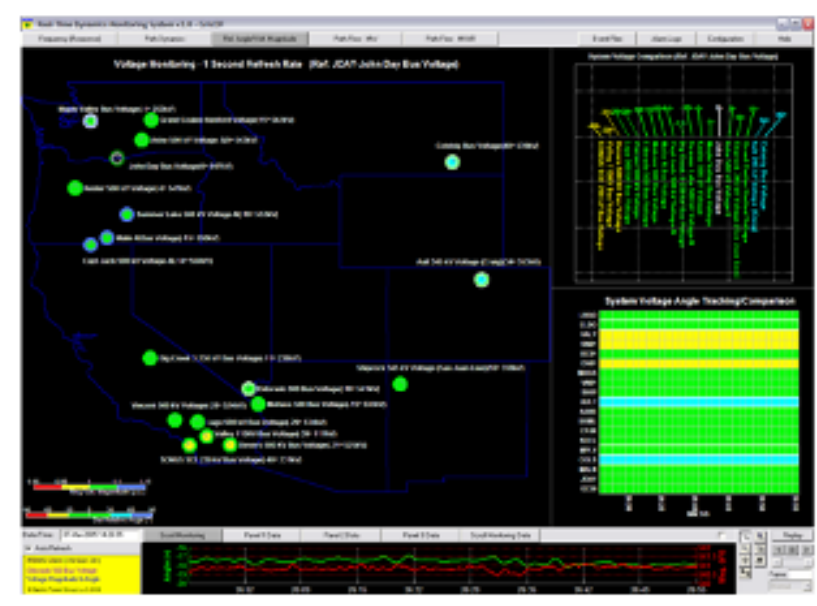

CERTS

䊝 Electric Power Group 


\section{MW and MVAR Monitoring Displays}

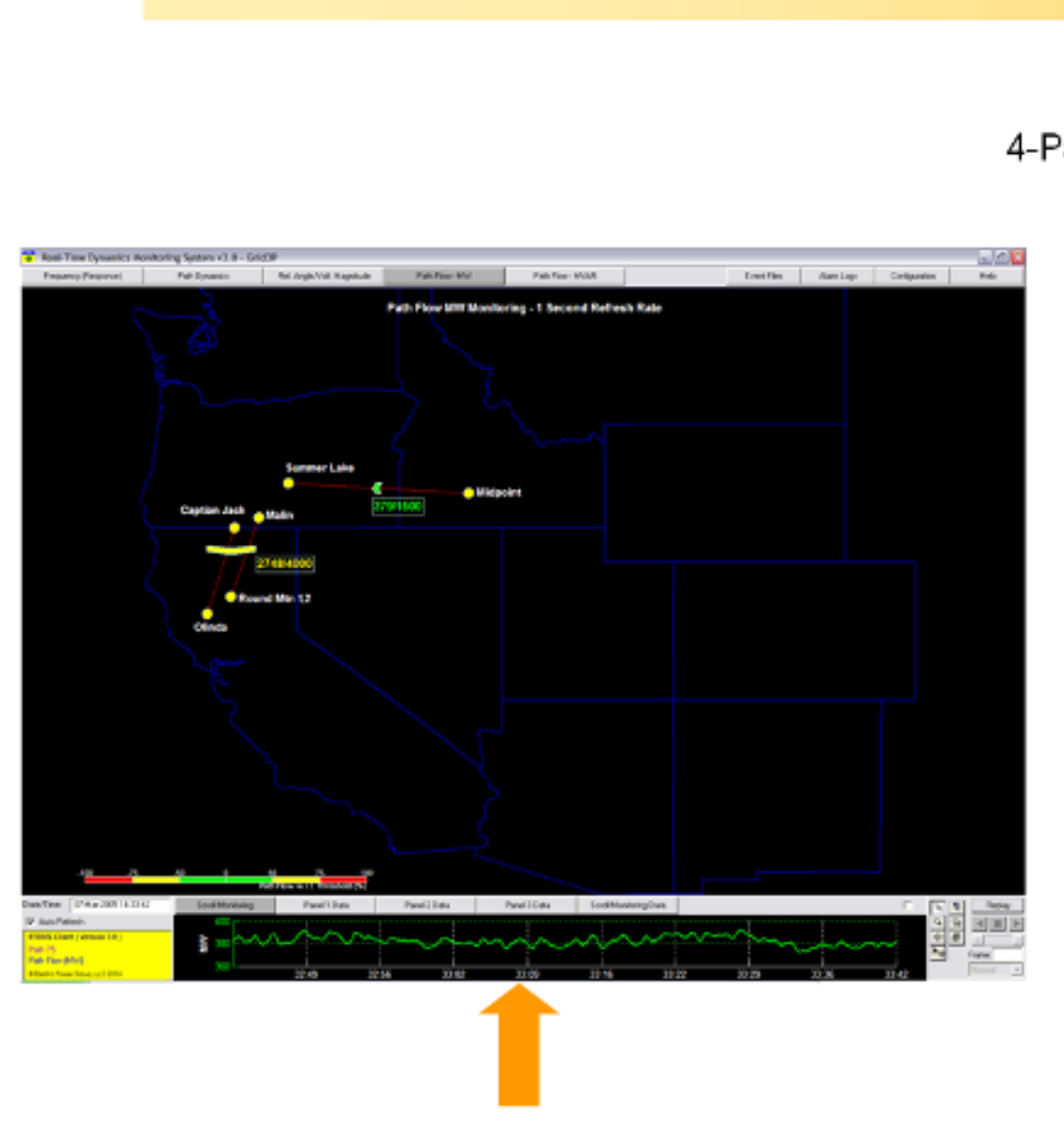

One Panel MW/MVAR
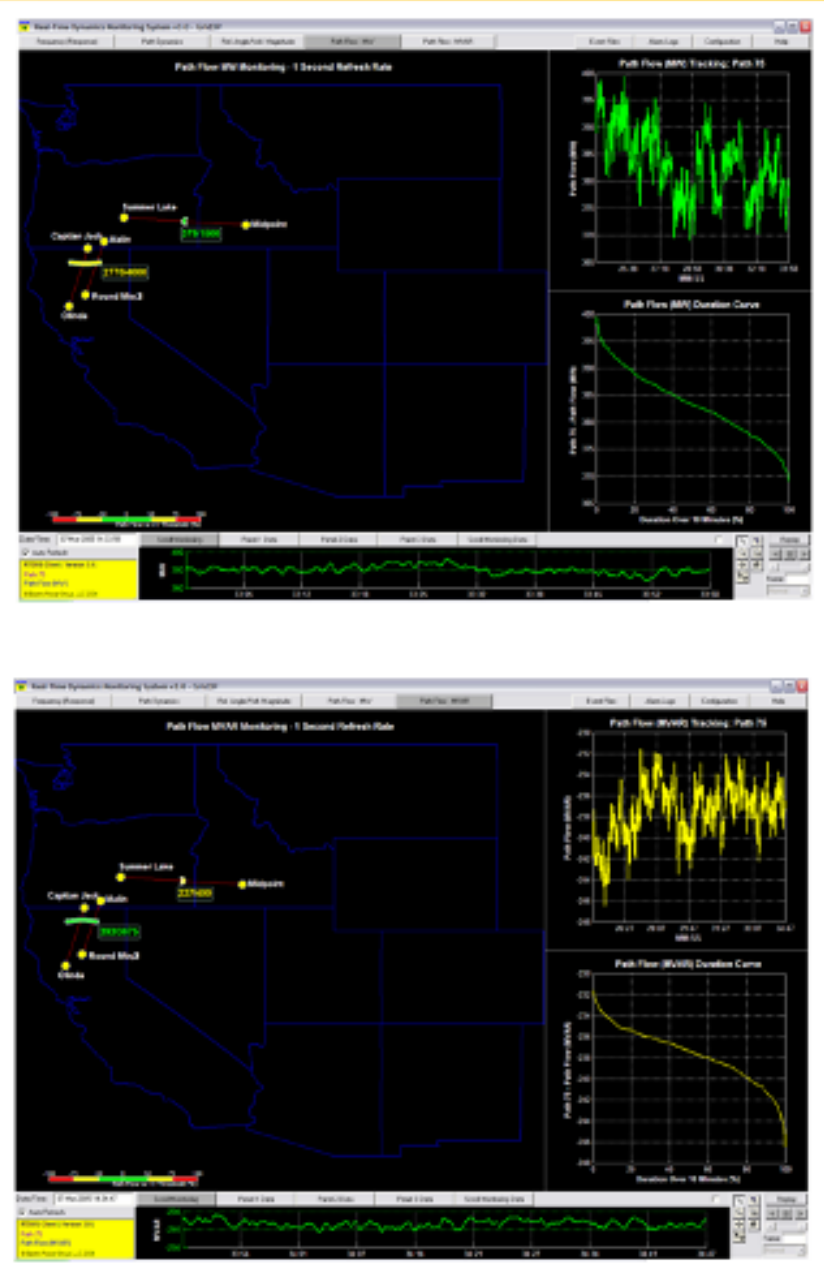

4-Panel View MVAR 


\section{RTDMS Version 4.0 - End of 1 Quarter 2006}




\section{RTDMS DASHBOARD SUMMARY}

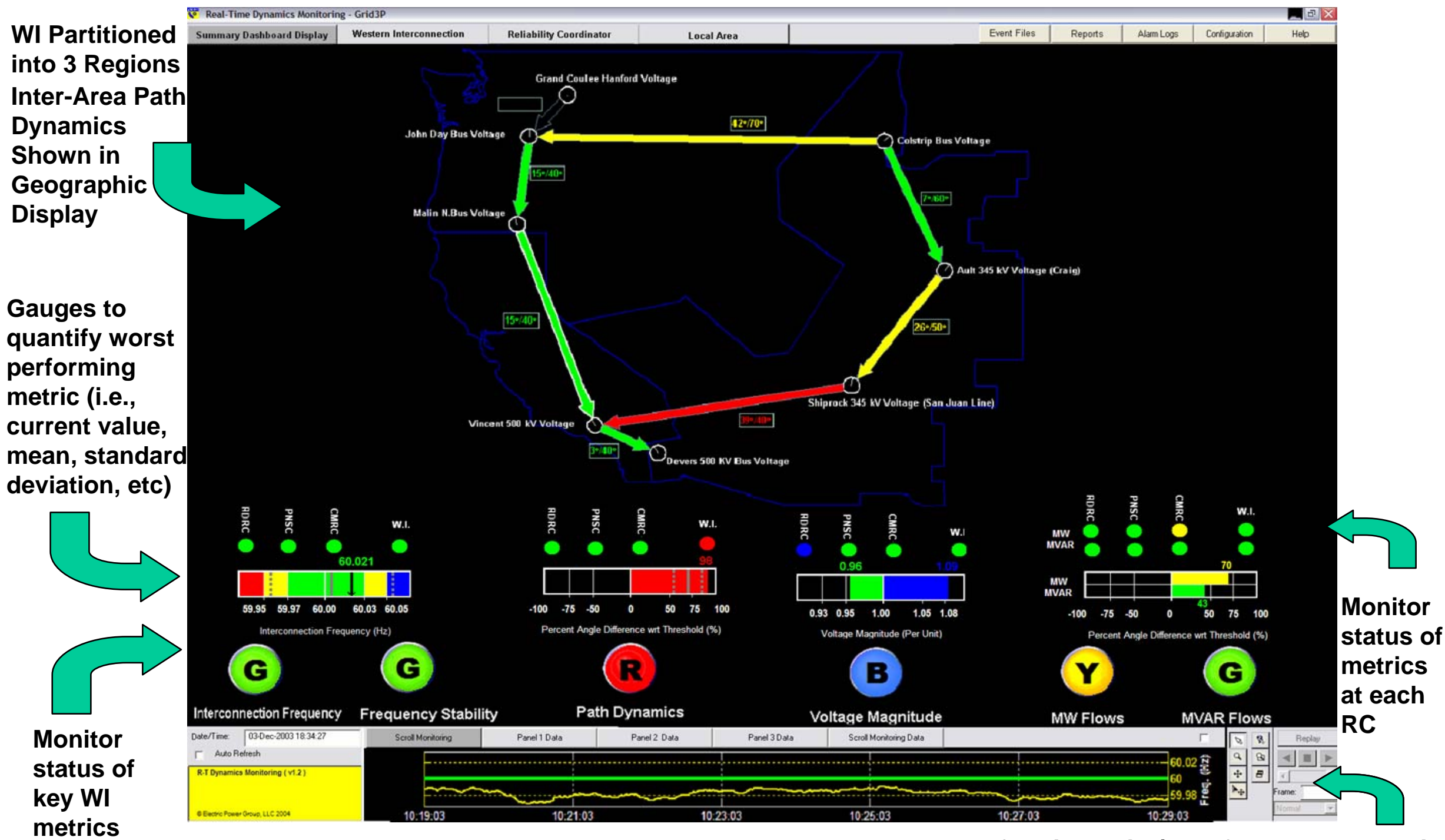

CERTS

Track worst performing point/path for selected metric 溇 Electric Power Group 


\section{RTDMS PMU PERFORMANCE MONITORING}

$>$ Real-time PMU status information provided within RTDMS displays

Historical PMU performance shown as charts/tables:

\section{PMU Data Unavailability Chart}

Friday, September 16, 2005 11:25 AM PST

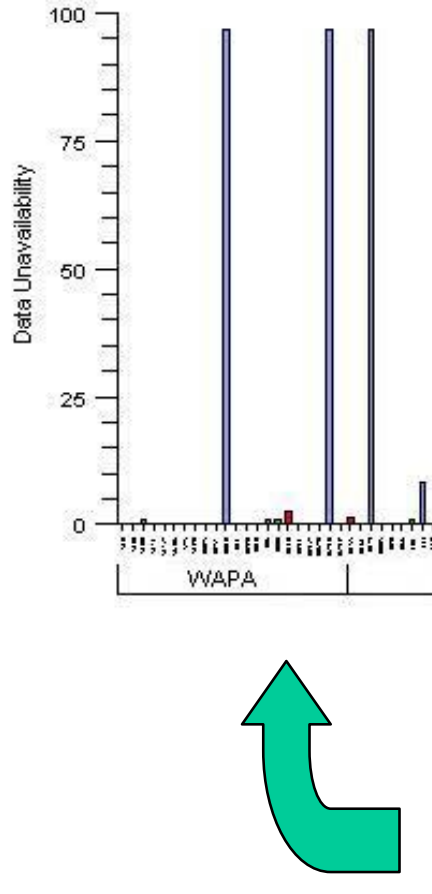

\section{$\square$ Drop out}

$\square$ Trans. Error

$\square$ Sync Error

\section{PMU Data Unavailability Report}

Friday, September 16, 2005 11:25 AM PST

\begin{tabular}{|c|c|c|c|c|c|c|}
\hline \multirow{2}{*}{\multicolumn{2}{|c|}{ PMU Name }} & \multirow{2}{*}{$\begin{array}{l}\text { Available } \\
(\%)\end{array}$} & \multicolumn{3}{|c|}{ Unavailable (\%) } & \multirow{2}{*}{$\begin{array}{c}\text { Contact } \\
\text { Information }\end{array}$} \\
\hline & & & $\begin{array}{c}\text { Drop } \\
\text { out }\end{array}$ & $\begin{array}{l}\text { Trans. } \\
\text { Error }\end{array}$ & $\begin{array}{l}\text { Sync. } \\
\text { Error }\end{array}$ & \\
\hline \multirow{5}{*}{$\frac{\pi}{3}$} & $\begin{array}{l}\text { WAPA } \\
\text { PMU1 }\end{array}$ & 99.10 & 0.70 & $\begin{array}{r}0.90 \\
0.20\end{array}$ & 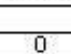 & (123) 456-7890 \\
\hline & WAPA & \multirow{2}{*}{0} & \multicolumn{3}{|c|}{100.00} & \multirow{2}{*}{ (123) 456-7890 } \\
\hline & PMU2 & & 100.00 & 0 & 0 & \\
\hline & WAPA & \multirow{2}{*}{98.00} & \multicolumn{3}{|c|}{2.00} & \multirow{2}{*}{ (123) 456-7890 } \\
\hline & & & 1.00 & 0 & 1.00 & \\
\hline \multirow{7}{*}{$\begin{array}{l}\text { 嚍 } \\
\end{array}$} & \multirow{2}{*}{ BPA PMU1 } & \multirow{2}{*}{100} & \multicolumn{3}{|c|}{0} & \multirow{2}{*}{ (123) 456-7890 } \\
\hline & & & 0 & 0 & 0 & \\
\hline & BPA. PMU2 & 100 & 0 & 0 & 0 & (123) 456-7890 \\
\hline & BPA $\mathrm{PM} / 3$ & \multirow[b]{2}{*}{0} & & 100.0 & & \multirow{2}{*}{ (123) 456-7890 } \\
\hline & GPA FIMUS & & 100.00 & 0 & 0 & \\
\hline & \multirow{2}{*}{ BPA. PMU4 } & \multirow{2}{*}{65.00} & \multicolumn{3}{|c|}{35.00} & \multirow{2}{*}{ (123) 456-7890 } \\
\hline & & & 34.00 & 0 & 1.00 & \\
\hline
\end{tabular}

Plots and Tables indicate data unavailability by:

'Dropouts', 'Transmission Errors', 'Synchronization Errors'

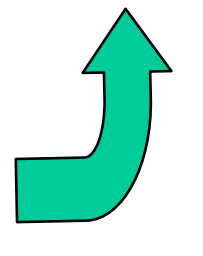




\section{RTDMS TRENDING AND REPORTING}
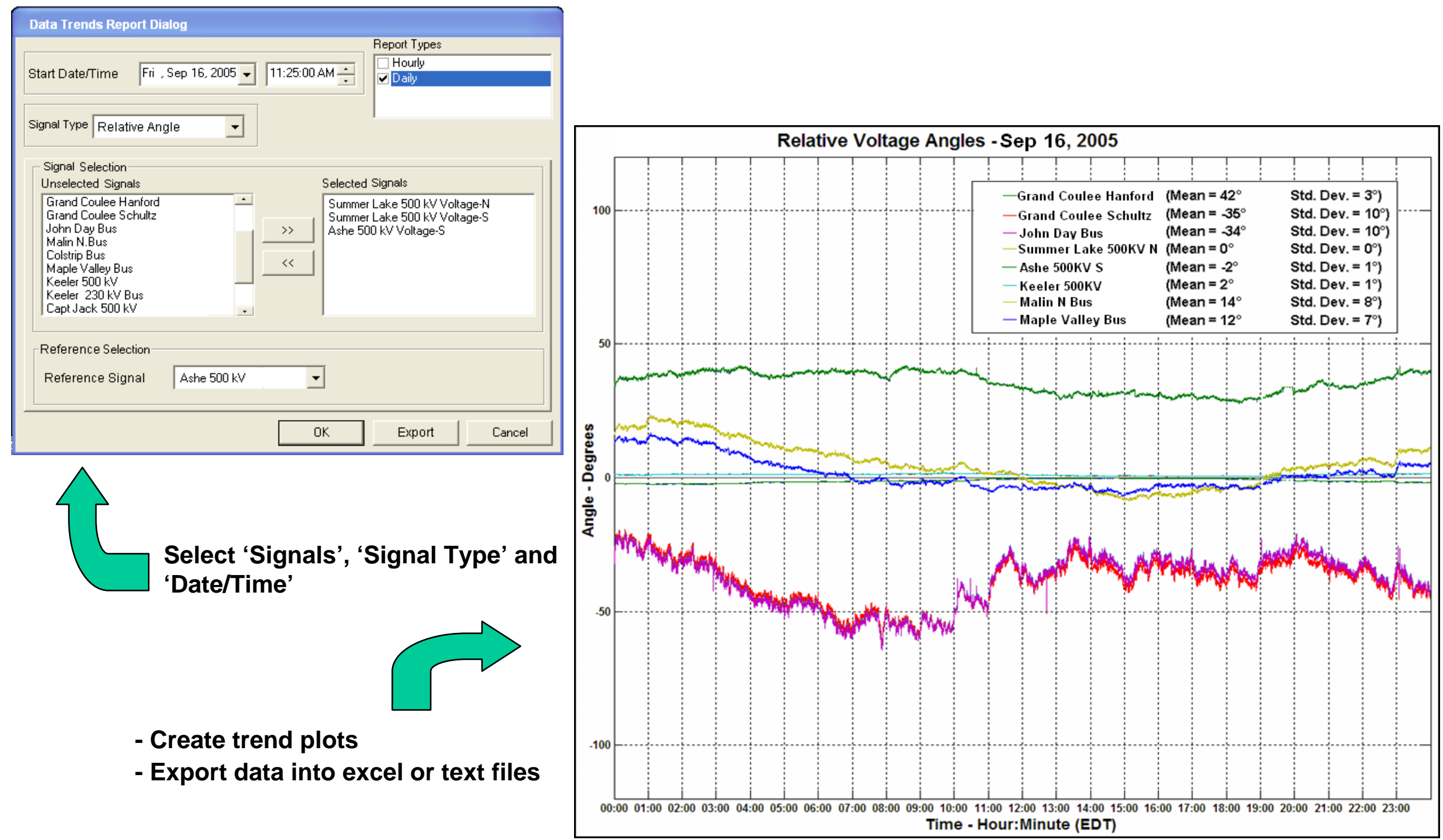

- Create trend plots

- Export data into excel or text files

Time - Hour:Minute (EDT)

CERTS

潾 Electric Power Group 
Appendix F

Task 3.0 Prototype Phasor-Based Real-Time Monitoring Software Tool Users Guide 


\section{Appendix G}

Deliverable Task 4.0 Scoping Study Report on Improving Load and Generator Response Models 
LBNL-59192

\title{
Improving Dynamic Load and Generator Response Performance Tools
}

\author{
Prepared for the \\ Public Interest Energy Research \\ California Energy Commission \\ Principal Author \\ Bernard C. Lesieutre \\ Ernest Orlando Lawrence Berkeley National Laboratory \\ 1 Cyclotron Road, MS 90R4000 \\ Berkeley CA 94720-8136
}

November 2005

The work described in this report was coordinated by the Consortium for Electric Reliability Technology Solutions and was funded by the California Energy Commission's Public Interest Energy Research Program through the University of California/California Institute for Energy Efficiency under Work for Others Contract No. 500-02-004. The Lawrence Berkeley National Laboratory is operated under U.S. Department of Energy Contract No. DE-AC02-05CH11231. 



\section{LEGAL NOTICE:}

This report was prepared as a result of work sponsored by the California Energy Commission (Energy Commission) and the University of California (UC). It does not necessarily represent the views of the Energy Commission, UC, their employees, or the State of California. The Energy Commission, the State of California, its employees, and EC make no warranty, express or implied, and assume no legal liability for the information in this report; nor does any party represent that the use of this information will not infringe upon privately owned rights. This report has not been approved or disapproved by the Energy Commission or UC, nor has the Energy Commission or UC passed upon the accuracy or adequacy of the information in the report.

The submitted manuscript has been authored by a contractor to the Regents of the University of California/California Institute for Energy and Environment. Accordingly, The Regents retains a non-exclusive, royalty-free license to publish or reproduce the published form of this contribution, for allow others to do so, for CIEE's purposes. 



\section{Table of Contents}

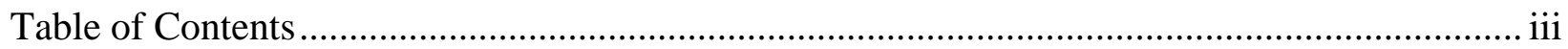

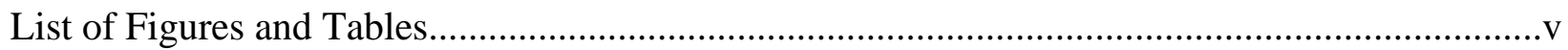

Acronyms and Abbreviations ............................................................................................... vii

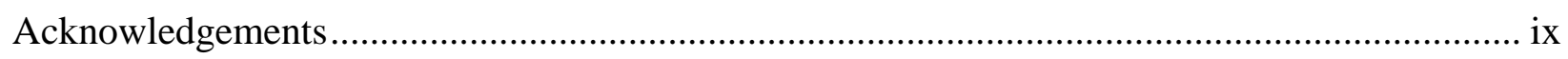

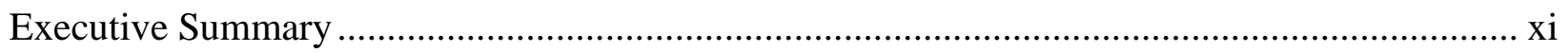

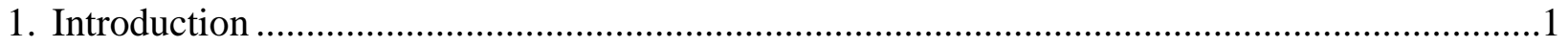

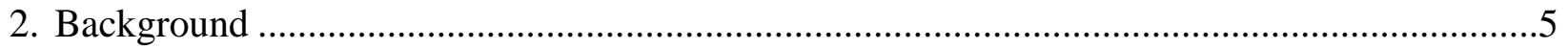

2.1 Application of Dynamic Models...................................................................................

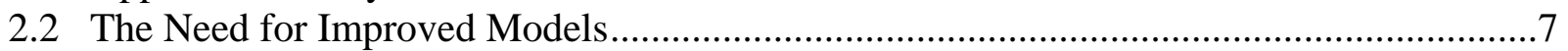

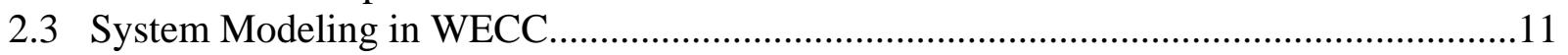

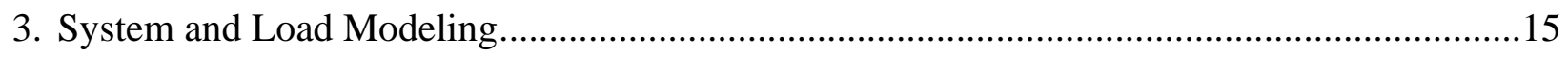

3.1 WECC Modeling Process …………………………................................................15

3.2 Load Modeling ..............................................................................................................17

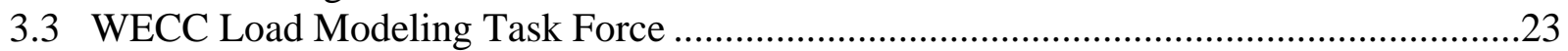

3.3.1 Direction of recommended load model......................................................................23

3.3.2 The load modeling process: Survey and Aggregation. .............................................26

3.4 Load Modeling Needs...............................................................................................2

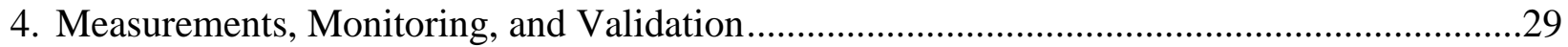

4.1 Measurements and System Dynamic Monitoring...........................................................29

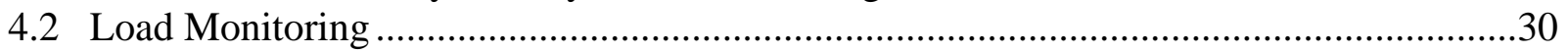

4.3 Measurement-Only Load Models .........................................................................................34

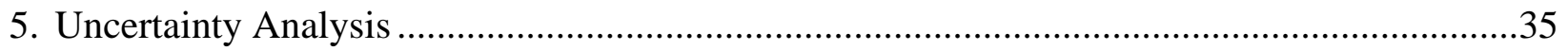

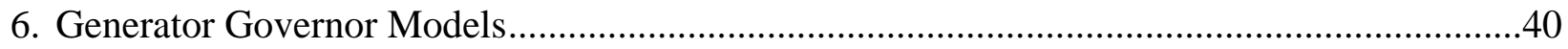

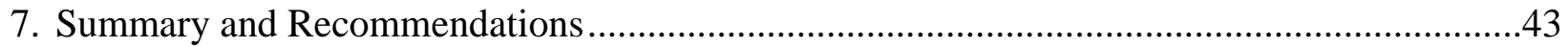

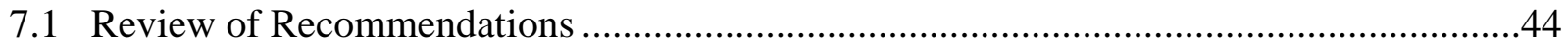

7.1.1 Load Model Development and Policies ....................................................................4

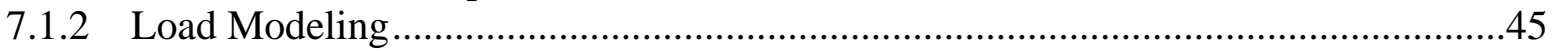

7.1.3 Measurement and Validation .................................................................................

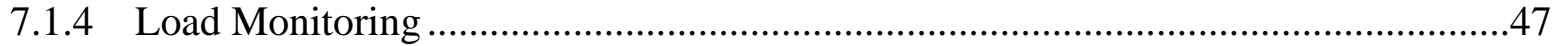

7.1.5 Measurement-Only (Black-Box) Models …………..................................................4

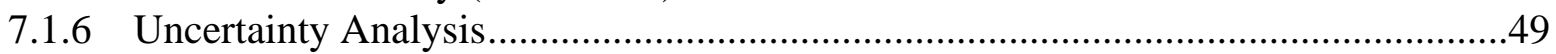

7.1.7 Generator Governor Models .............................................................................49

7.2 Summary table of recommendations ……………........................................................50 
Improving Dynamic Load and Generator Response Performance Tools

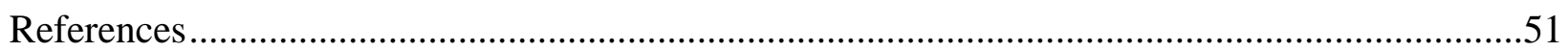




\section{List of Figures and Tables}

Figure 2-1. June 6, 2002 event. (courtesy of D. Kosterev) .................................................... 9

Figure 2-2. July 2004 Valley event . (courtesy of G. Chinn).................................................. 10

Figure 2-3 Simulation of the July 2004 Valley event using the standard model. Note that the voltage recovery is immediate after the fault is cleared. (courtesy of G. Chinn)............ 10

Figure 2-4. Organization chart for the Planning Coordination Committee ................................ 13

Figure 3-1 The introduction of a stylized distribution network.............................................. 24

Figure 3-2. An aggregate load model in which three representative motors are employed. ....... 25

Figure 4-1 Recorded and simulated response to a disturbance at a paper mill (courtesy D. Kosterev). 



\section{Acronyms and Abbreviations}

$\begin{array}{ll}\text { ACE } & \text { Area Control Error } \\ \text { AVR } & \text { Automatic Voltage Regulator } \\ \text { BPA } & \text { Bonneville Power Administration } \\ \text { CAISO } & \text { California Independent System Operator } \\ \text { CERTS } & \text { Consortium for Electric Reliability Technology Solutions } \\ \text { CIEE } & \text { California Institute for Energy Efficiency } \\ \text { DMWG } & \text { Disturbance Monitoring Working Group (WECC) } \\ \text { IEEE } & \text { Institute for Electrical and Electronic Engineers } \\ \text { HVAC } & \text { Heating, Ventilation, and Air Conditioning } \\ \text { LMTF } & \text { Load Modeling Task Force (WECC) } \\ \text { LTC } & \text { Load Tap Changing transformer } \\ \text { MIT } & \text { Massachusetts Institute of Technology } \\ \text { NERC } & \text { North American Electric Reliability Council } \\ \text { NILM } & \text { Non-Intrusive Load Monitor } \\ \text { PCM } & \text { Probabilistic Collocation Method } \\ \text { PIER } & \text { Public Interest Energy Research } \\ \text { PNM } & \text { Public Service Company of New Mexico } \\ \text { PSS } & \text { Power System Stabilizer } \\ \text { SCADA } & \text { Supervisory Control and Data Acquisition } \\ \text { SCE } & \text { Southern California Edison } \\ \text { WECC } & \text { Western Electricity Coordinating Council } \\ \text { WSCC } & \text { Western Systems Coordinating Council } \\ \text { ZIP } & \text { Constant Impedance (Z), Current (I), and Power (P) load model } \\ \end{array}$





\section{Acknowledgements}

The author would like to thank Merwin Brown, Jim Cole, and Virgil Rose of CIEE, and Joe Eto of LBNL for their support of this activity. I would like to thank the many people who have helped us gather information for this report, including Baj Agrawal, Jim Gronquist, Gerald Keenan, Dmitry Kosterev, Frank Puyleart, Irina Green, David Hawkins, Yuri Makarov, Anatoliy Meklin, Abraham Ellis, Henry Huang, Ning Lu, John Phillips, Garry Chinn, and Donald Davies. 



\section{Executive Summary}

This report is a scoping study to examine research opportunities to improve the accuracy of the system dynamic load and generator models, data and performance assessment tools used by CAISO operations engineers and planning engineers, as well as those used by their counterparts at the California utilities, to establish safe operating margins. Model-based simulations are commonly used to assess the impact of credible contingencies in order to determine system operating limits (path ratings, etc.) to ensure compliance with NERC and WECC reliability requirements. Improved models and a better understanding of the impact of uncertainties in these models will increase the reliability of grid operations by allowing operators to more accurately study system voltage problems and the dynamic stability response of the system to disturbances.

Secure operation of the grid relies on advanced planning for credible contingencies. Anticipatory studies are used to establish operating limits for secure operation as established by NERC and WECC reliability criteria. The criteria have very specific goals, for example, “The interconnected power system shall be operated at all times so that general system instability, uncontrolled separation, cascading outages, or voltage collapse, will not occur as a result of any single contingency or multiple contingencies of sufficiently high likelihood” ([23], III-119). This famous N-1 criterion, by itself, does not provide specific guidance to achieve this goal. Additional criteria are concretely specified in terms of measurable system quantities such as voltage, frequency, active and reactive power flows, and other measures derived from these. For the initial response after an event, reliability criteria specify the allowable ranges for transient deviations in voltage and frequency. Adherence to these criteria should prevent catastrophic failures. To study these transient events, model-based computer simulations are required to predict the system response to possible contingencies. It is the adequacy of these models that is the subject of this report.

Today, CAISO staff report growing concerns their load models have become inaccurate and no longer adequately reflect the actual states of the system that they are witnessing in real time, especially under stressed system conditions. They report that anticipated voltage problems sometimes do not occur, while other, unexpected voltage problems do. They have suggested that their models may no longer be accurately representing the actual behavior of load or performance of generator controls, especially during major disturbances on the system.

These suggestions are not un-founded. The load models in use today were first developed over 20 years ago and due to the difficulty of acquiring and maintaining accurate information, they are rarely updated to capture the changes that have taken place in the underlying composition of load (for example, increased saturation of power electronic devices, induction motors, etc.).

Moreover, the forms of the load models that are used throughout the year are unrealistically based on the estimated load composition during the summer peak period; they are not adapted to seasonal conditions. Likewise, until recently the models for governor frequency response control have not kept pace with changes in the industry. CAISO staff have noted decreased frequency response after large outages, and NERC has documented a persistent decline in frequency response in both the Eastern and Western Interconnects. As a result of restructuring, the settings on generator controls are no longer known to the transmission system operators, since different firms now operate the generators and transmission system. Newer generation technologies, especially, have plant controls that are believed to override the automatic governor controls on 
the generators, which transmission operators depend on to ensure system reliability. The assumption of availability of traditional governor response is optimistic and better information is required. Recent WECC modeling work has resulted in new plant control models that should improve the simulations of plant response.

The development and use of improved models will have a considerable impacts on system reliability, reducing the risk of costly-blackouts, and on long-term and short term (operational) planning, and will increase confidence in operator control of the grid.

The risk to blackouts exacerbated by inaccurate models was clearly illustrated by the August 10, 1996 blackout (and others) in which simulations after the event were unable to replicate the event. A study of that disturbance showed deficiencies in models of generator and plant controls, DC lines and their control, and in load models. Since that time WECC members have improved the DC line and generator and plant control models, and continue to work on improving load models, which remain deficient. "Close call" events in the West show the need for improvement in load models because of the concern that system damping to events is not precisely known, and observed slow voltage recoveries could propagate and lead to voltage collapse. We are reminded that the costs of blackouts are high. The August $14^{\text {th }} 2003$ blackout was estimated to have cost in the range of $\$ 4$ billon and $\$ 10$ billion in the United States (at least $\$ 2.3$ billion in Canada)[20], and a recent report estimates that power system disturbances cost $\$ 80$ billion annually in the United States[7].

The most direct manner in which more accurate models can improve security is to increase confidence in operational limits and operator controls. Secure operation of the grid is maintained through planning for credible contingencies, including the specification of path ratings and the deployment of remedial action schemes. Improved models may identify the need to curb optimistic ratings or may allow increases for overly conservative ratings. In either case, confidence in grid security will increase. Confidence for operator actions will also increase with the ability to accurately predict system responses to events and actions. (In the Introduction we present an example in which the observed voltage response in no way resembles the simulationbased prediction.)

In the longer-term improved models will benefit the decision process for capital investments, which must account for how operational limits affect the value of a proposed resource. For example, the motivation for one load modeling study was to gain a better understanding for system responses to contingencies to evaluate investment in components for remedial action schemes[6]. Likewise, since models impact operational path ratings, they will impact decisions for transmission and generation investment.

In this report we:

- Document the need for improved models.

- Review present modeling practices.

- Make recommendations for modeling research and development. 
We base our recommendations on interviews with participants at CAISO, members of WECC, and with academic researchers; a review of relevant research in the literature; and our own prior work and expertise in this area.

The scope of this report is broad in the research needs related to load modeling. Some of the recommendations do not require Energy Commission support to proceed. We feel PIER should be aware of all of these needs and activities. The focus of the research needs is limited to issues faced in the West, since this is the geographic area of primary interest to the PIER. In the West we emphasize the importance of WECC modeling activities. WECC maintains the standard models for the Western Interconnect that are used by all members for their studies. Significant change in models and modeling practices must undergo WECC analysis, scrutiny, and acceptance.

In our review of WECC modeling activities we report that the present load modeling practices are crude. The representations for load characteristics that are important for assessing transient voltage and frequency responses are not detailed; and, as comparison to many observed events show, there are legitimate questions about model adequacy. To examine these questions, WECC launched a Load Modeling Task Force in 2002. We detail many of their activities and results, and we draw on much of their experiences to identify many of the load modeling needs and recommendations in this report.

We find it useful to separate the modeling issues addressed in this report into the following topical categories:

1. Load Model Development and Policies

2. Load Modeling

3. Measurement and Validation

4. Load Monitoring

5. Measurement-Only (Black-Box) Models

6. Uncertainty Analysis

7. Generator Governor Models

We briefly summarize the issues within each category and present recommendations for research to address them.

\section{ES.1 Load Model Development and Policies.}

This category addresses higher-level issues with model development that are not covered by the subsequent categories that focus more on detailed deficiencies in the model themselves.

First we note that the characteristics of the load model are not adapted to seasonal changes when it is clear that the characteristics do change seasonally. Air conditioning load dominates the load in the Southwest in the summer, and heating load becomes important in the winter in the Northwest. The present load model characteristics are designed for summer conditions - even those used in the winter reliability assessments. 
The second topic in the category involves the use of the models in on-line state estimators to help validate the standard WECC models (and vice-versa). While the models serve different purposes, and do not completely overlap, it is likely that more than $90 \%$ of the electric grid representation is common to both and a comparison of the models would likely identify some inconsistencies that would benefit from further study.

The third and most important topic in this category is consideration of how more accurate models may require reevaluation of reliability criteria. Based on the study of slow-voltage recovery events (near voltage collapse), it is believed that stalled induction motors make up portion of the load that may be considered "voltage sensitive." The present reliability criteria that were developed for voltage sensitive loads were based on consideration of voltage-sensitive electronic load. Reevaluation of reliability criteria may be required in light of more accurate models that represent the voltage recovery characteristics of induction motor loads.

Summary of recommendations:

Recommendation: Include seasonal variations in load models. Recommendation: Incorporate state estimator models into the modeling process. Recommendation: Anticipate and support activities to review reliability criteria, taking into account better information provided by more detailed characteristics of new load models.

\section{ES.2 Load Modeling}

This category addresses specific deficiencies of the model to represent known physical characteristics. All three of the following recommendations involve the characterization of induction motor loads since it is widely believed that discrepancies between model-based simulations and observed behavior are largely due to inadequate induction motor representation.

The first observation is that induction motors perform mechanical work: they turn fans, pumps, and compressors. The mechanical characteristics of the motor-driven loads are crudely represented in load models and preliminary studies show that the results of simulations are sensitive to this representation.

The second observation is that there is large variation in the sizes and types of induction motors in use. All system models that include an induction motor representation assume a "three-phase" induction motor - as might be found in industrial and some commercial loads. On an average day three-phase motors constitute the major part of the total load. On hot days the residential airconditioning load can become the dominant part of the total load, and residential appliances use "single-phase" induction motors. Since many disruptive events occur on hot summer days, it is reasonable to study the effect of single-phase motors on system studies. There neither has been research on the use of single-phase motor models for this purpose nor the adequacy of threephase motors to represent single-phase motors in this context.

The third observation is that some induction motors trip off immediately due to contactor operation and other overloaded stalled motors eventually trip off-line through their own 
protective circuitry. There is some capability in simulation packages to account for this effect by including otherwise redundant motor models, some of which trip off-line during low voltage conditions. Research into a simpler model that performs this function could facilitate analysis and remove duplicity. Furthermore, improvements to low-voltage protection should be discussed with manufacturers. ${ }^{1}$

Summary of recommendations:

\section{Recommendation: Perform studies to determine mechanical load characteristics for induction motors.}

Recommendation: Compare the responses of singe-phase and three-phase motors under different disturbances.

Recommendation: Model motor load shedding behavior under low voltage conditions. Recommendation: Discuss improvements to low-voltage protection on residential air conditioners with manufacturers.

\section{ES.3 Measurement and Validation}

This category addresses the benefit of certain types of measurements for load modeling, and anticipates the near-term issue of how to efficiently perform model validation with increased availability of quality measurements.

To monitor load characteristics, it makes sense to measure voltage and frequency at points near the load. To this end, WECC Disturbance Monitoring Working Group (DMWG) has specified requirements for a new measuring device that monitors electrical quantities on feeders at substations. To gain benefit from these monitors, we should encourage their use.

It is expected that in the future there will be an increase use in wide area measurement systems, and we should anticipate how to incorporate the data in model validation studies. One can envision a mathematical tool akin to a state estimator that would estimate dynamic phenomena on a short timescale (cycles) instead of the quasi-steady state estimators in use today (minutes). ${ }^{1}$

Summary of recommendations:

Recommendation: Encourage the placement of the $\$ 10 \mathrm{~K}$ load monitors.

Recommendation: Support a basic research project to outline the challenges associated with automated model validation and developing a dynamic state monitor.

\section{ES.4 Load Monitoring}

This category addresses the goal of estimating physical load composition from measurements. Present load modeling practices are based largely on assumption of typical load composition (motor, lighting, heating ...) and not measurement. Consequently there is a large amount of uncertainty in the models that are used. We draw attention to three separate aspects of load

${ }^{1}$ This topic is not directly within the scope of this report, but we feel it is worth recommending. 
monitoring, although we expect that all three would be best addressed in a single project. These are developing and extending techniques for estimating load composition from measurements (we review prior work on this topic in the report), estimating model uncertainty as part of the analysis, and seeking additional information in the harmonics of electrical waveforms that may help identify load composition (harmonics are often filtered out of the data and their information is lost). The uncertainty also includes geographic extent: how well a model translates to different locations. That is, is a residential load model typical enough that the model can be substituted to other locations?

Decisions that depend on load models have tremendous impact. Operating limits (path ratings, nomograms), remedial action schemes, capital improvement projects may all be impacted by dynamic studies and presently the load models used in such studies are crude. Any support for decisions that may be gained though measurement (over assumption) and related uncertainty analysis should be pursued.

Summary of recommendations:

Recommendation: Support activities to estimate load composition from measurements. Recommendation: Support activities to test load model substitutability assumptions, and to characterize a range of uncertainty in models.

Recommendation: Assess the value of harmonic information for the purposes of estimating load composition.

\section{ES.5 Measurement-Only (Black-Box) Models}

A report on load modeling needs would be incomplete without consideration of so-called blackbox models. In contrast to physically based load models that seek to represent fundamental physical characteristics of the load, black box models set out to match observed system behavior without constraint on the form of the model. This is a powerful approach that has wide application. But it relies on having a wealth of measured data with which to estimate the model, and this data is not common in power systems. For this reason and details described in this report we do not recommend new research in this area at this time.

\section{Recommendation: Follow efforts to develop measurement-only (black-box) load models.}

\section{ES.6 Uncertainty Analysis}

The motivation for this scoping study is that there is presently considerable uncertainty in the models we use, and correspondingly reduced confidence in analyses performed using these models. The load is changing continually and a single dynamic representation may not identify adverse conditions under differing loading conditions. The load models are necessarily simplified models and the loss of accuracy may be accommodated with a characterization of model parameter uncertainties. We can improve the models by gathering more data and information, but we must always cope with some level of uncertainty. With load models, this level may be high. There are technical challenges that make traditional approaches to 
uncertainty analysis prohibitive (Monte Carlo and derivatives), and we need to adapt or develop new methods to this task.

Like the topic of model estimation, this topic is very important as it can be tied to decisionmaking processes in a natural way. Furthermore, it is uncertainty in load models that is widely believed to be the dominant cause for discrepancies between simulations and observed behavior.

\section{Recommendation: Initiate a program to develop methods to evaluate the effect of load model uncertainties on system studies.}

\section{ES.7 Generator Governor Models}

This category addresses issues associated with the observed decline in generator governor frequency response in the nation's electric grids. We review the reported reasons for this decline in the main text. Here we note that the WECC has a new plant control model with the capability of representing the reduced availability of "droop governor" response by introducing a higherlevel feedback control loop that regulates to a plant output set point. We recommend this model be used. The remaining issue is how to determine the specific parameters for each generator in the model. Ideally this information may be obtained voluntarily through survey, or a policy could make reporting this information mandatory. In the extreme, it is possible that the data may not be obtained voluntarily or that the average response reported in a survey may differ from actual response in important instances, and a new monitoring and analysis tool may need to be developed to estimate the characteristics of each generator.

\section{Recommendation: Use the governor model of [19][18] and support WECC activities to maintain a database of plant governor characteristics. \\ Recommendation: Develop or adapt tools to monitor supplier governor frequency response.}

\section{Summary of Recommendations}

In Table ES-1 we summarize the recommendations listed above and provide a qualitative comparison of significance and research requirements for each. Distinctions are made on level of effort, time required, need for PIER direction and support, and significance of the results. The difference between stated levels are presented next.

The level of effort required to complete the research, a relative measure encompassing the staffing, focus, design and installation of special equipment, and in a general sense, total cost.

- High: Multiple investigators and necessary installation of equipment and analysis of measured data.

- Moderate: Single lead investigator and need for development of new techniques for analysis. Or this designation may refer to an otherwise costly activity that is best combined with another recommendation.

- Low: Single investigator using established techniques to perform the study.

The expected time to completion: The distinction here is between an activity that should take one-year or less, and those that will require multiple years. A further note is made on those that 
will continue as on-going activities after the initial study (such as maintaining and updating data).

The level of PIER direction and support. Some projects require little direct PIER support, as they will be initiated and completed by others. Some projects involve long-term and basic research and might not be initiated without and PIER support.

- Low: will be initiated and completed by others.

- Moderate: may require some direction or support to supplement projects with others.

- High: Long-term or basic research that will require PIER direction and support to conduct the research.

Significance: An estimate of the impact on improving the models and tools.

- Low: the research will not likely have a significant impact.

- Moderate: will provide an incremental improvement in models and analysis tools.

- High: introduces a fundamental improvement in the models and analysis tools.

Table ES-1 appears on the next page. 
ES 1 Summary or Research Recommendations

\begin{tabular}{|c|c|c|c|c|}
\hline Recommendation & $\begin{array}{c}\text { Level of } \\
\text { Effort }\end{array}$ & Time Req'd & PIER Support & Significance \\
\hline \multicolumn{5}{|c|}{ Load Model Development and Policies } \\
\hline Develop seasonal models. & low & 1-year & low & moderate \\
\hline $\begin{array}{l}\text { Validate with state estimator } \\
\text { models. }\end{array}$ & low & 1-year & low & moderate \\
\hline Review reliability criteria. & low & multi-year & low & high \\
\hline \multicolumn{5}{|c|}{ Load Modeling } \\
\hline $\begin{array}{l}\text { Study motor mechanical load } \\
\text { characteristics and impact. }\end{array}$ & moderate & 1-year & moderate & high \\
\hline $\begin{array}{l}\text { Study impact of single-phase and } \\
\text { three-phase motors. }\end{array}$ & moderate & 1-year & high & high \\
\hline $\begin{array}{l}\text { Model motor load shedding and } \\
\text { low-voltage conditions. }\end{array}$ & moderate & 1-year & moderate & low \\
\hline Improve low-voltage protection. & low & 1-year & low & high \\
\hline \multicolumn{5}{|c|}{ Measurement and Validation } \\
\hline$\$ 10 \mathrm{~K}$ load monitor. & low & 1-year & low & high \\
\hline $\begin{array}{l}\text { Scoping study: research needs for } \\
\text { automatic validation } \\
\text { and dynamic state estimation. }\end{array}$ & moderate & 1-year & moderate & moderate \\
\hline \multicolumn{5}{|c|}{ Load Monitoring } \\
\hline $\begin{array}{l}\begin{array}{l}\text { Estimate load composition from } \\
\text { measurements. }\end{array} \\
\end{array}$ & high & multi-year & high & high \\
\hline $\begin{array}{l}\text { Characterize model uncertainties } \\
\text { using measurements. }\end{array}$ & moderate & multi-year & high & high \\
\hline $\begin{array}{l}\text { Use harmonic information in } \\
\text { measurements to enhance } \\
\text { load composition estimates. }\end{array}$ & moderate & multi-year & high & unknown \\
\hline \multicolumn{5}{|c|}{ Measurement-Only (Black Box) Models } \\
\hline $\begin{array}{l}\text { Follow research activities in this } \\
\text { area. }\end{array}$ & low & multi-year & low & low \\
\hline \multicolumn{5}{|c|}{ Uncertainty Analysis } \\
\hline $\begin{array}{l}\text { Develop methods to assess the } \\
\text { impact of load model } \\
\text { uncertainties on system studies. }\end{array}$ & high & multi-year & high & high \\
\hline \multicolumn{5}{|c|}{ Generator Governor Models } \\
\hline $\begin{array}{l}\text { Support WECC activities to } \\
\text { implement best model and } \\
\text { maintain data for generator } \\
\text { characteristics. }\end{array}$ & low & multi-year & Low & high \\
\hline $\begin{array}{l}\text { Develop tools to monitor individual } \\
\text { generator } \\
\text { frequency response. }\end{array}$ & high & multi-year & high & moderate \\
\hline
\end{tabular}





\section{Introduction}

This report is a scoping study to examine research opportunities to improve the accuracy of the system dynamic load and generator models, data and performance assessment tools used by CAISO operations engineers and planning engineers, as well as those used by their counterparts at the California utilities, to establish safe operating margins. Improved models and a better understanding of the likely impacts of remaining uncertainties in these models will increase the reliability of grid operations by allowing operators to more accurately study system voltage problems and the dynamic stability response of the system to disturbances.

Accurate models are necessary to help maintain reliability. These models are used by system planners and operators to analyze expected and worst-case operating conditions to determine limits to maintain secure operation of the grid.

Today, CAISO staff report growing concerns their load models have become inaccurate and no longer adequately reflect the actual states of the system that they are witnessing in real time, especially under stressed system conditions. They report that anticipated voltage problems sometimes do not occur, while other, unanticipated voltage problems do. They have suggested that their models may no longer be accurately representing the actual behavior of load or performance of generator controls, especially during major disturbances on the system.

These suggestions are not un-founded. The load models in use today were first developed over 20 years ago and are rarely updated to capture the dramatic changes that have taken place in the underlying composition of load (for example, increased saturation of power electronic devices, induction motors, etc.). Moreover, the forms of the load models that are used throughout the year are unrealistically based on the estimated load composition during the summer peak period; they do not adapt to seasonal conditions. Likewise, until recently, the models for governor frequency response control have not kept pace with changes in the industry. CAISO staff have noted decreased frequency response after large outages, and NERC has documented a persistent decline in frequency response in both the Eastern and Western Interconnects. As a result of restructuring, the settings on generator controls are no longer known to the transmission system operators, since different firms now operate the generators and transmission system. Newer generation technologies, especially, have plant controls that are believed to override the automatic governor controls on the generators, which transmission operators depend on to ensure system reliability. The assumption of traditional governor availability is optimistic and better information is required. Recent WECC modeling work has resulted in new plant control models that should improve the simulations of plant response.

The development and use of improved models will have a considerable impacts on system reliability, reducing the risk of costly-blackouts, and on long-term and short term (operational) planning, and will increase confidence in operator control of the grid.

The risk to blackouts exacerbated by inaccurate models was clearly illustrated by the August 10, 1996 blackout (and others) in which simulations after the event were unable to replicate the event. A study of that disturbance showed deficiencies in models of generator and plant controls, DC lines and their control, and in load models. Since that time WECC members have improved the DC line and generator and plant control models, and continue to work on 
improving load models, which remain deficient. "Close call” events in the West show the need for improvement in load models because of the concern that system damping to events is not precisely known, and observed slow voltage recoveries could propagate and lead to voltage collapse. We are reminded that the costs of blackouts are high. The August $14^{\text {th }} 2003$ blackout was estimated to have cost in the range of $\$ 4$ billon and $\$ 10$ billion in the United States (at least $\$ 2.3$ billion in Canada)[20], and a recent report estimates that power system disturbances cost $\$ 80$ billion annually in the United States [7].

The most immediate direct manner in which more accurate models can improve security is to increase confidence in operational limits and operator controls. Secure operation of the grid is maintained through planning for credible contingencies, including the specification of path ratings and the deployment of remedial action schemes. Improved models may identify the need to curb optimistic ratings or may allow increases for overly conservative ratings. In either case, confidence in grid security will increase. Confidence for operator actions will also increase with the ability to accurately predict system responses to events and actions. (In the Introduction we present an example in which the observed voltage response in no way resembles the simulationbased prediction.)

In the longer-term improved models will benefit the decision process for capital investments, which must account for how operational limits value the benefit of a proposed resource. For example, the motivation for one load modeling study was to gain a better understanding for system responses to contingencies to evaluate investment in components for remedial action schemes [6]. Likewise, since models impact operational path ratings, they will impact decisions for transmission and generation investment.

In this report we:

- Document the need for improved models.

- Review present modeling practices.

- Make recommendations for modeling research and development.

We base our recommendations on interviews with interested participants at CAISO, WECC members, and with academic researchers; a review of relevant research in the literature; and our own prior work and expertise in this area.

A few general observations are in order. The initial focus of this work was on load modeling and generator frequency response characteristics. Our research suggests that there are additional fundamental modeling issues of equal importance. There is concern that there are basic inconsistencies between planning models used to anticipate problems in the grid, and the stateestimator models used to monitor the operating state of the system. Some minor variations are expected, of course, as components in operation change due to maintenance schedules, however more pervasive and significant differences are believed to be present. Procedures for validating models could be improved, in addition to the models themselves. Basic information about transmission line and transformer characteristics should be validated in addition to the load and generator models. 
We also need to be realistic about the amount of modeling improvement that can be achieved or that may be needed. The load comprises all end-use electrical equipment in every home, business, and factory connected to the grid. It is impossible and unnecessary to include models for all individual components. As we will present below, dynamics seen in measurements from severe disturbances, while complex, do suggest that aggregate models can sufficiently capture the observed phenomena. Nevertheless, we must acknowledge that perfect knowledge of load dynamic behavior will be impossible to ascertain from present and likely future measurement technologies.

Given that imperfect knowledge of load characteristics is inevitable, we will need to speculate on the level of accuracy that can be achieved and the amount of uncertainty that must be accommodated. By specifically characterizing the uncertainty in load model parameters, we can develop tools to calculate the effect of this uncertainty on studies used to determine operating policies.

In the following Background chapter we review type of models considered, their application, some events that indicate the need for model improvement, and we give an overview of WECC structure and relevant activities. WECC is the primary source for modeling and model validation activities in the West, and WECC models are used by all its members.

This report is focused on the needs and activities in the West because they are most relevant to the Energy Commission's PIER program. The report is not limited solely to issues that may be addressed by PIER, but discusses a wide range of research needs. In our recommendations, we distinguish between those needs that will benefit from PIER involvement and those that will likely be addressed by others. 
Improving Dynamic Load and Generator Response Performance Tools 


\section{Background}

The load and generator models that are the subject of this report are used to assess system response immediately following a disturbance. It is expected that the models have sufficient fidelity to enable engineers and operators to determine whether post-fault voltages and frequency deviations satisfy specific reliability standards.

In the West, the Western Electricity Coordinating Council is responsible for developing and maintaining detailed models of the Western Interconnect. Is important to emphasize the role WECC takes in this modeling effort. As the NERC reliability region in the West, WECC has obvious interest in maintaining accurate models. Moreover, the models that WECC develops are shared among WECC members and form a common basis for analysis of grid. These are the standard models and all the members use these models. There is a committee process for evaluating and recommending improvements to the models as needs arise. We discuss this in more detail later; here we note that all general changes in the recommended form of models will benefit from WECC involvement in the process. We begin this background section by highlighting some of the uses of the dynamic models.

\subsection{Application of Dynamic Models}

The models discussed here for the evaluation of immediate system response after a network disturbance are used in:

- Anticipatory studies - typically used to establish operating limits such as transmission path ratings, operating nomograms, and designing and tuning of certain controllers such as remedial action schemes.

- Post-disturbance studies - for model validation, and to better understand the underlying cause and spread of a particular event.

- Individual studies of interest - to study specific events and operating conditions that may not be covered in typical anticipatory studies.

Secure operation of the grid relies on anticipation and advanced planning for credible contingencies. These anticipatory studies are used to establish operating limits for secure operation as established by NERC and WECC reliability criteria. The criteria have very specific goals, for example, "The interconnected power system shall be operated at all times so that general system instability, uncontrolled separation, cascading outages, or voltage collapse, will not occur as a result of any single contingency or multiple contingencies of sufficiently high likelihood" ([23], III-119). This famous N-1 criterion, by itself, does not provide specific guidance to achieve this goal. Additional criteria are concretely specified in terms of measurable system characteristics such as voltage, frequency, active and reactive power flows, and other measures derived from these (ACE for example). Because we are interested in the response of the system after some initiating event (generator trip, faulted line, etc.), we briefly present some of the representative requirements with respect to voltage and frequency.

NERC and WECC designate system performance into four categories: A. no outages, B. loss of a single element, C. loss of two or more elements, and D. severe disturbance arising from loss of two or more elements. The standards emphasize the prevention of uncontrolled loss of load. As 
a sample of engineering proxies for this goal, we list the frequency and voltage requirements for the effect of an event in one area on other areas ([24], XI-18) in the following table. Note that we don't list category A here - there is no contingency - or category D since this category corresponds to catastrophic failures where frequency and voltage levels are not assumed to be contained. In the sidebar we describe the voltage criteria more visually.

\begin{tabular}{|l|l|l|l|l|l|}
\hline \multirow{2}{*}{ Category } & Frequency & \multicolumn{2}{|l|}{ Voltage } \\
\cline { 2 - 6 } & Lower limit & Maximum dip & \multicolumn{2}{|l|}{$\begin{array}{l}\text { Dip not to exceed size for } \\
\text { duration }\end{array}$} & $\begin{array}{l}\text { Post- } \\
\text { disturbance } \\
\text { deviation }\end{array}$ \\
\cline { 3 - 6 } & & & size & duration & $5 \%$ \\
\hline $\begin{array}{l}\text { B: single } \\
\text { outement }\end{array}$ & $59.6 \mathrm{~Hz}$ & $25 \%$ & $20 \%$ & 20 cycles & $5 \%$ cycles \\
\hline $\begin{array}{l}\text { C: } \text { multiple } \\
\text { outages }\end{array}$ & $59.0 \mathrm{~Hz}$. & $30 \%$ & $20 \%$ & $40 \%$ \\
\hline
\end{tabular}

The system is expected to be designed to be able to meet these criteria (and many more) during operation. In turn, these criteria are intended to ensure that the grid remains stable and robust to outages. To analyze the system's ability to meet these criteria, and to establish appropriate operating limits, it is necessary to use model-based computer simulations. The most severe disturbances are simulated to determine if the system operating limits are stringent enough to ensure the system response satisfies these voltage and frequency criteria.

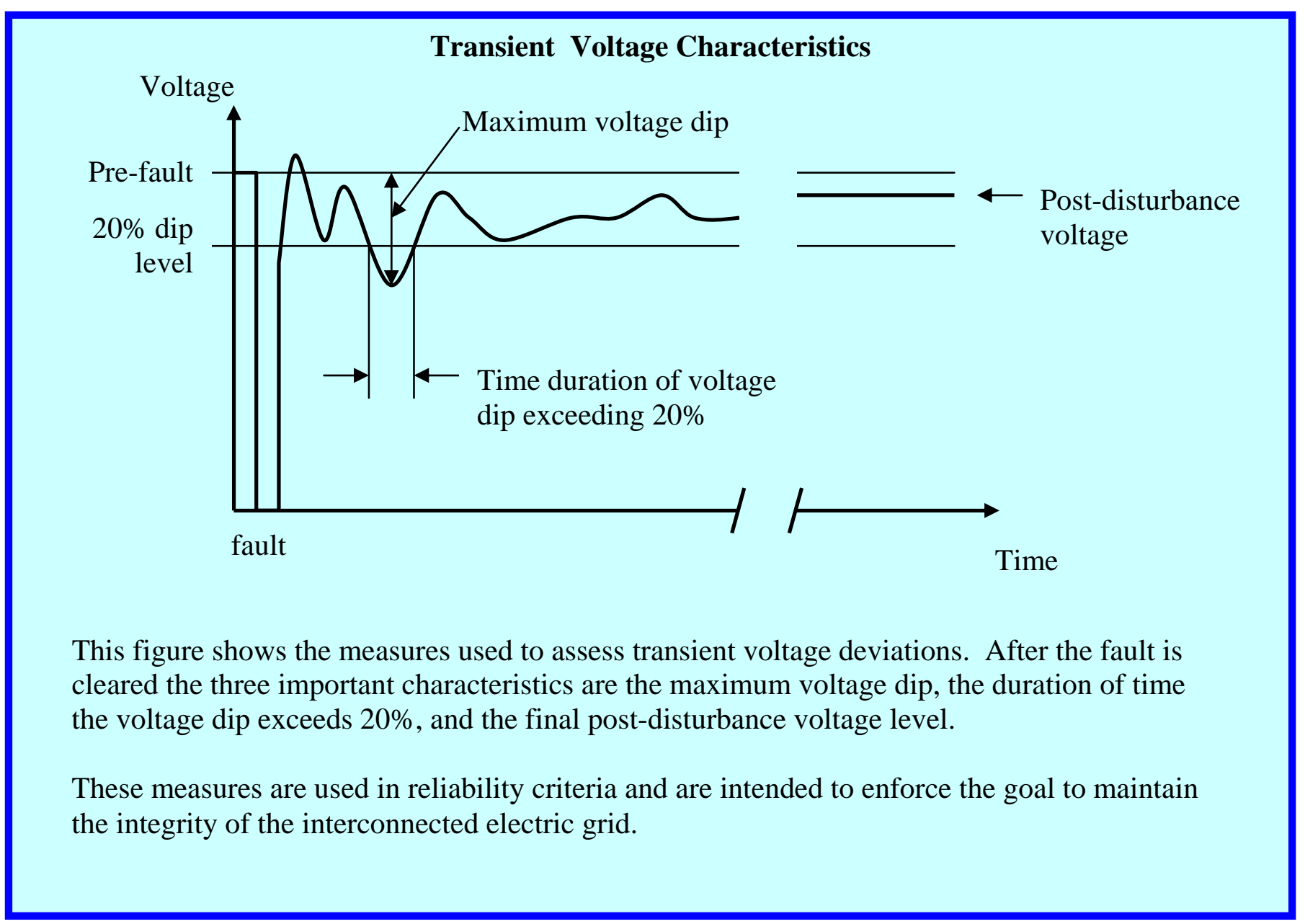


Another important application of these models is their use in evaluating themselves. Model validation is important activity to better models using all the information that is available. Measurements from disturbances are compared to those predicted by computer simulation to assess the accuracy of the models. Because the models are used to establish operating limits, the design of remedial action schemes and related decisions related to adjustment (expansion) of grid capabilities, it is important to use the most accurate models possible. In the next section we discuss evidence that suggests that models are in need of improvement.

\subsection{The Need for Improved Models}

A fundamental maxim of modeling is to use the simplest model that captures the phenomenon of interest. Given the difficulties in characterizing the dynamic characteristics of loads, the necessary aggregate nature of the model and the inability to perform tests to design and validate models, it is typical to employ very simple models for the loads, perhaps too simple. When can we tell that our models are not adequate? When are fundamental changes required and when can relatively mild adjustments tune the models?

During severe disturbances devices in the network will record frequency, voltage, current, active and reactive power. A comparison of these recordings to computed values using mathematical models can be used to assess the accuracy of the model for a given disturbance. A detailed mathematical study is required to identify and improve model shortcomings, whether fundamental or tuning. Let us consider a few historical disturbances that have led to changes in load modeling and then examine a few more recent and local disturbances that suggest renewed activity in load modeling is warranted.

An example of a disturbance that concluded in a fundamental change in modeling of load characteristics is the Swedish Blackout of 1983. The initiating event for this disturbance was the loss of a transmission line connecting the generation rich northern part of the country to the load centers in the south. There were a number of redundant lines connecting the north and south and it was a surprise that this event resulted in a complete blackout of the entire country. Modelbased simulations initially concluded that this event should not have caused such a severe disturbance. Clearly the models were deficient in some way. A detailed study concluded that the disturbance could not be replicated without a fundamental change in the load model, in this case the explicit incorporation of induction motor models [21]. With an induction motor load model in place, the event could be simulated.

We will discuss the form of load models in more detail in the next chapter. Here it is worth mentioning that models for induction motors differ from simpler static models for loads due to their different interaction with the network: because a motor stores energy in its rotational mass, it is possible for a motor to sometimes supply energy to the network, affecting overall system damping. It is also possible for induction motors to stall at low voltages, which will exacerbate low voltages. 
Another important example reported in the literature, also involving induction motors, is the August 10, 1996 WSCC $^{1}$ disturbance that resulted in a loss of more than 30GW of load [12]. This disturbance occurred on a hot day when the system was operating under stressed conditions. The initiating event involved a transmission line fault to a nearby tree. The loss of that line led to overloaded conditions on other lines, and subsequent trippings of generators and additional lines. The measurements from disturbance recorders indicate that the event was characterized by a dynamic instability leading up to an eventual cascading outage. The power flowing across key interfaces oscillated, initially undamped, and then began to oscillate in a negatively damped fashion (the magnitudes of the oscillations increased). The system became unstable.

The initial simulation of the disturbance using standard WSCC data set, adjusted for operating conditions, failed to capture the observed voltage and frequency deviations and the power flow oscillations. A detailed study showed five basic deficiencies in the standard model:

1. Inadequate DC line model.

2. Automatic Generation Controls were not in the standard model, and were important for some hydro plants during the disturbance.

3. Large thermal generators may take minutes to respond to frequency deviations and the revised model blocked governor controls for these plants throughout the WSSC system.

4. Voltage controls for some hydro generators were not adequately represented in the standard model.

5. Dynamic induction motor load models were introduced to represent the air conditioning and irrigation load that were present on the system. The standard model had employed a constant current representation for the load.

With these updates to the model, the simulation captured the basic voltage, frequency, and power oscillations of the disturbance.

We note here that the first four items involved updates to the model to better represent known characteristics of the components. The fifth item, load model adjustments, involved tuning unknown load characteristics to best match the data. Without the changes to the load model, the simulations did not show growing oscillations and system instability. The load model representation was crucial for replicating the disturbance.

The study of this disturbance was a major motivation for changing the standard load model in the Western Interconnect models to include $20 \%$ induction motor loads. Again, we will discuss the explicit forms of models in the next chapter. Here we note that the analysis of this disturbance required an adjustment to a standard load model.

Recent disturbances indicate that there is need for modifications to the standard load model. Recordings from two different representative disturbances in which load dynamic are believed to have considerable impact are shown in Figure 2-1 and Figure 2-2 below. In the June 2002 disturbance, voltage oscillations are observed. While these oscillations are slightly damped, the uncertainty in load characteristics in our models raises legitimate concern that we will not

\footnotetext{
${ }^{1}$ Now WECC. WSCC (Western Systems Coordinating Council) joined with the Southwest Regional Transmission Association and the Western Regional Transmission Association to form WECC in 2002.
} 
anticipate undamped, unstable conditions. In the July 2004 disturbance we observe a sustained voltage depression. In this case the depression is believed to be caused by air conditioning motors (induction motors) stalling. This plot shows what appears to be an incidence of near voltage collapse. In both cases the standard models fail to predict the observed response.

The July 2004 disturbance was initiated by fault on a $115 \mathrm{kV}$ line in the Southern California Edison (SCE) system. The fault was cleared within four cycles (67 milliseconds). The 30second voltage sag that followed the fault clearing was unexpected. In Figure 2-3 the simulated response (using the standard model) is shown. The simulated response shows no delay in voltage recovery; the voltage immediately returns to normal levels after the fault clearing. The observed behavior is quite different and is believed to be due to air conditioning motor stalls. A 1992 laboratory study of voltage recovery after motor stalls demonstrated this type of slow recovery[25]. That report also noted that this class of motors would stall for any voltage dip below 65\%, regardless of duration, with additional probability of stall at higher voltages for sustained dips. An analysis of the July 2004 event in which a stalled motor current model was imposed for the low voltage condition was able to adequately represent the event [4]. In the simulation, the motors began to trip-off line after some delay, allowing the system voltage to recover.

These and other disturbances are under study by WECC committees to help validate and improve models. (The LMTF is working on validation of eight events.) The problem of concern to us is that these disturbances are not well predicted using the standard WECC models, and load model deficiencies are considered to be important. The questions that need addressing are whether the existing form of models can represent the observed behavior or is some phenomenon not being modeled, how uncertain are our models and can we assess the effect of uncertainties in the models.

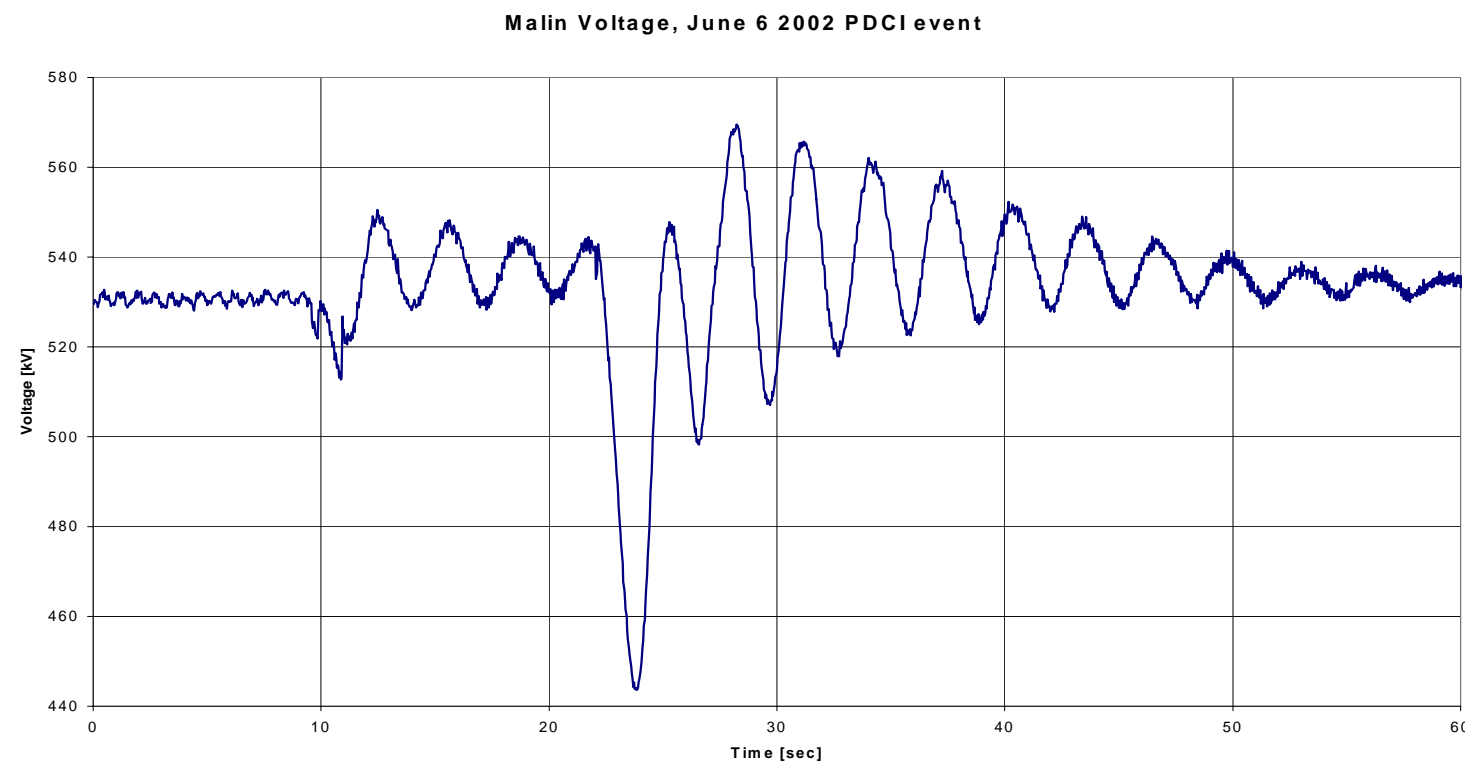

Figure 2-1. June 6, 2002 event. (courtesy of D. Kosterev) 


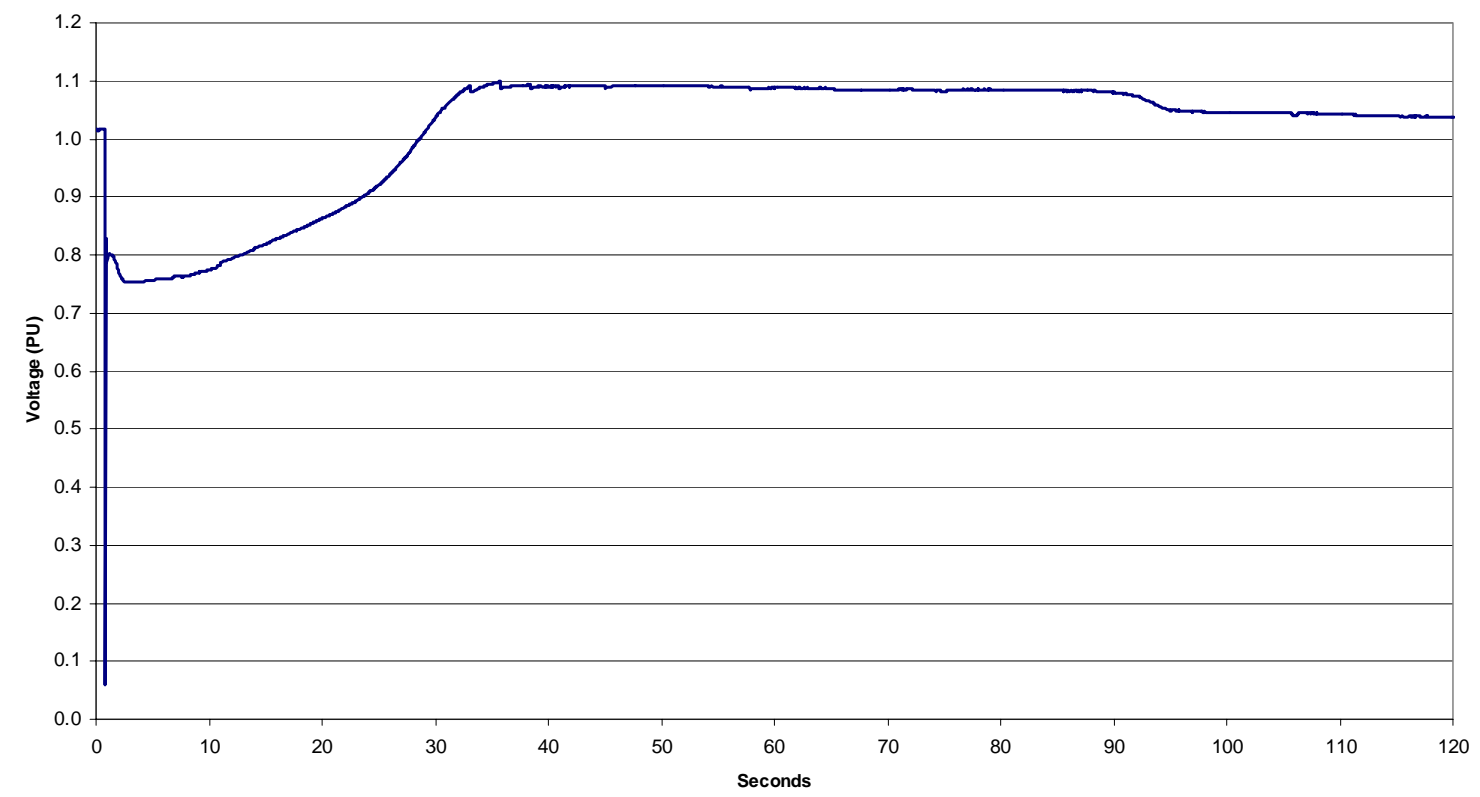

Figure 2-2. July 2004 Valley event . (courtesy of G. Chinn)

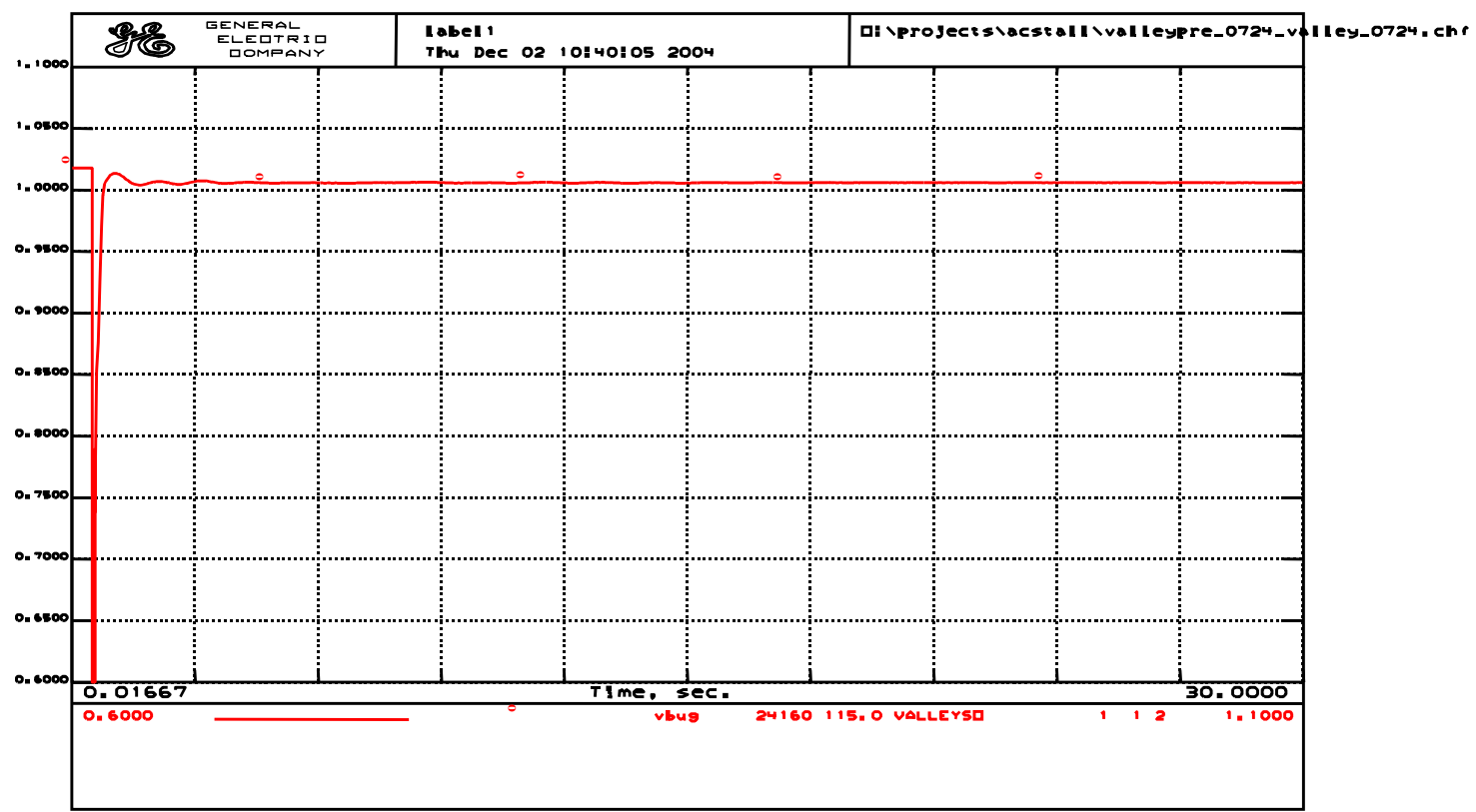

Figure 2-3 Simulation of the July 2004 Valley event using the standard model. Note that the voltage recovery is immediate after the fault is cleared. (courtesy of G. Chinn) 
As partial answers to these questions we note that the $20 \%$ induction motor load that worked well in the analysis of the 1996 outage has not proven to be accurate in all subsequent analyses. Surveys indicate that induction motors constitute around $60 \%$ of the load. It appears that the process of aggregation of loads and distribution system seems to reduce the impact of induction motor loads somewhat, but a complete understanding is lacking. Another phenomenon not well represented in aggregate induction motor loads, for large disturbances, is the discrete tripping of some motors while others remain connected. The creation of a simple model to represent these features may be important to explain the sustained dip, and eventual rise in the voltage profile shown in Figure 2-2. Finally, the models are uncertain and methods to assess uncertain models need to be explored.

The need for research on load modeling is clear. Studies of major past events indicate that simulations are sensitive to load models. Studies of more recent close-call events, demonstrate that the models require refinement. Research is being done on these issues. As mentioned above, WECC committees are actively conducting research on the both the analysis of disturbances and on general model techniques. They are an important source of information and expertise and in the next section we discuss their structure and activities in the modeling area.

\subsection{System Modeling in WECC}

System reliability in the West fall under the purview of the Western Electricity Coordinating Council (WECC), the NERC region comprising all or parts of 14 western states in the U.S., the provinces of Alberta and British Columbia, and part of Baja California, Mexico. Because WECC collects data and develops models for reliability studies, and has a responsibility to do so, it is useful to review the organization's goals, basic structure, and activities. In particular, in this report we will review the activities of the WECC Load Modeling Task Force and it is appropriate to provide the context for their work within WECC.

Membership in WECC includes participants and organizations with a common interest in maintaining the integrity of the grid. WECC's 167 members are classified by transmission providers, transmission customers, and state and provincial representatives. WECC conducts the business of applying NERC reliability criteria, developing its own system specific and sometime more stringent reliability criteria, performing various analyses to assess near- and long-term reliability, and developing common operating procedures to maintain system security.

As a NERC region, WECC is responsible for coordinating reliability in the West. The WECC organization has a number of administrative and policy committees, and two primary system specific committees, the Operating Committee and the Planning Coordination Committee. Generally, the Operating Committee is responsible for policies and procedures for secure operation of the grid, including (and not limited to) establishing reliability criteria, emergency procedures, interchange scheduling, overseeing compliance monitoring and operator training and certification, analyzing severe disturbances, and more. The Planning Coordination Committee is generally responsible for maintaining models and data to perform studies on the system, and to perform these studies. These include regional planning studies and specific evaluations of changes in system facilities. There is overlap between the two committees, and they coordinate on planning activities and system simulations. For details on the specific activities of these two 
committees the reader may refer to the WECC Operating Committee Handbook[23], and the WECC Planning Coordination Committee Handbook [24].

For our purposes we are interested in the models that are developed and used by WECC. NERC and WECC standards require that the electric system must be planned to withstand probable outages (Specific definitions and conditions related to system adequacy and security may be found in Chapter 3 of [23].) The process of evaluating system security in the planning process uses simulated testing of the system (page III-15, [23].) As a NERC region, WECC is required to perform winter and summer reliability assessments, and near-term (1-5 yrs) and long-term (6$10 \mathrm{yr}$ ) assessments. Consequently, WECC is required to maintain a working model and database with which to perform these studies.

The Planning Coordination Committee maintains the models and data to perform the required studies, and a committee structure to evaluate and make changes to recommended models as becomes necessary. The committee also reviews and suggests changes, as appropriate, to the NERC/WECC planning standards. There are two subcommittees and six working groups under the Planning Coordination Committee (see Figure 2-4). The Reliability Subcommittee and the Reliability Performance Evaluation Working Group are responsible for evaluating and recommending changes to the planning standards. The Technical Studies Subcommittee is responsible for developing and maintaining simulation models and data to be used in reliability studies and to perform these studies. The System Review working group is largely responsible for preparing case studies and performing the required reliability assessments. They maintain the system model database and coordinate data submissions from WECC members. The two computer programs used by the subcommittee have users groups to ensure that the programs meet WECC and members needs. Most important for the purposes of this report is the Modeling and Validation Working Group. This group compares data recorded from system disturbances to predictions from simulations to determine if modeling improvements are warranted. They will recommend new models and modeling techniques as appropriate. Under this working group is a task force dedicated to improving load modeling.

We will discuss the specific activities of the WECC Load Modeling Task Force in the next chapter of this report. Here we try to place their activities in the context of WECC goals and administrative organization. In summary, WECC uses model-based simulation to assess reliability. The models are constantly under review for opportunities for improvement, and load model improvement would be beneficial to more accurate evaluation of system security. Furthermore, these models are in common use among WECC members. 


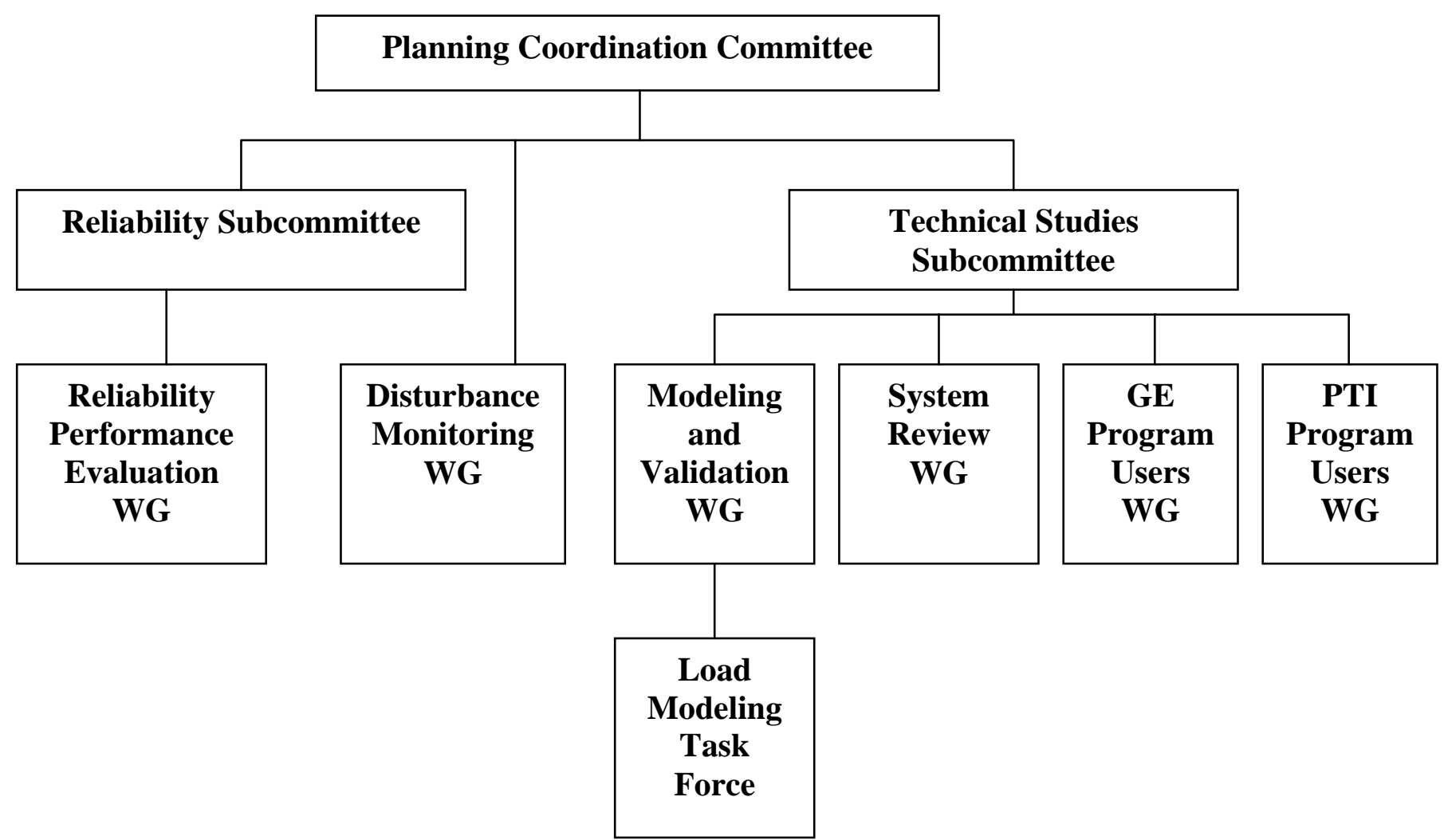

Figure 2-4. Organization chart for the Planning Coordination Committee 
Improving Dynamic Load and Generator Response Performance Tools 


\section{System and Load Modeling}

In this chapter we discuss details of system and load modeling and the relevant issues that may benefit from modeling research. We will review a typical modeling exercise to identify areas for improvement. In this case we discuss the WECC procedure for developing planning models. We will mention the benefit of consistency checking with state estimator models. The majority of this chapter is dedicated to the need for load modeling research. We provide a background in general approaches, what is done in practice, present research activities, and the areas of need for basic research in the load modeling.

The discussion in this chapter will be directed towards the specific forms models may take. We dedicate the next chapter to how measurements and monitoring may be used to improve models and the need to proactively consider how to handle data as appropriate metering equipment becomes available.

\subsection{WECC Modeling Process}

The System Review Working Group under the Technical Studies Subcommittee is responsible for developing and maintaining models in WECC, and for conducting reliability studies. They perform 11 studies per year including 5 operating studies for upcoming seasons, 2 scenario cases, 2 typical cases, and generic 5 and 10- year studies. The data required to perform these studies are collected from WECC members, roughly on a monthly basis as these studies are designed, scheduled, and completed.

Members of the System Review Working Group serve as “Area Coordinators” and develop models for designated regions in WECC (e.g. Northern California, Southern California, etc.). They collect study-specific information, such as expected load profiles and generator availability, that are important for power flow studies, and they maintain a database of system dynamic information used in stability studies. The database includes information on generator characteristics and controls, and dynamic load models. (We will comment more on this dynamic information shortly.) The Area Coordinators check their models for internal consistency and quality and pass along the models to WECC staff. WECC staff piece together the area model to form a complete model of the Western Interconnect. They also perform checks for consistency and quality. For example, they ensure that scheduled flows between regions are reported consistently. This model is then used to study the specific case it was designed for. Such cases are distinguished by season and loading characteristics, such as a heavy winter base case or light spring base case.

A Master Dynamics File, as used by the simulation software, is generally maintained and updated as members submit more accurate data, but this information is not required to be reviewed and updated with each case. Consequently it is updated less frequently. For example, NERC standards require that generators be tested at least once every five years. Between tests the dynamic data for a specific generator is not likely to be updated. Typical, generic representations are applied for generators that are in the planning stages and for which testing has never been done. Dynamic load models, which are important for stability studies, are also infrequently updated. Furthermore, the models submitted by WECC members typically use a single static load model for an entire region. While there are exceptions, this simplified load 
modeling assumption is generally the rule. Based on validation studies, WECC has adopted an interim model in which $20 \%$ of the load is represented by a generic induction motor model throughout the entire system. WECC staff does introduce this in the final model.

These models are then used to simulate credible contingencies to ensure the responses satisfy WECC reliability criteria. These criteria include restrictions on allowable transient voltage and frequency deviations from nominal after a fault has been cleared. The transient voltage conditions are defined in terms of an absolute minimum allowable voltage and a (higher) minimum voltage that the system may only go below for a defined duration. The frequency criteria are based on absolute deviations from nominal frequency. Four different event classifications are defined which dictate the appropriate load and generator shedding and network interruptions that may be needed to maintain operation of the grid. It is intended that such load loss should be handled in a controlled manner. For single failures (N-1), no load, generator, or network interruptions should be required (other than to remove the source of the disturbance).

Important to us here is the consideration given to load modeling when developing these reliability criteria. A discussion is provided in [11]. The voltage sensitive loads used to develop the transient voltage reliability criteria are assumed to be related to computer and electronic equipment. There is reasonable evidence in the recent events to suggest that induction motor loads also constitute a voltage sensitive load that needs consideration. The July 2004 event described in the introduction is believed to have an uncontrolled loss of load (air conditioners) and a suppressed voltage after the fault was cleared. As we will detail in this chapter, much of the research on load modeling is centered on the appropriate representation of motor loads. With this focus we need to recognize that consideration of induction motor voltage sensitive loads may require a review of transient voltage reliability criteria.

Based on our interviews we echo the following three recommendations concerning this type of modeling procedure.

\section{Recommendation: Include seasonal variations in load models.}

It is clear that the load changes substantially with season. Most dramatically is the airconditioning load in the Southwest that is present in the summer, but absent in the winter, and the heating-related load that is present in Northwest in the winter and largely absent in the summer. Some form of regional and seasonal variations in the models can be made as they presently exist, and will benefit from the improved models that we discuss in the next section.

\section{Recommendation: Incorporate state estimator models into the modeling process.}

State estimators are used in several regions in the West and the models that they use should be the most accurate available. It would be particularly valuable to compare the planning models with state estimator models as one check for consistency. The WECC Technical Studies Subcommittee is presently considering ways to use state estimator models in the modeling process. As WECC moves forward along this sensible path, it is possible that data confidentiality issues will arise that will need consideration. The Energy Commission may be positioned to aid in making useful state estimator data available to improve system models. 


\section{Recommendation: Anticipate and support activities to review reliability criteria, taking into account detailed characteristics of new load models.}

Recognition that induction motor loads constitute a portion of the load that may be considered voltage-sensitive, reliability criteria associated with voltage transients should be reviewed to account for this portion of the load.

\subsection{Load Modeling}

Here is it useful to begin with a review of load modeling issues and provide an abbreviated historical perspective before delving into the details of present models and in-depth discussion of future needs.

First we clarify what a "load" model is in order to contrast it from generator and network models. The greatest distinction is that a load model usually represents an aggregate of many components, often including low-voltage distribution networks. Generator models are specific mathematical models that represent physical operating characteristics of that component and its associated controls. Equations are used to model magnetic fluxes, rotor speed, field winding voltage and controls (AVR and PSS), turbines, governors, and more. Likewise, network models include mathematical representations for transmission lines, transformers, reactive power controls, and the like. These focus on the operation and characteristics of individual components. Load models are also mathematical models, but they do not represent individual components. Rather, they are used to represent entire portions of the power system that are not explicitly modeled. These tend to be low voltage networks to which the tens of thousands of end-use electrical appliances are ultimately connected. To be accurate, a load model must adequately represent not only the end-use characteristics of the energy supply, but also the effect of intervening lower-voltage grid with its transformers, capacitors, lines, and regulators.

Historically, very simple models have been used and they continue to be used. In many instances simple models are entirely adequate. In a power flow study with the purpose of evaluating whether projected generation and transmission is capable of serving projected loads, the power flow equations can be used with the load quantity specified as constant constraints in terms of active and reactive power. No sophisticated model is needed, simply accurate information about the projected demand is required (including effects of the lower-voltage network). For power flow studies with the purpose to assess the steady-state operating condition after a contingency (assuming a steady-state is reached!), it may sensible to include voltage-dependent models for certain loads to represent the change in demand with a change in voltage profile. There are often voltage regulating components in the lower-voltage network that will readjust voltages after a contingency, effectively restoring voltages and power levels. So even in this contingency scenario, the need for a sophisticated load model is not clear. The need for a detailed load model is for the analysis of the network after a contingency and before a steady state is achieved, especially to determine if the system will remain stable enough to return to a steady state.

The simplest model for the load (other than constant demand) is to assume that the load appears to react to voltage variations as a constant impedance would. This form of model was common in the more distant past when network analyzers were used to simulate the network for stability studies. The analyzer could then simulate the entire system, or with an impedance representation for the network and loads, the engineer could reduce the entire network down to generator buses 
and then perform a simulation of generator responses. A discussion of this usage is found in Kimbark's book [10]. While one might argue that the constant impedance load could represent the lower-voltage subtransmission and distribution networks and characteristics of resistive heating and lighting loads, undoubtedly the form was chosen for ease of implementation. It made the analysis practical. For reference to the more detailed models that followed, we write the constant impedance load model below in (1). $P, Q$, and $V$ represent the active power, reactive power, and voltage, and $P_{L}, Q_{L}$, and $V_{0}$ represent the pre-disturbance values for active power, reactive power, and voltage.

$$
P=P_{L}\left(\frac{V}{V_{0}}\right)^{2} \quad Q=Q_{L}\left(\frac{V}{V_{0}}\right)^{2}
$$

As tools for analysis progressed to the use of digital computers, the reliance on simple load models to facilitate analysis remained a practical issue. In their 1993 paper on "Load Representation for Dynamic Performance Analysis” [1], the IEEE task force of the same name, presented information on a survey of industry representatives that reported that the dominant load model for stability analysis was a "constant current" model for active power and a constant impedance model for reactive power ${ }^{2}$. Other respondents reported using impedance models for both active and reactive power, polynomial models of the powers in terms of voltages, and two respondents used induction motor models for some studies. In (2) we display a second-order polynomial model for active power in terms of voltage. A similar equation is used for the reactive power. This is commonly referred to as a ZIP model because the first term corresponds to constant impedance, the second term to constant current and the last term to constant power, and the common electrical engineering symbols for impedance, current, and power are Z, I, and $\mathrm{P}$ respectively.

$$
P=P_{2}\left(\frac{V}{V_{0}}\right)^{2}+P_{1}\left(\frac{V}{V_{0}}\right)+P_{0}
$$

The static models, such as the ZIP model, have the practical benefit of not increasing the computational complexity of simulations, whereas dynamic models such as induction motors, do tend to make simulation run slower. ${ }^{3}$ This was a concern up and through the 1980s but has become less a concern now with faster computers. The static models suffer from detail and cannot represent dynamics that many loads exhibit. Half of the respondents to the survey

\footnotetext{
2 "Constant current” is a slight misnomer since the load current will not remain constant with voltage changes in this case with the reactive power described as a constant impedance. Constant current refers to a model in which the power is directly proportional to voltage. When both the active and reactive powers are described this way, the model will enforce constant current magnitude at constant power factor.

${ }^{3}$ The adjectives "static" and "dynamic" refer both to types of studies and to the form of load models used in studies. Static studies, such as power flow, refer to steady-state analysis. Dynamic studies refer to stability studies involving the response of the system to disturbances. Static loads models have no "memory;" they respond instantaneously to changes in voltage frequency. Dynamic load models have memory and can store and exchange energy with the network.
} 
mentioned above stated dissatisfaction with the models they were using. Improved accuracy in models was warranted.

The IEEE Task Force on Load Representation for Dynamic Performance followed their 1993 paper with a paper in 1995 with recommended models for standard load models [2]. This task force comprising industry experts with common interest in the load modeling put significant effort into this study. Many of their observations are recommendations are valid today, ten years later. Their observations and recommendations include:

1. Load models should be physical and flexible.

2. Multiple load models should be placed at each bus to represent the phenomena to be captured.

3. They emphasize the need for induction motor models, including details of saturation and mechanical load characteristics.

4. Include models of synchronous machines as applicable.

5. Include representation for distribution transformer saturation.

6. Include control actions in LTCs.

7. Model load shedding.

In Item 1 it is clear without discussion that the models should be flexible enough to support accurate models as needed, and this is reiterated in Item 2 by stating that multiple important loads should be explicitly represented. The notion that models should be physically based is not a fundamental axiom. There are two-basic approaches to load modeling, measurement-based, and composition-based. The former approach is entirely based on fitting a model to data without restriction on the form of the model. Neural Net-modeling is common for developing such models. The risk with such models is that they are only guaranteed to be accurate for the data from which they were developed, and there is understandable concern that without a physical basis, the models will not be accurate under different conditions that may arise. Since data are relatively rare, there is little opportunity to construct and then validate such models. We will discuss this more in the next chapter, which we devote to measurement issues. Compositionbased models derive from assumed knowledge of the dominant types of loads present in network and direct incorporation of their characteristics or aggregation of similar characteristics. One has increased confidence in results using physically based models for disturbances since they can be accepted as physically possible based on the assumed load composition. And, of course, data from disturbances can be used to refine the model and assumptions.

The third item persists as a dominant modeling issues. Induction motors comprise at least 50\% of the load used, and under heavy summer loading conditions, air-conditioning load alone can reach this level. As we discussed in the Introduction of this report, inadequate representation for induction motor load contributes to discrepancies between simulations and observed disturbances. The 1995 IEEE recommended modeling practices suggest that consideration be given to distinguishing between large and small induction motors because they have different characteristics. (Large induction motors have magnetic flux dynamics that can often be neglected in small motors.) The mechanical load torque on the motor can have an impact on the dynamics and they recommend a particular form for modeling this, which we will discuss shortly as part of the WECC load modeling work. Other details of load models can include the different 
types of motors, a representation for magnetic flux saturation, and low-voltage protection that may be present.

The fifth item in the list emphasizes the importance of magnetic saturation in distribution transformers, especially for studies that involve potential overvoltages. Note, however, that distribution transformers are typically not included in large-scale studies. A more detailed model of the load with some representation of the distribution network may be warranted. We visit this issue shortly in the WECC load modeling work.

As they note in their paper, Item 6 concerning LTC control actions is usually a network modeling issues where models for these transformers may already be present. The voltage regulating action of these transformers has been shown to be critical for representing certain voltage instabilities.

The last item we listed above represents the actions of undervoltage and underfrequency relays to shed load. These may apply to certain loads represented at a bus, some portion of each load, or to the entire load.

This 1995 paper suggests the following static load model to be used as part of the load at a bus, along with more detailed models for induction motors:

$$
\begin{aligned}
\frac{P}{P_{f r a c} P_{0}}= & K_{p z}\left(\frac{V}{V_{0}}\right)^{2}+K_{p i}\left(\frac{V}{V_{0}}\right)+K_{p c} \\
& +K_{p 1}\left(\frac{V}{V_{0}}\right)^{n_{p v 1}}\left(1+n_{p f 1} \Delta f\right)+K_{p 2}\left(\frac{V}{V_{0}}\right)^{n_{p v 2}}\left(1+n_{p f 2} \Delta f\right) \\
K_{p z}=1- & \left(K_{p i}+K_{p c}+K_{p 1}+K_{p 2}\right)
\end{aligned}
$$

where $P, Q, V$, and $\Delta f$ are variables representing active power, reactive power, voltage, frequency difference from nominal. The remaining symbols denote various constants in the model to distribute the relative proportion of load to the ZIP model in the first three terms of (3) and the two frequency-dependent, voltage-exponential terms, their exponents, etc. There is an equivalent form for reactive power. While it may not be obvious from the form, the terms in the model are motivated by and related to physical characteristics of various tested load components, but remain general enough to allow for the aggregate modeling of different components within the same framework.

A dominant dynamic portion of the load model is the induction motor motel. One mathematical representation for a basic induction motor (three-phase, single cage, no saturation) is given by the equations: 


$$
\begin{aligned}
& V_{D}=E_{D}^{\prime}+R_{s} I_{D s}-X^{\prime} I_{Q s} \\
& V_{Q}=E_{Q}^{\prime}+R_{s} I_{Q s}+X^{\prime} I_{D s} \\
& T_{0}^{\prime} \frac{d E_{Q}^{\prime}}{d t}=-E_{Q}^{\prime}+\frac{X_{m}^{2}}{X_{r}} I_{D s}-s \frac{X_{r}}{R_{r}} E_{D}^{\prime} \\
& T_{0}^{\prime} \frac{d E_{D}^{\prime}}{d t}=-E_{D}^{\prime}-\frac{X_{m}^{2}}{X_{r}} I_{Q s}+s \frac{X_{r}}{R_{r}} E_{Q}^{\prime} \\
& 2 H \frac{d s}{d t}=T_{L}(1-s)-E_{Q}^{\prime} I_{Q s}-E_{D}^{\prime} I_{D s}
\end{aligned}
$$

where $V_{D}, V_{Q}, I_{D s}$ and $I_{Q s}$ are related to the terminal voltage and current, $E_{D}^{\prime}$ and $E_{Q}^{\prime}$ are related to rotor magnetic flux, and $s$ is the slip (difference from synchronous speed). The remaining symbols are constants. The first equation is algebraic representation of the stator circuit, the first two differential equations represent rotor magnetic flux dynamics, and the last differential equation is the rotor torque equation. The differential equations indicate that the model has memory; the motor characteristics are not instantaneous functions of the terminal voltage. The inclusion of an induction motor in the load model does increase the complexity, but it adds important physical characteristics that are not well represented by the static models.

The recommendations of the IEEE task force are excellent, but there remains the issue of how to gather the data and information with which to populate the model. The model is not simple. The static model described by (3) and (4) and the reactive power counterpart requires the definition of 20 parameter values. Each induction motor model will require the definition of approximately 10 parameter values. Inclusion of other detailed model for discharge lighting, transformer saturation, LTC controls, and synchronous motors, will require even more parameters.

The traditional approach, at least philosophically, is to develop representative models by load class and then appropriately aggregate these models to form a composite model. A procedure for developing a detailed load model begins with an estimate of different customer classes that are part of the load, with distinctions made between residential, commercial, and industrial loads. For each class a typical model is imposed. Industrial customers, for example, will have a high proportion of their energy consumed in large induction motors, some with power electronic drives. A composite model will then include a detailed model of an induction motor, a model for the power electronic drive load (constant power over typical voltages), and a representation for the rest of the load. Similar, but different models are assumed for residential and commercial load classes. Based on the residential, commercial, and industrial loads present at a location, an aggregate model is built. Software aids are available to help. The EPRI LOADSYN program is used, and the WECC Load Modeling Task Force is developing a complementary tool for this purpose.

As we noted in the beginning of this chapter, WECC models are initially derived from data submitted by members. The detail of the model then depends on the members' submission. Here we note that the computer software used by the WECC members is capable of representing the models suggested in the IEEE Task Force recommendations. The WECC recommended 
standard static model is based on the IEEE recommendations, although mildly simpler. It takes the following form [17]:

$$
\begin{aligned}
& P=P_{0}\left(P_{1} V^{2}+P_{2} V+P_{3}+P_{4}\left(1+K_{p d} f\right)\right) \\
& Q=Q_{0}\left(Q_{1} V^{2}+Q_{2} V+Q_{3}+Q_{4}\left(1+K_{q d} f\right)\right)
\end{aligned}
$$

This model is similar to (3) in that incorporates a ZIP model, but it differs in that in only includes a single frequency-dependent term (for each active and reactive power) and that this term is assumed to be insensitive to voltage.

Recognizing the importance of induction motor models, WECC imposes a $20 \%$ induction motor representation in the composite load model. The study forming the basis for this "interim" model is reported in [17]. In this study, the authors report on their efforts to replicate the observed behavior during the summer 1996 blackouts. In particular, they introduce an induction motor model, without which the simulations fail to capture the observed instabilities. From this lesson, they developed a default induction motor model that is intended to be a compromise between large and small motor models, and they considered some important sensitivity studies to test assumptions. The default motor model implemented at high voltage buses (for approximately $20 \%$ of the load) across the WECC system succeeds in replicating the basic features of the 1996 blackouts. Then, in this thoughtful piece, they consider sensitivity studies:

1. Connection of motor loads to new low-voltage buses. The results of this sensitivity study shows that motors thus connected increase system damping. They speculate that to achieve the same level of damping (or lack of damping) at a high-voltage bus, the percent of motor load would have to increase to $50 \%$. (Note that this is a reasonable percentage and is consistent with estimates of total motor load.)

2. Different motor load percentages. These sensitivity studies show that increasing motor load percentage results in a decrease in damping.

3. Different motor inertias. These studies suggest that the sensitivity of system damping is relatively modest compared to the sensitivity of motor load percentages. As might be expected, increased inertia resulted in decreased damping.

4. Large and small motor models. Keeping the percentage of motor loads constant, it appears that the small motor parameters resulted in less damping than large motor parameters.

5. Location. In this interesting scenario, the authors find that motors located in the electrical "middle" of the system have the greatest negative impact on system damping.

The authors conclude their analyses with the study of a fictitious event in which the two largest generating stations trip out during heavily loaded summer conditions. These types of hypothetical studies are what the models are designed for, and the inclusion of the induction motors models has a noticeable impact on the results. While the system remains stable, the results show that certain path loadings need to be adjusted to meet WECC reliability criteria. This is an important consequence of improved models; improved models will impact the results of reliability studies.

They recommend this model for interim period while the WECC Load Modeling Task Force is conducting research on how to improve load models. They are accounting for some practical 
limits of available information and at the same time determining the most sensitive characteristics of the model that need attention. We discuss their activities next, which will lead to some of our recommendations for continued and future research.

\subsection{WECC Load Modeling Task Force}

The WECC Load Modeling Task Force (LMTF) is engaged in activities to improve load models in WECC planning models. Formed in 2002, the LMTF is tasked with developing a load model form that can be implemented in the two programs used by WECC members, (GE PSLF, and PTI PSE/E), developing a tool to aid in the determining model parameters given information about the load, and to recommended generic models when little information is available.

This is an ongoing research and development activity and some of the advancements summarized here are courtesy of the LMTF and its members. By reviewing some of their current work and the gaps it identifies, we see where future work may be best directed. A recent paper summarizes some of their activities [5].

The model under design and development must meet some sensible criteria. It must be accurate enough to capture the load's effect on system damping during disturbances, and it must be able to reflect the loads impact on voltage dynamics initiated by faults and system voltage dips. Also, from a practical point of view given the paucity of detailed information about loads, it should be robust to parameter variations (uncertainty). The latter objective suggests that key sensitivities should be identified and included in the model. As a recurring theme in this report (and in the load modeling literature in general), motor loads have a significant impact on system response and need particular attention. A final concern is that the model, when implemented, should not tax the capabilities of the computer software. That is, the computational requirements should not dramatically increase, and the algorithms should remain numerically stable.

In their present activities, the LTMF has performed background work on distribution systems, characteristics of end-use loads, load composition by class and location, special models for industrial loads, and load monitoring. With this information they are developing a structure for a recommended load model and a means to determine parameters from information.

\subsubsection{Direction of recommended load model.}

Two improvements on the structure of the model are:

- The introduction of a basic form of distribution network. This is not a detailed representation of a particular distribution network associated with a specific location. Rather it is a model to capture the effect of the existence of a distribution network including transformers and electrical distance between substation and end use.

- A more detailed and careful use of motor models.

The distribution networks (and typically not-modeled lower voltage networks) can be complicated with many lateral lines and loads. In this effort we emphasize that the distribution network representation is only a stylized version that introduce some key features of the network. This is considered part of a load model, not a directed effort to incorporate details of the 
distribution network. The features that are included in this manner are transformers and the effect of lines and reactive compensation. The change this introduces in a typical load model is depicted below in Figure 3-1. A transformer and line are introduced to connect the substation bus to a new lower voltage bus to which the end-use load is connected.

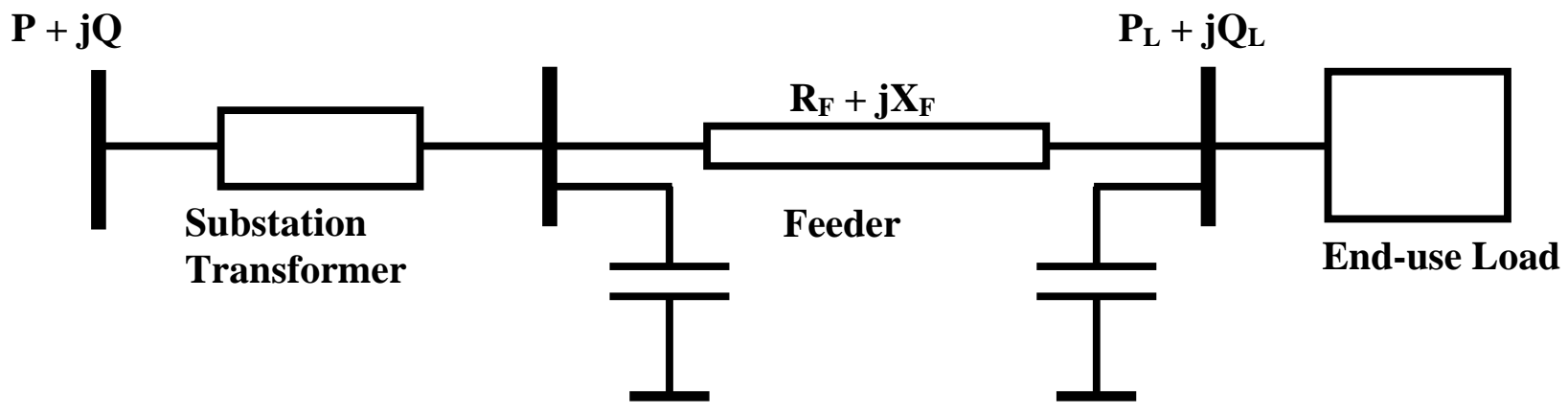

Figure 3-1. The introduction of a stylized distribution network.

The end-use load that is connected at the end of the line in Figure 3-1 will not have the same form as the rough and formerly used (and currently-used) models of loads at the substation bus. The end-use loads in the new model should be closer to those reported in surveys (which we discuss shortly). For example, in [17] the authors introduced a 20\% induction motor model (at substation bus) to replicated observed disturbances, while surveys indicate that actual induction motor loads exceed $50 \%$ of the load. With the new structure of the model, it is expected that known estimates for load composition can be used directly, which should ease the work of engineers and increase confidence in the models.

The addition of the new lines and transformers requires some assumptions about the specific characteristics these should take. Engineering judgment based on typical distribution feeder voltage drops can be used, recognizing that in some cases other distinctions may be applicable. For example, short urban distribution feeders differ from long rural feeders. LMTF studies show that in the case of a short feeder representation, the simulation results are insensitive to transformer and feeder impedances. However, the results are sensitive to these parameters for long feeder representations [9], and more care may be necessary for constructing those cases.

The second item listed above, motor modeling, remains a significant issue for load modeling. The first issue is that end-use motor loads constitute the dominant part of the energy demand. While under normal operation this is concentrated in the industrial sector, under the heavily-load conditions when most system problems occur, residential and commercial HVAC energy use becomes a primary factor. Neglecting motor loads is believed to be the main reason for discrepancies between observed disturbances and simulations of power system dynamics.

The second issue and challenge in modeling the motor load is that there is such a wide variety in types of motors performing different tasks with different controls that it is difficult to construct a simple model that captures all the necessary features. Some motors are large, others are small, and they have different characteristics. (Magnetic flux dynamics in large motors impact dynamic 
studies, but do not for small motors.) Some motors are designed to be operated from three-phase supplies, others use single-phase supplies. (The effect of this difference remains unstudied in dynamic simulations. It is a gap that needs investigation.) Some motors have sophisticated power electronic controls that make them appear as constant power demands to the system. Some motor have protective relays that automatically turn them off after sustained low voltages; others do not. Different motors serve different mechanical purposes, driving pumps, compressor, fans, etc. The different mechanical loads also impact the dynamic simulations. (The impact of different mechanical load types remains a gap that needs to be studied.) This large range of different types of motors and usage makes it challenging to design an accurate and simple model, especially when one considers that the aggregate model actually represents thousands of individual components.

Standard methods for aggregating motors appear to work well for combining the effect of motors with basically similar characteristics, but do not work well for combining the effects of fundamentally different motors. Thus an aggregation of a large class of large motors with similar mechanical loading conditions into a single motor model works well for system studies. But significant differences are noticed between a model with both large and small motors when they are combined into a single equivalent motor. Furthermore, the controls of motors, even similar motors, differs at low voltages, suggesting the need for more than one model for, say, small motor loads, to capture the effects at low voltages.

Such a fine distinction among the end use of energy is too detailed for practice given the information that is available. A simpler, but detailed, model will be developed and recommended, such as that shown below in Figure 3-2 in which three aggregate motor models are used.

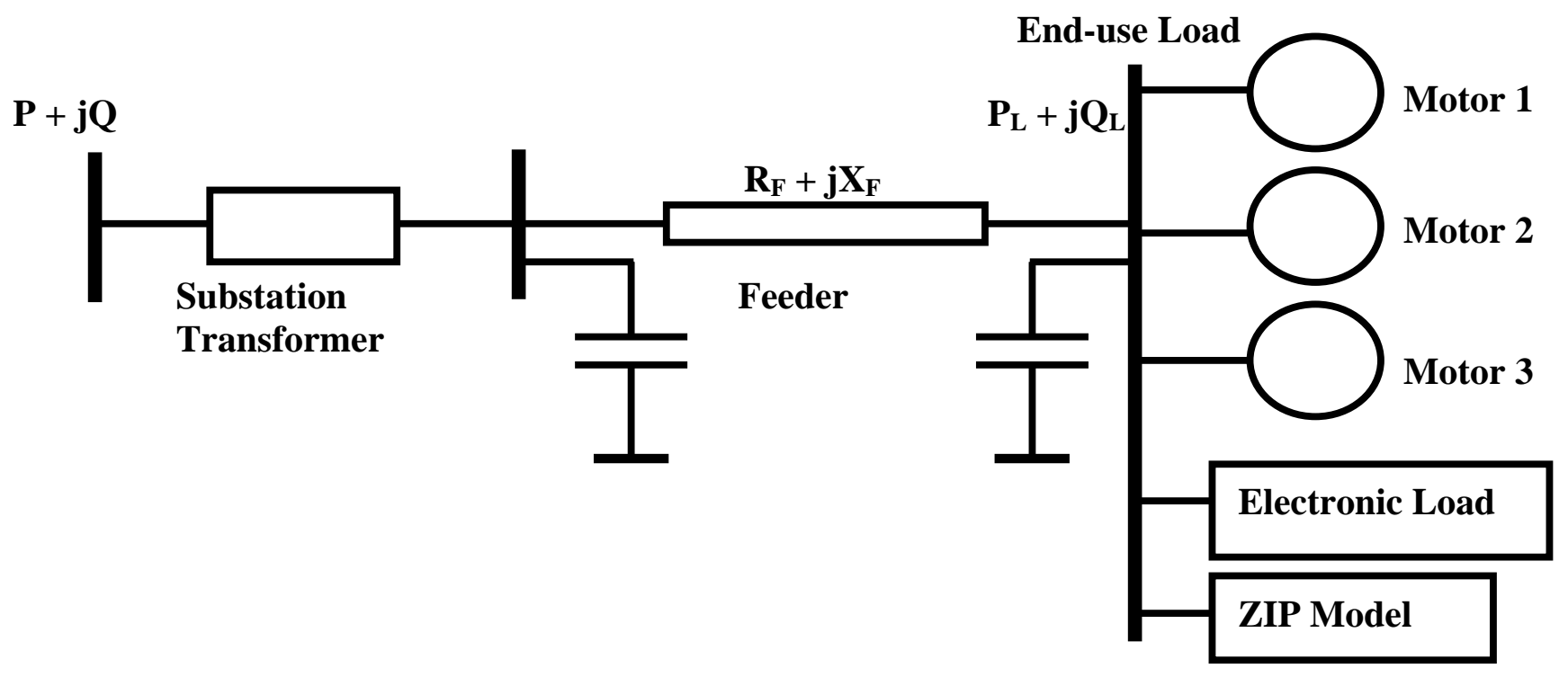

Figure 3-2. An aggregate load model in which three representative motors are employed.

The first two motors will represent three-phase motor load, differing by load shedding capability, and the third motor will represent single-phase motors. The remaining load includes representations for the electronic load and the remaining components using a ZIP model. 


\subsubsection{The load modeling process: Survey and Aggregation.}

The process for updating load models follows the steps of obtaining new estimates for load composition by class (industrial, commercial, etc.) and then performing an aggregation step to combine the effects of representative models for each class. In their efforts, the LMTF has conducted a survey of members to identify load composition by class and is developing a tool to construct and aggregate model in this fashion.

The basic procedure for load composition estimation is shown below in Figure 3-4. Data from substation load class is converted to more detailed descriptions of load components, which are then aggregated to fit the specified load model. In addition, a representation for substation transformer and feeder are added to complete the load models. Details of the present state of the Load Modeling Tool (LMT) that performs these tasks, taking data from substation load class and developing load models, are described in [15].

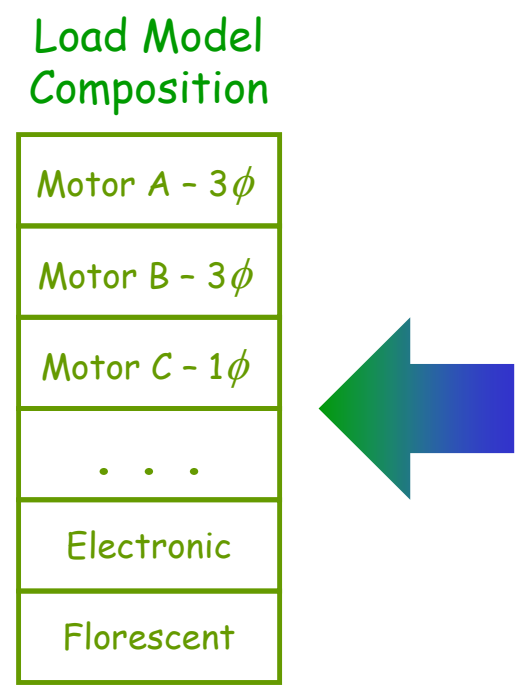

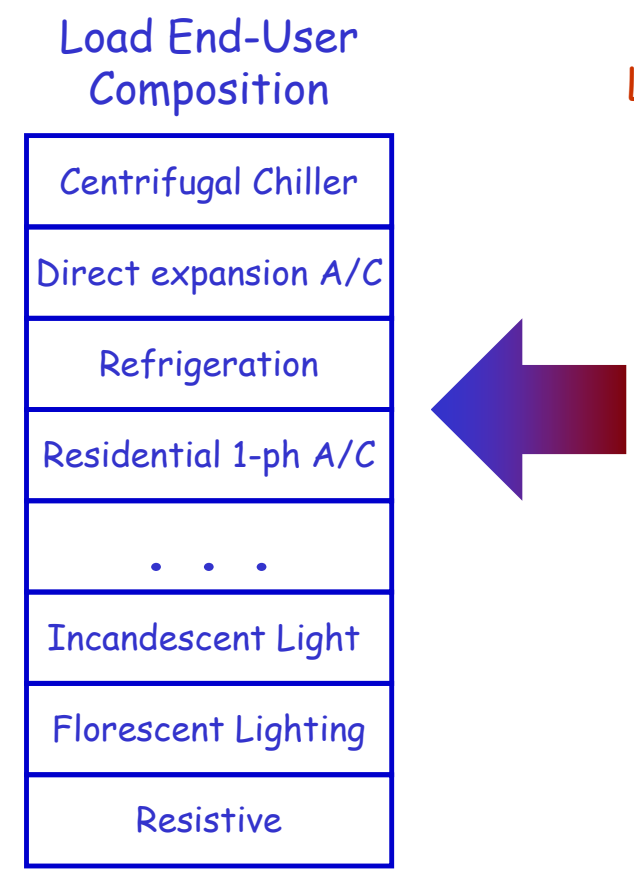

\section{Substation Load Class Mix}

Figure 3-3 A method for load model composition estimation from substation data. 
The final step in load model for dynamic performance is in validation. This is accomplished through comparisons of model-based simulations with recorded measurements in the field. The WECC performs model validation based on disturbances, and their experiences in these efforts are part of the motivation for the formation of the LMTF. Some validation studies are reported in the public literature such as [12] [17], while other reports are available only to WECC members.

Validation studies based on disturbances raises the issue of what information is and can be gathered from monitoring devices. This is the topic of the next chapter. Before embarking on that discussion and its own research needs, we summarize the research needs related to load modeling development.

\subsection{Load Modeling Needs}

In this section we summarize the load modeling needs with respect to the structure of the model and the information that may be gathered to enhance the models. Issues related to measurement, and validation from measurement we leave to the next chapter. We also recognize that significant work has been done by WECC committees already and that on-going research will continue within that structure. We emphasize here the issues that may not be done without some additional support, even for research that is best done by WECC committees and members.

The basic structure of the recommended load model that will come from the LMTF activities is likely to be adequate and serve simulations needs. No additional research is recommended for amending the structure as depicted by Figure 3-2. The models for the components require significant work yet, as well as information gathering for different operating seasons.

By far, the load component requiring the most attentions is the representation of induction motors. While this is long-recognized problem, there remain challenges for accurate modeling. We list items individually in our recommendations.

\section{Recommendation: Perform studies to determine mechanical load characteristics for} induction motors. These studies would best be performed in a testing laboratory. Preliminary LMTF studies show that simulation results depend on the representation of the mechanical load's characteristic. At the simplest level, the relation between torque and speed differs between pumps, fans, and compressors, and the models will be improved by quantifying these differences by laboratory measurements (instead of by assumption). It may be the case that more sophisticated dynamic models for the loads may be necessary, especially for compressors.

\section{Recommendation: Compare the responses of singe-phase and three-phase motors under different disturbances. This work should couple laboratory-setting experiments with detailed models and simulations.}

The issue here is that under the heavily loaded conditions of great interest, the percentage of single-phase motor load increases (in residential air conditions, for example). The models used in simulation studies all correspond to three-phase motors. There is an unstudied, basic question about how accurately a three-phase motor model can represent the response of a single-phase motor under disturbance conditions. This is a reasonable question in that three-phase and singlephase motors model differ, most notable in the energy conversion to torque. This concern 
complements the issue with motor mechanical load characteristics, which is also related to torque, and this recommendation could be studied at the same time as the previous recommendation

Recommendation: More work is required to model motor load shedding behavior under low voltage conditions. The aggregate model of induction motor loads requires a mechanism for allowing a portion of the load to trip off line during a disturbance. The problem here is the difficulty in representing all the different characteristics of motor loads in a simple aggregate model. Presently the overall model could be augmented with different motors, ones that trip off line during a disturbance, and others that do not. In the traditional spirit and practice of modeling, an aggregate model that represent this low voltage trip, would be beneficial.

\section{Recommendation: Discuss improvements to low-voltage protection on residential air conditioners with manufacturers.}

It is observed that the slow voltage recovery observed after a disturbance could be avoided by temporarily removing voltage-sensitive loads, such as air-conditioners, and the restarting them after a few minutes delay. This approach to mitigating the problem on the component level should be discussed with manufacturers. 


\section{Measurements, Monitoring, and Validation}

There is value in system measurements for load modeling. Measurements are crucial for validation work to confirm the accuracy of models, and furthermore, since load models affect operations and policy, justification for the models based on measurements in addition to reasonable assumptions should be required. In this chapter we review these issues, describe some of the uses of measurements, and project on what the uses will be, or can be in the future. Our recommendations will serve to improve load modeling capabilities.

Load models have an impact on reliability studies, planning studies, and even policies for reliability. A change in the load models used could result in a change in path ratings, transmission planning, and reliability criteria, and thus there is an economic value (and economic consequence) to using more accurate load models. Our stand in this report is that more accurate models are justified regardless of the positive or negative economic impact it may impart on planning. The issue that requires very serious consideration is whether the load modeling procedure as alluded to in the previous chapters is justifiable in light of possible consequences. The basic procedure presently being pursued is largely based on some knowledge and some assumptions. For example, the load model is built up from knowledge of load class (which is arguably known or can be known from utility billing records) and assumptions about typical behavior of loads within a class (residential, commercial, industrial). And then there is an imperfect aggregation step to simplify the model enough to allow practical numerical simulation. While the best information available may be used to justify the models, in light of the important impact they have, the results would benefit from use of measurement.

Measurements can be used to:

- validate models,

- form the basis of measurement-based models,

- test assumptions in the modeling procedure with staged testing or continual monitoring, and

- characterize uncertainty in the models.

We review these uses and consider what improvements can be made with existing, expected future measurement capabilities, and more sophisticated measuring equipment that does not presently exist.

\subsection{Measurements and System Dynamic Monitoring}

Existing data come from a variety of sources including state-estimator outputs, SCADA data, fault recorders, and phasor measurement units. For purposes of monitoring these all have benefits and drawbacks. State estimator data is useful for validation studies because it provides information on the state of they system prior and after a disturbance. Because it only runs every few minutes, a state estimator does not (and is not intended to) follow the fast dynamic responses of loads. Likewise, SCADA is somewhat limited in this regard, sampling every few seconds, but does provide information on relatively slow events that may persist for tens of seconds. Fault data recorders provide much of the information that is used in validation studies. These devices save information on voltages, frequency, and current, once a disturbance in noticed. They are 
limited for purposes of load modeling and validation in that their placement may not be optimal for any particular disturbance, are not typically placed near loads with the purpose of determining load characteristics. Phasor measurement units provide another source of information that is sampled at a rate consistent with fast response studies (30 samples reported per second). Their use is still not perfect (or designed) for load modeling, since they will also not be placed for this purpose, and they report positive sequence information (i.e., not information along individual phases to which loads are ultimately connected).

The IEEE Disturbance Monitoring Working Group (DMWG) has consider these issues and are seeking a vendor to develop a low cost (under $\$ 10 \mathrm{~K}$ ) monitor that is specifically intended to help identify load characteristics. It will be placed on distribution feeders and will measure phase currents and voltages. This provides direct information about what is happening at the interface where the (better-known) system model stops and the load model starts.

\section{Recommendation: Support the development and placement of \$10K load monitors.} Encourage the use of monitors, and staff time, to gather information beneficial to improved load modeling.

In the future, it is likely that there will be a proliferation of phasor measurement units and similar high-sampling rate real-time recorders. These offer the promise of improved disturbance validation. Presently, given the paucity of data and rarity of events, each disturbance validation exercise is handled uniquely. One can expect that better models and greater data will allow the creation of a nearly automated process of model validation from measured data. Conceptually, this will mimic the activities of a state estimator, except with more sophisticated, dynamic models. Initially, during a development stage, it might be run off-line with historical data, but should be developed with the intent of possible on-line implementation. While this task is not exclusively limited to the topic of load modeling, and might be considered outside the purview of this report, we make the recommendation here to begin the background research to plan for this future. Two basic questions could be addressed in an initial study. One, what are the basic mathematical tools that would be used and their data requirements. Two, what institutional issues related to data gathering will need consideration.

\section{Recommendation: Support a basic research project to outline the challenges associated} with automated model validation and developing a dynamic state monitor.

The issues to be addressed are the mathematical tools and data requirements, and institutional challenges with gathering and sharing data.

\subsection{Load Monitoring}

In the near term, load models need improvement and may benefit from measurements. There are examples in the WECC where this is the focus of research. We discuss a few of the efforts in the West and the lessons learned that can be applied to future research.

In a study of a specific industrial plant, the Bonneville Power Administration (BPA) developed a load model for a paper mill and validated the model using disturbance data. The model was developed with information on the electrical layout about the plant and its end-use components. In Figure 4-1 the simulated and recorded responses are provided for comparison. It is clear that 
the model represents the actual response very well. The encouraging lesson one may infer from this study is that it is possible to accurately characterize the behavior of the load when information and measurements are available. With an industrial plant the load is concentrated and information may be gathered (assuming cooperation with the load) to form a relatively simple model that captures the basic physical features of the load. If the operation of the plant is fairly consistent over time, it should be a useful representative model for the site. It is understood that a model formed in this manner is specific to the individual plant and care should be taken before replicating the form of the model to other industrial plants, especially of very different types (i.e. not paper mills).

- voltage

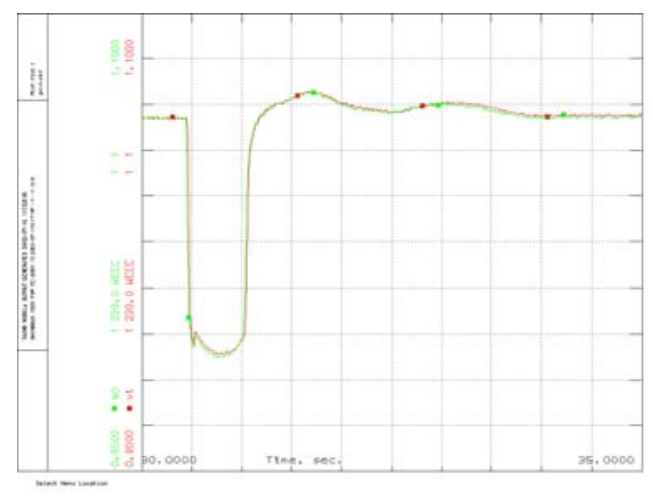

- current

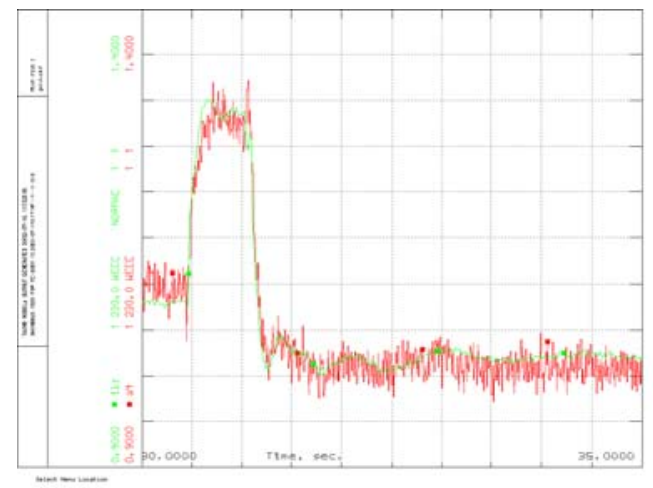

- power

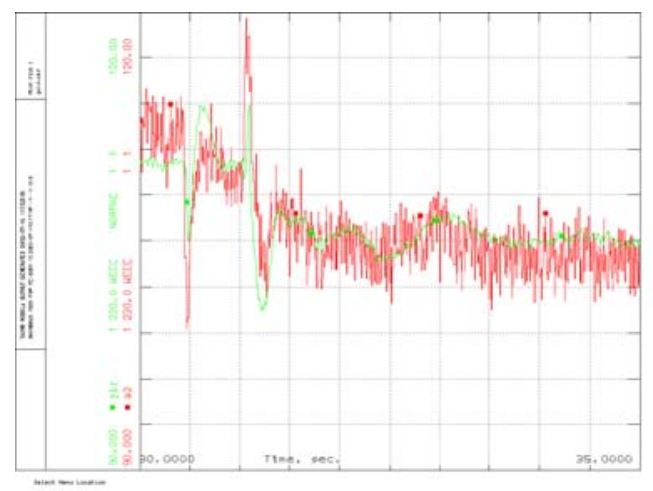

- reactive

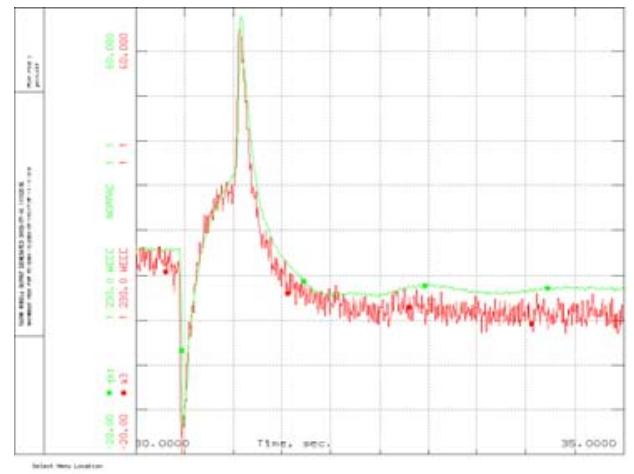

Figure 4-1 Recorded and simulated response to a disturbance at a paper mill (courtesy D. Kosterev).

Public Service Company of New Mexico (PNM) has also conducted research on characterization of loads in their system, from measurements. Some of their results are presented in [6]. Their approach is consistent with the physically based load modeling philosophy discussed in this report. They assume representative models for dominant electrical components and then use measurements to estimate the percentage of each component in load. The components include small, medium, and large motors, incandescent lighting, fluorescent lighting, thermostatic loads, electronics loads, and capacitors. They also designed custom measurement systems to record 
voltages and currents on individual feeders from a distribution substation. They collected data from natural disturbances, and from staged tests.

While the models are physically based, we emphasize the difference between this approach and the typical physically based modeling approach: the information comes from measurements. Presently model parameters are populated based on knowledge of load class, that is, the percentage of residential, commercial, and industrial load. In the PNM study, model is populated by assumptions of typical load components (not class) and measurement-based estimations of percentages of each component. The report shows that this approach is feasible and provides reasonable results.

The report and discussion with the author also highlight some important observations and lessons. These include the following:

- Any estimation technique will fit the data. It is important to use an estimation technique that respects the physical nature of the load. They noticed that simple least-squares estimation often resulted in coefficients that were not physical (unrealistically large numbers, and negative weights). They effectively employed a fuzzy regression scheme that resulted in physically meaningful coefficients.

- The measurements at the distribution level are noisy. Voltage disturbances of a minimum deviation of $2.5 \%$ are necessary to extract useful information from measurements.

- It is important to measure responses of individual phases to estimate single-phase load components.

- There are significant challenges to isolating load characteristic in measurements. These include the time frame of the measurement to exclude effects of load variation and the effect of automatic voltage controls that may be present in the network.

- Load composition changes with time, and data coming from natural disturbances may not yield the most appropriate model for general use.

This is very promising and reasonable work on load modeling. We recommend that it continue with a focus on gaining experience with this approach to load monitoring and how to apply it to system-wide studies, given that relatively few representative substations can be metered in this way. Of particular importance is the question of how to populate load models in general based on a few measurements.

Recommendation: Support activities to estimate load composition from measurements. This includes investigating refinement in metering equipment, assumed load models (such as adding stylized distribution feeder model as WECC moves in this direction), staged testing, and techniques for estimation that may expand conditions under which estimation is possible.

Recommendation: Support activities to test load model substitutability assumptions, and to characterize a range of uncertainty in models.

This addresses the issue of whether or not representative models are truly representative by comparing results from substations that would be assumed to have similar loading characteristics by load class. The matches will not be perfect, so there would be additional value to characterizing the range of values obtained for different load classes that may be evaluating 
using uncertainty analyses. Also, a calculation of uncertainty in estimates at each measured location will facilitate uncertainty simulations.

We also believe this measurement approach may benefit from ideas and techniques used in the Non-Intrusive Load Monitor (NILM) that was developed at MIT [13]. The NILM is a device placed at the service entry to a building and through sophisticated signal processing is able to detect when individual components in the building turn on and off. Furthermore, with information of how the equipment should operate, or has operated in the past, it is able to diagnose degradation of equipment and they systems they run. It has been employed and tested in several buildings in California under support from the Energy Commission [3].

The core of the NILM technology is a detection algorithm that correlates observed transients in the spectral components (harmonics) of voltage and currents to the signatures of equipment. The start-up waveforms for motors appear different from those of incandescent lighting, discharge lighting, etc., and at the level of a building many components can be distinguished. At the level of the power grid there is little hope that individual components could be identified in this manner (except, perhaps at industrial facilities). Nevertheless, it may be possible to distinguish in a course sense the operation of classes of particular components based on the analysis of steadystate harmonic characteristics of the load voltages and currents. An initial study of this approach was promising [14]. One could envision a load composition monitor that would attempt to estimate the percent of load in certain categories.

Such research would need to be considered high risk. It is yet unknown whether information in the harmonics will be useful. At the minimum, is very likely that information in the harmonics can improve the component estimates from disturbance data. Presently the best work relies on only the $60 \mathrm{~Hz}$ waveforms, and the harmonics can only add information. But in the case of steady-state analysis, it is not clear that harmonic information can be correlated to load composition.

The benefit of such research, if successful, could be large. The load changes continually and the best models now in use assume that load composition does not change; the models are prepared for a high summer load scenario. We have made a recommendation for the development of seasonal models, but these do not track hourly and daily changes in composition. An estimate of load composition, from measurement, may save the operators from surprises.

We think it would be prudent to design and install monitoring equipment at some substations with the capability of recording harmonics. This is for the expressed purpose of assessing the value of this extra information. If there appears to be information in steady state, a subsequent project may look into the development of a load composition monitor.

\section{Recommendation: Assess the value of harmonic information for the purposes of estimating load composition.}

The additional information in harmonics will augment the primary frequency data now used, and there may be useful information in steady-state waveforms. 


\subsection{Measurement-Only Load Models}

There is an alternative to the physically based load models that we have discussed in this report. One can make an argument for the use of measurement-based load models, the form of which do not necessarily have a physical basis. Neural net models are a common form of measurementbased models. The advantage of this type of model is that the form of the model is not restricted and can adapt to fit measured data. Furthermore, one must admit that physically based models are one only an approximation of the real network; they are designed to capture basic physical features, but only in a coarse sense. The aggregation and uncertainty in composition, etc., make the form inexact. It is entirely reasonable and appropriate to consider generic "black-box" modeling techniques that can accurately reproduce the observed behavior of loads.

The fatal flaw with this approach is the extreme lack of data with which to develop a model that can be expected to perform well (or better than physically-based models) over a wide range of operating conditions. Without any physical basis to the models, one cannot be certain the models will represent load characteristics for conditions outside those used to fit parameters of the model. Severe outages, which are the conditions of interest, are relatively rare and recorded data is scarce - at least from the perspective of having enough data to develop a comprehensive model. Of course it is possible to fit and/or validate black-box models using simulated data with loads modeled in a traditional manner. In doing so there is little doubt that measurement-only models can fit the characteristics of a physically based model. (But a model fit in this manner offers no advantage over a physically based model.) Most practitioners that we interviewed feel that there is not enough data to support such a modeling approach at this time (or in the foreseeable future - hopefully severe disturbances will continue to be relatively rare!).

Measurement efforts are likely to be the most successful and useful for estimates of load composition, and not for the development of black-box load models. However, we should be cognizant of such efforts and employ any breakthroughs if they occur.

Recommendation: Follow efforts to develop measurement-only (black-box) load models. In general, black-box modeling techniques are most valuable when the available data is rich enough to show all the operating characteristics of the modeled component. Presently, we believe that the data set does not support this approach. 


\section{Uncertainty Analysis}

The load models we have discussed are necessarily imperfect. It is not possible to model every end-use component in the system, and it is not reasonable to represent details of every distribution network in a model spanning a continent. And it isn't necessary. An engineer with a background in dynamic phenomena can look at the disturbance plots, such as those shown in Figure 2-1 and Figure 2-2, and recognize that an incredibly detailed model with thousands of components modeled is not required to capture the basic features of the disturbance. The challenge is to develop a parametric model that is flexible enough to be able to represent the observed disturbances, and then to consider parameters suitable for further analysis. Efforts are under way to improve the form of load models to capture some of the salient features of loads and distribution networks, and we have recommended continued research to examine important features that are not adequately featured in the models.

Completion of efforts to improve the form of the load models should yield a representation that is capable of capturing the important features of the load that affect dynamic studies. However, there will remain a significant amount of uncertainty. Even if there exists a particular set of parameters values for the model that will accurately represent the system loads, it is not possible to know the values of these parameters. For example, the form of the load model may allow for accurate analysis of the effect of induction motors on system damping and prediction of low voltage responses of compressor-loaded motors, but we will still not know, with certainty, the amount of motor load that should be present in the model. In a practical sense, we may have adequate models for components and low-voltage systems, but we cannot exactly know what percentage of the loads correspond to each component. Moreover, any aggregation step introduces uncertainty in the representation of the model.

There may be value in considering the effect of model uncertainties may have on the outcome of studies. This is a common practice in many areas of engineering, including power engineering. (For example, scenario analysis is used in transmission planning to evaluate future needs under different assumptions for resource availability, and the $\mathrm{N}-1$ reliability criterion effectively removes uncertainty in location of events by considering them all.) There are a few common methods for uncertainty analysis and their use depends on the information available and computation limits that may be encountered. These methods include:

1. Worst Case Study. In this case, one posits a worst-case scenario (a task in itself) under which a system is required to operate, and then designs the system to do so. Presumably this is sufficient to cover all other operating conditions.

2. Scenario Analysis. One posits a set of different scenarios under which the system is expected to operate, and then ensures the system can operate under these various conditions.

3. Weighted Scenario Analysis. This is identical to scenario analysis except weights are assigned to the different scenarios. These may represent the relative importance, or the probability of occurrence of the different conditions. In this case it may not be deemed necessary to operate under all conditions (otherwise there is no need for the weights), rather one may require that operation be expected with some (high) probability. Or, in the transmission planning context, we may expect that today's decisions will suffice to meet future needs with an acceptable (high) probability. 
4. Monte Carlo Simulation. This is a common technique for uncertainty analysis when uncertainty in models can be parameterized by distribution functions. It may be considered an automated weighted scenario analysis where the scenarios considered are randomly chosen based on the distribution functions. In this manner many thousands of scenarios may be considered. Like the weighted scenario analysis, it allows one to place decisions in a likelihood context.

In the context of uncertainty in load modeling, these approaches are not routinely applied. For the dynamic simulations that are of most interest in this report, WECC uses static model supplied by participants, augmented with a $20 \%$ induction motor load. This is largely due to results of model tuning from the 1996 disturbances. Presently the WECC LMTF is developing a new model with data that will be applicable to a heavy loaded summer condition. This might be considered akin to a worst-case analysis. The LMTF will likely conduct load modeling sensitivity studies as part of their research investigations. There is no plan to evaluate uncertainty in load models for standard planning and reliability studies.

It should be clear that many studies are (and should be) driven by worst-case analyses. It is a challenging task to pose the conditions and load models suitable for realistic worst-case analysis. Sensitivity studies are helpful for isolating the key characteristics that are represented in the worst case models, and can provide information about the sensitivity of to worst-case model assumptions. These same parametric models and sensitivity information can be used to evaluate model uncertainties in formal way, at least in theory.

Part of the problem is that there are several impediments to evaluating load model uncertainties, including:

- There are no quantitative data on load model uncertainties. One has to characterize uncertainty in order to evaluate it.

- There are few efficient tools to perform the uncertainty analysis. In the context of dynamic simulations this is computationally challenging. The analysis relies on evaluation of repeated simulations using different load modeling assumptions. A Monte Carlo study, for example, would require thousands of simulations and is computationally prohibitive.

- There has not been a study to quantify the value of such an uncertainty analysis.

The last point does not suggest that there is not value in improved models. It was noted in [17] that the inclusion of $20 \%$ induction motor load in the model would warrant changes in path ratings, and in [6] the purpose of the estimation of load model composition was for studies to design remedial action schemes. The last point differs slightly in that it suggests not only improved models, but also that there is value to an evaluation of the uncertainties in the models. While this has not been proven, it appears sensible. Since we cannot represent every component in the system, some uncertainty in the model is unavoidable. Moreover, it is generally felt that deficiencies in load modeling are dominant weakness in the model. We proceed with a discussion of this research topic assuming that there will be value in uncertainty analyses, and we expect that researchers will consider this question as work is done in this area. 
The first issue of availability of data on uncertainty is addressed by recommendations in the monitoring section of the last chapter. The load monitoring activities can be augmented to quantify uncertainty in two ways. One, uncertainty in the load composition estimates may be calculated from the data and calculation, and two, typical ranges for different substation locations may be compiled for use in models for which there is little data and no monitors.

\section{A visual description of uncertainty analysis}

Figure 5-1 shows the ideal: a complete uncertainty description of the input is available; an exact, trusted model is used to compute a complete uncertainty description of the output. This is typically done with a Monte Carlo simulation. If a tool analyzing the exact model is time consuming, the repeated sampling approach of the Monte Carlo will be computationally prohibitive. If a simulation set requires 100 simulations, each requiring 1 minute of processing and computation time and the Monte Carlo simulation requires 1000 repetitions, then (without parallel processors) the computations time will approach 100,000 minutes. While potentially computationally costly to compute, the output description is very useful. The output distribution obtained through this approach can be analyzed easily to answer most questions about the range of possible outcomes and their likelihood of occurrence.

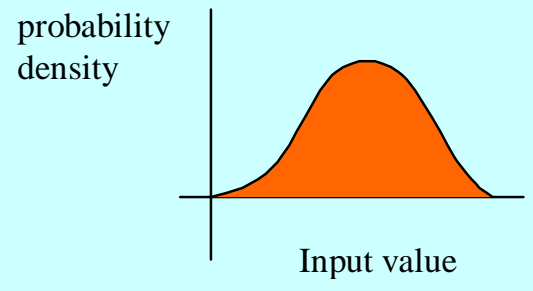

Input uncertainty description

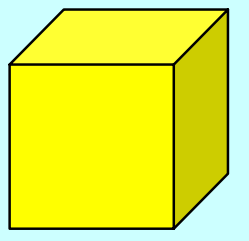

Exact Model

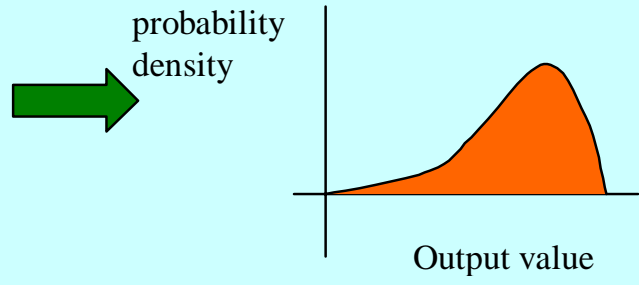

Output uncertainty description

Figure 5-1 A complete uncertainty analysis. The uncertainty is contained in a parameterized description of inputs into an exact model. Analysis of the uncertainty and exact model yield a description of the uncertainty of the outcome, shown on the right. Typically a Monte Carlo method is used for the analysis.

Scenario Analysis is commonly used. Instead of a detailed uncertainty description, selected scenarios are chosen to evaluate using the exact model. This does not inherently apply likelihood weights to the scenarios (Figure 5-2), but probabilities can be applied to scenarios if desired (Figure 53). The output descriptions aren't as valuable as that shown in Figure 1, but computationally, scenario analysis is practical.

An alternative approach is to maintain the exact uncertainty description and perform a Monte Carlo (or even better, analytic) analysis using an approximate model that runs very quickly, possibly several orders of magnitude more quickly than the original. If this can be done without losing too much accuracy, it is an attractive approach. It supplies the desired form of output uncertainty description. This is depicted in Figure 5-4. 


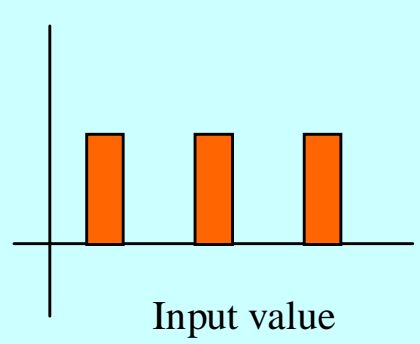

Input description

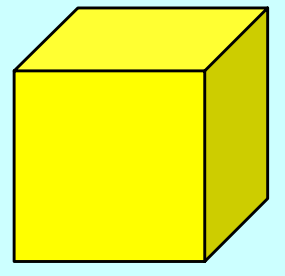

Exact Model
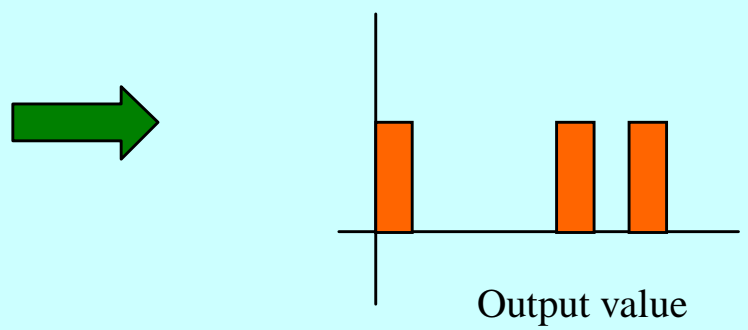

Output

description

Figure 5-2Scenario Analysis: select input scenarios are analyzed with an exact model yielding the output results. No likelihood is explicitly indicated.

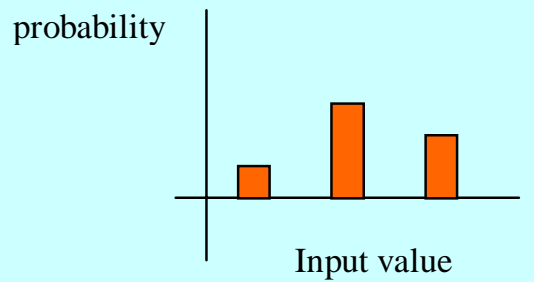

Input

uncertainty

description

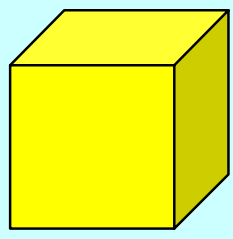

Exact Model

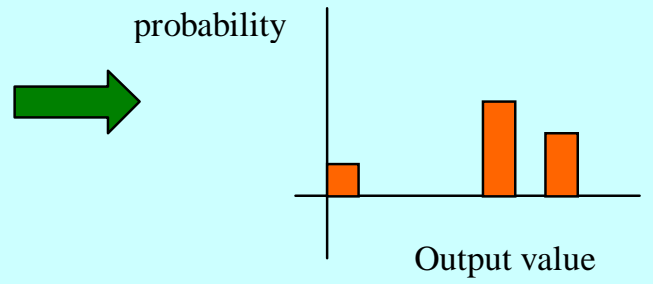

Output uncertainty description

Figure 5-3 Weighted scenario analysis: Likelihoods (probabilities) are assigned to each scenario.

probability
density

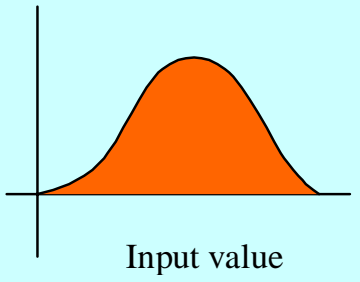

Input

uncertainty

description
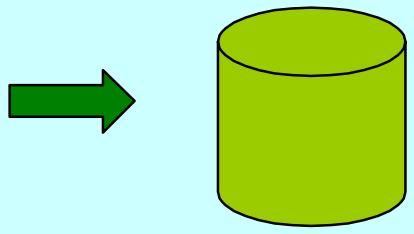

Approximate

Model

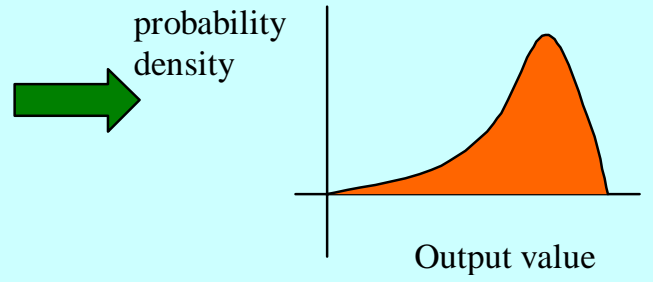

Output uncertainty description

Figure 5-2 Full uncertainty description with approximate model yields an approximation of the full uncertainty description of the output. 
Parallel research can be initiated to determine how to evaluate uncertain information if it were available. This research will need consider the type of analysis to use (worst case, scenarios, Monte Carlo) based on the information that is available and the purpose of the study. If it can be shown with confidence that there is a particular worst-case load model and that it can occur in the actual grid, then a worst case scenario study is the easiest to perform. On the other hand if no such worst-case model is known, or is deemed extremely unlikely, or there are several (or numerous) model combinations that may be consider worst-case, then scenario analysis or Monte Carlo simulation will be appropriate.

In the sidebar on uncertainty analysis, we give a visual presentation of the differences in these approaches. An "exact” evaluation of uncertainty typically requires significant computation, and presumes an accurate description of the uncertainty is available. Scenario analysis and weighted scenario analysis are computationally more efficient, but do not consider all possible uncertainties, or all possible outcomes. Care must be chosen when using scenario analysis to ensure that all uncertainties of interest and outcomes are considered.

The last portion of the sidebar depicts a relatively recent development in uncertainty analysis in which the detailed model is replaced by an approximate, reduced-order model. The premise is that the detailed model is difficult (or time consuming) to evaluate and that a well-designed approximate model is simpler. Furthermore, the approximate model can be designed specifically to support the uncertainty analysis. An example of this is the Probabilistic Collocation Method (PCM) (also known as the Deterministic Equivalent Modeling Method (DEMM)) that was introduced to evaluate global climate change models [22]. The global climate models can be very sophisticated and require up to a days worth of computation to evaluate a single scenario. A Monte Carlo simulation is out of the question. By gathering information for a few well-chosen simulation runs, an approximate model that maps the uncertain inputs to the outputs of interest is developed, and this simpler model is easily evaluated using Monte Carlo techniques. This approach has been applied to a power system model in an academic setting with promising results [8]. The authors evaluated uncertainties in load models and fault characteristics in dynamic simulations of power systems. Using the PCM method they were able to reduce the computation requirements by a factor of 300 (2 days reduced to 10 minutes). This and similar approaches are worth pursuing in this context.

\section{Recommendation: Initiate a program to develop methods to evaluate the effect of load model uncertainties on system studies.}

Load models and their parameters will never be known with certainty. Since they are believed to be a main source of discrepancy between simulations and observations, it is sensible to begin a program into studying the effect of uncertainties on outcomes of interest. 


\section{Generator Governor Models}

In the dynamic simulation tasks of interest, generators are modeled in great detail and the models are largely believed to be accurate. Unlike load models that are aggregate by necessity, detailed physical representations of individual generators are feasible. Mathematical models are developed for the physical generator itself and for all the supporting subsystems and controls.

In theory, all generators should be equipped with a droop governor control (see sidebar) that helps maintain the system energy balance after sudden, unanticipated changes. These often occur if a generating plant trips off-line and the remaining generators must increase output to compensate. As the frequency changes, the generators should respond by increasing (or decreasing) output to reestablish an energy balance. By setting all generators to the same droop setting (5\% in the West), they all should compensate in proportion to their relative sizes.

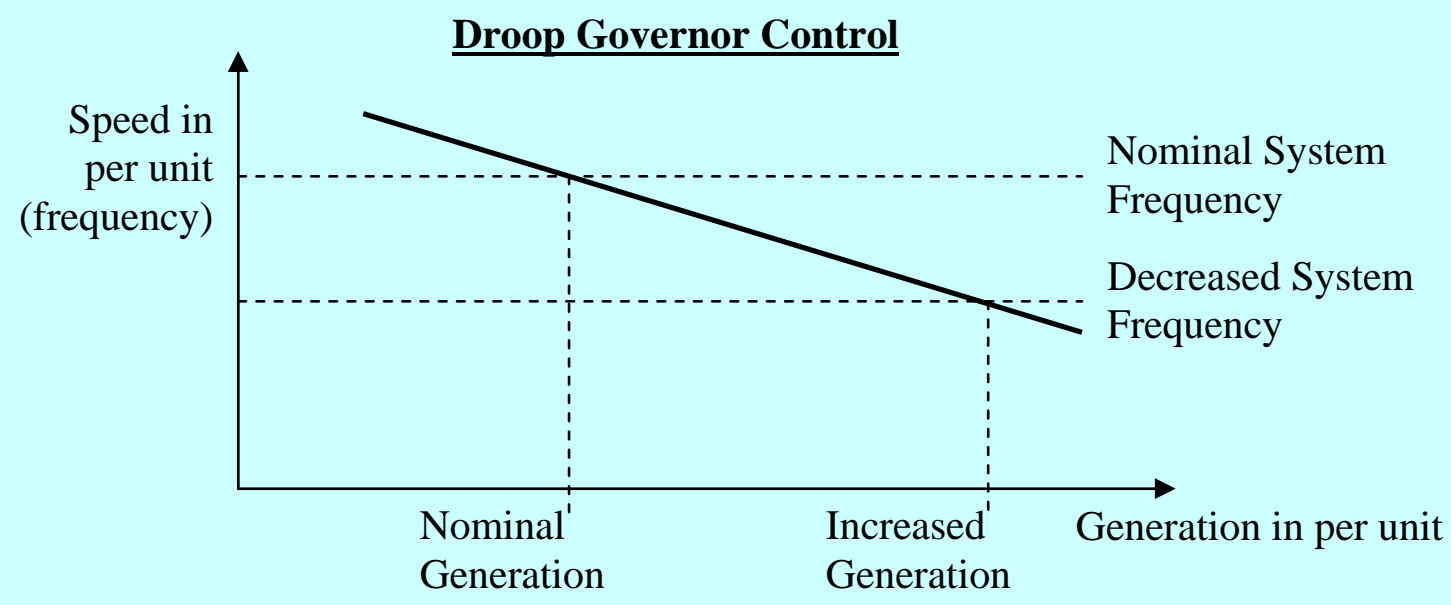

A disturbance in the system that results in a change in frequency signals a net power imbalance. If the load exceeds generation, the frequency will decrease (generators will slow down), and if left unchecked the frequency will continue to fall. Governor controls on generators will act to counter changes in frequency by increasing or decreasing output as needed. The characteristic of this control is shown in the figure above. The slope of the control line is called the droop and is commonly set to 5\%. (Note that the independent action - frequency change - is shown on the vertical axis, and the dependent action, generation output, is shown on the horizontal axis.)

To coordinate the response of the many generators, the droop of the governors are set to the same value. This automatically distributes the needed change in generation among the generators by the relative amount of nominal output. Thus a $100 \mathrm{MW}$ generator will contribute 10 times as much in generation change than a $10 \mathrm{MW}$ generator.

In practice it has been noticed by CAISO that after moderate and large disturbances the amount frequency response is smaller than expected and there is concern that this, in part, because some plant operational controls may override the needed governor response. NERC has documented a persistent decline in frequency response over recent years and suggest a number of reasons for it [16]. These include a fundamental change in load characteristics - induction motors that 
typically decrease energy use with a decrease in frequency supply may no longer do so if controlled by power electronics - and issues related to operating generators near capacity. A generator operating near its maximum supply limit will not be able supply additional energy if called upon to do so. Worse, the NERC document reports, certain combined-cycle generators may actually be operating on a temperature control loop when near the maximum output, and can exacerbate frequency problems by decreasing output with lower frequency. It may also be the case that plant operators simply disable droop governor controls, although they should not. One may also make the observation that in a centralized electricity market there is an economic motivation for suppliers to be fully dispatched, limiting their ability to increase output, and an economic incentive to not decrease output for loss of sales.

Droop governor response is intended to provide quick a quick response to maintain the integrity of the system. It is not intended to dictate long term dispatch and in a centralized control system frequency will be restored to its nominal value by an economic redispatch of generation. When there is not a centralized control system to dictate the output of all generators, then there may be plants with their own controls to maintain a generation set point. While they may be equipped with functioning droop governors that do react to a disturbance, their own controls to regulate output will work against the initial droop governor response.

This is a serious problem. It is not clear that it is a modeling problem as much as an information problem. Present models for generators and generator controls are probably adequate or can be adapted to account for actual generator operation. A recent addition to generator models controls includes a power setting that represents controls that adjust to maintain a specified power output [19] [18]. This effect tends to reduce the governor response. This model has been shown to increase the accuracy of simulations. In anticipating the response to a disturbance, CAISO needs to know how a generator's output will respond, and operate monitoring tools to verify that the response is what it should be. The issues involve mechanisms to get this information, and to maintain an up-to-date database of this information.

\section{Recommendation: Use the governor model of [19][18] and support WECC activities to maintain a database of plant governor characteristics.}

\section{Recommendation: Develop or adapt tools to monitor supplier governor frequency response. \\ WECC has adopted a governor model that captures the effect of reduced frequency response. They populate the model based on information from survey of generator owners, and have validated the model using historical data. In order to gain accurate model parameters based on measurements, one can develop a tool to identify the responses of individual units.}


Improving Dynamic Load and Generator Response Performance Tools 


\section{Summary and Recommendations}

Load models have an impact on dynamic studies and deficiencies in the models are believed to be a dominant source of discrepancy between observations and model-based simulations. Improved load models should increase confidence in operational limits for reliability. Changes in load models can result in operating limits, and may require reconsideration of reliability criteria. In a general sense, improvements to load models include:

1. The development and use of different models for different operating conditions. Most notable is the lack of different models for different seasons.

2. Improving the understanding and form of load models.

3. Improving confidence in models and usage through measurement and uncertainty analysis.

There is considerable work being done on load modeling already. In the West, WECC is actively engaged in research activity in this area. WECC models are widely used by all WECC members, and as a NERC region they have an interest and responsibility in ensuring reliability. Where there is overlap and existing expertise, it is sensible to support these activities and researchers as their results have a direct impact on operation in the West.

Throughout this report we have suggested recommendations by research topic. These are general recommendations to the field and all do not require direct backing and sponsorship by the PIER. Here we restate the recommendations still organized by research topic. Some recommendations require little PIER input and will likely be completed under existing work, while others suggest initiating completely new programs that may not be accomplished without PIER support. In addition to a summary of each recommendation, we provide an assessment of the necessary research in four categories: 1) the level of effort required to complete the research, 2) the expected time required, 3) the level of needed PIER direction and support, and 4) the significance of the research. We conclude with a summary table of recommendations.

Before proceeding with a summary of the recommendations, we further describe the distinctions used in the four categories of research requirements.

The level of effort required to complete the research, a relative measure encompassing the staffing, focus, design and installation of special equipment, and in a general sense, total cost.

- High: Multiple investigators and necessary installation of equipment and analysis of measured data.

- Moderate: Single lead investigator and need for development of new techniques for analysis. Or this designation may refer to an otherwise costly activity that is best combined with another recommendation.

- Low: Single investigator using established techniques to perform the study.

The expected time to completion: The distinction here is between an activity that should take one-year or less, and those that will require multiple years. A further note is made on those that 
will continue as on-going activities after the initial study (such as maintaining and updating data).

The level of PIER direction and support. Some projects require little direct PIER support, as they will be initiated and completed by others. Some projects involve long-term and basic research and might not be initiated without PIER support.

- Low: Will be initiated and completed by others.

- Moderate: May require some direction and support to supplement projects with others.

- High: Long-term and basic research that will require PIER direction and support to initiate the research.

Significance: An estimate of the impact on improving the models and tools.

- Low: the research will not likely have a significant impact.

- Moderate: will provide an incremental improvement in models and analysis tools.

- High: introduces a fundamental improvement in the models and analysis tools.

\subsection{Review of Recommendations}

Here we list the report recommendations. For reference we include the page number in the report where these recommendations originate, and we include a brief comment about the recommendation.

\subsubsection{Load Model Development and Policies}

Recommendation: Include seasonal variations in load models (pg 16).

It is clear that the load changes substantially with season. Most dramatically is the airconditioning load in the Southwest that is present in the summer, but absent in the winter, and the heating-related load that is present in Northwest in the winter and largely absent in the summer. Some form of regional and seasonal variations in the models can be made as they presently exist, and will benefit from the improved models that we discuss in the next section. This work is best done by WECC (LMTF initially), but they currently do not have plans to do so.

Level of Effort: Low. Requires new survey for seasonal load composition and application of tools and techniques already under development by the LMTF. Time Required: 1-year. Ongoing maintenance of data will be required.

PIER support: Low. A small amount of support may be required to initiate the activity. Significance: Moderate. Most of the significant events are observed during the summer conditions, which corresponds to the one seasonal model in use.

Recommendation: Incorporate state estimator models into the modeling process (pg 16). State estimators are used in several regions in the West and the models that they use should be the most accurate available. It would be particularly valuable to compare the planning models with state estimator models as one check for consistency. The WECC Technical Studies Subcommittee is presently considering ways to use state estimator models in the modeling 
process. As WECC moves forward along this sensible path, it is possible that data confidentiality issues will arise that will need consideration. The PIER may be positioned to aid in making useful state estimator data available to improve system models.

Level of Effort: Low. Likely to be initiated by WECC.

Time Required: 1-year initiate research followed by continual use.

PIER support: Low.

Significance: Moderate. A comparison of planning models and state estimator models will likely turn up a few inconsistencies. Fixing these will provide and incremental improvement to the models.

Recommendation: Anticipate and support activities to review reliability criteria, taking into account information provided by detailed characteristics of new load models (pg 17). Recognition that induction motor loads constitute a portion of the load that may be considered voltage-sensitive, reliability criteria associated with voltage transients should be reviewed to account for this portion of the load.

Level of Effort: Low. Will need to be conducted by WECC.

Time Required: multi-year. Reviewing and changing standards can be a long process. PIER support: Low.

Significance: High. A change in reliability standards by definition requires a fundamental change in analysis of the grid.

\subsubsection{Load Modeling}

Recommendation: Perform studies to determine mechanical load characteristics for induction motors (pg 27).

Preliminary LMTF studies show that simulation results depend on the representation of the mechanical load's characteristic. At the simplest level, the relation between torque and speed differs between pumps, fans, and compressors, and the models will be improved by quantifying these differences by laboratory measurements (instead of by assumption). It may be the case that more sophisticated dynamic models for the loads may be necessary, especially for compressors. This is unknown at this point, and this research is presently beyond the WECC LMTF supported activities. These studies would best be performed in a testing laboratory.

Level of Effort: Moderate. Can by carried out by single lead investigator, but it will require laboratory facilities, and equipment to test.

Time Required: single-year.

PIER support: Moderate. This is important project that the LMTF would like to see done.

Limited testing and modeling is likely to be initiated by some WECC members, but

supplemental PIER support will allow more comprehensive testing.

Significance: High. This involves a fundamental changing in accurate load model representation.

\section{Recommendation: Compare the responses of singe-phase and three-phase motors under different disturbances (pg 27).}

The issue here is that under the heavily loaded conditions of great interest, the percentage of single-phase motor load increases (in residential air conditions, for example). The models used in simulation studies all correspond to three-phase motors. There is an unstudied, basic question 
about how accurately a three-phase motor model can represent the response of a single-phase motor under disturbance conditions. This is a reasonable question in that three-phase and singlephase motors model differ, most notable in the energy conversion to torque. This concern complements the issue with motor mechanical load characteristics, which is also related to torque, and this recommendation could be studied at the same time as the previous recommendation. This work is not likely to be undertaken without additional support. This work should couple laboratory-setting experiments with detailed models and simulations.

Level of Effort: Moderate. Can by carried out by single lead investigator, but it will require laboratory facilities, and equipment to test.

Time Required: single-year.

PIER support: High. This is important project that the LMTF would like to see done. This is fundamental research and PIER support is likely to be needed to initiate this work. Significance: High. This involves a fundamental changing in structure of the load model and may have a fundamental impact on results.

\section{Recommendation: More work is required to model motor load shedding behavior under low voltage conditions. The aggregate model of induction motor loads requires a mechanism for allowing a portion of the load to trip off line during a disturbance (pg 28).} The problem here is the difficulty in representing all the different characteristics of motor loads in a simple aggregate model. Presently the overall model could be augmented with different motors, ones that trip off line during a disturbance, and others that do not. In the traditional spirit and practice of modeling, an aggregate model that represent this low voltage trip, would be beneficial. Such research is not currently being done.

Level of Effort: Moderate. Can by carried out by single lead investigator, but involves development of fundamentally new models, or novel mechanisms to switch between established models.

Time Required: single-year.

PIER support: Moderate. It is not likely to be initiated without PIER funds.

Significance: Low. While this research does result in a fundamental change in the structure of the load model, the same impact can be achieved with existing models if one is willing to accept more motors being represented at the end use load.

\section{Recommendation: Discuss improvements to low-voltage protection on residential air conditioners with manufacturers (pg 28).}

It is observed that the slow voltage recovery observed after a disturbance could be avoided by temporarily removing voltage-sensitive loads, such as air-conditioners, and the restarting them after a few minutes delay. This approach to mitigating the problem on the component level should be discussed with manufacturers.

Level of Effort: Low. Appropriate industry staff need to contact relevant industry representatives.

Time Required: single-year.

PIER support: Low. It does not require significant support. 
Significance: High. This change to protection could significantly reduce the chance of the propagation of a voltage disturbance.

\subsubsection{Measurement and Validation}

Recommendation: Support the development and placement of \$10K load monitors (pg 30.) Encourage the use of monitors, and staff time, to gather data beneficial to improved load modeling.

Level of Effort: Low. The specification work has already been completed by WECC. Time Required: single-year, and on going. Encourage use of the monitors. PIER support: Low.

Significance: High. Data is valuable. A special monitor for the purpose of monitoring loads will aid in all modeling and analysis activities.

Recommendation: Support a basic research project to outline the challenges associated with developing a dynamic state monitor (pg 30).

The issues to be addressed are the mathematical tools and data requirements, and institutional challenges with gathering and sharing data. This could evolve into a major research program and a scoping study or white paper on the issues would need to be prepared.

Level of Effort: Low. Single investigator and scoping study report

Time Required: single-year.

PIER support: Moderate: This will require PIER support to initiate, but it is a low-cost effort. Significance: Moderate: It will identify issues associated with the development of a new technology. Ultimate success and value will be in the subsequent research.

\subsubsection{Load Monitoring}

\section{Recommendation: Support activities to estimate load composition from measurements (pg} 32).

This includes investigating refinement in metering equipment, assumed load models (such as adding stylized distribution feeder model as WECC moves in this direction), staged testing, and techniques for estimation that may expand conditions under which estimation is possible. This requires significant effort and testing in the field. This work will need to be done with the cooperation of a utility in the West.

Level of Effort: High. This will require cooperation with a utility to install equipment to gather data. This is basic research that will require novel analysis techniques to complete the task. Time Required: multi-year, and on-going to maintain database.

PIER support: High. This is a significant project to be conducted with utility partnership. Significance: High. These measurements will provide a fundamental improvement in the accuracy of load models.

Recommendation: Support activities to test load model substitutability assumptions, and to characterize a range of uncertainty in models (pg 32).

This addresses the issue of whether or not representative models are truly representative by comparing results from substations that would be assumed to have similar loading characteristics 
by load class. The matches will not be perfect, so there would be additional value to characterizing the range of values obtained for different load classes that may be evaluating using uncertainty analyses. Also, a calculation of uncertainty in estimates at each measured locations will facilitate uncertainty simulations. This work can and should be done jointly with the research in the previous recommendation and may be grouped as a larger project. Again, it will require fieldwork and cooperation with a utility.

Level of Effort: Moderate. This will require cooperation with a utility to install equipment to gather data, however this should be combined with the previous recommendation to reduce redundancy in monitoring efforts.

Time Required: multi-year, and on-going to maintain database.

PIER support: High. This is basic research that should be performed with utility partnership. Significance: High. Assessment of the uncertainty in load models will allow the analysis of uncertainty in dynamic studies. This is not done now.

\section{Recommendation: Assess the value of harmonic information for the purposes of estimating load composition (pg 33).}

The additional information in harmonics will augment the primary frequency data now used, and there may be useful information in steady-state waveforms. This will require field testing and possible refinement of metering equipment. This work could be done in conjunction with the previous recommendations.

Level of Effort: Moderate. This will require cooperation with a utility to install equipment to gather data; however, this should be combined with the previous two recommendations to reduce redundancy in monitoring efforts.

Time Required: multi-year, and on-going to maintain database.

PIER support: High. This is basic research that should be performed with utility partnership. Significance: Unknown. It is not known how much information there is in the harmonics. Energy Commission-sponsored research was successful in finding useful information in harmonics at the level of a building or plant.

\subsubsection{Measurement-Only (Black-Box) Models}

\section{Recommendation: Follow efforts to develop measurement-only (black-box) load models (pg 34).}

In general, black-box modeling techniques are most valuable when the available data is rich enough to show all the operating characteristics of the modeled component. Presently, we believe that the data set does not support this approach.

Level of Effort: Low. Monitor on-going work or by others.

Time Required: multi-year, and on-going monitoring of research in this area.

PIER support: Low.

Significance: Low. 


\subsubsection{Uncertainty Analysis}

Recommendation: Initiate a program to develop methods to evaluate the effect of load model uncertainties on system studies (pg 39).

Load models and their parameters will never be known with certainty. Since they are believed

to be a main source of discrepancy between simulations and observations, it is sensible to begin a program into studying the effect of uncertainties on outcomes of interest.

Level of Effort: High. Requires the development of new techniques to analyze the impact of uncertainties in dynamic simulations.

Time Required: multi-year.

PIER support: High. This is basic research that may require PIER support to initiate.

Significance: High. The models will never be know with certainty, and evaluation of uncertainty will lead to greater confidence and better decision in the operations and planning.

\subsubsection{Generator Governor Models}

Recommendation: Use the new governor model [19] [18] and support WECC activities to maintain a database of plant governor characteristics (pg 41).

This activity will be handled by WECC.

Level of Effort: Low. Will be completed by WECC.

Time Required: multi-year, and on-going to maintain database

PIER support: Low.

Significance: High. Prior models overestimated governor response.

\section{Recommendation: Develop or adapt tools to monitor supplier governor frequency response (pg 45).}

WECC has adopted a governor model that captures the effect of reduced frequency response. They populate the model based on information from survey of generator owners, and have validated the model using historical data. In order to gain accurate model parameters based on measurements, one can develop a tool to identify the responses of individual units.

Level of Effort: High. Need to develop new tools to complete this task

Time Required: multi-year, and on-going to monitor generator characteristics PIER support: High. This involves basic research that may require PIER support to initiate. Significance: Moderate. This would be a valuable tool. The alternative is to seek ways to populate the model through surveys or mandatory reporting. 


\subsection{Summary table of recommendations}

Here we present a summary table of recommendations.

\begin{tabular}{|c|c|c|c|c|}
\hline Recommendation & $\begin{array}{c}\text { Level of } \\
\text { Effort }\end{array}$ & Time Req'd & $\begin{array}{c}\text { PIER } \\
\text { Support }\end{array}$ & Significance \\
\hline \multicolumn{5}{|c|}{ Load Model Development and Policies } \\
\hline Develop seasonal models. & low & 1-year & low & moderate \\
\hline Validate with state estimator models & low & 1-year & low & moderate \\
\hline Review reliability criteria. & low & multi-year & low & high \\
\hline \multicolumn{5}{|c|}{ Load Modeling } \\
\hline $\begin{array}{l}\text { Study motor mechanical load } \\
\text { characteristics and impact. }\end{array}$ & moderate & 1-year & moderate & high \\
\hline $\begin{array}{l}\text { Study impact of single-phase and } \\
\text { three-phase motors. }\end{array}$ & moderate & 1-year & high & high \\
\hline $\begin{array}{l}\text { Model motor load shedding and } \\
\text { low-voltage conditions. }\end{array}$ & moderate & 1-year & moderate & low \\
\hline Improve low-voltage protection. & Low & 1-year & Low & high \\
\hline \multicolumn{5}{|c|}{ Measurement and Validation } \\
\hline$\$ 10 \mathrm{~K}$ load monitor. & low & 1-year & low & high \\
\hline $\begin{array}{l}\text { Scoping study: research needs for } \\
\text { automatic validation } \\
\text { and dynamic state estimation. }\end{array}$ & moderate & 1-year & moderate & moderate \\
\hline \multicolumn{5}{|c|}{ Load Monitoring } \\
\hline $\begin{array}{l}\text { Estimate load composition from } \\
\text { measurements. }\end{array}$ & high & multi-year & high & high \\
\hline $\begin{array}{l}\text { Characterize model uncertainties } \\
\text { using measurements. }\end{array}$ & moderate & multi-year & high & high \\
\hline $\begin{array}{l}\text { Use harmonic information in } \\
\text { measurements to enhance } \\
\text { load composition estimates. }\end{array}$ & moderate & multi-year & high & unknown \\
\hline \multicolumn{5}{|c|}{ Measurement-Only (Black Box) Models } \\
\hline $\begin{array}{l}\text { Follow research activities in this } \\
\text { area. }\end{array}$ & low & multi-year & low & low \\
\hline \multicolumn{5}{|c|}{ Uncertainty Analysis } \\
\hline $\begin{array}{l}\text { Develop methods to assess the } \\
\text { impact of load model } \\
\text { uncertainties on system studies. }\end{array}$ & high & multi-year & high & high \\
\hline \multicolumn{5}{|c|}{ Generator Governor Models } \\
\hline $\begin{array}{l}\text { Support WECC activities to } \\
\text { implement best model and } \\
\text { maintain data for generator } \\
\text { characteristics. }\end{array}$ & low & multi-year & Low & high \\
\hline $\begin{array}{l}\text { Develop tools to monitor individual } \\
\text { generator. } \\
\text { frequency response. }\end{array}$ & high & multi-year & high & moderate \\
\hline
\end{tabular}




\section{References}

[1] “Load Representation for Dynamic Performance Analysis,” IEEE Transactions on Power Systems, Vol. 8, No. 2, pp 472-482, May 1993.

[2] “Standard Load Models for Power Flow and Dynamic Performance Simulation,” IEEE Transactions on Power Systems, Vol. 10, No. 3, pp1302-1313, August 1995.

[3] California Energy Commission, Final Report Compilation for Equipment Scheduling and Cycling, P-500-03-096-A2, October 2003.

[4] Chinn, G.L., "Modeling Stalled Induction Motors,” to be presented at the IEEE Transmission and Distribution Conference, October, 2005.

[5] Ellis, A., D. Kosterev, A. Meklin, “Dynamic Load Models: Where Are We?” to be presented at the IEEE Transmission and Distribution Conference, October 2005.

[6] EPRI and PNM, Advanced Load Modeling, Technical Report 1007318, September 2002.

[7] Hamachi LaCommare, K., and J.H. Eto, "Understanding the Cost of Power Interruptions to U.S. Electricity Consumers,” LBNL Report LBNL-55718, September 2004.

[8] Hockenberry, J., and B.C. Lesieutre, "Evaluation of Uncertainties in Dynamic Simulations: The Probabilistic Collocation Method,” IEEE Transactions on Power Systems, Vol. 19, No. 3, pp 1483-1491, August 1994.

[9] Huang, H., T. Nguyen, D. Kosterev, “Load Modeling and Validation,” presentation to the WECC Load Modeling Task Force, San Francisco, January 2005.

[10] Kimbark, E.W., Power System Stability, Volume 1: Elements of Stability Calculations, IEEE Press, 1995. (A reprint of the original 1948 version.)

[11] Kondragunta, J., and WSCC Reliability Subcommittee, "Supporting Document for Reliability Criteria for Transmission System Planning,” August 1994.

[12] Kosterev, D.N., C.W. Taylor, and W.A. Mittelstadt, "Model validation for the August 10, 1996 WSCC system outage,” IEEE Transactions on Power Systems, Vol. 14. No. 3, August 1999.

[13] Leeb, S.B., S.R. Shaw, and J.L. Kirtley, "Transient Event Detection in Spectral Envelope Estimates for Nonintrusive Load Monitoring,” IEEE Transactions on Power Delivery, Vol. 10, No. 3, pp. 1200-1210, July 1995.

[14] Leeb, S.B., B.C. Lesieutre, S.R. Shaw, "Determination of Load Composition Using Spectral Envelope Estimates," Proceedings of the $17^{\text {th }}$ Annual North American Power Symposium, Bozeman, MT, October 1995.

[15] Meklin, A., and D. Sutphin, "Dynamic Simulations with Improved Representation of Loads and their Connection to a Power System,” Australasian Universities Power Engineering Conference, Hobart, Australia, September 2005.

[16] NERC, Frequency Response Standard Whitepaper, April 6, 2004.

[17] Pereira, L., D. Kosterev, P. Mackin, D. Davies, J. Undrill, and W. Zhu, “An Interim Dynamic Induction Motor Model for Stability Studies in the WSCC,” IEEE Transactions on Power Systems, Vol. 17, No. 4, pp 1108-1115, November 2002.

[18] Pereira, L., D. Kosterev, D. Davies, S. Patterson, “New Thermal Governor Model Selection and Validation in the WECC.” IEEE Transactions on Power Systems, Vol. 19, No. 1, pp. 517-523, February 2004.

[19] Pereira, L., “New Thermal Governor Model Development,” IEEE Power and Energy Magazine, pp. 62-70, May/June 2005. 
[20] U.S.-Canada Power System Outage Task Force, "Final Report on the August 14, 2003 Blackout in the United States and Canada,” April 2004.

[21] Walve, K., “Modeling of power system components at severe disturbances,” in Proceedings of the International Conference on Large High Voltage Electric Systems (CIGRE), August 1986.

[22] Webster, M., M.A. Tatang, and G.J. McRae, “Application of the Probabilistic Collocation Method for an Uncertainty Analysis of a Simple Ocean Model,” Joint Program on the Science and Policy of Global Change, MIT, Cambridge, MA, Technical Report 4, January 1996.

[23] WECC, Operating Committee Handbook, revised July 2005.

[24] WECC, Planning Coordination Committee Handbook, revised December 2004.

[25] Williams, B.R., W.R. Schmus, D.C. Dawson, “Transmission Voltage Recovery Delayed by Stalled Air Conditioner Compressors,” IEEE Transactions on Power Systems, Vol. 7, No. 3, pp. 1173-1181, August 1992. 